\title{
OXYGEN MANAGEMENT IN NEW ZEALAND AND AUSTRALIAN INTENSIVE CARE UNITS: \\ A KNOWLEDGE TRANSLATION STUDY
}

BY

Diane Margaret Mackle

\begin{abstract}
A thesis
submitted to the Victoria University of Wellington in fulfilment of the requirements for the degree of Doctor of Philosophy
\end{abstract}

Victoria University of Wellington 


\section{ABSTRACT}

Background: Knowledge translation literature shows a delay between publication and uptake of research findings into clinical practice. There is uncertainty about whether this delay exists in the intensive care unit (ICU) context and whether participation in research influences changes in clinical practice. Knowing whether ICU participation in research increases the likelihood of research findings being incorporated into clinical care is important to funders, researchers and patients.

Aim: To investigate the effects of participating in the Intensive Care Unit Randomised Trial Comparing Two Approaches to Oxygen therapy (ICU-ROX) randomised controlled trial on attitudes and practices in relation to ICU oxygen therapy.

Methods: The research question was examined using three different methods: a survey, an inception cohort study, and a retrospective cohort study. The practitioner attitudes survey and inception cohort study were conducted before ICU-ROX started, after ICU-ROX finished but before the results were known, and, finally, after publication of the ICU-ROX results. The retrospective cohort study (using the Australian and New Zealand ICU adult patient database) compared data from 48 weeks before and 48 weeks after publication of the ICU-ROX trial.

Results: Practitioner attitudes survey: Responses were received from 112 specialist doctors and 153 ICU nurses from 11 ICU-ROX and 11 non-ICU-ROX sites. Before the ICU-ROX trial was conducted, respondents from ICU-ROX trial sites and those from non-ICU-ROX trial sites were similarly likely to indicate they would use a fraction of inspired oxygen $\left(\mathrm{F}_{1} \mathrm{O}_{2}\right)$ of 0.21 , in a ventilated patient with peripheral oxygen saturations $\left(\mathrm{SpO}_{2}\right)$ of $91-96 \%$. However, after participation in the ICU-ROX trial, and before the trial results were known, ICU-ROX trial site respondents were significantly more likely than their non-ICU-ROX trial site counterparts to state they would use an $\mathrm{F}_{1} \mathrm{O}_{2}$ of 0.21 in a patient with an $\mathrm{SpO}_{2}$ of $91-96 \%$. The differences in all patient admission categories, except the 'other ICU patients' category, persisted after the ICU-ROX trial was published. Nurses were generally less likely to report they would use an $\mathrm{F}_{1} \mathrm{O}_{2}$ of 0.21 in a ventilated patient than doctors. Following ICU-ROX publication, $41.1 \%$ of the doctors compared to $13.6 \%$ of the nurses knew the ICU-ROX results $(P<0.001)$, and $53.1 \%$ and $5.4 \%$, respectively, reported having read the publication $(P<0.001)$.

Inception cohort study: Data were analysed from 650 patients from 11 ICU-ROX and 11 nonICU-ROX sites. ICU-ROX site patients spent $8.2 \%$ of the time ventilated at $\mathrm{F}_{1} \mathrm{O}_{2}$ of 0.21 , before 
ICU-ROX started, 7.7\% after their participation in the ICU-ROX trial and $7.1 \%$ of the time after ICU-ROX publication, a change over time was not statistically significant. For the non-ICUROX site patients, time spent ventilated at $\mathrm{F}_{1} \mathrm{O}_{2}$ of 0.21 was $0.9 \%$ before ICU-ROX, $2.4 \%$ after ICU-ROX was conducted and 3.3\% after publication. This change for non-ICU-ROX site patients after ICU-ROX publication was statistically significant (relative rate $3.63 ; 95 \% \mathrm{CI} 1.39$ to $9.47, \mathrm{P}=0.01)$.

Retrospective cohort study: The $\mathrm{F}_{1} \mathrm{O}_{2}$ from the arterial blood gas associated with the worst Acute Physiology and Chronic Health Evaluation III-J score in the first 24 hours of admission to ICU was analysed for 51,498 pre-publication admissions and 47,523 post-publication admissions from 15 ICU-ROX sites and 188 non-ICU-ROX sites. Although the average $\mathrm{F}_{1} \mathrm{O}_{2}$ values varied considerably from week to week, there was a statistically significant reduction in the average $\mathrm{F}_{1} \mathrm{O}_{2}$ over time in ICU-ROX sites $(\mathrm{P}=0.01)$ but not in non-ICU-ROX sites $(\mathrm{P}=0.49)$. Conclusion: Differences between attitudes of practitioners in relation to oxygen in ICU-ROX sites compared with non-ICU-ROX sites changed over time (before and after the ICU-ROX trial, and after publication of the results). Differences in oxygen therapy practice changes for ICUROX versus non-ICU-ROX sites shown in the inception cohort study and retrospective cohort study were small, and are unlikely to be clinically important. These studies did not demonstrate a consistent effect of participation in ICU-ROX on knowledge translation.

Key words: Implementation Science; institutional participation; multiple methods; ICU-ROX; attitudes 


\section{Acknowledgements}

I was honoured to receive a Health Research Council of NZ Clinical Training Fellowship in 2016. This funding is incredibly important to support and develop novice researchers; building New Zealand's research team for the future.

There are a number of organisations who played a pivotal part in this study, and I would like to thank them all. Firstly, the ANZICS Clinical Trials Group (CTG) for supporting and endorsing this study, and offering the opportunity to speak about it at scientific meetings every year. The Intensive Care Research Coordinators Interest Group (IRCIG) which provides mentoring, education and networking opportunities to research coordinators in Australian and New Zealand ICUs. Attending the annual IRCIG and CTG meetings was a starting point for this journey. The team at ANZICS-CORE, who have supported this study by allowing use of the ANZICS Adult Patient Database for research - it is such an important resource. The ICU-ROX Management Committee for their support, in particular in the early stages of this study, by reviewing the protocol and survey.

I have worked in intensive care research for 14 years and know that research is only possible in this setting because of the hard work of research coordinators, nurses, and doctors. This study was no different. There is a wonderful tradition in the ANZICS CTG called doing 'love jobs', which essentially means participating in research studies (usually small and usually observational) for little or no money. I had participated in many of these over the years, often doing them in my own time, to ensure my ICU could contribute. When I put out the call for help with my study, research staff across the two countries raised their hands. This was overwhelming and I will be forever grateful for all the doctors and nurses, and in particular the research coordinators who made this study possible. The two Australian ICU-ROX project managers from the ANZIC-RC - Victoria King and Natalie Linke, made the initial approaches to Australian sites and helped me understand the complicated ethics application process in Australia. Special mention must go to Elizabeth Yarad at Royal North Shore Hospital, who freely and enthusiastically offered assistance, encouraging six NSW sites to participate in this knowledge translation study, in the 'comparator arm'. 
ICU patients contribute to ICU research every day. Their contribution enables us all to advance the care provided to future ICU patients. Thank you to those who assisted with my study.

I was privileged to have three amazing supervisors, each of whom brought something special to my PhD and this thesis. Professor Richard Beasley brought his wisdom and expertise of supervising many PhD students, as my primary supervisor. He is the director of the Medical Research Institute of NZ, where I am employed as a programme manager for ICU research. He is an outstanding researcher, and internationally recognised expert on oxygen and asthma. Dr Kathy Nelson, who had previously supervised my Master's thesis not only kept me on target, but acted as mentor, counsellor and friend. She brought her experience as an accomplished nursing academic with wide-ranging knowledge of multiple research methods, knowledge translation and supervising PhD students. Her devotion to her students and relentless determination to get me over the line was unsurpassed. Dr Paul Young (now Professor) is an inspirational and talented researcher, intensive care specialist, social media medical influencer, gifted orator and wordsmith. I first met Paul when he began work as a new ICU specialist at Wellington ICU, where he is now co-medical director. He is now a worldrenowned intensive care researcher, who it is a privilege to work alongside. Paul has been a driving force for this research and thesis, always available to set me on the right track with his insights, understanding and interpretation of the clinical questions, context, statistics and results.

I'm very grateful for the support of Allie Eathorne at MRINZ and Michael Bailey at ANZIC-RC for their statistical support, and Nick Shortt for his support with REDCAP. Thank you for your seemingly endless patience.

The team I work with at MRINZ have been extremely supportive, picking up extra work, to enable me to spend time on my PhD studies and thesis. Special mention goes to my friend and colleague, Sally Hurford, who over the past four years, has not only encouraged and supported me to devote more time to my thesis, but has taken on additional duties to enable this to happen. 
But most of all, I would like to thank my husband, Steve, and three children Ruby, Eva and Luke. I realise the sacrifice that all members of my family have made, but none more than my husband, Steve. When I began my academic journey with a Master's degree, all three kids were at primary school. As I finish this PhD, Ruby and Eva are at university and Luke is in Year 12 at college. I would like to thank you all, and I hope you can see what can be achieved with passion, hard work and a supportive team.

Throughout the writing of this thesis, many special people in my life have died. My friend Emma, an astute journalist and special woman. We met regularly at our monthly book group, and she never failed to check on the progress of my PhD. Peter Hicks, medical director at Wellington ICU, who first recognised and encouraged my passion for health data and research, and was genuinely interested in everyone he met. Michael-a wonderful Irish friend whose death made all who knew him want to grab life a little more. And my mother, who while still alive, is not the woman she was at the beginning of this journey, her brain having been slowly taken by Lewy Body dementia.

To all of you who have made me who I am, and this thesis what it is - thank you. 


\section{Relevant publications completed during PhD programme}

Beasley, R., Mackle, D., \& Young, P. (2019). Oxygen: a new look at an old therapy. Journal of the Royal Society of New Zealand, 49(2). https://doi.org/10.1080/03036758.2019.1566154

Beasley, Richard, \& Mackle, D. (2019). Increased risk of mortality with liberal oxygen therapy compared with conservative oxygen therapy in critically ill adults. BMJ EvidenceBased Medicine, 24(3), 113-114. https://doi.org/10.1136/bmjebm-2018-111054

Carr, A. C., Spencer, E., Mackle, D., Hunt, A., Judd, H., Mehrtens, J., Parker, K., Stockwell, Z., Gale, C., Beaumont, M., Kaur, S., Bihari, S., \& Young, P. J. (2020). The effect of conservative oxygen therapy on systemic biomarkers of oxidative stress in critically ill patients. Free Radical Biology and Medicine, In Press.(June), 13-18.

https://doi.org/10.1016/i.freeradbiomed.2020.06.018

Mackle, D., Bellomo, R., Bailey, M., Beasley, R., Deane, A., Eastwood, G., Finfer, S., Freebairn, R., King, V., Linke, N., Litton, E., McArthur, C., McGuinness, S., Panwar, R., P, Y., \& ICU-ROX Investigators and the Australian and New Zealand Intensive Care Society Clinical Trials. (2020). Conservative Oxygen Therapy during Mechanical Ventilation in the ICU. New England Journal of Medicine, 382(11), 989-998. https://doi.org/10.1056/nejmoa1903297

Mackle, D. M., Bailey, M. J., Beasley, R. W., Bellomo, R., Bennett, V. L., Deane, A. M., Eastwood, G. M., Finfer, S., Freebairn, R. C., Litton, E., Linke, N. J., McArthur, C. J., McGuinness, S. P., Panwar, R., Young, P. J., \& Australian and New Zealand Intensive Care Society Clinical Trials Group. (2018). Protocol summary and statistical analysis plan for the intensive care unit randomised trial comparing two approaches to oxygen therapy (ICUROX). Critical Care and Resuscitation, 20(1), 22-32.

http://www.ncbi.nlm.nih.gov/pubmed/29458318

Young, P., Mackle, D., Bellomo, R., Bailey, M., Beasley, R., Deane, A., Eastwood, G., Finfer, S., Freebairn, R., King, V., Linke, N., \& Litton, E., McArthur, C., McGuinness, S., \& Panwar, R. Conservative oxygen therapy for mechanically ventilated adults with suspected hypoxic 
ischaemic encephalopathy. Intensive Care Medicine, 46, 2411-2422.

https://doi.org/10.1007/s00134-020-06196-y

Young, P., Mackle, D., Bellomo, R., Bailey, M., Beasley, R., Deane, A., Eastwood, G., Finfer, S., Freebairn, R., King, V., Linke, N., Litton, E., McArthur, C., McGuinness, S., \& Panwar, R. (2020). Conservative oxygen therapy for mechanically ventilated adults with sepsis: a post hoc analysis of data from the intensive care unit randomized trial comparing two approaches to oxygen therapy (ICU-ROX). Intensive Care Medicine, 46(1), 17-26. https://doi.org/10.1007/s00134-019-05857-x

Young, P.J., Mackle, D. M., Bailey, M. J., Beasley, R. W., Bennett, V. L., Deane, A. M., Eastwood, G. M., Finfer, S., Freebairn, R. C., Litton, E., Linke, N. J., McArthur, C. J., McGuinness, S. P., Panwar, R., \& Bellomo, R. (2017). Intensive care unit randomised trial comparing two approaches to oxygen therapy (ICU-ROX): results of the pilot phase. Critical Care and Resuscitation, 19(4).

Young, Paul J., Bailey, M., Bellomo, R., Bernard, S., Bray, J., Jakkula, P., Kuisma, M., Mackle, D., Martin, D., Nolan, J. P., Panwar, R., Reinikainen, M., Skrifvars, M. B., \& Thomas, M. (2020). Conservative or liberal oxygen therapy in adults after cardiac arrest: An individuallevel patient data meta-analysis of randomised controlled trials. Resuscitation, 157(September 2020), 15-22. https://doi.org/10.1016/j.resuscitation.2020.09.036 


\section{TABLE OF CONTENTS}

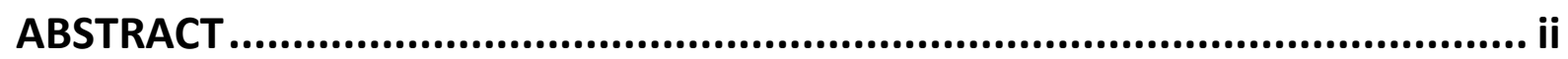

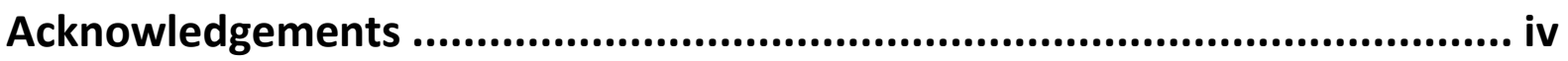

Relevant publications completed during PhD programme ......................... vii

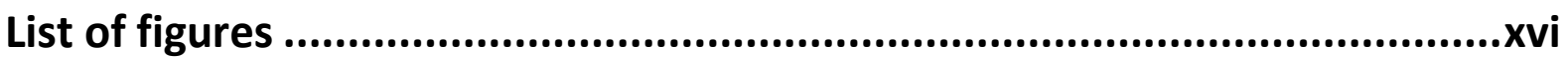

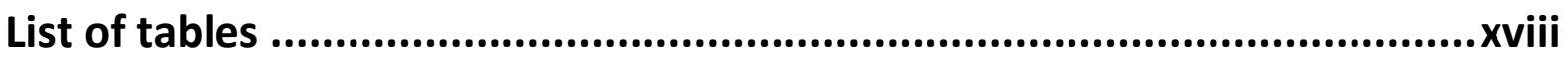

List of abbreviations .......................................................................................xx

Personal perspective......................................................................................xxii

CHAPTER $1 \quad$ INTRODUCTION................................................................ 1

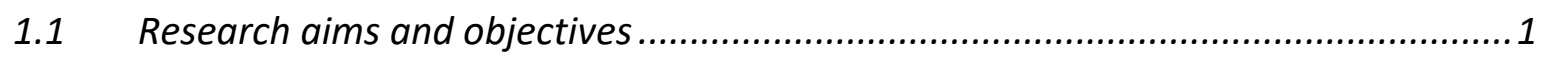

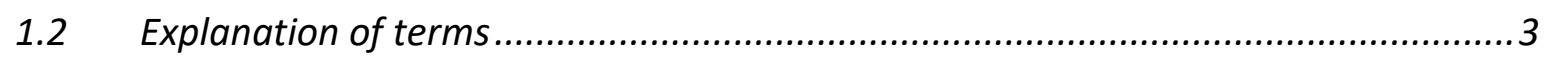

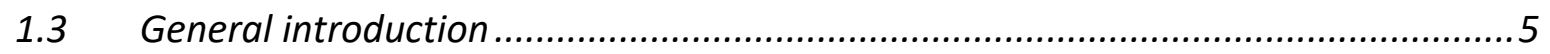

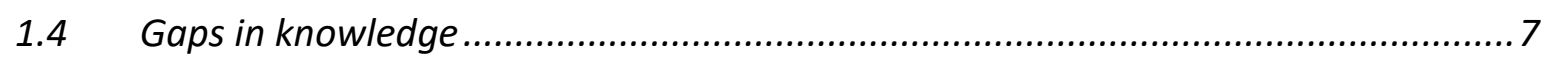

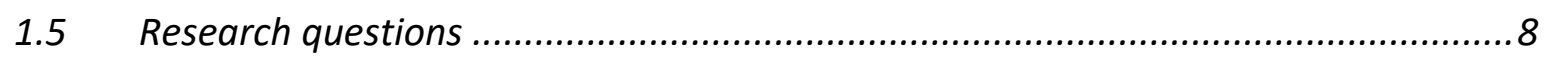

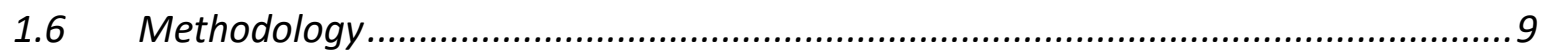

1.7 My role as researcher and ICU-ROX project manager ........................................10

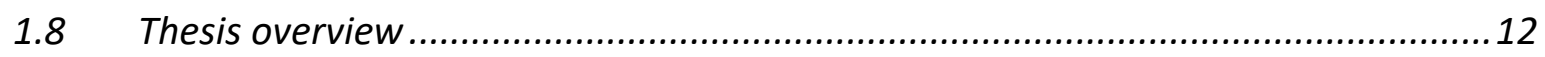

CHAPTER 2 OXYGEN AND ICU-ROX PUBLICATION .............................. 14

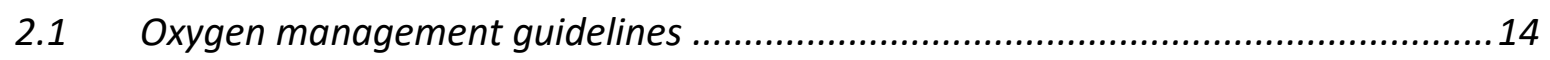

2.2 ICU oxygen management research prior to ICU-ROX ...................................... 15

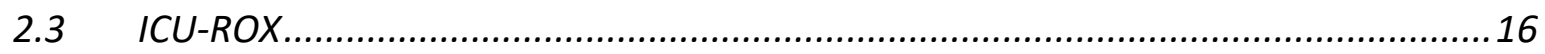

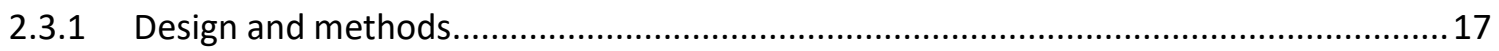




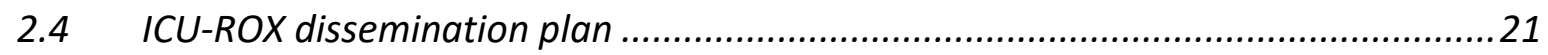

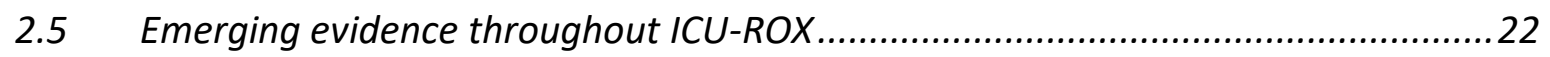

2.6 ICU-ROX contribution to knowledge and subsequent research ...........................22

CHAPTER $3 \quad$ KNOWLEDGE TRANSLATION.......................................... 25

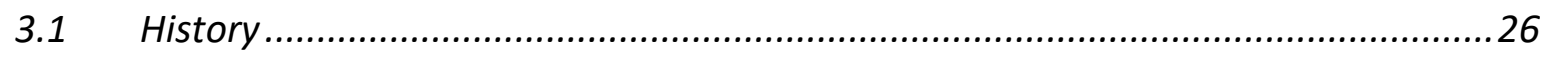

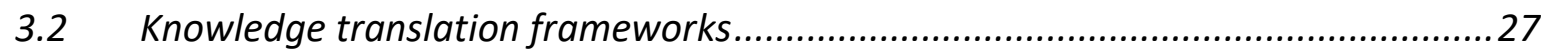

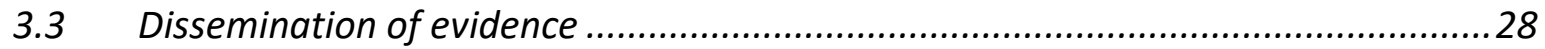

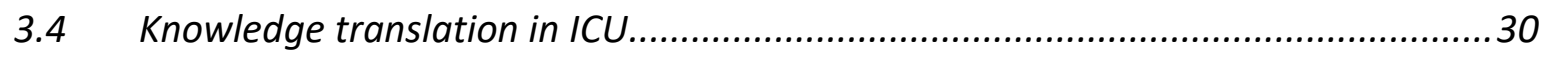

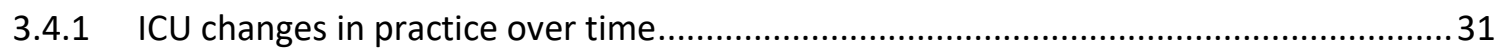

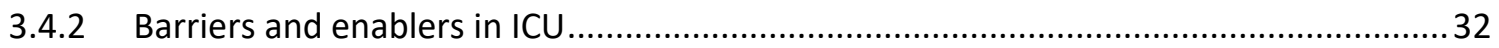

\section{CHAPTER $4 \quad$ LITERATURE REVIEW ..................................................... 34}

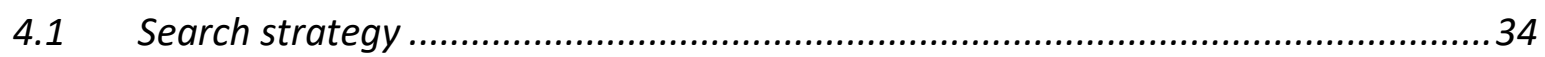

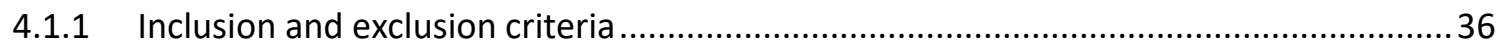

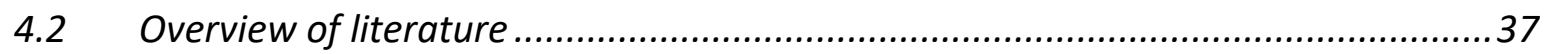

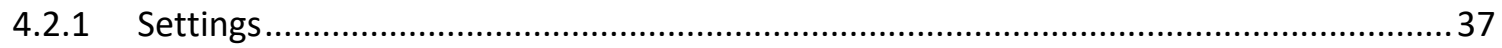

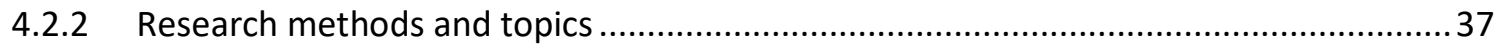

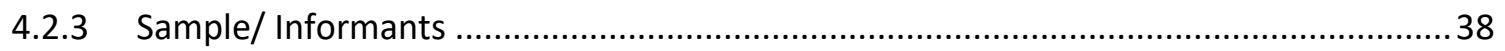

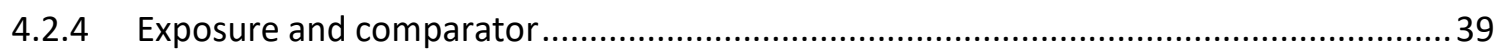

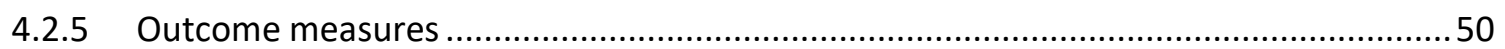

4.2.6 Timing of knowledge translation study in relation to published RCT .............................50

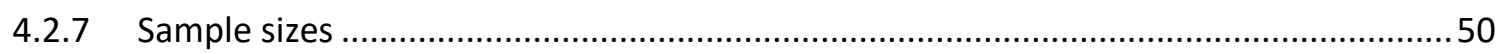

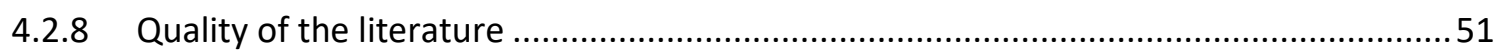

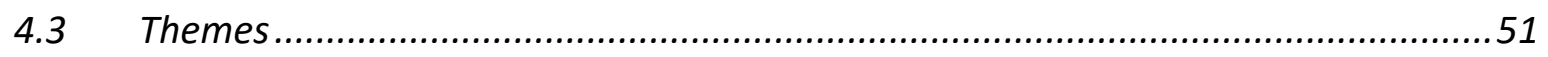

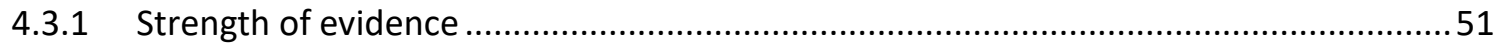

4.3.2 Effect of exposure to the trial protocol (before publication of results)........................... 54

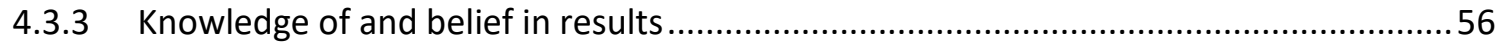

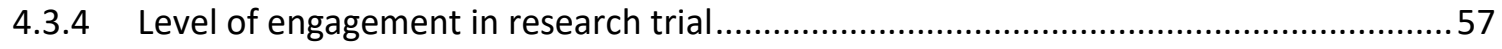

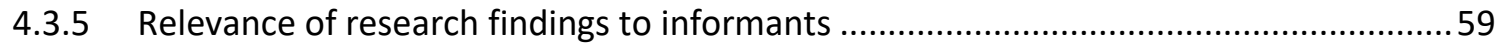

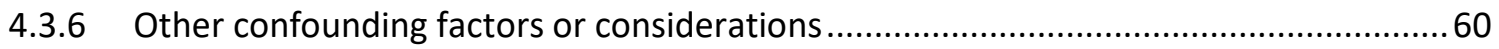


4.4 Summary, gaps and limitations in the literature

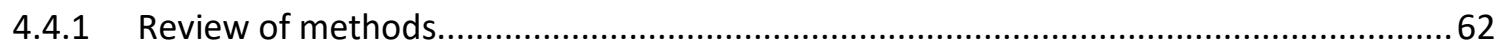

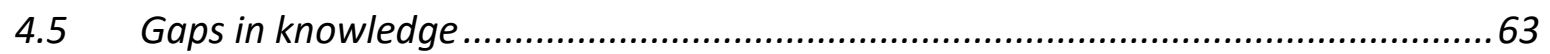

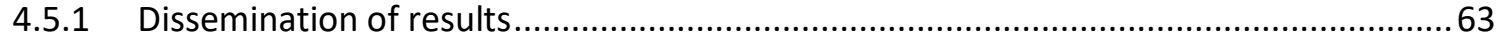

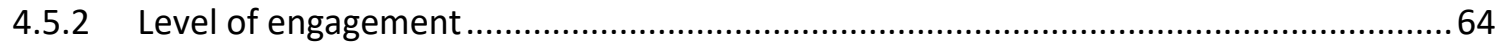

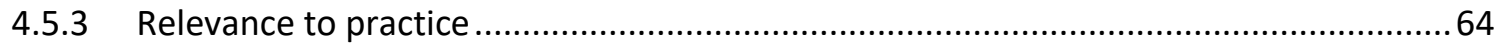

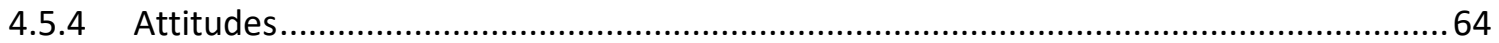

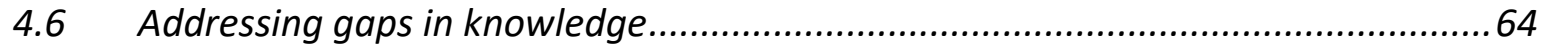

\section{CHAPTER 5 METHODOLOGY, FRAMEWORK AND METHODS OVERVIEW66}

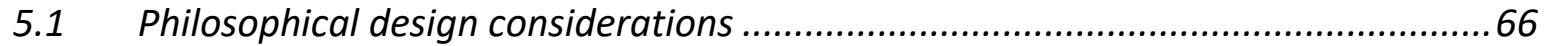

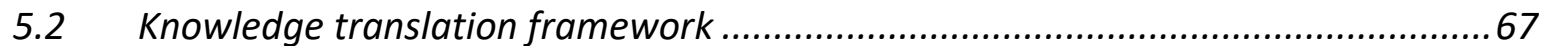

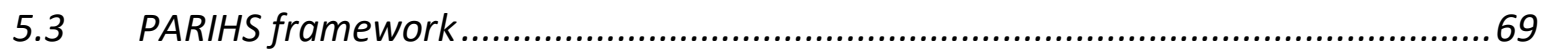

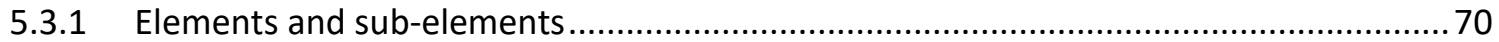

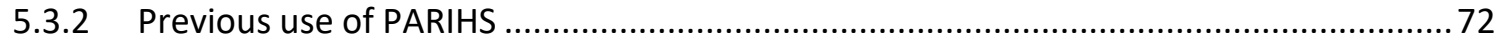

5.3.3 PARIHS Framework in relation to ICU-ROX TRIPS ...................................................... 73

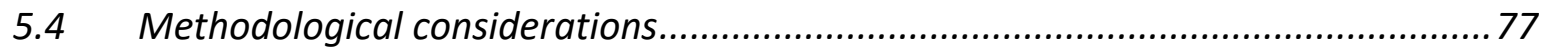

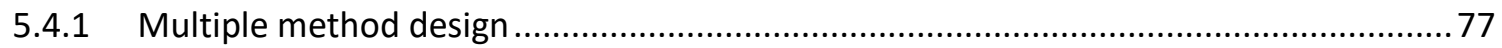

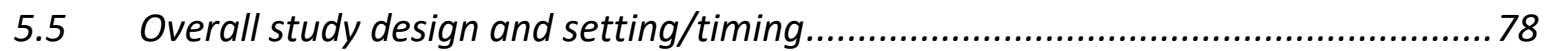

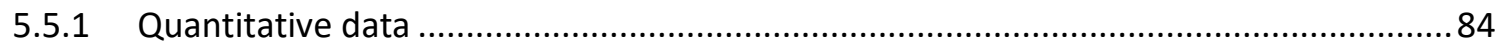

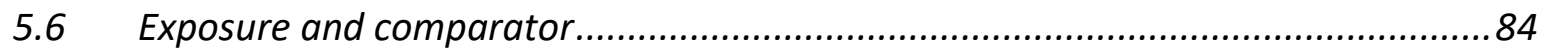

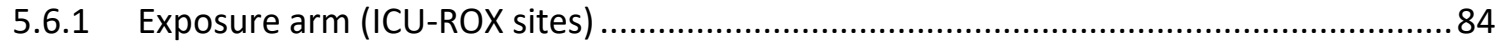

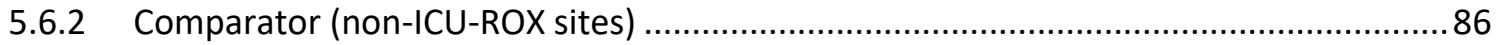

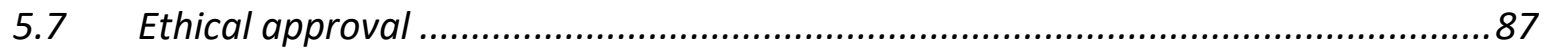

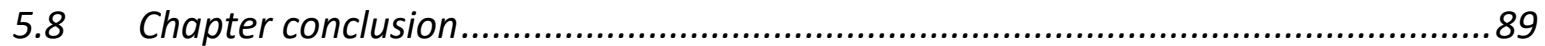

CHAPTER 6 PRACTITIONER ATTITUDES SURVEY ...................................91

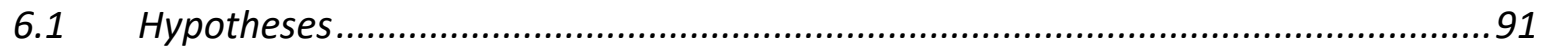

6.2 Study setting (practitioner attitudes surveys and inception cohort studies) ...........92

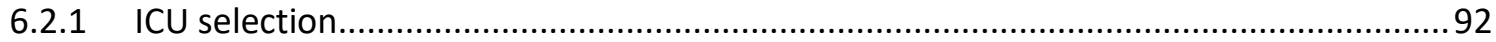




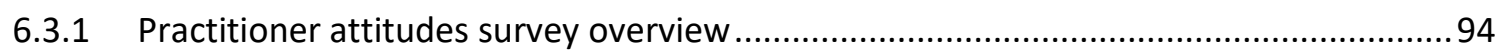

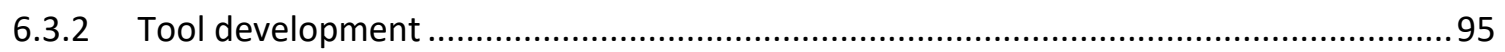

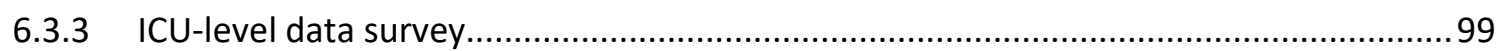

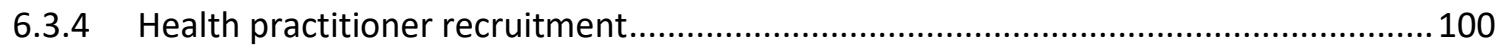

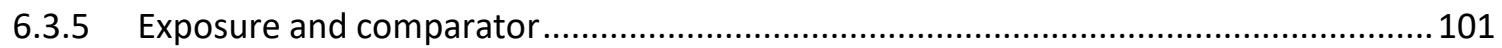

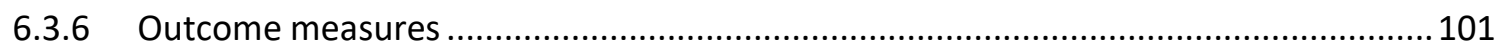

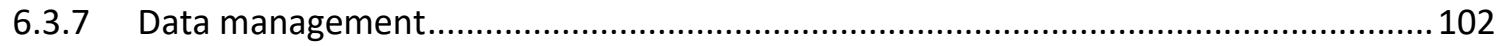

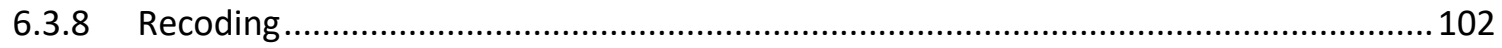

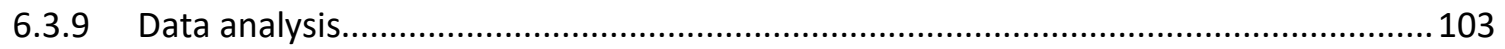

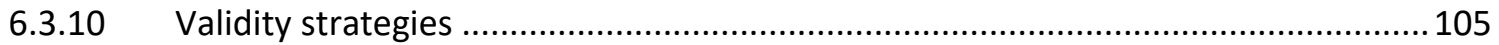

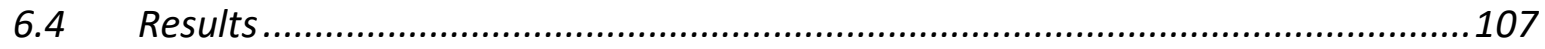

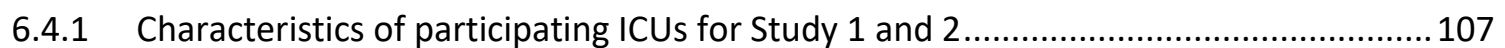

6.4.2 Comparisons by exposure group (ICU-ROX vs non-ICU-ROX) ......................................111

6.4.3 Comparisons by role (ICU specialist doctor vs ICU nurse) ............................................. 124

6.4.4 Findings from open-ended comments (all time points) ............................................139

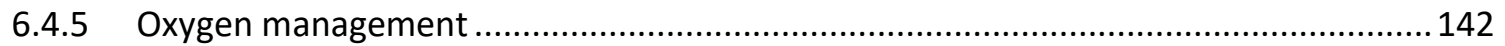

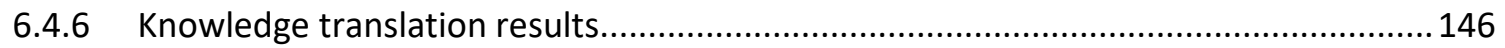

6.4.7 Level of engagement in ICU-ROX and knowledge of results ......................................150

6.4.8 Open comments about the influence of the ICU-ROX publication on practice .............151

6.4.9 ICU-level survey knowledge translation and dissemination practices..........................153

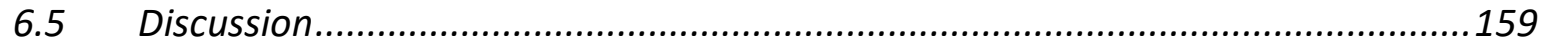

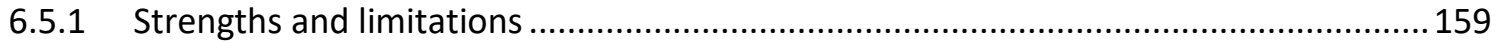

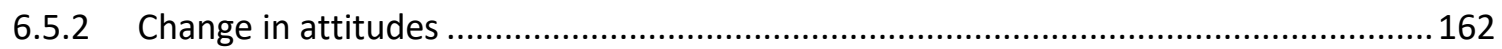

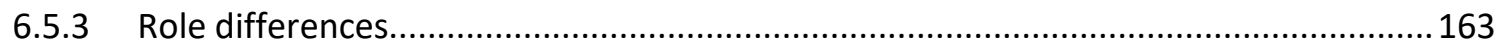

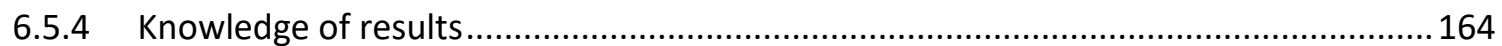

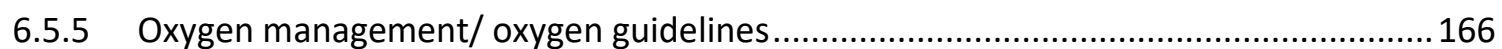

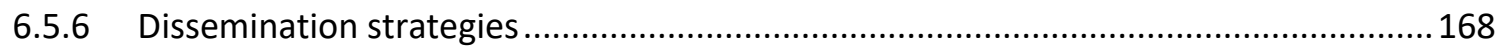

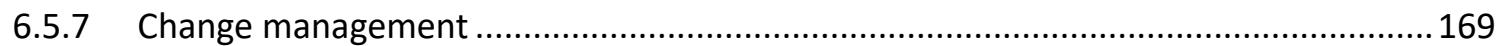

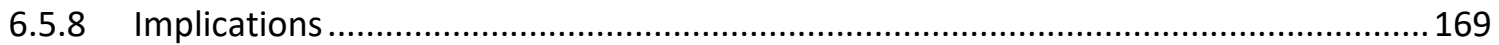

CHAPTER 7 INCEPTION COHORT STUDY.......................................... 170

$7.1 \quad$ Aims

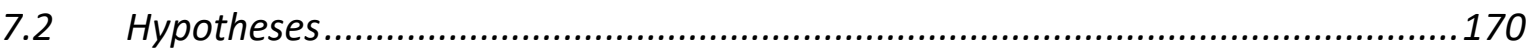




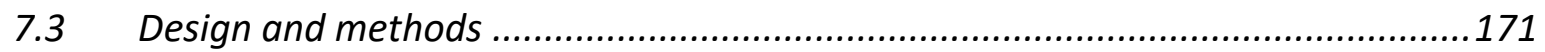

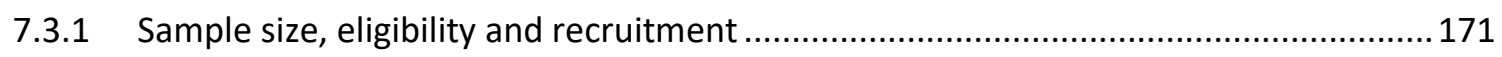

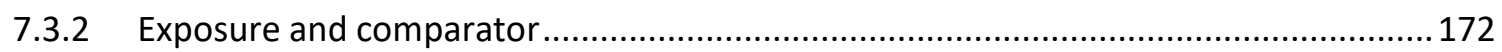

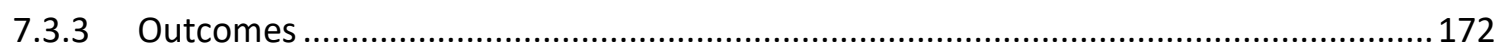

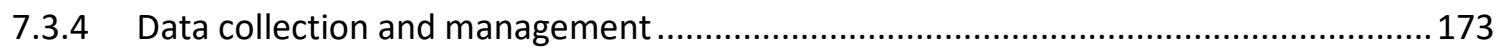

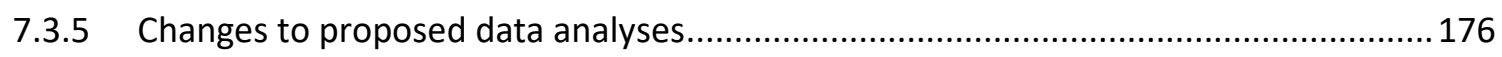

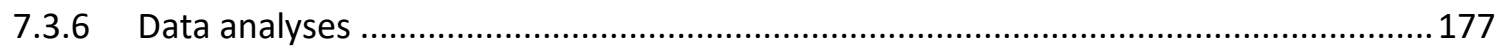

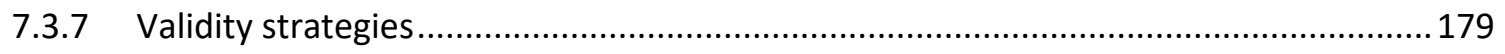

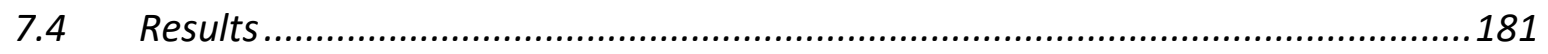

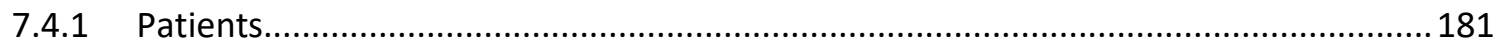

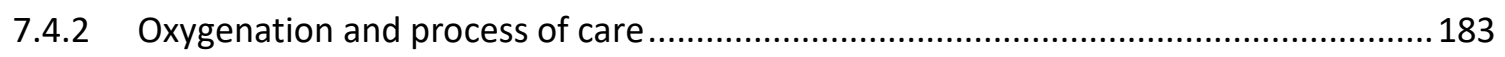

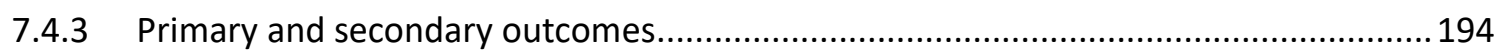

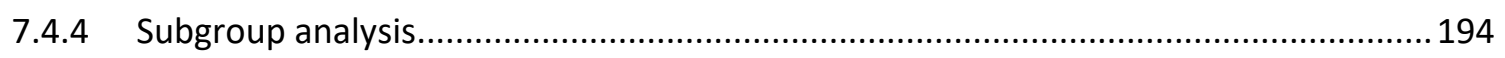

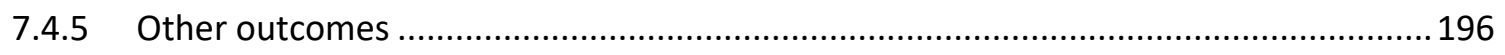

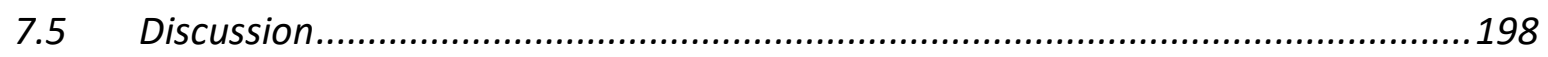

CHAPTER 8 RETROSPECTIVE COHORT STUDY .................................. 201

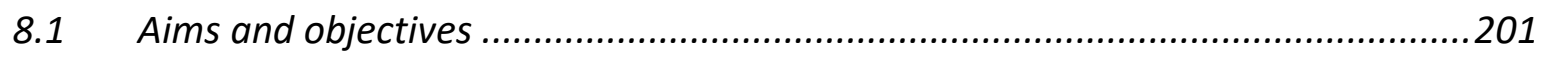

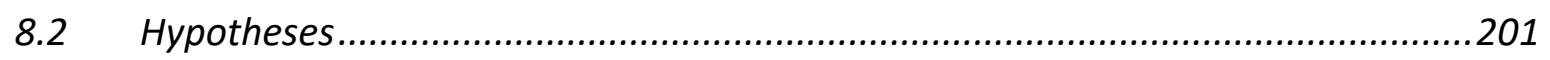

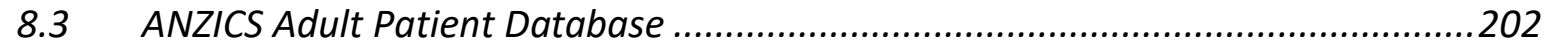

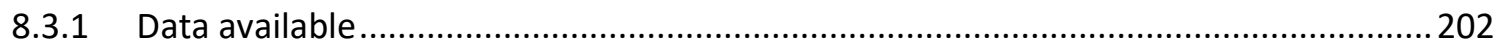

8.3.2 Adult Patient Database validity strategies ..................................................................204

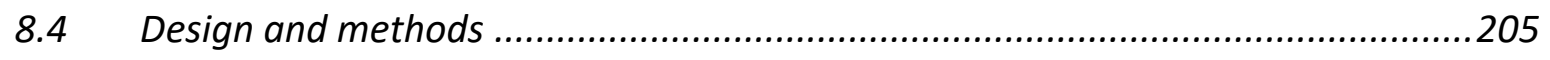

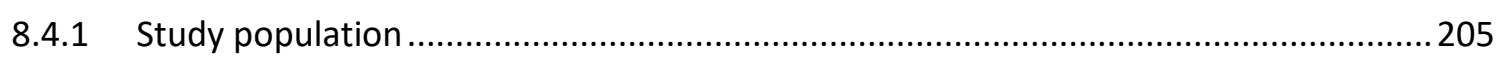

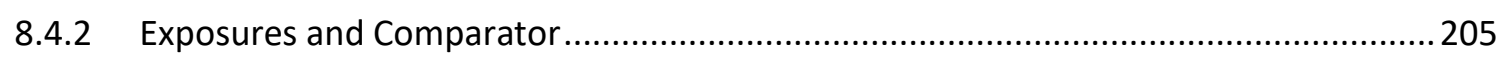

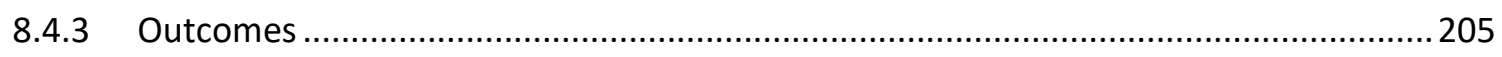

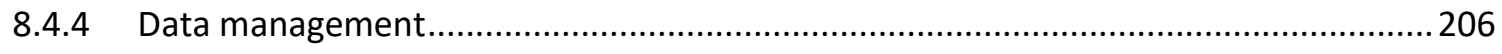

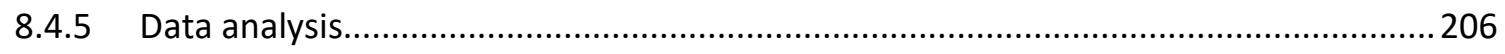

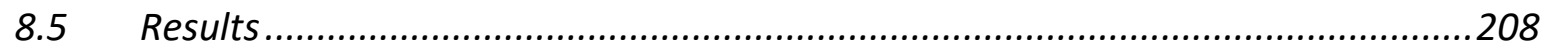

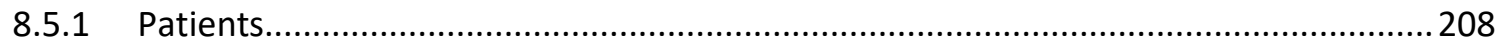

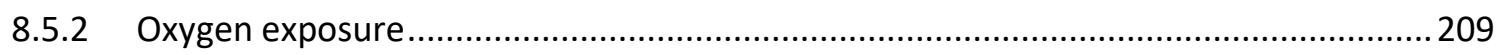

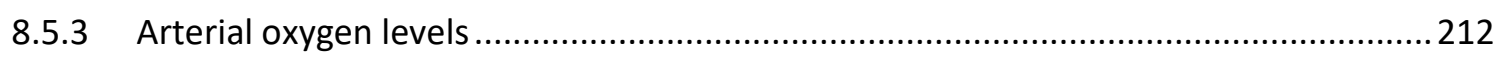


CHAPTER 9 INTEGRATED DISCUSSION AND CONCLUSION................... 221

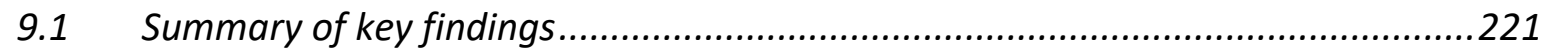

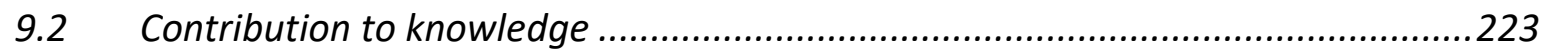

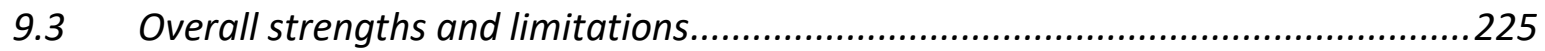

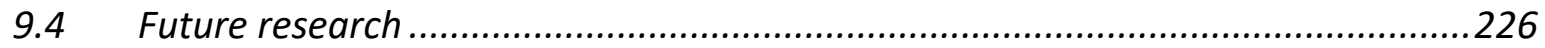

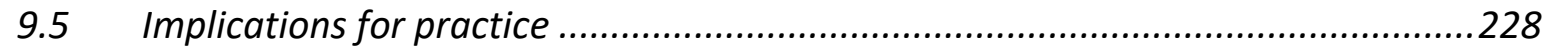

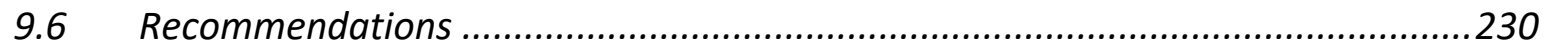

9.6.1 Health Research Council of NZ (and other funders) .................................................230

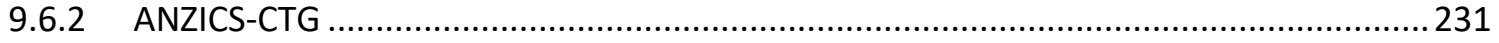

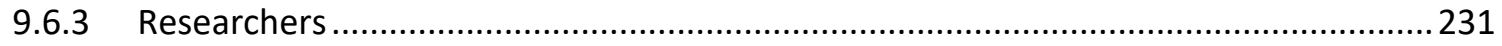

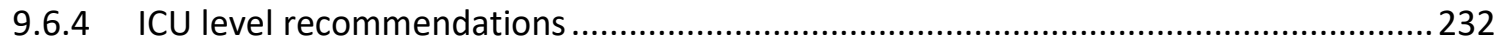

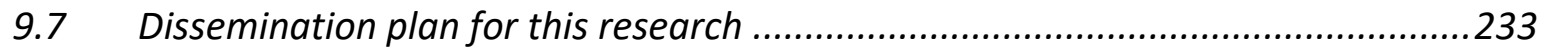

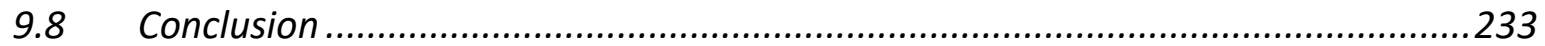

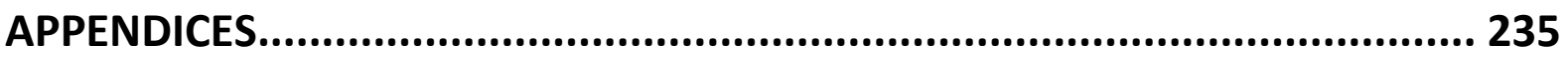

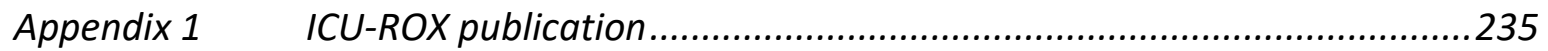

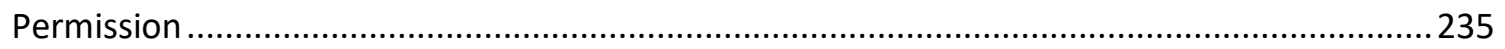

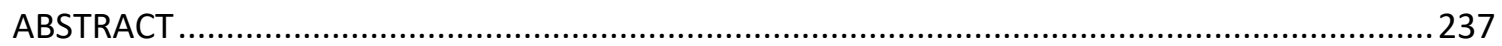

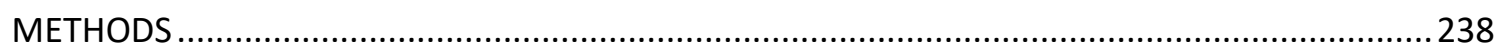

RESULTS

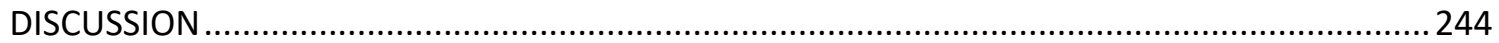

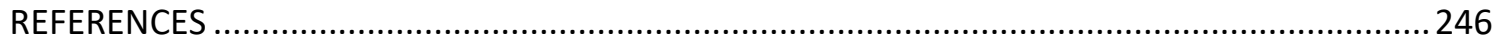

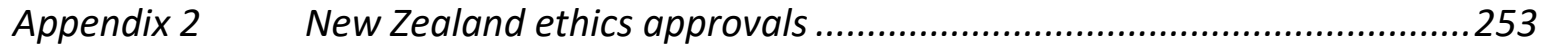

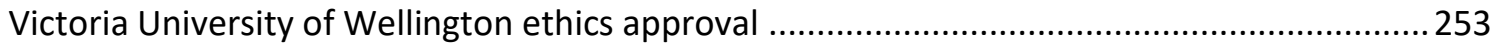

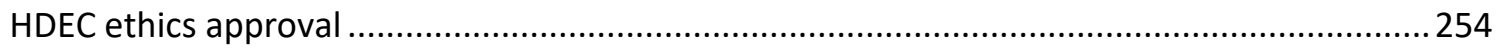

Appendix $3 \quad$ Australian state and territory ethics approvals .................................258

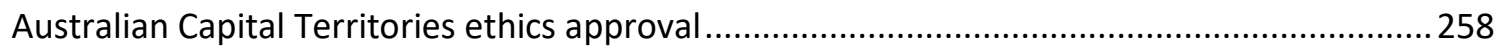

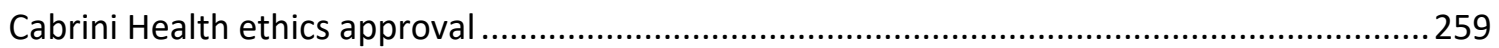

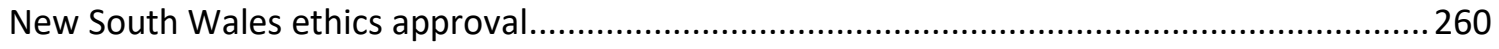




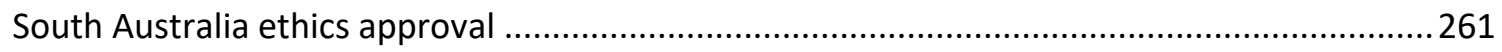

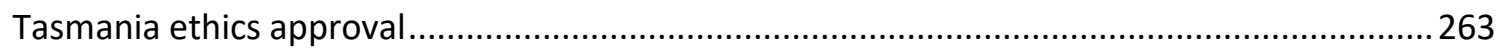

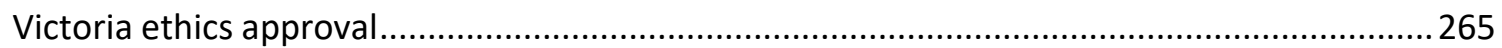

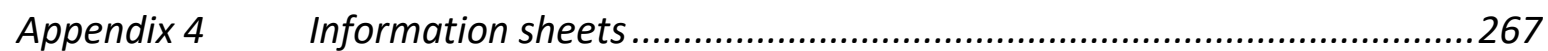

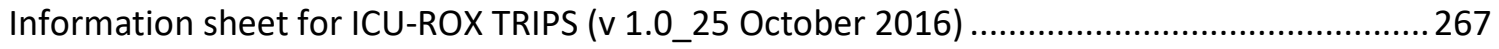

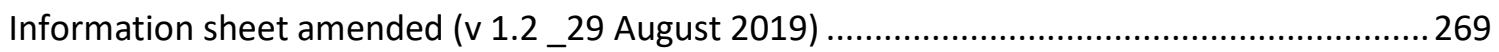

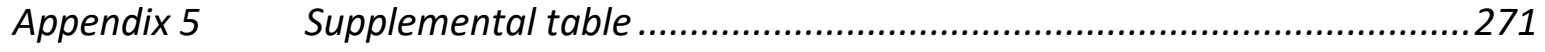

Appendix $6 \quad$ ANZICS Clinical Trials Group endorsement ......................................273

Appendix $7 \quad$ ANZICS Centre for Outcome and Resource Evaluation approval ..........2274

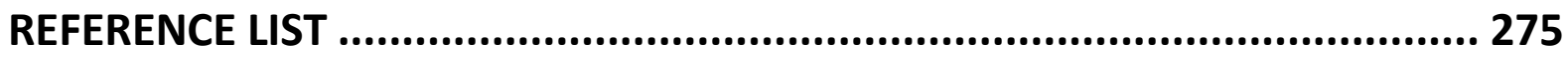




\section{List of figures}

Figure 1 Flow chart for management of ventilated patients allocated to the conservative oxygen therapy arm

Figure 2 Flow chart for management of ventilated patients allocated to the standard oxygen therapy arm .18

Figure 3 Overview of ICU-ROX TRIPS methods in relation to PARIHS Framework .73

Figure 4 Structure and timing of multiple methods for ICU-ROX TRIPS. 79

Figure 5 Site selection for ICU-ROX TRIPS (practitioner attitudes survey and inception cohort study) .93

Figure 6 Flow diagram of survey responses. 109

Figure 7 Likelihood of ICU-ROX vs non-ICU-ROX site respondents using $\mathrm{F}_{1} \mathrm{O}_{2}$ of 0.21 if $\mathrm{SpO}_{2}$ is 91-96\%, over time*

Figure 8 Likelihood of ICU-ROX vs non-ICU-ROX site respondents using different levels of $\mathrm{F}_{1} \mathrm{O}_{2}$ for patients with suspected Hypoxic Ischaemic Encephalopathy (T3) ${ }^{*}$ 118

Figure 9 Likelihood of ICU-ROX vs non-ICU-ROX site respondents reducing the $\mathrm{F}_{1} \mathrm{O}_{2}$ from 0.25 if $\mathrm{SpO}_{2} \geq 97 \%$, over time*

Figure 10 Likelihood of ICU-ROX vs non-ICU-ROX site respondents reducing the $\mathrm{F}_{1} \mathrm{O}_{2}$ from 0.3 if $\mathrm{SpO}_{2} \geq 97 \%$, over time*

Figure 11 Likelihood of ICU-ROX vs non-ICU-ROX site respondents reducing the $\mathrm{F}_{1} \mathrm{O}_{2}$ from 0.4 if $\mathrm{SpO}_{2} \geq 97 \%$, over time ${ }^{*}$

Figure 12 Likelihood of ICU-ROX vs non-ICU-ROX site respondents reducing the $\mathrm{F}_{1} \mathrm{O}_{2}$ from 0.6 if $\mathrm{SpO}_{2} \geq 97 \%$, over time*

Figure 13 Likelihood of ICU-ROX vs non-ICU-ROX site respondents reducing the $\mathrm{F}_{1} \mathrm{O}_{2}$ from 1.0 if $\mathrm{SpO}_{2} \geq 97 \%$, over time*

Figure 14 Likelihood of ICU doctor vs nurse respondents using $\mathrm{F}_{1} \mathrm{O}_{2}$ of 0.21 if $\mathrm{SpO}_{2}$ is $91-96 \%$, over time* 130

Figure 15 Likelihood of using ICU doctor vs nurse respondents using different levels of $\mathrm{F}_{1} \mathrm{O}_{2}$ for patients with suspected Hypoxic Ischaemic Encephalopathy (T3)*.... 131

Figure 16 Likelihood of ICU doctor vs nurse respondents reducing $\mathrm{F}_{1} \mathrm{O}_{2}$ from 0.25 if $\mathrm{SpO}_{2}$ is $\geq$ $97 \%$, over time* 132

Figure 17 Likelihood of ICU doctor vs nurse respondents reducing $\mathrm{F}_{1} \mathrm{O}_{2}$ from 0.3 if $\mathrm{SpO}_{2}$ is $\geq$ $97 \%$, over time* 
Figure 18 Likelihood of ICU doctor vs nurse respondents reducing $\mathrm{F}_{1} \mathrm{O}_{2}$ from 0.4 if $\mathrm{SpO}_{2}$ is $\geq 97 \%$, over time*

Figure 19 Likelihood of ICU doctor vs nurse respondents reducing $\mathrm{F}_{1} \mathrm{O}_{2}$ from 0.6 if $\mathrm{SpO}_{2}$ is $\geq 97 \%$, over time*

Figure 20 Likelihood of ICU doctor vs nurse respondents reducing $\mathrm{F}_{1} \mathrm{O}_{2}$ from 1.0 if $\mathrm{SpO}_{2}$ is $\geq 97 \%$, over time*

Figure 21 Likelihood of ICU specialist doctors (ICU-ROX vs non-ICU-ROX) using $\mathrm{F}_{1} \mathrm{O}_{2}$ of 0.21 if $\mathrm{SpO}_{2}$ is $91-96 \%$, over time ${ }^{*}$

Figure 22 Likelihood of ICU nurses (ICU-ROX vs non-ICU-ROX) using $\mathrm{F}_{1} \mathrm{O}_{2}$ of 0.21 if $\mathrm{SpO}_{2}$ is 91 $96 \%$, over time*

Figure 23 Flow diagram of patients

Figure $24 \mathrm{~F}_{1} \mathrm{O}_{2}$ while ventilated in ICU by time and exposure group (A: mean; B: highest; C:

lowest) 192

Figure $25 \mathrm{PaO}_{2}$ while ventilated in ICU by time and exposure group (A: mean, B: highest, C: lowest)

Figure 26 Flow of patients for retrospective cohort study 208

Figure $27 \mathrm{~F}_{1} \mathrm{O}_{2} *$ in the first 24 hours in the ICU by week (ICU-ROX sites) ${ }^{\dagger}$ 214

Figure $28 \mathrm{~F}_{1} \mathrm{O}_{2} *$ in the first 24 hours in the ICU by week (non-ICU-ROX sites) ${ }^{\dagger}$ 215

Figure $29 \mathrm{PaO}_{2} *$ in the first 24 hours in the ICU by week (ICU-ROX sites) ${ }^{+}$ 216

Figure $30 \mathrm{PaO}_{2}{ }^{*}$ in the first 24 hours in the ICU by week (non-ICU-ROX sites) ${ }^{\dagger}$ 217 


\section{List of tables}

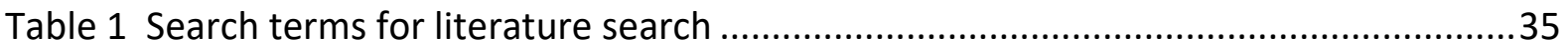

Table 2 Summary of reviewed studies (date order) .......................................................... 40

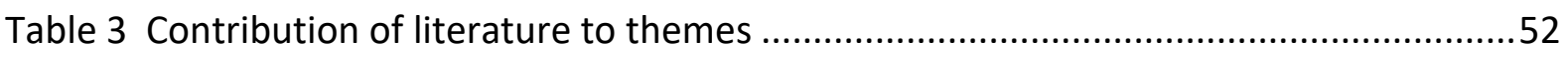

Table 4 Overview of methods used to meet objectives in relation to the PARIHS framework

Table 5 Differences in exposure to ICU-ROX between ICU-ROX and non-ICU-ROX sites.......85

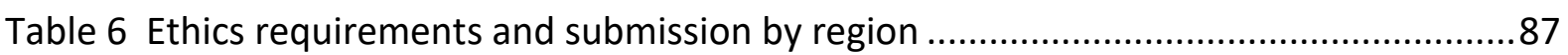

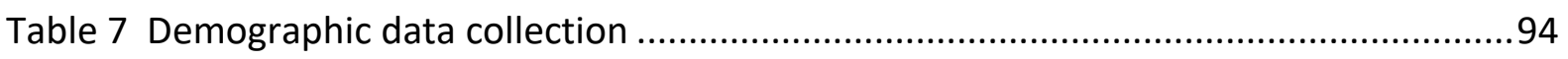

Table 8 Summary of oxygen-related questions and response options for TRIP Practitioner attitudes survey

Table 9 Additional survey questions included in the practitioner attitudes survey (after

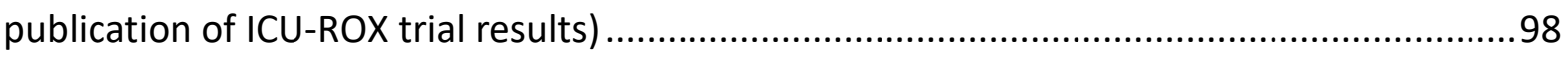

Table 10 Summary of $\mathrm{F}_{1} \mathrm{O}_{2}$ fields that required recoding...............................................103

Table 11 Characteristics of ICU-ROX vs non-ICU-ROX sites (practitioner attitudes survey and

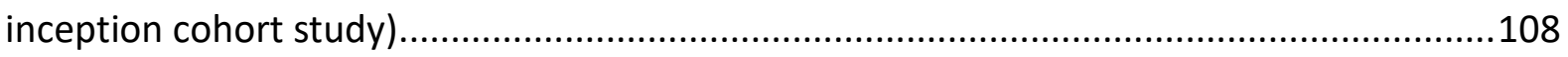

Table 12 Practitioner attitudes survey responses over time (by role and exposure) ..........110

Table 13 Respondent baseline characteristics for ICU-ROX vs non-ICU-ROX sites ..............111

Table 14 Respondent baseline characteristics for ICU-ROX vs non-ICU-ROX sites (for T3:

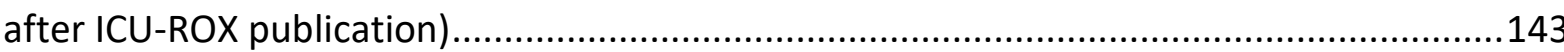

Table 15 Reported determinants of oxygen management (practitioners attitudes survey)144

Table 16 Reported determinants of oxygen management (ICU-level survey) .....................144

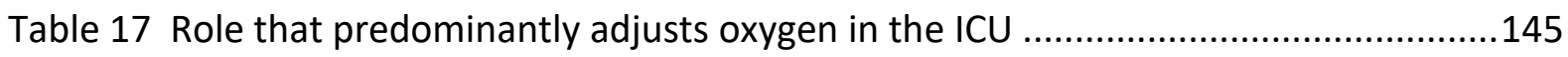

Table 18 Prompts for adjusting oxygen (practitioner attitudes survey) * ..........................146

Table 19 Respondent participation in the ICU-ROX trial ................................................147

Table 20 Mode of finding out ICU-ROX results (ICU-ROX vs non-ICU-ROX respondents) ....148

Table 21 Mode of finding out ICU-ROX results (ICU doctor vs nurse respondents)..............148

Table 22 Sections of ICU-ROX publication read (ICU-ROX vs non-ICU-ROX respondents) ...149

Table 23 Sections of the ICU-ROX publication read (ICU doctor vs nurse respondents) .....150

Table 24 Knowledge of results if enrolled a patient (ICU-ROX sites only)...........................150

Table 25 Knowledge of results if cared for a patient (ICU-ROX sites only) .........................150

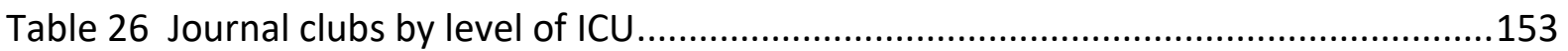


Table 27 Usual modes of dissemination for research that sites are involved in 154

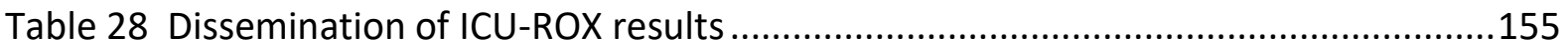

Table 29 Physiological descriptors and process of care measures ...................................173

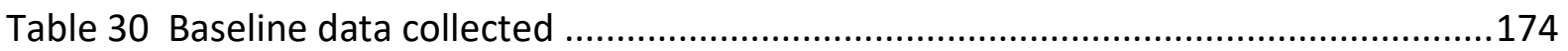

Table 31 Daily data collected for primary and secondary outcomes .................................176

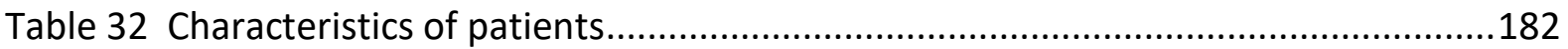

Table 33 Additional baseline characteristics of patients ......................................................183

Table 34 Descriptive data for $\mathrm{F}_{\mathrm{l}} \mathrm{O}_{2}$ of 0.21 hours while ventilated in ICU...........................184

Table 35 Primary and secondary outcome measures: percentage of time $\mathrm{F}_{1} \mathrm{O}_{2}$ is 0.21 while

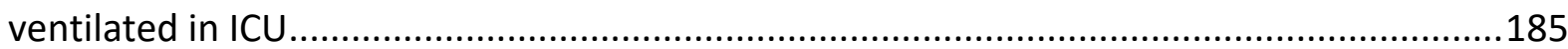

Table 36 Descriptive data for $\mathrm{SpO}_{2} \geq 97 \%$ hours while ventilated in ICU ...........................186

Table 37 Percentage of time $\mathrm{SpO}_{2} \geq 97 \%$ while ventilated in ICU ...................................187

Table 38 Descriptive data for $\mathrm{SpO}_{2}<91 \%$ hours while ventilated in ICU............................188

Table 39 Percentage of time $\mathrm{SpO}_{2}<91 \%$ while ventilated in ICU ....................................189

Table 40 Descriptive data for $\mathrm{SpO}_{2}<88 \%$ hours while ventilated in ICU ............................190

Table 41 Percentage of time $\mathrm{SpO}_{2}<88 \%$ while ventilated in ICU ......................................191

Table 42 Sensitivity analysis for percentage of time $\mathrm{F}_{1} \mathrm{O}_{2}$ is 0.21 while ventilated in ICU....195

Table 43 Subgroup analyses: and relative rate $\mathrm{F}_{1} \mathrm{O}_{2}$ is 0.21 while ventilated in ICU ............196

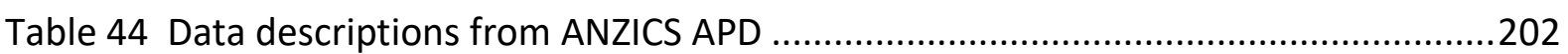

Table 45 Determining the worst APACHE-III-J score for oxygenation variables...................204

Table 46 Characteristics of patients in the ICU-ROX trial site cohort (pre-ICU-ROX

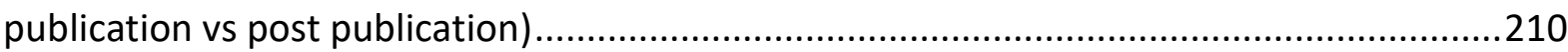

Table 47 Characteristics of patients in the non-ICU-ROX trial site cohort (pre-ICU-ROX

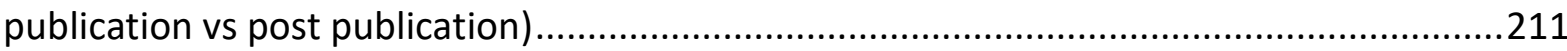

Table $48 \mathrm{~F}_{1} \mathrm{O}_{2}$ and $\mathrm{PaO}_{2}$ levels in patients' first 24 hours of ICU admission for ICU-ROX vs

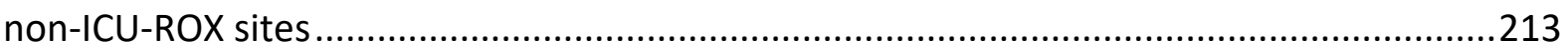

Table S1 Survey response rate by question over time (denominators) ............................... 270 


\section{List of abbreviations}

\begin{tabular}{|c|c|}
\hline Abbreviation & Meaning \\
\hline A-a gradient & Alveolar-arterial gradient \\
\hline ABG & Arterial blood gas \\
\hline ACE (inhibitors) & Angiotensin converting enzyme inhibitors \\
\hline ANZ & Australia and New Zealand \\
\hline ANZCTR & Australian New Zealand Clinical Trials Registry \\
\hline ANZIC-RC & Australian and New Zealand Intensive Care Research Centre \\
\hline ANZICS & Australia and New Zealand Intensive Care Society \\
\hline ANZICS CTG & $\begin{array}{l}\text { Australia and New Zealand Intensive Care Society Clinical } \\
\text { Trials Group }\end{array}$ \\
\hline ANZICS CORE & $\begin{array}{l}\text { Australia and New Zealand Intensive Care Society Centre for } \\
\text { Centre for Outcome and Resource Evaluation }\end{array}$ \\
\hline APACHE & Acute Physiology and Chronic Health Evaluation \\
\hline APD & Adult patient database \\
\hline ASPECT & Aspirin Esomeprazole Chemoprevention Trial \\
\hline BTS & British Thoracic Society \\
\hline CASP & Critical Appraisal Skills Programme \\
\hline $\mathrm{CICM}$ & College of Intensive Care Medicine \\
\hline CINAHL & Cumulative Index to Nursing and Allied Health Literature \\
\hline DCA & Directional coronary atherectomy \\
\hline DIGITAT & $\begin{array}{l}\text { Disproportionate Intra-Uterine Growth Intervention Trial at } \\
\text { Term }\end{array}$ \\
\hline $\mathrm{F}_{1} \mathrm{O}_{2}$ & Fraction of inspired oxygen \\
\hline GP & General Practitioner \\
\hline GUSTO-1 & $\begin{array}{l}\text { Global Utilization [sic] of Streptokinase and Tissue } \\
\text { Plasminogen Activator for Occluded Coronary Arteries }\end{array}$ \\
\hline HDEC (if used) & Health and Disability Ethics Committee \\
\hline HIE & Hypoxic Ischaemic Encephalopathy \\
\hline HOT or NOT & $\begin{array}{l}\text { HyperOxic Therapy OR NormOxic Therapy after out-of- } \\
\text { hospital cardiac arrest }\end{array}$ \\
\hline HRC & Health Research Council (of New Zealand) \\
\hline HYPITAT & Hypertension and Pre-eclampsia Intervention Trial at Term \\
\hline ICU & Intensive Care Unit \\
\hline ICU-ROX & $\begin{array}{l}\text { The Intensive Care Unit Randomised Trial Comparing Two } \\
\text { Approaches to OXygen therapy }\end{array}$ \\
\hline ICU-ROX sites & ICUs that participated in the ICU-ROX trial \\
\hline IRCIG & Intensive Care Research Coordinator Interest Group \\
\hline LMWH & Low molecular weight heparin \\
\hline $\mathrm{Ml}$ & Myocardial infarction \\
\hline MILS & Multicenter [sic] Investigation on Limitation of Infarct Size \\
\hline
\end{tabular}




\begin{tabular}{|c|c|}
\hline Abbreviation & Meaning \\
\hline MRINZ & Medical Research Institute of New Zealand \\
\hline $\mathrm{n} / \mathrm{a}$ & Not applicable \\
\hline n.d. & No date \\
\hline $\mathrm{np}$ & Statistical analysis not possible \\
\hline NEJM & New England Journal of Medicine \\
\hline NICE-SUGAR & $\begin{array}{l}\text { Normoglycemia in Intensive Care Evaluation Survival Using } \\
\text { Glucose Algorithm Regulation }\end{array}$ \\
\hline Non-ICU-ROX sites & ICUs that did not participate in the ICU-ROX trial \\
\hline NZ & New Zealand \\
\hline $\mathrm{PaO}_{2}$ & Partial pressure of arterial oxygen \\
\hline PARIHS & $\begin{array}{l}\text { Promoting Action on Research Implementation in Health } \\
\text { Services (framework) }\end{array}$ \\
\hline $\mathrm{PICO} / \mathrm{PECO}$ & $\begin{array}{l}\text { Population/ Problem, Intervention/ Exposure, Comparator } \\
\text { and Outcome }\end{array}$ \\
\hline $\mathrm{PPI}$ & Protein Pump Inhibitor \\
\hline RCT & Randomised controlled trial \\
\hline $\mathrm{SaO}_{2}$ & Arterial oxygen saturation (measured by arterial blood gas) \\
\hline SAS & Statistical Analysis Software \\
\hline SAVE & Survival and Ventricular Enlargement trial \\
\hline SD & Standard deviation \\
\hline $\mathrm{SpO}_{2}$ & $\begin{array}{l}\text { Arterial oxygen saturation measured by peripheral pulse } \\
\text { oximetry }\end{array}$ \\
\hline SPSS & Statistical Package for the Social Sciences \\
\hline SYNERGY & Superior Yield of the New Strategy of Enoxaparin \\
\hline T1 & First time period - before ICU-ROX began \\
\hline $\mathrm{T} 2$ & $\begin{array}{l}\text { Second time period - after ICU-ROX finished enrolment but } \\
\text { before results were known }\end{array}$ \\
\hline T3 & Third time period - 1 to 6 months after ICU-ROX publication \\
\hline TIMI & Thrombolysis in Myocardial Infarction \\
\hline TRIPS & Translating Research into Practice (study) \\
\hline TTM & Targeted Temperature Management \\
\hline UK & United Kingdom \\
\hline USA & United States of America \\
\hline VFD & Ventilator-free days \\
\hline
\end{tabular}




\section{Personal perspective}

When I started working as a staff nurse around 30 years ago, using the highest amount of oxygen possible was the default position in all actual and potential emergencies. At this time, pulse oximetry was not widely available, and not routinely measured in the general medical and surgical wards I worked in. In my practice, I was unaware of what a normal oxygen saturation or partial pressure of oxygen was. I took a break from nursing in the early 1990s, and when I returned four years later much had changed, including the frequent use of pulse oximetry in wards - with most wards having one pulse oximeter that was used for all patients.

When I began working in Wellington ICU in 1996, peripheral oxygen saturations were continuously monitored and arterial blood gases (ABGs) were assessed frequently. I was taught the meaning of pulse oximetry, partial pressure of oxygen and acid-base balance by a senior nurse during my six-week orientation to ICU, and more extensively about oxygenation and ventilation when I undertook the 'ICU course' in 1997. As I recall, the criteria for extubating a patient were that they were on less than $40 \%$ oxygen, were breathing spontaneously, and were able to maintain their own airway. Use of $100 \%$ oxygen was known to be harmful, causing damage to the lungs, and we reduced the oxygen down from $100 \%$ as soon as possible. It was not, however, unusual to see patients on $100 \%$ for prolonged periods of time and very common for patients to stay on $60 \%$ oxygen indefinitely. This was particularly evident when transporting patient outside of ICU as the transport ventilator at the time only had two settings $-60 \%$ or $100 \%$ oxygen. I remember the emphasis was on lower $\mathrm{SpO}_{2}$ alarm limits, and every effort to avoid hypoxia was made, as should be the case. However, apart from avoiding $100 \%$ oxygen, little attention was paid to avoiding hyperoxia, and I don't remember it ever being mentioned.

In my early years nursing, the emphasis was on 'reflective practice' not 'evidence-based practice'. I had heard the term 'evidence-based practice' but it was something that was spoken about but not really practiced. The first ward I worked in was 'continuing care' and as new graduate staff nurses, we learned from the more senior nurses. Practices that were common at that time, but not evidence-based were 'rubbing alcohol' on skin (to toughen it to prevent bedsores) and exposing bedsores to ultra violet light via a radiant lamp and oxygen (through piped oxygen being blown onto a wound through oxygen tubing). These practices 
were carried out 'because we've always done it that way'. It was really only when I started in ICU in 1996 that I started to hear about research and evidence. Some of the research that was quoted by our senior nurses and doctors was dated, and I got the impression that evidence was sparse. Over the years I heard more and more about evidence and research, but in truth most of my nursing practice was determined by the senior doctor who was in charge on the day.

My eyes were opened when I became a Research Nurse, and became actively involved in 'creating new knowledge', and attending meetings where ICU nurses and doctors from Australia and NZ discussed research. The first clinical trial I was involved in was the Normoglycemia in Intensive Care Evaluation Survival Using Glucose Algorithm Regulation (NICE-SUGAR) trial (The NICE-SUGAR Investigators, 2009). Prior to the publication of the NICESUGAR results being released to our staff, I ran a crude survey to see what the approximately 100 ICU staff thought the results would be (at the 90-day primary outcome). The results were evenly distributed between 'no difference' and 'strict glucose control would be better', apart from six people who thought strict glucose control would be worse - they were correct! The results showed that intensive glucose control increased mortality, 90 days after randomisation. This made me think there is something about being involved that biases the individual towards the 'new' being better. Also of importance was that staff in the ICU were invested in the results once the trial was published, the results were disseminated in our ICU, and our practice returned to a tolerance for higher glucose.

When I joined Dr Paul Young in running a feasibility study about titrating oxygen to peripheral oxygen saturation following cardiac arrest (HOT or NOT) in 2013 (Young et al., 2014), I found the concept of using less than the maximum possible oxygen a huge challenge to my instilled thinking, as it went against everything I had learned and practised for the previous 25 years. My practice (in alignment with guidelines) was to give the maximum amount of oxygen possible in any emergency, as my training had told me that flooding the body and brain with oxygen would improve the patient's outcome, although at that time, I had never read any evidence about it. Anecdotally, other nurses caring for these patients struggled with the concept of lower oxygen too. Ultimately, HOT or NOT was stopped early, as it was not feasible 
to titrate oxygen in the pre-hospital setting, thought to be due to patients' poor post arrest perfusion affecting the peripheral oxygen saturation monitoring.

When Dr Young first conceived of the ICU-ROX trial in 2015, my thinking about oxygen management had evolved since 'HOT or NOT' and I regarded using lower oxygen as a possibility and could imagine using limited oxygen, based on the emerging evidence. I became the project manager for this trial and we began the pilot trial in September 2015. Doing a PhD that also involved ICU-ROX was very enticing, and the idea of conducting a study about oxygen and knowledge translation was born. As an ICU nurse involved in research, I was not only interested in the differences between sites that participated in research (and those that did not) but also the differences between ICU nurses and doctors. 


\section{CHAPTER 1 INTRODUCTION}

Research is essential to expand knowledge and inform best practice in health care. Knowledge translation literature shows a delay between publication and uptake of research findings into clinical practice, and that many factors influence clinician decision-making (Grimshaw et al., 2012; Khera et al., 2018). However, there is uncertainty about whether this delay exists in the intensive care unit (ICU) context and whether participation in research changes attitudes and clinical practices in the research health practitioners are involved in. Conducting a large randomised controlled trial (RCT) that compared conservative with standard oxygen therapy in Australian and New Zealand (ANZ) ICUs, specifically the Intensive Care Unit Randomised Trial Comparing Two Approaches to Oxygen therapy (ICU-ROX) trial (Mackle et al., 2018) provided the opportunity to prospectively examine the effects of participation in an RCT on attitudes and practices. This thesis reports on research designed to examine the attitudes and practices concerning oxygen management of ICU doctors and nurses, before and after ICU-ROX was conducted, and again after publication of the results, and compares whether there was a difference between health practitioners from ICUs that participated in ICU-ROX and those that did not (non-ICU-ROX sites).

This chapter commences with the aims and objectives of the research, and an explanation of terminology used throughout the thesis. This is followed by an introduction to the research, including a brief overview of literature and gaps in knowledge. The importance of the research is presented, along with the research questions and methodology. Following this, is a description of the role I had for the knowledge translation research and for ICU-ROX. The chapter concludes with an overview of the remaining thesis chapters.

\subsection{Research aims and objectives}

The overarching purpose of this research is to increase the understanding of knowledge translation in the ICU setting. The specific aim was to establish whether there was a difference over time in healthcare practitioners' attitudes and practices with regard to oxygen management, who worked in an ICU that participated in a clinical trial about conservative oxygen management in ICU patients, compared to healthcare practitioners from an ICU that did not participate in the clinical trial. The secondary aim was to explore whether there was a difference between the attitudes of ICU specialist doctors and ICU nurses in relation to conservative oxygen therapy. 
The objectives of the research were to:

- Establish baseline attitudes about oxygen management of health practitioners from ANZ ICUs that were going to participate in ICU-ROX compared to those that were not

- Establish baseline practices regarding oxygen management of ANZ ICUs that were going to participate in ICU-ROX compared to those that were not

- Determine whether there was an attitude change regarding oxygen management in health practitioners from ANZ ICUs after participation in ICU-ROX but before the results were known, compared to those from ICUs that did not participate

- Determine whether there was a practice change regarding oxygen management in ANZ ICUs after participation in ICU-ROX but before the results were known, compared to ICUs that did not participate in ICU-ROX

- Determine whether there was an attitude change about oxygen management in health practitioners from ANZ ICUs that participated in ICU-ROX (compared to those that did not), 1 to 6 months after publication of the ICU-ROX results

- Determine whether there was a practice change regarding oxygen management in ANZ ICUs that participated in ICU-ROX (compared to those that did not), 1 to 6 months after publication of the ICU-ROX results

- Determine whether a practice change regarding oxygen management was seen in all ANZ ICUs over time, when reviewed 11 months after publication of the ICU-ROX results, compared to 11 months prior to publication, and whether this was different between ICU-ROX and non-ICU-ROX sites

- Determine whether participation in ICU-ROX was associated with greater knowledge of the published results by comparing health practitioners from participating ICUs' knowledge to those from non-ICU-ROX ICUs; and those who enrolled or cared for a patient with those who did not

- Determine whether there was a difference in attitudes regarding oxygen management between ICU specialist doctors and ICU nurses before ICU-ROX, after ICU-ROX but before the results were known and again after ICU-ROX was published

- Explore whether there was a difference between ICU specialist doctors and ICU nurses regarding their level of knowledge about the ICU-ROX results and publication, and the ways they found out about the results 
- Explore existing systems for knowledge translation and change management for each of the participating ICUs.

These aims and objectives were addressed using a multiple method design; namely a practitioner attitudes survey, inception cohort study and retrospective cohort study, which are outlined in section 1.6.

\subsection{Explanation of terms}

This doctoral study was conducted to assess the knowledge translation of a randomised controlled clinical trial called 'The Intensive Care Unit Randomised Trial Comparing Two Approaches to Oxygen therapy'. From this point on, this trial will be referred to as 'ICU-ROX'. The programme of research (three studies) for this thesis was collectively known as ICU-ROX Translating Research into Practice study (TRIPS) to participants and will be referred to as ICUROX TRIPS throughout the thesis.

ICU-ROX was a trial about oxygen therapy in patients receiving invasive mechanical ventilation in ICU. Invasive ventilation is commonly understood as 'life support' by the general public. Invasive mechanical ventilation means the patient is receiving positive-pressure ventilation through an endotracheal or tracheostomy tube. As this thesis is only about invasive mechanical ventilation (rather than non-invasive ventilation), for simplicity, this is referred to as 'ventilation' or 'mechanical ventilation'.

The amount of oxygen delivered via ventilation is shown as a fraction of inspired oxygen $\left(\mathrm{F}_{1} \mathrm{O}_{2}\right)$ in this research. Some people may be more used to the term 'percentage of oxygen'; however, $\mathrm{F}_{1} \mathrm{O}_{2}$ is the preferred terminology. As an example, 0.8 refers to $80 \%$ of the gas delivered being oxygen. Room air is $\mathrm{F}_{1} \mathrm{O}_{2}$ of 0.21 (or $21 \%$ oxygen). Unless in a specific quote, when referring to the amount of oxygen, the abbreviation $\mathrm{F}_{1} \mathrm{O}_{2}$ will be used throughout this thesis.

The term 'knowledge translation' referring to using research knowledge in clinical practice, is typically used in New Zealand (NZ) and Australia, and will be used throughout this thesis. Implementation Science is the term used in the United Kingdom (UK) and Canada to explain the concept. The term implementation science will be used when it directly relates to specific literature. Chapter 3 provides more detailed information about knowledge translation. 
The terms exposure and comparator are used throughout this thesis. When describing the design of the three studies carried out for this research, the PICO (Population/ Problem, Intervention/ Exposure, Comparator and Outcome) tool is used (Polit \& Beck, 2017). The term exposure, as opposed to intervention, is used in the current research because staff in ICU were not part of an intervention, rather they were exposed to the ICU-ROX protocolised treatment and all that entailed. The term comparator is used to describe those ICUs not exposed to the ICU-ROX protocolised treatment. Further details of what exposure and comparator involved are provided in Chapter 5 (Methodology). The terms exposure and comparator are also used when reporting on the literature in Chapter 4. When the term 'intervention' is used, this is more likely related to the ICU-ROX or other randomised controlled trial intervention.

Research co-ordinator is the role responsible for managing research at a site, in this case in the ICU (Mackle \& Nelson, 2018; Rickard et al., 2006). In the Australian and New Zealand (ANZ) ICU context, this is usually a nurse with ICU experience, although could be another suitably-trained person. There are many different terms for this role; 'Research Co-ordinator' is more commonly used in Australia and 'Research Nurse' in NZ (Mackle \& Nelson, 2018). As the term used in guidelines about ICU recommendations (College of Intensive Care Medicine [CICM], 2016) is research co-ordinator, this term (sometimes abbreviated to RC) is used throughout this thesis.

Due to the nature of this research, much is written about randomised controlled trials (RCT) and knowledge translation studies. In this thesis, randomised controlled trials are referred to as RCTs or (clinical) trials; and observational research as studies.

For consistency, the term 'health practitioner' is used to collectively refer to doctors and nurses. The exception to this is in reference to literature, or in the case of the survey, when 'clinician' is used. Where one particular role (e.g., doctor or nurse) is being referred to the specific term is used.

The abbreviations $\mathrm{T} 1, \mathrm{~T} 2$ and $\mathrm{T} 3$ are used to describe the three time points the survey and inception cohort study were conducted at. Specifically, T1 refers to the study period before 
ICU-ROX started; T2 refers to the period after ICU-ROX finished recruitment (but before results were known) and T3 is the period after ICU-ROX was published.

I refer to myself as 'the researcher', and the thesis is written in the third person, except for sections which position my role in the research.

\subsection{General introduction}

Intensive care doctors and nurses make decisions about clinical care daily. Evidence, while important, is only one of the drivers of health practitioners' attitudes and behaviours, which are also influenced by their previous experiences, key influential people, peers' views, awareness of and belief in new evidence, context, unit culture, and general attitudes to research (Grimshaw et al., 2012; Gupta et al., 2017; Livingston et al., 2020; Sackett et al., 1996; Squires et al., 2011a).

ICUs provide care for critically ill patients with life-threatening injuries and conditions. There are 181 ICUs in Australia and 29 in NZ, with over 200,000 patient admissions to ANZ ICUs in 2018/19, 32.9\% of them mechanically ventilated (Australian and New Zealand Intensive Care Society [ANZICS] Centre for Outcome and Resource Evaluation [CORE], 2020). While the mortality rate has decreased from $10 \%$ over the past 10 years, it remains at approximately eight percent (ANZICS CORE, 2020). ICU patients' overall management is overseen by a specialist intensive care medicine consultant and patients are cared for with a nurse to patient ratio of 1:1 if mechanically ventilated or 1:2 for lower acuity, non-ventilated patients (CICM, 2016; New Zealand Nurses Organisation. Critical Care Nurses' Section, 2014). General conduct and management in ANZ ICUs is guided by the policies and guidelines of the College of Intensive Care Medicine of Australia and NZ, which is the professional body for intensive care specialists (CICM, 2016). ICUs are categorised as Level I, II or III, with Level I providing only immediate resuscitation and short-term cardio-respiratory support. Level II are general ICUs which require the supervision of an intensive care specialist, and Level III are tertiary referral and training centres providing complex multi-system life support for an indefinite period.

Intensive care units in Australia and New Zealand have participated in a number of significant studies about key aspects of caring for critically care patients, many of which have been published in highly ranked medical journals (Chapman et al., 2018; Mackle et al., 2020; 
Myburgh et al., 2012; The NICE-SUGAR Investigators, 2009; Young , Saxena et al., 2015; Young, Bagshaw et al., 2020). Participation in an active research programme is encouraged in ANZ ICUs, regardless of the level, and Level II and III ICUs are required to have an active research programme and employ at least one full-time research co-ordinator (CICM, 2016).

The Australian and New Zealand Intensive Care Society Clinical Trials Group (ANZICS CTG) is a collaborative group which provides a research network within the ANZ ICU community, and has 74 member ICUs (ANZICS CTG, 2018). The CTG is a standing committee of ANZICS, which is an organisation that advocates for ICU patients, their families and the intensive care community (ANZICS, 2021). The ANZICS CTG provides a framework to "promote excellence in intensive care medicine through collaborative clinical research focussed on improving patient-centred outcomes" (ANZICS CTG, 2016, p. 3). At least two bi-national meetings per year provide an opportunity for research staff to collaborate and network, exchange ideas, and present new trials and research results.

One of the decisions ICU health practitioners make is the amount of oxygen to give mechanically ventilated patients. Oxygen is a ubiquitous treatment, administered to almost every patient mechanically ventilated in ICU (Panwar et al., 2013; Suzuki et al., 2013). Importantly, oxygen is not adjusted automatically, but by doctors and nurses who make decisions regarding oxygen management in ANZ ICUs (Henderwood, 2015; Livingston et al., 2020; Rose et al., 2008), with one study reporting that oxygen is sometimes adjusted independently by a nurse at the bedside (Rose et al., 2008). When this knowledge translation study began, there was little evidence about the best amount of oxygen to give, or whether giving no supplemental oxygen while ventilated was safe. Rather, oxygen management focused on avoiding hypoxia. An observational study with regard to oxygen management of mechanically ventilated patients in ANZ ICUs reported oxygen management was liberal and that it was uncommon for patients to be on room air (Panwar et al., 2016). Emerging evidence about the potential risks of hyperoxia, and not knowing the optimal amount of oxygen to give to ICU patients, formed the basis of the ICU-ROX trial.

ICU-ROX was an RCT that compared conservative oxygen therapy to standard oxygen therapy in mechanically ventilated adults in ICU (Mackle et al., 2018). Recruitment into the ICU-ROX pilot trial began in September 2015, and for randomised patients, oxygen was adjusted 
according to the trial protocol. For the conservative care arm, the intervention entailed reducing the oxygen to room air as quickly as possible, and having an upper peripheral oxygen saturation $\left(\mathrm{SpO}_{2}\right)$ alarm limit of $97 \%$, and for the standard care arm, the use of $\mathrm{F}_{1} \mathrm{O}_{2}$ less than 0.3 was discouraged while ventilated. Chapter 2 provides more information about the ICUROX trial and oxygen management generally.

\subsection{Gaps in knowledge}

The literature to date has not demonstrated a link between participation in research and subsequent knowledge use, and no research on this topic has been conducted in Australia or NZ or in ICU. Some studies reported no increased knowledge or use of results, despite personal or institutional involvement in a clinical trial even when the RCT showed very high quality evidence (Khera et al., 2018; Majumdar et al., 2002; Shah et al., 2010), while other studies showed mixed results (Kizer et al., 1999; Litjens et al., 2013; Sweeting et al., 2020). Conversely, some literature supported the view that institutional or regional participation in a particular research trial increased the knowledge and/or use of the results in clinical practice (Adler, 1978; Corrigan \& Glass, 2005; Ketley \& Woods, 1993; Meineche-Schmidt et al., 2006; Omoigui et al., 1998). Most studies examined the experience of doctors only, except three which included nurses, midwives and physiotherapists (Litjens et al., 2013; Sweeting et al., 2020; Tranmer et al., 2002). Only one study examined the attitudes of health practitioners, and that was not in relation to participation in an RCT (Tranmer et al., 2002). A dissemination plan was only referenced in one study, that investigated participation in an observational study and subsequent knowledge translation (Sweeting et al., 2020). In addition, the majority of research was retrospective, so the opportunity to conduct a study prospectively allowed the assessment of attitudes in 'real time'.

As such, a gap in the knowledge existed about participation in research in ANZ ICUs, which includes doctors and nurses. Additionally, there was an opportunity to examine attitudes and practices to oxygen management before, during and after the trial process, and examine the differences in attitudes between doctors and nurses. The work regarding level of engagement can be extended on by examining the level of knowledge of trial results related to the health practitioner's personal and unit-level engagement with the RCT.

This research will contribute to the knowledge about whether participation in research increases the likelihood of research findings being translated into practice in ANZ ICUs. 
Understanding whether participating in research, and being exposed to a trial intervention increases the use of evidence or shortens the time findings are translated into clinical practice, is important for critically ill patients and ICU health practitioners. In addition, knowing that participation in research might potentially speed up implementation would have implications for funding agencies who are increasingly focused on knowledge translation. If health practitioners who participate in research are more likely to know and use research they are involved in, this study could provide support for policies aimed at increasing institutional participation in clinical trials in Australian and NZ ICUs. In addition, understanding whether current dissemination practices are effective has major implications for future ICU trials.

\subsection{Research questions}

The following questions were decided at the outset of this study.

1. Is there a difference between the baseline attitudes about conservative oxygen therapy, of health practitioners from ICUs that will be participating in a trial that evaluates conservative oxygen therapy and health practitioners from ICUs that will not?

2. Does participation in a trial evaluating conservative oxygen therapy in ICU change health practitioners' attitudes about this therapy, before the results of that trial are known?

3. Does participation in a trial evaluating conservative oxygen therapy in ICU change health practitioners' attitudes about this therapy, once the results of that trial are known?

4. Is there a difference between the attitudes about oxygen management in ICU of ICU specialist doctors and ICU nurses before a trial evaluating conservative oxygen therapy?

5. Do the attitudes of ICU specialist doctors and ICU nurses change after a trial evaluating conservative oxygen therapy, and again once the results are known?

6. Is there a difference between the baseline practices of oxygen management in ICUs, that will be participating in a trial that evaluates conservative oxygen therapy and ICUs that will not?

7. Does participation in a trial evaluating conservative oxygen therapy in ICU change practices in relation to the provision of oxygen, before the results of that trial are known? 
8. Does participation in a trial evaluating conservative oxygen therapy in ICU change practices about this therapy, once the results of that trial are known?

9. Does oxygen management of mechanically ventilated patients in ICU change over time in all ANZs, does it change more after a trial evaluating conservative oxygen management is published, and is there a difference between sites that participated in ICU-ROX compared to those that did not?

10. Does a dissemination plan about an ICU trial that includes publication in a high-ranking journal and presentation at an international conference, reach the target audience?

11. Is there a difference in the way ICU specialist doctors and ICU nurses learn about the results of a trial evaluating conservative oxygen therapy, once the results of the trial are known?

12. What are the existing processes with respect to knowledge translation and change management in ICUs?

\subsection{Methodology}

To answer the research questions, the philosophical standpoint for this research was postpositivism. Aligned with the Promoting Action on Research Implementation in Health Services (PARIHS) framework (Kitson et al., 1998), multiple methods were used to prospectively investigate the effect of ICU participation in ICU-ROX. The PARIHS framework consists of three elements: Evidence, Context and Facilitation. For this knowledge translation research, the evidence is the results of ICU-ROX, the context is participation or non-participation in ICUROX, reviewed from three different levels of involvement and facilitation is operationalised as the assessment of dissemination. The three different perspectives collectively form a multiple-method approach of three observational studies in a programme of research collectively known as ICU-ROX TRIPS. A multiple method approach enables the research questions to be answered from a number of different perspectives through the use of related but standalone studies (Morse, 2010). Firstly; a series of surveys assessed the attitudes of health practitioners over time in ICUs participating in ICU-ROX compared to non-participating ICUs. In addition, attitudes were compared by role, using individual doctors and nurses as informants. Concurrently in the same ICUs, an inception cohort study was conducted to evaluate clinical practices with regard to oxygen management between sites that participated in ICU-ROX compared with those that did not, over time. The inception cohort study used a wider scope than the survey, incorporating the practices at an ICU level. The third study was a retrospective cohort study that assessed temporal trends of all ANZ ICUs. This study 
compared oxygen management from before and after publication of ICU-ROX in oxygen management for, and between ICUs that participated in ICU-ROX compared to nonparticipating ICUs, using an interrupted time-series analysis of existing registry data (with the ICU-ROX publication as the interruption).

Knowledge translation research usually researches the use of existing knowledge, however, for this study the knowledge was not known at the beginning of the study, so all possibilities for results of the ICU-ROX trial had to be considered. Specifically, at the beginning of this study, the findings of the ICU-ROX trial for the primary outcome of alive, ventilator-free days to 28 days after randomisation were unknown. The ICU-ROX outcome was considered to be one of three possibilities (assuming there were no adverse findings in the secondary outcomes):

1. Patients receiving conservative oxygen therapy have more alive, ventilator-free days (VFDs) than those receiving standard oxygen therapy

2. Patients receiving standard oxygen therapy have more VFDs than those receiving conservative oxygen therapy

3. No statistically significant difference in VFDs between patients receiving conservative oxygen compared to standard oxygen therapy.

ICU-ROX began with a pilot run-in phase of 100 patients in September 2015, that ran until June 2016. Following analysis of the pilot study (Young et al., 2017), the ICU-ROX trial restarted enrolment in March 2017, concluding in May 2018. This knowledge translation study was started in November 2016 and concluded in September 2020.

A comprehensive summary of the ICU-ROX trial and results is presented in Chapter 2, but in brief, ICU-ROX showed that conservative oxygen therapy did not improve alive, ventilatorfree days at day 28 compared to standard oxygen therapy (option 3). There were no adverse findings in the secondary outcomes and conservative oxygen therapy appeared to be safe in mechanically ventilated adults in ICU (Mackle et al., 2020). Hypotheses for ICU-ROX TRIPS were developed to cover each potential outcome of ICU-ROX and are included in the individual study chapters.

\subsection{My role as researcher and ICU-ROX project manager}

This thesis is about the knowledge translation of the ICU-ROX trial; assessing attitudes and practices of ICU health practitioners to oxygen management in ICU before and after ICU-ROX, 
and again after ICU-ROX publication. As a researcher studying the knowledge translation of ICU-ROX, I sought to observe, not influence, what happened. I was responsible for every aspect of the knowledge translation research including conceiving the idea, overall research design and protocol for the three studies, implementation, analysis, execution and reporting. Further details about the three studies are presented in Chapters 6, 7 and 8. I worked independently except when particular expertise was required, which is outlined throughout the thesis.

I was the project manager for ICU-ROX, and held a key role in managing the trial, including developing and executing the protocol; along with substantive intellectual input into the overall study and publications. However, in this role it was important for ICU-ROX and the knowledge translation studies that I did not have any undue influence on the ICU-ROX results, or alter the usual dissemination plan, and the following precautions were taken.

The trial design, protocol, statistical analysis and monitoring plans were the collective responsibility of the ICU-ROX Management Committee, with Dr Paul Young as Chair. I was a member of the management committee and my role as project manager was to manage every aspect of the study, on behalf of that committee. I supervised an Australian project manager who managed ICU-ROX in Australia. As project manager, I worked closely with Dr Young, who as Chief Investigator, had overall responsibility for the trial. Dr Young applied for the initial ethics and registered the trial in the Australian New Zealand Clinical Trials Registry (ANZCTR). ICU-ROX had an independent data monitoring committee, who adhered to a pre-determined charter. I conducted the start-up meetings, developed the tools necessary to run the study, ensured participating sites had all ethics and local governance requirements, worked closely with the software company in the development of the database, communicated with site research staff, monitored the data and consent process in NZ (according to a pre-specified monitoring plan), ensured protocol compliance (including setting up protocol compliance reports) and communicated with the Australian project management group. Dr Young and I collectively liaised with the data monitoring and management committees, and in collaboration with the management committee, wrote the manuscripts.

Site research co-ordinators collected and entered data into a bespoke trial database designed by Spiral software (Wellington, New Zealand). The ICU-ROX database was controlled by 
individual log-in and an audit trail in the database logged every entry made. I was responsible for reviewing data (internal consistency checks); issuing data queries to site research staff. I was not permitted to make any changes to individual patient data, as is usual practice for all trials we manage. A 'changes' report was used, which could be filtered by individual log-in, to record who had made changes. There was one instance when a change (apart from unlocking patient records for the site research staff to make changes or issuing queries) was logged against my log-in. This was an error that occurred early in the study, when a site staff member inadvertently made a change while I was logged in to demonstrate the database. The trial data were downloaded and collated by an independent information technology specialist at the Medical Research Institute of New Zealand. These data were sent directly to the trial statistician who conducted an independent analysis as per the pre-published statistical analysis plan (Mackle et al., 2018).

The ICU-ROX Chief Investigator and chair of the Management Committee, Dr Young, in conjunction with the ICU-ROX Management Committee, was responsible for the dissemination plan (outlined in Section 2.4), which was similar to his dissemination of previous ICU trials (Chapman et al., 2018; Young, Bagshaw et al., 2020). As project manager for ICU-ROX, it was my role to distribute the publication to ICU-ROX sites, which was done as usual. No effort was made to specifically disseminate this to the non-ICU-ROX sites (as was normal practice).

\subsection{Thesis overview}

This thesis is presented in nine chapters. This chapter has introduced the topic, context, the terms used throughout the thesis and my role in the research. While this thesis is primarily about knowledge translation, oxygen management is fundamental to the research. As such, the background to each of these topics is presented in separate chapters. Chapter 2 is about oxygen and begins with a brief summary of guidelines and evidence about oxygen management prior to ICU-ROX beginning. Following this is a summary of the design and results of ICU-ROX, followed by the dissemination plan, and a summary of the contribution ICU-ROX made to evidence about oxygen. The full ICU-ROX publication is in Appendix 1 to provide more detailed information about ICU-ROX. Chapter 3 provides background information about knowledge translation, including a brief history, worldwide and in NZ, and within the ICU context. In Chapter 4, the review of the literature is presented which describes what is already known about participation in research and the subsequent effect on using the 
knowledge created, and importantly what is not known and requires further exploration. The methodology is presented in Chapter 5 including how this study fits philosophically within the theoretical lens of postpositivism. In addition, this chapter outlines the PARIHS framework that informed the methodological decisions for this doctoral study. This framework is explained along with the rationale for using it and the multiple method approach is introduced. Included in this chapter is an overview of the methods for the three studies and ethical considerations.

Given the complex nature of this thesis, each of the three studies are presented in full (hypotheses, methods, design, results and discussion), separately in Chapters 6, 7 and 8. Specifically, the Practitioner Attitudes Study is in Chapter 6; the Inception Cohort study in Chapter 7, and the Retrospective Cohort Study in Chapter 8. The overview of the study setting for the Practitioner Attitudes Survey and Inception Cohort Study are in Chapter 6, as both studies were conducted concurrently in the same ANZ ICUs. The thesis concludes with Chapter 9, which summarises the three studies, how the findings relate to the literature, and what the findings mean. Strengths and limitations of the research are outlined; along with recommendations for future research. This final chapter also presents the contribution to the field of knowledge translation, and recommendations for practice. 


\section{CHAPTER 2 OXYGEN AND ICU-ROX PUBLICATION}

This chapter outlines the evolution of oxygen evidence in ICU. It does not aim to provide a full history of oxygen evidence but positions the ICU-ROX trial within recent evidence and guidelines. The chapter begins with an overview of oxygen guidelines, followed by the evidence prior to starting ICU-ROX to highlight the environment ICU-ROX was designed in. A summary of ICU-ROX is then provided, followed by the dissemination plan used, and the contribution of ICU-ROX to knowledge about oxygen management in ICU. Finally, research about oxygen management in ICU that was published during and after ICU-ROX is outlined.

\subsection{Oxygen management guidelines}

There are no oxygen management guidelines that specifically relate to the care of patients in ICU. Guidance regarding ICU patients with acute respiratory distress syndrome (ARDS) of a target peripheral oxygen saturation $\left(\mathrm{SpO}_{2}\right)$ of $88 \%$ to $95 \%$ and arterial oxygen $\left(\mathrm{PaO}_{2}\right)$ of 55 to $80 \mathrm{mmHg}$ is often referred to and was recommended by the ARDS network (ARDS-NET) group (2000; Schjørring et al., 2021).

First published by the British Thoracic Society (BTS) in 2008, landmark guidelines about emergency oxygen use in adult patients provided a comprehensive evidence-based summary about three areas of concern: risks associated with hypoxaemia, hyperoxaemia and exposure to high levels of $\mathrm{F}_{1} \mathrm{O}_{2}$ (O'Driscoll et al., 2008). Hypoxaemia is the abnormally low level of arterial oxygen $\left(\mathrm{PaO}_{2}\right)$ (Beasley et al., 2019; O'Driscoll et al., 2008). The evidence and guidelines are clear that severe hypoxaemia is associated with increased organ dysfunction and risk of death (de Jonge et al., 2008; Eastwood, Bellomo et al., 2012). Hyperoxaemia, is the abnormally high level of oxygen in arterial blood (O'Driscoll et al., 2008). At the time the guidelines were published, the harm associated with hyperoxaemia was less clear, due to insufficient clinical trial evidence, except for patients with chronic obstructive pulmonary disease (COPD). Exposure to high levels of $\mathrm{F}_{1} \mathrm{O}_{2}$ is considered harmful because of direct toxicity at a cellular level (Beasley et al., 2015, 2019).

The BTS oxygen guidelines recommended a peripheral oxygen saturation $\left(\mathrm{SpO}_{2}\right)$ target in acutely ill patients of $94 \%$ to $98 \%$ (O'Driscoll et al., 2008). For patients with COPD, a target peripheral saturation range of $88-92 \%$ was recommended. This evidence-based approach has been adopted, with modification, by the Thoracic Society of Australia and NZ which published 
oxygen management guidelines in 2015, recommending a target $\mathrm{SpO}_{2}$ of 92 to $96 \%$ be prescribed for critically ill patients (Beasley et al.). Overall, oxygen guidelines advise titrating to a prescribed oxygen saturation range to avoid hypoxia, hyperoxia and high concentrations of $\mathrm{F}_{1} \mathrm{O}_{2}$.

\subsection{ICU oxygen management research prior to ICU-ROX}

Prior to beginning ICU-ROX trial, there was limited evidence about the best amount of oxygen to use in ICU patients. In retrospective reviews of large databases, hypoxia was associated with organ dysfunction and death (de Jonge et al., 2008; Eastwood, Bellomo, et al., 2012). De Jonge et al. found in their study of data from 50 ICUs in the Netherlands that a high fraction of inspired oxygen $\left(\mathrm{F}_{1} \mathrm{O}_{2}\right)$ in the first 24 hours of ICU stay was associated with increased inhospital mortality both for low partial pressure of arterial oxygen $\left(\mathrm{PaO}_{2}\right)$ (hypoxia) and high $\mathrm{PaO}_{2}$ (hyperoxia). However, an analysis using the Australia and NZ Intensive Care Society (ANZICS) adult patient database, reported the average $\mathrm{F}_{1} \mathrm{O}_{2}$ in the first 24 hours of ICU admission was 0.62 , and there was an increased risk of mortality with hypoxia, but not hyperoxia (Eastwood, Bellomo et al., 2012). A meta-analysis of 24 studies from 2008 to 2015 suggested that hyperoxia was associated with increased mortality (Helmerhorst et al., 2015). Subsequently, a observational study of arterial blood gases for 14,441 ICU patients from three hospitals in the Netherlands found hyperoxia was associated with increased mortality and fewer alive, ventilator-free days (Helmerhorst et al., 2017).

Observational studies reported tolerance for high $\mathrm{F}_{1} \mathrm{O}_{2}$ (De Graaff et al., 2011) and $\mathrm{SpO}_{2}$ (Young, Beasley et al., 2015) by ICU staff, highlighting the need for further research about oxygen practices. In a retrospective study, De Graaff et al. (2011) reviewed 126,778 arterial blood gas results (ABGs) from 5,498 mechanically ventilated patients, conducted in a single ICU in the Netherlands. Analysis showed that $22 \%$ of $\mathrm{PaO}_{2}$ results were $>120 \mathrm{mmHg}$, classified as hyperoxia. Only $25 \%$ of these $A B G$ s resulted in adjusting the $\mathrm{F}_{1} \mathrm{O}_{2}$ on the ventilator to 0.4 or less. Oxygen use throughout this period was liberal, and a point-prevalence study conducted in Australian and NZ (ANZ) ICUs in 2013 reported tolerance of high $\mathrm{SpO}_{2}$ levels. There was low use of target oxygen ranges with lower $\mathrm{SpO}_{2}$ monitor alarms prescribed by doctors in $39 \%$ of mechanically ventilated patients, and upper limit $\mathrm{SpO}_{2}$ limits prescribed in $5.2 \%$ of ventilated patients (Young, Beasley et al., 2015). 
Self-reported views about oxygen management highlighted variability in practice both for ICU specialist doctors (Eastwood et al., 2011) and ICU nurses (Eastwood, Reade et al., 2012) in ANZ ICUs. In the study of ICU doctors, there were differences in views about oxygen toxicity and tolerance of hypoxaemia, depending on the type of ICU (metropolitan or regional) and length of time since speciality training (Eastwood et al., 2011). In the study of ICU nurses, there was significant variability between respondents with respect to oxygen management, specifically in relation to their management of hypoxaemia in mechanically ventilation of a patient with ARDS (Eastwood, Reade et al., 2012). These studies highlighted the need for development of better guidance about oxygen management in ICU.

Another issue was that clinicians' reported views about oxygen management differed from their observed practices (Helmerhorst et al., 2014). Doctors and nurses from three ICUs in the Netherlands were surveyed and the majority of the 215 respondents reported the minimal arterial oxygen saturation $\left(\mathrm{SaO}_{2}\right)$ range they would accept was $85 \%$ to $95 \%$. For $\mathrm{PaO}_{2}$ the reported acceptable range was $52.5 \mathrm{mmHg}$ to $75 \mathrm{mmHg}$. Subsequent analysis of 107,888 $A B G$ results showed that $73.4 \%$ of $\mathrm{PaO}_{2}$ results were higher than the doctors' and nurses' defined acceptable range (Helmerhorst et al., 2014).

Research exploring the feasibility of conducting a trial about conservative oxygen therapy emerged throughout this period. One study was a 'before and after' study set in a single Australian ICU (Suzuki et al., 2014). This study compared usual practice (before) with a protocolised treatment that required clinicians to prescribe an $\mathrm{SpO}_{2}$ level of $90 \%$ to $92 \%$ using the lowest possible $\mathrm{F}_{1} \mathrm{O}_{2}$ (after). The researchers concluded conservative oxygen therapy was feasible, and did not result in increased adverse clinical outcomes. Subsequently, a pilot study in four ANZ ICUs randomised patients to a conservative (titrating $\mathrm{SpO}_{2} 88-92 \%$ ) or liberal $\left(\mathrm{SpO}_{2} \geq 96 \%\right)$ oxygen strategy (Panwar et al., 2016). The researchers found that a larger trial comparing conservative and liberal oxygen was feasible and warranted, with no additional adverse effects in either treatment arm. Similar findings of feasibility and the need for further research about conservative oxygen therapy in ICU were reported in the Netherlands (Helmerhorst et al., 2016).

\subsection{ICU-ROX}

ICU-ROX was a randomised clinical trial that compared conservative with standard oxygen therapy in 21 ANZ ICUs between September 2015 and May 2018 (Mackle et al., 2020). The 
trial began with a 100-participant vanguard pilot phase (Young et al., 2017) leading to minor changes to the protocol for the subsequent 900 participants. The protocol and statistical analysis plan were published in advance of the final analysis (Mackle et al., 2018). The full ICU-ROX publication is provided in Appendix 1.

\subsubsection{Design and methods}

Mechanically ventilated patients 18 years of age or older who were expected to remain mechanically ventilated in ICU beyond the calendar day after recruitment were eligible for inclusion. Exclusion criteria included: hypoxia or hyperoxia clinically indicated, pregnancy, drug overdose, treatment limitations, conditions which meant the patient could not be liberated from the ventilator, previous enrolment in ICU-ROX and not being in the patient's best interests. Enrolment was restricted to patients who had received less than two hours of invasive mechanical ventilation and/or non-invasive ventilation in an ICU.

For patients randomised to conservative oxygen therapy, the $\mathrm{F}_{1} \mathrm{O}_{2}$ was decreased to 0.21 , or supplemental oxygen was discontinued in patients who had been extubated, according to the flow diagram (Figure 1), provided the $\mathrm{SpO}_{2}$ was above the acceptable lower limit. To minimise exposure to high levels of oxygen, an upper $\mathrm{SpO}_{2}$ monitor alarm set at $97 \%$ was mandated, whenever supplemental oxygen was administered in the ICU.

For patients randomised to standard oxygen therapy, no upper $\mathrm{SpO}_{2}$ alarm was permitted, and the use of $\mathrm{FiO}_{2}<0.3$ was discouraged while they were mechanically ventilated (Figure 2).

In both groups, the acceptable lower limit for oxygen saturation measured by pulse oximetry $\left(\mathrm{SpO}_{2}\right)$ was monitored with an alarm set at an $\mathrm{SpO}_{2}$ of $90 \%$ or less. This alarm limit could be altered at the discretion of the treating clinician. If an arterial blood gas showed a $\mathrm{PaO}_{2}$ of less than $60 \mathrm{mmHg}$ or an arterial oxygen saturation $\left(\mathrm{SaO}_{2}\right)$ lower than the acceptable $\mathrm{SpO}_{2}$, $\mathrm{F}_{1} \mathrm{O}_{2}$ could be increased, irrespective of $\mathrm{SpO}_{2}$, at the discretion of the treating clinician. 


\section{ROX CONSERVATIVE OXYGEN THERAPY}

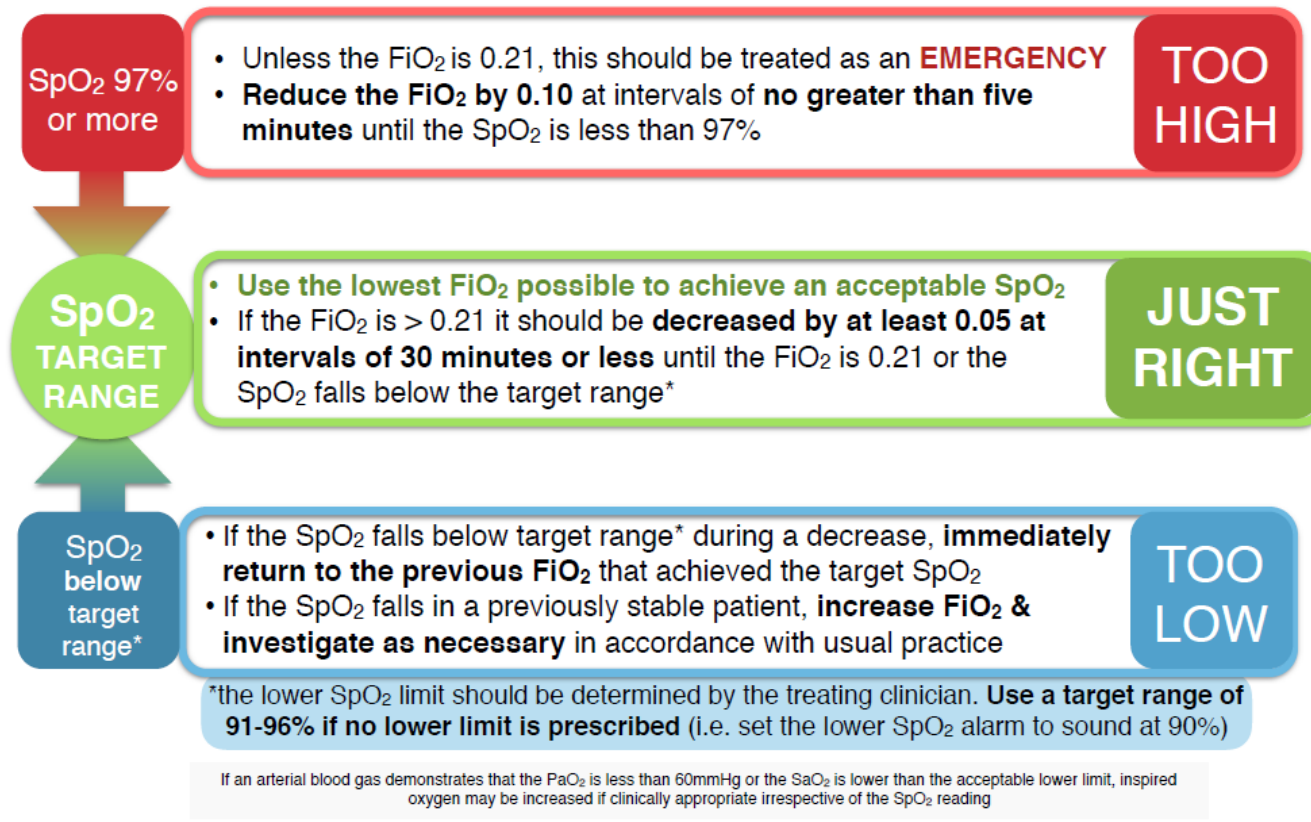

Figure 1 Flow chart for management of ventilated patients allocated to the conservative oxygen therapy arm

(Reproduced with permission from Dr Paul Young, ICU-ROX Chief Investigator)

\section{OU ROX STANDARD OXYGEN THERAPY}

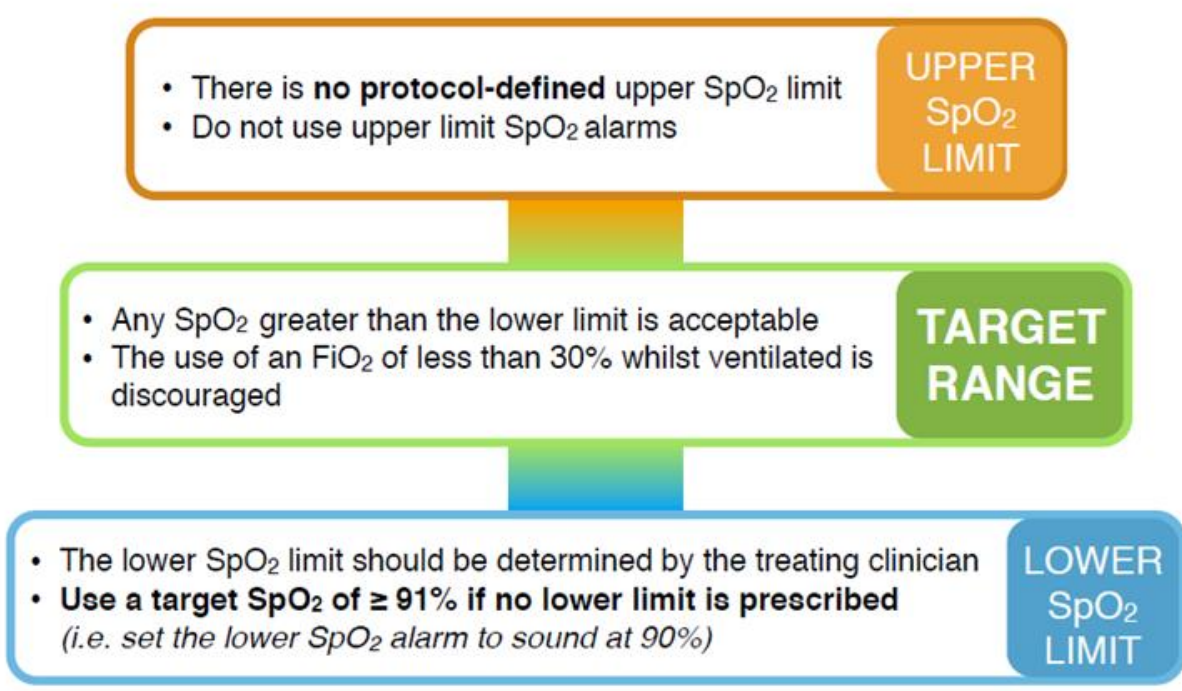

If an arterial blood gas demonstrates that the $\mathrm{PaO}_{2}$ is less than $60 \mathrm{mmHg}$ or the $\mathrm{SaO}_{2}$ is lower than the acceptable lower limit, inspired oxygen may be increased if clinically appropriate irrespective of the $\mathrm{SpO}_{2}$ reading

Figure 2 Flow chart for management of ventilated patients allocated to the standard oxygen therapy arm

(Reproduced with permission from Dr Paul Young, ICU-ROX Chief Investigator) 
The primary outcome for ICU-ROX was ventilator-free days to day 28 (Schoenfeld \& Bernard, 2002). Ventilator-free days were defined as the total number of calendar days or part calendar days of unassisted breathing during the first 28 days after randomisation; all patients who died by day 28 were assigned zero ventilator-free days.

Key secondary outcomes were death from any cause at day 90 and day 180 after randomisation, the duration of survival, the proportion of patients in paid employment at baseline who were unemployed at day 180 , and cognitive function and health-related quality of life at day 180. Cause-specific mortality was also recorded.

The main physiological descriptors of potential relevance to this knowledge translation study were:

- Total number and proportion of hours when $\mathrm{SpO}_{2} \geq 97 \%$

- Total number and proportion of hours when $\mathrm{SpO}_{2}<91 \%$

- Proportion of hours when $\mathrm{SpO}_{2}<88 \%$

- Lowest, highest and mean daily $\mathrm{PaO}_{2}$

The main process of care measures, potentially of relevance to this knowledge translation study were:

- Total number and proportion of hours $\mathrm{F}_{1} \mathrm{O}_{2}$ is 0.21

- Lower, highest, mean daily $\mathrm{F}_{1} \mathrm{O}_{2}$ while ventilated

Data were collected from the time of randomisation until 28 days after randomisation.

\subsubsection{Results}

From September 2015 through May 2018, 1000 patients in 21 adult medical-surgical ICUs in Australia and New Zealand were enrolled, with 499 patients assigned to conservative oxygen and 501 to standard oxygen therapy. Consent was withdrawn by 35 participants, resulting in an intention-to-treat population of 965 , of whom 484 were assigned to conservative oxygen and 481 to standard oxygen. The study groups had similar characteristics at baseline. Figures and tables are available with the publication in Appendix 1.

\section{Oxygenation and process of care}


Patients allocated to conservative oxygen spent more time receiving an $\mathrm{F}_{1} \mathrm{O}_{2}$ of 0.21 in the ICU than patients allocated to standard oxygen ( 29 hours [interquartile range (IQR), 5 to 78] vs. 1 hour [IQR, 0 to 17]; absolute difference, 28 hours; $95 \% \mathrm{Cl}, 22-34$ ), and less time in the ICU with an $\mathrm{SpO}_{2}$ of $97 \%$ or higher (27 hours [IQR, 11 to 63.5] vs. 49 hours [IQR, 22 to 112]; absolute difference, 22 hours; $95 \% \mathrm{Cl}, 14-30)$. The number and percentage of hours with $\mathrm{SpO}_{2}$ less than $91 \%$ and with $\mathrm{SpO}_{2}$ less than $88 \%$ were similar between groups.

\section{Primary outcome}

Ventilator-free days to day 28 did not differ significantly between the conservative oxygen group and the standard oxygen group: 21.3 days (IQR, 0 to 26.3) for patients assigned to conservative oxygen and 22.1 days (IQR, 0 to 26.2) for patients assigned to standard oxygen (absolute difference, -0.3 days; $95 \% \mathrm{Cl},-2.1$ to $1.6 ; \mathrm{P}=0.80$ ).

\section{Secondary outcomes}

Final study follow-up to assess secondary outcomes occurred a median of 186 days after randomisation [IQR, 181 to 197 days]. A total of 170 of 476 patients (35.7\%) assigned to conservative oxygen and 164 of 475 patients (34.5\%) assigned to standard oxygen had died by day 180 (relative risk; 1.03; 95\% Cl, 0.87 to 1.23; hazard ratio; $1.05 ; 95 \% \mathrm{Cl}, 0.85$ to 1.30) Among survivors, no evidence of difference between groups in employment status (among participants in paid employment at baseline) was found. For conservative oxygen therapy patients, 77 of 112 (68.8\%) were in paid employment at final follow-up, compared to 66 of $108(61.1 \%)$ standard oxygen patients. Cognitive function was similar by treatment group with 5 of 203 (2.5\%) conservative oxygen patients and 6 of 206 (2.9\%) standard oxygen patients having severe cognitive impairment at final follow-up. In mobility and personal-care components of the quality of life assessment, estimates indicated a relatively greater proportion of patients had moderate problems and a relatively smaller proportion of patients had severe problems in the conservative group. No evidence of differences in other domains of the quality of life assessment was found.

\section{Subgroup analysis}

There was substantial heterogeneity in the effect of conservative oxygen on ventilator-free days in patients with vs. without suspected hypoxic ischemic encephalopathy but not in other pre-specified subgroups. Among patients with suspected hypoxic ischemic 
encephalopathy, median ventilator free days to day 28 were 21.1 (IQR, 0 to 26.1 ) and 0 (IQR, 0 to 26) for conservative oxygen and standard oxygen groups respectively (absolute difference, 21.1 days; $95 \% \mathrm{Cl}, 10.4$ to 28.0 ). In post-hoc analyses of the suspected hypoxic ischemic encephalopathy subgroup, 37 of 86 (43\%) conservative oxygen patients and 46 of $78(59 \%)$ standard oxygen patients had died by day 180 (relative risk; $0.73 ; 95 \% \mathrm{Cl}, 0.54$ to 0.99; hazard ratio; $0.67 ; 95 \% \mathrm{Cl}, 0.43$ to 1.03$)$ with 43 of 78 (55.1\%) and 49 of 72 (68.1\%) respectively having an unfavourable outcome on the GOS-E (relative risk; $0.81 ; 95 \% \mathrm{Cl}, 0.63$ to 1.05$)$.

\subsection{ICU-ROX dissemination plan}

The ICU-ROX trial results were widely disseminated, as planned, within Australia and New Zealand, and globally through multiple avenues, including publication in a high-ranking medical journal, as a plenary presentation at the World Congress of Intensive Care, via social media, in editorials and directly to the ANZ ICU research community and ICU-ROX sites. The first presentation of results was to the site investigators and research coordinators, via Zoom, in February 2019. Soon after, the results were presented at a closed session at the ANZICS CTG Scientific Meeting in March 2019, to approximately 200 delegates, most of whom were ICU research doctors and nurses. As these presentations preceded the publication, the results were embargoed, meaning research staff were not permitted to disseminate the results to their ICU staff until the study was published. The ICU-ROX results were presented publicly for the first time at the Intensive Care World Congress in Melbourne in October 2019, with a simultaneous publication in the New England Journal of Medicine (Mackle et al., 2020). The New England Journal of Medicine is the highest ranking general medical journal in the world with an impact factor of 74.699 (on 3 June 2021) (Massachusetts Medical Society, 2021). The ICU-ROX results were widely tweeted immediately after the simultaneous publication and presentation of results, including a 'tweetorial' by Paul Young, summarising the trial design, results and implications (Young, 2019). A link to the full article was posted on the Medical Research Institute of New Zealand Facebook page in the following week. The full publication was emailed to all participating site research staff in Australia and NZ immediately after the presentation, although no direction was given about how to disseminate the results to staff. The ICU-ROX results were presented again, by invitation, in January 2020 at the Critical Care Review meeting in Belfast. This presentation was live-streamed and the results again widely tweeted. The article was published in the paper version of the New England Journal of 
Medicine in March 2020, and was later recognised as one of 14 notable articles of 2020 (NEJM, 2020).

\subsection{Emerging evidence throughout ICU-ROX}

Research about oxygen management in ICU continued to emerge throughout the period ICUROX was conducted. The impact of these publications on attitudes to oxygen management in ICU was not tested in the knowledge translation study.

A single centre Italian study was published online in October 2016 (Girardis et al., 2016), after recruitment into the ICU-ROX pilot was complete, and before ICU ROX TRIPS began. Girardis et al. (2016) reported that conservative oxygen therapy resulted in lower ICU mortality. However, a critical appraisal of this article identified some significant issues with the design. The study had stopped recruiting due to difficulties enrolling patients following an unplanned interim analysis in the absence of a pre-defined stopping point, and included non-ventilated patients. After the pre-planned ICU-ROX pilot was completed and before ICU-ROX restarted, the pilot results were published (Young et al., 2017). This showed that ICU-ROX was feasible, with separation between conservative and standard oxygen therapy arms, and eligibility and recruitment targets were met; ICU-ROX restarted in March 2017. Published approximately one month prior to completion of ICU-ROX recruitment, a meta-analysis of 25 RCTs comparing conservative with liberal oxygen showed a liberal oxygen strategy increased the risk of mortality, and recommended conservative oxygen therapy (Chu et al., 2018). This metaanalysis was heavily weighted by the aforementioned study (Girardis et al., 2016) and only eight of the trials included patients who were invasively ventilated.

\subsection{ICU-ROX contribution to knowledge and subsequent research}

The results of ICU-ROX restored equipoise about the level of oxygen that should be used for oxygen management in ventilated ICU patients. The ICU-ROX conclusion that there was no difference in alive ventilator-free days at day 28 between conservative and liberal, reversed the previous findings of Girardis et al. (2016) and meta-analysis by Chu et al. (2018) which had reported that conservative oxygen therapy decreased mortality. ICU-ROX was the largest oxygen therapy trial conducted at the time and was considered robust evidence (multi-centre, blinded outcome, peer-reviewed). It also showed that both conservative and liberal approaches to oxygen management appeared to be safe. However, the findings were heterogeneous and did not provide evidence to guide clinicians about the optimal level of 
oxygen for ICU patients (Young, Bagshaw et al., 2019). Two post-hoc analyses were triggered from pre-specified definitions about conservative oxygen in sepsis and hypoxic ischaemic encephalopathy (Young et al., 2020a; Young et al., 2020b). In the analysis of the sepsis subgroup, point estimates favoured usual care (liberal oxygen); however, were not sufficiently robust to influence recommendations for clinical practice; highlighting the need for a larger trial (Young et al., 2020a). For patients admitted following cardiac arrest with suspected hypoxic ischaemic encephalopathy, conservative oxygen was not associated with a statistically significant reduction in death or unfavourable neurological outcomes; however, an important benefit could not be excluded (Young et al. 2020b). The hypoxic ischaemic encephalopathy data were included in an individual patient meta-analysis which concluded that conservative oxygen therapy was associated with a reduction in mortality compared to liberal oxygen therapy but the evidence was of low certainty; and further research was required (Young, Bailey, Bellomo et al., 2020).

Since the publication of ICU-ROX and conduct of this knowledge translation study, two studies about the attitudes and practices regarding ICU oxygen management in the Netherlands were published (Grim, 2020; Grim et al., 2020). An update of their earlier work (Helmerhorst et al., 2014), Grim et al. (2020) reported an increase in the percentage of doctors and nurses who reported that oxygen-induced lung injury was a major concern, from $59 \%$ to $64 \%$. The survey had 180 respondents, with the largest group being nurses (79.4\%), followed by intensive care specialists (10.6\%). Practices were assessed using review of 101, 964 ABG results and showed a change in practice following the introduction of a conservative oxygen management guideline, resulting in lower $\mathrm{PaO}_{2}$, fewer hyperoxia episodes and a decrease in $\mathrm{F}_{1} \mathrm{O}_{2}$ levels used. A second study used a self-reported, scenario-based survey of attitudes and practices to oxygen management (Grim, 2020). Responses were received from 1361 ICU doctors and nurses from 28 ICUs in the Netherlands. Overall, clinicians reported preference for lower ranges of both arterial oxygen saturations (80-85\%) and arterial oxygen levels $(45 \mathrm{mmHg})$. Nurses were reportedly significantly less tolerant of the lower $\mathrm{SaO}_{2}$ and $\mathrm{PaO}_{2}$ ranges than doctors.

Recently, an international, multicentre study of 2888 ICU patients compared a low oxygen target $\left(\mathrm{PaO}_{2} 60 \mathrm{mmHg}\right)$ with a high oxygen target $\left(\mathrm{PaO}_{2}\right.$ of $\left.90 \mathrm{mmHg}\right)$ (Schjørring et al., 2021). The researchers found no statistically significant difference in mortality at 90 days, supporting 
the current stance of equipoise about conservative oxygen therapy. The optimal oxygen regimen to give mechanically ventilated ICU patients remains uncertain with treatment effect estimates from prior RCTs encompassing the possibility of a clinically important increase or decrease in mortality with the use of conservative oxygen therapy.

The Mega Randomised Registry Trial Comparing Conservative vs. Liberal OXygenation Targets (Mega-ROX), a global study of 40,000 ICU patients with mortality as the primary outcome, is currently underway. Further information about Mega-ROX is available on the Australian and New Zealand Clinical Trials Registry (reference ACTRN12620000391976). 


\section{CHAPTER 3 KNOWLEDGE TRANSLATION}

This chapter begins with a definition and background information about knowledge translation, followed by the history, including in New Zealand (NZ). Following this is a detailed explanation of dissemination, and finally knowledge translation in ICU.

Knowledge translation research involves the investigation of strategies that contribute to research findings being used in clinical practice. The commonest definition for knowledge translation, and the one recently adopted in the New Zealand Health Research Strategy (Ministry of Business, Innovation and Employment \& Ministry of Health [MBIE \& MOH], 2017) is:

a dynamic and iterative process that includes synthesis, dissemination, exchange and ethically sound application of knowledge to improve the health of people, provide more effective health services and products and strengthen the health care system. (p. 40, MBIE \& MIE, 2017, from [Canadian Institutes of Health Research, 2020])

There are multiple terms for knowledge translation used throughout the literature. These terms include: implementation science, translating research into practice study (TRIPS), knowledge utilisation and knowledge transfer (Graham et al., 2006). Along with different terms, there are two types of knowledge translation: biomedical research into clinical science and knowledge, and translating new research evidence into clinical practice (Grimshaw et al., 2012; Rabin et al., 2008; Woolf, 2008). This thesis is about translating evidence into clinical practice, and all references to knowledge translation throughout the thesis are about this.

Failure to translate research findings into practice and policy was identified as a weakness in 2017 in the NZ health system (MBIE \& MOH, 2017). This combined ministry document, which outlined the vision, plan and framework for improving the health and well-being of New Zealanders, identified knowledge translation of research findings into policy and practices as a key objective, and highlighted the need for increased dissemination of research findings. Recognition of the importance of knowledge translation is also acknowledged by NZ's principal health research funding agency which requires investigators applying for grants to outline their proposed dissemination and knowledge transfer strategy, including how it will be done, the target audience and by whom (Health Research Council of New Zealand [HRC], 
n.d.). In 2020, research impact accounts for $22.5 \%$ of scoring for HRC project grant applications (HRC, 2020).

Internationally, recognition of a widespread failure to use research findings and current best practice guidelines in the clinical setting has persisted for at least two decades (Hunt et al., 2012; McGlynn et al., 2003; Seddon et al., 2001). A 2003 study in the United States of America (USA) showed that patients received the recommended healthcare for their health condition an average of $54.9 \%$ of the time, although this rate varied considerably between clinical areas and conditions (McGlynn et al., 2003). An Australian study of 1154 patients in two states found that recommendations were followed an average of $57 \%$ of the time, also depending on the clinical area (Hunt et al., 2012). As part of a larger systematic review (90 articles), predominantly from the United Kingdom (UK), four NZ research publications from 1995 to 1996 were included in a study about quality of health care, and whether it was evidencebased (Seddon et al., 2001). While acknowledging it was difficult to draw conclusions about care in NZ due to the small number of publications, overall quality of care was not only found to not reach acceptable standards, but also to have been ignored as an issue in the healthcare system.

There are varying findings regarding the delay between research being conducted and the subsequent adoption into clinical practice. This delay in using evidence in practice is commonly reported as 17 years (Morris et al., 2011); however a more recent study reported a lag time of three years in the oncology setting (Khera et al., 2018). The time to adopt findings in the ICU environment is unknown; however one study showed almost immediate change (Salter et al., 2018), and given the rapid change of technology and the critical health status of patients in ICU, understanding factors that drive knowledge uptake in this environment is important.

\subsection{History}

Knowledge translation as a discipline grew out of the realisation that clinical practice in healthcare was often delivered without an evidence base. Much of the literature in the late 1980s and 1990s focussed on identifying best evidence, synthesising the evidence and creating guidelines, with the expectation that these activities would influence clinical practice (Grimshaw et al., 1995; Sackett, 1989). Sackett published work in 1989, which ranked evidence into five levels, with randomised controlled trials ranked highest. Also in 1989, the 
term 'systematic review' was first used by Archie Cochrane (Chalmers et al., 2002). The term describes the methodology of synthesising, comparing and combining research results on a topic; partially addressing the problem of doctors lacking time to synthesise all relevant research information on a topic, or determining which evidence to use when results were conflicting. The Cochrane Collaboration was established in 1993, as a repository for systematic reviews and, further increasing access to current, synthesised evidence (Cochrane, 2016). Despite these developments, there was recognition throughout the 1990s that evidence alone was insufficient to change clinical practice. In a review of guideline adoption, Cabana et al. (1999) identified the main reasons doctors did not use clinical guidelines were lack of awareness, lack of familiarity, and lack of agreement from colleagues. The researchers also identified that the sequence for using evidence was firstly an awareness of the knowledge, leading to an attitude change and finally to a behaviour change. Two years later Grol (2001) identified that more research was needed about factors that would increase guideline use, as use involved more than simply providing evidence to clinicians.

The early focus regarding evidence-based healthcare in New Zealand was about quality improvement and standardisation of care through guideline use. The NZ Guidelines Group, an independent organisation established in 1999, encouraged use of evidence in clinical practice and provided evidence-based guidance and implementation approaches (Ministry of Health, n.d.). Around this time the Ministry of Health (2002) published a document Toward Clinical Excellence. This document outlined the levels of evidence described by Sackett (1989), and provided guidance on peer review, clinical audit, using clinical pathways and guidelines. As part of the audit process, a section was included about using a dissemination and implementation strategy, the latter highlighting that the strategy needed to be multifactorial, and health professionals needed to receive information in a way they understood. The Ministry explicitly stated that only distributing printed material was an ineffective strategy. More recently, the NZ Health Research Strategy (MBIE \& MOH, 2017), discussed above, named knowledge translation as one of four strategic priorities for NZ to adopt to address this failure.

\subsection{Knowledge translation frameworks}

Alongside the recognition that synthesis of evidence was insufficient, theoretical frameworks to assist in translating knowledge translation began to emerge (Eccles et al., 2005; Estabrooks et al., 2006; Kitson et al., 1998). Knowledge translation frameworks are considered essential 
for the process of translating knowledge and for researching knowledge translation. One of the first theories was Diffusion of Innovation in 1962 in the rural sector, and focussed on understanding the rate of change and characteristics of the people new information was diffused to (Rogers, 1995). Many frameworks have since been developed and some are discussed in Chapter 4; however, the framework chosen for this study was Promoting Action on Research Implementation in Health Services (PARIHS) (Kitson et al., 1998). The key PARIHS framework elements of Evidence, Context and Facilitation resonated with the research questions and provided a framework for developing the methods.

\subsection{Dissemination of evidence}

Dissemination of new evidence is a key part of knowledge translation, and an essential requirement for grant funding. Early guidance on knowledge translation identified the following considerations when disseminating evidence to decision makers: what to disseminate, how, to whom, by whom and review of effectiveness (Lavis et al., 2003). However, even when research findings are disseminated, there can still be a failure to translate knowledge into clinical practice. Identifying barriers and potential enablers to knowledge translation is part of knowledge translation and are woven throughout this section. Barriers can be structural, organisational, financial, regulators, professional, individual clinician and patient level (Grimshaw et al., 2012). Identification and understanding barriers of knowledge use, as described above, enables strategies to be developed to manage those barriers.

The strength of evidence and believability are important for knowledge to be used in clinical care. Throughout literature, attributes of evidence are highlighted as a key factor in what information should be used by the end user (Grimshaw et al., 2012; Grol \& Grimshaw, 2003; Sackett, 1989). Grimshaw et al. (2012) argued that the evidence from a single study was insufficient to change practice. However, funding organisations require dissemination of all research conducted, even a single study (HRC, n.d.), and it is plausible that the level of evidence from a single study could be significant enough to recommend a practice change. Each trial adds to the overall evidence, and in some cases reverses previous knowledge, necessitating de-adoption of practices on the basis of new evidence, making effective dissemination even more important (Herrera-Perez et al., 2019; Prasad et al., 2013; Sauro et al., 2019). 
Ensuring that provision of research findings is targeted to the people who need them is key to effective knowledge translation. Specifically, in the clinical context, ensuring that research findings get to the staff caring for patients, and the information is delivered in a way they understand. Potential barriers to knowledge translation specific to staff have been identified as lack of awareness of research, insufficient time or ability to read and synthesise research, deciding which evidence to use, prevailing opinion, key person disagreeing with the evidence, desire to maintain the status quo and disagreement between healthcare professionals (Cabana et al., 1999; Grimshaw et al., 1995, 2012; Grol \& Grimshaw, 2003). While much of this literature focuses on doctors, similar issues were seen in nursing (MacGuire, 1990; Squires et al., 2011b). Early nursing commentary reported that the problem was a lack of awareness of research, but also the overwhelming amount of research which nurses found difficult to synthesise (MacGuire, 1990). Seeking to understand the influences of research use by nurses, a systematic review of 22 articles was conducted in 2003 (Estabrooks et al.). The researchers found the only significant indicator of nurses' use of evidence in practice was the individual nurse's beliefs and attitudes to research. An update of this review in 2011 found that in addition to the earlier identified attitudes to research, having a graduate degree in nursing, working in a specialty area, job satisfaction and attendance at conferences or inservice education increased research use in clinical practice (Squires et al., 2011a). Similarly, in an Australian mixed methods study concerning the use of evidence-based practice of 50 allied health professionals and 10 managers, the barriers identified included lack of time, reading research not seen as part of clinical role, and difficulty searching and understanding literature (Harding et al., 2014). Furthermore, in a recent study of 259 physicians, nurses and pharmacists from nine Canadian ICUs, the commonest barrier to adoption of a pharmacological venous thromboembolism prophylaxis was insufficient knowledge or understanding of the evidence (Sauro et al., 2019). In this same article, the commonest barrier to the de-adoption of using albumin for fluid resuscitation was having a clinical leader who had a strong preference.

Other studies have identified that one of the issues for nurses not using new evidence may be their learning style. Specifically, Thompson et al. (2001) used a qualitative approach to assess the preferred mode of finding out new knowledge of 122 nurses in three large hospitals in the UK. The researchers reported that nurses preferred to learn information from people, rather than from reading text-based or electronic sources of publications. This finding was 
later confirmed in an Australian qualitative study set in a single Level III ICU (Marshall et al., 2011) and by a review of the literature from 1998 to 2014, reporting that nurses preferred interactive and human sources of information for learning (Ebenezer, 2015). Conversely, a single centre qualitative study set in a Norway ICU, identified that multiple information sources were used by nurses to inform clinical practice, including research findings, theoretical knowledge and ward culture, in addition to experiential knowledge and learning from nursing and medical colleagues (Bringsvor et al., 2014).

Knowledge translation literature consistently reports that new evidence should be disseminated, and that it requires a multi-faceted and context-specific approach. The End of Grant Knowledge Translation Plan Worksheet in the Canadian Institutes of Health Research (2012) Guide to Knowledge Translation lists multiple ways to communicate study results with stakeholders. These include by passive diffusion such as conference presentations, publications and podcasts, and actively by dissemination including education, media, targeted social media campaign, brief plain language summaries along with consideration of barriers and facilitators. In a scoping review about disseminating systematic review findings, Tricco et al. (2006) also recommended researchers write a single page of the important study findings and implications, tailored to the audience.

Enablers (called facilators in some publications) are strategies identified that may enhance the translation of knowledge. These include education, having a key influential person (knowledge broker or champion), more time and more resources, and use of systematic reviews and meta-analyses of research to overcome the individual having to synthesise research findings (Grol \& Grimshaw, 2003; Kitson et al., 1998). The most frequent enabler identified by Sauro et al. (2019) was on-site education. Recently a study set in a single Australian ICU (Grant \& Booth, 2009) identified contextual factors which were positively related to research use, as informal interactions with other staff, positive culture, resources and time to learn.

\subsection{Knowledge translation in ICU}

Knowledge translation research in ICU has steadily increased over the past 20 years and three significant studies have been published since this doctoral study began (Hammond et al., 2017; Salter et al., 2018; Sauro et al., 2019). ICU knowledge translation research falls into two broad categories. Firstly, assessing change in practice over time and secondly, identification 
of barriers and enablers in ICU. Examples of these in the ICU context are discussed in the next section.

\subsubsection{ICU changes in practice over time}

The change in practice following a significant ICU trial about glycaemic control was assessed in ANZ ICUs using a retrospective analysis (Kaukonen et al., 2013). In the study, the researchers used the ANZ adult patient database to review glycaemic control in 49 ANZ ICUs before and after the publication of a significant RCT about intensive insulin therapy (Kaukonen et al., 2013). The RCT was the Normoglycemia in Intensive Care Evaluation Survival Using Glucose Algorithm Regulation (NICE-SUGAR) trial that found that tight glycaemic control increased mortality 90 days after randomisation (The NICE-SUGAR Investigators, 2009); further investigation later attributed this to increased incidents of hypoglycaemia (The NICESUGAR Investigators, 2012). NICE-SUGAR, which was conducted to test the results of an earlier Belgian study which found lower mortality with tight glycaemic control in a trial set predominantly in surgical ICUs (Van den Berghe et al., 2006), reversed the evidence about tight glycaemic control in ICU. Kaukonen et al. (2013) compared the mean glucose reading from the first 24 hours of ICU stay over time from two years before the NICE-SUGAR publication with two years after, using segmented regression analysis of an interrupted time series. There was no difference in practice following the NICE-SUGAR publication and the researchers concluded this was because most ANZ ICUs had not adopted the intensive insulin therapy advocated in the years following the initial Van den Berghe et al. trial. Conversely, a similar knowledge translation study set in the USA showed that after publication of the initial intensive insulin therapy trial (Van den Berghe et al., 2006), tight glycaemic control was steadily adopted over time, however de-adoption was not seen following the publication of the NICE-SUGAR trial (Niven et al., 2015), suggesting that knowledge translation may be region or context-specific.

A fairly recent observational study compared the choice of intravenous fluids for resuscitation in intensive care patients between 2007 and 2014 (Hammond et al., 2017). An earlier knowledge translation study had shown that the predominant intravenous fluid of choice for resuscitation was colloid with significantly more resuscitation episodes than crystalloid fluids (Finfer et al., 2010). When this international study was repeated in 2014, it showed that the use of crystalloids had increased and colloids decreased, however geographical location was a significant predictor of choice. In the years between the two studies, evidence showed 
more adverse outcomes from the colloid under investigation (hydroxy ethyl starch), than normal saline (Myburgh et al., 2012). This change in practice is important; however, it could not be attributed to any specific publication. The researchers went further and conducted a study of 'drivers of choice' for fluid selection, in which using randomly selected patient scenarios, doctors and nurses were asked how they would assess which fluid to give, from a pre-specified list (Taylor et al., 2017). The doctors ranked safety concerns as their top driver of choice followed by patient type (including severity of illness) and fluid type. Nurses selected the same drivers, but ranked patient type highest, followed by safety and fluid type.

The final example of practice changing over time in ICU, is a retrospective cohort study evaluating temperature management practices in patients admitted to ICU following an outof-hospital cardiac arrest (Salter et al., 2018). These researchers specifically sought to evaluate whether practice had changed following the publication of a trial about targeted temperature management (TTM) following cardiac arrest (Nielsen et al., 2013). The TTM trial showed that that there was no mortality difference between cooling this group of patients to $36^{\circ} \mathrm{C}$ compared to the previous recommendation of $33^{\circ} \mathrm{C}$. Salter et al. used data from the ANZ ICU adult patient database to evaluate the change in the lowest temperature in the first 24 hours of ICU stay, over time, using segmented regression comparing before the TTM publication with after the publication. The researchers found that lowest temperature in patients admitted to ANZ ICUs following cardiac arrest increased significantly after publication of the TTM trial (i.e. patients were cooled less than before). This finding suggested practice had changed in ANZ ICUs, in response to the TTM trial results publication.

\subsubsection{Barriers and enablers in ICU}

In a 2013 literature review, Sinuff et al. identified issues which may enable the uptake of knowledge in ICU. The review of 119, predominantly single-centre, observational studies, from 1986 to 2010 found that the factors that positively affected knowledge uptake included protocols, guidelines and bundles (of care) and staff education. The addition of reminders, audits and feedback to staff also assisted. More recently, a scoping review of implementation science in adult critical care, reviewed 82 articles between 1999 and 2019 (McNett et al., 2020). The commonest implementation strategy was educational meetings, followed by audit and feedback, then to a lesser degree, development of tools, presence of a local opinion leader and development of effective teaching materials. The review did not evaluate the effectiveness of the strategies. 
An examination of the barriers to using a therapy (selective digestive decontamination) was conducted in Australia, Canada, NZ and UK ICUs (Cuthbertson et al., 2013). At the time of the study, selective digestive decontamination had moderately convincing evidence in reducing hospital acquired infections, including meta-analyses of 28 RCTs, yet had not been adopted in ICUs internationally. The researchers undertook a multiple method study to identify the beliefs, views and perceived barriers about selective digestive decontamination, based on the opinions of four key stakeholder group; ICU physicians, pharmacists, clinical leads, and clinical microbiologists/ infectious disease physicians. Primarily, the stakeholders were not convinced about the evidence despite the meta-analyses, and had concerns about potential antibiotic resistance. The researchers concluded that factors other than scientific evidence influence the uptake of evidence. As previously discussed, barriers and facilitators were also examined in a recent Canadian ICU study (Sauro et al., 2019). This latter study used a multiple method approach to examine the barriers for adopting pharmaceutical prophylaxis for venous thromboembolism and de-adoption of albumin for resuscitation, and found the most common barriers were insufficient knowledge, ICU culture and lack of clinical guidelines and clinical leaders with a strong preference. 


\section{CHAPTER 4 LITERATURE REVIEW}

In this chapter, the literature explicitly relevant to the research questions is explored. Specifically, whether the attitudes and practices of health practitioners at institutions that participated in a research trial were more likely to use the results of that trial, than institutions that did not participate in that research. The chapter commences with the search strategy and appraisal approach. Secondly, an overview and critique of the 14 relevant studies (history, setting, population, intervention, methodology) is presented. The third section presents the six themes that emerged from the review findings. Finally, the gaps in literature are identified as well as the methodological factors important to consider in this current research.

Two previously published literature reviews on similar topics were examined and found to be out of scope for the current topic (Boaz et al., 2015; Clarke \& Loudon, 2011). Specifically, they aimed to evaluate whether engagement in research correlated in better patient outcomes or healthcare performance. However, in the absence of relevant literature through exhaustive searching, these two systematic reviews were invaluable starting points for finding literature. Clarke and Loudon (2011) noted the heterogeneity of the 15 studies they reviewed, and concluded that there was no evidence that patients in trial sites had better outcomes than patients in sites that did not participate in specific trials. Of relevance, they also determined that there was no difference in the use of research findings in the trial-sites compared to nontrial sites. Secondly, Boaz et al. (2015) identified 33 papers to answer the question 'does research engagement (by clinicians and organisations) improve health care performance?'. This paper was outside the scope of the current research because it did not specifically report on a study designed to test whether participating in a specific trial impacts the use of findings from that trial. These researchers gave a guarded conclusion that there was some evidence indicating engagement in research improved healthcare performance. They identified areas for future research such as understanding at a more granular level what whether organisational determinants increase healthcare performance and participation in research.

\subsection{Search strategy}

A systematic review was initially proposed using the synonyms for knowledge translation and other terms relevant to the project. When this search was undertaken it became apparent that the nomenclature for this research topic was problematic. Assistance was sought from 
two different librarians at Victoria University of Wellington, over the duration of the thesis, who were also unable to find an easy way to conduct a literature review systematically.

The decision to adopt an integrative approach was made when the paucity and disparity of literature was found. An integrative review is a review that is representative of and important to a topic (Florczak, 2013; Souza et al., 2010; Torraco, 2005). This type of review is best suited to either an emerging topic or when there is a need for a new perspective on a mature topic with disparate literature. The literature can be broad-ranging and use both experimental and non-experimental sources and include manual searches in relevant journals (Souza et al., 2010; Torraco, 2005). Integrative reviews sometimes include theoretical literature (Florczak, 2005); however, a decision was made not to do this in the present research.

A search was conducted in four databases - PubMed, CINAHL Complete, Cochrane Central Register and EMBASE - using different combinations of the terms listed in Table 1, for the period 1950 to current, and limited to English language. Each line of search terms listed in Table 1 was initially completed separately, then the searches were combined with the Boolean AND. Articles were assessed for relevance using the inclusion and exclusion criteria. Searches were conducted at the beginning of the research (February 2017), throughout the early study period (2018 and 2019) and at the final write up (March 2021), with alerts throughout the duration of the research period. Manual searches of key articles and review of journals such as Implementation Science were also undertaken, and these methods were the main source of the reviewed literature. Articles for inclusion in the review had to meet the pre-set inclusion criteria.

Table 1 Search terms for literature search

\begin{tabular}{|c|}
\hline Terms \\
\hline $\begin{array}{l}\text { Knowledge translation OR evidence-base* medicine OR evidence-base* practice OR } \\
\text { knowledge exchange OR research translation OR research utilization OR knowledge } \\
\text { transfer OR 'TRIPS' OR translat* research into practice OR implement* OR 'EBM' }\end{array}$ \\
\hline Institution OR hospital OR site OR organisation \\
\hline Participation OR involvement OR engagement \\
\hline Research OR clinical trial OR RCT OR trial OR 'randomised controlled trial' \\
\hline Impact OR change OR improve OR effect OR affect OR influence OR benefit \\
\hline Attitudes OR practice OR belief OR behaviour \\
\hline $\begin{array}{l}\text { Doctor OR nurse OR clinician OR health professional OR health practitioner OR } \\
\text { health personnel OR staff }\end{array}$ \\
\hline
\end{tabular}

*This is a 'wildcard' search term, used to search for multiple endings to the word being searched Abbreviations: EBM: Evidence-based medicine; TRIPS: Translating Research Into Practice 


\subsubsection{Inclusion and exclusion criteria}

Studies were included in the review if they evaluated the knowledge translation of a specific research trial, in which sites, institutions or regions had participated. All relevant articles were included, regardless of the quality of the study $(n=13)$. Additionally, one study was included because it specifically addressed the level of engagement in research, and therefore was considered important to the research question about practitioner attitudes, despite not testing whether participating in research translated into a resultant increase in knowledge use (Tranmer et al., 2002).

Three areas of literature were excluded. Firstly, a large number of articles about knowledge translation that assessed the impact, knowledge translation or currency of practice of existing evidence or guidelines were excluded. While articles such as these provided important background information, they were not included in this review unless they assessed the effect of site or health practitioner participation in an RCT on subsequent knowledge translation. Included in this category were significant studies that reviewed practice over time in ICU; but did not compare sites that participated with those that did not (Kaukonen et al., 2013; Salter et al., 2018). Secondly, literature that focused on the benefits to patients when they participate in research (Janni et al., 2006; Krzyzanowska et al., 2011; Webster et al., 2017) was excluded as the current research concerned health practitioner change. Finally, studies about the relationship between the level of institutional research activity and patient-centred outcomes such as overall mortality have been excluded if the study did not address whether the participation linked to a specific trial (Bennett et al., 2012; Ozdemir et al., 2015).

Few articles were identified via the searches with the majority identified through manual review of reference lists and citations. Two studies were published after the initial search (Khera et al., 2018; Sweeting et al., 2020), one of which (Khera et al., 2018) used a similar multiple method design to that chosen for this PhD study.

A systematic approach to article appraisal was adopted. Details, findings and a critique of each article was undertaken and summarised in table format (Table 2). Critique was undertaken using the Critical Appraisal Skills Programme (CASP) tools (Oxford Centre for Triple Value Healthcare, n.d.). The specific headings in the summary table were chosen to align with to the PICO framework (Polit \& Beck, 2017); specifically design; informants, 
exposure, comparator, and outcomes measures. In addition, the RCT which the knowledge translation study was conducted about and results, along with the knowledge translation study methods, outcome measures and overall findings were included. Findings from the table were then analysed to determine points of convergence and difference in the design, and the agreement or disagreement with each other, and whether studies reinforced or contradicted each other. Findings from this analysis were then synthesised to present a picture about what is currently known about knowledge translation and health practitioner engagement in clinical trials research, and the strength or quality of this knowledge.

\subsection{Overview of literature}

The first research identified, which assessed whether institutional trial participation translated into a clinical practice change, was more than 40 years ago (Adler, 1978). Studies have been published sporadically since this time and have predominantly been about interventions that can easily be studied. Included in this category are multiple studies about whether doctors' participation in a drug trial changed their prescribing that drug, by reviewing prescription records (Corrigan \& Glass, 2005; Ketley \& Woods, 1993; Majumdar et al., 2002; Shah et al., 2010). A few studies of this nature emerged in the late 1990s and early 2000s, possibly related to the increasing evidence about drugs to successfully manage cardiac disease. Because of the paucity of literature, much of what is critiqued in this review is more than 10 years old, and was included in the two previous literature reviews (Boaz et al., 2015; Clarke \& Loudon, 2011). The overview presented next covers research methods, topics, settings, sample/ informants, exposure/comparator and outcome measures.

\subsubsection{Settings}

The 14 studies were conducted in five countries: United States of America $(n=6)$, United Kingdom $(n=4)$, Denmark $(n=2)$, Canada $(n=1)$, and Netherlands $(n=1)$. The studies were conducted in various clinical settings including cardiology $(n=6)$, primary care $(n=3)$, gastroenterology $(n=1)$, obstetrics and gynaecology $(n=1)$, oncology $(n=1)$, mixed medical and surgical unit $(n=1)$ and surgical $(n=1)$.

\subsubsection{Research methods and topics}

As befits the topic of knowledge translation, all studies included in this review were observational (Table 2). The methods that were used in the 14 articles were retrospective review of registry or other database $(n=7)$, survey only $(n=5)$, and survey and observational 
study $(n=2)$. With respect to retrospective database review studies, six of these evaluated prescribing practices over time by reviewing existing drug prescription databases, with a predominance of studies conducted in cardiology patients.

Self-reported surveys used either existing or created survey tools. Four researchers created bespoke survey tools to answer their research questions (Das et al., 2008; Khera et al., 2018; Litjens et al., 2013; Sweeting et al., 2020), while Tranmer et al. (2002) used a research utilisation questionnaire (RUQ) which asked respondents to rate their use of research in the clinical setting. Three of the bespoke surveys focussed on respondents' knowledge of trial results, and self-reported impact of the results on clinical practice (Khera et al., 2018; Litjens et al., 2013; Sweeting et al., 2020). A combination of closed questions and Likert scale responses were included, with some questions leading to open-ended responses (Khera et al., 2018; Sweeting et al., 2020). In addition, views about being involved in the original research were included (Sweeting et al., 2020) and respondents' opinions regarding strategies to increase knowledge translation (Khera et al., 2018). Litjens et al. (2013) included a single line summary of the results for the nine RCTs they were investigating. Das et al.'s survey focused on respondents' practices with regard to the research topic, although the RCT results were unknown at the time of the survey. Two studies used more than one method to assess knowledge translation; in one study a physician survey and registry review (Khera et al., 2018), and in the other an observational study and patient satisfaction survey (Meineche-Schmidt et al., 2006).

\subsubsection{Sample/ Informants}

Doctors were the professional group studied most in the literature reviewed (Table 2). Many of the studies related to medical interventions such as the prescription of drugs or procedures which at the time of the study could only be conducted by doctors. Using registry data, some studies focussed on the practices of the overall institution (Andersen et al., 2006; Ketley \& Woods, 1993; Majumdar et al., 2002; Shah et al., 2010), while others were conducted about the individual practitioner (Corrigan \& Glass, 2005; Kizer et al., 1999). Midwives and nurses were included in a study about multiple RCTs set in obstetrics and gynaecology clinics, however they only represented a small proportion of the respondents $(23 \%$ and $2 \%$, respectively) (Litjens et al., 2013). The small number of midwives and nurses may, in part, be because this study relied on gynaecologists sending the survey to two residents and/ or nurses if they could not be readily identified by the researchers. One study included 21 
physiotherapists (Sweeting et al., 2020), and in the only study that focussed solely on nurses, the researchers created a scenario-based study to assess nurse engagement in research at six medical surgical units, designing a study about sleep (Tranmer et al., 2002). Six studies assessed effect of participation at a site, hospital or district level (Adler, 1978; Andersen et al., 2006; Ketley \& Woods, 1993; Majumdar et al., 2002; Omoigui et al., 1998; Shah et al., 2010), six at an individual practitioner level (Corrigan \& Glass, 2005; Das et al., 2008; Kizer et al., 1999; Meineche-Schmidt et al., 2006; Sweeting et al., 2020; Tranmer et al., 2002), and two at both individual and site level (Khera et al., 2018; Litjens et al., 2013).

\subsubsection{Exposure and comparator}

The exposure for most $(n=9)$ studies was participation in a clinical trial and the comparator, where presented, was non-participation (Table 2). One study compared before a trial commenced as comparator and after participation in a trial as exposure (Das et al., 2008), and one a combination of before, after, participation and non-participation (Majumdar et al., 2002). There were two exceptions where studies did not investigate a randomised controlled trial. Tranmer et al. (2002) randomised six medical-surgical units into three levels of engagement in the design of a study about sleep which had not been conducted, and the purpose was to assess engagement in research. In addition, Sweeting et al. (2020) measured knowledge translation following participation in an observational study, and along with one other study did not have a comparator (Ketley \& Woods, 1993). 
Table 2 Summary of reviewed studies (date order)

\begin{tabular}{|c|c|c|c|c|c|}
\hline $\begin{array}{l}\text { Author, Year } \\
\text { Setting, Country, } \\
\text { Informants }\end{array}$ & $\begin{array}{l}\text { Research trial or } \\
\text { study }\end{array}$ & Trial or study result & Exposure/ Comparator & $\begin{array}{c}\text { Method/s } \\
\text { Outcome measure/s }\end{array}$ & $\begin{array}{l}\text { Knowledge translation } \\
\text { study results (summary) }\end{array}$ \\
\hline $\begin{array}{l}\text { Adler (1978) } \\
\text { Surgical } \\
\text { UK } \\
\text { Informants } \\
\text { Surgeons* }\end{array}$ & $\begin{array}{l}\text { Clinical trial: } \\
\text { Length of time } \\
\text { from discharge for } \\
\text { varicose vein repair } \\
\text { and inguinal hernia } \\
\text { repair (single site) } \\
\text { (Adler et al., 1978) }\end{array}$ & $\begin{array}{l}\text { Early discharge (48 } \\
\text { hours) non-inferior } \\
\text { to usual care (6-7 } \\
\text { days) } \\
=>\text { recommended }\end{array}$ & $\begin{array}{l}\text { Exposure } \\
\text { District where study was } \\
\text { conducted } \\
\mathrm{N}=1 \text { site } \\
\text { Comparator Adjacent (non- } \\
\text { study) districts } \mathrm{N}=2 \text { sites }\end{array}$ & $\begin{array}{l}\text { Method } \\
\text { Record review of discharge } \\
\text { Outcome } \\
\text { Change in length of stay over } \\
\text { time (before and after) } \\
\text { amongst sites and between } \\
\text { districts }\end{array}$ & Exposure $>$ Comparator \\
\hline $\begin{array}{l}\text { Ketley \& Woods } \\
\text { (1993) } \\
\text { Cardiology } \\
\text { USA } \\
\text { Informants } \\
\text { Physicians }\end{array}$ & $\begin{array}{l}\text { Four RCTs about } \\
\text { thrombolytic drugs } \\
\text { in patients with } \\
\text { acute myocardial } \\
\text { infarction (1989- } \\
\text { 1991) } \\
\text { (AIMS Trial Study } \\
\text { Group, 1988; GISSI, } \\
\text { 1987; ISIS-2 } \\
\text { Collaborative } \\
\text { Group, 1988; } \\
\text { Wilcox et al., 1988) }\end{array}$ & $\begin{array}{l}\text { Increasing evidence } \\
\text { over time, indicating } \\
\text { positive results for } \\
\text { thrombolytic agents } \\
\text { following myocardial } \\
\text { infarction } \\
=>\text { recommended }\end{array}$ & $\begin{array}{l}\text { Exposure } \\
\text { After publication } \\
\text { (Districts which participated } \\
\text { in trials) } \\
\mathrm{N}=12 \text { districts } \\
\text { Comparator } \\
\mathrm{n} / \mathrm{a}\end{array}$ & $\begin{array}{l}\text { Method } \\
\text { Registry review of } \\
\text { prescribing practices } \\
\text { Outcome } \\
\text { Association between } \\
\text { hospital trial participation } \\
\text { and use of thrombolytics }\end{array}$ & Exposure $>$ Comparator \\
\hline
\end{tabular}




\begin{tabular}{|c|c|c|c|c|c|}
\hline $\begin{array}{l}\text { Author, Year } \\
\text { Setting, Country, } \\
\text { Informants }\end{array}$ & $\begin{array}{l}\text { Research trial or } \\
\text { study }\end{array}$ & Trial or study result & Exposure/ Comparator & $\begin{array}{c}\text { Method/s } \\
\text { Outcome measure/s }\end{array}$ & $\begin{array}{l}\text { Knowledge translation } \\
\text { study results (summary) }\end{array}$ \\
\hline $\begin{array}{l}\text { Omoigui et al. } \\
\text { (1998) } \\
\text { Cardiac } \\
\text { intervention } \\
\text { USA } \\
\\
\text { Informants } \\
\text { Cardiac } \\
\text { interventionalists* }\end{array}$ & $\begin{array}{l}\text { RCT: } \\
\text { Coronary } \\
\text { Angioplasty versus } \\
\text { excision } \\
\text { atherectomy trial } \\
\text { (CAVEAT-I) } \\
\text { (Topol et al., 1993) }\end{array}$ & $\begin{array}{l}\text { Use of directional } \\
\text { coronary } \\
\text { atherectomy (DCA) } \\
\text { showed more } \\
\text { adverse events than } \\
\text { balloon } \\
\text { => not } \\
\text { recommended }\end{array}$ & $\begin{array}{l}\text { Pre-trial: } \\
\text { Site-level participation in } \\
\text { CAVEAT-1(1992) } \\
\mathrm{N}=34 \text { sites } \\
\mathrm{n}=643 \text { patients } \\
\text { Post-trial: Site-level } \\
\text { participation in CAVEAT-1 } \\
(1994) \\
\mathrm{N}=33 \text { sites } \\
\mathrm{N}=647 \text { patients } \\
\text { Control: } \\
\mathrm{N}=24 \text { Non-CAVEAT-1 sites } \\
\mathrm{N}=393 \text { patients }\end{array}$ & $\begin{array}{l}\text { Method } \\
\text { Survey to site coordinators } \\
\text { about patient medical } \\
\text { history and coronary } \\
\text { procedures } \\
\text { Outcome } \\
\text { Reported use of trial device } \\
\text { (DCA), balloon or other for } \\
\text { coronary angioplasty }\end{array}$ & Exposure $>$ Comparator \\
\hline $\begin{array}{l}\text { Kizer et al. } \\
\text { (1999) } \\
\text { Cardiology } \\
\text { USA } \\
\text { Informants } \\
\text { Physicians } \\
\\
\text { Kizer et al. } \\
\text { continued }\end{array}$ & $\begin{array}{l}\text { RCTs: } \\
\text { MILIS, TIMI-1, TIMI- } \\
\text { 2, TIMI-4, TIMI-5, } \\
\text { TIMI-6, TIMI-9B } \\
\text { (Antman, 1996; } \\
\text { Cannon, McCabe, } \\
\text { Diver, et al., 1994; } \\
\text { Cannon, McCabe, } \\
\text { Henry, et al., 1994; } \\
\text { Chesebro et al., } \\
\text { 1987; Lee \& TIMI } 6 \\
\text { Investigators, 1995; } \\
\text { Roberts et al., }\end{array}$ & $\begin{array}{l}\text { Increasing and } \\
\text { changing evidence } \\
\text { over time } \\
\text { Aspirin, beta } \\
\text { blockers, } \\
\text { ACE-inhibitors } \\
\text { =>recommended }\end{array}$ & $\begin{array}{l}\text { Exposure } \\
\text { Physician participation in } \\
\text { RCT as assessed by } \\
\text { prescribing practices at } \\
\text { discharge following } \mathrm{MI} \text { (and } \\
\text { enrolment in an RCT) } \\
\text { Comparator } \\
\text { Routine clinical practice as } \\
\text { assessed by prescribing } \\
\text { practices for Ml at } \\
\text { enrolment of patients in an } \\
\text { RCT }\end{array}$ & $\begin{array}{l}\text { Method } \\
\text { Database (MILIS-TIMI) } \\
\text { review of prescribing } \\
\text { practices } \\
\text { Outcomes } \\
\text { Prescription rates of Aspirin, } \\
\text { beta blockers, ACE-inhibitors } \\
\text { and calcium channel } \\
\text { blockers, as assessed }\end{array}$ & $\begin{array}{l}\text { Aspirin: } \\
\text { Exposure > Comparator } \\
\text { Beta-blockers: } \\
\text { Exposure > Comparator } \\
\text { ACE-inhibitors: } \\
\text { Exposure > Comparator }\end{array}$ \\
\hline
\end{tabular}




\begin{tabular}{|c|c|c|c|c|c|}
\hline $\begin{array}{c}\text { Author, Year } \\
\text { Setting, Country, }\end{array}$ & $\begin{array}{l}\text { Research trial or } \\
\text { study }\end{array}$ & Trial or study result & Exposure/ Comparator & $\begin{array}{c}\text { Method/s } \\
\text { Outcome measure/s }\end{array}$ & $\begin{array}{l}\text { Knowledge translation } \\
\text { study results (summary) }\end{array}$ \\
\hline & $\begin{array}{l}\text { 1984; The TIMI } \\
\text { Study Group, 1989) }\end{array}$ & $\begin{array}{l}\text { Calcium channel } \\
\text { blockers } \\
=>\text { not } \\
\text { recommended }\end{array}$ & & & $\begin{array}{l}\text { Calcium channel blockers: } \\
\text { Exposure = Comparator }\end{array}$ \\
\hline \multirow[t]{2}{*}{$\begin{array}{l}\text { Majumdar, Chang, } \\
\text { \& Armstrong } \\
\text { (2002) } \\
\text { Cardiology } \\
\text { USA } \\
\text { Informants } \\
\text { Physicians }\end{array}$} & \multirow[t]{2}{*}{$\begin{array}{l}\text { RCT: } \\
\text { SAVE trial } \\
\text { (Pfeffer et al., } \\
\text { 1992) }\end{array}$} & \multirow[t]{2}{*}{$\begin{array}{l}\text { ACE-inhibitor } \\
\Rightarrow>\text { recommended }\end{array}$} & $\begin{array}{l}\text { Exposure } 1 \\
\text { Site level participation in } \\
\text { SAVE trial } \\
\mathrm{N}=22 \text { sites } \\
\mathrm{N}=1415 \text { patients } \\
\text { Comparator } 1 \\
\text { Participation non-SAVE trial } \\
\text { sites } \\
\mathrm{N}=637 \text { sites } \\
\mathrm{N}=24,471 \text { patients }\end{array}$ & $\begin{array}{l}\text { Method } \\
\text { Database (GUSTO-1) review } \\
\text { of prescribing practices } \\
\text { Outcome } \\
\text { Prescription of ACE-inhibitor } \\
\text { at hospital discharge }\end{array}$ & $\begin{array}{l}\text { SAVE vs non-SAVE sites: } \\
\text { Exposure = Comparator }\end{array}$ \\
\hline & & & $\begin{array}{l}\text { Exposure } 2 \\
\text { Publication } \\
\text { Pre-SAVE trial } \\
\text { (SAVE trial site and non-trial } \\
\text { sites) } \\
\text { Comparator } \mathbf{2} \\
\text { Publication } \\
\text { Post SAVE trial } \\
\text { (SAVE trial site and non-trial } \\
\text { sites) }\end{array}$ & $\begin{array}{l}\text { Method } \\
\text { Database (GUSTO-1) review } \\
\text { of prescribing practices } \\
\text { Outcome } \\
\text { Prescription of ACE-inhibitor } \\
\text { at hospital discharge }\end{array}$ & $\begin{array}{l}\text { Pre vs post-SAVE trial: } \\
\text { Exposure = Comparator }\end{array}$ \\
\hline
\end{tabular}




\begin{tabular}{|c|c|c|c|c|c|}
\hline $\begin{array}{l}\text { Author, Year } \\
\text { Setting, Country, } \\
\text { Informants }\end{array}$ & $\begin{array}{l}\text { Research trial or } \\
\text { study }\end{array}$ & Trial or study result & Exposure/ Comparator & $\begin{array}{c}\text { Method/s } \\
\text { Outcome measure/s }\end{array}$ & $\begin{array}{l}\text { Knowledge translation } \\
\text { study results (summary) }\end{array}$ \\
\hline $\begin{array}{l}\text { Majumdar } \\
\text { continued }\end{array}$ & $\begin{array}{l}\text { RCT: } \\
\text { MDPIT } \\
\text { (The Multicenter } \\
\text { Diltiazem } \\
\text { Postinfarction Trial } \\
\text { Research Group, } \\
\text { 1988) }\end{array}$ & $\begin{array}{l}\text { Diltiazem } \\
=>\text { not } \\
\text { recommended }\end{array}$ & $\begin{array}{l}\text { Exposure } 3 \\
\text { MDPIT sites } \\
N=9 \text { sites } \\
N=551 \text { patients } \\
\text { Comparator } 3 \\
\text { Non-MDPIT sites } \\
N=650 \text { sites } \\
N=25,335 \text { patients }\end{array}$ & $\begin{array}{l}\text { Method } \\
\text { Database (GUSTO-1) review } \\
\text { of prescribing practices } \\
\text { Outcome } \\
\text { Prescription of a calcium } \\
\text { channel blocker at hospital } \\
\text { discharge }\end{array}$ & $\begin{array}{l}\text { MDPIT vs non-MDPIT sites: } \\
\text { Exposure = Comparator }\end{array}$ \\
\hline $\begin{array}{l}\text { Tranmer et al., } \\
\text { (2002) } \\
\text { Medical surgical } \\
\text { units } \\
\text { Canada } \\
\text { Informants } \\
\text { Nurses }\end{array}$ & $\begin{array}{l}\text { n/a } \\
\text { Descriptive study } \\
\text { about sleep } \\
\text { patterns in medical } \\
\text { surgical patients } \\
\text { (Tranmer et al., } \\
\text { 2003) }\end{array}$ & $\mathrm{n} / \mathrm{a}$ & $\begin{array}{l}\text { Exposure } \\
\text { Low and high level } \\
\text { participation in research } \\
\text { protocol development } \\
\text { Comparator } \\
\text { Usual level participation in } \\
\text { research protocol } \\
\text { development } \\
\text { Before } \\
\text { Control: } n=34 \\
\text { Low: } n=21 \\
\text { High: } n=37 \\
\text { After } \\
\text { Control: } n=24 \\
\text { Low: } n=39 \text { [sic] } \\
\text { High } n=29\end{array}$ & $\begin{array}{l}\text { Method } \\
\text { RUQ survey } \\
\text { Outcome } \\
\text { Effect of direct and indirect } \\
\text { research participation on } \\
\text { staff nurses' attitude } \\
\text { towards research }\end{array}$ & $\begin{array}{l}\text { High exposure > low } \\
\text { exposure or usual } \\
\text { comparator }\end{array}$ \\
\hline
\end{tabular}




\begin{tabular}{|c|c|c|c|c|c|}
\hline $\begin{array}{l}\text { Author, Year } \\
\text { Setting, Country, } \\
\text { Informants }\end{array}$ & $\begin{array}{l}\text { Research trial or } \\
\text { study }\end{array}$ & Trial or study result & Exposure/ Comparator & $\begin{array}{c}\text { Method/s } \\
\text { Outcome measure/s }\end{array}$ & $\begin{array}{l}\text { Knowledge translation } \\
\text { study results (summary) }\end{array}$ \\
\hline $\begin{array}{l}\text { Corrigan and Glass } \\
\text { (2005) } \\
\text { Cardiology } \\
\text { USA } \\
\text { Informants } \\
\text { Physicians }\end{array}$ & $\begin{array}{l}\text { Multiple RCTs (not } \\
\text { stated) }\end{array}$ & $\mathrm{n} / \mathrm{a}$ & $\begin{array}{l}\text { Exposure } \\
\text { Investigator physicians (at } \\
\text { least one } R C T \text { ) } \\
\mathrm{N}=2108 \\
\text { Comparator } \\
\text { Matched non-investigator } \\
\text { physicians } \mathrm{N}=2108\end{array}$ & $\begin{array}{l}\text { Method } \\
\text { Registry review of } \\
\text { prescription practices } \\
\text { Outcome } \\
\text { Prescription of trial drug } 3,6 \\
\text { and } 18 \text { months after launch }\end{array}$ & Exposure $>$ Comparator \\
\hline $\begin{array}{l}\text { Meineche-Schmidt, } \\
\text { Hvenegaard, \& Juhl } \\
\text { (2006) } \\
\text { General Practice } \\
\text { Denmark } \\
\text { Informants } \\
\text { General } \\
\text { practitioners }\end{array}$ & $\begin{array}{l}\text { RCT: } \\
\text { The ONE study - } \\
\text { management of } \\
\text { GERD with } \\
\text { esomeprazole, } \\
\text { (cost of on-demand } \\
\text { treatment vs } \\
\text { courses) } \\
\text { (Meineche-Schmidt } \\
\text { et al., 2004) }\end{array}$ & $\begin{array}{l}\text { On-demand } \\
\text { prescription of } \\
\text { esomeprazole was } \\
\text { cheaper than } \\
\text { treatment courses } \\
=>\text { recommended }\end{array}$ & $\begin{array}{l}\text { Exposure } \\
\text { ONE-GP patients: } \\
\text { ONE-patients } \\
\text { Group A (ONE-patients): } \\
\text { patients participated in ONE } \\
\text { study: } \mathrm{N}=247 \\
\text { Group B ('Other patients'): } \\
\text { Patients who did not } \\
\text { participate in ONE: N=451 } \\
\text { Comparator } \\
\text { Non-ONE-GPs: } \\
\text { Group C: ('Other patients') } \\
\text { N=469 }\end{array}$ & $\begin{array}{l}\text { Methods } \\
\text { Observational study } \\
\text { Patient satisfaction survey } \\
\text { Outcome } \\
\text { Management strategies for } \\
\text { GERD } \\
\text { Patient satisfaction }\end{array}$ & $\begin{array}{l}\text { GPs: } \\
\text { Exposure > Comparator } \\
\text { Patients: } \\
\text { Exposure > Comparator }\end{array}$ \\
\hline
\end{tabular}




\begin{tabular}{|c|c|c|c|c|c|}
\hline $\begin{array}{l}\text { Author, Year } \\
\text { Setting, Country, } \\
\text { Informants }\end{array}$ & $\begin{array}{l}\text { Research trial or } \\
\text { study }\end{array}$ & Trial or study result & Exposure/ Comparator & $\begin{array}{c}\text { Method/s } \\
\text { Outcome measure/s }\end{array}$ & $\begin{array}{l}\text { Knowledge translation } \\
\text { study results (summary) }\end{array}$ \\
\hline $\begin{array}{l}\text { Andersen, } \\
\text { Kragstrup, \& } \\
\text { Søndergaard } \\
\text { (2006) } \\
\text { General Practice } \\
\text { Denmark } \\
\\
\text { Informants } \\
\text { General } \\
\text { Practitioners }\end{array}$ & $\begin{array}{l}\text { RCT: } \\
\text { Symbicort Asthma } \\
\text { Control Plan } \\
\text { (SymbiAC) }\end{array}$ & $\begin{array}{l}\text { Unknown at time of } \\
\text { KT study }\end{array}$ & $\begin{array}{l}\text { Exposure } \\
\text { Site level participation in } \\
\text { SymbiAC } \\
N=10 \text { practices } \\
N=5439 \text { patients } \\
\text { Comparator } \\
\text { Non-participation in } \\
\text { SymbiAC } \\
N=165 \text { practices } \\
N=59,574 \text { patients }\end{array}$ & $\begin{array}{l}\text { Method } \\
\text { Retrospective cohort study } \\
\text { Outcomes } \\
\text { Adherence to guidelines for } \\
\text { asthma patient (measured } \\
\text { by use of inhaled } \\
\text { corticosteroids) } \\
\text { Use of sponsor drugs }\end{array}$ & $\begin{array}{l}\text { Use of RCT sponsored drugs: } \\
\text { Exposure > Comparator } \\
\text { Guideline adherence: } \\
\text { Exposure = Comparator }\end{array}$ \\
\hline $\begin{array}{l}\text { Das et al. } \\
\text { (2008) } \\
\text { Gastroenterology } \\
\text { UK } \\
\text { Informants } \\
\text { Gastroenterologists } \\
\text { Physicians } \\
\text { Surgeons }\end{array}$ & $\begin{array}{l}\text { RCT: } \\
\text { Aspiring } \\
\text { Esomeprazole } \\
\text { Chemoprevention } \\
\text { Trial (ASPECT) } \\
\text { (Jankowski et al., } \\
\text { 2018) }\end{array}$ & $\begin{array}{l}\text { Unknown at time of } \\
\text { KT study }\end{array}$ & $\begin{array}{l}\text { Exposure } \\
\text { After or during site } \\
\text { participation in after } \\
\text { ASPECT } \\
\text { Survey } 2 \text { : } \\
N=57 \text { ASPECT sites } \\
N=50 \text { sites about to start } \\
N=57 \text { respondents } \\
\text { Comparator } \\
\text { Before site participation in } \\
\text { ASPECT } \\
\text { Survey } 1 \text { : } \\
n=288 \text { respondents }\end{array}$ & $\begin{array}{l}\text { Method } \\
\text { Survey about management } \\
\text { of Barrett's oesophagus } \\
\text { Outcomes } \\
\text { Adherence to ACG guidelines } \\
\text { including surveillance and } \\
\text { biopsies }\end{array}$ & Exposure $>$ Comparator \\
\hline
\end{tabular}




\begin{tabular}{|c|c|c|c|c|c|}
\hline $\begin{array}{l}\text { Author, Year } \\
\text { Setting, Country, } \\
\text { Informants }\end{array}$ & $\begin{array}{l}\text { Research trial or } \\
\text { study }\end{array}$ & Trial or study result & Exposure/ Comparator & $\begin{array}{c}\text { Method/s } \\
\text { Outcome measure/s }\end{array}$ & $\begin{array}{l}\text { Knowledge translation } \\
\text { study results (summary) }\end{array}$ \\
\hline $\begin{array}{l}\text { Shah et al. } \\
(2010) \\
\text { Cardiology } \\
\text { USA } \\
\text { Informants } \\
\text { Physicians }\end{array}$ & $\begin{array}{l}\text { RCT: } \\
\text { SYNERGY trial } \\
\text { (Ferguson et al., } \\
\text { 2004) }\end{array}$ & $\begin{array}{l}\text { LMWH non-inferior } \\
\text { to UFH in non-ST } \\
\text { elevation but higher } \\
\text { bleeding rates in } \\
\text { LMWH (and } \\
\text { LMWH/UFH } \\
\text { combined) } \\
\text { = enoxaparin } \\
\text { (LMWH) } \\
\text { recommended in } \\
\text { guideline (2002) }\end{array}$ & $\begin{array}{l}\text { Exposure } \\
\text { Site participation in } \\
\text { SYNERGY } \\
\text { Before publication:24,440 } \\
\text { patients } \\
\text { After publication: } 15,431 \\
\text { patients } \\
\text { Comparator } \\
\text { Site non-participation in } \\
\text { SYNERGY Before } \\
\text { publication: } 48,860 \text { patients } \\
\text { After publication: } 34,033 \\
\text { patients }\end{array}$ & $\begin{array}{l}\text { Method } \\
\text { Registry review of } \\
\text { prescribing practices } \\
\text { Outcome } \\
\text { Prescription of LMWH and } \\
\text { UFH as recorded in CRUSADE } \\
\text { registry }\end{array}$ & Exposure $=$ Comparator \\
\hline \multirow{4}{*}{$\begin{array}{l}\text { Litjens, Oude } \\
\text { Rengerink, Danhof, } \\
\text { Kruitwagen, \& Mol } \\
\text { (2013) } \\
\text { Obstetrics and } \\
\text { Gynaecology } \\
\text { Netherlands } \\
\text { Informants } \\
\text { Gynaecologists } \\
\text { Resident doctors } \\
\text { Midwives } \\
\text { Research nurses }\end{array}$} & Multiple RCTs & & \multirow{4}{*}{$\begin{array}{l}\text { Exposure } \\
\text { Site or individual } \\
\text { participation in specific } \\
\text { research trials } \\
\text { Comparator } \\
\text { Non-participation of site in } \\
\text { particular trial } \\
\text { Overall: } n=202 \\
\text { Gynaecologists: } n=117 \\
\text { Resident (doctors) } n=34 \\
\text { Midwives: } n=46 \\
\text { Research nurses: } n=4\end{array}$} & \multirow{4}{*}{$\begin{array}{l}\text { Method } \\
\text { Survey } \\
\text { Outcomes } \\
\text { Knowledge of studies } \\
\text { 1.Recruited vs non-recruiters } \\
\text { 2.Participation vs non- } \\
\text { participation }\end{array}$} & \multirow{4}{*}{$\begin{array}{l}\text { Knowledge: } \\
\text { Exposure > comparator }\end{array}$} \\
\hline & $\begin{array}{l}\text { LEO (Bayram et al., } \\
\text { 2004) }\end{array}$ & $\begin{array}{l}\text { No difference } \\
\text { between } \\
\text { electrocautery or } \\
\text { follicle stimulating } \\
\text { hormone in PCOS }\end{array}$ & & & \\
\hline & $\begin{array}{l}\text { Metformin (Moll et } \\
\text { al., 2006) }\end{array}$ & $\begin{array}{l}\text { No difference in } \\
\text { ovulation between } \\
\text { clomiphene alone } \\
\text { compared to } \\
\text { clomiphene plus } \\
\text { metformin }\end{array}$ & & & \\
\hline & $\begin{array}{l}\text { ECV (Kok et al., } \\
\text { 2008) }\end{array}$ & $\begin{array}{l}\text { Niphedipine did not } \\
\text { increase successful } \\
\text { ECV }\end{array}$ & & & \\
\hline
\end{tabular}




\begin{tabular}{|c|c|c|c|c|c|}
\hline $\begin{array}{l}\text { Author, Year } \\
\text { Setting, Country, }\end{array}$ & $\begin{array}{l}\text { Research trial or } \\
\text { study }\end{array}$ & Trial or study result & Exposure/ Comparator & $\begin{array}{c}\text { Method/s } \\
\text { Outcome measure/s }\end{array}$ & $\begin{array}{l}\text { Knowledge translation } \\
\text { study results (summary) }\end{array}$ \\
\hline \multirow{6}{*}{ Litjens continued } & $\begin{array}{l}\text { HYPITAT } \\
\text { (Koopmans et al., } \\
\text { 2009) }\end{array}$ & $\begin{array}{l}\text { Induction (for } \\
\text { women with } \\
\text { hypertension and } \\
\text { pre-eclampsia) } \\
\text { better for mother; } \\
\text { no different for baby }\end{array}$ & & $\begin{array}{l}\text { Outcome } \\
\text { Belief (if knew the results) }\end{array}$ & $\begin{array}{l}\text { Belief in results: } \\
\text { Exposure = comparator }\end{array}$ \\
\hline & $\begin{array}{l}\text { Bed Rest (Custers } \\
\text { et al., 2009) }\end{array}$ & $\begin{array}{l}\text { Immobilisation after } \\
\text { insemination better } \\
\text { outcomes }\end{array}$ & & \multirow[t]{5}{*}{$\begin{array}{l}\text { Outcome } \\
\text { Implementation } \\
\text { Recruited vs non-recruited }\end{array}$} & \multirow{5}{*}{$\begin{array}{l}\text { Reported implementation: } \\
\text { Trial dependent but higher in } \\
\text { centres that recruited for } \\
\text { any trial: } \\
\text { Mixed (trial specific) }\end{array}$} \\
\hline & $\begin{array}{l}\text { IUPC (Bakker et al., } \\
\text { 2010) }\end{array}$ & $\begin{array}{l}\text { IUPC did not improve } \\
\text { outcome if induced } \\
\text { or augmented labour }\end{array}$ & & & \\
\hline & $\begin{array}{l}\text { ALIFE (Kaandorp et } \\
\text { al., 2010) }\end{array}$ & $\begin{array}{l}\text { Anticoagulants did } \\
\text { not decrease } \\
\text { miscarriage } \\
\end{array}$ & & & \\
\hline & $\begin{array}{l}\text { DIGITAT (Boers et } \\
\text { al., 2011) }\end{array}$ & $\begin{array}{l}\text { No difference in } \\
\text { induction of labour } \\
\text { with } \\
\text { disproportionate } \\
\text { intra-uterine growth }\end{array}$ & & & \\
\hline & $\begin{array}{l}\text { TLH (Mourits et al., } \\
\text { 2010) }\end{array}$ & $\begin{array}{l}\text { No advantage for } \\
\text { hysterectomy and } \\
\text { BSPO conducted } \\
\text { laparoscopically vs } \\
\text { laparotomy }\end{array}$ & & & \\
\hline
\end{tabular}




\begin{tabular}{|c|c|c|c|c|c|}
\hline $\begin{array}{l}\text { Author, Year } \\
\text { Setting, Country, } \\
\text { Informants }\end{array}$ & $\begin{array}{l}\text { Research trial or } \\
\text { study }\end{array}$ & Trial or study result & Exposure/ Comparator & $\begin{array}{c}\text { Method/s } \\
\text { Outcome measure/s }\end{array}$ & $\begin{array}{c}\text { Knowledge translation } \\
\text { study results }\end{array}$ \\
\hline \multirow[t]{2}{*}{$\begin{array}{l}\text { Khera et al. (2018) } \\
\text { Oncology } \\
\text { USA } \\
\text { Informants } \\
\text { Physicians } \\
\text { Medical directors }\end{array}$} & \multirow[t]{2}{*}{$\begin{array}{l}\text { RCT: } \\
\text { Comparison of } \\
\text { peripheral blood } \\
\text { and bone marrow } \\
\text { stem cells in } \\
\text { unrelated donor } \\
\text { haemopoietic cell } \\
\text { transplantation } \\
\text { (BMT CTN 0201) } \\
\text { (Anasetti et al., } \\
\text { 2012) }\end{array}$} & \multirow[t]{2}{*}{$\begin{array}{l}\text { Bone marrow (BM) } \\
\text { was not inferior to } \\
\text { peripheral blood (PB) } \\
\text { and may reduce } \\
\text { graft failure } \\
\text { Bone Marrow graft } \\
\text { => recommended }\end{array}$} & $\begin{array}{l}\text { Exposure } \\
\text { Centre participation in BMT } \\
\text { CTN } 0201 \text { study } \\
\mathrm{N}=160 \text { physicians } \\
\text { Comparator Centre non- } \\
\text { participation in BMT CTN } \\
0201 \\
N=137 \text { physicians }\end{array}$ & $\begin{array}{l}\text { Method } \\
\text { Survey } \\
\text { Outcome } \\
\text { Physician-reported personal } \\
\text { preferences and institutional } \\
\text { preference for PB to GM }\end{array}$ & $\begin{array}{l}\text { Knowledge of results: } \\
\text { Exposure = Comparator }\end{array}$ \\
\hline & & & $\begin{array}{l}\text { Exposure } \\
\text { Centre participation in BMT } \\
\text { CTN } 0201 \text { study } \\
\text { Comparator Centre non- } \\
\text { participation in BMT CTN } \\
0201 \\
N=4598 \text { participants } \\
N=292 \text { centres }\end{array}$ & $\begin{array}{l}\text { Method } \\
\text { Observational registry-based } \\
\text { study } \\
\text { Outcome } \\
\text { Temporal trends of BM and } \\
\text { PB graft use }\end{array}$ & $\begin{array}{l}\text { Observational study: } \\
\text { Exposure = Comparator }\end{array}$ \\
\hline $\begin{array}{l}\text { Sweeting et al. } \\
\text { (2020) } \\
\text { Hospital and } \\
\text { community, NHS } \\
\text { UK } \\
\text { Informants } \\
\text { Physiotherapists }\end{array}$ & $\begin{array}{l}\text { Observational } \\
\text { study } \\
\text { (Chester et al., } \\
\text { 2018) }\end{array}$ & $\begin{array}{l}\text { Ten predictors of } \\
\text { SPADI and } \\
\text { QuickDASH were } \\
\text { identified }\end{array}$ & $\begin{array}{l}\text { Exposure } \\
\text { Data collection for study } \\
\text { about musculoskeletal } \\
\text { shoulder pain } \\
\mathrm{N}=21 \text { physiotherapists } \\
\text { Comparator } \\
\mathrm{n} / \mathrm{a}\end{array}$ & $\begin{array}{l}\text { Method } \\
\text { Survey about impact of } \\
\text { publication } \\
\text { Outcome } \\
\text { Knowledge of results } \\
\text { Used results in practice }\end{array}$ & Mixed (no comparator) \\
\hline
\end{tabular}

${ }^{*}$ not explicitly stated, but inferred from method used 
Abbreviations: ACE-inhibitors: angiotensin converting enzyme inhibitors; ACG: American College of Gastroenterology; ALIFE: Anticoagulants for Living Fetus; ASPECT: Aspiring Esomeprazole Chemoprevention Trial; BM: bone marrow; BMT CTN0201: study comparing bone marrow with peripheral blood transplant; BSPO: bilateral salpingo-

oophorectomy; CAVEAT: Coronary Angioplasty Versus Excisional Atherectomy Trial; CRUSADE registry: Can Rapid Risk Stratification of Unstable Angina Patients Suppress Adverse Outcomes with Early Implementation of the ACC/AHA (American College of Cardiology/ American Heart Association) Guidelines registry; DCA: directional coronary atherectomy; DIGITAT: Disproportionate Intra-Uterine Growth Intervention Trial at Term; ECV: External Cephalic Version- nifedipine did not increase; GERD: gastro-oesophageal reflux disease; GP: general practitioner; GUSTO-1: Global Utilization [sic]of Streptokinase and Tissue Plasminogen Activator for Occluded Coronary Arteries; HYPITAT:

Hypertension and Pre-eclampsia Intervention Trial at Term; IUPC: Intra-Uterine Pressure Catheter; KT: Knowledge translation; LEO: Laparoscopic Electrocautery of the Ovaries;

LMWH: low molecular weight heparin; MDPIT: Multicenter Diltiazem Postinfarction Trial; MI: myocardial infarction; MILIS: Multicenter Investigation on Limitation of Infarct Size; n/a: not applicable; NHS: National Health Service: Non-ST: non ST segment; ONE (study, GPs \& patients): management of gastro-oesophageal reflux disease with esomeprazole, testing the cost of on-demand treatment vs treatment courses; PB: peripheral blood; RCT: Randomised controlled trial; PCOS: polycystic ovarian syndrome; RUQ: Research

Utilisation Questionnaire; QuickDASH: Quick Disability of the Arm, Shoulder and Hand Questionnaire; SAVE: Survival and Ventricular Enlargement trial; SPADI: Shoulder Pain and Disability Index; SymbiAC: Symbicort Asthma Control Plan; SYNERGY trial: Superior Yield of the New Strategy of Enoxaparin, Revascularization, and Glycoprotein IIb/IIIA

Inhibitors; TIMI: Thrombolysis in Myocardial Infarction; TLH: Total Laparoscopic Hysterectomy; UFH: unfractionated heparin; UK: United Kingdom; USA: United States of America 


\subsubsection{Outcome measures}

Most studies compared the difference between the use of the intervention (drug, procedure or guideline) examined in the original trial or study (Table 2). The Litjens et al. (2013) study extended this by measuring knowledge and belief in the results and another study (Tranmer et al., 2002) was conducted specifically to assess nurse attitudes to research following differing levels of engagement in designing a research study.

\subsubsection{Timing of knowledge translation study in relation to published RCT}

The length of time between the RCT and the knowledge translation study varied considerably. The results for two RCTs were unknown at the time of the knowledge translation study (Andersen et al., 2006; Das et al., 2008), while two studies were conducted almost immediately after publication of the RCT (0-6 months) (Corrigan \& Glass, 2005; Majumdar et al., 2002). Sweeting et al.(2020) surveyed physiotherapists four years after their involvement in study, reflecting that this length of time had the potential for recall bias. For three studies timing was unclear, but is estimated as two to three years after publication of the original research (Adler, 1978; Ketley \& Woods, 1993; Meineche-Schmidt et al., 2006; Shah et al., 2010). The time variability was greatest when the impact of multiple study results were investigated, as in Litjens et al. (2013), where the survey about the knowledge, convincement and implementation of nine RCTs was conducted two to 10 years after the RCTs. Additionally, two studies reviewed practice more than once over time. Corrigan and Glass reviewed practice three, six and 18 months after product launch and Kizer et al. (1999) multiple times over several years.

\subsubsection{Sample sizes}

There was large variation in sample sizes participants, and in institutions and regions involved, making comparison difficult (Table 2). As all studies were observational, sample sizes were not calculated. Studies that analysed existing registry data about patients or prescriptions tended to be relatively large $(4598$ to 122,764) (Andersen et al., 2006; Khera et al., 2018; Majumdar et al., 2004; Shah et al., 2010). However, where the method was survey, observational or the informants were health practitioners, the sample sizes were much smaller (21-1683) (Das et al., 2008; Khera et al., 2018; Litjens et al., 2013; MeinecheSchmidt et al., 2006; Omoigui et al., 1998; Sweeting et al., 2020; Tranmer et al., 2002). 


\subsubsection{Quality of the literature}

The Critical Appraisal Skills Programme (CASP) tools were used to critically appraise the publications (Oxford Centre for Triple Value Healthcare, n.d.). CASP tools are a set of checklists used to provide a framework for assessing quality of literature. There are eight different checklists which are specific to each study design. All studies in this review used an observational design, and the CASP 'cohort study' checklist was used to assess all. While observational research ranks them low on the hierarchy of evidence (Polit \& Beck, 2017), it would be very difficult to assess this topic in any other way, and observation generates the best evidence in this field. The quality of the articles increased over time, with early publications (Adler, 1978; Ketley \& Woods, 1993; Kizer et al., 1999) providing little detail of the respondents' characteristics or number of participants. There was no one issue with all studies. Where there is a specific issue with the quality of a study, this has been highlighted in the analysis of themes.

\subsection{Themes}

Six themes were identified in the literature (Table 3). The themes are strength of evidence, effect of participation before results were known, knowledge of results, level of engagement in original research, relevance of results, and other confounding factors. No one study contributed to all themes, with most studies contributing to one to three themes.

\subsubsection{Strength of evidence}

There is no consistency in the 14 articles about whether the clinical trial being examined recommended a practice change or not and whether sites that participated were more likely to use the findings or not (knowledge translation). Specifically, sites that participated in a trial with a positive result were found to change practice (Adler, 1978) and not change practice (Khera et al., 2018; Majumdar et al., 2002), and one study reported no change following a clinical trial that showed non-inferiority, which means the treatment was 'not worse' than the control (Shah et al., 2010). Conversely, the Omoigui et al. (1998) study showed that trial sites increased use of an investigational device, despite the clinical trial results reporting more adverse effects from this device than an alternative procedure. 
Table 3 Contribution of literature to themes

\begin{tabular}{|c|c|c|c|c|c|c|}
\hline & $\begin{array}{l}\text { Strength of } \\
\text { evidence }\end{array}$ & \begin{tabular}{|c|} 
Effect of \\
participation \\
before RCT \\
results \\
known
\end{tabular} & $\begin{array}{l}\text { Knowledge } \\
\text { of and/or } \\
\text { belief in } \\
\text { results }\end{array}$ & $\begin{array}{c}\text { Level of } \\
\text { engagement }\end{array}$ & $\begin{array}{c}\text { Relevance of } \\
\text { research }\end{array}$ & $\begin{array}{c}\text { Other } \\
\text { confounding } \\
\text { factors }\end{array}$ \\
\hline $\begin{array}{l}\text { Adler } \\
(1978)\end{array}$ & $X$ & & & & & $x$ \\
\hline $\begin{array}{l}\text { Ketley \& Woods } \\
\text { (1993) }\end{array}$ & $X$ & & & & & \\
\hline $\begin{array}{l}\text { Omoigui et al. } \\
\text { (1998) }\end{array}$ & $x$ & & & & & \\
\hline $\begin{array}{l}\text { Kizer et al. } \\
\text { (1999) }\end{array}$ & $X$ & & & $x$ & & \\
\hline $\begin{array}{l}\text { Majumdar et al. } \\
(2002)\end{array}$ & $X$ & & & & & \\
\hline $\begin{array}{l}\text { Tranmer et al. } \\
(2002)\end{array}$ & & $x$ & & $X$ & & \\
\hline $\begin{array}{l}\text { Corrigan \& Glass } \\
(2005)\end{array}$ & & & & & & $X$ \\
\hline $\begin{array}{l}\text { Meineche- } \\
\text { Schmidt et al. } \\
(2006)\end{array}$ & & & & & & $x$ \\
\hline $\begin{array}{l}\text { Andersen et al. } \\
(2006)\end{array}$ & & $x$ & & & & $x$ \\
\hline \begin{tabular}{|l} 
Das et al. \\
$(2008)$
\end{tabular} & & $X$ & & & & \\
\hline $\begin{array}{l}\text { Shah et al. } \\
(2010)\end{array}$ & $X$ & & & & & $x$ \\
\hline \begin{tabular}{|l} 
Litjens et al. \\
$(2013)$
\end{tabular} & & & $X$ & $X$ & $x$ & \\
\hline $\begin{array}{l}\text { Khera et al. } \\
(2018)\end{array}$ & $X$ & & $x$ & & & $X$ \\
\hline $\begin{array}{l}\text { Sweeting et al. } \\
(2020)\end{array}$ & & & $X$ & & & \\
\hline
\end{tabular}

Abbreviations: RCT: randomised controlled trial

The earliest study about implementing trial knowledge concluded that sites that participated in a RCT with a positive result changed practice to a greater extent compared to nonparticipation (Adler, 1978). In the trial, patients undergoing surgery for varicose veins or inguinal hernia repair were randomised to being discharged home either 48 hours postsurgery or standard care ( 6 to 7 days). The RCT showed no difference in post-operative complications or length of convalescence in either group, and no difference in reoccurrence with inguinal hernia repair, resulting in cost savings due to the shorter stay (Adler et al., 1978). While the result appears convincing, the trial was conducted in a single-centre. 
Adler's (1978) sole authored publication about the impact of the results alludes to a local policy change in discharge practice as a result of the RCT findings. Adler conducted a retrospective review two years after the RCT and found that the mean length of stay had significantly decreased in the study region, compared to surrounding geographical regions. He concluded that participation in the study influenced the knowledge uptake, although acknowledged that confounding factors such as availability of district nursing care were not accounted for. Likewise, Ketley and Woods (1993) found that the use of thrombolytic drugs following myocardial infarction was related to the level of involvement of 12 districts that had participated in four inter-related research trials. Some of the patients included in the analysis may have been enrolled in one of the RCTs at the time of the observational study, however the researchers accounted for this.

A positive result for an RCT was not always a determining factor in whether the results were used by the sites that helped create the evidence (Khera et al., 2018; Majumdar et al., 2002). The Survival and Ventricular Enlargement (SAVE) trial showed a significant benefit of angiotensin-converting enzyme (ACE) inhibitors for patients with asymptomatic left ventricular enlargement following myocardial infarction (Pfeffer et al., 1992). Majumdar et al. (2002) conducted a retrospective review of prescribing practices for 25,886 patients following myocardial infarction in 659 North American hospitals, who were enrolled in the Global Utilization of Streptokinase and Tissue Plasminogen Activator for Occluded Coronary Arteries (GUSTO-1) study. Of these 659 hospitals, 22 (3\%) had also participated in the SAVE trial. Review of the GUSTO-1 database showed that use of ACE-inhibitors at discharge increased overall from $14 \%$ to $18 \%(P=0.001)$ after publication of the SAVE results. However, there was no statistical difference $(P=0.67)$ between sites that had participated in SAVE (21\%) and those that had not (20\%). The researchers concluded that participating in a research trial did not increase the likelihood of the findings of that trial being used in practice, compared to non-participation. Similarly, a more recent study comparing peripheral blood with bone marrow in unrelated donor transplantation showed no overall difference in change in practice; either at participating sites or non-participating sites (Khera et al., 2018). The results of this RCT showed that bone marrow transplantation was not inferior to peripheral blood transplantation, and the graft-versus-host disease at two years was significantly less with bone marrow transplantation ( $P=0.01$ ) (Anasetti et al., 2012). 
Physician participation had previously been highlighted as a potential facilitator for change in practice (Khera, 2015), however when examined, this was not the case (Khera et al., 2018). Although $92 \%$ of physicians were aware of the RCT result, few had changed their practice $(18 \%)$ or perceived their centre had changed practice $(16 \%)$.

Sites participating in a study investigating non-inferiority of an anticoagulant in heart disease also did not show a change in practice (Shah et al., 2010). A pharmaceutical company sponsored RCT called Superior Yield of the New Strategy of Enoxaparin, Revascularization and Glycoprotein IIb/IIla Inhibitors (SYNERGY) was conducted in 118 sites in the USA over four years (Ferguson et al., 2004). The results showed that the low-molecular-weight heparin (LMWH), enoxaparin, was non-inferior compared to unfractionated heparin in patients with non-ST-elevation myocardial infarction (Ferguson et al., 2004). Existing registry data were used to examine trends in $\mathrm{LMWH}$ use before and after publication of SYNERGY in all trial sites compared to non-trial 270 registry sites (Shah et al., 2010). LMWH use increased slightly during the trial, but this was not sustained. Use of LMWH did not increase after publication and there was no increase of LMWH at SYNERGY sites compared to non-SYNERGY sites.

There was increased use of a study device by investigators after their participation in a procedural cardiac trial, despite the RCT showing increased adverse effects than an alternative device (Omoigui et al., 1998). Directional coronary atherectomy (DCA) was trialled in patients with ischaemic heart disease who were suitable for atherectomy or angioplasty. The publication showed that DCA increased adverse clinical outcomes, had higher cost and had no clinical benefit, compared to balloon angioplasty (Topol et al., 1993). Despite this finding, a review of investigators' practice before and after participation in the RCT revealed trial-site investigators had increased their use of DCA one year after publication (Omoigui et al., 1998).

\subsubsection{Effect of exposure to the trial protocol (before publication of results)}

Two studies reported a change in practice even before the results of the RCT were known (Andersen et al., 2006; Das et al., 2008), suggesting that participation in research alone may be sufficient to change practice. Andersen et al. (2006) conducted a retrospective cohort study of the use of inhaled steroid in Danish general practices that had participated in an 
open-label, pharmaceutical-sponsored trial comparing two dosing regimens of an asthma drug (Symbicort) $(n=10)$ with general practices that had not $(n=165)$. Despite results of the RCT being unknown at the time of this study, use of the company sponsored drugs increased after the trial, however there was no impact on practices' adherence to international treatment recommendations. The researchers highlighted that not all physicians at the trial sites participated in the RCT, and it was not possible to account for this in their analysis. Similarly, a survey of UK gastroenterologists was conducted before and after sites started a study about the management of Barrett's oesophagus but before the RCT results were known (Das et al., 2008). The Aspirin Esomeprazole Chemoprevention Trial (ASPECT) was a multi-factorial trial assessing the use of proton pump inhibitors (PPI) and aspirin, and required increased surveillance with an emphasis on histological sampling (Jankowski et al., 2018). The study of practices which consisted of three surveys was conducted before ASPECT started recruitment, and again after some sites had started (Das et al., 2008). The first survey was sent to 401 senior gastroenterologists and trainees in 2002, of whom 288 (57\%) responded. This survey served as a baseline of self-reported practice and screened for those sites that would be suitable to participate in ASPECT. The second survey was sent to the same respondents in $2005 / 2006$, by which time 57 centres had commenced ASPECT and a further 50 were due to start. The third survey was sent only to the largest ASPECT recruiting site, specifically asking about histological sampling, and is not reported in this review. Prior to the ASPECT trial, the UK British Society of Gastroenterologists did not have specific screening guidelines for Barrett's oesophagus. Following the second survey, the increase in the percentage of patients receiving the recommended biopsy had increased by $18.8 \%$ from baseline $(\mathrm{P}<0.05)$; however, for patients enrolled in the ASPECT trial the number receiving a biopsy had increased by $37.7 \%$ compared to baseline $(P<0.01)$. The researchers also reported that overall adherence to guidelines had improved in the ASPECT sites. The finding that participation in ASPECT increased adherence to guidelines is somewhat misleading, as many of the patients in which the guidelines had been adhered to, were enrolled in ASPECT, and would have been subject to the trial protocol. The results of the ASPECT trial were published 10 years after this knowledge translation study, and showed that high-dose PPI and aspirin chemoprevention therapy improved outcomes for patients with Barrett's oesophagus (Jankowski et al., 2018). 


\subsubsection{Knowledge of and belief in results}

Three of the reviewed studies addressed the issue of whether health practitioners knew about the evidence of a study or trial they or their department had participated in (Khera et al., 2018; Litjens et al., 2013; Sweeting et al., 2020), and Litjens et al. also addressed the belief in the results. All three research teams found that health practitioners who participated in a trial were more likely to know about the trial results than their non-trial counterparts (if a comparator was included).

For the 2013 study by Litjens et al. the researchers distributed a survey to all 89 Obstetrics and Gynaecology departments in the Netherlands, along with the results of nine obstetricrelated RCTs (Table 2). All studies that were included had a clear result of either proving or disproving a hypothesis. Health practitioners (gynaecologists, resident [trainee] doctors, midwives and nurses) were invited to participate, although a maximum of two responses per professional group, per hospital were included in the analysis. Respondents were surveyed about whether they already knew the trial results prior to receiving the survey (and RCT summaries) and how convinced they were by the findings. They were asked about their current practice in relation to the results, whether they worked in a department that had participated in each trial and whether they had recruited for the trial. A total of 286 questionnaires were received from 83 departments; although 84 (29\%) were excluded because they exceeded the maximum per department, were incomplete or other issues. Allowing for a maximum number of respondents of 534, based on two gynaecologists, two residents, one midwife and one research nurse per site, the return rate was $38 \%(202 / 534)$. Health practitioners from sites that had participated in the trials were significantly more likely to know the trial results (range $84-100 \%$, depending on the RCT), than those from nontrial sites (range 38-86\%). The overall result (for all nine RCTs) was significantly higher when respondents had participated in one of the trials (Relative risk [RR]: 1.5, 95\% Cl: 1.3-1.8, $\mathrm{P}<0.01)$. Those who knew the trial results were almost always convinced of the trial results (average 94\%; range 89-97\%), and there was no difference between those who recruited into the trial and those who did not.

More recently, Khera et al. (2018) surveyed bone marrow transplant physicians about their knowledge of the results of a trial that compared peripheral blood graft with bone marrow 
graft (Anasetti et al., 2012). The survey respondents included 160 transplant physicians who worked in a site that had participated in the original trial, and 137 from non-trial sites. The survey, which was designed to assess personal and centre changes in practice, was part of a multiple methods study that also reviewed registry data to review practices. A large proportion of the respondents (93\%) knew the trial result, and of these, $88 \%$ had read the trial publication. Only a small percentage of physicians in both trial and non-trial sites reported a personal $(18 \%)$ or centre $(16 \%)$ change of practice. The observational study reported no difference in the recommended practice of using bone marrow graft at either trial or non-trial sites.

Similarly, a small study involving physiotherapists reviewed whether respondents were aware of the results of an observational study about predictors of musculoskeletal shoulder pain (Chester et al., 2018) which they had collected data for. The survey was sent to 34 of the 115 physiotherapists who assisted in the original study four years previously, of whom 21 responded (Sweeting et al., 2020). Fifteen of the 21 physiotherapists (71\%) knew of and understood the results, and reported that their beliefs about physiotherapy of shoulder pain had changed as a result of the study. Only five $(24 \%)$ had read the publication, which was sent directly to physiotherapists who had participated. In addition to the publication, the physiotherapists had found out the results via post-graduate courses $(n=4)$, direct contact with the author $(n=4)$, colleagues $(n=2)$ and social media $(n=1)$, with one respondent finding out through multiple modes.

\subsubsection{Level of engagement in research trial}

Two studies reported a positive association between a higher level of engagement in a clinical trial and using the results of the RCT (Kizer et al., 1999; Litjens et al., 2013). Taking a different stance, Tranmer et al. (2002) specifically set up a study to assess whether the level of engagement of nurses in the design of a study affected their attitude to research. The study was set in six mixed medical and surgical units in a single hospital in Ontario, Canada. The units were cluster-randomised to three levels of exposure to research (low, high and usual), with two units in each cluster, and were involved in the design and implementation of a study protocol about patients' sleep patterns. Nurses in the low group ( $n=10$ members) met once, while the high participation group ( $n=20$ members) met several times and received in-depth training. For the two usual engagement level units, no research group 
was formed, no additional activities organised, and no research protocol was developed. Nurses in the six units were surveyed using a self-reported research utilisation questionnaire prior to the commencement of the study and again after participation in the groups. Respondent rate to the questionnaire was $39 \%$ at baseline and $37 \%$ a year after data collection was finished in the sleep study. Participation in either the high or low groups had a positive effect on staff nurses' attitudes about research utilisation at an individual level but not a unit level. The nurses in the high group had a statistically higher score in attitude to research before the study started, and again after participating $(P<0.05)$. The numbers were small for this study and sleep study had not been conducted at the time the engagement study was published (Tranmer et al., 2003).

Using a retrospective review of prescribing practices, Kizer (1999) compared physicians' use of aspirin, beta blockers, ACE-inhibitors and calcium channel blockers on enrolment and at discharge for trial participants with ST elevation, compared to routine clinical practice. This 1999 study compared prescribing practices of physicians in the USA who were investigators in a series of RCTs about myocardial infarction, with physicians in routine clinical practice. The RCTs were Multicenter Investigation on Limitation of Infarct Size (MILS) and a series of studies about thrombolysis in myocardial infarction (TIMI), and evaluated various combinations of aspirin, beta-blockers, ACE-inhibitors and calcium antagonists. The existing MILS-TIMI database was used to review prescribing practices of each of the trial drugs. The prescribing practices at hospital discharge for patients with a myocardial infarction were used as the exposure, researchers inferring that this indicated the prescribing physician's (investigator's) beliefs about the current evidence. This was compared to routine practice, which was defined as the prescribing practices patients were subject to at enrolment into one of the RCTs. The researchers concluded that physicians who designed or participated in research were more likely and quicker to use current best practice than routine care, although it was unclear how involved in research design these investigators were. Similar to Das et al. (2008), patients would have been subject to the drugs prescribed as per the trial protocol throughout admission and this may have influenced prescribers' behaviours at patient discharge. 
As part of their overall study about knowledge and use of the results of RCTs, Litjens et al. (2013) found that the level of engagement in an RCT increased the likelihood of knowing about, being convinced of the results, and using the results in practice. The level of engagement in research was assessed as recruiting a patient or not, as well as hospital level participation. Knowledge of the results was greater if they had recruited for the trial; however, there was no difference in how convinced of the trial results they were. When comparing the knowledge of the trial results, based on whether they had recruited or not, this ranged from $29 \%$ to $86 \%$ in the non-recruiting respondents; compared to $84 \%$ to $100 \%$ in the respondents from hospitals that had recruited (pooled RR: 1.8, 95\% Cl: 1.7-1.9, $\mathrm{P}<0.01)$. The biggest difference was for a trial about whether metformin in addition to clomiphene increased ovulation with $94 \%$ of recruiting respondents knowing the results, compared to $29 \%$ of non-recruiters (RR 3.29; $95 \% \mathrm{Cl}: 2.54-4.24)$. However, the number who recruited was very small $(n=17)$ compared to non-recruiters $(n=185)$. For the only trial in which the number of recruiters $(n=143)$ was larger than non-recruiters $(n=59)$, the percentage who knew the result was $97 \%$ and $86 \%$ respectively. Implementation of trial results was assessed at a site level and hospitals that had recruited into a trial, but the overall increase was minimal.

\subsubsection{Relevance of research findings to informants}

One study analysed knowledge of specific RCTs by role (Litjens et al., 2013); although they failed to account for the relevance of RCTs to the health practitioners' role. Nurses and midwives had a lower level of knowledge about eight of the nine RCTs reviewed, than gynaecologist and trainee doctors. The specific numbers were not reported for all studies, but an example was given about a trial that showed induction of labour in women (after 36 weeks gestation) with pre-eclampsia or hypertension, improved outcomes for women (and no difference for babies), than not intervening (HYPITAT) (Koopmans et al., 2009). For HYPITAT, $96 \%$ of specialists and $100 \%$ of trainees compared to $89 \%$ of midwives, knew the

results of a trial that was relevant to all roles involved in antenatal care. The only RCT in which more nurses and midwives knew the results more than doctors showed no clear advantage or disadvantage for induction of labour when disproportionate intra-uterine grown is present (DIGITAT) (Boers et al., 2011). Three of the RCTs appeared to be relevant only to doctors or nurses working in an infertility setting (Bayram et al., 2004; Custers et al., 2009; Moll et al., 2006), and two involved surgical procedures (Bayram et al., 2004; Mourits 
et al., 2010), one of which was about laparoscopic hysterectomy in endometrial cancer, which would be of no or little relevance to midwives. Two further trials (Kaandorp et al., 2010; Kok et al., 2008), while relevant to antenatal care, were regarding drug prescription, which would more likely be relevant to doctors than midwives or nurses. Reported implementation differed for some trials including HYPITAT which was used significantly more by resident doctors (79\%) and gynaecologists (66\%) than midwives (45\%). There was no difference between roles for implementation of the results from trials about anticoagulants in miscarriage (Kaandorp et al., 2010), using niphedipine to assist in turning breech babies (Kok et al., 2008), bedrest after insemination or intra-uterine pressure catheters in induced or augmented labour (Bakker et al., 2010).

Other studies included in this literature review did not specifically assess relevance, although as the informants were from the same specialties as the original trials, the majority of the knowledge translation studies would be highly relevant. For example Majumdar et al., (2002) studied the participation of cardiac physicians in an RCT about medication in myocardial infarction; likewise Khera et al., (2018) studied the participation of transplant physicians involved in an RCT comparing peripheral blood with bone marrow grafts.

\subsubsection{Other confounding factors or considerations}

There were other confounding factors when considering whether research results were used in clinical practice, following participation in a trial. In particular, the impact of commercial sponsorship (Andersen et al., 2006; Corrigan \& Glass, 2005; Meineche-Schmidt et al., 2006; Shah et al., 2010), patient preference (Meineche-Schmidt et al., 2006) and cost benefits of research therapies (Adler, 1978). Four studies identified that the sites had participated in a trial sponsored or supported by a commercial pharmaceutical company, about a new drug or a new regimen for an existing drug. The purpose of each of these knowledge translation studies was to assess the influence that participating in pharmaceutical trials had on prescribing practices of doctors after participation in these trials. This was shown to be the case in three of the four studies, including both studies in primary care (Andersen et al., 2006; Meineche-Schmidt et al., 2006).

Of the four pharmaceutically sponsored trials, three found participating doctors were more likely to use the trial drug. Firstly, Corrigan and Glass (2005) conducted a matched case 
control study of 4216 physicians in the USA. Half of the physicians (2108) had participated in at least one Phase III clinical trial between 1 January 1995 and 31 December 1996. The remaining 2108 investigators were matched on a one-to-one matched group according to geographic location, specialty and prescribing behaviours in the previous three months. The 38 drugs studied included drugs for: asthma, allergic rhinitis, hypertension, osteoarthritis, rheumatoid arthritis, depression, pneumonia, hypercholesterolaemia and diabetes. A retrospective review of a database of individual physicians investigated drug-specific prescribing data, three months before their participation in a trial, and three, six and 18 months after the product launch of the trial drug. At three months after product launch investigators were more likely to use the trial drug than control physicians ( $26 \%$ and $16 \%$, respectively $(P \leq 0.001)$. This effect persisted over the following 15 months. The study concluded that physicians who had participated in the drug trials (Investigators) were more like to prescribe the investigational product, especially if it was a 'first in class' drug, than physicians who had not participated. Secondly, in a study reviewing general practitioners' (GPs) practices following participation in a sponsored drug trial about asthma, Andersen et al. (2006) found GPs were more likely to prescribe the pharmaceutical company's drug; although adherence to international guidelines did not change. This suggested that participation influenced specific prescribing practice for the drug that had been studied but not overall adherence to guidelines. In the third example, GPs were more likely to use the drug following participation in a trial about PPIs in gastro-oesophageal reflux, although this was complicated by patients' preferences (Meineche-Schmidt et al., 2006). Approximately one third of the exposure arm included patients who had participated in the trial, which also compared patient-driven (on-demand) prescriptions, compared to a course of the PPI. Conversely, Shah et al. (2010)found no increase in prescription of low molecular weight heparin for either SYNERGY or non-SYNERGY sites following participation or publication.

One study reported the reason for adoption of a trial result was the cost benefit (Adler, 1978). Specifically, Adler highlighted that reducing the length of stay of patients after surgery was a significant cost-saving for the participating district.

\subsection{Summary, gaps and limitations in the literature}

The existing literature about whether site participation in clinical research increases the likelihood of translating the findings of that research into practice is inconsistent and 
inconclusive. In addition, none of the studies cited using a knowledge translation framework to design their research. It was not possible to combine the literature in a meta-analysis due to variations in size methods and disparity of results. This review highlighted areas that have been considered in a limited way, and could be extended on, and gaps in knowledge, indicating further investigation is warranted.

\subsubsection{Review of methods}

Methodologically, there was a predominance of retrospective review of prescribing practices, and self-reported survey. While the use of surveys and retrospective reviews have strengths, the combination of survey and observational studies is stronger. Multiple (two) methods were used by Khera et al. (2018), in the oncology setting, allowing the researchers to survey the transplant physicians' beliefs about their practice, and review their actual practice by registry-review.

All studies were conducted in the northern hemisphere, with a predominance in North America, where the health system differs greatly from Australia and NZ. There was a predominance of literature set in cardiology, reviewing advances in management and drug therapy from the 1980 s to 2010 . Other studies were distributed throughout other specialities including primary care, obstetrics and gynaecology, gastroenterology and surgery. Although knowledge translation studies have been conducted in ICU (Kaukonen et al., 2013; Salter et al., 2018), none compared the effect of participation versus nonparticipation in the research.

Doctors were the professional group studied most in the research. Many of the studies related to medical interventions such as the prescription of drugs or procedures which could only be conducted by doctors. Nurses, midwives and allied health professionals were underrepresented in the literature. While this approach was relevant for the research conducted, such as prescription of drugs or surgery, the situation in the ICU setting requires inclusion of nurses. ICU nurses experience a high level of autonomy in many ANZ ICUs, and adjusting ventilation parameters, including oxygen (Henderwood, 2015; Rose et al., 2008)

The trial interventions being studied were, once again, predominantly drug prescribing. Additionally, procedures that can only be conducted by doctors were studied, including 
those from cardiac, obstetrics and gynaecology, oncology and surgery. Oxygen use is ubiquitous in ICU, with almost every ventilated patient receiving oxygen (Panwar et al., 2013; Suzuki et al., 2013). No literature was found about the effect of participation in research about oxygen on clinical practice. With respect to exposure and comparator, there were mixed results, with several studies combining both a 'before and after' trial approach, or comparing participating sites with non-participating.

There was considerable variation about the timing of the knowledge translation studies compared to when the RCT was undertaken (from before results known to more than 10 years post result publication). Some of the studies reported that participation in the RCT alone was enough to make a change, showing the effect of participation and exposure to the trial protocol, but then failed to assess this again once the results of the trial were known (Andersen et al., 2006; Das et al., 2008). Others only reviewed the practices after results were known (although due to their retrospective nature were able to see trends in drug prescription over time, including throughout the trial period). Only two of the studies collected baseline data of practice prior to a trial starting (Das et al., 2008; Tranmer et al., 2002). Collecting data prior to participation in a study or trial, allows examination of existing practice in real time therefore allowing assessment of variables that cannot be measure retrospectively, such as staff attitudes to an intervention.

\subsection{Gaps in knowledge}

In the thematic analysis, no single element was identified that could reliability predict knowledge translation after participation in an RCT. Examination of the themes did not show a relationship between the strength of evidence or participation and use of that evidence.

\subsubsection{Dissemination of results}

Three studies assessed whether respondents' knew of the RCT or study results (Khera et al., 2018; Litjens et al., 2013; Sweeting et al., 2020). Only Sweeting et al. (2020) stated a dissemination plan, which was to send a link to the publication to physiotherapists who had assisted with the original study. They also reported the way the 15 physiotherapists found out the results of a study they collected data for. Understanding how health practitioners find out the results of RCTs offers information about ways to disseminate findings in future research. 


\subsubsection{Level of engagement}

The level of participation in research may affect the findings, as can be seen in the study where health practitioners were involved in the development of research protocols (Tranmer et al., 2002). Engagement in the research process appeared to make a difference at an individual level but not a site level (Tranmer et al., 2002). Additionally, Litjens et al. (2013) examined the level of engagement in a study by asking respondents whether they had enrolled patients in the study, and showed an association between enrolment and knowledge translation; an idea that could be extended in future research.

\subsubsection{Relevance to practice}

There was limited reporting about whether relevance of research findings resulted in a greater use of those findings. Tranmer et al. (2002) and Litjens et al. (2013) both show an indication that this may be the case, but in cardiac studies such as Majumdar et al. (2002), where the finding could be considered highly relevant to cardiologist investigators, the findings were used no more in sites that participated than those that did not.

\subsubsection{Attitudes}

Only one study explicitly assessed staff attitudes to participation in research, and this was limited to their attitude about research (Tranmer et al., 2002). While engagement in research reportedly changed the individual nurses' attitudes to research, it did not result in an institutional change. Additionally, Khera et al. (2018), reported a change in personal practice about bone marrow transplant, similar to a change in attitude.

\subsection{Addressing gaps in knowledge}

The ICU-ROX TRIPS programme of research aimed to address some of these gaps in the literature by incorporating the following, which will be described in more depth in the methodology chapter:

- Multiple method approach - addressing what people think (survey of attitudes), what people do (observational study of practices), and what people do from a distance (retrospective review of existing database)

- Australia and New Zealand ICUs as a setting

- ICU doctors and nurses as informants 
- Exposure and comparator (participating in the ICU-ROX trial compared to nonparticipation, and before ICU-ROX and after)

- Use of PARIHS framework in evaluation of an ICU study and dissemination evaluation

- Collection of data prior to start of ICU-ROX trial and comparing it at different time points (after ICU-ROX but before the result is known and again after ICU-ROX is published)

- Collection of data about attitudes and knowledge of ICU-ROX results from health practitioners

- Examination of oxygen as an intervention

- Examination of success of the dissemination plan by assessing whether people knew the results and how they found out

- Assessment of factors that inform informants' practice with regard to oxygen management (to assess relevance of ICU-ROX findings)

- Review ICU-level practices with regard to practice change, and knowledge translation processes within ICU. 


\section{CHAPTER 5 METHODOLOGY, FRAMEWORK AND METHODS OVERVIEW}

In view of the uncertainty and lack of consensus in the literature about whether institutional participation in research affects the use of the research findings in clinical practice, this chapter addresses the philosophical, theoretical and methodological approach to extend the understanding on this topic. The methodological approach is determined by the research questions to be answered, which were outlined in Chapter 1, as well as the ethical considerations.

This chapter begins with the theoretical paradigm or philosophical worldview for ICU-ROX TRIPS, which is postpositivism. Following this, the conceptual framework that underpins this study is discussed with an overview of the purpose and importance of such a framework. The chosen framework of Promoting Action on Research Implementation in Health Services (PARIHS) (Kitson et al., 1998) is discussed, followed by the rationale for this choice, how PARIHS has previously been operationalised and finally how ICU-ROX TRIPS aligns with and was informed using this framework. Following this, is the rationale for choosing a multiplemethod approach, which is discussed in relation to the philosophical and conceptual approaches, and an overview of the multiple methods chosen, ethical issues and a description of the study exposure and comparator.

\subsection{Philosophical design considerations}

The research paradigm (Guba, 1990) or philosophical worldview (Creswell, 2014) for this study is postpositivism which is the view that there are multiple interpretations of the truth, rather than a single truth (Letourneau \& Allen, 1999). A paradigm is the set of beliefs adopted by a particular group which provide the lens, structure and guidance to research (Guba, 1990; Weaver \& Olson, 2006), and informs the methodology and methods for the research.

Postpositivism arose out of criticism of positivism, which is most associated with sciencebased research and is hallmarked by absolute truths or laws (Phillips \& Burbules, 2000). Positivism dates back to the $19^{\text {th }}$ century with philosophers such as Auguste Comte arguing there was a hierarchy of evidence with the sciences at the top and social sciences at the 
bottom (Smith, 1983). Critique of positivism arose in the mid-20 $20^{\text {th }}$ century by philosophers such as Karl Popper (Houghton et al., 2012; Phillips \& Burbules, 2000) who developed postpositivism to address this critique and the limitations of positivism. These philosophers challenged the need to use scientific methods to explain human behaviours and more recently, Phillips \& Burbules (2000) argue there is uncertainty of results if research involves human behaviours and actions.

Postpositivism is usually associated with the social sciences and a postpositivist lens incorporates context, multiple realities and complexity (Phillips \& Burbules, 2000). Intrinsically linked to its positivist origins, postpositivism uses the scientific method and testing, is deterministic, and argues causal link (Creswell, 2014). Postpositivism is usually associated with objective and quantitative data; however, qualitative data can also be used. The decision about whether qualitative or quantitative is used in postpositivism should be based on the method best suited to the research (Crotty, 1998; Letourneau \& Allen, 1999).

Postpositivism is well aligned to a multiple method approach as it requires multiple measures or data sources to adequately answer the research question (Houghton et al., 2012). Houghton et al. argue that a multiple method approach in postpositivism improves the overall quality of the data and reduces bias. The combination of knowledge translation being a social science in which people apply their own values and experiences to evidence, yet using a scientific method to answer the research questions, make the postpositivist paradigm particularly suitable for ICU-ROX TRIPS.

\subsection{Knowledge translation framework}

A framework is an important component of a research paradigm (Weaver \& Olson, 2006) and within the postpositivist paradigm using a knowledge translation framework is an appropriate lens for ICU-ROX TRIPS. The use of a conceptual or theoretical framework in knowledge translation research provides a construct or architecture for the research, and enables research to be conducted in a consistent and structured manner, allowing comparison with other knowledge translation studies.

There is inconsistency in terminology and language in the models, frameworks and theories for implementation science and knowledge translation. Rapport et al. (2018) advised that 
this inconsistency is a key problem and recommended a return to first principles. Extending on earlier work by Rabin et al. (2008), Rapport et al. (2018) argued there are five key concepts of implementation science, namely diffusion, dissemination, implementation, adoption and sustainability. The concepts are part of a continual process called the diffusion-dissemination-implementation continuum. Specifically, diffusion is the passive spread of new information or evidence over time, while dissemination is a targeted approach to impart new evidence to an identified audience. Implementation is the active process to incorporate new evidence into practice through a deliberate act and adoption is the degree to which those new ideas are taken up. Finally, sustainability is where new knowledge has been applied. This then cycles back to further diffusion.

There are a myriad of theoretical frameworks for knowledge translation and selecting the most appropriate one involves careful analysis of the available options. Birken et al. (2017) argued that selecting the most suitable framework involves assessing and comparing the characteristics of theories for usability, testability, applicability and acceptability. These researchers found the most commonly used frameworks were the Theoretical Domains Framework, Consolidated Framework for Implementation Research (CFIR) and the Diffusion of Innovations theory. Additionally, the Promoting Action on Research Implementation in Health Services (PARIHS) framework was found to be widely used in the healthcare setting (Mckillop et al., 2012; O’Keefe-McCarthy et al., 2008; Powrie et al., 2014).

Although the use of theoretical frameworks has increased over time (Nilsen, 2015), they remain underused and misused in knowledge translation research (Colquhoun et al., 2010; Davies et al., 2010; Liang et al., 2017). There has been minimal use of theory in ICU knowledge translation studies, although Cuthbertson et al. (2013) employed the Theoretical Domains Framework in their assessment of selective decontamination of the digestive tract use in ICU. It is common for researchers to use part of a knowledge translation theory, rather than a theory in its entirety. In an evaluation of research using the Reach, Efficacy, Adoption, Implementation and Maintenance (RE-AIM) framework, less than 10 percent of grant applicants who claimed to use RE-AIM, used all dimensions of the framework (Kessler et al., 2013). Rapport et al. (2018) argue that only using some aspects of a theory is acceptable, as researchers must choose the most appropriate concepts to answer their question. 
Diffusion of Innovation is one of the earliest frameworks, developed in 1962 in the area of farming (Rogers, 1995). The five main elements of this framework are: the innovation itself, innovativeness of the adopter, social system and communication channels, individual adoption process and diffusion system. Rogers (1995) refers to different types of adopters of new information on a continuous categorisation scale of innovators, early adopters, early and late majority, and laggards. The level of adoption was a useful concept for ICU-ROX TRIPS, as it was plausible that institutions that participate in research were more likely to be early adopters of that research, however Diffusion of Innovation did not fully resonate with the study aims.

Drawing on elements from the diffusion-dissemination-implementation continuum by Rabin et al. (2008) and Rapport et al. (2018), the ICU-ROX results were both diffused and disseminated using a structured approach to ensure evidence was imparted to the people most likely to need or use it. ICU-ROX TRIPS investigated whether participation in ICU-ROX changed the attitudes and practices and level of knowledge health practitioners had about the findings. Because ICU-ROX was published less than six months prior to ICU-ROX TRIPS final data collection, and the result did not offer an answer that would necessarily change practice, the implementation strategy of ICU-ROX ends at dissemination of the results.

While none of the existing frameworks were entirely suitable for ICU-ROX TRIPS, PARIHS was chosen as it was the 'best fit'. The essence of PARIHS is the interaction between the three elements of Evidence, Context and Facilitation. These elements resound with the philosophical argument of postpositivism and the decision to use a multiple method study. Using these elements allowed the researcher flexibility to use the framework for a study where the evidence was not known at the outset. Additionally, PARIHS was developed for healthcare, and did not preclude research in an acute clinical setting such as ICU.

\subsection{PARIHS framework}

The PARIHS framework was first developed as a conceptual framework in 1998 using the collective experience of the authors as researchers, and following theoretical and retrospective review of four studies at the Royal College of Nursing Institute, UK (Kitson et al., 1998). Each of the elements of Evidence, Context and Facilitation contain sub-elements 
which provide clarity and detailed guidance of specifically what needs to be considered is in section 5.3.1. Each element is subject to a continuum scale from low to high. All three elements are weighted equally and successful implementation (SI) is described as the function (f) of the nature and type of evidence $(E)$, qualities of the context $(C)$ and the way it is facilitated (F) expressed as: $\mathrm{SI}=\mathrm{f}(\mathrm{E}, \mathrm{C}, \mathrm{F}$ ) (Kitson et al., 1998).

The PARIHS framework was reviewed, and a concept analysis conducted to strengthen its' theoretical underpinning, resulting in several publications between 2001 and 2004 (McCormack et al., 2002; Rycroft-Malone, Harvey, et al., 2002, 2004; Rycroft-Malone, Kitson, et al., 2002; Rycroft-Malone, Seers, et al., 2004). A guide and critique was published in 2004 which further clarified the framework (Rycroft-Malone, 2004). The PARIHS framework which has continued to be refined, validated and reviewed over time, making it still current despite the more than 20 years since its inception (Harvey \& Kitson, 2016; Kitson et al., 2008). Researchers who were not part of the original PARIHS development group published a critique and guide to using a revised version of the framework (named r-PARIHS) suitable for use by the Veterans Association in the USA (Helfrich et al., 2010; Stetler et al., 2011). These researchers provided some changes to terminology, added sub-elements and provided more detail about how to use the framework. PARIHS has been used extensively in the evaluation of the implementation of knowledge into practice, and has been used in the ICU setting (Hill et al., 2017; O’Keefe-McCarthy et al., 2008; Qin et al., 2020; Stetler et al., 2011).

The original framework rather than the revised version was chosen for ICU-ROX TRIPS, as it offered more flexibility for this study; however aspects and definitions from the revised framework and guide by Stetler et al. (2011) provided clarity to some ambiguous situations. A change in practice was not purported, but rather, observing whether change was more likely to happen given a specific context in relation to exposure to knowledge creation (i.e. institutional participation in ICU-ROX).

\subsubsection{Elements and sub-elements}

Evidence incorporates the sub-elements of research, clinical experience, patient experience and local information. Aligning with the well-accepted hierarchy of evidence (Polit \& Beck, 2017), systematic reviews and high quality randomised controlled trials are at the high end 
of the scale of evidence for 'Research', while poorly conceived, designed and/or executed research is at the low end. Clinical experience refers to experiences of the health practitioners and to be considered high quality evidence, needs to be critiqued, evaluated and have consensus between groups. Low quality clinical experience evidence would include anecdote or views which lack consensus between groups. The patient experience element typically relates to whether patients can adhere to a specific treatment or intervention. For example, if an intervention was very effective but untenable because of unpleasant side effects, this could make it difficult to implement due to a lack of patient compliance. Rycroft clarified in 2002 that if clinical and patient experience sub-elements were used, these experiences should be subject to the same rigour that high quality research evidence is (Rycroft-Malone, Kitson, et al., 2002). Patient experience would be assessed as high-quality evidence if it had been validated between patient groups, in areas where a partnership with healthcare is important, while low quality might be an implementation strategy where the patient experience is not considered. Local evidence at the high end of the scale may include local data that have been collected and systematically reviewed, whereas local data that have not been reflected on would rank as low quality.

Context is defined as the environment in which healthcare will take place, or the environment in which the evidence will be implemented (McCormack et al., 2002), with the sub-elements being receptive context, culture, leadership and evaluation (Rycroft-Malone, 2004). The environment includes not only the physical environment but also leadership, organisational culture and individuals' beliefs and attitudes to change (Kitson et al., 1998; Rycroft-Malone, 2004; Rycroft-Malone et al., 2013; Stetler et al., 2011).

First articulated as a sub-element in 2002, receptive context (also called contextual readiness) includes physical, social and professional boundaries, decentralised decision making, organisational context, receptiveness to change and sufficient resource allocation for a practice change (Rycroft-Malone, 2004). A high rank for cultural context would incorporate valuing of individual staff and patients, promotion of learning, role consistency and the change being aligned with the strategic focus of the organisation. Low ranking for the cultural context includes unclear values, task-driven change, inconsistency and lack of resourcing. The sub-element of leadership ranks transformational leadership, teamwork, 
role clarity, inclusiveness, enabling and empowering approach to teaching and learning as high. Conversely, a low rank includes autocratic decision making, lack of teamwork and role clarity, and didactic approaches to teaching, learning and managing. A high rank for the evaluation sub-element, means feedback is provided using multiple methods at an individual, team and system level, while a low rank is either an absence of feedback or single rather than multiple methods of feedback.

Facilitation in the PARIHS framework refers to the role of a facilitator who enables others to implement a change (Kitson et al., 1998; Rycroft-Malone, 2004). The sub-elements of Facilitation relate to that facilitator's role, skills and attributes, and the characteristics of a facilitator, and the ability to support and enable practitioners to change are considered significant (Harvey et al., 2002). A high rank includes adult learning techniques and an inclusive approach to learning and teaching, while a low ranking would, once again, be task orientated and traditional approaches to teaching new information. In the revised framework, Stetler et al. (2011) states that the facilitator can be the same leader as in the context element.

\subsubsection{Previous use of PARIHS}

A revised PARIHS framework was successfully used to evaluate the uptake of a guideline to reduce the frequency, severity and duration of post-operative delirium in orthopaedic patients (Powrie et al., 2014). A multidisciplinary group appraised the evidence about postoperative delirium, including characteristics of high-risk patients, prevention, assessment and treatment strategies. The strategies they identified for prevention of delirium included promotion of sleep, optimisation of hydration, nutrition, pain control mobility and using the delirium assessment tool Confusion Assessment Method (CAM). CAM was considered the gold standard for identification of delirium. Following this exploration of evidence, the group were able to identify their target population as patients 65 years and older who had undergone orthopaedic surgery. Through their assessment of context, the group identified barriers of insufficient time to incorporate the new evidence into practice, and inadequate signalling to conduct a CAM assessment. In the facilitation phase the group were proactive, and ensured that physicians, nurse champions and pharmacists were available. The group also incorporated other ways of overcoming barriers by adding cues for the CAM assessment and physicians initiating delirium prevention strategies. In addition to the standard PARIHS 
elements, the researchers included the additional element of assessing whether the guideline was successfully implemented into practice. Despite including additional support and resources, the adoption of CAM was not consistently achieved, however there was a reduction in length of stay for the target population following delirium, and a decrease in mortality rate.

\subsubsection{PARIHS Framework in relation to ICU-ROX TRIPS}

The PARIHS framework had not previously been used in ICU research to evaluate the effect of dissemination and participation from different contexts. An overview of how this framework was used in this research is described in this section and shown in Figure 3.

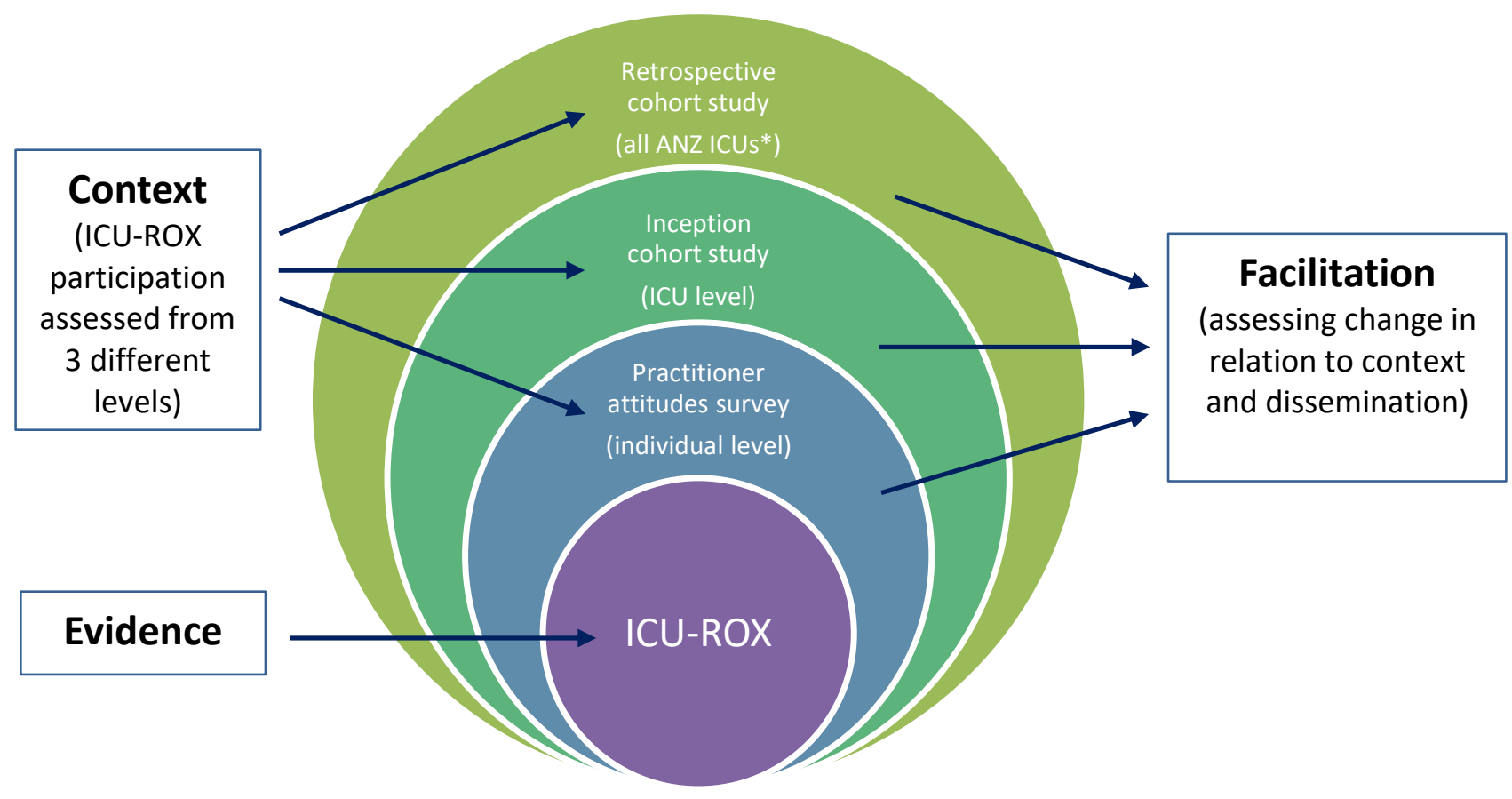

Figure 3 Overview of ICU-ROX TRIPS methods in relation to PARIHS Framework

\subsubsection{Evidence}

The research evidence for ICU-ROX TRIPS is the ICU-ROX trial, and resulting publication in October 2019 (Mackle et al., 2020). ICU-ROX was a well-designed and conducted clinical trial about oxygen management in ICU and can be considered robust evidence because it was multi-centred, fully recruited, is replicable and there was separation between the two treatment groups (Boaz et al., 2015). The ICU-ROX results have contributed to the evidence about oxygen management, however there was no statistically significant difference in the 
primary endpoint of 'alive Ventilator free days to day 28 ' between conservative oxygen therapy and usual oxygen therapy in mechanically ventilated ICU patients.

ICU-ROX TRIPS aimed to assess whether participating in ICU-ROX would influence attitudes and practices about oxygen, irrespective of the findings, compared to non-participation in the ICU-ROX. Grimshaw et al. (2012) argue that evidence from a single trial is not sufficient to change practice, although conceded that funders and other researchers may expect this to happen, as is the case in NZ HRC-funded studies. The evidence about oxygen management in ICU evolved throughout the period of the ICU-ROX TRIPS study was seen in Chapter 2.

As well as empirical evidence, PARIHS incorporates clinical and patient experience, and local information. While these sub-elements do not change the evidence of high quality RCTs and meta-analyses, understanding whether clinical and/or patient experience, and local information affect the attitudes and practices of health practitioners is important when assessing the effects of institutional participation in ICU-ROX.

Clinical experience was assessed in this research by ascertaining the health practitioners' knowledge and understanding of the ICU-ROX results and whether they thought it would change their practice. Because the ICU-ROX results concluded there was no difference in the primary outcome between conservative and usual oxygen therapy, assessing practitioners' interpretation of them in the context of ICU-ROX participation was important. Understanding how they found out about the ICU-ROX results, and how much of the publication they read offered an insight.

Patient experience was not able to be assessed in ICU-ROX TRIPS as all patients eligible for ICU-ROX were unconscious and therefore unable to control the delivery of the oxygen intervention. Local (site-specific) knowledge and barriers to change were not fully assessed in this study; however, this is discussed more in the context element. 


\subsubsection{Context}

In the PARIHS framework, context is equally weighted with the evidence and facilitation elements. However, in ICU-ROX TRIPS, context is the most important element because institutional participation in ICU-ROX is a different context to non-participation in ICU-ROX.

\section{Receptive context}

The receptive context or contextual readiness for knowledge translation could be extended to include the setting the research was conducted in, and the similarity to the setting the research findings are introduced into. The physical environment for ICU-ROX TRIPS was similar between the two groups being compared, and all three ICU-ROX TRIPS studies were set in ANZ, where ICU-ROX was conducted. Organisational context is operationalised through examination of the ICU environments (staffing, funding, bed numbers, metropolitan or rural), engagement in ICU-ROX research (at a site and individual level) and geographical location. The overall context for ICU in the ANZ setting is well supported by a research structure and collaborative network (the ANZICS CTG).

Clinical role (professional boundaries) may be important when assessing the uptake of the ICU-ROX findings. It was not known whether ICU doctors and nurses were equally versed with current evidence about oxygen management, or how they learn this information. Nurses generally experience a high level of autonomy in the ICU setting (Rose et al., 2008), continuously monitoring and assessing patients, and adjusting care and interventions accordingly, including oxygen. These changes are usually made in response to parameters and oxygen saturation targets pre-defined by doctors, protocols or ICU practices, along with nurses' level of clinical expertise. ICU-ROX TRIPS assessed which role was responsible for adjusting oxygen in ICU, and how they decided to make changes to the oxygen level.

A consideration for ICU-ROX TRIPS was the role of health practitioners as researchers because of their participation in ICU-ROX. This study assessed the different levels of participation in ICU-ROX by the respondents (whether they worked in an ICU that participated in ICU-ROX or not, enrolled a patient or cared for a patient in ICU-ROX or no participation). It compared ICUs that participated with those that did not and assessed 
whether participation increased their investment in the ICU-ROX findings and meant they were more likely to have knowledge of or use the results.

\section{Culture}

Participation in research may add to the culture of knowledge use and change in an ICU. ICU culture was operationalised in ICU-ROX TRIPS through assessing each ICU's level of participation in research. This included collecting and analysing data about the research activity and the number of research staff employed, along with whether the findings of research they participate in is disseminated to ICU staff. It is plausible that health practitioners in an ICU where research is active, would be more aware of and receptive to new research than in other ICUs. Research is reportedly used more compliantly in acute care than for chronic settings (Grol \& Grimshaw, 2003). A recent study from Salter et al., (2018) showed the speed with which practice can change in response to new evidence. In their study which reviewed the use of targeted temperature management (TTM) following cardiac arrest, Salter et al. showed that the average lowest temperature in the first 24 hours of ICU, rose after the publication of a TTM RCT which reported no difference between cooling to $33^{\circ} \mathrm{C}$ or $36^{\circ} \mathrm{C}$ (Nielsen et al., 2013). This early adoption of new evidence may reflect an embedded culture of research, based on the College of Intensive Care Medicine (2016), requirement for Level II and III ANZ ICUs to have an active research programme.

\section{Leadership / structure}

Whether leadership was transformational was difficult to evaluate in this study. The structure of change management and whether decision-making is at an ICU level (decentralised) was not known. Data were gathered regarding change makers, and ways that information about ICU-ROX was received, and the organisational structure of research departments within the ICUs. Individual beliefs and attitudes to research in general are not included in this research, however staff attitudes to ICU-ROX specifically are assessed through their knowledge and use of the ICU-ROX results, within the context of institutional participation or non-participation. 


\subsubsection{Facilitation}

ICU-ROX TRIPS diverges from PARIHS with regard to facilitation as PARIHS specifically refers to the Facilitation element being a facilitator role or person. Rather, facilitation was operationalised to examine the research and knowledge translation infrastructures used within ICUs, staff awareness of the ICU-ROX results and dissemination of ICU-ROX results.

\subsubsection{Balance between the elements}

The original authors of the PARIHS framework stated that successful implementation of an intervention is determined by the balance and equal weighting of the three elements (Kitson et al., 1998). For ICU-ROX TRIPS, there is considerable crossover between evidence, context and facilitation. For example, while dissemination of results sits within the facilitation element, whether an ICU actively disseminated the results is also about context and specifically, culture. Likewise, the facilitation and role of facilitator is unlikely to make any difference if the overall culture and leadership of a clinical area were opposed to change. While quality of evidence is extremely important, it was unknown whether the quality of the ICU-ROX evidence would have less impact on whether that evidence was implemented than the context of participating in ICU-ROX. Likewise, the results of ICU-ROX are evidence, yet the value of that evidence is related to the context in which it is received.

\subsection{Methodological considerations}

Incorporating the best design features identified in the literature review, a multiple method approach using three separate studies was adopted for ICU-ROX TRIPS as this enabled the overall study aim to be addressed and the research questions to be answered rigorously. A multiple method design aligns with both postpositivism and the PARIHS framework.

\subsubsection{Multiple method design}

A multiple method approach uses two or more methods that gather either all qualitative or all quantitative data and contribute to an overall programme of research (Hess-Biber \& Johnson, 2015; Morse, 2010). Each study of a multiple method project is complete within itself and can be published individually, each contributing to part or all of the overall research question/s (Morse, 2010). Using multiple methods provides different perspectives to the overall research question, reducing the risk of inherent bias and strengthening the overall 
findings (Letourneau \& Allen, 1999; Morse, 2010). There may have been earlier studies, but the term was first found in the literature in 1959 by Campbell and Fiske. Multiple method research is often confused with mixed methods design which uses both quantitative data and qualitative data in a way which, when combined, uses the strength of each design to validate the findings (Creswell, 2014).

A multiple methods approach was chosen for ICU-ROX TRIPS because it enabled the research questions to be answered from different inter-related viewpoints. The multiple methods employed for ICU-ROX TRIPS were survey of health practitioner attitudes, inception cohort study and retrospective cohort study of oxygen practices. The surveys and inception cohort studies were conducted concurrently in each of the ICU-ROX TRIPS study ICUs, and their relationship can be considered to be complementary to each other, intended to enhance each other and be treated with the same weighting (Davis et al., 2010). The retrospective cohort study added depth to the overall argument of the research by analysing routinely collected data from all ANZ ICUs that contribute to the ANZICS adult patient database (APD). This layering of information, from a small group of health practitioners completing the survey, to a larger cohort affecting patient care in the inception cohort study and an even larger cohort in the retrospective cohort study, aimed to strengthen the overall findings. While all three studies are separate, standalone and could be published separately, they all contribute to the primary aim which is to understand the effect of ICU-level participation in ICU-ROX on the knowledge translation of that trial.

\subsection{Overall study design and setting/timing}

Figure 4 shows the sequence and timing of the three methods. The practitioner attitudes survey and inception cohort study commenced prior to ICU-ROX starting to provide baseline data about attitudes and practices (T1). These studies were conducted again after ICU-ROX had finished enrolling, but prior to publication or knowledge of the ICU-ROX results (T2) to assess the change in attitudes and practices after participating in ICU-ROX (or not). The practitioner attitudes survey and inception cohort study were conducted again one to six months after the ICU-ROX results were published (T3) to compare attitudes and practices between the two groups, after the ICU-ROX results were known. The retrospective cohort study was conducted at the end of the study period and using existing registry data, reviewed oxygen management 48 weeks before and 48 weeks after ICU-ROX was published. 


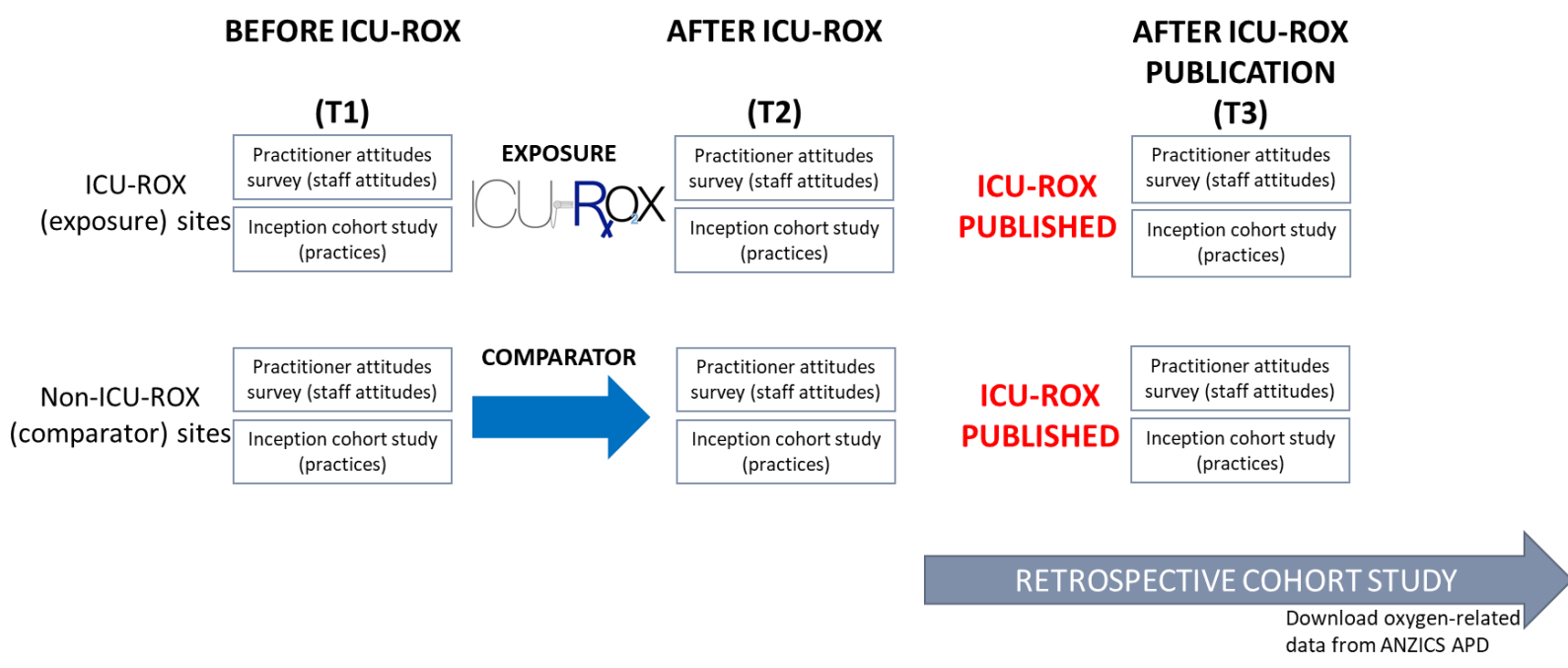

Figure 4 Structure and timing of multiple methods for ICU-ROX TRIPS

Abbreviations: ANZICS APD: Australian and New Zealand Intensive Care Society adult patient database; ICUROX: Intensive Care Unit Randomised Trial Comparing Two Approaches to Oxygen therapy trial; ICU-ROX sites: sites that participated in the ICU-ROX trial; non-ICU-ROX sites: sites that did not participate in the ICUROX trial; T1: before ICU-ROX commenced; T2: after ICU-ROX was completed enrolment but before results were known; T3: after ICU-ROX publication.

Comparing attitudes and practices using multiple exposure and comparators (ICU-ROX vs non-ICU-ROX sites and before and after ICU-ROX) were drawn from the literature to show how the effects of participation may change over time. With no consistent timeframe in the literature to guide this study the decision was made to collect data both immediately after the trial was finished, but before the results were known and again one to six months after publication of ICU-ROX.

The series of surveys were designed to investigate the research questions about the attitudes of healthcare practitioners with regard to oxygen management. They involved self-reported data using survey design as used in five of the publications discussed in the literature review (Das et al., 2008; Khera et al., 2018; Litjens et al., 2013; Sweeting et al., 2020; Tranmer et al., 2002). In addition, the use of bespoke surveys in the literature informed the decision to use this approach in the current research. The series of inception cohort studies, were designed to answer the research question about oxygen practices in ventilated patients in ICU. These observational data captured oxygen management (conducted concurrently but independently of the survey), and provided evidence about what happens in practice. The retrospective cohort study used existing registry data, similar to four studies reviewed in Chapter 4 (Andersen et al., 2006; Kizer et al., 1999; Majumdar et 
al., 2002; Shah et al., 2010). This method has also been used in previous ICU studies (Kaukonen et al., 2013; Salter et al., 2018). In this current research the aim was to analyse temporal trends in ICU oxygen management over time in ANZ ICUs, in relation to the ICUROX publication. The work about engagement outlined in the literature (Litjens et al., 2013) was built on in ICU-ROX TRIPS, by including a question about whether respondents enrolled a patient into ICU-ROX or not.

As described in Chapter 4, effects of participation in research trials on knowledge translation has mainly been limited to doctors. Oxygen is considered very relevant to all health practitioners who work in ICU. To ascertain how relevant the findings of ICU-ROX were, a question was incorporated into the survey to find out who adjusts oxygen in the ICU, and how respondents thought the ICU-ROX results would change their practice.

Table 4 shows how the decision to use the three methods chosen to answer meet the study objectives in a comprehensive way, linking these decisions to the PARIHS framework. 
Table 4 Overview of methods used to meet objectives in relation to the PARIHS framework

\begin{tabular}{|c|c|c|c|c|c|}
\hline & Study objective & Relationship to PARIHS & Method & Data generated & Setting \\
\hline 1. & $\begin{array}{l}\text { Establish baseline attitudes about } \\
\text { oxygen management of health } \\
\text { practitioners }\end{array}$ & $\begin{array}{l}\text { Context } \\
2 \text { groups of respondents } \\
\text { (ICU-ROX sites and non-ICU- } \\
\text { ROX sites) }\end{array}$ & Survey (T1) & $\begin{array}{l}\text { Likelihood of adjusting } \\
\text { oxygen in series of clinical } \\
\text { scenarios and levels of } \\
\text { oxygen. }\end{array}$ & $\begin{array}{l}\text { ANZ ICUs } \\
\text { Staff from: } \\
\text { ICU-ROX sites } \\
\text { (exposure) } \\
\text { Non-ICU-ROX sites } \\
\text { (comparator) }\end{array}$ \\
\hline 2. & $\begin{array}{l}\text { Establish baseline practices regarding } \\
\text { oxygen management }\end{array}$ & $\begin{array}{l}\text { Context } \\
2 \text { groups of respondents } \\
\text { (ICU-ROX sites and non-ICU- } \\
\text { ROX sites) }\end{array}$ & $\begin{array}{l}\text { Inception cohort } \\
\text { study (T1) }\end{array}$ & $\begin{array}{l}\text { Time spent at } \mathrm{F}_{1} \mathrm{O}_{2} 0.21 \\
\text { (room air) for } \mathrm{ICU}-\mathrm{ROX} \text { and } \\
\text { non-ICU-ROX site patients }\end{array}$ & $\begin{array}{l}\text { ANZ ICUs } \\
\text { Patient data from: } \\
\text { ICU-ROX sites } \\
\text { (exposure) } \\
\text { Non-ICU-ROX sites } \\
\text { (comparator) }\end{array}$ \\
\hline 3. & $\begin{array}{l}\text { Establish whether there was an } \\
\text { attitude change about oxygen } \\
\text { management in health practitioners } \\
\text { from ANZ ICUs after participation in } \\
\text { ICU-ROX (but before the results are } \\
\text { known), compared to those from } \\
\text { ICUs that did not participate }\end{array}$ & $\begin{array}{l}\text { Evidence (clinician } \\
\text { experience) } \\
\text { Context - participation in } \\
\text { ICU-ROX vs non-participation }\end{array}$ & $\begin{array}{l}\text { Survey } \\
\text { T1->T2 }\end{array}$ & $\begin{array}{l}\text { Difference in likelihood of } \\
\text { adjusting oxygen in clinical } \\
\text { scenarios. Responses at T1 } \\
\text { compared with responses } \\
\text { at T2; and between ICU- } \\
\text { ROX and non-ICU-ROX sites }\end{array}$ & $\begin{array}{l}\text { ANZ ICUs } \\
\text { Staff from: } \\
\text { ICU-ROX sites } \\
\text { Non-ICU-ROX sites }\end{array}$ \\
\hline 4. & $\begin{array}{l}\text { Establish whether there was a } \\
\text { practice change regarding oxygen } \\
\text { management in ANZ ICUs after } \\
\text { participation in ICU-ROX (but before } \\
\text { the results are known), compared to } \\
\text { ICUs that did not participate in ICU- } \\
\text { ROX }\end{array}$ & $\begin{array}{l}\text { Evidence (clinician } \\
\text { experience) } \\
\text { Context - participation in } \\
\text { ICU-ROX vs non-participation }\end{array}$ & $\begin{array}{l}\text { Inception cohort } \\
\text { study } \\
\text { T1->T2 }\end{array}$ & $\begin{array}{l}\text { Change in the time spent at } \\
\mathrm{F}_{1} \mathrm{O}_{2} 0.21 \text { between } \\
\mathrm{T} 1 \text { and } \mathrm{T} 2 \text { for ICU-ROX } \\
\mathrm{T} 1 \text { and } \mathrm{T} 2 \text { non-ICU-ROX }\end{array}$ & $\begin{array}{l}\text { ANZ ICUs } \\
\text { Patient data from: } \\
\text { ICU-ROX sites } \\
\text { Non-ICU-ROX sites }\end{array}$ \\
\hline
\end{tabular}




\begin{tabular}{|c|c|c|c|c|c|}
\hline & Study objective & Relationship to PARIHS & Method & Data generated & Setting \\
\hline 5. & $\begin{array}{l}\text { Establish whether there is an attitude } \\
\text { change about oxygen management in } \\
\text { health practitioners from ANZ ICUs } \\
\text { that participated in ICU-ROX } \\
\text { (compared to those that did not) 1-6 } \\
\text { months after publication of the ICU- } \\
\text { ROX results }\end{array}$ & $\begin{array}{l}\text { Evidence - ICU-ROX results } \\
\text { Context - participation in } \\
\text { ICU-ROX vs non-participation } \\
\text { Facilitation - dissemination } \\
\text { of ICU-ROX results }\end{array}$ & Survey T2->T3 & $\begin{array}{l}\text { Difference in likelihood of } \\
\text { adjusting oxygen in clinical } \\
\text { scenarios. Responses at T2 } \\
\text { compared with responses } \\
\text { at T3; and between ICU- } \\
\text { ROX and non-ICU-ROX sites }\end{array}$ & $\begin{array}{l}\text { ANZ ICUs } \\
\text { Staff from: } \\
\text { ICU-ROX sites } \\
\text { Non-ICU-ROX sites }\end{array}$ \\
\hline 6. & $\begin{array}{l}\text { Establish whether there is a practice } \\
\text { change regarding oxygen } \\
\text { management in ANZ ICUs that } \\
\text { participated in ICU-ROX (compared to } \\
\text { those that did not) 1-6 months after } \\
\text { publication of the ICU-ROX results }\end{array}$ & $\begin{array}{l}\text { Evidence - ICU-ROX results } \\
\text { Context - participation in } \\
\text { ICU-ROX vs non-participation } \\
\text { Facilitation - dissemination } \\
\text { of ICU-ROX results }\end{array}$ & $\begin{array}{l}\text { Inception cohort } \\
\text { study T1->T3 }\end{array}$ & $\begin{array}{l}\text { Change in the time spent at } \\
\mathrm{F}_{1} \mathrm{O}_{2} 0.21 \\
\mathrm{~T} 1 \text { and } \mathrm{T} 3 \text { for ICU-ROX } \\
\mathrm{T} 1 \text { and } \mathrm{T} 3 \text { non-ICU-ROX }\end{array}$ & $\begin{array}{l}\text { ANZ ICUs } \\
\text { Patient data from: } \\
\text { ICU-ROX sites } \\
\text { Non-ICU-ROX sites }\end{array}$ \\
\hline 8. & $\begin{array}{l}\text { Determine whether participation ICU- } \\
\text { ROX influenced the knowledge about } \\
\text { the ICU-ROX publication by } \\
\text { comparing health practitioners from } \\
\text { participating ICUs to those from non- } \\
\text { ICU-ROX ICUs; and those who } \\
\text { enrolled or cared for a patient and } \\
\text { those who did not }\end{array}$ & $\begin{array}{l}\text { Evidence - ICU-ROX results } \\
\text { Context - participation in } \\
\text { ICU-ROX vs non-participation } \\
\text { Facilitation - dissemination } \\
\text { of ICU-ROX results }\end{array}$ & Survey (T3) & $\begin{array}{l}\text { Knowledge of results } \\
\text { Enrolled a patient (or not) } \\
\text { Cared for a patient (or not) }\end{array}$ & $\begin{array}{l}\text { ANZ ICUs } \\
\text { Staff from: } \\
\text { ICU-ROX sites and } \\
\text { Non-ICU-ROX sites }\end{array}$ \\
\hline 9. & $\begin{array}{l}\text { Determine whether there was a } \\
\text { difference in attitudes regarding } \\
\text { oxygen management between ICU } \\
\text { specialist doctors and ICU nurses } \\
\text { before ICU-ROX, after ICU-ROX but } \\
\text { before the results were known and } \\
\text { again after ICU-ROX was published }\end{array}$ & $\begin{array}{l}\text { Evidence (clinician } \\
\text { experience) - ICU-ROX } \\
\text { results } \\
\text { Context-doctors vs nurses } \\
\text { Facilitation - dissemination } \\
\text { of ICU-ROX results }\end{array}$ & $\begin{array}{l}\text { Survey } \\
\text { T1->T2 } \\
\text { T2-> T3 }\end{array}$ & $\begin{array}{l}\text { Difference in likelihood of } \\
\text { adjusting oxygen in clinical } \\
\text { scenarios. Responses at T1 } \\
\text { compared to T2; Responses } \\
\text { at T2 compared with } \\
\text { responses at T3; and } \\
\text { between ICU doctors and } \\
\text { ICU Nurses }\end{array}$ & $\begin{array}{l}\text { ANZ ICUs } \\
\text { Staff from: } \\
\text { ICU-ROX sites and } \\
\text { Non-ICU-ROX sites }\end{array}$ \\
\hline
\end{tabular}




\begin{tabular}{|c|c|c|c|c|c|}
\hline & Study objective & Relationship to PARIHS & Method & Data generated & Setting \\
\hline 10. & $\begin{array}{l}\text { Determine whether a practice change } \\
\text { regarding oxygen management was } \\
\text { seen in all ANZ ICUs over time, when } \\
\text { reviewed } 48 \text { weeks after publication } \\
\text { of the ICU-ROX results, compared to } \\
48 \text { weeks prior to publication, and } \\
\text { whether this was different between } \\
\text { ICU-ROX and non-ICU-ROX sites }\end{array}$ & $\begin{array}{l}\text { Context } \\
\text { Comparing changes between } \\
\text { groups near where evidence } \\
\text { was created and distant }\end{array}$ & $\begin{array}{l}\text { Retrospective } \\
\text { cohort study }\end{array}$ & $\begin{array}{l}\mathrm{F}_{1} \mathrm{O}_{2} \text { and } \mathrm{PaO}_{2} \text { from the } \mathrm{ABG} \\
\text { taken during the first } 24 \\
\text { hours of the } \mathrm{ICU} \text { admission } \\
\text { that produces the highest } \\
\text { (worst) score using the } \\
\text { APACHE III-J scoring } \\
\text { algorithm }\end{array}$ & $\begin{array}{l}\text { All ANZ ICUs (except } \\
\text { pilot sites) } \\
\text { ICU-ROX sites } \\
\text { Non-ICU-ROX sites }\end{array}$ \\
\hline 11. & $\begin{array}{l}\text { Explore whether there was a } \\
\text { difference between ICU specialist } \\
\text { doctors and ICU nurses regarding } \\
\text { their level of knowledge about the } \\
\text { ICU-ROX results and publication, and } \\
\text { the ways they found out about the } \\
\text { results }\end{array}$ & $\begin{array}{l}\text { Evidence - ICU-ROX results } \\
\text { Context - doctors vs nurses } \\
\text { Facilitation - dissemination } \\
\text { of ICU-ROX results }\end{array}$ & Survey (T3) & $\begin{array}{l}\text { Knowledge of results } \\
\text { Ways of finding out results }\end{array}$ & $\begin{array}{l}\text { ANZ ICUs } \\
\text { Staff from: } \\
\text { ICU-ROX sites and } \\
\text { Non-ICU-ROX sites }\end{array}$ \\
\hline 12. & $\begin{array}{l}\text { Explore existing systems for } \\
\text { knowledge translation and change } \\
\text { management for each participating } \\
\text { ICUs. }\end{array}$ & Facilitation & $\begin{array}{l}\text { Survey (ICU- } \\
\text { level) }\end{array}$ & $\begin{array}{l}\text { Dissemination strategies } \\
\text { Open-ended questions } \\
\text { about: } \\
\text { Research champion } \\
\text { Knowledge leader } \\
\text { Change management }\end{array}$ & $\begin{array}{l}\text { ANZ ICUs } \\
\text { Research staff from: } \\
\text { ICU-ROX sites and } \\
\text { non-ICU-ROX sites }\end{array}$ \\
\hline
\end{tabular}

Abbreviations: ABG: arterial blood gas; ANZ: Australian and New Zealand; APACHE-III-J: Acute Physiology and Chronic Health Evaluation; FiOz: fraction of inspired oxygen; ICU: Intensive Care Unit; ICU-ROX sites: ICUs that participated in the ICU-ROX trial; non-ICU-ROX sites: ICUs that did not participate in the ICU-ROX trial; non-ICU-ROX; PaO2: partial pressure of arterial oxygen; T1: before ICU-ROX commenced; T2: after ICU-ROX was complete but before results were known; T3: after ICU-ROX publication. 


\subsubsection{Quantitative data}

All three studies for ICU-ROX TRIPS were observational and used quantitative data. The survey has one open question for any comments and at the third time point included openended questions about change management and knowledge translation practices including dissemination, oxygen management, and knowledge of ICU-ROX. Although open-ended and therefore text data were collected, ICU-ROX TRIPS could not be considered a mixed method study because the survey questions were designed to obtain specific information and did not use the depth of what would be achieved with a truly qualitative approach.

\subsection{Exposure and comparator}

Key to this study are the exposure and comparator and the differences between them. Although many staff in both the exposure (ICU-ROX sites) and comparator (non-ICU-ROX) arms may have been aware of the ICU-ROX trial and the results, the level of exposure to the trial protocol differed (Table 5).

\subsubsection{Exposure arm (ICU-ROX sites)}

ICUs in the exposure arm of ICU-ROX TRIPS participated in ICU-ROX. This participation in ICU-ROX required detailed knowledge of the ICU-ROX protocol and comprised training, daily engagement with the protocol, and frequent communication, before, during and after ICUROX was conducted.

Research staff at the ICU-ROX ICUs had access to, and in-depth knowledge of, the ICU-ROX trial protocol before ICU-ROX started. These research staff were trained in all aspects of the ICU-ROX protocol by attending start-up meetings prior to initiating the trial in their ICUs. They were also responsible for applying for ethics and local governance approval, including consultation with Māori for the NZ sites involved. Successful participation in a clinical trial such as ICU-ROX requires engagement of all staff in the ICU, and site research staff ran extensive education programmes for ICU doctors and nurses prior to ICU-ROX starting and ongoing training during the trial.

During the ICU-ROX trial, research staff ensured adherence to the protocolised trial treatments. Patients were randomised to either conservative or usual oxygen therapy, and doctors and nurses caring for them needed to understand both interventions. Research staff 
produced or adapted tools (provided by the coordinating centre) for use at the bedside to ensure ICU-ROX protocol understanding and adherence in their ICU, and reviewed protocol compliance and data collection regularly.

Table 5 Differences in exposure to ICU-ROX between ICU-ROX and non-ICU-ROX sites

\begin{tabular}{|c|c|c|}
\hline Nature of exposure & $\begin{array}{c}\text { Exposure } \\
\text { (ICU-ROX sites) }\end{array}$ & $\begin{array}{c}\text { Comparator } \\
\text { (Non-ICU-ROX sites) }\end{array}$ \\
\hline Provided with ICU-ROX trial protocol & Yes (research staff) & No \\
\hline $\begin{array}{l}\text { Administer ICU-ROX protocolised treatment } \\
\text { (conservative and usual care) }\end{array}$ & Yes & No \\
\hline $\begin{array}{l}\text { Education about ICU-ROX at beginning and } \\
\text { throughout study }\end{array}$ & Yes & No \\
\hline $\begin{array}{l}\text { Bedside tools - adjusting oxygen according to } \\
\text { protocol }\end{array}$ & Yes & No \\
\hline $\begin{array}{l}\text { Received feedback about protocol adherence and } \\
\text { recruitment }\end{array}$ & Yes & No \\
\hline Remote monitoring & Yes (research staff) & No \\
\hline On-site monitoring & Yes (research staff) & No \\
\hline Regular recruitment updates and newsletters & Yes (research staff) & No \\
\hline $\begin{array}{l}\text { Closed investigator results meeting } \\
\text { (February 2019) }\end{array}$ & Yes (research staff) & No \\
\hline $\begin{array}{l}\text { Closed ANZICS CTG results meeting } \\
\text { (March 2019) }\end{array}$ & Yes (research staff) & Yes (research staff) \\
\hline $\begin{array}{l}\text { World Congress presentation } \\
\text { (October 2019) }\end{array}$ & Yes & Yes \\
\hline $\begin{array}{l}\text { Critical Care Review presentation } \\
\text { (January 2020) }\end{array}$ & Yes & Yes \\
\hline Provided with NEJM publication & Yes (research staff) & No \\
\hline Access to NEJM publication & Yes & Yes \\
\hline $\begin{array}{l}\text { Encouraged to follow twitter handle \#ICU- } \\
\text { ROXTRIAL throughout study }\end{array}$ & Yes & No \\
\hline $\begin{array}{l}\text { Able to follow twitter handle \#ICUROXtrial } \\
\text { throughout study }\end{array}$ & Yes & Yes \\
\hline Tweetorial - summary of results & Yes & Yes \\
\hline Regular ICU-ROX updates at CTG meetings & Yes (research staff) & Yes (research staff) \\
\hline Regular ICU-ROX TRIPS updates at CTG meetings & Yes (research staff) & Yes (research staff) \\
\hline
\end{tabular}

Abbreviations: ANZICS CTG: Australian and New Zealand Intensive Care Society Clinical Trials Group; ICU-ROX: Intensive Care Unit Randomised Trial Comparing Two Approaches to Oxygen therapy; ICU-ROX TRIPS: ICUROX Translating Research Into Practice Study; NEJM: New England Journal of Medicine.

Research staff at ICU-ROX sites received frequent communication from the co-ordinating centre/s. This included regular updates about recruitment, emails about study milestones and newsletters. This communication was sent only to site research staff, who were 
expected to pass important information onto the clinical staff if required. Site research staff were encouraged to follow the \#ICUROXtrial hashtag and the chief investigator's twitter handle (@dogICUma), which included regular recruitment updates.

ICU-ROX site staff were contacted regularly by coordinating centre staff regarding monitoring requirements. ICU-ROX was monitored according to a pre-specified monitoring plan, requiring both regular on-site visits and remote contact for data clarification via the study database and email. Throughout ICU-ROX, data were reviewed for internal validity, through a series of pre-specified logic queries. Site research staff were required to investigate data queries and respond. Coordinating centre staff also contacted site research staff about protocol adherence and recruitment rates in particular when either of these fell below an acceptable standard, identified through reporting and site self-identification of issues.

After ICU-ROX completed recruitment, site research staff continued to be contacted frequently. This was to verify data from the data cleaning process, ensure all patient consents had been obtained or notified to the local ethics committees, where required. Once data had been analysed, site research staff were invited to attend a 'results' meeting in February 2019, prior to publication of the ICU-ROX results, conducted via videoconferencing. Meeting attendees were not permitted to disseminate the results until ICUROX had been published, six months later. ICU-ROX sites were notified that the results would be announced publicly at the World Congress in Melbourne and published simultaneously in October 2019. Following this presentation, a copy of the publication was sent to the sites that had participated in ICU-ROX. No direction was given about how, or whether this should be disseminated to staff. All ICU-ROX site research staff were included as authors in the supplementary appendix of the ICU-ROX publication (Mackle et al., 2020).

\subsubsection{Comparator (non-ICU-ROX sites)}

The ICU-ROX TRIPS comparator arm was non-participation in the ICU-ROX trial. Both research and other staff from ICUs in the non-ICU-ROX arm may have been exposed to some presentations about ICU-ROX if they had attended meetings at which an update or results were presented (Table 5). All staff were able to 'follow' the trial progress on Twitter, although this was not actively promoted to them. 


\subsection{Ethical approval}

Ethics approval was required in New Zealand and Australia for the ICU-ROX TRIPS studies. Copies of all approvals are in Appendices 2 and 3, and summarised in Table 6. Ethical issues that were identified concerned the survey not being anonymous, and the inception cohort study being conducted on vulnerable patients. All ethical and local governance approvals were completed prior to the commencement of any site. Ethical approval for the ICU-ROX trial was approved separately and does not affect this study.

Table 6 Ethics requirements and submission by region

\begin{tabular}{|c|c|c|c|c|c|}
\hline State/ Country & Lead site & $\begin{array}{l}\text { Number } \\
\text { of sites }\end{array}$ & $\begin{array}{c}\text { Practitioner } \\
\text { attitudes } \\
\text { survey }\end{array}$ & $\begin{array}{l}\text { Inception } \\
\text { cohort study }\end{array}$ & $\begin{array}{l}\text { Retrospective } \\
\text { cohort study }\end{array}$ \\
\hline \multicolumn{6}{|l|}{ New Zealand } \\
\hline $\begin{array}{l}\text { National } \\
\text { application }\end{array}$ & $\begin{array}{c}\text { MRINZ } \\
\text { (Researcher) }\end{array}$ & 7 & $\begin{array}{c}\text { Victoria } \\
\text { University of } \\
\text { Wellington }\end{array}$ & $\begin{array}{c}\text { HDEC } \\
\text { Northern B } \\
\text { Committee }\end{array}$ & $\begin{array}{c}\text { HDEC } \\
\text { Northern B } \\
\text { Committee }\end{array}$ \\
\hline \multicolumn{6}{|l|}{ Australia } \\
\hline $\begin{array}{l}\text { Australian Capital } \\
\text { Territories }\end{array}$ & $\begin{array}{l}\text { Canberra } \\
\text { Hospital }\end{array}$ & 1 & $\begin{array}{c}\text { Low risk } \\
\text { application }\end{array}$ & $\begin{array}{c}\text { Low risk } \\
\text { application }\end{array}$ & $\begin{array}{c}\text { Low risk } \\
\text { application }\end{array}$ \\
\hline New South Wales & $\begin{array}{l}\text { Royal North } \\
\text { Shore } \\
\text { Hospital }\end{array}$ & 6 & $\begin{array}{l}\text { Low-negligible } \\
\text { risk application }\end{array}$ & $\begin{array}{l}\text { Low-negligible } \\
\text { risk application }\end{array}$ & $\begin{array}{l}\text { Low-negligible } \\
\text { risk application }\end{array}$ \\
\hline South Australia & $\begin{array}{c}\text { Lyell McEwin } \\
\text { Hospital }\end{array}$ & 3 & $\begin{array}{l}\text { Low-negligible } \\
\text { risk application }\end{array}$ & $\begin{array}{l}\text { Low-negligible } \\
\text { risk application }\end{array}$ & $\begin{array}{l}\text { Low-negligible } \\
\text { risk application }\end{array}$ \\
\hline Tasmania & $\begin{array}{c}\text { Launceston } \\
\text { Hospital }\end{array}$ & 1 & $\begin{array}{l}\text { Low-negligible } \\
\text { risk application }\end{array}$ & $\begin{array}{l}\text { Low-negligible } \\
\text { risk application }\end{array}$ & $\begin{array}{l}\text { Low-negligible } \\
\text { risk application }\end{array}$ \\
\hline Victoria & $\begin{array}{c}\text { Alfred } \\
\text { Hospital }\end{array}$ & 3 & $\begin{array}{c}\text { Victoria } \\
\text { University of } \\
\text { Wellington }\end{array}$ & $\begin{array}{l}\text { Low-negligible } \\
\text { risk application }\end{array}$ & $\begin{array}{l}\text { Low-negligible } \\
\text { risk application }\end{array}$ \\
\hline Victoria & $\begin{array}{l}\text { Cabrini } \\
\text { Hospital } \\
\text { (private) }\end{array}$ & 1 & $\begin{array}{l}\text { Low-negligible } \\
\text { risk application }\end{array}$ & $\begin{array}{l}\text { Low-negligible } \\
\text { risk application }\end{array}$ & $\begin{array}{l}\text { Low-negligible } \\
\text { risk application }\end{array}$ \\
\hline
\end{tabular}

Abbreviations: HDEC: Health and Disability Ethics Committee; MRINZ: Medical Research Institute of New Zealand.

The practitioner attitudes survey could not be conducted anonymously because the same individuals were contacted to conduct the survey at the second and third time periods. An information sheet (Appendix 4) outlining this was sent to all prospective respondents, and completion of the survey presumed consent. As an additional measure, RCs at each ICU coordinating this study advised the staff they needed to provide an email address. Data were de-identified prior to analysis. Confidentiality was maintained by emailing respondents using a 'blind copy' function, so that although their email addresses were known by the researcher, other respondents did not have this information. 
As the inception cohort study was being conducted in a vulnerable patient group (unconscious ICU patients), both ethics approval and local governance approval were required at each site conducting the study. Data were de-identified, with participants identified by a unique study identifier, and only data that are routinely collected as part of clinical care were used. Sites kept a patient enrolment log, which links the patient unique identifier to the study identifier. This log was retained at the site, and never shared with the researcher or supervisors. The retrospective cohort study used data from the ANZICS APD, which are de-identified and routinely collected as part of ICU clinical care and benchmarking. The researcher had no access to identifiable information, and only pooled data were used for analysis.

The practitioner attitudes survey was submitted to Victoria University of Wellington Ethics Committee, and approval was granted on 27 October 2016 (reference \#23657) (Appendix 2). An amendment to the Information Sheet (Appendix 4) and survey (for the $3^{\text {rd }}$ time point) was approved on 10 September 2019. The purpose of this was amendment was that the third time point was changed from ' 6 to 12 months' to ' 1 to 12 months', to increase the retention of respondents by shortening the period after ICU-ROX publication to contact them for the third time. The full 12 months was not required and the studies finished six months after publication. Because the inception cohort study was collecting data on vulnerable patients without patient consent, a full ethics application was required by the Health and Disability Ethics committee (HDEC) in New Zealand. All three studies were included in the study protocol which was submitted with acknowledgement by HDEC that the practitioner attitudes study did not meet their minimum requirement for ethics approval, and acknowledgement that it had been approved by Victoria University of Wellington. The remaining two methods (inception cohort study and retrospective cohort study) were approved on 8 December 2016 (reference 16/NTA/177) (Appendix 2).

The ethics process in Australia varies between states. The five states/territories involved in this study were Victoria, New South Wales, South Australia, Tasmania and Australian Capital Territories. The ethics committee at Monash University, Victoria, Australia (where the ANZIC-Research Centre is situated) was consulted about whether ethics approval would be required for the practitioner attitudes survey in Australia, and they advised that as this had been approved in NZ, and was considered low risk (with no patient involvement), it would 
not be necessary. The initial ethics application in Australia was made by the Alfred Hospital in Victoria. All other states elected to submit the practitioner attitudes survey again as part of the whole protocol, to avoid confusion for the ethics committees. This study was able to be submitted as low-negligible risk in Australia because it was an observational (noninterventional) study. A lead site research coordinator in each Australian state volunteered to submit the study to ethics. Once the lead site had ethics approval, each participating ICU obtained approval from their local research governance office.

\subsection{Chapter conclusion}

This chapter has shown that postpositivism is the best paradigm for this research, allowing for the multiple realities associated with, or interpretations of, results, whilst acknowledging the evidence (ICU-ROX results) is based in science. Postpositivism is an appropriate worldview for both the PARIHS knowledge translation framework and the multiple method approach used for ICU-ROX TRIPS. Multiple methods allowed the research questions to be answered from different viewpoints, using different data, and reducing bias.

Critical to the design of this study was the choice of the PARIHS framework, which provides a lens and a framework to structure this knowledge translation study. PARIHS encompasses the evidence, context and facilitation elements, and can be used retrospectively in an acute care setting. The evidence for ICU-ROX TRIPS is predominantly the ICU-ROX trial results, along with the clinical experience of participating in ICU-ROX, and facilitation is dissemination of those results. Context is considered the most important element, encompassing the main variable being analysed which is institutional participation or nonparticipation in ICU-ROX, along with the context in which ICU-ROX was conducted, compared with the ICUs in which the results were received.

Multiple methods were required to answer the research questions, making it a sound choice. The methods chosen have been outlined, and their relationship to each other and the study objectives shown. Differences between the exposure and comparator have been explained and ethical considerations and approvals provided. 
The next three chapters are standalone chapters, including the hypotheses, design and methods, results and discussion for each of the three studies: practitioner attitudes survey, inception cohort study and retrospective cohort study. 


\section{CHAPTER 6 PRACTITIONER ATTITUDES SURVEY}

This chapter begins with the hypotheses, design, methods, results and discussion for the practitioner attitudes survey. The survey and the inception cohort study (Chapter 7 ) were conducted concurrently in the same ICUs, and site selection is described in this chapter, followed by the details specific to the survey.

The primary aim of the practitioner attitudes survey was to understand whether attitudes changed regarding oxygen therapy management in ICU-ROX sites compared to non-ICUROX sites. The survey was administered to the same people three times. To assess their baseline attitudes, the survey was administered before the ICU-ROX trial started at each site for the ICU-ROX group, and as soon as ethics was approved and prior to overall ICUROX enrolment completion for the non-ICU-ROX group, resulting in a staggered start time (T1). The survey was administered again from 28 days after ICU-ROX had finished, but before the results were known (T2). The final survey was sent to respondents again one month after the ICU-ROX results were published (T3); pending ethical and governance approvals for a protocol amendment. The final survey was closed on 8 April 2020, due to dwindling responses associated with the COVID-19 pandemic.

\subsection{Hypotheses}

The hypotheses for the practitioner attitudes survey were:

1. In ANZ ICUs, prior to enrolment in ICU-ROX commencing, the attitudes of health practitioners towards conservative oxygen therapy will be more favourable in ICUs that participate in ICU-ROX (ICU-ROX ICUs) than in ICUs that do not participate in ICU-ROX (non-ICU-ROX ICUs).

2. In ICU-ROX ICUs, attitudes towards conservative oxygen therapy will become even more favourable after enrolment has completed (and before results are known) than they were before enrolment began; such a change in attitudes over time will not be observed in non-ICU-ROX ICUs.

3a. In the event that ICU-ROX demonstrates that conservative oxygen therapy improves outcomes, attitudes towards this therapy will be similar in ICU-ROX and non-ICU-ROX ICUs following publication of the trial results.

3b. In the event that ICU-ROX demonstrates no difference in outcomes for patients who are treated with conservative oxygen therapy compared with usual oxygen therapy, attitudes towards this therapy continue to be more conservative in ICU-ROX than non-ICU-ROX ICUs following publication of the trial results. 
3c. In the event that ICU-ROX demonstrates that conservative oxygen therapy worsens patient outcomes compared with usual oxygen therapy, attitudes towards oxygen will be similar in ICU-ROX and non-ICU-ROX ICUs.

\subsection{Study setting (practitioner attitudes surveys and inception cohort studies)}

The practitioner attitudes surveys (Study 1) and inception cohort studies (Study 2) were conducted concurrently in 22 ANZ ICUs. Eleven of these were in the ICU-ROX (exposure) arm and 11 were in the non-ICU-ROX (comparator) arm.

\subsubsection{ICU selection}

The decision about which ICUs would participate in the ICU-ROX trial was made prior to this knowledge translation study (ICU-ROX TRIPS). An email asking for expressions of interest to participate in both the ICU-ROX trial and ICU-ROX TRIPS was sent to ANZ ICUs via the ANZICS clinical trials group (CTG) and Intensive Care Research Coordinators Interest Group (IRCIG) email lists in September 2016. This letter of invitation was sent to all ANZ ICUs on the email lists, including academic and non-academic ICUs. In NZ, all ICUs for which an expression of interest in ICU-ROX was received were invited to participate in ICU-ROX, while in Australia, only ICUs in the three states in which the ICU-ROX pilot study had been conducted were able to participate. This was a logistical decision made by management at the ANZIC Research Centre (ANZIC-RC) in Melbourne, who were contracted to coordinate ICU-ROX in Australia. This decision meant all ICUs from Victoria, Western Australia and South Australia from which an expression of interest was received were invited to participate in ICU-ROX.

ICUs for ICU-ROX TRIPS were selected using purposeful sampling. All non-pilot ICU-ROX sites were invited to participate in the exposure arm. Of the 21 ICUs that participated in ICU-ROX, 11 agreed to participate in ICU-ROX TRIPS; five of these were in NZ, and six in Australia (Figure 5). The six sites (3 Australian and 3 New Zealand) that had participated in the ICUROX pilot study were excluded from ICU-ROX TRIPS because they had already administered the ICU-ROX protocol at the time ICU-ROX TRIPS was designed.

For the comparator arm of ICU-ROX TRIPS, purposeful sampling was used for site selection, by inviting ANZ ICUs that were not able to participate in the main ICU-ROX trial to participate. In Australia, six ICUs in New South Wales, and one site in each of Victoria, Western Australia, Canberra and Tasmania agreed to participate in the non-ICU-ROX (comparator) arm, 
although the Western Australian site was withdrawn prior to starting because they were unable to obtain timely ethics approval. Two NZ sites that had initially expressed interest in ICU-ROX but later decided against participating were invited and agreed to participate in ICU-ROX TRIPS as non-ICU-ROX sites.

EXPOSURE arm

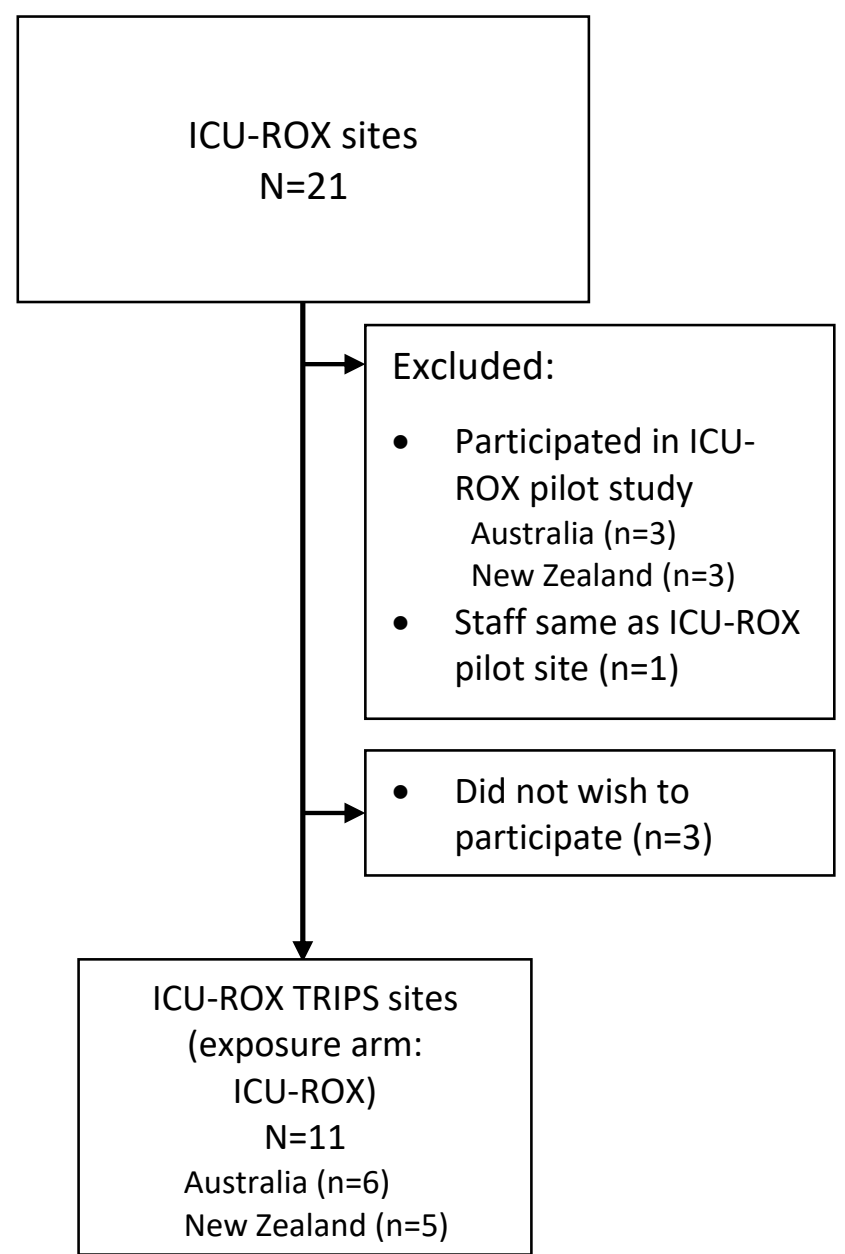

\section{COMPARATOR arm}

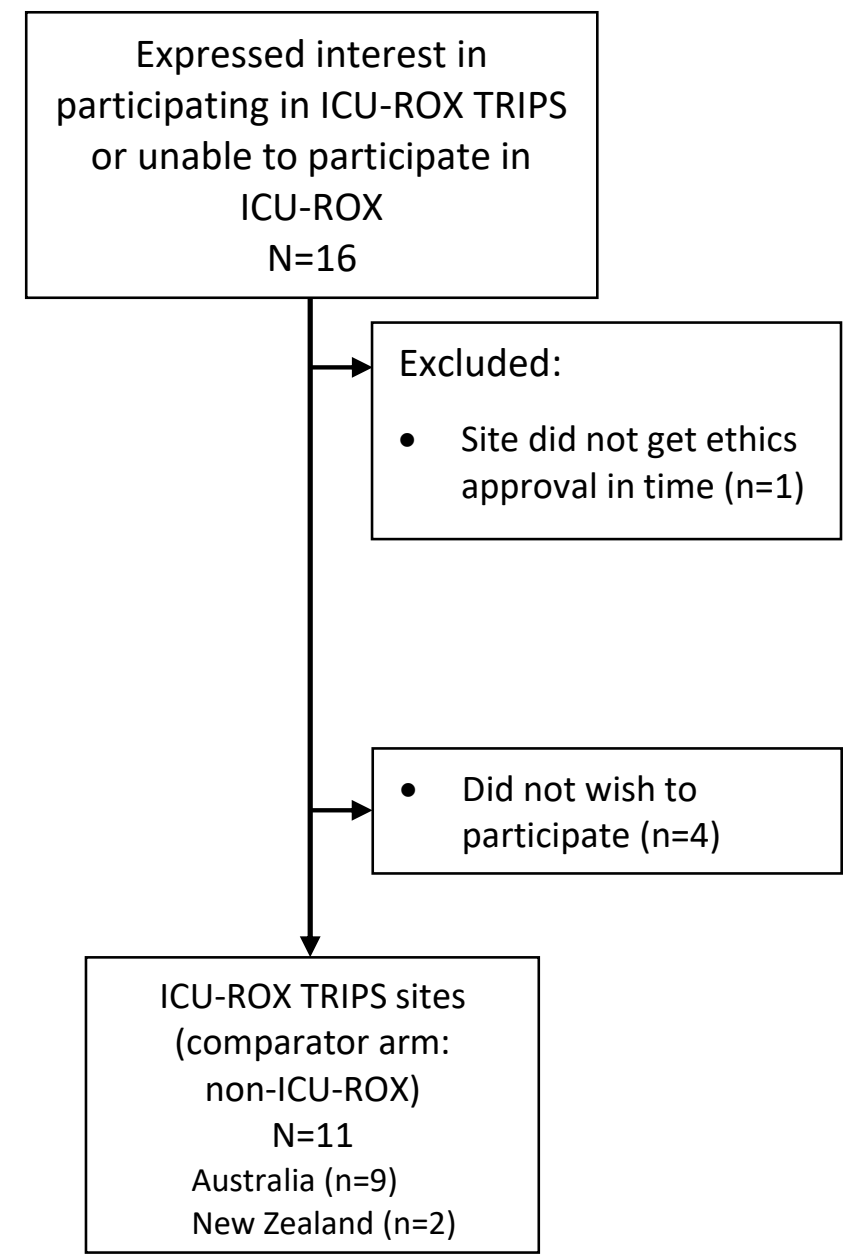

Figure 5 Site selection for ICU-ROX TRIPS (practitioner attitudes survey and inception cohort study)

Abbreviations: ICU-ROX: Intensive Care Unit Randomised Trial Comparing Two Approaches to OXygen therapy trial; ICU-ROX sites: ICUs that participated in the ICU-ROX trial; ICU-ROX TRIPS: ICU-ROX Translating Research into Practice study; non-ICU-ROX sites: ICUs that did not participate in the ICU-ROX trial 


\subsection{Design and methods}

\subsubsection{Practitioner attitudes survey overview}

The practitioner attitudes survey used a self-administered, quantitative design, developed specifically for ICU-ROX TRIPS. The survey began with an information page advising respondents of the purpose of the survey, and that it was expected to take approximately five minutes to complete, a reiteration to respondents they could opt out, and contact details for the researcher. The survey consisted of four clinical profile questions about respondents (name of ICU, whether they were an ICU specialist doctor or nurse, how long had been working as an ICU specialist or nurse (grouped into ranges 0-5 years, 6-10 years, $>10$ years] and the respondent's email address for contact (Table 7).

Table 7 Demographic data collection

\begin{tabular}{|c|c|c|c|c|}
\hline Survey & Q no. & Question & Variable & Comments \\
\hline $\mathrm{T} 1, \mathrm{~T} 2, \mathrm{~T} 3$ & $1(2)$ & Name of ICU & Free text & \\
\hline $\mathrm{T} 1, \mathrm{~T} 2, \mathrm{~T} 3$ & $2(3)$ & $\begin{array}{l}\text { Are you an ICU specialist or } \\
\text { nurse? }\end{array}$ & $\begin{array}{ll}\text { - } & \text { ICU specialist } \\
& \text { doctor } \\
\text { - } & \text { ICU nurse } \\
\end{array}$ & \\
\hline $\mathrm{T} 1, \mathrm{~T} 2, \mathrm{~T} 3$ & $3(4)$ & $\begin{array}{l}\text { How long have you been } \\
\text { working as an ICU specialist } \\
\text { or ICU nurse? }\end{array}$ & $\begin{array}{ll}\cdot & <5 \text { years } \\
\text { - } & 6-10 \text { years } \\
\text { - } & >10 \text { years } \\
\end{array}$ & \\
\hline $\mathrm{T} 1, \mathrm{~T} 2, \mathrm{~T} 3$ & $4(5)$ & Email address & Free text & $\begin{array}{l}\text { For re-contacting to } \\
\text { complete survey at } \mathrm{T} 2 \\
\text { and } \mathrm{T} 3 \text {, and to match that } \\
\text { only one survey per } \\
\text { person had been } \\
\text { collected. }\end{array}$ \\
\hline T2, T3 & 1 & $\begin{array}{l}\text { Are you still working in an } \\
\text { ICU? }\end{array}$ & - $\quad$ Yes or No & $\begin{array}{l}\text { If Yes, go to question ( } 2 \text { ) } \\
\text { If No, go to question } 6\end{array}$ \\
\hline $\mathrm{T} 2, \mathrm{~T} 3$ & 6 & $\begin{array}{l}\text { Which ICU were you } \\
\text { working in most recently? }\end{array}$ & Free text & \\
\hline $\mathrm{T} 2, \mathrm{~T} 3$ & 7 & $\begin{array}{l}\text { Were you an ICU specialist } \\
\text { or nurse }\end{array}$ & $\begin{array}{ll}\text { - } & \text { ICU specialist } \\
& \text { doctor } \\
\text { - } & \text { ICU nurse } \\
\end{array}$ & \\
\hline $\mathrm{T} 2, \mathrm{~T} 3$ & 8 & $\begin{array}{l}\text { How long were you } \\
\text { working as an ICU specialist } \\
\text { or ICU nurse? }\end{array}$ & $\begin{array}{ll}\cdot & <5 \text { years } \\
\text { - } & 6-10 \text { years } \\
\text { - } & >10 \text { years } \\
\end{array}$ & \\
\hline $\mathrm{T} 2, \mathrm{~T} 3$ & 9 & $\begin{array}{l}\text { How long ago did you leave } \\
\text { ICU (in months?) }\end{array}$ & Free text & \\
\hline $\mathrm{T} 2, \mathrm{~T} 3$ & 10 & $\begin{array}{l}\text { Does your current role } \\
\text { involve oxygen } \\
\text { management? }\end{array}$ & - Yes or No & \\
\hline
\end{tabular}

Abbreviations: ICU: Intensive Care Unit; Q. no.: question number T1: before ICU-ROX commenced; T2: after ICU-ROX was completed but before results were known; T3: after ICU-ROX publication. 
Six scenario-based questions about attitudes to oxygen management in mechanically ventilated patients in ICU were included (Table 8) and the final question was an open-ended question providing respondents with an opportunity to write comments. Responses were entered by respondents directly into the SurveyMonkey tool. Minor modifications were made to the survey for T2 and T3 to adjust for change, such as asking the question 'Are you still working in an ICU?' (Table 7) being added and a significant addition was made to the survey at T3 which is detailed later in this section.

Table 8 Summary of oxygen-related questions and response options for TRIP Practitioner attitudes survey

\begin{tabular}{|c|c|c|}
\hline Question & Categories & Options \\
\hline $\begin{array}{l}\text { How likely would you be to } \\
\text { use an } \mathrm{F}_{1} \mathrm{O}_{2} \text { of } 0.21 \text { (room air) } \\
\text { while a patient is invasively } \\
\text { mechanically ventilated, } \\
\text { providing the } \mathrm{SpO}_{2} \text { remains } \\
91-96 \% \text { in each of the } \\
\text { following situations? }\end{array}$ & $\begin{array}{l}\text { - Post-operative surgical patient } \\
\text { - } \quad \text { Patient with an admission diagnosis } \\
\text { of acute brain pathology (e.g., TBI, } \\
\text { stroke, CNS infection) } \\
\text { - } \quad \text { Patient admitted following a cardiac } \\
\text { arrest with suspected Hypoxic } \\
\text { ischaemic encephalopathy (T3 only) } \\
\text { - } \quad \text { Patient with pneumonia } \\
\text { - An ICU patient with none of the } \\
\text { above conditions and no specific } \\
\text { clinical indication for either a low } \\
\mathrm{F}_{1} \mathrm{O}_{2} \text { or high } \mathrm{F}_{1} \mathrm{O}_{2}\end{array}$ & $\begin{array}{ll}\text { - } & \text { Very unlikely } \\
\text { - } & \text { Unlikely } \\
\text { - } & \text { Neither likely } \\
& \text { nor unlikely } \\
\text { - } & \text { Likely } \\
\text { - } & \text { Very likely }\end{array}$ \\
\hline $\begin{array}{l}\text { If a patient's } \mathrm{F}_{1} \mathrm{O}_{2} \text { is } \\
{[1.0 / 0.6 / 0.4 / 0.3 / 0.25]} \\
\text { and their } \mathrm{SpO}_{2} \text { is } \geq 97 \% \text {, how } \\
\text { likely would you be to reduce } \\
\text { the } \mathrm{F}_{1} \mathrm{O}_{2} \text { in the next } 5 \text { minutes } \\
\text { in an invasively mechanically } \\
\text { ventilated patient in the } \\
\text { following circumstances? }\end{array}$ & 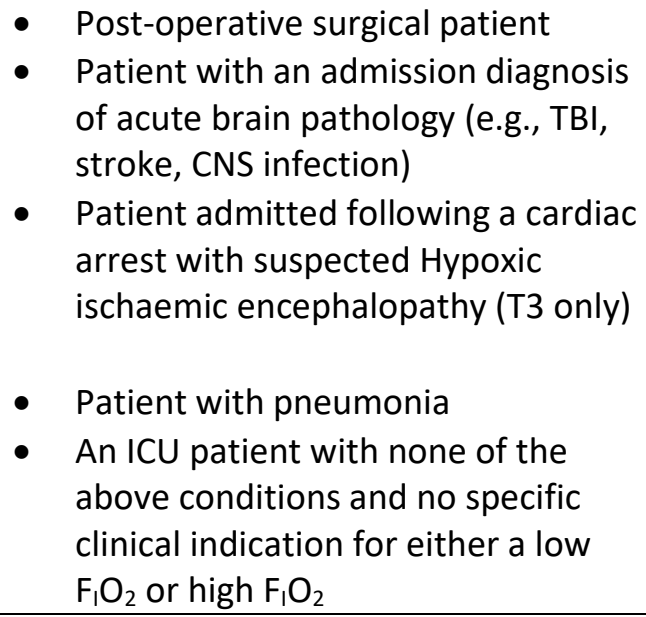 & $\begin{array}{ll}\text { - } & \text { Very unlikely } \\
\text { - } & \text { Unlikely } \\
\text { - } & \text { Neither likely } \\
& \text { nor unlikely } \\
\text { - } & \text { Likely } \\
\text { - } & \text { Very likely }\end{array}$ \\
\hline
\end{tabular}

Abbreviations: $\mathrm{F}_{1} \mathrm{O}_{2}$ : fraction of inspired oxygen; ICU: Intensive Care Unit; $\mathrm{SpO}_{2}$ : peripheral oxygen saturation; TBI: traumatic brain injury; CNS: central nervous system; T3: survey conducted after ICU-ROX was published.

\subsubsection{Tool development}

The practitioner attitudes survey was initially developed based on discussions with the ICUROX management committee. A scenario-based, closed question method which would take less than five minutes to complete was chosen because this approach was considered the 
most likely to be completed by health practitioners. Scenario-based surveys are not atypical in the ICU setting (Taylor et al., 2017).

Content validity of the survey was established by a panel of subject matter experts. Specifically, this panel consisted of members of the ICU-ROX management committee (ICU specialist doctors and nurses and a statistician), two of my PhD supervisors, and members of the research team at Wellington ICU who were not able to participate in the ICU-ROX TRIPS as they were in the ICU-ROX pilot. No formal qualitative analyses were conducted; however, informal discussions with the Wellington ICU team formed the basis of the survey. The draft survey was developed and sent to the expert panel for review and modified in response to their feedback until all were satisfied with the final survey. These modifications included changing one of the ICU-ROX a priori subgroups $\left(\mathrm{PaO}_{2} / \mathrm{F}_{1} \mathrm{O}_{2}\right.$ ratio $<$ or $\left.>300\right)$ to 'patient with pneumonia', which is a common lung condition in ICU as this was considered easier to identify, and both terms capture patients with lung disease. The lungs are the organ most exposed to oxygen and health practitioners' views about what oxygen regimen is optimal might differ in patients where lung pathology is present. The panel also agreed on the final levels of oxygen, removing 0.8 which had initially been included, as it was considered to be similar to 1.0. They recommended including both 0.25 and 0.3 , because in their clinical judgment and the observational data, either of these values may be considered the lowest acceptable level of oxygen for ventilated patients, by most ICU health practitioners (Young, Beasley et al., 2015).

The final survey (before ICU-ROX) approved by the expert panel captured four patient diagnostic groups and six levels of oxygen (Table 8). Specifically, designed to align with the ICU-ROX trial subgroups, the diagnoses were post-operative surgical patient, admission diagnosis of acute brain pathology, pneumonia or an ICU patient with none of the other conditions. The levels of oxygen in the final survey were: primary measure 0.21 ; then 1.0 , $0.6,0.4,0.3$ and 0.25 . The order of the oxygen questions was chosen so the primary measure of interest was answered first, followed by the questions about reducing $\mathrm{F}_{1} \mathrm{O}_{2}$, in descending order from 1.0 down to 0.25 . Health practitioners were asked to indicate their likelihood of reducing oxygen in mechanically ventilated patients, given the particular patient diagnostic group and level of oxygen the patient was receiving. Likelihood was assessed using a fivelevel Likert scale (Polit \& Beck, 2017). 
The survey was amended for the 'after ICU-ROX publication' period to include details about the ICU-ROX publication and oxygen management, as well as inclusion of a fifth patient admission category, of suspected hypoxic ischaemic encephalopathy. This patient condition was omitted in error from the survey for the first two time periods, but had been added as a subgroup to the ICU-ROX trial protocol after the pilot study because an admission diagnosis of cardiac arrest was unexpectedly found to be the largest (21\%) group of patients enrolled in the ICU-ROX pilot (Young et al., 2017). Patients with suspected hypoxic ischaemic encephalopathy were patients admitted to ICU following a cardiac arrest who did not obey commands after return of spontaneous circulation and before administration of sedation (Mackle et al., 2018).

Questions about the respondents' knowledge of the ICU-ROX results and oxygen management were also added to the survey administered after ICU-ROX publication (Table 9). These included an open question about how respondents thought the ICU-ROX results would influence their practice with regard to oxygen management in ICU. The updated survey was once again tested and piloted by an expert panel, which comprised the research team at Wellington ICU, and two of my PhD supervisors. As the revised survey was longer, the expert panel was specifically asked to comment on: content, length, clarity including the readability and wording, how well the online survey 'worked' and whether there were any additional questions they considered important. Questions about oxygen management, which were initially considered confusing, were amended in response to the panel's feedback and all panel members were satisfied with the final survey tool (Table 9). 
Table 9 Additional survey questions included in the practitioner attitudes survey (after publication of ICU-ROX trial results)

\begin{tabular}{|c|c|c|}
\hline Question & Options & Instructions \\
\hline \multicolumn{3}{|l|}{ ICU-ROX Results } \\
\hline $\begin{array}{l}\text { What was your level of } \\
\text { participation in the ICU-ROX } \\
\text { trial? }\end{array}$ & $\begin{array}{ll}\text { - } & \text { None - my ICU did not participate in } \\
& \text { ICU-ROX } \\
\text { - } & \text { Enrolled a patient in ICU-ROX } \\
\text { - } & \text { Patient/s under my care were in ICU- } \\
& \text { ROX } \\
\text { - } & \text { My ICU participated in ICU-ROX but I } \\
\text { had no involvement with a patient } \\
\text { enrolled in ICU-ROX }\end{array}$ & Select one \\
\hline $\begin{array}{l}\text { How did you hear about the } \\
\text { ICU-ROX trial results? }\end{array}$ & $\begin{array}{l}\text { - I have not heard the ICU-ROX results } \\
\text { - } \text { Read the published paper } \\
\text { - } \quad \text { Conference presentation } \\
\text { - } \quad \text { Results meeting } \\
\text { - } \quad \text { Social media } \\
\text { - } \quad \text { Media } \\
\text { - } \quad \text { Someone at work } \\
\text { - } \quad \text { Editorial } \\
\text { - } \quad \text { Other (please specify) }\end{array}$ & Tick all that apply \\
\hline $\begin{array}{l}\text { Which sections of the ICU-ROX } \\
\text { publication did you read? }\end{array}$ & 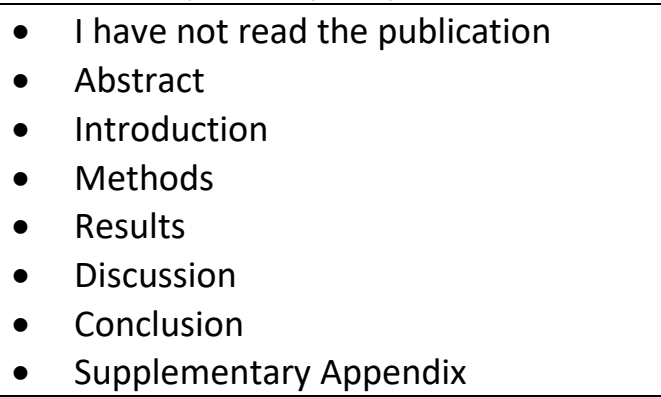 & Tick all that apply \\
\hline $\begin{array}{l}\text { In what ways do you think the } \\
\text { ICU-ROX results will influence } \\
\text { your practice with regard to } \\
\text { oxygen management in ICU? }\end{array}$ & $\mathrm{n} / \mathrm{a}-$ open question & \\
\hline \multicolumn{3}{|l|}{ Oxygen Management } \\
\hline $\begin{array}{l}\text { How is the overall oxygen } \\
\text { management in your ICU } \\
\text { determined? }\end{array}$ & $\begin{array}{l}\text { - Guideline - national or international } \\
\text { - Guideline - hospital level } \\
\text { - Guideline - ICU level } \\
\text { - Clinician preference } \\
\text { - } \quad \text { Don't know } \\
\text { - Other (please specify) }\end{array}$ & Tick all that apply \\
\hline $\begin{array}{l}\text { Who predominantly adjusts } \\
\text { the oxygen for patients in } \\
\text { your ICU? }\end{array}$ & $\begin{array}{ll} & \text { Doctors } \\
\text { - } & \text { Nurses } \\
\text { - } & \text { Other (please specify) }\end{array}$ & Tick one \\
\hline $\begin{array}{l}\text { What prompts you and/or } \\
\text { your colleagues to adjust the } \\
\text { oxygen level for patients? }\end{array}$ & $\begin{array}{l}\text { - Standing order or guideline } \\
\text { - An oxygen saturation }\left(\mathrm{SpO}_{2} \text { or } \mathrm{SaO}_{2}\right) \\
\text { range is prescribed } \\
\text { - An arterial oxygen }\left(\mathrm{PaO}_{2}\right) \text { range is } \\
\text { prescribed } \\
\text { - A minimum } \mathrm{F}_{1} \mathrm{O}_{2} \text { or oxygen flow rate } \\
\text { is prescribed }\end{array}$ & Tick all that apply \\
\hline
\end{tabular}




\begin{tabular}{|l|l|l|}
\hline Question & Options & Instructions \\
\hline & $\begin{array}{l}\text { A maximum } \mathrm{F}_{1} \mathrm{O}_{2} \text { or oxygen flow rate } \\
\text { is prescribed }\end{array}$ & \\
& $\begin{array}{l}\text { No oxygen-related targets are } \\
\text { prescribed } \\
\text { Other (please specify) }\end{array}$ & \\
\hline $\begin{array}{l}\text { Any other comments } \\
\text { regarding oxygen } \\
\text { management in the ICU? }\end{array}$ & $\mathrm{n} / \mathrm{a}$ - open question & \\
\hline
\end{tabular}

Abbreviations: ICU: Intensive Care Unit; ICU-ROX trial: Intensive Care Unit Randomised Trial Comparing Two Approaches to Oxygen therapy; $\mathrm{F}_{1} \mathrm{O}_{2}$ : fraction of inspired oxygen; $\mathrm{n} / \mathrm{a}$ : not applicable; $\mathrm{PaO}_{2}$ : partial arterial oxygen; $\mathrm{SpO}_{2}$ : peripheral oxygen saturations; $\mathrm{SaO}_{2}$ : arterial oxygen saturations.

\subsubsection{ICU-level data survey}

In addition to the practitioner attitudes survey, an ICU-level survey was developed to collect data about each ICU. This ICU survey was based on previous ICU surveys (Eastwood et al., 2011) and asked for information that would routinely be reported to the ANZICS adult patient database (ANZICS Centre for Outcome and Resource Evaluation, 2021). Specifically, information about the characteristics of ICU (level, location, funding), admissions (number, categories, source, ventilation) and staffing levels was included. In addition, questions about oxygen management, research activity, journal clubs, and dissemination of research results, practice change processes, knowledge leader and research champions were included. These latter questions were to ascertain the level of knowledge translation and research dissemination activity in the ICUs, and to assess whether a role such as a facilitator or facilitation process, previously described in the PARIHS framework emerged (Kitson et al., 1998). This survey was pre-tested by a subject matter expert panel including two supervisors, and the research manager at Wellington ICU. Amendments were made in response to feedback, until all panel experts were satisfied with the survey.

One person from each site was asked to complete this survey at the beginning of the third study time point. The survey was sent to the $\mathrm{RC} / \mathrm{s}$ and investigator at each site for them to select the most appropriate person to complete the survey. Data were entered into the SurveyMonkey tool by the site RC or returned to the researcher via email. In this case, it was entered into SurveyMonkey by the researcher, and the original form retained. Internal validity checking was conducted, and the Research Coordinator contacted for clarification if required. 


\subsubsection{Health practitioner recruitment}

Purposeful sampling was used for the practitioner attitudes survey. A research coordinator (RC) at each of the participating ICUs was asked to approach 5-10 nurses and 5-10 senior doctors in their ICU to participate in the survey. Staff who indicated they were not willing or not able to participate in the survey for all three time points were excluded. Once the doctors and nurses had expressed interest in participating, the RC sent them an email with the survey link. An information sheet was included with the email and consent was implied by completion of the survey. RCs were informed regularly of the number of doctors and nurses from their ICU who had responded. The RCs were asked to send one reminder to the doctors and nurses who had agreed to participate but were not required to follow them up any further. The RCs provided the number of doctors and nurses they had approached to participate in the study for use as the denominator for the response rate.

For the survey at subsequent time-periods, the researcher contacted the respondents from the initial survey directly. Specifically, 28 days after ICU-ROX enrolment was completed, the researcher contacted the respondents by email. The respondents from the first survey were contacted again from one month after publication of ICU-ROX results, irrespective of whether they had responded to the second survey. This contact was staggered, pending approval of a protocol amendment for the modified survey. At each time point, reminder emails were sent after the initial email.

The sample size of at least five nurses and five doctors from each ICU was a pragmatic decision, considered realistic for each site to achieve, and to account for many smaller ICUs having few ICU specialist doctors. The rationale for including both doctors and nurses was because the attitudes of both groups are important. While the practices and policies of individual ICUs was not known, typically both doctors and nurses play a key role in the oxygen practices for ventilated ICU patients (Young, Beasley et al., 2015). ICU nurses are known to work both collaboratively with doctors and independently, in the mechanical ventilation weaning process in some ICUs, of which oxygen adjustment is a key component (Henderwood, 2015; Rose et al., 2008). The decision to include only senior doctors (intensive care specialists), rather than all doctors was based on the issue of retention and exposure. Trainee doctors (registrars) usually work in ICU for periods of three, six or 12 months, then move to another clinical area or another ICU. For this study, it was important that staff were 
likely to remain in the same ICU setting for the duration of the study, to reduce the influence of working in other ICUs.

\subsubsection{Exposure and comparator}

As discussed in section 5.6, the exposure for the practitioner attitudes survey was ICU-level participation in ICU-ROX and the comparator was non-participation in ICU-ROX.

\subsubsection{Outcome measures}

The primary measures of interest were the difference in attitudes (expressed as likelihood) between respondents from ICU-ROX sites, and non-ICU-ROX sites, by comparing the indicated likelihood of using $\mathrm{F}_{1} \mathrm{O}_{2} 0.21$ (if the $\mathrm{SpO}_{2}$ was 91-96\%), in each patient admission diagnostic group at each time point. This primary measure was intended to align with the ICU-ROX trial, in which the patients in the conservative arm were required to have their oxygen reduced to $\mathrm{F}_{1} \mathrm{O}_{2}(0.21)$ room air as quickly as possible (Mackle et al., 2018).

Secondary measures of interest were the difference between ICU-ROX and non-ICU-ROX site respondents, of the likelihood of using reducing $\mathrm{F}_{1} \mathrm{O}_{2}$ from different levels $\left(\mathrm{F}_{1} \mathrm{O}_{2}\right.$ of 1.0, 0.6, $0.4,0.3,0.25)$ in each of the patient admission diagnostic groups. The change in reported likelihood over time within the ICU-ROX site respondents, and within the non-ICU-ROX respondents were also measured. Further measures of interest were a comparison between ICU specialist doctors and ICU nurses of the likelihood of using $\mathrm{F}_{1} \mathrm{O}_{2}$ 0.21, and the likelihood of reducing $\mathrm{F}_{1} \mathrm{O}_{2}$ from $1.0,0.6,0.4,0.3$ and 0.25 , for each of the patient admission diagnostic groups at each time point. The change in reported likelihood was also compared within the doctor respondents, and within the nurse respondents over time for all patient admission diagnoses.

If a difference was detected between doctors and nurses, with respect to their likelihood of using $\mathrm{F}_{1} \mathrm{O}_{2}$ of 0.21 in patients with an $\mathrm{SpO}_{2}$ 91-96\%, two further measures of interest were: comparison between ICU-ROX-site doctors and non-ICU-ROX-site doctors, and comparison between ICU-ROX site nurses and non-ICU-ROX nurses. 


\subsubsection{Data management}

Surveys were completed by the respondents directly into SurveyMonkey. The data were initially downloaded from SurveyMonkey into an excel spreadsheet, data cleaning performed, and the respondents de-identified. The cleaned database was uploaded to SPSS.

Data cleaning involved each survey being checked for duplication and completeness. To ensure only one survey for each respondent was included in the final analysis, a log was kept by the researcher. Where a respondent had completed the survey more than once at each time point, the first, most complete survey was used. If the respondent did not provide an email address, and the site was not able to be identified so the researcher could contact them for clarification, the response was omitted. No attempt was made to follow-up respondents who had not answered all questions. Demographic data were checked across the three time points, to ensure the same role had been selected over time.

Survey responses were de-identified prior to any analyses being conducted. Each health practitioner who responded at the first time point was assigned a unique study identifier (Study ID) which was used in the analysis and the database was deidentified by removing email addresses prior to undertaking any analyses. The Study identifier (ID) was used to link the three studies to review attrition rate.

\subsubsection{Recoding}

Recoding of data was undertaken to increase the number of analyses that could be undertaken. Chi-square requires a minimum expected cell size of five in at least $80 \%$ of cells, and no cell should have less than one (McHugh, 2013). On initial review, there were several cells that did not meet these criteria. Recoding was undertaken where there was a clinical or theoretical rationale to do so. Where necessary, adjacent 'like' groups were combined and recoded; Likely and Very likely were combined into a new category of Very likely/Likely, and Unlikely and Very Unlikely combined into a new category of Very unlikely/Unlikely (Table 10). In the situations where this recoding still did not reach sufficient cell-size to conduct Chi-square, the decision was made not to include 'neither likely nor unlikely' into the recoding, as it indicates a standalone category of 'not knowing', and has no clinical or theoretical rationale. Table 10 shows that recoding was mainly required at $\mathrm{F}_{1} \mathrm{O}_{2}$ levels of 0.4 and above, and occurred in all surveys. 
Table 10 Summary of $\mathrm{F}_{1} \mathrm{O}_{2}$ fields that required recoding for analysis

\begin{tabular}{|l|c|c|c|c|c|c|}
\hline & $\mathbf{0 . 2 1}$ & $\mathbf{0 . 2 5}$ & $\mathbf{0 . 3}$ & $\mathbf{0 . 4}$ & $\mathbf{0 . 6}$ & $\mathbf{1 . 0}$ \\
\hline Post-operative & $\mathrm{T} 3$ & - & $\mathrm{T} 2, \mathrm{~T} 3$ & $\mathrm{~T} 1, \mathrm{~T} 2, \mathrm{~T} 3$ & $\mathrm{~T} 1, \mathrm{~T} 2, \mathrm{~T} 3$ & $\mathrm{~T} 1, \mathrm{~T} 2, \mathrm{~T} 3$ \\
\hline $\begin{array}{l}\text { Acute brain } \\
\text { pathology }\end{array}$ & - & - & $\mathrm{T} 3$ & $\mathrm{~T} 1, \mathrm{~T} 2, \mathrm{~T} 3$ & $\mathrm{~T} 1, \mathrm{~T} 2, \mathrm{~T} 3$ & $\mathrm{~T} 1, \mathrm{~T} 2, \mathrm{~T} 3$ \\
\hline $\begin{array}{l}\text { Hypoxic ischaemic } \\
\text { encephalopathy }\end{array}$ & - & - & $\mathrm{T} 3$ & $\mathrm{~T} 3$ & $\mathrm{~T} 3$ & $\mathrm{~T} 3$ \\
\hline Pneumonia & - & - & $\mathrm{T} 3$ & $\mathrm{~T} 1, \mathrm{~T} 2, \mathrm{~T} 3$ & $\mathrm{~T} 1, \mathrm{~T} 2, \mathrm{~T} 3$ & $\mathrm{~T} 1, \mathrm{~T} 2, \mathrm{~T} 3$ \\
\hline Other ICU patient & $\mathrm{T} 3$ & - & $\mathrm{T} 2, \mathrm{~T} 3$ & $\mathrm{~T} 1, \mathrm{~T} 2, \mathrm{~T} 3$ & $\mathrm{~T} 1, \mathrm{~T} 2, \mathrm{~T} 3$ & $\mathrm{~T} 1, \mathrm{~T} 2, \mathrm{~T} 3$ \\
\hline
\end{tabular}

Abbreviations: ICU: Intensive Care Unit; T1: before ICU-ROX commenced; T2: after ICU-ROX was complete but before results were known; T3: after ICU-ROX publication.

In addition, for the open questions, where respondents had selected 'other' as a response, this was recoded where possible and obvious (e.g., when a respondent had written 'both', this was added as a new category).

\subsubsection{Data analysis}

A statistical analysis plan was written and agreed prior to any analyses taking place; the contents of which are included in this thesis. Statistical analyses were conducted by the researcher using SPSS version 27, with guidance from a statistician and supervisors. Site level demographic data were analysed by a statistician, under the guidance of the researcher using SAS version 9.4.

All quantitative data were initially assessed for normality and frequency statistics undertaken, then between-group analysis, then within-group for ICU-ROX and non-ICU-ROX. In addition, data were analysed for differences between role (between-group and withingroup). The numerator was expressed as a percentage of the total response for each category. Analysis was conducted without imputation of missing data. Likert scale data were treated as ordinal data because although there is an order, the difference between categories was not known.

Comparisons between groups were performed using Chi-squared $\left(X^{2}\right)$ tests for proportions, Student $t$-tests for normally distributed data and Wilcoxon rank sum tests otherwise, with results reported as $n$ with percentages, mean \pm standard deviations or median [interquartile range (IQR)] respectively. Graphs were also generated to illustrate the results. 
The data for length of time in the role provided at the first survey were used for the summary table. In addition, for respondents who had changed their place of work, the exposure or comparator (ICU-ROX or non-ICU-ROX) at the beginning of the study ('before ICU-ROX') was reported. For the ICU level survey, site characteristics, oxygen management data, and knowledge translation data are presented as descriptive data.

\subsubsection{Between and within-group analyses}

The following comparisons were undertaken: between group by exposure or comparator (ICU-ROX vs non-ICU-ROX); within-group by exposure or comparator (ICU-ROX site respondents over time; non-ICU-ROX respondents over time); between-group by role (ICUROX and non-ROX site doctors vs ICU-ROX and non-ICU-ROX site nurses); within-group by role (ICU-ROX and non-ICU-ROX doctors over time; ICU-ROX and non-ICU-ROX nurses over time). Between-group comparisons were conducted at each of the predefined time points: before ICU-ROX started at each site (T1); after ICU-ROX finished, but before the results were known (T2) and between one and six months after publication of the ICU-ROX results (T3). Within-group comparisons were conducted between 'before ICU-ROX' (T1) and 'after ICUROX (but before the results were known) (T2); and again between 'after ICU-ROX' (T2) and 'after ICU-ROX results were published' (T3).

The pre-specified comparisons at each time point were:

- Likelihood of health practitioners from each of the two cohorts (ICU-ROX and nonICU-ROX) using an $\mathrm{F}_{1} \mathrm{O}_{2}$ of 0.21 for each of the categories: post-operative surgical patient, admission diagnosis of acute brain pathology, a suspected hypoxic ischaemic encephalopathy (T3 only), pneumonia or an ICU patient with none of the other conditions.

- Likelihood of health practitioners from each of the two study groups reducing the $\mathrm{F}_{1} \mathrm{O}_{2}$ at each predefined level $(1.0,0.6,0.4,0.3,0.25)$ for each of the patient groups (post-operative, admission diagnosis of acute brain pathology, patient admitted following cardiac arrest a suspected hypoxic ischaemic encephalopathy (T3 only), pneumonia or any mechanically ventilated ICU patient with none of the other conditions and no clinical indication for a low or high $\left.\mathrm{F}_{1} \mathrm{O}_{2}\right)$. 
Additionally, a between-group comparison was made for only nurses (comparing ICU-ROX site nurses and non-ICU-ROX nurses), and only doctors (comparing ICU-ROX-site doctors and non-ICU-ROX-site doctors), for likelihood of using $\mathrm{F}_{1} \mathrm{O}_{2} 0.21$.

In the final survey, after ICU-ROX, respondents were asked about their knowledge of the ICU-ROX results. Between-group analyses were initially by exposure (ICU-ROX vs non-ICUROX), then by role (doctors vs nurses).

\subsubsection{Qualitative data}

Open-ended comments from the three practitioner attitudes surveys and the ICU-site level survey were coded by hand for content analysis. The following method for content analysis was used:

1. Read through comments as a whole

2. Identify common topics and themes and generate codes

3. Code comments

4. Review coding to ensure analysis captured all coding

5. Summarise findings.

The coding was conducted by the researcher and verified by a supervisor. Any disagreements were discussed, until consensus was reached.

\subsubsection{Validity strategies}

Several validity strategies were considered and used at the design, recruitment, data management and analysis stages of this study. Specifically, validation of survey design, recognition of the potential for bias in recruitment, management of duplicate surveys, deidentification and use of a pre-specified data management and statistical analysis plan.

As outlined in section 6.3.2, validation of the practitioner attitudes and ICU-level surveys involved subject matter experts as well as pre-testing and piloting. The decision for site RCs to be responsible for recruiting health practitioners was made to increase the likelihood of responses from all sites; however, this did have the potential to introduce selection bias. It is unknown whether RCs asked only staff they thought would participate, and survey respondents may have been people with a greater interest in research than those who did not. 
As outlined in section 6.3.7, measures were taken to ensure that there was only one survey for each respondent at each of the three time points. This process was defined prior to any analyses taking place, and used a specific hierarchy based on completeness and timeliness of survey completion. A comparison of role data was undertaken between the three time points to ensure no typographical errors had been made.

To reduce any bias by the researcher, data were de-identified prior to any analyses being undertaken. It was not possible to blind the sites allocation as these were known to the researcher; however, the respondents were not known, and were identified by Study ID only. Data management and statistical analysis was undertaken by the researcher, according to a pre-specified plan. Tables were checked against the raw results by others to avoid transcription errors. Qualitative data were coded by both the researcher and her supervisor. 


\subsection{Results}

Presentation of the survey results begin with the characteristics of the ICUs participating in the survey and inception cohort study. Then the characteristics of the practitioner attitudes survey are presented, followed by the effects of participation or non-participation in ICUROX on attitudes and the effects of role on attitudes. Figures showing the results are at the end of each section (participation and role). Following this the results from open-ended comments are presented. The final sections are about self-reported oxygen, management practices knowledge of the ICU-ROX publication, dissemination and knowledge translation activities within in the participating ICUs.

\subsubsection{Characteristics of participating ICUs for Study 1 and 2}

ICU-level survey responses were received from 21 of the 22 participating ICUs between December 2019 and November 2020. One non-ICU-ROX site withdrew from this study prior to the third time point and did not provide site-level data. There were no significant differences between the ICUs that participated in ICU-ROX and those that did not (non-ICUROX) (Table 11).

The link to the practitioner attitudes survey was sent to 169 doctors and 243 nurses from 22 Australian and New Zealand ICUs. Of these, 112 doctors (66.3\%) and 153 nurses (63.0\%) completed the survey (Table 12). Before ICU-ROX started (T1), 265 valid partially or fully completed responses were received between November 2016 and November 2017 (Figure 6). Of these 265 respondents, 142 were from ICU-ROX sites, and 123 from non-ICU-ROX sites (Table 12). After ICU-ROX finished (but before results were known) (T2), 252 valid responses were received, (June 2018-December 2018), and 193 valid responses between November 2019 and March 2020, following ICU-ROX publication in October 2019 (T3). 
Table 11 Characteristics of ICU-ROX vs non-ICU-ROX sites (practitioner attitudes survey and inception cohort study)

\begin{tabular}{|c|c|c|c|}
\hline Characteristic & $\begin{array}{c}\text { ICU-ROX sites } \\
(\mathrm{N}=11)\end{array}$ & $\begin{array}{l}\text { Non-ICU-ROX } \\
\text { sites }(\mathrm{N}=10)^{*}\end{array}$ & $P$ value \\
\hline \multicolumn{4}{|l|}{ Country, n (\%) } \\
\hline New Zealand & $5(45.5 \%)$ & $2(20.0 \%)$ & $\mathrm{np}$ \\
\hline Australia & $6(54.5 \%)$ & $8(80.0 \%)$ & \\
\hline \multicolumn{3}{|l|}{ ICU Type, n (\%) } & 0.62 \\
\hline Level III (Tertiary) & $8(72.7 \%)$ & $8(80.0 \%)$ & \\
\hline Level II & $2(18.2 \%)$ & $2(20.0 \%)$ & \\
\hline Level I & $1(9.1 \%)$ & $0(0.0 \%)$ & \\
\hline \multicolumn{3}{|l|}{ Location, n (\%) } & 0.48 \\
\hline Metropolitan & $11(100.0 \%)$ & $9(90.0 \%))$ & \\
\hline Rural & $0(0.0 \%)$ & $1(10.0 \%)$ & \\
\hline Private & $0(0.0 \%)$ & $1(10.0 \%)$ & \\
\hline \multicolumn{4}{|l|}{ ICU Characteristics, mean (SD) } \\
\hline Number of funded beds per ICU & $19.0 \pm 12.7$ & $20.1 \pm 10.5$ & 0.83 \\
\hline Mechanically ventilated + & $775.3 \pm 654.6$ & $705.3 \pm 392.7$ & 0.78 \\
\hline Total admissions (2018) & $1662.00 \pm 903.2$ & $1591.4 \pm 770.6$ & 0.85 \\
\hline Elective & $654.2 \pm 575.0$ & $548.7 \pm 301.9$ & 0.61 \\
\hline Emergency & $1005.5 \pm 719.1$ & $1042.7 \pm 612.3$ & 0.90 \\
\hline \multicolumn{4}{|l|}{ Admission source } \\
\hline Ward & $308.9 \pm 188.3$ & $317.1 \pm 220.5$ & 0.93 \\
\hline Emergency Department & $529.0 \pm 364.7$ & $418.2 \pm 225.4$ & 0.42 \\
\hline Operating theatre & $743.5 \pm 559.8$ & $722.2 \pm 402.8$ & 0.92 \\
\hline Other hospital or ICU & $137.9 \pm 134.9$ & $120.2 \pm 73.0$ & 17.7 \\
\hline \multicolumn{4}{|l|}{ Staffing, median (IQR) } \\
\hline ICU specialists & $8.2[6$ to 10$]$ & $8.5[5$ to 10$]$ & 0.55 \\
\hline Registrars & 10.0 [7 to 15$]$ & 11 [6 to 9 ] & 1.00 \\
\hline Senior nursest ${ }^{\dagger}$ & 11 (4 to 12.1$)$ & $18[7$ to 21$]$ & 0.41 \\
\hline Staff nurses & 91 (38 to 179$)$ & 78.5 [69 to 120$]$ & 0.94 \\
\hline Research coordinators & $2[1$ to 3$]$ & $1[1$ to 3$]$ & 0.36 \\
\hline \multicolumn{4}{|l|}{ Research studies } \\
\hline ANZICS CTG-endorsed, $\mathrm{n}(\%)$ & $7.3 \pm 3.4$ & $5.4 \pm 2.1$ & 0.15 \\
\hline Pharmaceutical, median (IQR) & $0[0$ to 1$]$ & $0[0$ to 1$]$ & 0.67 \\
\hline Other, median (IQR) & $1.5[0$ to 4$]$ & $1[0$ to 4$]$ & 0.80 \\
\hline
\end{tabular}

* No data provided by one site ${ }^{\dagger}$ data from 2019 or FY 2018 for 2 sites

Abbreviations: ANZICS-CTG: Australia New Zealand Intensive Care Society Clinical Trials Group; ICU: Intensive Care Unit; ICU-ROX: sites that participated in the ICU-ROX trial; IQR: interquartile range; non-ICU-ROX: sites that did not participate in ICU-ROX; $n$ (\%): number and percentage of total; np: analysis not possible; SD: standard deviation; Study 1: practitioner attitudes survey; Study 2: inception cohort study. 


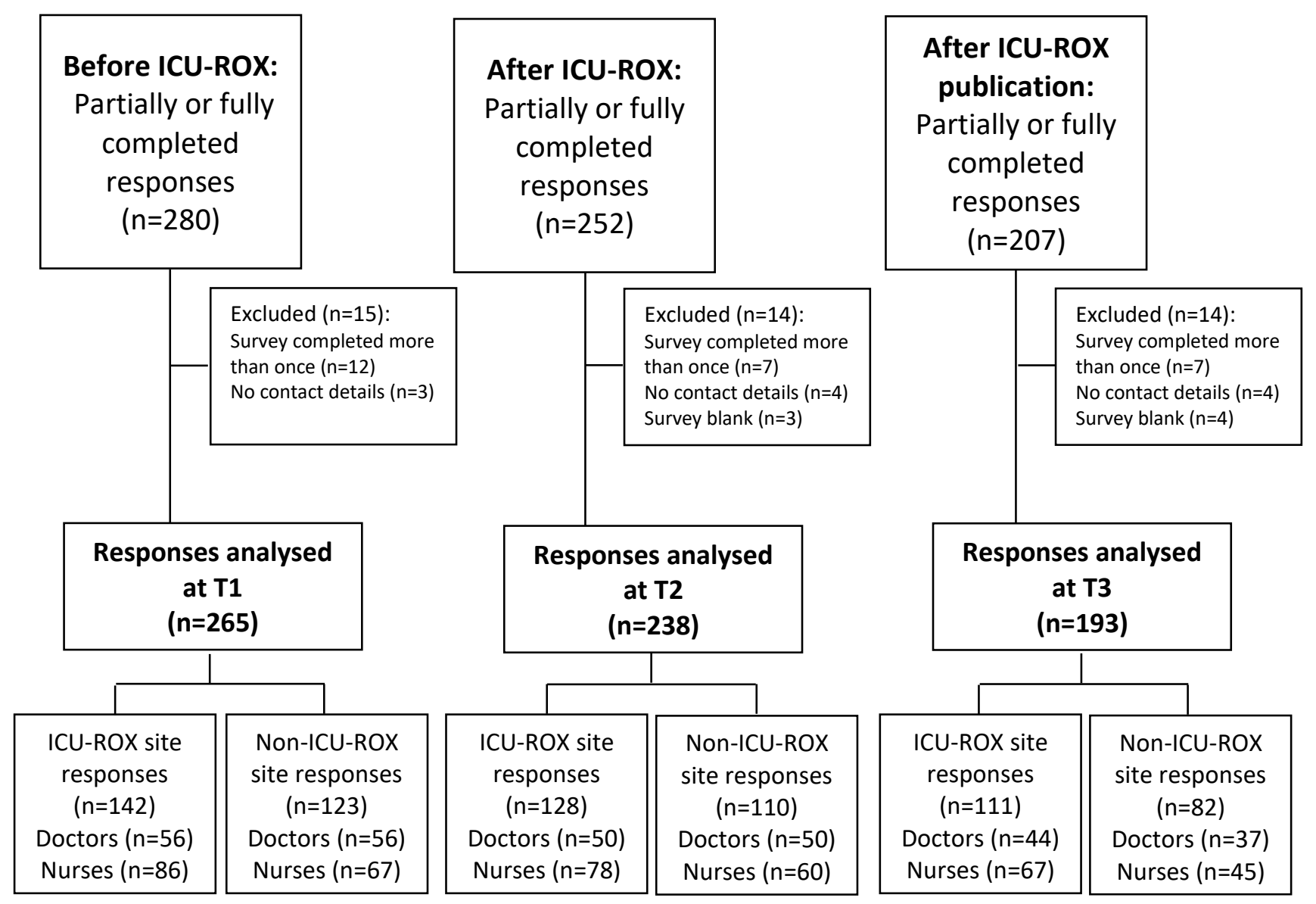

Figure 6 Flow diagram of survey responses

Abbreviations: ICU-ROX: Intensive Care Unit Randomised Trial Comparing Two Approaches to Oxygen therapy; ICUROX sites: ICUs that participated in the ICU-ROX trial; non-ICU-ROX sites: ICUs that did not participate in the ICU-ROX trial; T1: before ICU-ROX commenced; T2: after ICU-ROX completed enrolment but before results were known; T3: after ICU-ROX publication.

Many respondents did not answer the survey at all three time points, and the number of responses decreased over time (Figure 6, Table 12). Most of the loss was between the second and third time points, and $33.3 \%$ was respondents from non-ICU-ROX sites compared to $21.8 \%$ from ICU-ROX sites. This difference was, in part, due to the withdrawal of one nonICU-ROX site. The overall drop-out rate was similar for doctors and nurses $(27.7 \%$ and $26.8 \%$, respectively). 
Table 12 Practitioner attitudes survey responses over time (by role and exposure)

\begin{tabular}{|c|c|c|c|}
\hline & $\begin{array}{l}\text { ICU-ROX } \\
\text { respondents }\end{array}$ & $\begin{array}{l}\text { Non-ICU-ROX } \\
\text { respondents }\end{array}$ & $P$ value \\
\hline \multicolumn{4}{|c|}{ T1: Before ICU-ROX (N=265) } \\
\hline ICU specialist doctors & 56 & 56 & \multirow{3}{*}{0.37} \\
\hline ICU nurses & 86 & 67 & \\
\hline Total & $142(53.6 \%)$ & $123(46.4 \%)$ & \\
\hline \multicolumn{4}{|c|}{ T2: After ICU-ROX (n=238) } \\
\hline ICU specialist doctors & 50 & 50 & \multirow{3}{*}{0.32} \\
\hline ICU nurses & 78 & 60 & \\
\hline Total & $128(53.8 \%)$ & $110(46.2 \%)$ & \\
\hline \multicolumn{4}{|c|}{ T3: After ICU-ROX publication ( $n=193)$} \\
\hline ICU specialist doctors & 44 & 37 & \multirow{3}{*}{0.45} \\
\hline ICU nurses & 67 & 45 & \\
\hline Total & $111(57.5 \%)$ & 82 (42.5\%) & \\
\hline
\end{tabular}

Abbreviations: ICU: Intensive Care Unit; ICU-ROX site respondents: respondents from ICUs that participated in the ICU-ROX trial; non-ICU-ROX sites: respondents from ICUs that did not participate in the ICU-ROX trial; T1: before ICU-ROX commenced; T2: after ICU-ROX completed enrolment but before results were known; T3: after ICU-ROX publication

The characteristics of respondents from ICU-ROX sites and the non-ICU-ROX sites were similar (Table 13). The exception to this was that nurses from the non-ICU-ROX sites had been in their role for significantly longer than the ICU-ROX site nurses ( $P=0.002)$. After ICUROX was completed, eight respondents reported they no longer worked in the same ICU as before ICU-ROX, and for three of them, their new role did not involve oxygen management. The number who no longer worked at the same ICU increased to 17 after publication of ICUROX; with 10 no longer in a role involving oxygen management. At the time of analysis, it was realised that one respondent was a physiotherapist, and this person's responses were included in the nurses' group.

Few respondents answered all questions $(n=20)$. Typically, respondents completed the survey about $\mathrm{F}_{1} \mathrm{O}_{2} 0.21$ for most patient categories; however, the number of responses varied, resulting in variable denominators for the results (Table S1 in Appendix 5). Respondents from ICU-ROX sites answered $96.6 \%$ to $97.9 \%$ of possible questions and nonICU-ROX was $95.3 \%$ to $97 \%$. 
Table 13 Respondent baseline characteristics for ICU-ROX vs non-ICU-ROX sites

\begin{tabular}{|c|c|c|c|}
\hline Characteristic & $\begin{array}{l}\text { ICU-ROX } \\
\text { respondents } \\
(N=142)\end{array}$ & $\begin{array}{c}\text { Non-ICU-ROX } \\
\text { respondents } \\
(\mathrm{N}=123)\end{array}$ & $P$ value \\
\hline Doctors, n (\%) & $56(39.4 \%)$ & $56(45.5 \%)$ & \multirow{2}{*}{0.32} \\
\hline Nurses, n (\%) & $86(60.6 \%)$ & $67(54.5 \%)$ & \\
\hline $\begin{array}{l}\text { Completed survey at } \mathrm{T} 1 \text {, } \\
\mathrm{T} 2 \text { and } \mathrm{T} 3, \mathrm{n}(\%)\end{array}$ & $104(73.2 \%)$ & $77(62.6 \%)$ & 0.50 \\
\hline \multicolumn{4}{|c|}{ Length of time in role at $\mathrm{T} 1$ (doctors), $\mathrm{n}(\%)$} \\
\hline$<5$ years & $24(42.9 \%)$ & $15(26.8 \%)$ & \multirow{3}{*}{0.18} \\
\hline $6-10$ years & $15(26.8 \%)$ & $17(30.4 \%)$ & \\
\hline$>10$ years & $17(30.4 \%)$ & $23(41.1 \%)$ & \\
\hline \multicolumn{4}{|c|}{ Length of time in role at $\mathrm{T} 1$ (nurses), $\mathrm{n}(\%)$} \\
\hline$<5$ years & $25(29.1 \%)$ & $9(13.4 \%)$ & \multirow{3}{*}{0.002} \\
\hline $6-10$ years & $28(32.6 \%)$ & $13(19.4 \%)$ & \\
\hline$>10$ years & $33(38.4 \%)$ & $45(67.2 \%)$ & \\
\hline
\end{tabular}

Abbreviations: ICU: Intensive Care Unit; ICU-ROX respondents: respondents from ICUs that participated in the ICU-ROX trial; $\mathrm{n}(\%)$ : number and percentage; non-ICU-ROX respondents: respondents from ICUs that did not participate in the ICU-ROX trial; T1: before ICU-ROX commenced; T2: after ICU-ROX was complete but before results were known; T3: after ICU-ROX publication.

\subsubsection{Comparisons by exposure group (ICU-ROX vs non-ICU-ROX)}

\subsubsection{Primary measure of interest $\left(\mathrm{F}_{1} \mathrm{O}_{2}\right.$ of 0.21$)$}

Before the ICU-ROX trial was conducted, respondents from ICU-ROX trial sites and those from non-ICU-ROX trial sites were similarly likely to indicate they would likely use an $\mathrm{F}_{1} \mathrm{O}_{2}$ of 0.21 , in a ventilated patient with an $\mathrm{SpO}_{2}$ of 91-96\%, irrespective of a patient's diagnosis (Figure 7). However, after their participation in the ICU-ROX trial, and before the trial results were known, the reported preferences of ICU-ROX-site respondents in relation to this aspect of oxygen management differed from those of non-ICU-ROX respondents. In particular, for post-operative surgical patients $\left(X^{2}[4, N=233]=17.26, P=0.002\right)$, patients with an acute brain pathology $\left(X^{2}[4, N=236]=18.87, \mathrm{P}=0.001\right)$, pneumonia $\left(X^{2}[4, N=127]=14.82, \mathrm{P}=0.005\right)$ and 'other ICU' patients $\left(X^{2}[4, N=236]=13.34, P=0.01\right)$, respondents from ICU-ROX trial sites were significantly more likely than their non-ICU-ROX trial counterparts to indicate they would use an $\mathrm{F}_{1} \mathrm{O}_{2}$ of 0.21 in a patient with an $\mathrm{SpO}_{2}$ of $91-96 \%$. The differences in indicated likelihood in all patient categories remained after the ICU-ROX trial was published, with the exception that no difference was present in the 'other ICU patients' category. Specifically, for post-operative surgical patients $\left(X^{2}[3, N=193]=11.85, P=0.008\right)$, admission diagnosis of 
acute brain pathology $\left(X^{2}[4, N=192]=15.36, P=0.004\right)$ and pneumonia $\left(X^{2}[4, N=193]=14.80\right.$, $\mathrm{P}=0.005)$, ICU-ROX respondents reported they were more likely to use an $\mathrm{F}_{1} \mathrm{O}_{2}$ of 0.21 than their non-ICU-ROX counterparts. This difference was also present for patients admitted with a suspected hypoxic ischaemic encephalopathy $\left(X^{2}[4, N=193]=17.89, \mathrm{P}=0.001\right)$, which was only included in the survey after ICU-ROX was published (Figure 8).

ICU-ROX respondents' indicated likelihood of using an $\mathrm{F}_{1} \mathrm{O}_{2}$ of 0.21 (when $\mathrm{SpO}_{2}$ is $91-96 \%$ ) increased over time (Figure 7). The likelihood was statistically significantly different after participating in ICU-ROX, compared to prior to their participation in ICU-ROX, irrespective of patient admission category. Specifically, for post-operative surgical patients, $\left(X^{2}[4, N=267]\right.$ $=24.90, \mathrm{P}<0.001)$, patients with an admission diagnosis of acute brain pathology, $\left(X^{2}[4\right.$, $N=268]=27.07, \mathrm{P}<0.001)$, pneumonia $\left(X^{2}[4, N=267]=24.88, \mathrm{P}<0.001\right)$ and 'other ICU' patients $\left(X^{2}[4, N=269]=16.60, P=0.002\right)$ respondents became significantly more likely to indicate that they would use an $\mathrm{F}_{1} \mathrm{O}_{2}$ of 0.21 . These trial-site respondents remained similarly likely to reduce to an $\mathrm{F}_{1} \mathrm{O}_{2}$ of 0.21 after the publication of the ICU-ROX results; however, the distribution of their indicated likelihood did not change further.

In the non-ICU-ROX respondents, a change in likelihood of using $\mathrm{F}_{1} \mathrm{O}_{2}$ of 0.21 in a patient with an $\mathrm{SpO}_{2}$ of 91-96\% was evident after ICU-ROX was conducted than before, for some patient categories (Figure 7). Specifically, for patients with pneumonia $\left(X^{2}[4, N=229]=10.97\right.$, $\mathrm{P}=0.03)$ and 'other ICU patients' $\left(X^{2}[4, N=231]=10.19, \mathrm{P}=0.04\right)$, the non-ICU-ROX respondents became more likely to indicate they would use an $\mathrm{F}_{1} \mathrm{O}_{2}$ of 0.21 after ICU-ROX had been conducted. This change in distribution of indicated likelihood was not evident in post-operative surgical patients or those with an admission diagnosis of acute brain pathology. There was no further change in likelihood of using $\mathrm{F}_{1} \mathrm{O}_{2}$ of 0.21 after the ICU-ROX trial was published, irrespective of patient diagnosis.

\subsubsection{Comparisons by exposure for $\mathrm{F}_{1} \mathrm{O}_{2}$ of $\mathbf{0 . 2 5}$}

Even before ICU-ROX started, there was a difference in reported likelihood of reducing $\mathrm{F}_{1} \mathrm{O}_{2}$ from 0.25 (if the $\mathrm{SpO}_{2} \geq 97 \%$ ) between ICU-ROX and non-ICU-ROX respondents (Figure 9). In particular, respondents from ICU-ROX sites indicated they were more likely to reduce the $\mathrm{F}_{1} \mathrm{O}_{2}$ from 0.25 than their non-trial site counterparts, irrespective of patient admission diagnosis. However, after participating in ICU-ROX, this difference was no longer apparent, 
and respondents from both ICU-ROX and non-ICU-ROX sites were similarly likely to indicate they would reduce the $\mathrm{F}_{1} \mathrm{O}_{2}$. This similarity persisted after publication of the ICU-ROX trial results in all patient categories except those admitted with a suspected hypoxic ischaemic encephalopathy (Figure 8). For this patient category, 50\% of respondents from the non-ICUROX sites indicated they were either 'Unlikely' or 'Very Unlikely' to reduce the oxygen from $\mathrm{F}_{1} \mathrm{O}_{2}$ of 0.25 compared to $30.6 \%$ of those from ICU-ROX sites.

For ICU-ROX respondents, their reported likelihood of reducing the $\mathrm{F}_{1} \mathrm{O}_{2}$ from 0.25 (if $\mathrm{SpO}_{2} \geq$ 97\%) changed after they participated in ICU-ROX, for some patient groups (Figure 9). Specifically, for 'post-operative surgical' patients $\left(X^{2}[4, N=266]=15.40, P=0.004\right)$ and 'other ICU patient' categories $\left(X^{2}[4, N=264]=10.28, P=0.04\right)$, trial-site respondents became more likely to indicate they would reduce the $\mathrm{F}_{1} \mathrm{O}_{2}$ from 0.25 . There was no further change for these two patient admission diagnoses after the publication of ICU-ROX. The likelihood of reducing $\mathrm{F}_{1} \mathrm{O}_{2}$ from 0.25 remained similar after participation and again after ICU-ROX publication, for patients with an admission diagnosis of acute brain pathology and for those with pneumonia.

Non-ICU-ROX respondents' indicated likelihood of reducing $\mathrm{F}_{1} \mathrm{O}_{2}$ from 0.25 , if the $\mathrm{SpO}_{2} \geq 97 \%$, changed significantly over time, irrespective of a patient diagnosis (Figure 9). In particular, for post-operative surgical patients $\left(X^{2}[4, N=226]=13.50, P=0.01\right)$, patients with an acute brain pathology $\left(X^{2}[4, N=226]=11.62, P=0.02\right)$, patients with pneumonia $\left(X^{2}[4, N=225]\right.$ =13.97, $\mathrm{P}=0.007)$ and 'other ICU' $\left(X^{2}[4, N=226]=10.81, \mathrm{P}=0.03\right)$ patients, non-ICU-ROX respondents were significantly more likely to indicate they would to decrease the $\mathrm{F}_{1} \mathrm{O}_{2}$ from 0.25, after ICU-ROX concluded compared to before ICU-ROX. These differences remained after the ICU-ROX trial was published, although no further change was evident in any patient group.

\subsubsection{Comparisons by exposure for $\mathrm{F}_{1} \mathrm{O}_{2}$ of $\mathbf{0 . 3}$}

Before ICU-ROX started, respondents from the ICU-ROX and non-ICU-ROX sites similarly indicated they would reduce the $\mathrm{F}_{1} \mathrm{O}_{2}$ from 0.3 , if a patient's $\mathrm{SpO}_{2} \geq 97 \%$, irrespective of the patient's diagnosis (Figure 10). This similarity remained after ICU-ROX was conducted, with no statistical differences between respondents from ICU-ROX and non-ICU-ROX sites. After publication of the ICU-ROX results, this similarity persisted in all patient categories except 
post-operative surgical patients $\left(X^{2}[3, N=255]=8.91, \mathrm{P}=0.03\right)$, for which ICU-ROX respondents indicated they were more likely to reduce $\mathrm{F}_{1} \mathrm{O}_{2}$ from 0.3 , than their non-ICUROX counterparts.

Respondents from ICU-ROX sites reported a change in their likelihood of reducing $\mathrm{F}_{1} \mathrm{O}_{2}$ from 0.3 (if the $\mathrm{SpO}_{2} \geq 97 \%$ ) over time, for some patient categories. Specifically, for post-operative surgical $\left(X^{2}[4, N=259]=11.05, P=0.03\right)$, acute brain pathology $\left(X^{2}[4, N=260]=11.17, P=0.03\right)$ and pneumonia $\left(X^{2}[4, N=259]=10.28, P=0.04\right)$ patient categories, they indicated they were more likely to reduce the $\mathrm{F}_{1} \mathrm{O}_{2}$ (Figure 10). This change persisted after publication for postoperative surgical patients with respondents reportedly even more likely to reduce the $\mathrm{F}_{1} \mathrm{O}_{2}$ from 0.3. No further changes were evident for the admission diagnoses of acute brain pathology or pneumonia, and no change was apparent at any time for the 'other ICU patient' category. In contrast, the non-ICU-ROX respondents remained similarly likely to indicate they would reduce $\mathrm{F}_{1} \mathrm{O}_{2}$ from 0.3 if the $\mathrm{SpO}_{2} \geq 97 \%$. No change was seen after ICU-ROX was conducted or after the ICU-ROX publication compared to before ICU-ROX was began, irrespective of patient admission diagnosis.

\subsubsection{Comparisons by exposure for $\mathrm{F}_{1} \mathrm{O}_{2}$ of $\mathbf{0 . 4}$}

The indicated likelihood of respondents reducing $\mathrm{F}_{1} \mathrm{O}_{2}$ from 0.4 (if the $\mathrm{SpO}_{2} \geq 97 \%$,) from ICUROX and non-ICU-ROX sites was generally very similar before the ICU-ROX trial was conducted (Figure 11). This similarity in likelihood was present for all patient categories, with the exception that a difference was evident in the 'other ICU patients' category $\left(X^{2}[3\right.$, $N=251]=7.84, \mathrm{P}=0.049$ ), when the 'unlikely' and 'very unlikely' categories were combined. After the ICU-ROX trial was conducted, respondents from ICU-ROX trial sites and those from non-ICU-ROX trial sites were similarly likely to indicate they would reduce an $\mathrm{F}_{1} \mathrm{O}_{2}$ from 0.4 , except for patients with an admission diagnosis of acute brain pathology ( $X^{2}[3, N=223]$ $=7.96, \mathrm{P}=0.047)$. For this group of patients, the ICU-ROX respondents were more likely to indicate would reduce the $\mathrm{F}_{1} \mathrm{O}_{2}$ than the non-ICU-ROX respondents. After ICU-ROX was published, there were no significant differences between the ICU-ROX and non-ICU-ROX respondents for any patient categories (Figure 8, Figure 11).

The ICU-ROX respondents' indicated likelihood of reducing $\mathrm{F}_{1} \mathrm{O}_{2}$ from 0.4 (if $\mathrm{SpO}_{2} \geq 97 \%$ ) while ventilated remained similar for most patient groups over time (Figure 11). However, for 
patients with an acute brain pathology the likelihood of reducing $\mathrm{F}_{1} \mathrm{O}_{2}$ increased $\left(X^{2}[3\right.$, $N=259]=10.43, P=0.02$ ), after participation in ICU-ROX. No differences over time were seen for any other patient categories for the ICU-ROX respondents. Respondents from non-ICUROX sites remained similarly likely to reduce the $\mathrm{F}_{1} \mathrm{O}_{2}$ from 0.4 after ICU-ROX was completed, and after publication of the ICU-ROX trial compared to before ICU-ROX, irrespective of patient diagnosis.

\subsubsection{Comparisons by exposure for $\mathrm{F}_{1} \mathrm{O}_{2}$ of $\mathbf{0 . 6}$}

Before the ICU-ROX trial was conducted, respondents from both ICU-ROX and non-ICU-ROX sites similarly indicated that they would reduce the $\mathrm{F}_{1} \mathrm{O}_{2}$ from 0.6 if the $\mathrm{SpO}_{2}$ was $\geq 97 \%$ (Figure 12) in most patient groups. The exception was for patients with pneumonia, for which there was a statistically significant difference $\left(X^{2}[3, N=251]=9.99, P=0.02\right)$, when the 'Unlikely' and 'Very unlikely' responses were combined, with the non-ICU-ROX respondents being more likely to reduce the $\mathrm{F}_{1} \mathrm{O}_{2}$ than the ICU-ROX respondents. Statistical comparison was not possible after ICU-ROX or after publication because the number of responses in the 'Very likely', 'Likely' and 'Neither likely nor unlikely' categories were too small. However, visually the distribution of responses appears similar for ICU-ROX and non-ICU-ROX respondents (Figure 8, Figure 12).

For ICU-ROX respondents, the indicated likelihood of reducing $\mathrm{F}_{\mathrm{l}} \mathrm{O}_{2}$ from 0.6 (if $\mathrm{SpO}_{2} \geq 97 \%$ ) changed significantly for patients with an admission diagnosis of acute brain pathology $\left(X^{2}\right.$ $[3, N=257]=9.84, P=0.02$ ) after they had participated in ICU-ROX (Figure 12). No other significant changes were seen or it was not possible to conduct chi-square for any other patient groups, and none could be conducted for non-ICU-ROX respondents. For non-ICU$\mathrm{ROX}$ respondents' the indicated likelihood of reducing $\mathrm{F}_{1} \mathrm{O}_{2}$ from 0.6 appears similar over time (Figure 12).

\subsubsection{Comparisons by exposure for $\mathrm{F}_{1} \mathrm{O}_{2}$ of $\mathbf{1} .0$}

The indicated likelihood of reducing $\mathrm{F}_{1} \mathrm{O}_{2}$ from 1.0 if $\mathrm{SpO}_{2} \geq 97 \%$ appeared similar for respondents from ICU-ROX and non-ICU-ROX sites before and after ICU-ROX was conducted, irrespective of patient admission diagnosis (Figure 13). Chi-square was not conducted for several patient categories due to insufficient responses in the 'Very unlikely' and 'Unlikely' categories. The distribution appeared to differ after ICU-ROX was published with a higher 
proportion of non-ICU-ROX respondents selecting 'Very Likely' to reduce the $\mathrm{F}_{1} \mathrm{O}_{2}$ compared to their trial-site counterparts (Figure 13). Over time, reported likelihood of reducing $\mathrm{F}_{1} \mathrm{O}_{2}$ from 1.0 (if $\mathrm{SpO}_{2} \geq 97 \%$ ), appeared very similar in both the ICU-ROX and non-ICU-ROX site respondents. 


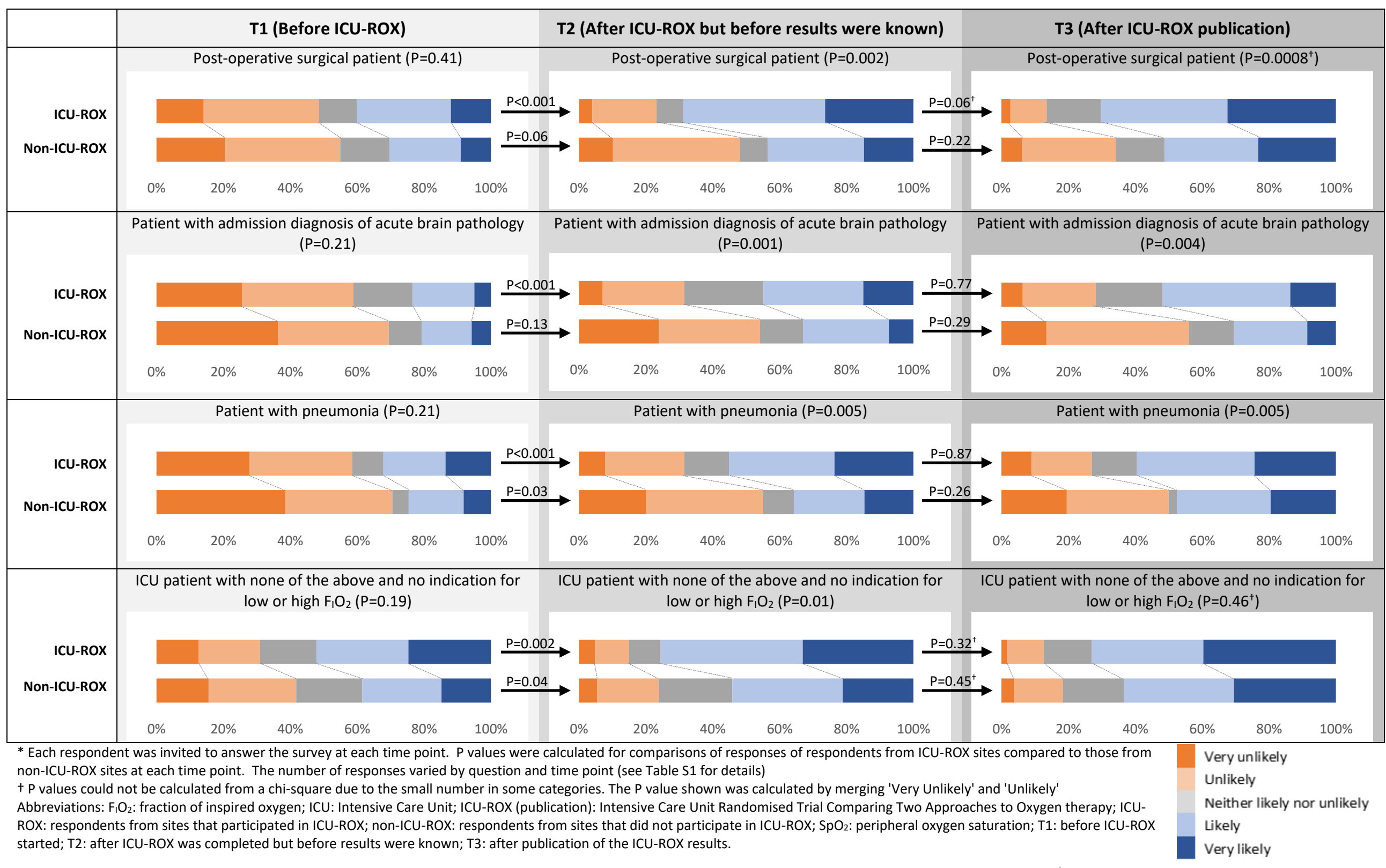

Figure 7 Likelihood of ICU-ROX vs non-ICU-ROX site respondents using $\mathrm{F}_{1} \mathrm{O}_{2}$ of 0.21 if $\mathrm{SpO}_{2}$ is $91-96 \%$, over time* 


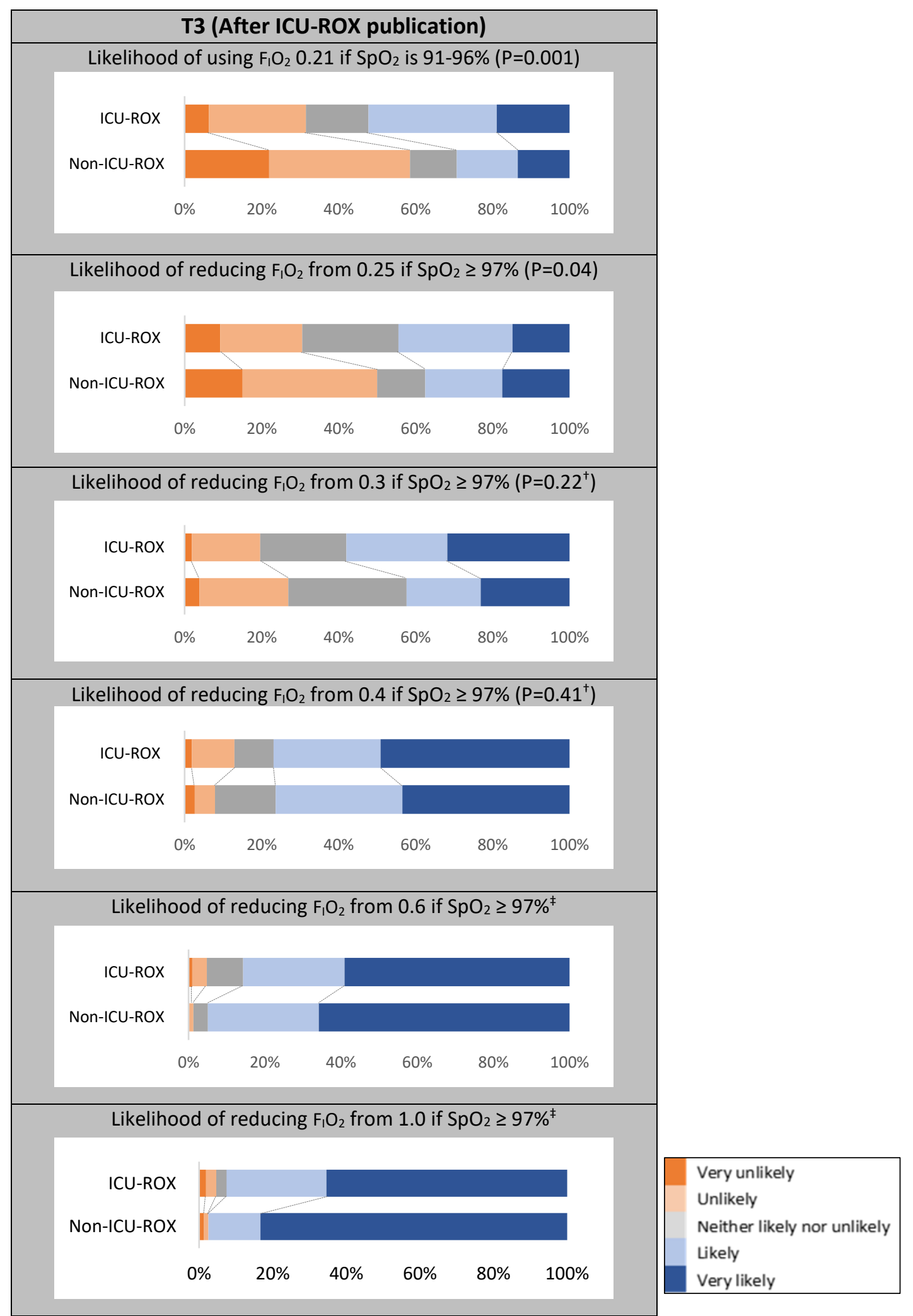

\section{Figure 8 Likelihood of ICU-ROX vs non-ICU-ROX site respondents using different levels of $\mathrm{F}_{1} \mathrm{O}_{2}$ for patients with suspected Hypoxic Ischaemic Encephalopathy (T3) ${ }^{*}$}

\footnotetext{
* Each respondent was invited to answer the survey at each time point. P values were calculated for comparisons of responses of respondents from ICU-ROX sites compared to those from non-ICU-ROX sites at each time point. The number of responses varied by question and time point (see Table S1 for details)

† $\mathrm{P}$ values could not be calculated from a chi-square due to the small number in some categories. The P value shown was calculated by merging 'Very Unlikely' and 'Unlikely'

$\ddagger P$ values could not be calculated from chi-square due to the small number in some categories (even when categories were merged). Abbreviations: $\mathrm{F}_{1} \mathrm{O}_{2}$ : fraction of inspired oxygen; ICU-ROX (publication): Intensive Care Unit Randomised Trial Comparing Two Approaches to Oxygen therapy; ICU-ROX: respondents from sites that participated in ICU-ROX; non-ICU-ROX: respondents from sites that did not participate in ICU-ROX; T3: after publication of ICU-ROX results.
} 


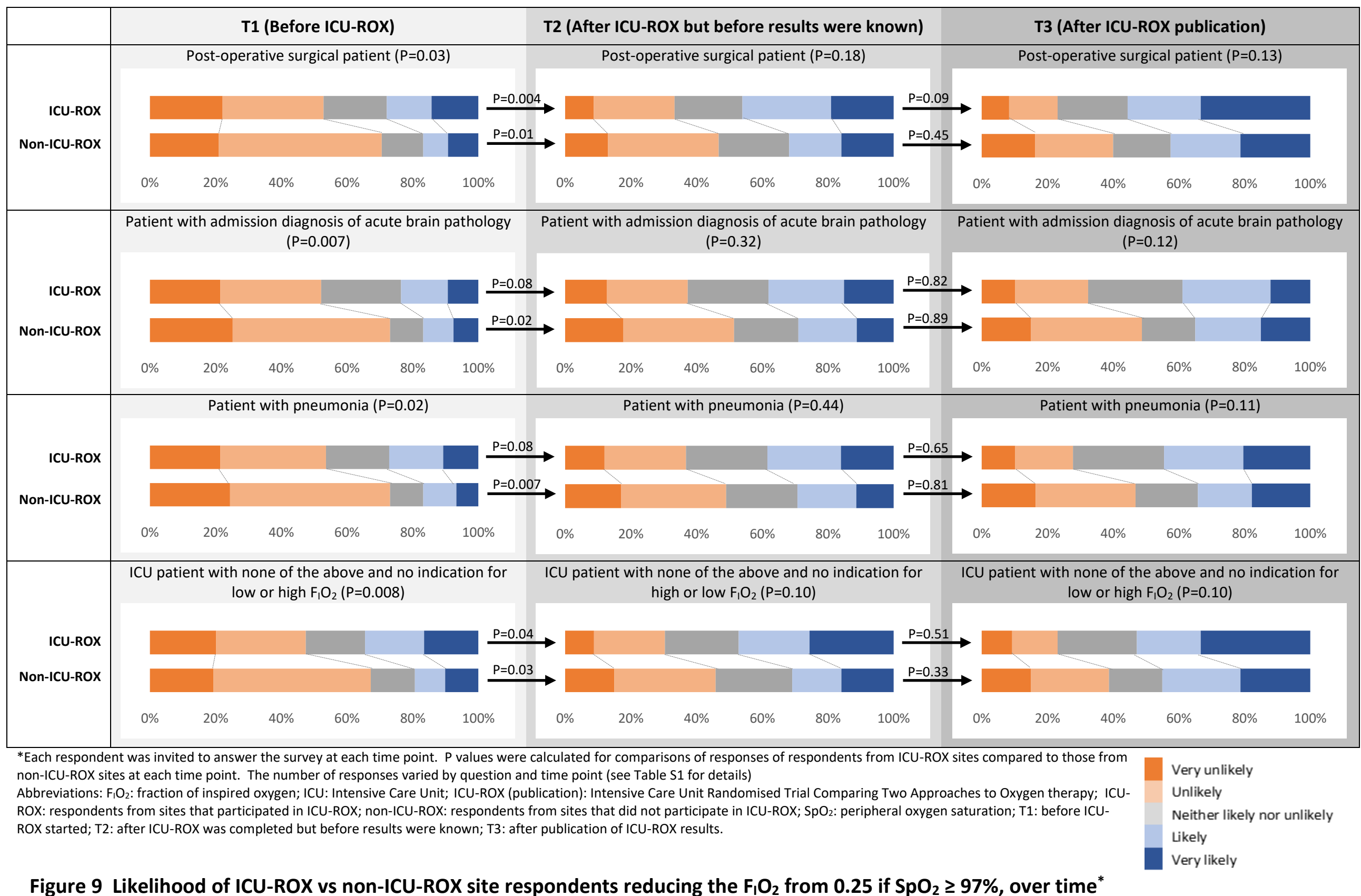




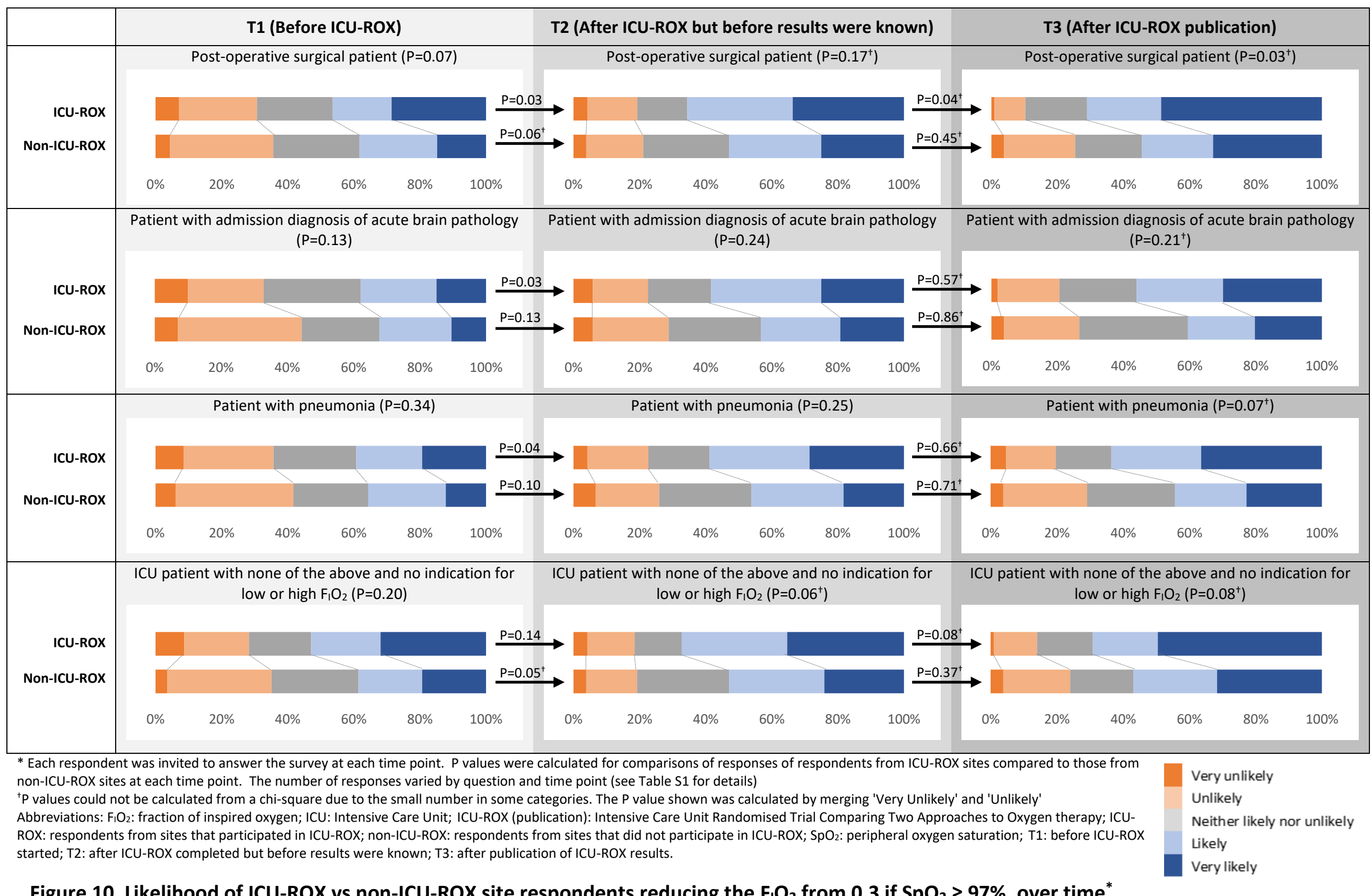




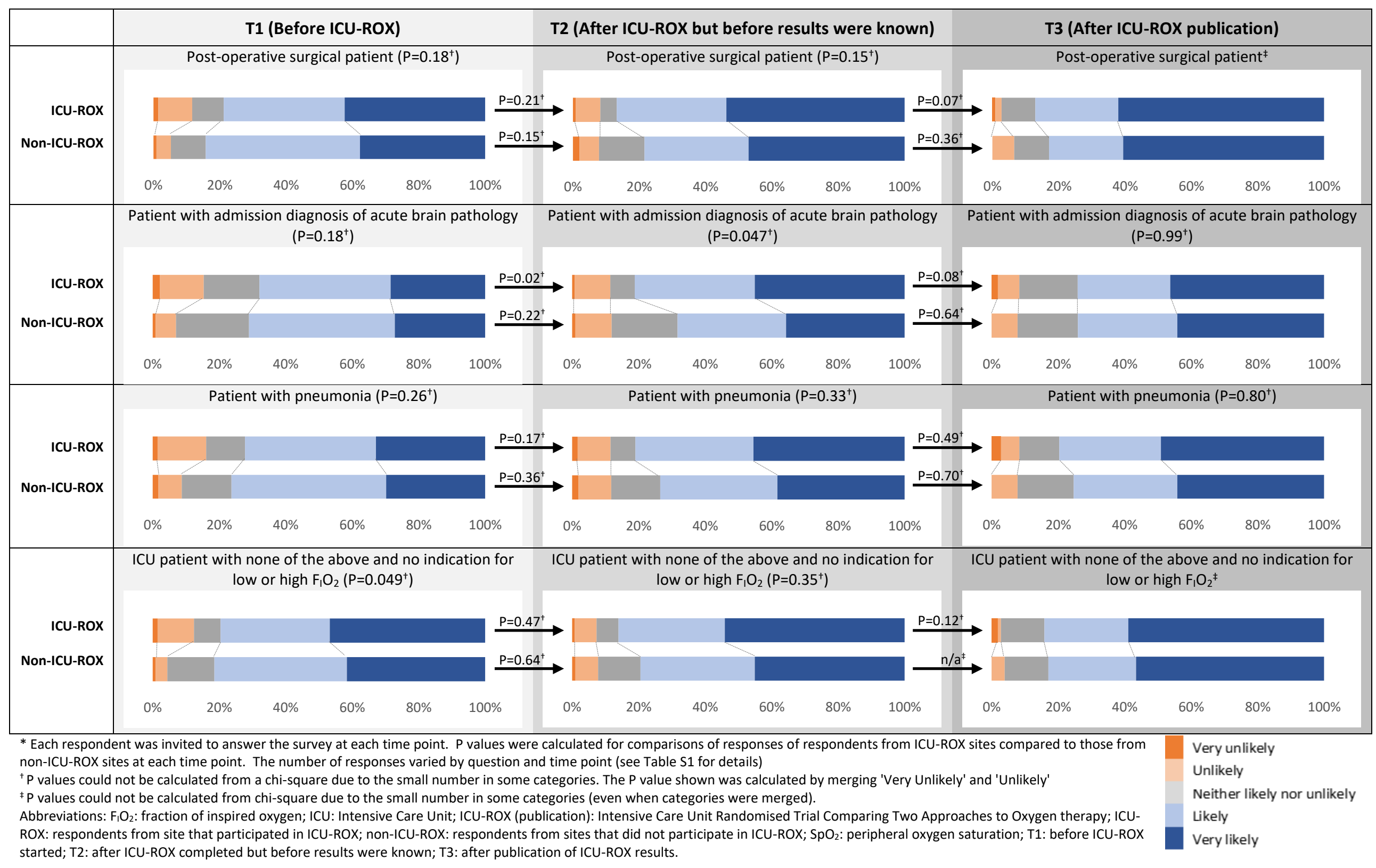

Figure 11 Likelihood of ICU-ROX vs non-ICU-ROX site respondents reducing the $\mathrm{F}_{1} \mathrm{O}_{2}$ from 0.4 if $\mathrm{SpO}_{2} \geq 97 \%$, over time* 


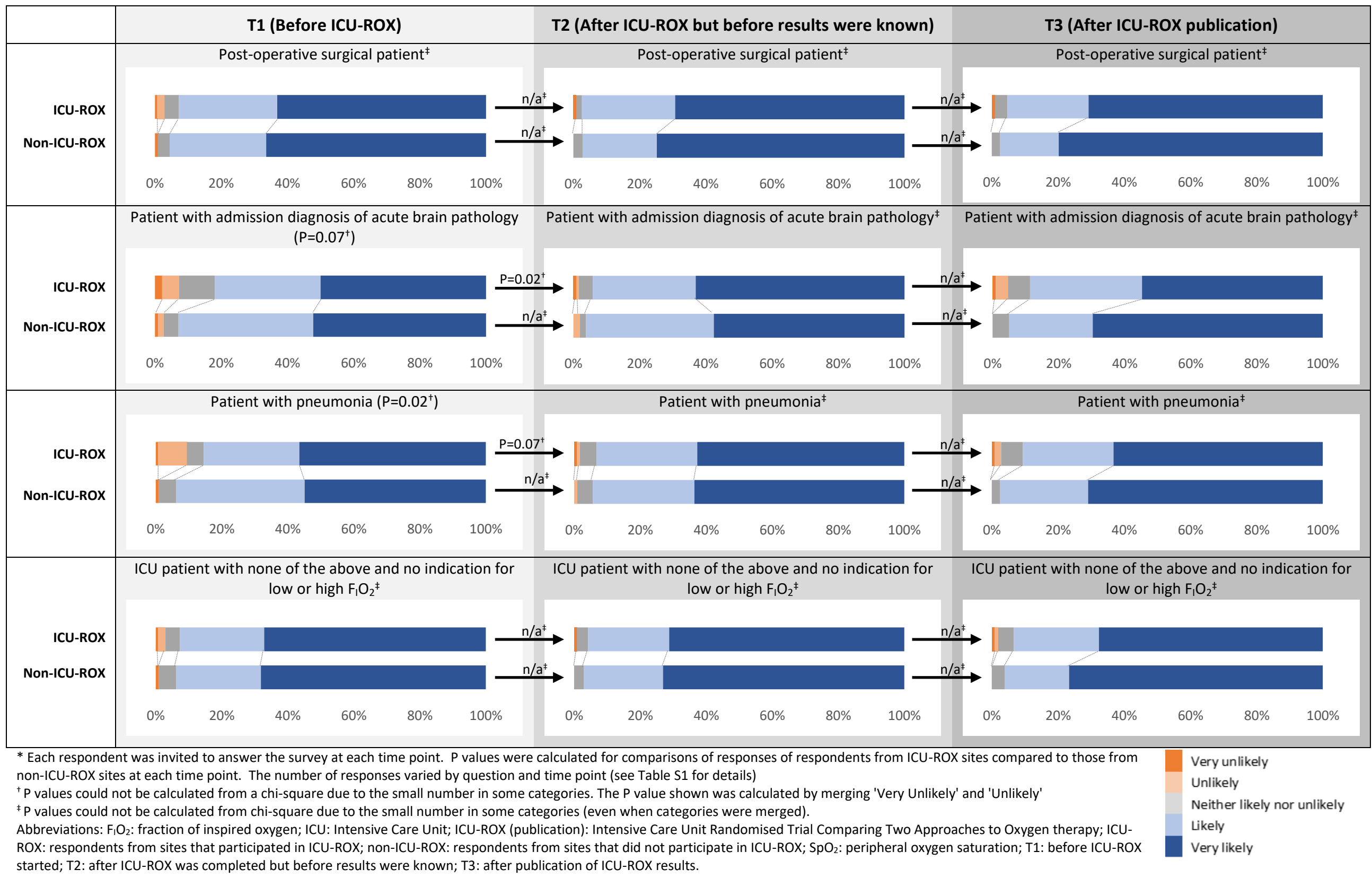

started; T2: after ICU-ROX was completed but before results were known; T3: after publication of ICU-ROX results.

Figure 12 Likelihood of ICU-ROX vs non-ICU-ROX site respondents reducing the $\mathrm{F}_{1} \mathrm{O}_{2}$ from 0.6 if $\mathrm{SpO}_{2} \geq 97 \%$, over time* 


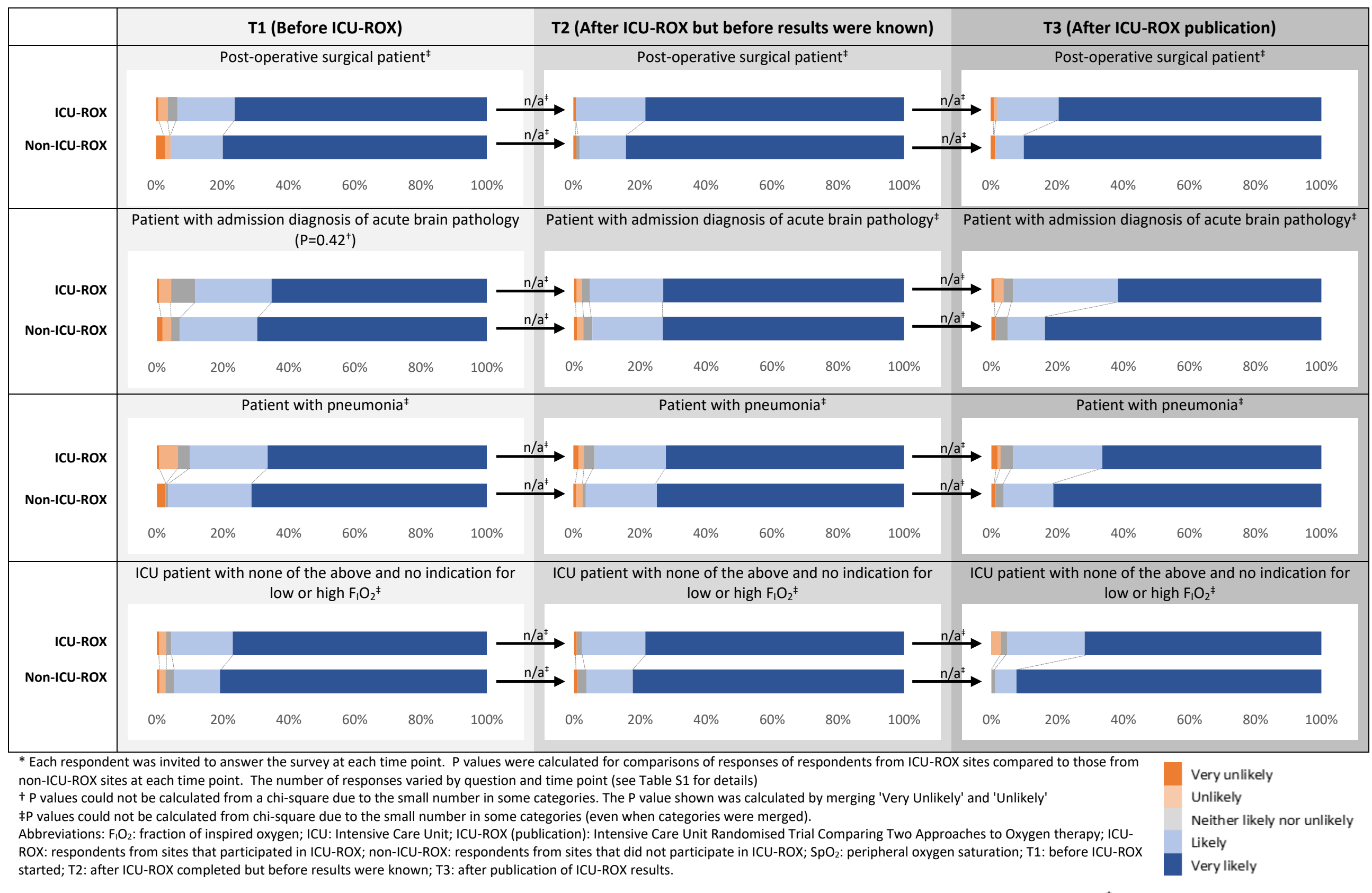

Figure 13 Likelihood of ICU-ROX vs non-ICU-ROX site respondents reducing the $\mathrm{F}_{1} \mathrm{O}_{2}$ from 1.0 if $\mathrm{SpO}_{2} \geq 97 \%$, over time* 


\subsubsection{Comparisons by role (ICU specialist doctor vs ICU nurse)}

\subsubsection{Primary measure of interest $\left(\mathrm{F}_{1} \mathrm{O}_{2}\right.$ of 0.21$)$}

Even before the ICU-ROX trial started, there was a significant difference in the reported likelihood of using $\mathrm{F}_{1} \mathrm{O}_{2}$ of 0.21 in ventilated patients with an $\mathrm{SpO}_{2}$ of $91-96 \%$ between ICU specialist doctor respondents and ICU nurse respondents (Figure 14). In particular, for postoperative surgical patients $\left(X^{2}[4, N=264]=28.01, P<0.001\right)$, patients with an admission diagnosis of acute brain pathology $\left(X^{2}[4, N=262]=11.89, P=0.02\right)$, and patients with pneumonia $\left(X^{2}[4, N=262]=25.13, P<0.001\right)$, nurses indicated they were less likely to use an $\mathrm{F}_{1} \mathrm{O}_{2}$ of 0.21 , than the doctors. The exception to this was for 'other ICU patients', in which there was no difference.

This difference in likelihood persisted after ICU-ROX had been conducted, but before the results were known (Figure 14). Specifically, for post-operative surgical patients $\left(X^{2}[4\right.$, $N=233]=25.28, P<0.001)$, patients with pneumonia $\left(X^{2}[4, N=233]=15.98, P=0.003\right)$ and 'other ICU' patients $\left(X^{2}[4, N=236]=9.86, p=0.04\right)$, nurses remained less likely than doctors to state they would use an $\mathrm{F}_{1} \mathrm{O}_{2}$ of 0.21 for most patient groups. This difference remained for post-operative surgical patients and patients with pneumonia, after publication of the ICU-ROX results; however, there was no difference for 'other ICU patients', or patients with an acute brain pathology at any time. A difference in reported likelihood was seen for patients with a suspected hypoxic brain encephalopathy $\left(X^{2}[4, N=193]=18.70, P=0.001\right)$, with nurses indicating they were less likely to use an $\mathrm{F}_{1} \mathrm{O}_{2}$ of 0.21 with these patients than the doctors (Figure 15).

Doctors indicated they would be more likely to use an $\mathrm{F}_{1} \mathrm{O}_{2}$ of 0.21 (when the $\mathrm{SpO}_{2}$ was 9196\%) after ICU-ROX was completed, than before ICU-ROX started, for some patient diagnostic groups (Figure 12). This change was evident for post-operative surgical $\left(X^{2}[4\right.$, $N=211]=9.69, \mathrm{P}=0.046)$ and 'other ICU' patients $\left(X^{2}[4, N=211]=11.03, \mathrm{P}=0.03\right)$. The doctors appeared similarly likely to use an $\mathrm{F}_{1} \mathrm{O}_{2}$ of 0.21 after the ICU-ROX results were published, as they did after ICU-ROX was completed, but did not change further.

Nurses' indicated likelihood of using $\mathrm{F}_{1} \mathrm{O}_{2}$ of 0.21 (when the $\mathrm{SpO}_{2}$ was 91-96\%) changed significantly after ICU-ROX was conducted, compared to before ICU-ROX, irrespective of patient diagnosis (Figure 14). Specifically, nurses reportedly became more likely to indicate 
they would reduce $\mathrm{F}_{1} \mathrm{O}_{2}$ from 0.21 in post-operative surgical patients $\left(X^{2}[4, N=286]=21.69\right.$, $\mathrm{P}<0.001)$, acute brain pathology $\left(X^{2}[4, N=290]=25.53, \mathrm{P}<0.001\right)$, pneumonia $\left(X^{2}[4, N=287]\right.$ $=27.51, \mathrm{P}<0.001)$ and 'other ICU' patient $\left(X^{2}[4, N=289]=16.39, \mathrm{P}=0.003\right)$. In general, these differences remained after the ICU-ROX trial was published, however did not change further, with the exception of postoperative surgical patients. For this patient admission category, the nurses became even more likely to use an $\mathrm{F}_{1} \mathrm{O}_{2}$ of $0.21\left(X^{2}[4, N=245]=15.25, \mathrm{P}=0.004\right)$.

\subsubsection{Comparisons by role for $\mathrm{F}_{1} \mathrm{O}_{2}$ of $\mathbf{0 . 2 5}$}

Before ICU-ROX was conducted, there was a difference in reported likelihood of reducing $\mathrm{F}_{1} \mathrm{O}_{2}$ from 0.25 (if $\mathrm{SpO}_{2} \geq 97 \%$ ) between respondents who were doctors, and their nursing counterparts, for some patient diagnostic groups (Figure 16). Specifically, doctors were significantly less likely to indicate they would reduce the $\mathrm{F}_{1} \mathrm{O}_{2}$ for patients with an admission diagnosis of acute brain pathology $\left(X^{2}[4, N=259]=10.27, \mathrm{P}=0.04\right)$ and for 'other ICU patients' $\left(X^{2}[4, N=258]=11.05, P=0.03\right)$, than nurses. Doctors and nurses were similarly unlikely to indicate they would reduce the $\mathrm{F}_{1} \mathrm{O}_{2}$ from 0.25 for post-operative surgical patients and patients with pneumonia. After ICU-ROX was conducted and again after publication of ICUROX the indicated preferences for both doctor and nurse respondents indicated they were similarly likely to reduce $\mathrm{F}_{1} \mathrm{O}_{2}$ from 0.25 , irrespective of patient diagnosis (Figure 15, Figure 16).

Doctor respondents' indicated likelihood of reducing $\mathrm{F}_{1} \mathrm{O}_{2}$ from 0.25 (if $\mathrm{SpO}_{2} \geq 97 \%$ ) changed after ICU-ROX was completed, compared to before ICU-ROX, irrespective of patient diagnosis (Figure 16). Specifically, they indicated they were more likely to reduce the $\mathrm{F}_{1} \mathrm{O}_{2}$ for post-operative surgical $\left(X^{2}[4, N=208]=13.30, P=0.01\right)$; acute brain pathology $\left(X^{2}[4\right.$, $N=207]=11.60, \mathrm{P}=0.02)$, pneumonia $\left(X^{2}[4, N=208]=10.88, \mathrm{P}=0.03\right)$ and 'other' ICU patients $\left(X^{2}[4, N=206]=12.00, P=0.02\right)$. This apparent change remained after the ICU-ROX trial was published, but did not change further.

The indicated likelihood of nurses reducing the $\mathrm{F}_{1} \mathrm{O}_{2}$ from 0.25 (if $\mathrm{SpO}_{2} \geq 97 \%$ ) changed over time for some patient groups (Figure 16). Specifically, the distribution of likelihood was statistically significantly different after ICU-ROX was conducted compared to before ICUROX for post-operative surgical patients $\left(X^{2}[4, N=284]=14.01, P=0.007\right)$ and those with pneumonia $\left(X^{2}[4, N=282]=11.88, P=0.02\right)$. However, the changes in likelihood for these two 
patient diagnoses did not change further after the publication of ICU-ROX. The indicated likelihood remained similar for other patient admission diagnoses over time.

\subsubsection{Comparisons by role for $\mathrm{F}_{1} \mathrm{O}_{2}$ of 0.3}

Doctor and nurse respondents reported they were similarly likely to reduce the $\mathrm{F}_{1} \mathrm{O}_{2}$ from 0.3 if $\mathrm{SpO}_{2} \geq 97 \%$, irrespective of patient diagnosis, before ICU-ROX was conducted (Figure 17). This similarity remained after the ICU-ROX trial was conducted, but before the trial results were known, in relation to this aspect of oxygen management for all except patients with acute brain pathology $\left(X^{2}[4, N=224]=9.94, P=0.04\right)$. For these patients, nurses reported they were more likely to reduce the $\mathrm{F}_{1} \mathrm{O}_{2}$ than the doctors. There was no difference between doctors and nurses after the ICU-ROX results had been published, irrespective of patient diagnosis (Figure 15, Figure 17).

For doctor respondents, the indicated likelihood of reducing $\mathrm{F}_{1} \mathrm{O}_{2} 0.3$ if $\mathrm{SpO}_{2} \geq 97 \%$, changed in most patient categories after ICU-ROX was completed, compared to before ICU-ROX started (Figure 17). In particular, for post-operative surgical patients $\left(X^{2}[4, N=201]=12.79\right.$, $\mathrm{P}=0.01)$, patients with pneumonia $\left(X^{2}[4, N=201]=12.41, \mathrm{P}=0.02\right)$ and 'other ICU patients' $\left(X^{2}\right.$ $[4, N=201]=13.21, P=0.04)$, doctors became significantly more likely to indicate they would reduce the $\mathrm{F}_{1} \mathrm{O}_{2}$ than previously. These apparent differences in likelihood remained after publication of the ICU-ROX results, however, there was no further change.

Nurse respondents were similarly likely to indicate they would reduce $\mathrm{F}_{1} \mathrm{O}_{2}$ from 0.3 if $\mathrm{SpO}_{2}$ $\geq 97 \%$ over time, for most patient diagnoses (Figure 17). The exception to this was for patients with an admission diagnosis of acute brain pathology $\left(X^{2}[4, N=278]=11.18, P=0.03\right)$. There was a difference in the 'Very unlikely' and 'Unlikely' categories from before ICU-ROX ( $14.1 \%$ and $25.5 \%$, respectively) to after ICU-ROX $(27.1 \%$ and $30.2 \%$, respectively). No further changes were evident after publication of the ICU-ROX results.

\subsubsection{Comparisons by role for $\mathrm{F}_{1} \mathrm{O}_{2}$ of 0.4}

At every time point, the doctor and nurse respondents similarly indicated they would reduce the $\mathrm{F}_{1} \mathrm{O}_{2}$ from 0.4 if $\mathrm{SpO}_{2}$ was $\geq 97 \%$, irrespective of patient diagnosis (Figure 15, Figure 18). Respondents in both groups were predominantly 'Likely' or 'Very Likely' to reduce the $\mathrm{F}_{1} \mathrm{O}_{2}$ from 0.4 for all patient categories. No difference over time was apparent for doctors 
regarding their likelihood of reducing $\mathrm{F}_{1} \mathrm{O}_{2}$ from 0.4 (if $\mathrm{SpO}_{2} \geq 97 \%$ ), irrespective of patient diagnosis (Figure 18). The nurses were similarly likely to indicate they would reduce $\mathrm{F}_{1} \mathrm{O}_{2}$ from 0.4 (if $\mathrm{SpO}_{2} \geq 97 \%$ ), after ICU-ROX as they were before ICU-ROX was started, with the exception of patients with an acute brain pathology $\left(X^{2}[3, N=273]=8.01, P=0.046\right)$. For this group of patients, the percentage of nurses who responded with 'Very likely' increased from $25.9 \%$ before ICU-ROX to $40 \%$ after ICU-ROX. There was no further change after ICU-ROX publication.

\subsubsection{Comparisons by role for $\mathrm{F}_{1} \mathrm{O}_{2}$ of 0.6}

Before ICU-ROX was conducted, there were some differences in indicated likelihood of reducing $\mathrm{F}_{1} \mathrm{O}_{2}$ from 0.6 (if $\mathrm{SpO}_{2} \geq 97 \%$ ) between doctors and nurses (Figure 19). Specifically, for patients with an acute brain pathology $\left(X^{2}[3, N=251]=12.20, P=0.007\right)$ and pneumonia $\left(X^{2}[3 N=251]=17.74, P<0.001\right)$, doctors answered with 'very likely' more than the nurses. Chi-square could not be calculated for other patient categories or at any other time points, even after recoding. The doctor respondents' likelihood appeared very similar over time for all patient categories. Nurse respondents appeared to become more likely to reduce $\mathrm{F}_{1} \mathrm{O}_{2}$ from 0.6 (if $\mathrm{SpO}_{2} \geq 97 \%$ ) over time. This change was statistically significant for patients with pneumonia $\left(X^{2}[3, N=273]=11.78, P=0.008\right)$ after ICU-ROX compared to before, and it was not possible to conduct chi-square for any other categories.

\subsubsection{Comparisons by role for $\mathrm{F}_{1} \mathrm{O}_{2}$ of $\mathbf{1} .0$}

The predominant category for reported likelihood of reducing $\mathrm{F}_{1} \mathrm{O}_{2}$ from 1.0 (if $\mathrm{SpO}_{2} \geq 97 \%$ ) was 'Very likely' for both doctor and nurse respondents at each time point, irrespective of patient diagnosis (Figure 20). It was not possible to conduct chi-square in any patient category at any time point, even after recoding, due to insufficient responses in the 'Very unlikely', 'Unlikely' and Neither likely nor unlikely' categories. However, the doctors responded with 'very likely' more than their nurse counterparts in every patient category at every time point. The doctor respondents' likelihood of reducing $\mathrm{F}_{1} \mathrm{O}_{2}$ from 1.0 appeared similar over time; as did the responses of the nurses.

\subsubsection{ICU specialist doctors and ICU nurses (ICU-ROX vs Non-ICU-ROX)}

Doctor respondents from ICU-ROX trial sites and those from non-ICU-ROX trial sites similarly indicated would use an $\mathrm{F}_{1} \mathrm{O}_{2}$ of 0.21 in a patient with an $\mathrm{SpO}_{2}$ of $91-96 \%$, before ICU-ROX was 
conducted, irrespective of patient admission diagnosis (Figure 21). It was not possible to conduct analyses on patients with pneumonia, even when 'Very unlikely' and 'Unlikely' categories were combined. After the ICU-ROX trial finished but before the trial results were known, the indicated preference of trial-site doctor respondents, in relation to this aspect of oxygen management appeared to differ from their non-ICU-ROX counterparts. In particular for 'post-operative surgical' ( $\left.X^{2}[3, N=100]=8.55, P=0.04\right)$ and 'other ICU' patients $\left(X^{2}[3, N=100]=9.35, P=0.03\right)$. Doctors from ICU-ROX sites were more likely to indicate they would use an $\mathrm{F}_{1} \mathrm{O}_{2}$ of 0.21 compared to non-ICU-ROX doctors. This difference was not present for patients with an acute brain pathology and it was not possible to test for a difference for patients with pneumonia. There was a trend towards a difference between doctor respondents from ICU-ROX and non-ICU-ROX sites for post-operative surgical patients after the ICU-ROX trial had been published $\left(X^{2}[4, N=81]=7.76, P=0.051\right)$; however, this did not reach significance.

Before ICU-ROX was conducted, nurse respondents from ICU-ROX sites and those from nonICU-ROX sites indicated similarly they were unlikely to use an $\mathrm{F}_{1} \mathrm{O}_{2}$ of 0.21 in a patient with $\mathrm{SpO}_{2}$ of 91-96\%, irrespective of patient diagnosis, (Figure 22). In particular, for patients with an acute brain pathology and 'other ICU patients', there was no difference and there were insufficient responses in some categories to conduct an analysis for post-operative surgical patient or patients with pneumonia. After participating in the ICU-ROX trial, even before the trial results were known, indicated preferences of trial-site nurses differed from those of non-ICU-ROX nurses for some variables. In particular, for patients admitted with acute brain pathology $\left(X^{2}[4, N=137]=18.05, P=0.001\right)$ and those with pneumonia $\left(X^{2}[4, N=136]\right.$ $=10.58, P=0.03$ ) nurses from ICU-ROX sites were significantly more likely than their non-ICU$\mathrm{ROX}$ trial counterparts to indicate they would use an $\mathrm{F}_{1} \mathrm{O}_{2}$ of 0.21 . In these patient categories, nurses from than ICU-ROX sites, were more likely to respond as 'Likely' or 'Very likely' (acute brain pathology 43.9\%; patient with pneumonia 45.5\%) compared to the non-ICU-ROX sites ( $25.4 \%$ and $22.1 \%$, respectively). In general, it was not possible to conduct analyses for the nurse respondents for most patient groups after the ICU-ROX trial was published, due to the small number of responses in some categories. The exception to this is a difference in indicated likelihood for post-operative surgical patients $\left(X^{2}[3, N=112]=8.83, P=0.03\right)$, with nurse respondents from ICU-ROX sites significantly more likely to report they would use an $\mathrm{F}_{1} \mathrm{O}_{2}$ of 0.21 , than non-ICU-ROX site nurses. 
Due to the limited number of responses in many categories, no further analyses were undertaken. 


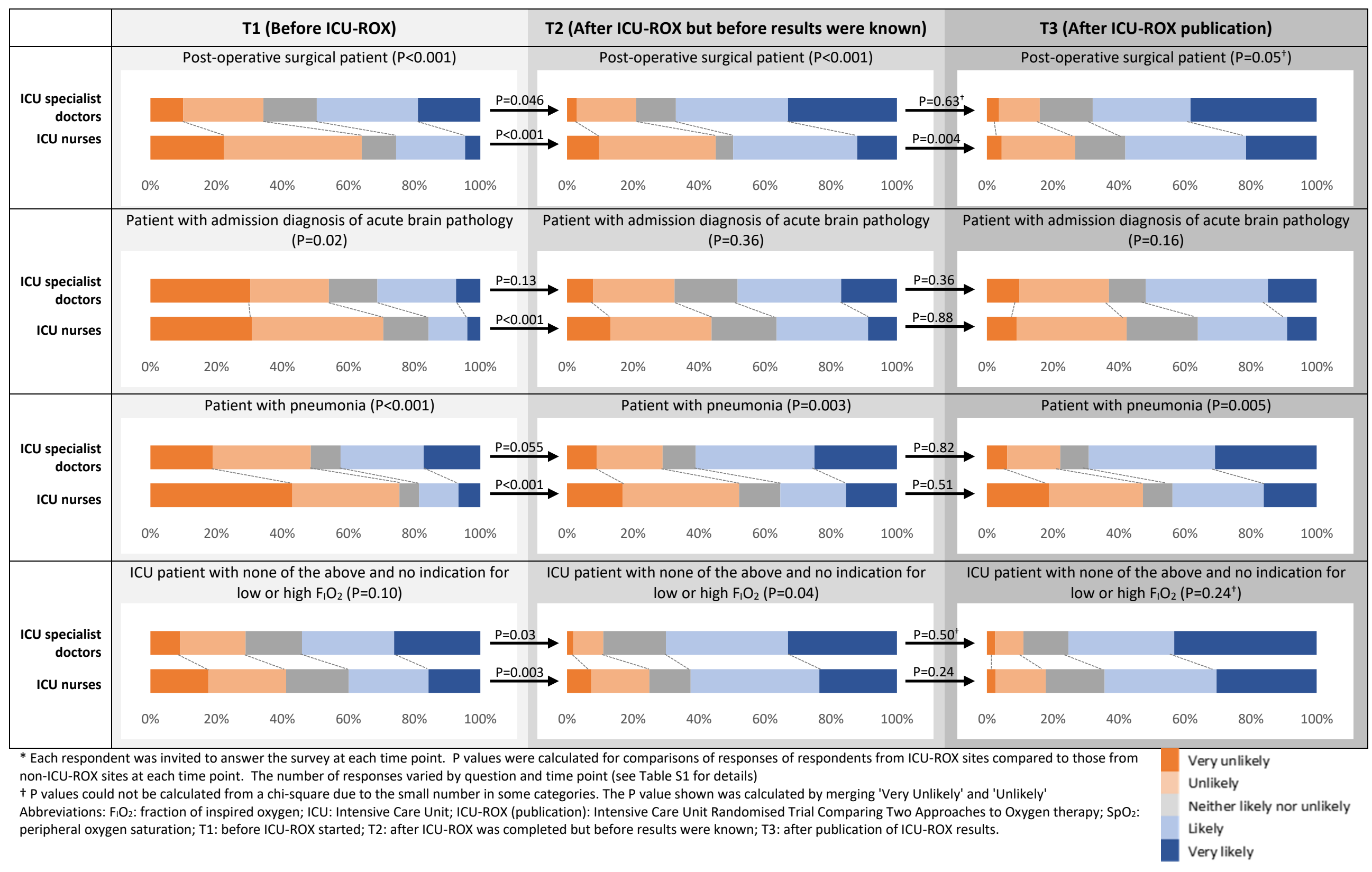

Figure 14 Likelihood of ICU doctor vs nurse respondents using $\mathrm{F}_{1} \mathrm{O}_{2}$ of 0.21 if $\mathrm{SpO}_{2}$ is $91-96 \%$, over time* 


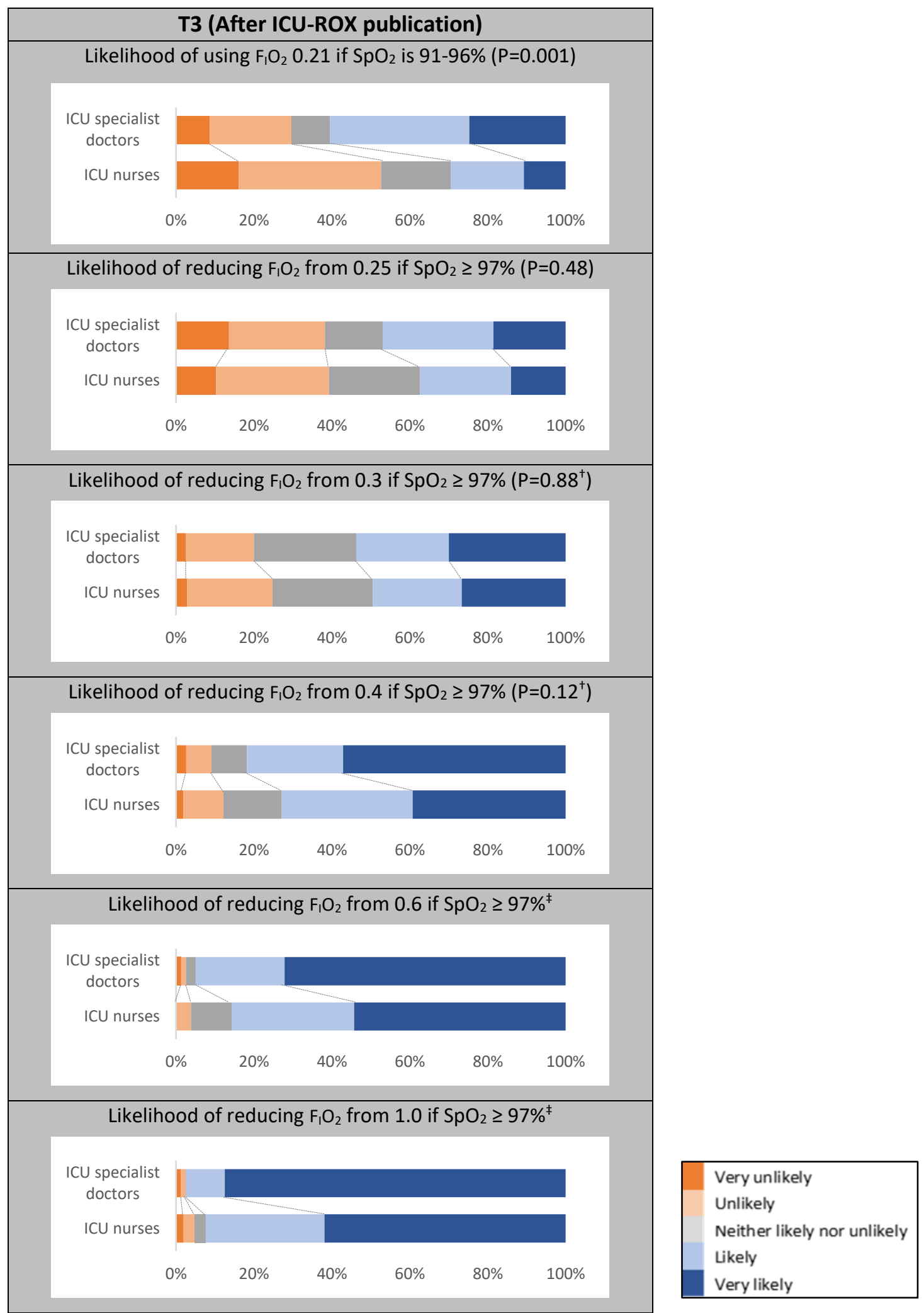

* Each respondent was invited to answer the survey at each time point. P values were calculated for comparisons of responses of respondents from ICU-ROX sites compared to those from non-ICU-ROX sites at each time point. The number of responses varied by question and time point (see Table S1 for details)

$+P$ values could not be calculated from a chi-square due to the small number in some categories. The $P$ value shown was calculated by merging 'Very Unlikely' and 'Unlikely'

$\ddagger P$ values could not be calculated from chi-square due to the small number in some categories (even when categories were merged). Abbreviations: $\mathrm{F}_{1} \mathrm{O}_{2}$ : fraction of inspired oxygen; ICU: Intensive Care Unit; ICU-ROX (publication): Intensive Care Unit Randomised Trial Comparing Two Approaches to Oxygen therapy; $\mathrm{SpO}_{2}$ : peripheral oxygen saturation; T1: before ICU-ROX started; T2: after ICU-ROX completed but before results were known; T3: after publication of ICU-ROX results.

Figure 15 Likelihood of using ICU doctor vs nurse respondents using different levels of $\mathrm{F}_{1} \mathrm{O}_{2}$ for patients with suspected Hypoxic Ischaemic Encephalopathy (T3) ${ }^{*}$ 


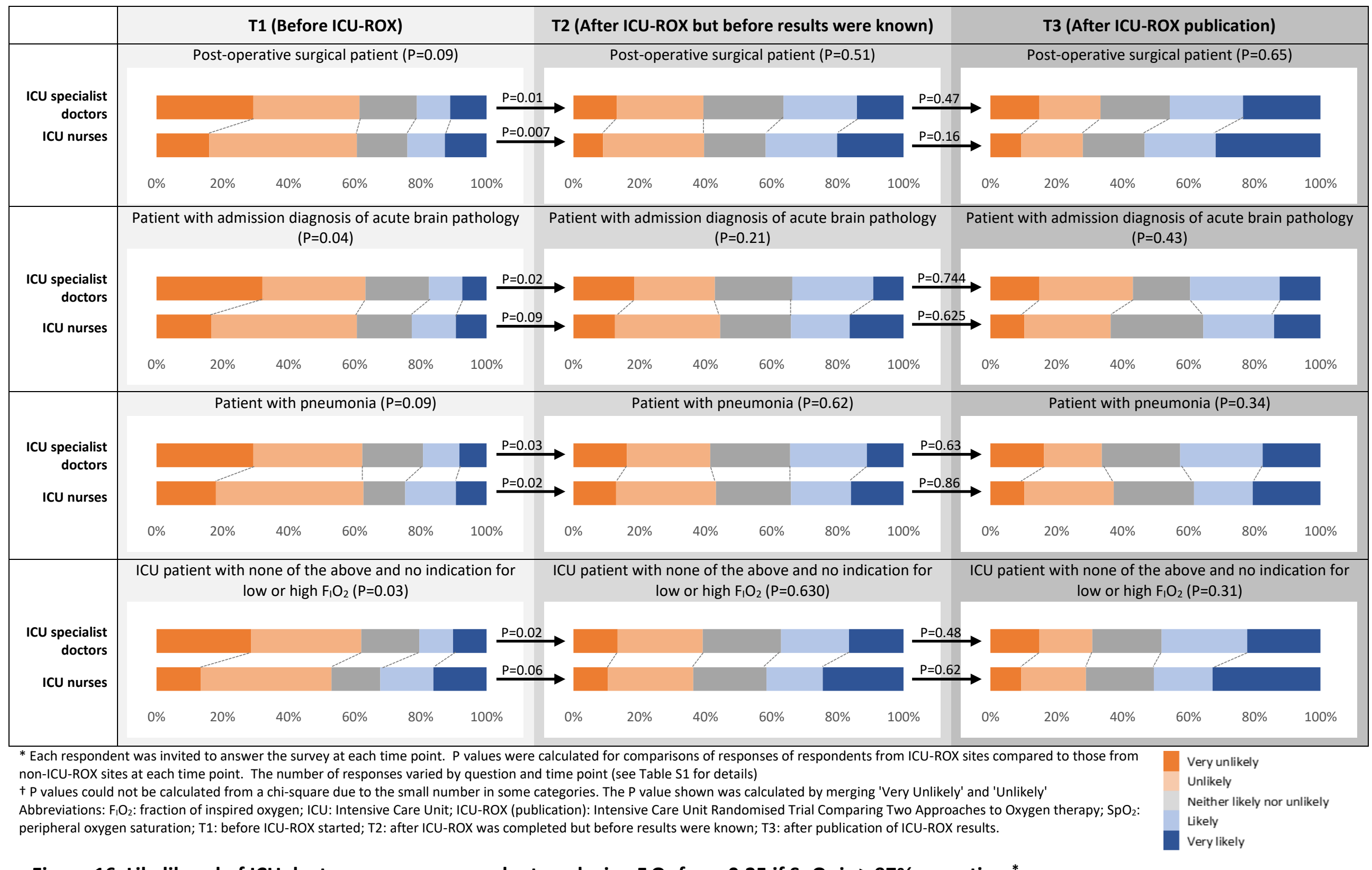

Figure 16 Likelihood of ICU doctor vs nurse respondents reducing $\mathrm{F}_{1} \mathrm{O}_{2}$ from 0.25 if $\mathrm{SpO}_{2}$ is $\geq 97 \%$, over time* 


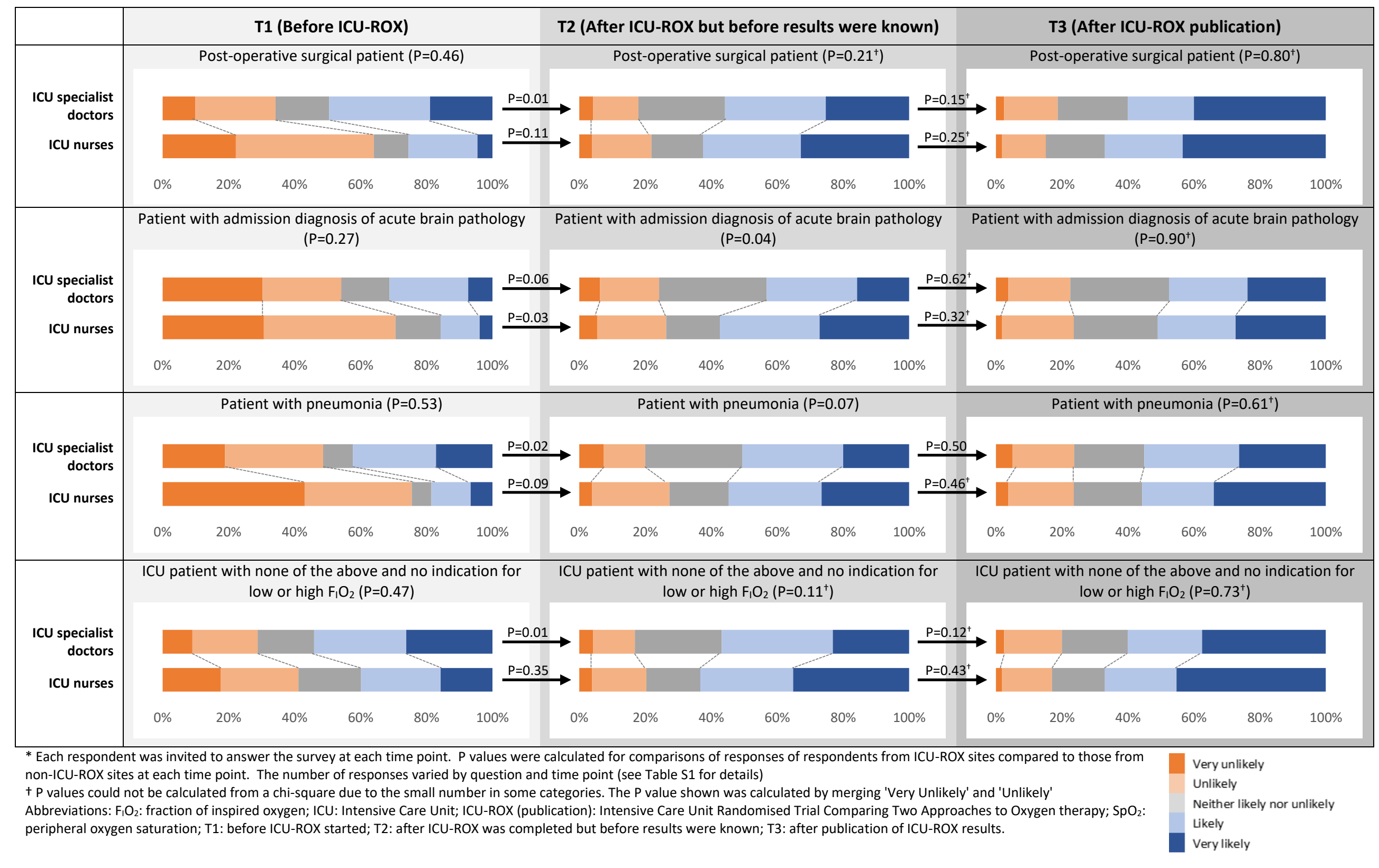

Figure 17 Likelihood of ICU doctor vs nurse respondents reducing $\mathrm{F}_{1} \mathrm{O}_{2}$ from 0.3 if $\mathrm{SpO}_{2}$ is $\geq 97 \%$, over time 


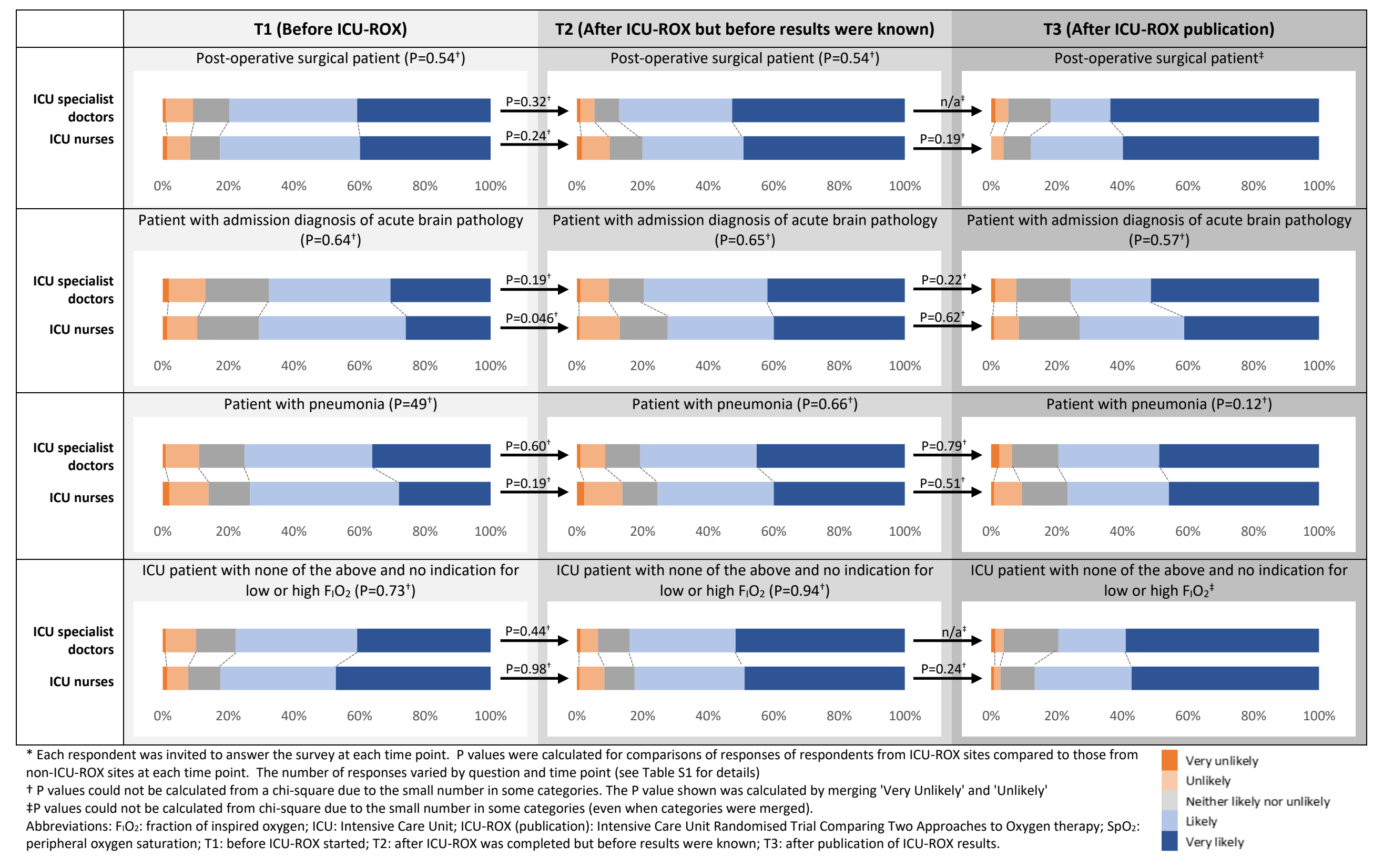

Figure 18 Likelihood of ICU doctor vs nurse respondents reducing $\mathrm{F}_{1} \mathrm{O}_{2}$ from 0.4 if $\mathrm{SpO}_{2}$ is $\geq 97 \%$, over time* 


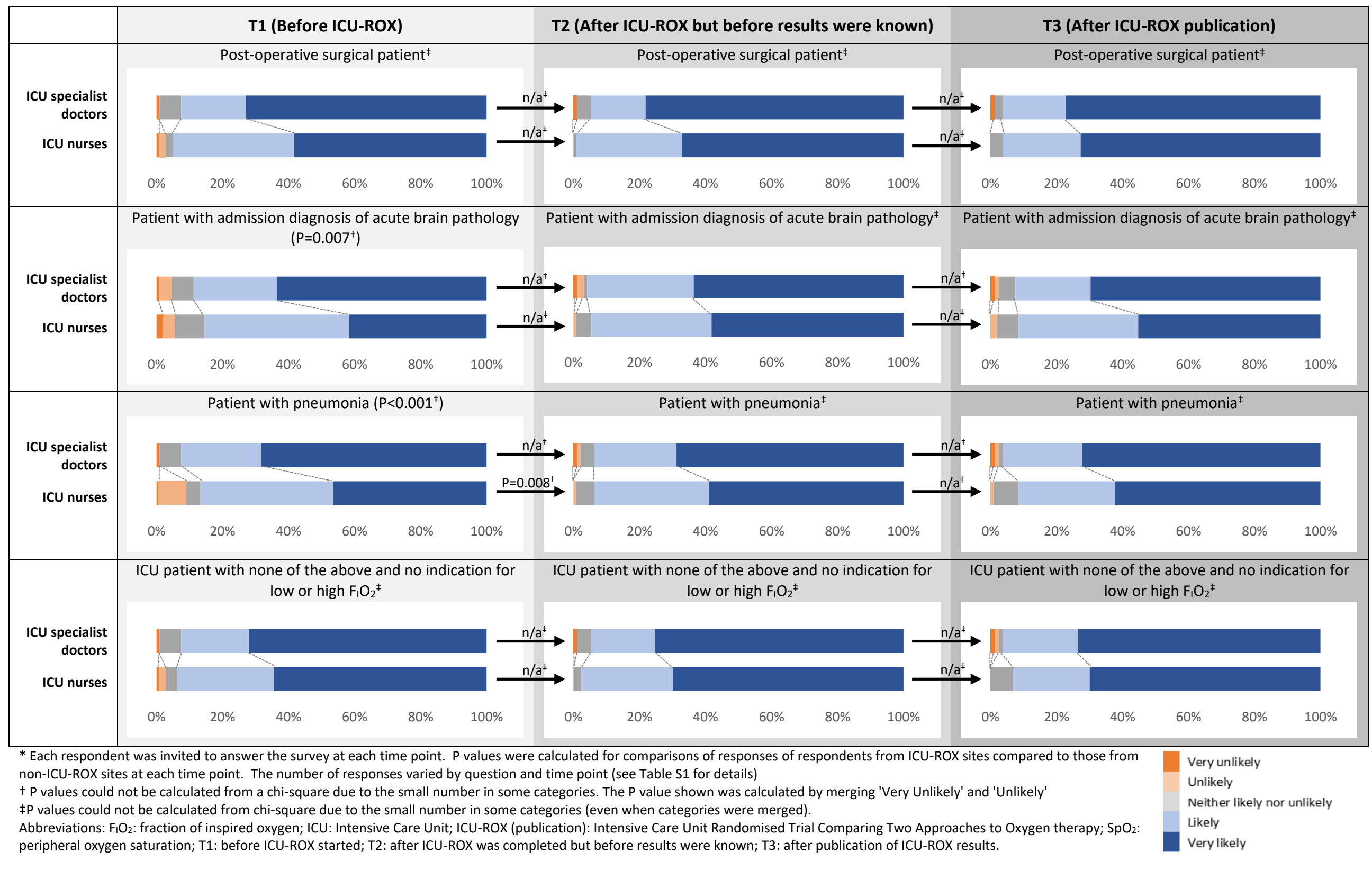

Figure 19 Likelihood of ICU doctor vs nurse respondents reducing $\mathrm{F}_{1} \mathrm{O}_{2}$ from 0.6 if $\mathrm{SpO}_{2}$ is $\geq 97 \%$, over time* 


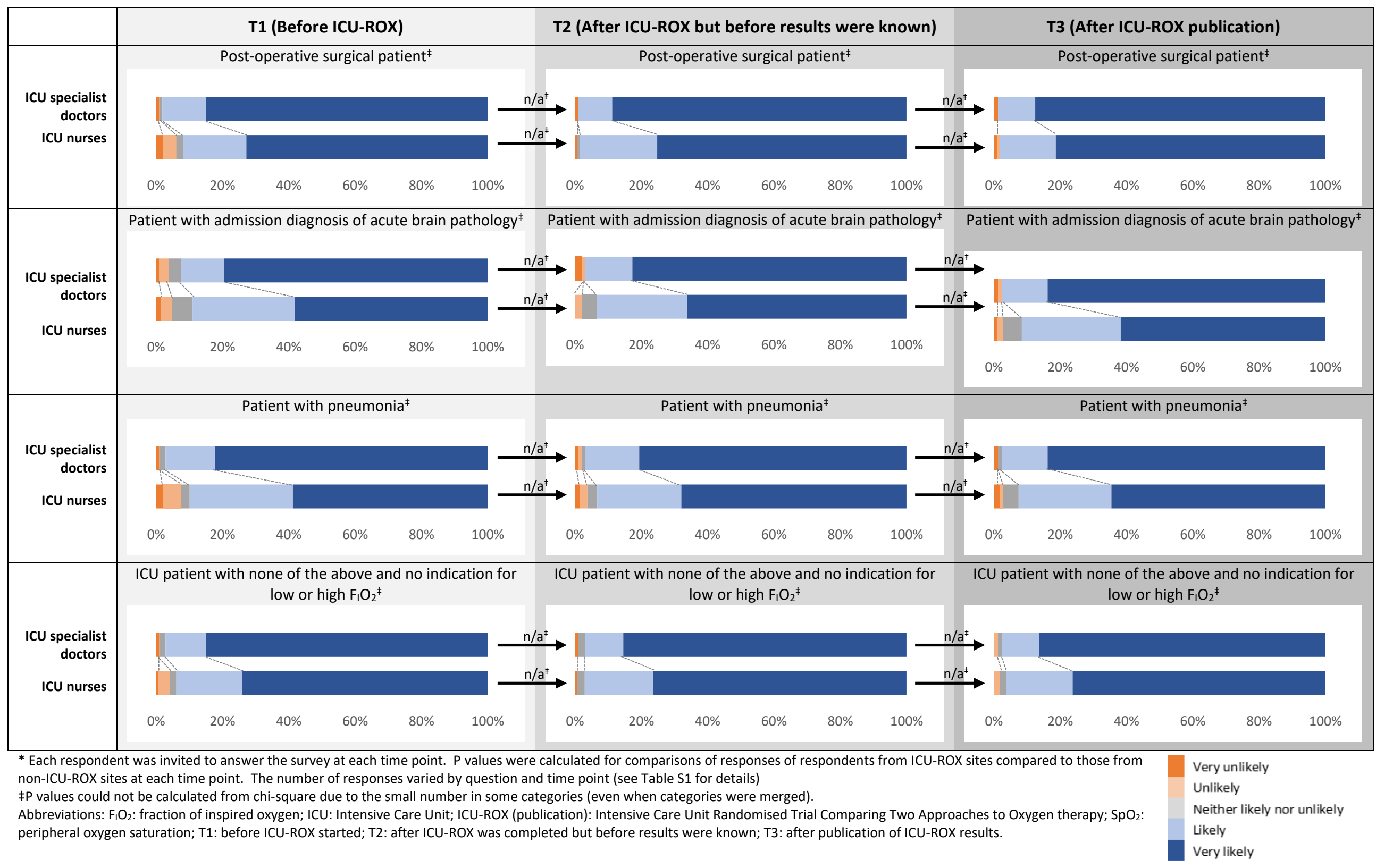

Figure 20 Likelihood of ICU doctor vs nurse respondents reducing $\mathrm{F}_{1} \mathrm{O}_{2}$ from 1.0 if $\mathrm{SpO}_{2}$ is $\geq 97 \%$, over time* 


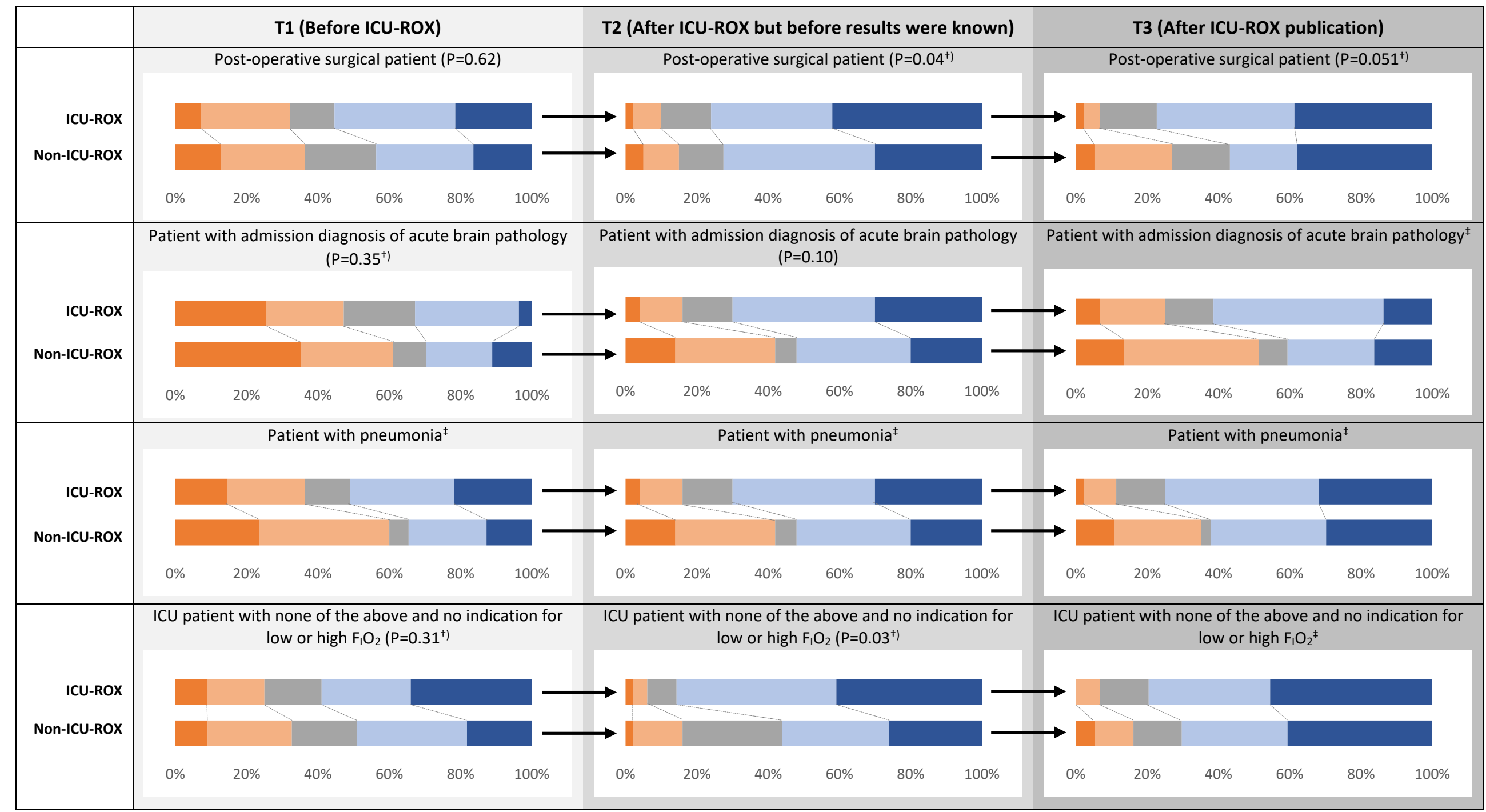

* Each respondent was invited to answer the survey at each time point. P values were calculated for comparisons of responses of respondents from ICU-ROX sites compared to those from non-ICU-ROX sites at each time point. The number of responses varied by question and time point (see Table S1 for details)

† P values could not be calculated from a chi-square due to the small number in some categories. The P value shown was calculated by merging 'Very Unlikely' and 'Unlikely' or 'Very likely' with 'Likely'. $\ddagger P$ values could not be calculated from chi-square due to the small number in some categories (even when categories were merged).

Abbreviations: $\mathrm{F}_{1} \mathrm{O}_{2}$ : fraction of inspired oxygen; ICU: Intensive Care Unit; ICU-ROX (publication): Intensive Care Unit Randomised Trial Comparing Two Approaches to Oxygen therapy; ICU-ROX: respondents from sites that participated in ICU-ROX; non-ICU-ROX: respondents from sites that
ICU-ROX was completed but before results were known; T3: after publication of ICU-ROX results.

\section{Figure 21 Likelihood of ICU specialist doctors (ICU-ROX vs non-ICU-ROX) using $\mathrm{F}_{1} \mathrm{O}_{2}$ of 0.21 if $\mathrm{SpO}_{2}$ is $91-96 \%$, over time}




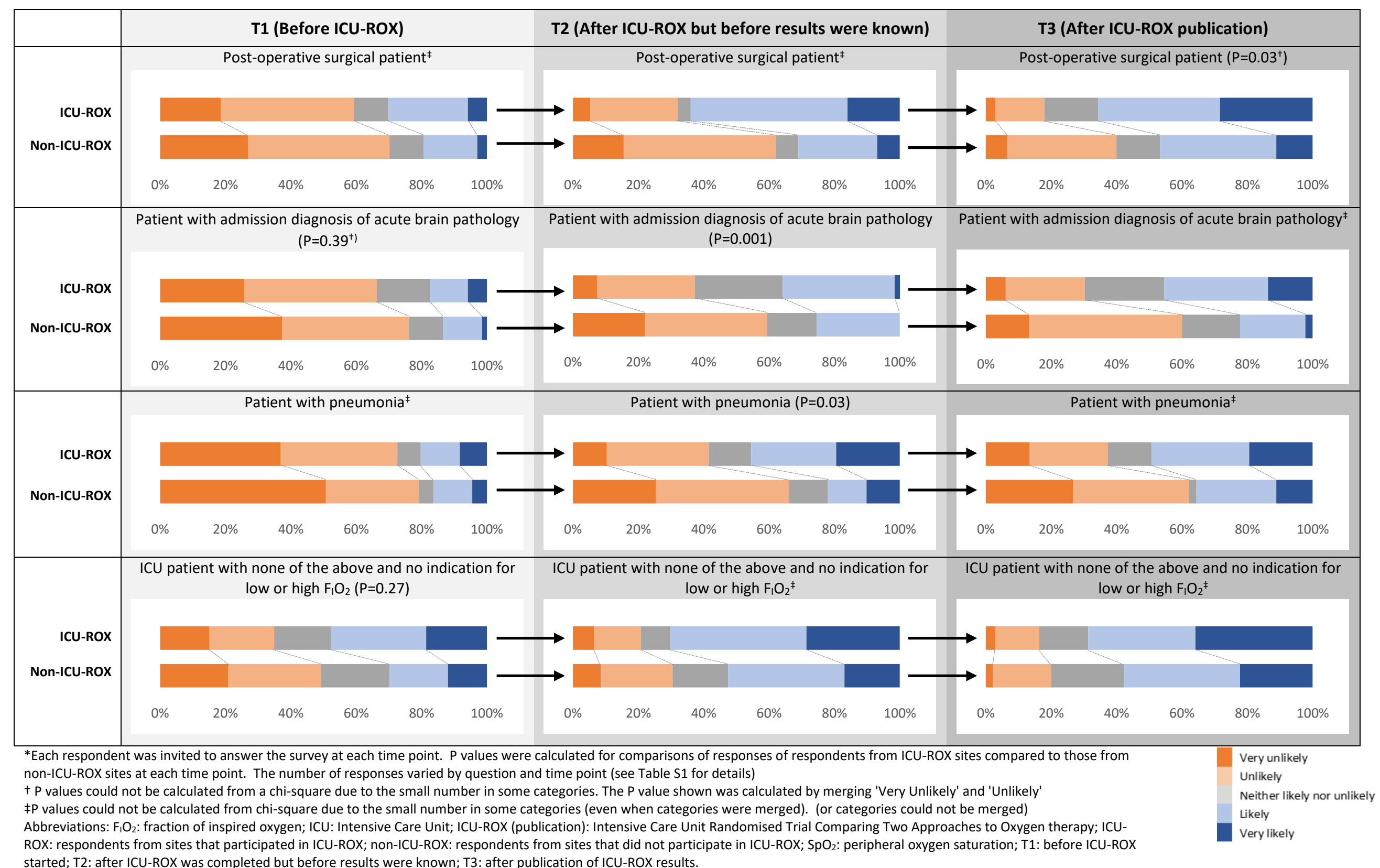

Figure 22 Likelihood of ICU nurses (ICU-ROX vs non-ICU-ROX) using $\mathrm{F}_{1} \mathrm{O}_{2}$ of 0.21 if $\mathrm{SpO}_{2}$ is $91-96 \%$, over time ${ }^{*}$ 


\subsubsection{Findings from open-ended comments (all time points)}

Of the 265 health practitioners surveyed, 70 provided at least one comment. Forty-three provided comments were from before ICU-ROX was conducted (T1), 26 after ICU-ROX but before results were known (T2), and 20 after ICU-ROX was published (T3). Nineteen people left comments on more than one occasion. Comments were used by respondents to justify how they answered the survey and to outline their experience and perspectives of oxygen management generally. As there were no obvious differences in the comments from respondents from ICU-ROX sites compared to non-ICU-ROX sites, a decision was made to present the findings from the content analysis of these data collectively. In presenting the findings, attention is given to the different perspectives of doctors and nurses where this is evident in the analysis, and some quotations are used to give 'voice' to the analysis. Three topics were evident: oxygen management generally, the setting and roles in oxygen management.

\subsubsection{Oxygen management}

The comments highlighted that oxygen management in ICU patients is complex and there is not a good evidence base for guiding many clinical decisions regarding this therapy. One ICU specialist wrote "there is broad recognition of the harmful effects of oxygen toxicity... [but] the threshold for harm is unclear" (doctor, 6-10 years, SITE21PS09 [non-ICU-ROX], T1), and another commented, "I am still not convinced that there is an important difference between an $\mathrm{FiO}_{2}$ of 0.21 and 0.4 irrespective of sats. ie. I don't believe .21 is "better"' (doctor, 5-10 years, SITE03PSO3 [ICU-ROX], T1). A third specialist shared that they thought the ICU-ROX trial would present useful findings; "For too long ICU professionals have accepted $\mathrm{SpO}_{2}>=98 \%$ as 'normal' or even 'good'. This clearly may not be the case. Roll on ICU-ROX!" (doctor, 0-5 years, SITE07PSO7 [non-ICU-ROX], T2). Nurses also noted the lack of evidence, with one nurse noting that practice was shaped by tradition; "We don't usually reduce the $\mathrm{FiO}_{2}$ below 0.3 on any patient. Nil clinical reasoning - we just tend not to go that low. ICU patients are unwell and intubated for a reason. Our practice hasn't changed within the past year" (nurse, 6-10 years, SITE11PS04 [non-ICU-ROX], T2).

Decisions regarding adjusting oxygen therapy were made based on multiple factors. Respondents frequently commented that decisions regarding oxygen management were 
primarily based on the individual patient's condition and diagnosis and treating the patient as a whole, rather than adjusting the oxygen due to an oxygen saturation alone.

Question 5 asked how we would allow .21 in ventilated patients, mostly likely if they stable I would allow that, but $91 \%$ and $96 \%$ is a big range from marginally acceptable to quite acceptable. It's difficult to generalise an answer actually. Cheers (nurse, 0-5 years, SITE17PS05 [non-ICU-ROX], T1)

Many nurses commented that their decision to adjust oxygen levels was dependent on the results of an arterial blood gas; "Before changing $\mathrm{FiO}_{2}$ setting on a ventilated patient I would always Do an $A B G$ prior to any $\mathrm{O}_{2}$ changes" (nurse, 0-5 years, SITE03PS02 [ICU-ROX], T1). In addition, many respondents commented that while they would reduce the oxygen level, they would not adjust it immediately (referring to the survey question asking whether they would reduce $\mathrm{F}_{1} \mathrm{O}_{2}$ 'in the next 5 minutes'), but would consider the patient profile. "I would consider co-morbidities, rate of weaning, and discuss with ICU consultant before making changes with $\mathrm{FIO}_{2} .4$ or less, so I would be longer than 5 minutes" (nurse, >10 years, SITE06PS11 [non-ICUROX], T1). Doctors and nurses both commented that oxygen was not weaned in isolation; they also adjusted positive end expiry pressure (PEEP) when weaning ventilatory support. "If PEEP remained high in the pneumonia patient then I'd adjust that before $\mathrm{FiO}_{2}$ if this was 4 or lower" (doctor, 0-5 years, SITE04PS05 [ICU-ROX], T2).

\subsubsection{Setting}

Three settings were noted as impacting oxygen management; country, hospital and ICU. Differences were noted by one consultant regarding oxygen management in Australia and India. "I have different practices in Australia where oxygen is, I guess, fixed cost, and India where there is a huge cost with increasing use" (doctor, 6-10 years, SITE15PS05 [ICU-ROX site], T1). For a few respondents it was hospital policy that guided how oxygen was managed; "I find that practice at this hospital tends to keep a minimum $\mathrm{FiO}_{2}$ of .3, whereas others I have worked at routinely go below this to 21\% if able" (nurse, 6-10 years, SITE13PS01 [non-ICUROX], T1). But it was ICU unit culture and practice that was mainly commented on.

At an ICU level, several respondents discussed the boundaries for oxygen management such as a generally accepted, often informal, lower level of oxygen used in their ICU; "Unit practice 
has been not to wean below $\mathrm{FiO}_{2}$ of 0.3 unless requested by medical staff - no policy states it, just practice" (nurse, 0-5 years, SITE01PS07 [ICU-ROX], T1). There were also protocols for specific patient groups that guided practice, irrespective of a patient's clinical condition. "Cardiothoracic patients who have had open heart surgery will remain on $1 \mathrm{FiO}_{2}$ for the first 2 hours!" (nurse, >10 years, SITE10PS04 [non-ICU-ROX), T1). Other considerations for oxygen management were the perceived cost of oxygen and equipment limitations; "Current ventilator only go to 25\%" (nurse, 6-10 years, SITE10PS05 [non-ICU-ROX], T1 and T2). A number of respondents commented that while there was a desire for change within their setting, achieving this was difficult; "The hardest thing about $\mathrm{FiO}_{2}$ settings is to change a unit culture" (doctor, >10 years, SITE12PS10 [non-ICU-ROX], T2). It was evident in some comments that change was underway;

I think the perception of getting the $\mathrm{FiO}_{2}$ below 5 [0] percent [sic] is changing and that staff are understanding the benefits of weaning all aspects of ventilator support as early as possible. However, I also think that there is room for significant improvement in terms of educating staff on the implications of ineffective ventilator weaning and the complications brought about by excessive 02 delivery. (Nurse, >10 years, SITE19PS14 [ICU-ROX], T1)

\subsubsection{Role}

The third topic that was evident was who makes decisions regarding oxygen management. These comments indicated that role and experience were drivers of decisions in some ICUs and thus management of oxygen. Comments indicated that in some ICUs, decisions were made by either doctors or nurses or were made jointly, and in others doctors set limits, and nurses adjusted oxygen accordingly.

I am more than happy with anyone with sats greater than 94\%, however sats aims are set by consultant- therefore will only aggressively wean down to set aim, whether that be 88,92 , or 97. (Nurse, 0-5 years SITE18PS11 [ICU-ROX] T1)

Patient safety underpinned the decisions.

In cases where $\mathrm{SpO}_{2}$ is over $97 \%, \mathrm{FiO}_{2}$ is above .6, would catch my attention quite quickly to reduce the $\mathrm{FiO}_{2}$. Between .4-.6 it probably would depend on patients condition and how stable they are. Less than .4 I probably would 
not concern too much till later on that shift usually would reduce $\mathrm{FiO}_{2}$ more towards later of the shift to make sure it's safe to do so since maybe there's no so much harm. (Nurse, 0-5 years SITE17PS05 [non-ICU-ROX], T1)

Not all respondents agreed with the current arrangements and practice in their ICU, and both doctors and nurses had some critique of each other's practice.

Mainly nurse managed but they are much better at turning things up than turning things down. It drives me crazy seeing patient with $\mathrm{SpO}_{2} 100 \%$ and still on unnecessary supplementary oxygen. (Doctor, >10 years SITE12S05 [non-ICU-ROX], T3)

As nurses at [SITE05], we are not allowed to change the ventilator settings in regards to the $\mathrm{FiO}_{2}$, and we have some consultants who are very reluctant to be challenged in their practice. (Nurse, 6-10 years, SITE05PS09 [ICU-ROX], T3)

The freedom to adjust the oxygen was also dependent on setting, and even experienced nurses in some ICUs were not able to adjust oxygen. While the nurses in some other ICUs had autonomy to initiate change and often led practice, doctors noted they would not hesitate to step in.

i am happy for nurses to weane [sic] FiO2 to as low as they like. unless they fail to do so, where i then step in and prescribe based on $\mathrm{SaO}_{2}$. If $\mathrm{FiO}_{2}$ is $<31 \%$ I'm usually comfortable as that seems to be the goal the nurses work towards. (Doctor, 0-5 years SITE18PS02 [ICU-ROX], T3)

\subsubsection{Oxygen management}

Table 14 provides the characteristics of the 193 respondents who completed the survey after the publication of the ICU-ROX trial (T3). Although the sample size was smaller, the general characteristics of the overall sample were similar to the original survey respondents (Table 13). Respondents from ICU-ROX sites and non-ICU-ROX sites remained similar, including the difference in length of time in the role between ICU-ROX and non-ICU-ROX site nurses. Nurses from the non-ICU-ROX sites were more experienced than those from the ICU-ROX sites. 
Table 14 Respondent baseline characteristics for ICU-ROX vs non-ICU-ROX sites (for T3: after ICU-ROX publication)

\begin{tabular}{|c|c|c|c|}
\hline Characteristic & $\begin{array}{l}\text { ICU-ROX } \\
\text { respondents } \\
(n=111)\end{array}$ & $\begin{array}{c}\text { Non-ICU-ROX } \\
\text { respondents } \\
\quad(n=82)\end{array}$ & $P$ value \\
\hline ICU Specialist doctors & $44(39.6 \%)$ & $37(45.1 \%)$ & \multirow{2}{*}{0.45} \\
\hline ICU Nurses & $67(60.4 \%)$ & 45 (54.9\%) & \\
\hline \multicolumn{4}{|c|}{ Length of time in role at $\mathrm{T} 1$ (doctors) } \\
\hline$<5$ years & $21(47.7 \%)$ & $12(32.4 \%)$ & \multirow{3}{*}{0.27} \\
\hline $6-10$ years & $10(22.7 \%)$ & $8(21.6 \%)$ & \\
\hline$>10$ years & $13(29.5 \%)$ & $17(45.9 \%)$ & \\
\hline \multicolumn{4}{|c|}{ Length of time in role at $\mathrm{T} 1$ (nurses) } \\
\hline$<5$ years & $17(25.4 \%)$ & $3(6.7 \%)$ & \multirow{3}{*}{0.004} \\
\hline $6-10$ years & $20(29.9 \%)$ & $8(17.8 \%)$ & \\
\hline$>10$ years & 30 (44.8\%) & 34 (75.6\%) & \\
\hline
\end{tabular}

Abbreviations: ICU-ROX respondents: respondents from ICUs that participated in ICU-ROX; non-ICU-ROX

respondents: respondents from ICUs that did not participate in ICU-ROX; T1: before ICU-ROX started; T2: after ICU-ROX was completed but before results were known; T3: after publication of ICU-ROX results.

Oxygen management in ICU is complex, and many factors determine its management in ICU, what roles doctors and nurses have and what prompts health practitioners to adjust oxygen. The most commonly identified determinant of oxygen management in ICU was clinician preference (Table 15).

Clinician preference was solely or partially included in $75 \%(n=145)$ of responses, and for $56.5 \%$ of respondents it was the only option selected. The second commonest determinant was 'ICU guideline' either in combination with clinician preference or alone. Responses from the site level survey were similar to the individual survey with clinician preference being the commonest response to the question what guides overall oxygen management (Table 16). For 13 of the 21 site respondents, 'clinician preference' was identified as the only factor guiding overall oxygen management. 
Table 15 Reported determinants of oxygen management (practitioners attitudes survey)

\begin{tabular}{|l|c|}
\hline & $\begin{array}{c}\text { All respondents } \\
\mathbf{N}=193\end{array}$ \\
\hline One preference (n=152) & $109(56.5 \%)$ \\
\hline Clinician preference only & $20(10.4 \%)$ \\
\hline Guideline - ICU level & $2(1.0 \%)$ \\
\hline Guideline - hospital level & $2(1.0 \%)$ \\
\hline Guideline - national or international & $8(4.1 \%)$ \\
\hline Other (only) & \\
\hline Selected 2 preferences (n=39) & $28(14.5 \%)$ \\
\hline Clinician preference and ICU guideline & $3(1.6 \%)$ \\
\hline Guideline - national or international and ICU guideline & $2(1.0 \%)$ \\
\hline Clinician preference and hospital level guideline & $2(1.0 \%)$ \\
\hline Clinician preference and national or international guideline & $3(1.6 \%)$ \\
\hline Other and ICU level guideline & $1(0.5 \%)$ \\
\hline Other and clinician preference & \\
\hline Selected 4 preferences (n=3) & $3(1.6 \%)$ \\
\hline Clinician preference and national, plus hospital, plus ICU level & $11(5.7 \%)$ \\
\hline Don't know or did not provide an answer &
\end{tabular}

Abbreviation: ICU: Intensive Care Unit.

Table 16 Reported determinants of oxygen management (ICU-level survey)

\begin{tabular}{|l|c|}
\hline & $\begin{array}{c}\text { All ICUs } \\
\mathrm{N}=21\end{array}$ \\
\hline One preference ( $\mathbf{n}=\mathbf{1 7})$ & \\
\hline Clinician preference only & $13(61.9 \%)$ \\
\hline Guideline - ICU level & $2(9.5 \%)$ \\
\hline Guideline - hospital level & $2(9.5 \%)$ \\
\hline Guideline - national or international & $0(0.0 \%)$ \\
\hline Selected 2 preferences ( $\mathbf{n}=\mathbf{2})$ & \\
\hline Clinician preference plus national or international guideline & $2(9.5 \%)$ \\
\hline Selected 3 preferences ( $\mathbf{n}=\mathbf{2})$ & \\
\hline Clinician preference plus hospital level, plus ICU level & $1(4.8 \%)$ \\
\hline Clinician preference plus national level, plus ICU level & $1(4.8 \%)$ \\
\hline
\end{tabular}

Abbreviation: ICU: Intensive Care Unit.

Nurses were reported as the main adjuster of oxygen in the ICU (Table 17). While doctors and nurses were involved in oxygen management, the commonest response by doctors (74.1\%) and nurses (63.5\%) was that the nurse adjusts oxygen in ICU. A small percentage (22\%) indicated it was a doctor's role. Comments indicated that there was uncertainty in 
some ICUs; "depends on what week it is. Some consultants don't like nurses touching too much, others don't care" (nurse, >10 years SITE10PS07 [non-ICU-ROX]). In contrast, two ICU specialist doctors noted "Nurses put it up, doctors put it down" (doctor, 0-5 years, SITE07PS02, [non-ICU-ROX]), and "mostly nursing, I would think in practice, but I do believe it is a significant medical consideration beyond the simple initial set-up" (doctor, >10 years SITE10PS11 [nonICU-ROX]). Two nurses specified that it was only nurses with "more experience" who would adjust the oxygen.

Table 17 Role that predominantly adjusts oxygen in the ICU

\begin{tabular}{|l|c|c|c|}
\hline & $\begin{array}{c}\text { ICU specialist } \\
\text { doctors } \\
\mathrm{n}=81\end{array}$ & $\begin{array}{c}\text { ICU } \\
\text { nurses } \\
\mathrm{n}=104\end{array}$ & Total \\
\hline Doctors & $16(19.8 \%)$ & $25(24.0 \%)$ & $41(22.2 \%)$ \\
\hline Nurses & $60(74.1 \%)$ & $66(63.5 \%)$ & $126(68.1 \%)$ \\
\hline Both doctors and nurses & $5(6.2 \%)$ & $7(6.7 \%)$ & $12(6.5 \%)$ \\
\hline $\begin{array}{l}\text { Nurses, but doctors make final } \\
\text { decision }\end{array}$ & $0(0.0 \%)$ & $4(3.8 \%)$ & $4(2.2 \%)$ \\
\hline Other & $0(0.0 \%)$ & $2(1.9 \%)$ & $2(1.1 \%)$ \\
\hline
\end{tabular}

Abbreviation: ICU: Intensive Care Unit.

Several oxygen-related targets were identified as a prompt for adjusting oxygen (Table 18). The commonest prompt listed ( $85.5 \%$ of respondents) was a prescribed peripheral or arterial oxygen saturation target. Nurses more commonly answered that an arterial oxygen range, that can only be obtained from an arterial blood gas, was prescribed (51.8\%). In contrast only $22.2 \%$ of doctors responded in this way. Ten comments, all from nurses, stated they would use an $A B G$, rather than an oxygen saturation, when assessing whether to adjust oxygen. In addition, one doctor commented "it would be good for if our pulse oximeters were reliable!" (>10years, SITE01PS08 [ICU-ROX]). Of the 185 respondents who provided an answer to this question, 88 selected only one option, 64 selected two options and 27 selected three or more options and a further six reported 'no oxygen targets prescribed' as their only answer. 
Table 18 Prompts for adjusting oxygen (practitioner attitudes survey) *

\begin{tabular}{|l|c|c|c|}
\hline & $\begin{array}{c}\text { ICU specialist } \\
\text { doctors } \\
\mathrm{n}=81\end{array}$ & $\begin{array}{c}\text { ICU } \\
\text { nurses } \\
\mathrm{n}=104\end{array}$ & $\mathrm{n}=193$ \\
\hline $\begin{array}{l}\text { An oxygen saturation range } \mathrm{SpO}_{2} \text { or } \\
\left.\mathrm{SaO}_{2}\right) \text { is prescribed }\end{array}$ & $73(90.1 \%)$ & $92(82.1 \%)$ & $165(85.5 \%)$ \\
\hline $\begin{array}{l}\text { An arterial oxygen }\left(\mathrm{PaO}_{2}\right) \text { range is } \\
\text { prescribed }\end{array}$ & $18(22.2 \%)$ & $58(51.8 \%)$ & $76(39.4 \%)$ \\
\hline Standing order or guideline & $6(7.4 \%)$ & $22(19.6 \%)$ & $28(14.5 \%)$ \\
\hline $\begin{array}{l}\text { A minimum } \mathrm{F}_{1} \mathrm{O}_{2} \text { or oxygen flow rate is } \\
\text { prescribed }\end{array}$ & $2(2.5 \%)$ & $13(11.6 \%)$ & $15(7.8 \%)$ \\
\hline $\begin{array}{l}\text { A maximum } \mathrm{F}_{1} \mathrm{O}_{2} \text { or oxygen flow rate } \\
\text { is prescribed }\end{array}$ & $4(4.9 \%)$ & $9(8.0 \%)$ & $13(6.7 \%)$ \\
\hline $\begin{array}{l}\text { No oxygen-related targets are } \\
\text { prescribed }\end{array}$ & $4(4.9 \%)$ & $6(5.4 \%)$ & $10(5.2 \%)$ \\
\hline Other & $2(2.5 \%)$ & $9(8.0 \%)$ & $10(5.2 \%)$ \\
\hline \multicolumn{1}{|c|}{ Clinical judgement } & $0(0.0 \%)$ & $1(0.9 \%)$ & $3(1.6 \%)$ \\
\hline \multicolumn{1}{|c|}{ Doctor's orders } & $1(1.2 \%)$ & $0(0.0 \%)$ & $1(0.5 \%)$ \\
\hline Cardiac output & $4(4.9 \%)$ & $7(6.3 \%)$ & $11(5.7 \%)$ \\
\hline No answer provided & & & \\
\hline
\end{tabular}

*respondents could select more than one option

Abbreviations: $\mathrm{F}_{1} \mathrm{O}_{2}$ : fraction of inspired oxygen; ICU: Intensive Care Unit; $\mathrm{PaO}_{2}$ : partial pressure of arterial oxygen; $\mathrm{SpO}_{2}$ : peripheral oxygen saturations; $\mathrm{SaO}_{2}$ : arterial oxygen saturations.

\subsubsection{Knowledge translation results}

\subsubsection{Level of involvement in ICU-ROX trial}

The majority of respondents from ICU-ROX sites had either enrolled a patient in ICU-ROX, or cared for a patient in ICU-ROX (Table 19). Doctors reported enrolling a patient into the ICUROX trial more than nurses ( $30.9 \%$ and $13.4 \%$, respectively), while the number caring for an ICU-ROX patient was similar for doctors and nurses (29.6\% and $28.6 \%$, respectively), including two doctors and one nurse from non-ICU-ROX sites. Nine respondents from five ICUs that participated in ICU-ROX reported they were from an ICU that did not participate in ICU-ROX, $22(n=25 \%)$ people from eight ICUs that did not participate in ICU-ROX reported that their ICU had participated in ICU-ROX. 
Table 19 Respondent participation in the ICU-ROX trial

\begin{tabular}{|l|c|c|c|}
\hline & $\begin{array}{c}\text { ICU-ROX } \\
\text { respondents } \\
\mathrm{n}=111\end{array}$ & $\begin{array}{c}\text { Non-ICU-ROX } \\
\text { respondents } \\
\mathrm{n}=82\end{array}$ & P value \\
\hline $\begin{array}{l}\text { None - my ICU did not participate in ICU- } \\
\text { ROX }\end{array}$ & $9(8.1 \%)$ & $55(67.1 \%)$ & $<0.001$ \\
\hline Enrolled a patient in ICU-ROX & $40(36.0 \%)$ & $0(0.0 \%)$ & $<0.001$ \\
\hline Patients under my care were in ICU-ROX & $53(47.4 \%)$ & $3(3.7 \%)$ & $<0.001$ \\
\hline $\begin{array}{l}\text { ICU participated in ICU-ROX but no } \\
\text { individual involvement with patient } \\
\text { enrolled in ICU-ROX }\end{array}$ & $27(24.3 \%)$ & $22(26.8 \%)$ & 0.69 \\
\hline
\end{tabular}

Abbreviations: ICU-ROX: Intensive Care Unit Randomised Trial Comparing Two Approaches to Oxygen therapy; ICU-ROX respondents: respondents from ICUs that participated in ICU-ROX; non-ICU-ROX respondents: respondents from ICUs that did not participate in ICU-ROX.

\subsubsection{Health practitioner knowledge of ICU-ROX results}

There were no differences between respondents from ICU-ROX and non-ICU-ROX sites when asked how they heard about the ICU-ROX trial results (Table 20). However, there was a difference between doctors and nurses, and significantly more nurses than doctors had not heard the ICU-ROX results at the time of the survey $(P<0.001)$. ICU specialist doctor respondents were statistically more likely to have read the publication, heard the presentation at a conference or had learned of the ICU-ROX results on social media than their nursing colleagues (Table 21). The commonest way respondents heard about the results was that "someone at work" told them, and there was no difference between ICU-ROX and nonICU-ROX site, or by role (Table 20, Table 21). Although it is unknown who at work reported this to staff, one nurse commented "intensivist in the unit bringing in new practice for lower saturation parameters" (nurse, 0-5 years SITE17PS05 [non-ICU-ROX]). 
Table 20 Mode of finding out ICU-ROX results (ICU-ROX vs non-ICU-ROX respondents)

\begin{tabular}{|l|c|c|c|}
\hline & $\begin{array}{c}\text { ICU-ROX } \\
\text { respondents } \\
\mathrm{n}=111\end{array}$ & $\begin{array}{c}\text { Non-ICU-ROX } \\
\text { respondents } \\
\mathrm{n}=82\end{array}$ & P value \\
\hline Someone at work & $41(36.9 \%)$ & $29(35.4 \%)$ & 0.82 \\
\hline Read the ICU-ROX publication & $32(28.8 \%)$ & $17(20.7 \%)$ & 0.20 \\
\hline Conference presentation & $23(20.7 \%)$ & $16(19.5 \%)$ & 0.84 \\
\hline Results meeting & $8(7.2 \%)$ & $2(2.4 \%)$ & $\mathrm{np}$ \\
\hline Social media & $10(9.0 \%)$ & $11(13.4 \%)$ & 0.33 \\
\hline Media & $0(0 \%)$ & $1(1.2 \%)$ & $\mathrm{np}$ \\
\hline Editorial & $4(3.6 \%)$ & $2(2.4 \%)$ & $\mathrm{np}$ \\
\hline In-service or journal club & $1(0.7 \%)$ & $1(0.8 \%)$ & $\mathrm{np}$ \\
\hline Research team ${ }^{+}$ & $5(3.5 \%)$ & $0(0.0 \%)$ & $\mathrm{np}$ \\
\hline The Bottom Line & $1(0.7 \%)$ & $1(0.8 \%)$ & $\mathrm{np}$ \\
\hline Other/ can't remember & $1(0.7 \%)$ & $2(2.4 \%)$ & $\mathrm{np}$ \\
\hline Had not heard the results & $31(27.9 \%)$ & $26(31.7 \%)$ & 0.57 \\
\hline
\end{tabular}

*The Bottom Line is a critical appraisal website

${ }^{\dagger}$ Research team includes both the Research nurse from the ICU, and the trials management team Abbreviations: ICU-ROX: Intensive Care Unit Randomised Trial Comparing Two Approaches to Oxygen therapy; ICU-ROX respondents: respondents from ICUs that participated in ICU-ROX; np: statistical analysis was not possible; non-ICU-ROX respondents: respondents from ICUs that did not participate in ICU-ROX.

Table 21 Mode of finding out ICU-ROX results (ICU doctor vs nurse respondents)

\begin{tabular}{|l|c|c|c|}
\hline & $\begin{array}{c}\text { ICU specialist } \\
\text { doctors } \\
\mathrm{n}=81\end{array}$ & $\begin{array}{c}\text { ICU } \\
\text { nurses } \\
\mathrm{n}=112\end{array}$ & P value \\
\hline Someone at work & $25(30.9 \%)$ & $45(40.2 \%)$ & 0.18 \\
\hline Read the ICU-ROX publication & $43(53.1 \%)$ & $6(5.4 \%)$ & $<0.001$ \\
\hline Conference presentation & $30(37.0 \%)$ & $9(8.0 \%)$ & $<0.001$ \\
\hline Results meeting & $7(8.6 \%)$ & $3(2.7 \%)$ & $\mathrm{np}$ \\
\hline Social media & $20(24.7 \%)$ & $1(0.9 \%)$ & 0.001 \\
\hline Media & $1(1.2 \%)$ & $0(0.0)$ & $\mathrm{np}$ \\
\hline Editorial & $4(4.9 \%)$ & $2(1.8 \%)$ & $\mathrm{np}$ \\
\hline In-service or journal club & $1(1.2 \%)$ & $1(0.9 \%)$ & $\mathrm{np}$ \\
\hline Research team ${ }^{\dagger}$ & $0(0.0 \%)$ & $5(4.5 \%)$ & $\mathrm{np}$ \\
\hline The Bottom Line & $1(1.2 \%)$ & $1(0.9 \%)$ & $\mathrm{np}$ \\
\hline Other/ can't remember & $2(2.4 \%)$ & $1(0.7 \%)$ & $\mathrm{np}$ \\
\hline Had not heard the results & $11(13.6 \%)$ & $46(41.1 \%)$ & $<0.001$ \\
\hline
\end{tabular}

${ }^{*}$ The Bottom Line is a critical appraisal website

${ }^{+}$Research team includes both the Research nurse from the ICU, and the trials management team

Abbreviations: ICU: Intensive Care Unit; ICU-ROX: Intensive Care Unit Randomised Trial Comparing Two Approaches to Oxygen therapy; $\mathrm{np}$ : statistical analysis was not possible. 
Excluding the supplementary appendix, 41 (21.2\%) respondents reported reading the whole publication, 11 of whom also read the supplementary appendix. The responses were similar between the ICU-ROX and non-ICU-ROX respondents, with the majority of respondents in both groups indicating they had not read the publication at all (Table 22). The doctor's responses differed significantly from the nurses with regard to the sections of the publication they had read (Table 23). Of the 41 respondents who read the full publication (excluding supplementary appendix), 37 (45.6\%) were doctors and four (3.5\%) were nurses. None of the 11 respondents who had also read the supplementary appendix were nurses, although one nurse who had participated in ICU-ROX read all sections (including the supplementary appendix) but not the methods. The majority of nurses had not read any part of the publication, with less than $10 \%$ reading any section.

Table 22 Sections of ICU-ROX publication read (ICU-ROX vs non-ICU-ROX respondents)

\begin{tabular}{|l|c|c|c|}
\hline & $\begin{array}{c}\text { ICU-ROX } \\
\text { respondents } \\
\mathrm{n}=111\end{array}$ & $\begin{array}{c}\text { Non-ICU-ROX } \\
\text { respondents } \\
\mathrm{n}=82\end{array}$ & P value \\
\hline Abstract & $40(36.0 \%)$ & $27(32.9 \%)$ & 0.65 \\
\hline Introduction & $35(31.5 \%)$ & $16(19.5 \%)$ & 0.06 \\
\hline Methods & $29(26.1 \%)$ & $17(20.7 \%)$ & 0.39 \\
\hline Results & $36(32.4 \%)$ & $18(22 \%)$ & 0.11 \\
\hline Discussion & $37(33.3 \%)$ & $20(24.4 \%)$ & 0.18 \\
\hline Conclusion & $39(35.1 \%)$ & $20(24.4 \%)$ & 0.11 \\
\hline Supplementary appendix & $8(7.2 \%)$ & $5(6.1 \%)$ & 0.76 \\
\hline Did not read the publication & $55(49.5 \%)$ & $45(58.5 \%)$ & 0.22 \\
\hline
\end{tabular}

Abbreviations: ICU-ROX trial: Intensive Care Unit Randomised Trial Comparing Two Approaches to Oxygen therapy; ICUROX respondents: respondents from ICUs that participated in ICU-ROX; non-ICU-ROX respondents: respondents from ICUs that did not participate in ICU-ROX. 
Table 23 Sections of the ICU-ROX publication read (ICU doctor vs nurse respondents)

\begin{tabular}{|l|c|c|c|}
\hline & $\begin{array}{c}\text { ICU specialist } \\
\text { doctors } \\
\mathrm{n}=81\end{array}$ & $\begin{array}{c}\text { ICU } \\
\text { nurses } \\
\mathrm{n}=112\end{array}$ & P value \\
\hline Abstract & $59(72.8 \%)$ & $8(7.1 \%)$ & $<0.001$ \\
\hline Introduction & $44(54.3 \%)$ & $7(6.3 \%)$ & $<0.001$ \\
\hline Methods & $40(49.4 \%)$ & $6(5.4 \%)$ & $<0.001$ \\
\hline Results & $45(55.6 \%)$ & $9(8.0 \%)$ & $<0.001$ \\
\hline Discussion & $46(56.8 \%)$ & $11(9.8 \%)$ & $<0.001$ \\
\hline Conclusion & $48(59.3 \%)$ & $11(9.8 \%)$ & $<0.001$ \\
\hline Supplementary appendix & $12(14.8 \%)$ & $1(0.9 \%)$ & $<0.001$ \\
\hline Did not read the publication & $18(22.2 \%)$ & $85(75.9 \%)$ & $<0.001$ \\
\hline
\end{tabular}

Abbreviations: ICU: Intensive Care Unit; ICU-ROX trial: Intensive Care Unit Randomised Trial Comparing Two Approaches to Oxygen therapy.

\subsubsection{Level of engagement in ICU-ROX and knowledge of results}

The level of engagement in ICU-ROX, measured by whether respondents had enrolled or cared for a patient in the ICU-ROX trial, were mixed. While enrolling a patient into ICU-ROX, appeared to make a difference in respondents' knowledge of the results, caring for a patient did not differ from not caring for a patient in ICU-ROX (Table 24, Table 25).

Table 24 Knowledge of results if enrolled a patient (ICU-ROX sites only)

\begin{tabular}{|l|c|c|c|}
\hline & $\begin{array}{c}\text { Enrolled a patient } \\
\text { into ICU-ROX } \\
(\mathrm{n}=40)\end{array}$ & $\begin{array}{c}\text { Did not enrol a } \\
\text { patient into ICU-ROX } \\
(\mathrm{n}=71)\end{array}$ & P value \\
\hline Knew of ICU-ROX results* & $35(87.5 \%)$ & $45(63.4 \%)$ & 0.007 \\
\hline Read the ICU-ROX publication & $22(55.0 \%)$ & $10(14.1 \%)$ & $<0.001$ \\
\hline
\end{tabular}

* respondent indicated at least one mode of finding out about the results

Abbreviation: ICU-ROX: Intensive Care Unit Randomised Trial Comparing Two Approaches to Oxygen therapy.

Table 25 Knowledge of results if cared for a patient (ICU-ROX sites only)

\begin{tabular}{|l|c|c|c|}
\hline & $\begin{array}{c}\text { Cared for a patient } \\
\text { enrolled in } \\
\text { ICU-ROX } \\
(\mathrm{n}=53)\end{array}$ & $\begin{array}{c}\text { Did not care for a } \\
\text { patient enrolled in } \\
\text { ICU-ROX } \\
(\mathrm{n}=58)\end{array}$ & P value \\
\hline Knew of ICU-ROX results* & $37(69.8 \%)$ & $43(74.1 \%)$ & 0.61 \\
\hline Read the ICU-ROX publication & $19(35.8 \%)$ & $13(22.4 \%)$ & 0.12 \\
\hline
\end{tabular}

* respondent indicated at least one mode of finding out about the results

Abbreviation: ICU-ROX: Intensive Care Unit Randomised Trial Comparing Two Approaches to Oxygen therapy. 


\subsubsection{Open comments about the influence of the ICU-ROX publication on practice}

Of the 193 people who responded to the survey after publication of the ICU-ROX results, 126 wrote a comment relating to how the ICU-ROX publication would or had influenced their practice. Of these, 11 indicated they had not read the publication and one had read it but could not remember the results. Forty-one (21.2\%) said they would not change practice, 38 shared that they were more likely to reduce oxygen than previously, and six were unsure how the results would impact their practice. Of significance to clinical roles, of the 60 ICU specialists who made a comment, $55 \%$ said they would not change; in contrast $9.1 \%$ of the 66 nurses said their practice would not change.

The commonest reason people gave for not changing practice was that respondents said their practice was already to reduce the $\mathrm{F}_{1} \mathrm{O}_{2}$ as much as possible. "I always tried to get $\mathrm{FIO}_{2}$ to 0.21 if possible, especially if $\mathrm{SaO}_{2}$ was > or= to 95\%", (nurse, >10years, SITE18PS07 [ICU-ROX],). Respondents in this group often commented that the paper was important as it provided evidence to justify their current practice, "Our pre-existing practice was to decrease the $\mathrm{FiO}_{2}$ where possible to lower target sats. I think this reinforces our ability to do this." doctor, 6-10 years, SITE04PS01 [ICU-ROX]).

The respondents who wrote that the findings would or had already changed their practice mainly commented how the results influenced their oxygen management generally. The commonest change $(n=28)$ was that they would be more likely to use less oxygen now, or would use the least amount necessary to maintain oxygenation targets; "Make me think that I can reduce the $\mathrm{FiO}_{2}$ down to .21 in the right circumstances as in past mostly drop to .3 then stay at that number!" (nurse, >10years, SITE18PS08 [ICU-ROX]). Many nurse respondents expressed that the publication had increased their awareness or 'opened their mind' to how they currently managed oxygen and the concept of using a more conservative approach ( $n=11)$. "Definately [sic] made me more aware of how much we over oxygenate at times, and am now more proactive in weaning fio" (nurse, 6-10 years, SITE10PSO2 [non-ICU-ROX]). Others ( 5 nurses and 1 doctor) saw the publication as a way to strengthen the argument that it was safe to use less oxygen than previously, and this could empower nurses; "will hopefully assist in the empowerment of nursing staff to reduce oxygen on mechanically ventilated patients with justification" (nurse, >10years, SITE14PSO2 [non-ICU-ROX]). Not all nurses ( $n=5)$ 
considered it would necessarily change their practice - their rationale being such decisions were made by the doctors or sometimes a specific doctor; "Unfortunately it is influenced by the most senior $\mathrm{Dr}$ on shift" (nurse,>10 years SITE11PS05 [non-ICU-ROX]). Some ICU specialists considered that the new evidence should bring about change in nursing practice; "With dissemination of the results we should be able to make bedside ICU nurses more comfortable dropping $\mathrm{FiO}_{2} "$ (doctor, 6-10 years, SITE19PS15 [ICU-ROX]).

Conversely, three doctors and one nurse stated they remained unconvinced, or had become 'less conservative' with oxygen, and would be less concerned about hyperoxia after the publication of ICU-ROX.

- "I think I am less convinced now that a $\mathrm{SpO}_{2}<93 \%$ is OK than I was previously", (nurse, $>10$ years, SITE02PS07 [ICU-ROX])

- "I am reassured that if patients inadvertently are left hyperoxic - that they don't suffer. It would be good if our pulse oximeters were reliable!" (doctor, >10 years, SITE01PS08 [ICU-ROX])

Four respondents stated that more research is needed, and anticipated an upcoming large Phase III study (referring to the Mega Randomised Registry Trial Comparing Conservative vs. Liberal OXygenation Targets [reference ACTRN12620000391976]).

Five respondents (all doctors) commented on the subgroup difference for patients with suspected hypoxic ischaemic encephalopathy and thought their practice would change in accordance with these hypothesis-generating findings. For example, respondent SITE19PS07 stated "Less likely to use higher $\mathrm{FiO}_{2}$ in hypoxic ischaemic encephalopathy", (doctor, 0-5 years, non-ICU-ROX).

A small but vocal thread throughout all comments across the time periods was about 'ICU culture', with both doctors and nurses reporting the difficulty of changing the culture within an ICU regarding oxygen.

- "Change culture around the delivery of oxygen in ICU by emphasising the importance of using as little as is needed for that patient" (doctor, 0-5 years, SITE10PS09 [non-ICU$\mathrm{ROX}])$ 


\subsubsection{ICU-level survey knowledge translation and dissemination practices}

Journal clubs were present in all but one ICU (Table 26), with one ICU having more than one journal club. The journal clubs were predominantly for doctors $(n=9)$; multidisciplinary $(n=6)$ or for doctors and nurses $(n=4)$.

Table 26 Journal clubs by level of ICU

\begin{tabular}{|c|c|c|c|c|}
\hline & $\begin{array}{c}\text { Level I } \\
\text { ICU } \\
(\mathrm{n}=1)\end{array}$ & $\begin{array}{c}\text { Level II } \\
\text { ICU } \\
(\mathrm{n}=4)\end{array}$ & $\begin{array}{c}\text { Level III } \\
\text { ICU } \\
(\mathrm{n}=16)\end{array}$ & $\begin{array}{c}\text { Total } \\
(\mathrm{n}=21)\end{array}$ \\
\hline Single journal club (n=19) & & & & \\
\hline Doctors only journal club* & 0 & 2 & 7 & 9 \\
\hline Nurses only journal club & 0 & 0 & 0 & 0 \\
\hline Doctors and nurses journal club & 0 & 0 & 4 & 4 \\
\hline Multidisciplinary journal club & 0 & 1 & 5 & 6 \\
\hline Multiple journal clubs (n=1) & & & & 1 \\
\hline $\begin{array}{c}\text { Doctors, nurses and } \\
\text { multidisciplinary journal club }\end{array}$ & 0 & 1 & 0 & 1 \\
\hline No journal clubs (n=1) & 1 & 0 & 0 & 1 \\
\hline
\end{tabular}

* For one site, nurses were welcome to join the doctors journal club, although this reportedly was not advertised.

Abbreviation: ICU: Intensive Care Unit.

When asked about usual dissemination practices, 16 of the 21 ICUs used more than one mode of dissemination for research the ICU was involved in, with six reporting using four modes (Table 27). With regards to disseminating the results of the ICU-ROX trial, all ICUs that participated in the trial had either disseminated the results or had a plan in place to do so (Table 28). The commonest mode of dissemination of the ICU-ROX results was by emailing staff $(n=10)$, followed by placing the publication on a noticeboard in the ICU $(n=7)$. Of the nine ICU-ROX sites that had already disseminated the results, five had used more than one mode of dissemination, with one site using four different modes of dissemination. For sites that did not participate in ICU-ROX, three had not disseminated the results, and did not report a plan to do so. Of the six non-ICU-ROX sites in that the results had been disseminated, three had used one mode and three used two modes of dissemination. 
Table 27 Usual modes of dissemination for research that sites are involved in

\begin{tabular}{|c|c|c|c|}
\hline & $\begin{array}{c}\text { ICU-ROX } \\
\text { sites } \\
(n=11)\end{array}$ & $\begin{array}{l}\text { Non-ICU-ROX } \\
\text { sites } \\
(\mathrm{n}=10)\end{array}$ & $\begin{array}{l}\text { All sites } \\
(n=21)\end{array}$ \\
\hline 1 mode & & & $5(23.8 \%)$ \\
\hline Email only & & 1 & 1 \\
\hline ICU newsletter only & 1 & 1 & 2 \\
\hline Noticeboard in ICU only & 1 & 1 & 2 \\
\hline 2 modes & & & $7(23.3 \%)$ \\
\hline Email plus journal club or meeting & 1 & & 1 \\
\hline Email plus noticeboard in ICU & 2 & 1 & 3 \\
\hline ICU Newsletter plus noticeboard in ICU & 1 & 1 & 2 \\
\hline Journal club plus ICU Newsletter & 1 & & 1 \\
\hline 3 modes & & & $3(14.3 \%)$ \\
\hline $\begin{array}{l}\text { Email, noticeboard in ICU plus either ICU } \\
\text { website, ICU Newsletter or shift handover }\end{array}$ & 2 & 1 & 3 \\
\hline 4 modes & & & $6(28.6 \%)$ \\
\hline $\begin{array}{l}\text { Communication book, noticeboard in ICU, } \\
\text { ward meeting, registrar and consultant } \\
\text { meetings }\end{array}$ & & 1 & 1 \\
\hline $\begin{array}{l}\text { Email, Facebook, ICU Newsletter and ICU } \\
\text { website }\end{array}$ & & 1 & 1 \\
\hline $\begin{array}{l}\text { Email, general correspondence, meetings, } \\
\text { noticeboard }\end{array}$ & 1 & & 1 \\
\hline $\begin{array}{l}\text { Email, ICU newsletter, ICU website and } \\
\text { noticeboard }\end{array}$ & & 2 & 2 \\
\hline $\begin{array}{l}\text { Email, hard copy of publication around } \\
\text { ICU, in-service education, Noticeboard }\end{array}$ & 1 & & 1 \\
\hline
\end{tabular}

Abbreviations: ICU: Intensive Care Unit; ICU-ROX sites: ICUs that participated in ICU-ROX; non-ICU-ROX sites: ICUs that did not participate in ICU-ROX. 
Table 28 Dissemination of ICU-ROX results

\begin{tabular}{|c|c|c|c|}
\hline & $\begin{array}{c}\text { ICU-ROX } \\
\text { sites } \\
(n=11) \\
\end{array}$ & $\begin{array}{c}\text { Non-ICU-ROX } \\
\text { sites } \\
(n=10) \\
\end{array}$ & $\begin{array}{l}\text { All sites } \\
(n=21)\end{array}$ \\
\hline 1 mode & & & $6(28.6 \%)$ \\
\hline Noticeboard in ICU only & 1 & 0 & 1 \\
\hline Email staff only & 1 & 1 & 2 \\
\hline Informal presentation & 1 & 0 & 1 \\
\hline Journal club & 0 & 2 & 2 \\
\hline 2 modes & & & $6(28.6 \%)$ \\
\hline $\begin{array}{l}\text { In-service and hard-copy publication for } \\
\text { staff to read }\end{array}$ & 1 & 0 & 1 \\
\hline Email plus ICU Newsletter & 0 & 1 & 1 \\
\hline Email plus journal club or meeting & 1 & 0 & 1 \\
\hline Noticeboard plus ICU Newsletter or email & 1 & 2 & 3 \\
\hline 3 modes & & & $2(9.5 \%)$ \\
\hline $\begin{array}{l}\text { Noticeboard, email, meeting or journal } \\
\text { club }\end{array}$ & 2 & 0 & 2 \\
\hline 4 modes & & & $1(4.7 \%)$ \\
\hline $\begin{array}{l}\text { Noticeboard, ICU newsletter, ICU } \\
\text { website, email }\end{array}$ & 1 & 0 & 1 \\
\hline Results not disseminated & & & $6(28.6 \%)$ \\
\hline Not disseminated as not in ICU-ROX & 0 & 3 & 3 \\
\hline Not disseminated yet (but intending to) & 2 & 1 & 3 \\
\hline
\end{tabular}

Abbreviations: ICU: Intensive Care Unit; ICU-ROX sites: ICUs that participated in ICU-ROX; non-ICU-ROX sites: ICUs that did not participate in ICU-ROX.

\subsubsection{Research champion}

For the majority of ICUs, the research champion/s was described as being a member of the research team or department. Four sites reported they did not have a research champion. The other 17 sites reported there was at least one person who fulfilled this role by encouraging staff to participate in research, including their own research. In most cases, this was the research nurse/co-ordinator $(n=10)$ and the ICU consultant in charge of research for the ICU $(n=11)$. In addition to this senior doctor, three sites reported that registrars or a research fellow were also research champions and assisted with practical elements of research, such as obtaining consent from patients for research studies. In addition to the research nurses, some respondents stated that nurses who were keen to participate in research were trained in screening for patients for research studies; "Informal - 
nursing/medical staff that attend education sessions with research coordinator to encourage research screening and enrolments in the unit" (SITE14).

There was a formally recognised position in one ICU (SITE19) which had a 'Research mentor' as well as the 'head of research' who assisted and encouraged novice researchers of any profession. Others reported similar roles that were both formally and informally recognised 'Deputy Director of ICU and Director of Medical Education. Publishes regularly in peer reviewed journals and assists novices in research studies. Formal and informal role' (SITE22). For those that stated this was a formal position, this was often in the form of research forums for nursing and medical development, or discussing recent publications.

\subsubsection{Knowledge leader}

In response to the questions about a knowledge leader or practice change champion, 18 of the 21 sites responded someone fulfilled this role in their ICU. Eight sites reported this role was filled by both doctors and nurses; in three it was only a doctor, and in four only a nurse. In most cases the doctors identified were intensive care specialists, usually the head of research, although one site responded that registrars presented to journal club. Where a nurse was reportedly a knowledge leader, this was usually a combination of the research nurses, clinical nurse consultant and nurse educators. Education was usually informal through bedside discussion and informal teaching; and by communicating about research studies and results, updating policy and procedures and updating staff about new evidence. One site articulated the different roles in their ICU;

Nurse Consultant - Clinical - formal - keeps up to date with best practice and informs staff of current articles, $R C$ - provides study results from studies we participated in, Senior registrars or PI will present at journal club. (SITE14)

\subsubsection{Change management}

Of the 19 responses regarding the process for changing ICU-wide clinical practices, 11 articulated a process for change management. Although each account had different steps in the process, they all identified some or most of the following: Identify issue, review current practice, discuss with stakeholders, review evidence, investigate feasibility of change, consensus from senior staff, test impact, change protocol or policy, endorse change (e.g., sign 
off), disseminate change, communicate to staff and evaluation or monitoring of change. In most ICUs, this process appeared to be a formal process $(n=17)$, although less formal in one, and described as ad hoc in another.

The process of change was commonly by a team of people, either formal or informal. Three sites reported having a quality team, one had a clinical practice committee and one had a clinical practice director. Other sites reported senior doctors and senior nurses as collaboratively involved in change management; "Definitely work as a team" (SITE13), or involved staff who had an interest in the change. In contrast, one ICU reported "Charge Nurse Manager decides change - informs staff" (SITE 03). These change management teams drafted the new or revised policy, which was reviewed by stakeholders. Other processes included during this consultation phase were assessing the feasibility for a change and any financial implications. Identification of an issue could be raised by "any level of staff" (SITE19), and any profession ("medical, nursing or allied health" [site 18]). In addition, the identification of an issue came from the education team, risk monitoring, due to new evidence in journal clubs, and one site stated change was usually initiated by senior medical staff.

Stakeholders were consulted either before or after a practice change, the timing depending on the change in question. Stakeholders were predominantly identified as senior doctors $(n=11)$ and the senior nursing team, including the charge nurse manager and educators $(n=10)$. In addition to this, members of the multidisciplinary team and heads of specific departments or portfolios were involved. Some sites specifically detailed obtaining feedback from "nursing staff" or "all staff", prior to a practice change being made, with one site clarifying this as "if relevant".

After drafting a change, and consulting with stakeholders, the decision about whether a change would be implemented was generally an ICU leadership decision. While many different medical and nursing roles were involved in the discussion and development of change, the ultimate decision in two sites was the ICU medical director. Additionally, many ICUs required endorsement, or 'final sign-off' of the change, such as by each of the leadership or quality groups or a clinical governance department before the change could be implemented. Once a policy or process was changed, staff were informed of the change. 
Dissemination of the new policy was by email, group or individual education, issuing of the new policy on a policy or general ICU website, meetings such as clinical practice meetings, daily handover or journal club. One site stated change was introduced by a "lead by example [from consultants] approach" (SITE22).

The amount of time for a change to be implemented varied by ICU, and the size of the change. Many respondents reported it "depended on the specific change". A small change could take as little as one week, although most reported the minimum time was one to two months. For several sites it would take up to 12 months to change a policy, with one site reporting "different groups look after different areas, then all report in one meeting quartly [sic] takes [a] long time" (SITE13). 


\subsection{Discussion}

The key findings for the practitioner attitudes survey were there were differences in attitudes to conservative oxygen therapy between health practitioners over time and from sites that participated in ICU-ROX compared to non-ICU-ROX sites. In addition, there was a difference in attitudes to conservative oxygen therapy between ICU doctors and nurses, and a difference in their knowledge of the ICU-ROX results, and the way they found out about the results.

\subsubsection{Strengths and limitations}

Before discussing the findings in detail, it is necessary to consider the strengths and limitations of this study. Purposeful recruitment was used to ensure representation of both doctors and nurses from all participating sites (Polit \& Beck, 2017). Purposive recruitment is, however, subject to researcher bias, which was mitigated by asking site research coordinators to approach staff to participate in the survey. Research coordinators may also have been subject to researcher bias, as they were probably most likely to ask staff who they thought would respond to the survey. Despite this purposive recruitment, the response rate of $67 \%$ indicates that there were many staff who said they would respond, and then did not. There are three main explanations for this: either they did not fully comprehend they would need to provide an email address for further contact from the researcher or they did not want the research coordinator to know they intended leaving ICU within 2-3 years, or they decided not to respond. An alternative to collecting an email address to contact staff over time would have been to conduct the study at a site level, without contacting the same people multiple times; however, a large sample would have been required, and it would have increased the chance of variability. This self-selection increases the chance of selection bias; however the baseline characteristics showed there were few differences between the ICU-ROX and non-ICU-ROX respondents (Polit \& Beck, 2017).

The baseline differences in respondents were about country and nurses' level of experience. Specifically, a higher proportion (82\%) of ICUs in the non-ICU-ROX sites were from Australia, compared to NZ (18\%) (Table 11). In addition, nurses from non-ICU-ROX sites were significantly more experienced than the nurses from ICU-ROX sites (Table 13). These differences are an inherent weakness in non-randomised observational research, and it not 
known whether having more similar sample characteristics in both groups would have made a difference to the attitudes to oxygen management of either group.

The comparison of ICU specialist doctors and nurses was inequitable as doctors and nurses have different training. However, it is often the nurse at the bedside making decisions about oxygen management and adjusting the $\mathrm{F}_{1} \mathrm{O}_{2}$ accordingly, and therefore, imperative that both roles were included in this research. To increase the respondent retention rate, only the views of ICU specialist doctors were obtained; it is unknown whether inclusion of registrars, in addition to specialist doctors would have changed the results of this study.

As discussed in section 6.3.10 (Validity strategies), the survey was validated by a panel of subject matter experts. There was an assumption that everyone who completed the survey understood the questions, which generally seemed to be the case, as evidenced by respondents answering differently for the different levels of oxygen and very few respondents answering the question about reducing oxygen from $\mathrm{F}_{1} \mathrm{O}_{2}$ of 1.0 as 'unlikely' or 'very unlikely'. However, at least one question appeared to have caused confusion. In response to the question 'What was your level of participation in the ICU-ROX trial?', 22 people from non-ICUROX sites selected 'my ICU participated in ICU-ROX but I had no involvement with a patient enrolled in ICU-ROX'. While this may represent staff changing ICUs throughout the study period, the most likely explanation may be confusion between the terms ICU-ROX and ICUROX TRIPS.

Other terminology in the survey that may have confused some respondents was the use of the word 'clinician', as in 'clinician preference'. The intention of the meaning was 'any clinician' (either doctor or nurse); however, some may have interpreted this as meaning only a doctor. Any future survey should provide clearer instructions and be more specific. A helpful addition to the survey may have been to ascertain who the 'someone at work' was, and whether it was via a formal or informal interaction, as this would be useful in designing future dissemination strategies.

A major limitation of the practitioner attitudes survey was the inadvertent omission of the hypoxic ischaemic encephalopathy subgroup at the first two time periods. There was a 
hypothesis-generating finding in this subgroup of patients in ICU-ROX; that conservative oxygen therapy may have benefited this group of patients. While this was not substantiated in a post-hoc analysis (Young et al., 2020b), the finding may have changed the attitudes of some health practitioners, and five health practitioners commented in the survey conducted after publication of ICU-ROX that they would change their practice for these patients.

As discussed in Chapter 2, there were other publications about oxygen management in ICU at the time this study was undertaken (Chu et al., 2018; Girardis et al., 2016; Grim, 2020; Grim et al., 2020). The influence of these external sources, is likely to be equally prevalent in both ICU-ROX and non-ICU-ROX sites; however, this was not tested in this knowledge translation research, and no conclusions can be drawn.

Many things affect the way people answer open-ended questions; their level of interest in the project, knowledge of the subject area, time available and other stressors. Where openended questions were included for both the individual and site level survey, these were answered with different levels of detail. The quality of the data analysis is, in part, limited to the quality of the data entered. The 'comments' questions were optional for each of the three surveys, and generally used for explaining answers respondents made. This was a helpful addition for issues such as two nurses from the same ICU explaining why they had to answer that they would be very unlikely to reduce oxygen from $100 \%$.

With regard to the open-ended questions about research champion, change leader and practice change process, some site research staff answered these in brief (e.g., one word), while others answered them comprehensively - this is not an indication of how robust, or not, their actual system is, only the reporting of it. This was not a qualitative study, and the answers people gave were adequate for the aim of this study.

The sites that participated in the survey and inception cohort study were not randomised, and no effort was made to match them. Table 11, however, demonstrated that the two cohorts were very similar. As stated at the beginning of this chapter, there were management reasons for selecting the ICU-ROX sites, beyond the control of the researcher. Finally, multiple 
statistical testing was conducted without adjustment for multiplicity of testing so that all results should strictly be considered exploratory.

\subsubsection{Change in attitudes}

There was clear evidence of differences in respondents' indicated attitude to oxygen management, over time and in ICU-ROX versus non-ICU-ROX sites. In particular, there was a difference in the primary measure of interest; attitudes about using $\mathrm{F}_{1} \mathrm{O}_{2}$ of 0.21 in mechanically ventilated patients. Practitioner attitudes were similar before ICU-ROX started, but changed after participation in ICU-ROX, and after publication for the ICU-ROX site respondents.

A possible explanation for the change after participation even before the results were known, is that participation itself influenced practitioners' attitudes. It is plausible that the exposure to conservative oxygen therapy changed their attitudes or that practitioners involved in ICUROX saw no harm from their experience with conservative oxygen therapy. Another explanation is that attitudes were based on evidence that emerged while ICU-ROX was being conducted that conservative oxygen therapy decreased mortality (Chu et al., 2018; Girardis et al., 2016); however this does not explain the difference between respondents from ICUROX sites changing differently to non-ICU-ROX site respondents. Alternatively, change could be an example of the Hawthorne effect where people change their attitudes and behaviours because they know they're being studied (McCambridge et al., 2014). This also seems unlikely because there no difference in attitudes prior to ICU-ROX, and change would have been evident in both ICU-ROX and non-ICU-ROX respondents. No literature was found that specifically addressed practitioner attitudes to an intervention following participation in a specific research trial. Tranmer et al. (2002) however, showed that attitudes to research in general improved with a higher level of engagement in research by designing a trial about sleep.

The differences between ICU-ROX and non-ICU-ROX respondents were sustained after publication of ICU-ROX trial results. An explanation for this is that increased awareness of evidence, after participating in research may have positively influenced health practitioners to read and accept the results of a trial they participated in because of their role in 
contributing to the evidence. In the case of the ICU-ROX publication, a change in attitude towards conservative oxygen therapy, would not necessarily have been expected as the primary outcome showed no difference in ventilator-free days (Mackle et al., 2020). While the finding that ICU-ROX showed no harm was caused by conservative oxygen therapy may have influenced some practitioners, the most likely explanation remains that participation, and exposure to conservative oxygen therapy, influenced health practitioners' attitudes. Previous literature about health practitioners' attitudes to oxygen therapy reported inconsistency in the self-reported opinions and practices about oxygen management in ICU for both intensive care specialists and ICU nurses in ANZ (Eastwood et al., 2011, Eastwood, Reade et al., 2012) and the Netherlands (Grim, 2020; Grim et al., 2020; Helmerhorst et al., 2014). More recently Livingston et al. (2020) reported clinicians' experience and decision making about management of supplemental oxygen therapy in mechanically ventilated patients in one Australian ICU were driven more by unit culture than evidence. However, none of the previous research about attitudes to oxygen management in ICU assessed the effect of participation in a research trial.

\subsubsection{Role differences}

A significant unexpected finding was the difference between doctors and nurses. Reported at every time point, nurses' attitudes indicated they would be less likely to use conservative oxygen therapy than doctors. Specifically, nurses were less likely to indicate using an $\mathrm{F}_{1} \mathrm{O}_{2}$ of 0.21 in a ventilated patient than doctors before ICU-ROX, after ICU-ROX, and again after publication. The finding that nurses were reportedly less likely to use 'conservative oxygen therapy' than doctors aligns with research from the Netherlands, where nurses were reportedly less accepting of low target ranges for $\mathrm{SaO}_{2}(80-85 \%)$ and $\mathrm{PaO}_{2}(45 \mathrm{mmHg})$, compared to doctors (Grim, 2020). The nurses' attitudes changed significantly after ICU-ROX (but before results were known), and they were more likely to indicate they would use an $\mathrm{F}_{1} \mathrm{O}_{2}$ of 0.21 than before. There were too few data to analyse whether nurses from ICU-ROX sites were more likely to change than their non-ICU-ROX counterparts (Figure 21). Amongst ICU nurse respondents, $58 \%$ of ICU nurses commented their practice would change after publication of the ICU-ROX results, and several commented that participating in ICU-ROX had 'opened their mind' to the idea of conservative oxygen. No literature was found about role and changing attitudes in response to participation in a specific trial, apart from the 
aforementioned Tranmer et al. (2002) study, that surveyed nurses' attitudes to research after involvement in designing a research trial.

In contrast to the nurses, when asked how the ICU-ROX results would change their practice, $55 \%$ of ICU specialists who commented stated the results would not change their practice. This was reportedly because they already avoided hyperoxia, and the findings confirmed their current attitude to conservative oxygen therapy, a sentiment noted in the comment by one doctor that "Nurses put it up, doctors put it down". It is plausible that doctors were more aware of previous literature that recommended conservative oxygen management in critically ill patients (Chu et al., 2018; Girardis et al., 2016). Several doctors commented that the evidence regarding oxygen management in ICU remained uncertain, and they relied on their personal beliefs and experiences. This view is reflective of the PARIHS framework, that includes clinical experience in the evidence element, arguing 'agreement amongst peers' as a way of validating clinical experience (Rycroft-Malone, Harvey, et al., 2002). Additionally, in the early evidence-based medicine research, Sackett et al. (1996) advocated integrating clinical expertise with the best available evidence.

\subsubsection{Knowledge of results}

There was no difference in knowledge of the ICU-ROX results between ICU-ROX and non-ICUROX site respondents. However, one important finding was that nurses were significantly less likely than doctors to have heard the ICU-ROX results. In addition, a higher level of engagement, based on whether respondents had enrolled a patient into ICU-ROX or not, was associated with increased knowledge of the ICU-ROX results, although 'caring for a patient' compared to those who did not (but worked in an ICU that participated in ICU-ROX), did not make a difference.

This research does not support two previous studies that site level participation in a trial increased the knowledge of the trial results (Khera et al., 2018; Litjens et al., 2013). In a knowledge translation study of a trial comparing bone marrow graft vs peripheral blood graft, $55 \%$ of trial-site respondents knew the results compared to $37 \%$ of non-trial site respondents (Khera et al., 2018). This compares with the current study in which $72 \%$ of trial-site respondents and $68 \%$ of non-trial site respondents had heard the results. Litjens et al. 
reported that health practitioners from sites that had participated in the nine trials they assessed were significantly more likely to know the trial results than those from non-trial sites; however, this varied considerably depending on the trial. The biggest difference was for a trial about fertility in which $94 \%$ of trial site respondents compared to $29 \%$ of non-trial site respondents knew the result. The current research is most similar to Litjens' findings regarding an RCT about hypertension and pre-eclampsia, in which $97 \%$ of trial site and $86 \%$ of non-trial-site respondents knew the results.

The current research suggests that ICU nurses were less likely to know the ICU-ROX trial results than their medical colleagues. Specifically, $41.1 \%$ of nurses had not heard the ICUROX results at all, compared to $13.6 \%$ of doctors. They were also less likely to have heard the ICU-ROX results at a conference, on social media or to have read the publication. Squires et al. (2011) reported attendance at conferences and in-services, increased research use by nurses, yet the current research indicated only nine (8\%) nurses heard the ICU-ROX result at a conference, although whether these nurses had the opportunity to attend a conference was not explored. Another surprising finding was that only one nurse $(0.9 \%)$ found out the ICUROX on social media (specifically Twitter), compared to $24.7 \%$ of doctors, indicating the nurses did not follow the \#ICUROXTRIAL hashtag. Little literature was found about the effectiveness of social media as a vehicle for translation research, specifically to nurses, although the finding in the current research concurred with Sweeting et al. (2020), in which only one physiotherapist (4.7\%) found out the result of a study they assisted with via social media.

The finding that $76 \%$ of nurses had not read any part of the ICU-ROX publication, was not anticipated in the ICU context. This finding was, however, similar to Sweeting et al.'s study, in which only $24 \%$ of participating physiotherapists had read the published paper (2020). Few nurses reading the ICU-ROX publication confirms what is already well-stated in the literature; that nurses are more likely to find new evidence, or seek clarification about clinical uncertainty, from practitioners they trust rather than from reading written evidence (Ebenezer, 2015; Marshall et al., 2011; Thompson et al., 2001). A suggestion that nurses do not have access to recent publications (Ebenezer, 2015), was not explored in the current study. The current research finding that $45(40 \%)$ nurses found out the results from "someone 
at work" aligns with the literature, and was a similar percentage to the doctors (31\%); however, doctors were also more likely to have read the ICU-ROX publication as well (53.1\%). While Litjens et al. (2013) also found that gynaecologists and resident doctors were more likely to know trial results than midwives or nurses, there were far fewer midwives than doctors, and only two research nurses in their sample. They also did not assess the relevance of research to the individual roles and many of the RCTs they examined, such as those about cancer treatment or infertility, were not relevant to midwifery practice.

Unlike some of the examples from Litjens et al. (2013), oxygen management and the ICU-ROX trial results are highly relevant to both ICU doctors and nurses (Eastwood, Reade et al., 2012; Henderwood, 2015; Rose et al., 2008; Young, Beasley et al., 2015). While respondents were not specifically asked about the relevance of the ICU-ROX trial to their role, $77 \%$ reported that nurses had a role in adjusting oxygen at the bedside. In addition, the open-ended comments suggested both doctors and nurses were involved in decisions about oxygen management, confirming the finding in Livingston et al. (2020) that oxygen management involves both roles.

Respondents who enrolled a patient in ICU-ROX were more likely to know the ICU-ROX results, suggesting that an increased level of engagement increased the likelihood of knowing the trial results, as seen in the 2013 study of multiple RCTs by Litjens et al (2013). However, the finding in the current research is confounded by the influence of role. Amongst ICU-ROX site respondents, $30.9 \%$ of doctors had enrolled a patient, compared to $13.4 \%$ of nurses, suggesting that role may have had a greater bearing on knowledge of results, than level of engagement when measured this way.

\subsubsection{Oxygen management/ oxygen guidelines}

The key findings about oxygen management were that the main determinant of oxygen management was clinician preference, the commonest prompt for adjusting oxygen was a prescribed arterial or peripheral oxygen saturation range and oxygen was predominantly adjusted by nurses, although the open comments suggested this was a shared decision.

Oxygen management was predominantly determined by 'clinician preference', and the use of ICU, hospital, national or international guidelines to guide practice was uncommon, a finding 
which was reflected in the ICU-level study. A possible explanation is that 'clinician preference' was based on oxygen guidelines or current evidence, however this study did not test this stance. This finding is similar to a Canadian ICU knowledge translation study that showed that de-adoption of albumin for fluid resuscitation was predominantly related to clinician preference (Sauro et al., 2019). This differs from Taylor et al. (2017), that indicated that choice of resuscitation fluid was primarily driven by safety; however, they did not include 'clinician' or 'personal preference' as an option for drivers of decision-making. Another explanation is that clinician experience may be a significant contributor to decisions about oxygen management, as recognised in the PARIHS framework (Rycroft-Malone, Seers, et al., 2004).

The commonest reported prompt for adjusting oxygen was a prescribed oxygen saturation range (either arterial or peripheral). That $85.5 \%$ of respondents reporting an $\mathrm{SpO}_{2}$ range was prescribed was higher than the previously reported rate in a point prevalence of practice conducted in 48 ANZ ICUs in 2013 (Young, Beasley et al., 2015). Young, Beasley et al. found a documented lower $\mathrm{SpO}_{2}$ target in $39.6 \%$ of mechanically ventilated patients and an upper $\mathrm{SpO}_{2}$ in $5.2 \%$. Details about $\mathrm{SpO}_{2}$ range were not sought in the current research, and as data are self-reported, they may not be an accurate reflection of practice (Helmerhorst et al., 2014).

The second commonest prompt for adjusting oxygen was an arterial oxygen range. Over half of nurses (51.8\%) reported this was prescribed compared to $22.2 \%$ of doctors. In addition, some nurses commented that decisions about oxygen management were made based on an arterial blood gas $(A B G)$ result, rather than $\mathrm{SpO}_{2}$. One doctor commented that $\mathrm{SpO}_{2}$ monitoring was "unreliable", providing one explanation for the reported reliance on ABGs to aid decisions about oxygen management in preference to $\mathrm{SpO}_{2}$ in some ICUs. Peripheral oxygen saturation measurement is less invasive than arterial blood sampling, and a recent study set in two ANZ ICUs found a high level of congruency between $\mathrm{SpO}_{2}$ and $\mathrm{PaO}_{2}$, and in most cases $\mathrm{SpO}_{2}$ was sufficient and accurate (Pilcher et al., 2020).

Although the majority of respondents indicated that oxygen was adjusted by a nurse, comments indicated that decisions were shared. Additionally, this division of responsibility 
was variable, and depended on the specific ICU, "physician of the day" and the experience of the nurse. These findings confirm previous studies that reported that while nurses participated actively in ventilation decisions, including oxygen adjustment; this depended on the individual ICU, education level and experience of the nurses (Rose et al., 2008). Livingston et al. (2020) reported that in addition to multi-disciplinary decisions regarding oxygen management, $\mathrm{F}_{1} \mathrm{O}_{2}$ levels were also influenced more by unit culture, rather than evidence, reported by some in this current research.

\subsubsection{Dissemination strategies}

Despite the extensive ICU-ROX dissemination plan outlined in section 2.4 , almost a third (27.9\%) of individual respondents from ICU-ROX sites had not heard the results, suggesting the dissemination strategies had not been effective. Only one previous study reported their dissemination plan, which involved sending a link of the publication to the participating physiotherapists (Sweeting et al., 2020). That study found that $71 \%$ of the 21 physiotherapists knew of the result, although only five (23.8\%) had read the publication. Even though ICU-ROX was published in a high-ranking journal and presented at an international conference, these are considered 'diffusion' rather than dissemination, as they are considered passive modes of delivering information to end users (CIHR, 2012). Although posting a summary on Twitter may be considered dissemination, it did not specifically target the audience, and relied on self-selection of interested people following the \#ICUROXTRIAL hashtag. Dissemination was achieved by targeted presentation of the results to research networks via the ANZ CTG, site investigator meetings and by sending the publication of the results to participating ICUs. However, these methods generally only ensured the ICU-ROX trial results got to the research teams at participating ICUs. How, and whether, these ICUs distributed the findings was decided by the individual ICU research teams. In some cases, passive dissemination strategies were used, including placing a copy of the publication on a noticeboard, webpage or leaving in a staff area. In other ICUs, research staff used more active, targeted means such as emailing staff with the results, presenting and discussing the results in journal club or in-service education to disseminate the results. An assessment of barriers or facilitators was not specifically conducted about the best way to disseminate the ICU-ROX trial results. 


\subsubsection{Change management}

The findings from the ICU level survey about change management indicated that most participating ICUs had a process for change management, either formal or informal. Many of the sites indicated both medical and nursing knowledge leaders, which may be of assistance when bringing new information to nurses, in particular. This aligns with the Facilitation role outlined in PARIHS (Harvey et al., 2002), however the level of facilitating of new research remains unknown in this setting.

It was not possible, from the current research and state of evidence about oxygen management in ICU, to assess how long it takes to change practice. However, there were comments from the site level data indicating that within one ICU, it could still take a full year to bring about change. In some cases, this was as little as one week. While only suggestive, these time frames are considerably shorter than the frequently cited 17 years (Morris et al., 2011) and more recent three years (Khera et al., 2018).

\subsubsection{Implications}

The current study raised the possibility that participation in clinical research is an independent variable affecting attitudes to oxygen management. Although far from conclusive, this may be more important to nurses than doctors. Participation in a trial about conservative oxygen therapy made a difference to attitudes about oxygen management, despite there being no difference between ICU-ROX and non-ICU-ROX site respondents' knowledge of results.

While dissemination of the ICU-ROX results was extensive, it failed to reach nurses, who were the role most likely to adjust oxygen in ICU. Strategies to increase nurses' knowledge of research for clinical activities they are involved in, and in some cases, drive, must be adopted for future studies. Recognition of the different learning styles, and ways they understand new evidence, is imperative for this to happen. This research has created an opportunity to improve the way research findings are disseminated in the future. Future dissemination strategies should ensure that these key roles know and understand the results from research, and how it fits into literature and clinical practice. 


\section{CHAPTER 7 INCEPTION COHORT STUDY}

This chapter is about the inception cohort study, an observational study about oxygen practices in ICU. Site selection was discussed in the previous chapter, as the same ICU-ROX and non-ICU-ROX sites participated in this study as for the survey. The timing of this study was also the same as for the survey: before ICU-ROX (T1), after ICU-ROX but before the results are known (T2) and one to six months after publication of the ICU-ROX results (T3). The chapter outlines the aims and hypotheses, study design and methods, results and finally the discussion specific to this study.

\subsection{Aims}

The main aim of the inception cohort study was to evaluate practices with regard to oxygen management in mechanically ventilated ICU patients, over time and in relation to the ICUROX trial and results.

\subsection{Hypotheses}

The hypotheses for the inception cohort studies were:

1. Prior to enrolment commencing prior to enrolment in ICU-ROX commencing, clinical practice in relation to oxygen therapy would be more favourable in ICUs that were going to participate in ICU-ROX (ICU-ROX ICUs) than in ICUs that were not going to participate in ICU-ROX (non-ICU-ROX ICUs).

2. Before trial results were known, clinical practice in relation to oxygen therapy would become more conservative in the ICU-ROX ICUs after enrolment had completed compared to before enrolment began; such a change in practice over time would not be observed in non-ICU-ROX ICUs.

3a. In the event that ICU-ROX demonstrates that conservative oxygen therapy improves outcomes, implementation of trial results as reflected by more conservative approaches to provision of oxygen therapy, will be more evident in participating ICUs than non-participating ICUs.

3b. In the event that ICU-ROX demonstrated no difference in outcomes for patients who were treated with conservative oxygen therapy compared with usual oxygen therapy, ICU-ROX ICUs would continue to be more conservative in their approaches to provision of oxygen than non-participating ICUs.

3c. In the event that ICU-ROX demonstrates that conservative oxygen therapy worsens patient outcomes compared with usual oxygen therapy, use of oxygen will be similar in non-participating and participating ICUs. 


\subsection{Design and methods}

An inception cohort study design was used to assess oxygen management practices over time by evaluating the exposure to oxygen in groups of mechanically ventilated patients in ICU. A cohort study is a non-experimental, prospective, observational method used to observe a group of people over time (Polit \& Beck, 2017). An inception cohort study follows patients at the same stage in the course of their disease or other variable, which in the case of this study was their course of invasive mechanical ventilation. In this study, there are six cohorts of patients; before ICU-ROX (ICU-ROX and non-ICU-ROX), after ICU-ROX (ICU-ROX and non-ICUROX) and after ICU-ROX publication (ICU-ROX and non-ICU-ROX). This study was endorsed by ANZICS Clinical Trials Group (Appendix 6).

\subsubsection{Sample size, eligibility and recruitment}

The sample size of 214 patients for each of the three time points was based on preliminary ICU-ROX pilot data comparing the conservative and usual therapy groups (Young et al., 2017). Based on the baseline percentage of time patients received $\mathrm{F}_{1} \mathrm{O}_{2} 0.21$ (room air) of $15.6 \%$ in the usual care group, compared to $33 \%$ of the time in the conservative group, a sample size of 214 patients provided $80 \%$ power to detect a difference, with an alpha of 0.05 using a twotailed $t$ test.

Data were collected for 10 consecutive eligible adult ( $\geq 18$ years) patients who were mechanically ventilated in each ICU at each time point. Patients were identified daily by the research coordinator $(\mathrm{RC})$ at each site, at commencement of invasive mechanical ventilation or ICU admission (if they were admitted already ventilated). Patients were excluded from data collection if they were mechanically ventilated for less than 12 hours or were receiving extracorporeal membrane oxygenation (ECMO). Sites were not required to keep a log of patients who were deemed ineligible.

These inclusion and exclusion criteria aligned with those used for the ICU-ROX trial (Mackle et al., 2018). Specifically, the ICU-ROX trial investigated invasively mechanically ventilated adults in the ICU. However, patients in ICU-ROX were required to be ventilated 'beyond the next calendar day', to ensure that a sicker group of patients was enrolled and would be exposed to the randomised treatment group. As this study was an observational design, the 
exclusion criterion of 'ventilated less than 12 hours' was used. Patients receiving ECMO were excluded because in ICU-ROX they were not subject to the treatment protocol while they were on ECMO, and most ICUs were not able to enrol patients on ECMO due to the treating clinician's decision. Consecutive eligible patients were chosen to reduce selection bias (Polit \& Beck, 2017).

\subsubsection{Exposure and comparator}

As discussed in section 5.5.1, the exposure for the study was ICU-level participation in ICUROX and the comparator was non-participation in ICU-ROX.

\subsubsection{Outcomes}

The primary outcome measure was the number of hours patients spent at $\mathrm{F}_{1} \mathrm{O}_{2}$ of 0.21 from the time of ventilation (or ICU admission if the patient was ventilated at admission) until the earliest of: extubation, ICU discharge, 10 days of data collection or death. The primary outcome was the difference in time spent at $\mathrm{F}_{1} \mathrm{O}_{2}$ of 0.21 after ICU-ROX was published (T3) compared to the time spent at $\mathrm{F}_{1} \mathrm{O}_{2}$ of 0.21 , before ICU-ROX was conducted (T1), for patients from sites that participated in ICU-ROX.

The key secondary outcome was the difference in time spent at $\mathrm{F}_{1} \mathrm{O}_{2}$ of 0.21 after ICU-ROX was published (T3) compared to the time spent at $\mathrm{F}_{1} \mathrm{O}_{2}$ of 0.21 , before ICU-ROX was conducted (T1), for patients from sites that did not participate in ICU-ROX (non-ICU-ROX sites). In addition, comparisons of the time spent at $\mathrm{F}_{1} \mathrm{O}_{2}$ of 0.21 were made for patients from both ICUROX and non-ICU-ROX sites after ICU-ROX publication (T3) compared to after ICU-ROX was completed, but before the results were known (T2). A number of process of care measures and physiological outcomes were reported for descriptive purposes (Table 29).

All outcomes were selected to align with the process of care and physiological outcomes and descriptors from the ICU-ROX trial (Mackle et al., 2018). Specifically, the time patients spent ventilated at $\mathrm{F}_{1} \mathrm{O}_{2}$ of 0.21 aligned with the ICU-ROX conservative intervention arm. Likewise, the $\mathrm{SpO}_{2}$ levels were also used to align with the ICU-ROX trial. $\mathrm{SpO}_{2} \geq 97 \%$ was the upper limit of $\mathrm{SpO}_{2}$ allowed in the conservative arm of the trial; $\mathrm{SpO}_{2}<91 \%$ was the default lower limit

of $\mathrm{SpO}_{2}$ recommended for both conservative and usual care in ICU-ROX and $\mathrm{SpO}_{2}<88 \%$ was collected to assess any difference in hypoxic episodes between groups. The physiological 
descriptors and process of care measures were closely aligned to those collected for the ICUROX trial (Mackle et al., 2018).

Table 29 Physiological descriptors and process of care measures

\begin{tabular}{|l|l|}
\hline Process of care & - Mean daily $\mathrm{F}_{1} \mathrm{O}_{2}$ (calculated from 6 hourly recordings) \\
& - Mean highest daily $\mathrm{F}_{1} \mathrm{O}_{2}$ \\
\hline Physiological & - Mean lowest daily $\mathrm{FO}_{2}$ \\
descriptors & - Mean daily $\mathrm{PaO}_{2}$ (calculated from 6 hourly recordings) \\
& - Mean highest daily $\mathrm{PaO}_{2}$ \\
& - Mean lowest daily $\mathrm{PaO}_{2}$ \\
& - Number of hours $\mathrm{with}_{\mathrm{SpO}} \geq 97 \%$ \\
& - Number of hours $\mathrm{SpO}_{2}<91 \%$ \\
\hline
\end{tabular}

Abbreviations: $\mathrm{F}_{1} \mathrm{O}_{2}$ : fraction of inspired oxygen; $\mathrm{PaO}_{2}$ : partial pressure of arterial oxygen; $\mathrm{SpO}_{2}$ : peripheral oxygen saturations.

\subsubsection{Data collection and management}

Data were collected by trained site RCs for consecutive ventilated patients that met the inclusion criteria and did not meet any exclusion criteria. Only data routinely collected and recorded as part of standard ICU care were used. Three paper worksheets were created and provided to sites, along with a 'data dictionary' to provide exact definitions of each data point to be collected, to reduce the risk of error. Baseline data comprised variables up until the study period began and daily data provided data during the 10-day study period (or until extubation, discharge or death). A REDCAP database was developed and site RCs were provided with individual access to enter data.

Data were collected after ethics approval but before the ICU-ROX trial started at each of the sites participating in ICU-ROX. In the non-ICU-ROX sites, data collection for the 'before ICUROX' time period began as soon as ethics was approved and prior to completion of overall ICU-ROX enrolment. Data collection for all participating sites began again 28 days after ICUROX had finished, but before the results were known. The final study period was between one and six months after the ICU-ROX results were published.

Baseline data were aligned with the ICU-ROX trial and provide de-identified, grouped information about patients to show the characteristics of patients from the ICU-ROX sites compared with those from the non-ICU-ROX sites (Table 30). A question was included about whether the patient would have been eligible for ICU-ROX. For this data point, sites were 
asked about inclusion criteria for ICU-ROX, and if they answered 'yes' to this and 'no', to any exclusion criterion (other than 'enrolled greater than two hours ago), they were deemed to have been eligible for ICU-ROX.

Table 30 Baseline data collected

\begin{tabular}{|c|c|c|}
\hline Section & Data collected & Options (if applicable) \\
\hline $\begin{array}{l}\text { Patient } \\
\text { demographics }\end{array}$ & $\begin{array}{l}\text { Age } \\
\text { Gender } \\
\text { Ethnicity (NZ only) }\end{array}$ & \\
\hline \multirow[t]{2}{*}{ ICU admission } & $\begin{array}{l}\text { Date and time of ICU } \\
\text { admission }\end{array}$ & \\
\hline & ICU admission source & $\begin{array}{ll}\text { - } & \text { Emergency department } \\
\text { - } & \text { Hospital floor (ward) } \\
\text { - } & \text { Other ICU } \\
\text { - } & \text { Other hospital } \\
\text { - } & \text { Operating theatre following } \\
& \text { EMERGENCY surgery } \\
\text { - } & \text { Operating theatre following ELECTIVE } \\
& \text { surgery }\end{array}$ \\
\hline \multirow[t]{2}{*}{$\begin{array}{l}\text { APACHE/ IIIness } \\
\text { severity }\end{array}$} & $\begin{array}{l}\text { APACHE-III admission } \\
\text { diagnostic code }\end{array}$ & $\begin{array}{l}\text { A list of operative and non-operative codes } \\
\text { was provided in the data dictionary }\end{array}$ \\
\hline & APACHE-II score & $\begin{array}{l}\text { - Score 0-70 } \\
\text { - Worksheet provided in data dictionary, } \\
\text { or could use a routinely collected score } \\
\text { - Calculated from worst score in first } 24 \\
\text { hours of ICU admission }\end{array}$ \\
\hline $\begin{array}{l}\text { Chronic illness co- } \\
\text { morbidities }\end{array}$ & Select all that apply & $\begin{array}{l}\text { - } \text { Respiratory } \\
\text { - } \text { Cardiovascular } \\
\text { - Hepatic } \\
\text { - } \text { Renal } \\
\text { - Immunosuppression by disease } \\
\text { - Immunosuppression by therapy } \\
\text { - } \text { Metastatic cancer } \\
\text { OR No chronic co-morbidities }\end{array}$ \\
\hline $\begin{array}{l}\text { Mechanical } \\
\text { Ventilation }\end{array}$ & $\begin{array}{l}\text { Date and time of initiation of } \\
\text { mechanical ventilation }\end{array}$ & \\
\hline $\begin{array}{l}\text { Acute brain } \\
\text { pathology }\end{array}$ & $\begin{array}{l}\text { What (if any) acute brain } \\
\text { pathology did this patient have } \\
\text { documented at baseline? }\end{array}$ & $\begin{array}{ll}\text { - } & \text { No acute brain pathology } \\
\text { - } & \text { Acute traumatic brain injury } \\
\text { - } & \text { CNS infection } \\
\text { - } & \text { Haemorrhagic stroke } \\
\text { - } & \text { Ischaemic stroke } \\
\text { - } & \text { Subarachnoid haemorrhage } \\
\text { - } & \text { (Suspected) Hypoxic brain injury } \\
\text { - } & \text { Other (please specify) }\end{array}$ \\
\hline $\begin{array}{l}\text { Eligibility for the } \\
\text { ICU-ROX trial }\end{array}$ & $\begin{array}{l}\text { Would this patient have met } \\
\text { this inclusion criteria for the } \\
\text { ICU-ROX trial? 'Expected to } \\
\text { remain mechanically }\end{array}$ & - Yes or No \\
\hline
\end{tabular}




\begin{tabular}{|c|c|c|}
\hline Section & Data collected & Options (if applicable) \\
\hline & $\begin{array}{l}\text { ventilated beyond the next } \\
\text { calendar day' }\end{array}$ & \\
\hline & $\begin{array}{l}\text { Would this patient have met } \\
\text { any exclusion criteria for the } \\
\text { ICU-ROX trial? } \\
\text { (apart from the 2-hour time- } \\
\text { frame for enrolment) }\end{array}$ & $\begin{array}{l}\text { - Hyperoxia clinically indicated } \\
\text { - } \quad \text { Avoidance of hyperoxia clinically } \\
\text { indicated } \\
\text { - Pregnancy } \\
\text { - } \quad \text { Death is deemed inevitable (current } \\
\text { acute illness) } \\
\text { - Life expectancy < } 90 \text { days } \\
\text { - Drug overdose (including alcohol) } \\
\text { - Long-term ventilator dependence } \\
\text { - Confirmed or suspected GBS, cervical } \\
\text { - } \text { dystrophy, or Motor Neurone Disease } \\
\text { - } \quad \text { Ent in patient's best interests } \\
\text { - } \text { oxygen therapy } \\
\text { OR No exclusiously enrolled in the ICU-ROX trial } \\
\text { timeframe) }\end{array}$ \\
\hline
\end{tabular}

Abbreviations: APACHE: Acute Physiology and Chronic Health Evaluation; C5: $5^{\text {th }}$ cervical vertebrae; CNS: central nervous system; GBS: Guillain-Barré syndrome; ICU: Intensive Care Unit; ICU-ROX trial: Intensive Care Unit Randomised Trial Comparing Two Approaches to Oxygen therapy; NZ: New Zealand.

Oxygen level, peripheral oxygen saturations and partial arterial oxygen data were collected daily from the time of ventilation or ICU admission (if the patient was admitted to ICU already ventilated) until day 10, extubation, ICU discharge or the patient died (Table 31). Finally, the date, time and reason for cessation of data collection were collected. 
Table 31 Daily data collected for primary and secondary outcomes

\begin{tabular}{|c|c|}
\hline Data point/s & Rationale for inclusion \\
\hline \multicolumn{2}{|l|}{ Fraction of inspired oxygen $\left(\mathrm{F}_{1} \mathrm{O}_{2}\right)$} \\
\hline $\mathrm{F}_{1} \mathrm{O}_{2}: 0600,1200,1800,2400$ & $\begin{array}{l}\text { Process of care descriptive data } \\
\text { To calculate mean } \mathrm{F}_{1} \mathrm{O}_{2} \text { per day }\end{array}$ \\
\hline $\mathrm{F}_{1} \mathrm{O}_{2}$ (lowest) & Process of care descriptive data \\
\hline $\mathrm{F}_{1} \mathrm{O}_{2}$ (highest) & Process of care descriptive data \\
\hline Number of hours $\mathrm{F}_{1} \mathrm{O}_{2}$ is 0.21 & Primary outcome \\
\hline Total number of hours $\mathrm{F}_{1} \mathrm{O}_{2}$ recorded & To calculate percentages for $\mathrm{F}_{1} \mathrm{O}_{2} 0.21$ \\
\hline \multicolumn{2}{|c|}{ Partial pressure of oxygen $\left(\mathrm{PaO}_{2}\right)(\text { in } \mathrm{mmHg})^{*}$} \\
\hline $\mathrm{PaO}_{2}: 0600,1200,1800,2400$ & $\begin{array}{l}\text { Physiological descriptive data } \\
\text { To calculate mean } \mathrm{PaO}_{2} \text { per day }\end{array}$ \\
\hline $\mathrm{PaO}_{2}$ (lowest) & Physiological descriptive data \\
\hline $\mathrm{PaO}_{2}$ (highest) & Physiological descriptive data \\
\hline \multicolumn{2}{|l|}{ Peripheral oxygen saturations $\left(\mathrm{SpO}_{2}\right)$} \\
\hline Number of hours $\mathrm{SpO}_{2} \geq 97 \%$ & Physiological descriptive data \\
\hline Number of hours $\mathrm{SpO}_{2}<91 \%$ & Physiological descriptive data \\
\hline Number of hours $\mathrm{SpO}_{2}<88 \%$ & Physiological descriptive data \\
\hline Total number of $\mathrm{SpO}_{2}$ hours recorded & To calculate percentages for $\mathrm{SpO}_{2}$ \\
\hline
\end{tabular}

*if collected in kilopascals ( $\mathrm{kPa}$ ), $\mathrm{PaO}_{2}$ is converted to $\mathrm{mmHg}$ by multiplying $\mathrm{kPa}$ value by 7.5

Abbreviations: $\mathrm{F}_{1} \mathrm{O}_{2}$ : fraction of inspired oxygen; $\mathrm{mmHg}$ : millimetres of mercury (standard unit of measure for $\left.\mathrm{PaO}_{2}\right) ; \mathrm{PaO}_{2}$ : partial pressure of arterial oxygen; $\mathrm{SpO}_{2}$ : peripheral oxygen saturations.

\subsubsection{Changes to proposed data analyses}

After first review of the data a number of issues were identified, which meant data analyses could not be undertaken as intended prior to the study starting. Specifically, these issues were with respect to: analysing proportion of hours, treating patients from 'before ICU-ROX' as 'baseline' and too many analyses. All issues were discussed, and decisions made in collaboration with the statistician, researcher and her supervisors. The final statistical analysis plan was agreed prior to any analyses being undertaken, and the final analyses are outlined in section 7.3.6.

The outcomes outlined earlier in this chapter differed from those intended when the study proposal and protocol were written. Specifically, the intention was to compare the proportion of hours at $\mathrm{F}_{1} \mathrm{O}_{2} 0.21$ between ICU-ROX and non-ICU-ROX sites at each of the three time points, and to compare the change over time. Initial review of the data at the end of data collection showed there were insufficient hours when patients were ventilated with an $\mathrm{F}_{1} \mathrm{O}_{2}$ of 0.21 , the data were not continuous due to missing data, and that missingness could 
not be accounted for. Missing data are common in ICU studies, as data are collected only as recorded for clinical care. Although clinical data are routinely documented in ICU each hour on the ICU chart, data may be missing from the ICU chart for a number of reasons, including the patient being absent from ICU (for surgery or radiological procedures), or for lower acuity patients because hourly data were not deemed necessary. Because systematic bias could not be ruled out (i.e. that for a particular patient group, data were not recorded), proportions could not be used in the final analysis. For example, if one hour was missing from a total of 24 hours in one day, that is not the same as one hour missing from a total of five hours. The decision was made to analyse data by the 'count' of the hours spent at $\mathrm{F}_{1} \mathrm{O}_{2}$ of 0.21 (rather than percentage), and calculate an estimated percentage.

The data from patients at T1 (before ICU-ROX) could not be considered baseline for statistical analyses. The time patients spent at $\mathrm{F}_{1} \mathrm{O}_{2}$ of 0.21 for the period 'before ICU-ROX' was initially intended to provide baseline data about oxygen practices in ICU-ROX versus non-ICU-ROX sites. While it is possible to observe the time spent ventilating patients at 0.21 , the patients at T1 could not be considered 'baseline', as the same group of patients were not being assessed at T2 and T3. This issue was overcome by comparing data for patients within ICUROX sites over time, and patients within non-ICU-ROX sites over time.

Finally, the number of analyses proposed had to be reduced. Specifically, in addition to those outlined in section 7.3.3 (T3 vs T1, and T3 vs T2, for ICU-ROX and non-ICU-ROX sites), it was initially intended that comparisons of all outcome data $\left(\mathrm{F}_{1} \mathrm{O}_{2}\right.$ of 0.21 and $\mathrm{SpO}_{2} \geq 97 \%,<91 \%$ and $<88 \%$ ) would also be conducted between the periods 'before ICU-ROX' (T1), and 'after ICU-ROX was conducted' (T2). It was determined that so many analyses would increase the risk of a Type 1 error, due to multiple testing and insufficient degrees of freedom and the decision was made prior to analysis to reduce the number of analyses for each of the outcome data.

\subsubsection{Data analyses}

Data were analysed on an intention-to-treat basis without imputation of missing data. All analyses were performed using SAS version 9.4 (SAS Institute, Inc., Cary, NC) with a two-sided $P$ value of 0.05 used to indicate statistical significance. 
Patient characteristics, physiological descriptors, and process of care measures are presented as descriptive data and graphs. Categorical data are described by counts and percentages and continuous data by mean and standard deviation (SD), median and 25th and 75th percentiles as the inter-quartile range (IQR), and minimum $(\min )$ to maximum (max) as the range.

The primary analysis was comparison of the relative rate of hours spent at $\mathrm{F}_{1} \mathrm{O}_{2}$ of 0.21 per patient per hour for ICU-ROX sites by Poisson regression with an offset for observed time (hours). Over-dispersion was evaluated prior to analysis and a corrected analysis applied if necessary. A sensitivity analysis was also undertaken to include potential confounding variables (age, gender, illness severity (APACHE II score), ICU admission diagnosis of respiratory pathology, respiratory co-morbidity, ICU-ROX eligibility, suspected hypoxic ischaemic encephalopathy and emergency vs elective admission). Patients were nested in ICU site and site was treated as a random variable. Analyses were performed on one pre-defined pair (eligible vs not eligible for the ICU-ROX trial) for the primary outcome variable and secondary outcome variables in relation to $\mathrm{F}_{1} \mathrm{O}_{2}$. Analysis of the secondary outcome variables $\left(\mathrm{F}_{1} \mathrm{O}_{2}\right.$ of 0.21 for non-ICU-ROX sites, $\mathrm{SpO}_{2} \geq 97 \%, \mathrm{SpO}_{2}<91 \%$ and $\mathrm{SpO}_{2}<88 \%$ ) were likewise by Poisson regression with an offset for observed time (hours). If a significant difference in $\mathrm{F}_{1} \mathrm{O}_{2} 0.21$ had not been detected, $\mathrm{SpO}_{2}$ conditions would have been reported as descriptive statistics only.

Descriptive statistics were conducted for mean daily $\mathrm{F}_{1} \mathrm{O}_{2}$, mean lowest and mean highest $\mathrm{F}_{1} \mathrm{O}_{2}$ by day for ICU-ROX and non-ICU-ROX sites. The mean daily $\mathrm{F}_{1} \mathrm{O}_{2}$ was calculated from recordings of $\mathrm{F}_{1} \mathrm{O}_{2}$ taken six-hourly while the patient was invasively ventilated in the ICU up until day 10, for ICU-ROX sites and non-ICU-ROX sites at each time point (before ICU-ROX, after ICU-ROX and after ICU-ROX publication). The mean highest $\mathrm{F}_{1} \mathrm{O}_{2}$ and lowest $\mathrm{F}_{1} \mathrm{O}_{2}$ were calculated daily, using the highest $\mathrm{F}_{1} \mathrm{O}_{2}$ and lowest $\mathrm{F}_{1} \mathrm{O}_{2}$, respectively.

Descriptive analyses were conducted for mean daily $\mathrm{PaO}_{2}$, mean lowest and mean highest $\mathrm{PaO}_{2}$ by day for ICU-ROX and non-ICU-ROX sites. The mean daily $\mathrm{PaO}_{2}$ was calculated from recordings of $\mathrm{PaO}_{2}$ taken six-hourly while the patient is invasively ventilated in the ICU up until day 10, for ICU-ROX sites and non-ICU-ROX sites at each time point (before ICU-ROX, after 
ICU-ROX and after ICU-ROX publication). The mean highest $\mathrm{PaO}_{2}$ and lowest $\mathrm{PaO}_{2}$ were calculated daily, using the highest $\mathrm{PaO}_{2}$ and lowest $\mathrm{PaO}_{2}$, respectively.

\subsubsection{Validity strategies}

A number of strategies were employed throughout the study to ensure it was conducted in a valid and reliable way. These strategies were evident with design, data collection, data management and statistical analysis. These included clearly defined inclusion and exclusion criteria, consecutive ventilated patients to reduce selection bias (Polit \& Beck, 2017) and provision of a data dictionary with definitions of all data points, to ensure consistency of data collection.

Experienced RCs at each site were responsible for identification of patients, data collection and entry into a secure REDCAP database. Each RC had an individual log-in. RCs were provided with a paper case report form to collect data and a data dictionary with a clear definition for each field. The researcher was available by email to answer any questions RCs had with regard to eligibility and data collection. Site RCs kept a 'patient enrolment log' that linked the Study ID and identifying information about the patient (such as a national health index number). This log was kept at each site and the researcher did not have access to this information and all data in the study database was by identified by Study ID only. Study IDs were numbered sequentially by site, using a site-specific prefix; for example: SITE1-10 for the first time period, SITE11-20 for the second time period and SITE21-30 for the third time period.

All statistical analyses were undertaken by an impartial statistician under the direction of the researcher. A statistical analysis plan was documented and agreed prior to any analyses being undertaken. The statistician remained blinded to the exposure allocation until after all analyses had been conducted.

Data were reviewed for internal consistency at the end of each study period by the researcher, and again at the end of the study by the researcher and statistician. Data checks were determined prior to the study and assessed the following: completeness of data, extraneous values and internal validity. Queries arising from this internal consistency checking were addressed by the RCs at the study ICUs. Where data were considered missing or incomplete, $\mathrm{RCs}$ were asked to complete them. For some fields, such as $\mathrm{PaO}_{2}$, missing data 
were not queried, as they were only included if collected as part of routine care. With regards to extraneous values, these were reviewed for all clinical data, and any considered to be outside the normal range for ICU patients were queried. RCs were requested to either confirm the value or correct it. Internal consistency queries were issued when data were not possible (e.g.; if the highest value was lower than the lowest value). RCs were once again asked to correct the data accordingly.

For one site that withdrew from the study prior to resolution of data queries, a plan was written prior to final data review. For this site, two experienced impartial ICU researchers from the Medical Research Institute of NZ reviewed all data queries and if they could agree on a logical resolution, the data were changed accordingly. Any variable for which they could not reach consensus, was left as entered. Specifically, missing acute brain pathology data (based on admission diagnosis) was added, lowest and highest values that had been entered the wrong way around were corrected and where $\mathrm{F}_{1} \mathrm{O}_{2}$ hours had incorrectly been entered as ' $n / a$ ', these were changed to ' 0 ' hours. Data that were not able to be resolved, and were left as entered, were two instances of very high (but possible) $\mathrm{PaO}_{2}$ and one instance of low $\mathrm{PaO}_{2}$, and missing values could not be added. These were determined to have negligible effect on the overall quality of the data. 


\subsection{Results}

The characteristics for the participating ICUs were presented in Chapter 5 (Table 11).

\subsubsection{Patients}

From February 2017 to April 2020, 650 patients from 22 ICUs in Australia and NZ were enrolled in the inception cohort study. These enrolments were in three distinct periods: February 2017 to November 2017 (before ICU-ROX); June 2018 to November 2018 (After ICUROX but before results were known); and November 2019 to April 2020 (after publication of ICU-ROX results). Of these, 330 were from ICUs that participated in ICU-ROX and 320 from ICUs that did not participate (Figure 23). All participants were included in the final analysis; although two were ineligible, because they were mechanically ventilated for less than 12 hours.
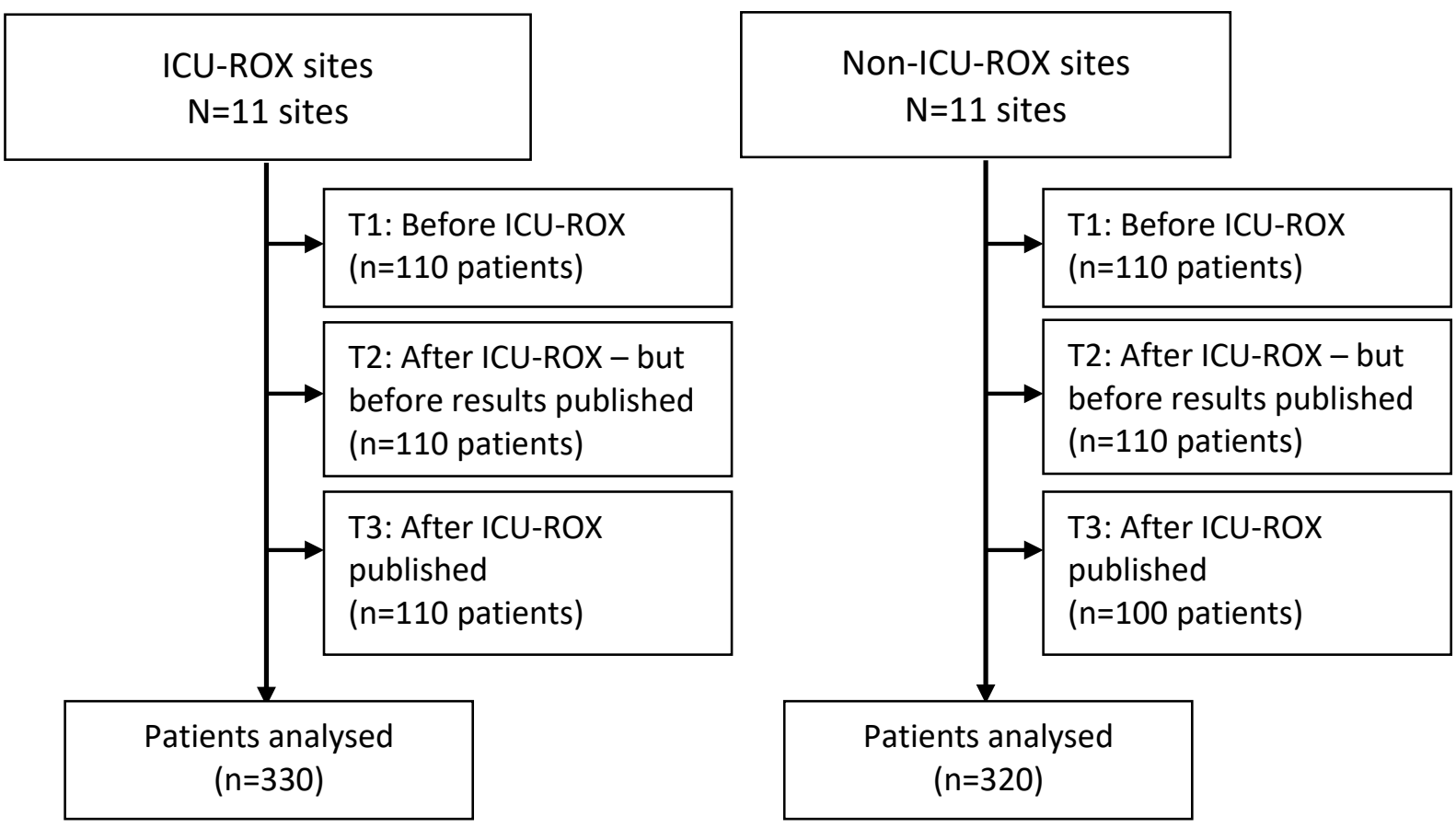

Figure 23 Flow diagram of patients

Abbreviations: ICU-ROX: Intensive Care Unit Randomised Trial Comparing Two Approaches to Oxygen therapy ICU-ROX sites: ICUs that participated in ICU-ROX; non-ICU-ROX sites: ICUs that did not participate in ICU-ROX; T1: before ICU-ROX started; T2: after ICU-ROX was completed but before results were known; T3: after publication of ICUROX results.

Comparisons of the characteristics of all patients who were enrolled showed that the ICUROX and non-ICU-ROX samples were similar (Table 32, Table 33). The exception is that a higher proportion of patients in the ICU-ROX sites were admitted from the operating theatre electively than from non-ICU-ROX sites $(19.7 \%$ and $12.8 \%$, respectively, $\mathrm{P}=0.02)$ and conversely, a higher proportion of patients from non-ICU-ROX sites were admitted 
emergently from the operating theatre (25.6\%) compared to $16.1 \%$ of ICU-ROX site patients $(P=0.003)$. In addition, there was a statistically significant difference in ethnicity between ICUROX and non-ICU-ROX site patients for European ( $54.8 \%$ and $74.4 \%$, respectively, $\mathrm{P}=0.002$ ) and Pacific Peoples (13.7\% and 1.1\%, respectively, $P=0.001$ ). Māori represented $20.2 \%$ of patients for whom ethnicity was collected.

Table 32 Characteristics of patients

\begin{tabular}{|c|c|c|c|}
\hline Characteristic & $\begin{array}{l}\text { ICU-ROX } \\
\text { patients } \\
\mathrm{N}=330^{*}\end{array}$ & $\begin{array}{c}\text { Non-ICU-ROX } \\
\text { patients } \\
\mathrm{N}=320^{*}\end{array}$ & $P$ value \\
\hline Male, sex, n (\%) & $220(66.7)$ & $216(67.5)$ & 0.82 \\
\hline Age, mean, SD & $57.6(16.5)$ & $59.7(20.1)$ & 0.12 \\
\hline APACHE II score, mean, SD & $19.4(8.4)$ & $20.1(8)$ & 0.27 \\
\hline Ethnicity, n (\%) & $n=168$ & $\mathrm{n}=90$ & 0.006 \\
\hline \begin{tabular}{l|l} 
& European \\
\end{tabular} & $92(54.8)$ & $67(74.4)$ & 0.002 \\
\hline Māori & $36(21.4)$ & $16(17.8)$ & 0.49 \\
\hline Pacific Peoples & $23(13.7)$ & $1(1.1)$ & 0.001 \\
\hline Asian & $13(7.7)$ & $4(4.4)$ & 0.31 \\
\hline Middle Eastern/ Latin American/ African & $2(1.2)$ & $0(0)$ & 0.30 \\
\hline Other & $2(1.2)$ & $2(2.2)$ & 0.52 \\
\hline \multicolumn{4}{|l|}{ Chronic co-morbid conditions, n (\%) } \\
\hline \begin{tabular}{l|l} 
& Respiratory \\
\end{tabular} & $11(3.3)$ & $18(5.6)$ & 0.16 \\
\hline Cardiovascular & $6(1.8)$ & $13(4.1)$ & 0.09 \\
\hline Hepatic & $7(2.1)$ & $15(4.7)$ & 0.07 \\
\hline Renal & $6(1.8)$ & $13(4.1)$ & 0.09 \\
\hline Immunosuppression by disease & $8(2.4)$ & $14(4.4)$ & 0.17 \\
\hline Immunosuppression by therapy & $12(3.6)$ & $21(6.6)$ & 0.09 \\
\hline Metastatic cancer & $6(1.8)$ & $13(4.1)$ & 0.09 \\
\hline \multicolumn{4}{|l|}{ Source of admission, $\mathrm{n}(\%)$} \\
\hline Emergency Department & $120(36.4)$ & $114(35.6)$ & 0.84 \\
\hline Hospital Floor & $45(13.6)$ & $47(14.7)$ & 0.70 \\
\hline Other ICU & $9(2.7)$ & $12(3.8)$ & 0.46 \\
\hline Other Hospital & $38(11.5)$ & $24(7.5)$ & 0.08 \\
\hline $\begin{array}{l}\text { Operating Theatre following EMERGENCY } \\
\text { surgery }\end{array}$ & $53(16.1)$ & $82(25.6)$ & 0.003 \\
\hline \begin{tabular}{l|l} 
& $\begin{array}{l}\text { Operating Theatre following ELECTIVE } \\
\text { surgery }\end{array}$ \\
\end{tabular} & $65(19.7)$ & $41(12.8)$ & 0.02 \\
\hline Eligible for ICU-ROX trial ${ }^{\dagger}, \mathrm{n}(\%)$ & $189(57.3)$ & $194(60.6)$ & 0.39 \\
\hline
\end{tabular}

*unless otherwise stated

+ To meet eligibility for the ICU-ROX trial, patients had to meet the inclusion criteria of adult, ventilated in and ICU and anticipated to remain ventilated until beyond the next calendar day. In addition, they had to have no exclusion criteria except for 'not enrolled within 2 hours'.

Abbreviations: APACHE II score: Acute Physiology and Chronic Health Evaluation score; ICU: Intensive Care Unit; ICU-ROX patients: patients from ICUs that participated in the ICU-ROX trial; ICU-ROX trial: Intensive Care Unit Randomised Trial Comparing Two Approaches to Oxygen therapy; $\mathrm{n}(\%)$ : number and percentage; non-ICU-ROX patients: patients from ICUs that did not participate in the ICU-ROX trial; SD: standard deviation. 
Table 33 Additional baseline characteristics of patients

\begin{tabular}{|c|c|c|c|}
\hline Characteristic & $\begin{array}{c}\text { ICU-ROX } \\
\text { patients } \\
\mathrm{N}=330\end{array}$ & $\begin{array}{c}\text { Non-ICU-ROX } \\
\text { patients } \\
\mathrm{N}=320\end{array}$ & $P$ value \\
\hline Acute brain pathology at baseline, $\mathrm{n}(\%)$ & & & 0.51 \\
\hline \begin{tabular}{l|l} 
& No acute brain pathology \\
\end{tabular} & $242(73.3)$ & $239(74.7)$ & \\
\hline Acute TBI & $18(5.5)$ & $20(6.3)$ & \\
\hline CNS infection & $6(1.8)$ & $5(1.6)$ & \\
\hline Haemorrhagic stroke & $13(3.9)$ & $12(3.8)$ & \\
\hline Ischaemic stroke & $5(1.5)$ & $4(1.3)$ & \\
\hline Subarachnoid haemorrhage & $7(2.1)$ & $1(0.3)$ & \\
\hline Hypoxic ischaemic encephalopathy & $35(10.6)$ & $31(9.7)$ & \\
\hline Other & $4(1.2)$ & $8(2.5)$ & \\
\hline Operative admission diagnoses, $\mathrm{n}(\%)$ & & & 0.15 \\
\hline \begin{tabular}{l|l} 
& Cardiovascular \\
\end{tabular} & $50(15.2)$ & $35(10.9)$ & \\
\hline Gastrointestinal & $21(6.4)$ & $34(10.6)$ & \\
\hline Gynaecological & $0(0.0)$ & $2(0.6)$ & \\
\hline Haematological & $0(0.0)$ & $1(0.3)$ & \\
\hline Metabolic & $0(0.0)$ & $2(0.6)$ & \\
\hline Musculoskeletal/Skin & $5(1.5)$ & $4(1.3)$ & \\
\hline Neurological & $8(2.4)$ & $16(5)$ & \\
\hline Renal & $2(0.6)$ & $2(0.6)$ & \\
\hline Respiratory & $17(5.2)$ & $13(4.1)$ & \\
\hline Trauma & $15(4.5)$ & $13(4.1)$ & \\
\hline Non-operative admission diagnoses, $\mathrm{n}(\%)$ & & & 0.18 \\
\hline \begin{tabular}{l|l|} 
& Cardiovascular \\
\end{tabular} & $42(12.7)$ & $37(11.6)$ & \\
\hline Gastrointestinal & $9(2.7)$ & $13(4.1)$ & \\
\hline Haematological & $0(0.0)$ & $5(1.6)$ & \\
\hline Metabolic & $12(3.6)$ & $21(6.6)$ & \\
\hline Musculoskeletal/skin & $2(0.6)$ & $1(0.3)$ & \\
\hline Neurological & $39(11.8)$ & $31(9.7)$ & \\
\hline Renal & $0(0.0)$ & $3(0.9)$ & \\
\hline Respiratory & $56(17)$ & $46(14.4)$ & \\
\hline Sepsis & $24(7.3)$ & $19(5.9)$ & \\
\hline Trauma & $27(8.2)$ & $21(6.6)$ & \\
\hline Other & $1(0.3)$ & $1(0.3)$ & \\
\hline
\end{tabular}

Abbreviations: CNS: Central nervous system; ICU: Intensive Care Unit; ICU-ROX patients: patients from ICUs that participated in the ICU-ROX trial; ICU-ROX trial: Intensive Care Unit Randomised Trial Comparing Two Approaches to Oxygen therapy; $\mathrm{n}(\%)$ : number and percentage; non-ICU-ROX patients: patients from ICUs that did not participate in the ICU-ROX trial; SD: standard deviation; TBI: traumatic brain injury.

\subsubsection{Oxygenation and process of care}

\subsubsection{1 $\mathrm{F}_{1} \mathrm{O}_{2}$ of 0.21}

Before ICU-ROX began, patients in the ICU-ROX sites spent more time receiving an $\mathrm{F}_{1} \mathrm{O}_{2}$ of 0.21 while ventilated (mean 6 hours, standard deviation [SD] 20.5) than those from the non-ICUROX sites (mean 0.8 hours, SD 5.4) (Table 34). After ICU-ROX enrolment was completed, but 
before the results were known, ICU-ROX site patients continued to spend more time ventilated at $\mathrm{F}_{1} \mathrm{O}_{2}$ of 0.21 , than those from non-ICU-ROX sites (mean 6.9 hours, SD 23.3 and 2.2 hours, SD 11.3, respectively). After publication of the ICU-ROX results, patients from ICUROX sites, spent a mean of 5 hours (SD 11.3) receiving $\mathrm{F}_{1} \mathrm{O}_{2} 0.21$ compared to their non-ICUROX site counterparts (mean 2.3 hours, SD 8.4). The estimated percentage of hours ventilated at an $\mathrm{F}_{1} \mathrm{O}_{2}$ of 0.21 decreased over time in the ICU-ROX site patients, and increased over time in the non-ICU-ROX site patients (Table 35).

Table 34 Descriptive data for $\mathrm{F}_{1} \mathrm{O}_{2}$ of 0.21 hours while ventilated in ICU

\begin{tabular}{|c|c|c|c|c|}
\hline Variable & Mean (SD) & Median (IQR) & $\begin{array}{l}\text { Minimum to } \\
\text { Maximum }\end{array}$ & Sum \\
\hline \multicolumn{5}{|l|}{ Before ICU-ROX (T1) } \\
\hline \multicolumn{5}{|l|}{ ICU-ROX sites $(n=110)$} \\
\hline Number of hours $\mathrm{F}_{1} \mathrm{O}_{2}$ is 0.21 & $6(20.5)$ & 0 (0 to 0$)$ & 0 to 143 & 656 \\
\hline Observed time (hours) & $71.4(66.1)$ & 44.5 (23 to 107$)$ & 7 to 254 & 7852 \\
\hline \multicolumn{5}{|l|}{ Non-ICU-ROX sites $(n=110)$} \\
\hline Number of hours $\mathrm{F}_{1} \mathrm{O}_{2}$ is 0.21 & $0.8(5.4)$ & 0 (0 to 0$)$ & 0 to 53 & 85 \\
\hline Observed time (hours) & $86.2(75.6)$ & $60(20$ to 137$)$ & 3 to 254 & 9478 \\
\hline \multicolumn{5}{|c|}{ After ICU-ROX but before results known (T2) } \\
\hline \multicolumn{5}{|l|}{ ICU-ROX sites $(n=110)$} \\
\hline Number of hours $\mathrm{F}_{1} \mathrm{O}_{2}$ is 0.21 & $6.9(23.3)$ & 0 (0 to 0$)$ & 0 to 157 & 762 \\
\hline Observed time (hours) & $90.3(74.1)$ & 66 (33 to 134$)$ & 6 to 261 & 9928 \\
\hline \multicolumn{5}{|l|}{ Non-ICU-ROX sites $(n=110)$} \\
\hline Number of hours $\mathrm{F}_{1} \mathrm{O}_{2}$ is 0.21 & $2.2(11.3)$ & $0(0$ to 0$)$ & 0 to 103 & 245 \\
\hline Observed time (hours) & $92.2(78.1)$ & 64.5 (29 to 144$)$ & 7 to 263 & 10137 \\
\hline \multicolumn{5}{|l|}{ After ICU-ROX publication (T3) } \\
\hline \multicolumn{5}{|l|}{ ICU-ROX sites $(n=110)$} \\
\hline Number of hours $\mathrm{F}_{1} \mathrm{O}_{2}$ is 0.21 & $5(11.3)$ & 0 (0 to 2$)$ & 0 to 58 & 548 \\
\hline Observed time (hours) & $70.6(66.6)$ & 40.5 (20 to 96$)$ & 6 to 259 & 7765 \\
\hline \multicolumn{5}{|l|}{ Non-ICU-ROX sites $(n=100)$} \\
\hline Number of hours $\mathrm{F}_{1} \mathrm{O}_{2}$ is 0.21 & $2.3(8.4)$ & $0(0$ to 0$)$ & 0 to 52 & 233 \\
\hline Observed time (hours) & $71.7(71.8)$ & 37 (19 to 95.5$)$ & 11 to 262 & 7165 \\
\hline
\end{tabular}

Abbreviations: $\mathrm{F}_{1} \mathrm{O}_{2}$ : fraction of inspired oxygen; ICU-ROX sites: patients from ICUs that participated in the ICUROX trial; IQR: interquartile range; non-ICU-ROX sites: patients from ICUs that did not participate in the ICUROX trial; SD: standard deviation; T1: before ICU-ROX started; T2: after ICU-ROX completed but before results were known; T3: after publication of ICU-ROX results. 
Table 35 Primary and secondary outcome measures: percentage of time $\mathrm{F}_{1} \mathrm{O}_{2}$ is 0.21 while ventilated in ICU

\begin{tabular}{|c|c|c|c|}
\hline Time period & $\begin{array}{c}\text { Percentage of time } \\
\mathrm{F}_{1} \mathrm{O}_{2} \text { is } 0.21 \\
(95 \% \mathrm{Cl})\end{array}$ & $\begin{array}{c}\text { Relative rate } \mathrm{F}_{1} \mathrm{O}_{2} \text { is } 0.21 \\
(95 \% \mathrm{Cl})\end{array}$ & $P$ value \\
\hline \multicolumn{4}{|l|}{ ICU-ROX sites } \\
\hline \multirow[t]{2}{*}{ T1: Before ICU-ROX } & $8.2 \%(6.2$ to $11 \%)$ & & \\
\hline & & T1 to T3: 0.85 (0.54 to 1.30$)$ & $0.45^{*}$ \\
\hline \multirow[t]{2}{*}{ T2: After ICU-ROX } & $7.7 \%$ (5.8 to $10 \%)$ & & \\
\hline & & T2 to T3: 0.92 (0.60 to 1.41$)$ & 0.70 \\
\hline T3: After ICU-ROX publication & $7.1 \%(5.1$ to $10 \%)$ & & \\
\hline \multicolumn{4}{|l|}{ Non-ICU-ROX sites } \\
\hline \multirow[t]{2}{*}{ T1: Before ICU-ROX } & $0.9 \%$ (0.4 to $2.0 \%)$ & & \\
\hline & & T1 to T3: 3.63 (1.39 to 9.47 ) & 0.01 \\
\hline \multirow[t]{2}{*}{ T2: After ICU-ROX } & $2.4 \%$ (1.5 to $3.9 \%)$ & & \\
\hline & & T2 to T3: 1.35 (0.67 to 2.69 ) & 0.40 \\
\hline T3: After ICU-ROX publication & $3.3 \%(2.0$ to $5.3 \%)$ & & \\
\hline
\end{tabular}

* Primary outcome measure

Abbreviations: $\mathrm{Cl}$ : confidence interval; $\mathrm{F}_{1} \mathrm{O}_{2}$ : fraction of inspired oxygen; ICU-ROX: Intensive Care Unit Randomised Trial Comparing Two Approaches to Oxygen therapy; ICU-ROX sites: ICUs that participated in ICU-ROX; non-ICU-ROX sites: ICUs that did not participate in ICU-ROX; T1: before ICU-ROX started; T2: after ICU-ROX completed but before results were known; T3: after publication of ICU-ROX results.

\subsubsection{2 $\mathrm{SpO}_{2} \geq 97 \%$}

Patients from ICU-ROX sites spent less time ventilated with an $\mathrm{SpO}_{2} \geq 97 \%$, (mean 44.4 hours, SD 46) than non-ICU-ROX site patients (mean 56.0 hours, SD 58.3) before ICU-ROX (Table 36). The time spent ventilated at $\mathrm{SpO}_{2} \geq 97 \%$ was similar after ICU-ROX enrolment was completed compared to before ICU-ROX for both ICU-ROX and non-ICU-ROX site patients. After publication of the ICU-ROX results, time spent ventilated with an $\mathrm{SpO}_{2} \geq 97 \%$ decreased in both ICU-ROX (mean 33.8 hours, SD 38.6) and non-ICU-ROX cohorts (mean 39.7 hours, SD 43.3). Similarly, the estimated percentage of hours at an $\mathrm{SpO}_{2} \geq 97 \%$ decreased over time in patients from both ICU-ROX and non-ICU-ROX sites (Table 37). 
Table 36 Descriptive data for $\mathrm{SpO}_{2} \geq 97 \%$ hours while ventilated in ICU

\begin{tabular}{|c|c|c|c|c|}
\hline Variable & Mean (SD) & Median (IQR) & $\begin{array}{c}\text { Minimum to } \\
\text { Maximum }\end{array}$ & Sum \\
\hline \multicolumn{5}{|l|}{ Before ICU-ROX (T1) } \\
\hline \multicolumn{5}{|l|}{ ICU-ROX sites $(n=110)$} \\
\hline Number of hours $\mathrm{SpO}_{2} \geq 97 \%$ & $44.5(46)$ & 23.5 (13 to 58$)$ & 0 to 214 & 4897 \\
\hline Observed time (hours) & $70.6(65.5)$ & 44.5 (23 to 107$)$ & 9 to 254 & 7768 \\
\hline \multicolumn{5}{|l|}{ Non-ICU-ROX sites ( $n=110)$} \\
\hline Number of hours $\mathrm{SpO}_{2} \geq 97 \%$ & $56(58.3)$ & 33 (14 to 78$)$ & 0 to 242 & 6164 \\
\hline Observed time (hours) & $86(75)$ & 57.5 (21 to 135$)$ & 3 to 254 & 9455 \\
\hline \multicolumn{5}{|c|}{ After ICU-ROX but before results known (T2) } \\
\hline \multicolumn{5}{|l|}{ ICU-ROX sites $(n=110)$} \\
\hline Number of hours $\mathrm{SpO}_{2} \geq 97 \%$ & $44.3(49.2)$ & 27 (13 to 60$)$ & 0 to 238 & 4870 \\
\hline Observed time (hours) & $90.3(74.3)$ & 65 (31 to 130$)$ & 6 to 261 & 9932 \\
\hline \multicolumn{5}{|l|}{ Non-ICU-ROX sites $(n=110)$} \\
\hline Number of hours $\mathrm{SpO}_{2} \geq 97 \%$ & $56.5(57.5)$ & 36.5 (15 to 73 ) & 2 to 245 & 6219 \\
\hline Observed time (hours) & $92.3(78.1)$ & 66 (28 to 147$)$ & 9 to 263 & 10151 \\
\hline \multicolumn{5}{|l|}{ After ICU-ROX publication (T3) } \\
\hline \multicolumn{5}{|l|}{ ICU-ROX sites $(n=110)$} \\
\hline Number of hours $\mathrm{SpO}_{2} \geq 97 \%$ & $33.8(38.6)$ & 19.5 (12 to 45$)$ & 0 to 224 & 3713 \\
\hline Observed time (hours) & $70.3(66.9)$ & 40.5 (20 to 96$)$ & 6 to 253 & 7736 \\
\hline \multicolumn{5}{|l|}{ Non-ICU-ROX sites $(n=100)$} \\
\hline Number of hours $\mathrm{SpO}_{2} \geq 97 \%$ & $39.7(43.3)$ & 20 (11 to 61$)$ & 0 to 215 & 3967 \\
\hline Observed time (hours) & $71.3(71.6)$ & 38.5 (19 to 95$)$ & 10 to 262 & 7134 \\
\hline
\end{tabular}

Abbreviations: ICU-ROX: Intensive Care Unit Randomised Trial Comparing Two Approaches to Oxygen therapy ICU-ROX sites: patients from ICUs that participated in the ICU-ROX trial; IQR: interquartile range; non-ICU-ROX sites: patients from ICUs that did not participate in the ICU-ROX trial; $\mathrm{SpO}_{2}$ : peripheral saturation of oxygen; SD: standard deviation; T1: before ICU-ROX started; T2: after ICU-ROX completed but before results were known; T3: after publication of ICU-ROX results. 
Table 37 Percentage of time $\mathrm{SpO}_{2} \geq 97 \%$ while ventilated in ICU

\begin{tabular}{|c|c|c|c|}
\hline Time period & $\begin{array}{l}\text { Percentage of time } \\
\mathrm{SpO}_{2} \geq 97 \%(95 \% \mathrm{Cl})\end{array}$ & $\begin{array}{c}\text { Relative rate } \mathrm{SpO}_{2} \geq 97 \% \\
(95 \% \mathrm{Cl})\end{array}$ & $P$ value \\
\hline \multicolumn{4}{|l|}{ ICU-ROX sites } \\
\hline \multirow[t]{2}{*}{ T1: Before ICU-ROX } & $63.0 \%$ (56.4 to $70.4 \%$ ) & & \\
\hline & & T1 to T3: np & $\mathrm{np}$ \\
\hline \multirow[t]{2}{*}{ T2: After ICU-ROX } & $49.0 \%$ (43.9 to $54.8 \%$ ) & & \\
\hline & & T2 to T3: 0.98 (0.82 to 1.16$)$ & 0.80 \\
\hline T3: After ICU-ROX publication & $48.0 \%$ (42.3 to $54.5 \%)$ & & \\
\hline \multicolumn{4}{|l|}{ Non-ICU-ROX sites } \\
\hline \multirow[t]{2}{*}{ T1: Before ICU-ROX } & $65.2 \%$ (59.1 to $72.0 \%$ ) & & \\
\hline & & T1 to T3: np & $\mathrm{np}$ \\
\hline \multirow[t]{2}{*}{ T2: After ICU-ROX } & $61.3 \%$ (55.5 to $67.6 \%)$ & & \\
\hline & & T2 to T3: 0.91 (0.78 to 1.06$)$ & 0.23 \\
\hline T3: After ICU-ROX publication & $55.6 \%$ (49.2 to $62.9 \%)$ & & \\
\hline
\end{tabular}

Abbreviations: Cl: confidence interval; ICU-ROX sites: ICUs that participated in ICU-ROX; np: statistical analysis was not possible; non-ICU-ROX sites: ICUs that did not participate in ICU-ROX; T1: before ICU-ROX started; T2: after ICU-ROX completed but before results were known; T3: after publication of ICU-ROX results.

\subsubsection{3 $\mathrm{SpO}_{2}<91 \%$}

Before ICU-ROX started, ICU-ROX site patients spent a mean of 1.7 hours (SD 4.5) at $\mathrm{SpO}_{2}<$ 91\%, compared to non-ICU-ROX patients with a mean of 1.8 hours (SD 5.6) (Table 38). After ICU-ROX enrolment was completed but before the results were known, the mean time increased in both the ICU-ROX and non-ICU-ROX cohort to 4.1 hours (SD 10.4) and 2.8 hours (SD 13.0), respectively. After the ICU-ROX results had been published, ICU-ROX site patients spent a mean of 2.2 hours (SD 7.9) compared to 3.4 hours (SD 10.7) for their non-ICU-ROX sites counterparts. The estimated percentage of hours spent at an $\mathrm{SpO}_{2}<91 \%$ increased over time in the non-ICU-ROX site cohort and decreased for ICU-ROX site patients (Table 39). 
Table 38 Descriptive data for $\mathrm{SpO}_{2}<91 \%$ hours while ventilated in ICU

\begin{tabular}{|c|c|c|c|c|}
\hline Variable & Mean (SD) & Median (IQR) & $\begin{array}{c}\text { Minimum to } \\
\text { Maximum }\end{array}$ & Sum \\
\hline \multicolumn{5}{|l|}{ Before ICU-ROX (T1) } \\
\hline \multicolumn{5}{|l|}{ ICU-ROX sites $(n=110)$} \\
\hline Number of hours $\mathrm{SpO}_{2}<91 \%$ & $1.7(4.5)$ & $0(0$ to 1$)$ & 0 to 26 & 186 \\
\hline Observed time (hours) & $70.6(65.5)$ & 44.5 (23 to 107$)$ & 9 to 254 & 7768 \\
\hline \multicolumn{5}{|l|}{ Non-ICU-ROX sites $(n=110)$} \\
\hline Number of hours $\mathrm{SpO}_{2}<91 \%$ & $1.8(5.6)$ & $0(0$ to 1$)$ & 0 to 51 & 196 \\
\hline Observed time (hours) & $86(75)$ & 57.5 (21 to 135$)$ & 3 to 254 & 9455 \\
\hline \multicolumn{5}{|c|}{ After ICU-ROX but before results known (T2) } \\
\hline \multicolumn{5}{|l|}{ ICU-ROX sites $(n=110)$} \\
\hline Number of hours $\mathrm{SpO}_{2}<91 \%$ & $4.1(10.4)$ & $0(0$ to 2$)$ & 0 to 70 & 452 \\
\hline Observed time (hours) & $90.3(74.3)$ & 65 (31 to 130$)$ & 6 to 261 & 9932 \\
\hline \multicolumn{5}{|l|}{ Non-ICU-ROX sites $(n=110)$} \\
\hline Number of hours $\mathrm{SpO}_{2}<91 \%$ & $2.8(13)$ & $0(0$ to 1$)$ & 0 to 119 & 307 \\
\hline Observed time (hours) & $92.3(78.1)$ & 66 (28 to 147$)$ & 9 to 263 & 10151 \\
\hline \multicolumn{5}{|l|}{ After ICU-ROX publication (T3) } \\
\hline \multicolumn{5}{|l|}{ ICU-ROX sites $(n=110)$} \\
\hline Number of hours $\mathrm{SpO}_{2}<91 \%$ & $2.2(7.9)$ & 0 (0 to 1$)$ & 0 to 64 & 238 \\
\hline Observed time (hours) & $70.3(66.9)$ & 40.5 (20 to 96$)$ & 6 to 253 & 7736 \\
\hline \multicolumn{5}{|l|}{ Non-ICU-ROX sites $(n=100)$} \\
\hline Number of hours $\mathrm{SpO}_{2}<91 \%$ & $3.4(10.7)$ & $0(0$ to 1$)$ & 0 to 70 & 344 \\
\hline Observed time (hours) & $71.3(71.6)$ & 38.5 (19 to 95$)$ & 10 to 262 & 7134 \\
\hline
\end{tabular}

Abbreviations: ICU-ROX: Intensive Care Unit Randomised Trial Comparing Two Approaches to Oxygen therapy; ICU-ROX sites: patients from ICUs that participated in the ICU-ROX trial; IQR: interquartile range; non-ICU-ROX sites: patients from ICUs that did not participate in the ICU-ROX trial; SD: standard deviation; $\mathrm{SpO}_{2}$ : peripheral saturation of oxygen; T1: before ICU-ROX started; T2: after ICU-ROX completed but before results were known; T3: after publication of ICU-ROX results. 
Table 39 Percentage of time $\mathrm{SpO}_{2}<91 \%$ while ventilated in ICU

\begin{tabular}{|c|c|c|c|}
\hline Time period & $\begin{array}{l}\text { Percentage of time } \\
\mathrm{SpO}_{2}<91 \%(95 \% \mathrm{Cl})\end{array}$ & $\begin{array}{c}\text { Relative rate } \mathrm{SpO}_{2}<91 \% \\
(95 \% \mathrm{Cl})\end{array}$ & $P$ value \\
\hline \multicolumn{4}{|l|}{ ICU-ROX sites } \\
\hline \multirow[t]{2}{*}{ T1: Before ICU-ROX } & $2.4 \%$ (1.6 to $3.6 \%)$ & & \\
\hline & & T1 to T3: 1.28 (0.76 to 2.18$)$ & 0.35 \\
\hline \multirow[t]{2}{*}{ T2: After ICU-ROX } & $4.6 \%$ (3.5 to $5.9 \%)$ & & \\
\hline & & T2 to T3: 0.68 (0.44 to 1.04$)$ & 0.08 \\
\hline T3: After ICU-ROX publication & $3.1 \%(2.2$ to $4.4 \%)$ & & \\
\hline \multicolumn{4}{|l|}{ Non-ICU-ROX sites } \\
\hline \multirow[t]{2}{*}{ T1: Before ICU-ROX } & $2.1 \%$ (1.4 to $3.1 \%)$ & & \\
\hline & & T1 to T3: 2.33 (1.43 to 3.78 ) & $<0.001$ \\
\hline \multirow[t]{2}{*}{ T2: After ICU-ROX } & $3.0 \%(2.2$ to $4.1 \%)$ & & \\
\hline & & T2 to T3: 1.59 (1.04 to 2.44$)$ & 0.03 \\
\hline T3: After ICU-ROX publication & $4.8 \%$ (3.6 to $6.5 \%)$ & & \\
\hline
\end{tabular}

Abbreviations: Cl: confidence interval; ICU-ROX trial: Intensive Care Unit Randomised Trial Comparing Two Approaches to Oxygen therapy; ICU-ROX sites: ICUs that participated in ICU-ROX; non-ICU-ROX sites: ICUs that did not participate in ICU-ROX; SpO 2: peripheral oxygen saturations; T1: before ICU-ROX started; T2: after ICU-ROX completed but before results were known; T3: after publication of ICU-ROX results.

\subsubsection{4 $\mathrm{SpO}_{2}<88 \%$}

Patients from both ICU-ROX and non-ICU-ROX sites spent a very small amount of time ventilated with an $\mathrm{SpO}_{2}<88 \%$ (Table 40). Before ICU-ROX, the mean time was 0.5 hour (SD 2.2) and 0.4 hour (SD 1.0), respectively. After ICU-ROX enrolment was complete, the mean time at $\mathrm{SpO}_{2}<88 \%$ for ICU-ROX site patients was 1 hour (SD 2.3) and non-ICU-ROX patients 1 hour (SD 4). After publication of the ICU-ROX results, the time spent at an $\mathrm{SpO}_{2}<88 \%$ remained similar and low in both ICU-ROX (mean 0.6 hour [SD 2.7]) and non-ICU-ROX patients (mean 1.1 hour [SD 3.4]). The estimated percentage of time spent at $\mathrm{SpO}_{2}<88 \%$ was low in both ICU-ROX and non-ICU-ROX site patients across all time periods (Table 41). 
Table 40 Descriptive data for $\mathrm{SpO}_{2}<88 \%$ hours while ventilated in ICU

\begin{tabular}{|c|c|c|c|c|}
\hline Variable & Mean (SD) & Median (IQR) & $\begin{array}{c}\text { Minimum to } \\
\text { Maximum }\end{array}$ & Sum \\
\hline \multicolumn{5}{|l|}{ Before ICU-ROX (T1) } \\
\hline \multicolumn{5}{|l|}{ ICU-ROX sites $(n=110)$} \\
\hline Number of hours $\mathrm{SpO}_{2}<88 \%$ & $0.5(2.2)$ & $0(0$ to 0$)$ & 0 to 19 & 60 \\
\hline Observed time (hours) & $70.6(65.5)$ & 44.5 (23 to 107$)$ & 9 to 254 & 7768 \\
\hline \multicolumn{5}{|l|}{ Non-ICU-ROX sites $(n=110)$} \\
\hline Number of hours $\mathrm{SpO}_{2}<88 \%$ & $0.4(1.0)$ & $0(0$ to 0$)$ & 0 to 7 & 42 \\
\hline Observed time (hours) & $86(75)$ & 57.5 (21 to 135$)$ & 3 to 254 & 9455 \\
\hline \multicolumn{5}{|c|}{ After ICU-ROX but before results known (T2) } \\
\hline \multicolumn{5}{|l|}{ ICU-ROX sites $(n=110)$} \\
\hline Number of hours $\mathrm{SpO}_{2}<88 \%$ & $1(2.3)$ & $0(0$ to 1$)$ & 0 to 14 & 108 \\
\hline Observed time (hours) & $90.3(74.3)$ & 65 (31 to 130$)$ & 6 to 261 & 9932 \\
\hline \multicolumn{5}{|l|}{ Non-ICU-ROX sites ( $n=110)$} \\
\hline Number of hours $\mathrm{SpO}_{2}<88 \%$ & $1(4)$ & $0(0$ to 0$)$ & 0 to 29 & 105 \\
\hline Observed time (hours) & $92.3(78.1)$ & 66 (28 to 147$)$ & 9 to 263 & 10151 \\
\hline \multicolumn{5}{|l|}{ After ICU-ROX publication (T3) } \\
\hline \multicolumn{5}{|l|}{ ICU-ROX sites ( $n=110)$} \\
\hline Number of hours $\mathrm{SpO}_{2}<88 \%$ & $0.6(2.7)$ & 0 (0 to 0$)$ & 0 to 26 & 63 \\
\hline Observed time (hours) & $70.3(66.9)$ & 40.5 (20 to 96$)$ & 6 to 253 & 7736 \\
\hline \multicolumn{5}{|l|}{ Non-ICU-ROX sites ( $n=100)$} \\
\hline Number of hours $\mathrm{SpO}_{2}<88 \%$ & $1.1(3.4)$ & $0(0$ to 0$)$ & 0 to 19 & 107 \\
\hline Observed time (hours) & $71.3(71.6)$ & 38.5 (19 to 95) & 10 to 262 & 7134 \\
\hline
\end{tabular}

Abbreviations: Cl: confidence interval; ICU-ROX sites: patients from ICUs that participated in the ICU-ROX trial; IQR: interquartile range; non-ICU-ROX sites: patients from ICUs that did not participate in the ICU-ROX trial; SD: standard deviation; $\mathrm{SpO}_{2}$ : peripheral saturation of oxygen; T1: before ICU-ROX started; T2: after ICU-ROX completed but before results were known; T3: after publication of ICU-ROX results.

\subsubsection{Mean, lowest and highest daily $\mathrm{F}_{1} \mathrm{O}_{2}$}

The mean, highest and lowest $\mathrm{F}_{1} \mathrm{O}_{2}$ for both ICU-ROX and non-ICU-ROX sites appeared very similar across all time points (Figure 24). For patients from ICU-ROX sites, the lowest $\mathrm{F}_{1} \mathrm{O}_{2}$ on Day 0 was lower after ICU-ROX was published with a mean $\mathrm{F}_{1} \mathrm{O}_{2}$ of 0.42 , compared to 0.46 before ICU-ROX and 0.45 after ICU-ROX participation. No other differences were apparent for either ICU-ROX or non-ICU-ROX site patients. There were few observations for days 7- 10, accounting for the wide dispersion of data at days 7 to 10 . 
Table 41 Percentage of time $\mathrm{SpO}_{2}<88 \%$ while ventilated in ICU

\begin{tabular}{|c|c|c|c|}
\hline Time period & $\begin{array}{l}\text { Percentage of time } \\
\mathrm{SpO}_{2}<88 \%(95 \% \mathrm{Cl})\end{array}$ & $\begin{array}{c}\text { Relative rate } \mathrm{SpO}_{2}<88 \% \\
(95 \% \mathrm{Cl})\end{array}$ & $P$ value \\
\hline \multicolumn{4}{|l|}{ ICU-ROX sites } \\
\hline \multirow[t]{2}{*}{ T1: Before ICU-ROX } & $0.7 \%(0.5$ to $1.2 \%)$ & & \\
\hline & & T1 to T3: 1.05 (0.59 to 1.89$)$ & 0.86 \\
\hline \multirow[t]{2}{*}{ T2: After ICU-ROX } & $1.1 \%$ (0.8 to $1.5 \%)$ & & \\
\hline & & T2 to T3: 0.75 (0.45 to 1.25$)$ & 0.27 \\
\hline T3: After ICU-ROX publication & $0.8 \%(0.5$ to $1.2 \%)$ & & \\
\hline \multicolumn{4}{|l|}{ Non-ICU-ROX sites } \\
\hline \multirow[t]{2}{*}{ T1: Before ICU-ROX } & $0.4 \%$ (0.3 to $0.7 \%)$ & & \\
\hline & & T1 to T3: 3.38 (1.87 to 6.09$)$ & $<0.001$ \\
\hline \multirow[t]{2}{*}{ T2: After ICU-ROX } & $1.0 \%$ (0.8 to $1.4 \%)$ & & \\
\hline & & T2 to T3: 1.45 (0.93 to 2.26 ) & 0.10 \\
\hline T3: After ICU-ROX publication & $1.5 \%$ (1.1 to $2.15 \%)$ & & \\
\hline
\end{tabular}

Abbreviations: Cl: confidence interval; ICU-ROX trial: Intensive Care Unit Randomised Trial Comparing Two Approaches to Oxygen therapy; ICU-ROX sites: ICUs that participated in ICU-ROX; non-ICU-ROX sites: ICUs that did not participate in ICU-ROX; $\mathrm{SpO}_{2}$ : peripheral oxygen saturations; T1: before ICU-ROX started; T2: after ICU-ROX completed but before results were known; T3: after publication of ICU-ROX results.

\subsubsection{Mean, lowest and highest daily $\mathrm{PaO}_{2}$}

The mean, highest and lowest $\mathrm{PaO}_{2}$ for both ICU-ROX and non-ICU-ROX sites were generally similar across all time points (Figure 25). Before ICU-ROX, after ICU-ROX and again after publication, the mean, highest and lowest $\mathrm{PaO}_{2}$ was highest on Day 0 (day of ICU admission or initiation of ventilation). The $\mathrm{PaO}_{2}$ for patients from sites that participated in ICU-ROX did not appear to change over the three time periods. However, for non-ICU-ROX sites patients, the mean $\mathrm{PaO}_{2}$ at Day 0 was higher before ICU-ROX (mean 132.7) was conducted than after ICU-ROX or after ICU-ROX publication (mean 116.6 and 116.1, respectively). The mean highest $\mathrm{PaO}_{2}$ at Day 0 was higher in the patients from ICU-ROX sites than the non-ICU-ROX sites before ICU-ROX. The number of $\mathrm{PaO}_{2}$ observations dropped from 107 at day 0 to $<55$ per day on Day 3, and to $<20$ observations per day in any group after day 7 for both groups across all time periods. 


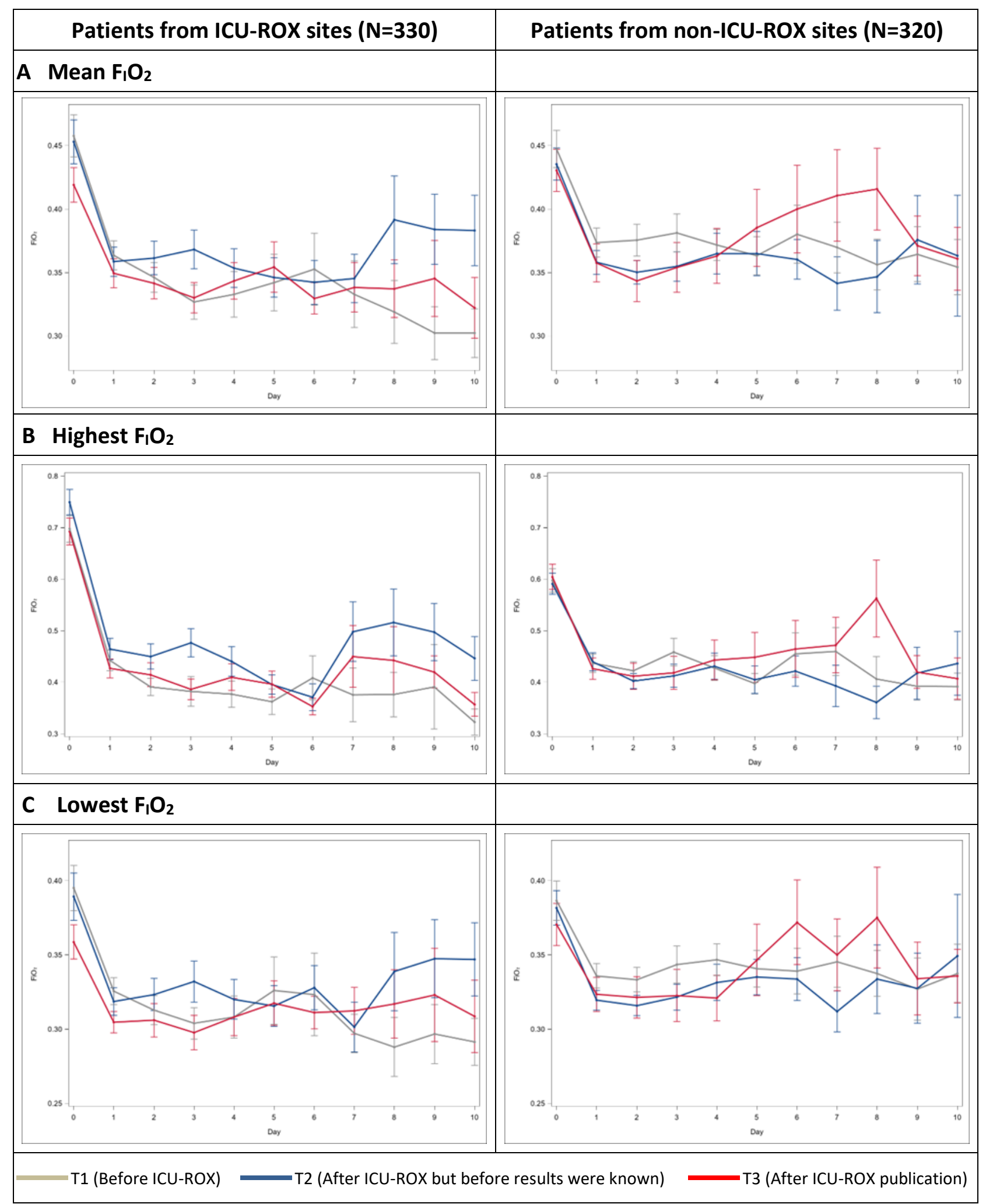

Figure $24 \mathrm{~F}_{1} \mathrm{O}_{2}$ while ventilated in ICU by time and exposure group (A: mean; B: highest; C: lowest)

Abbreviations: $\mathrm{F}_{1} \mathrm{O}_{2}$ : fraction of inspired oxygen; ICU-ROX trial: Intensive Care Unit Randomised Trial Comparing Two Approaches to Oxygen therapy; ICU-ROX sites: ICUs that participated in ICU-ROX; non-ICU-ROX sites: ICUs that did not participate in ICU-ROX. 


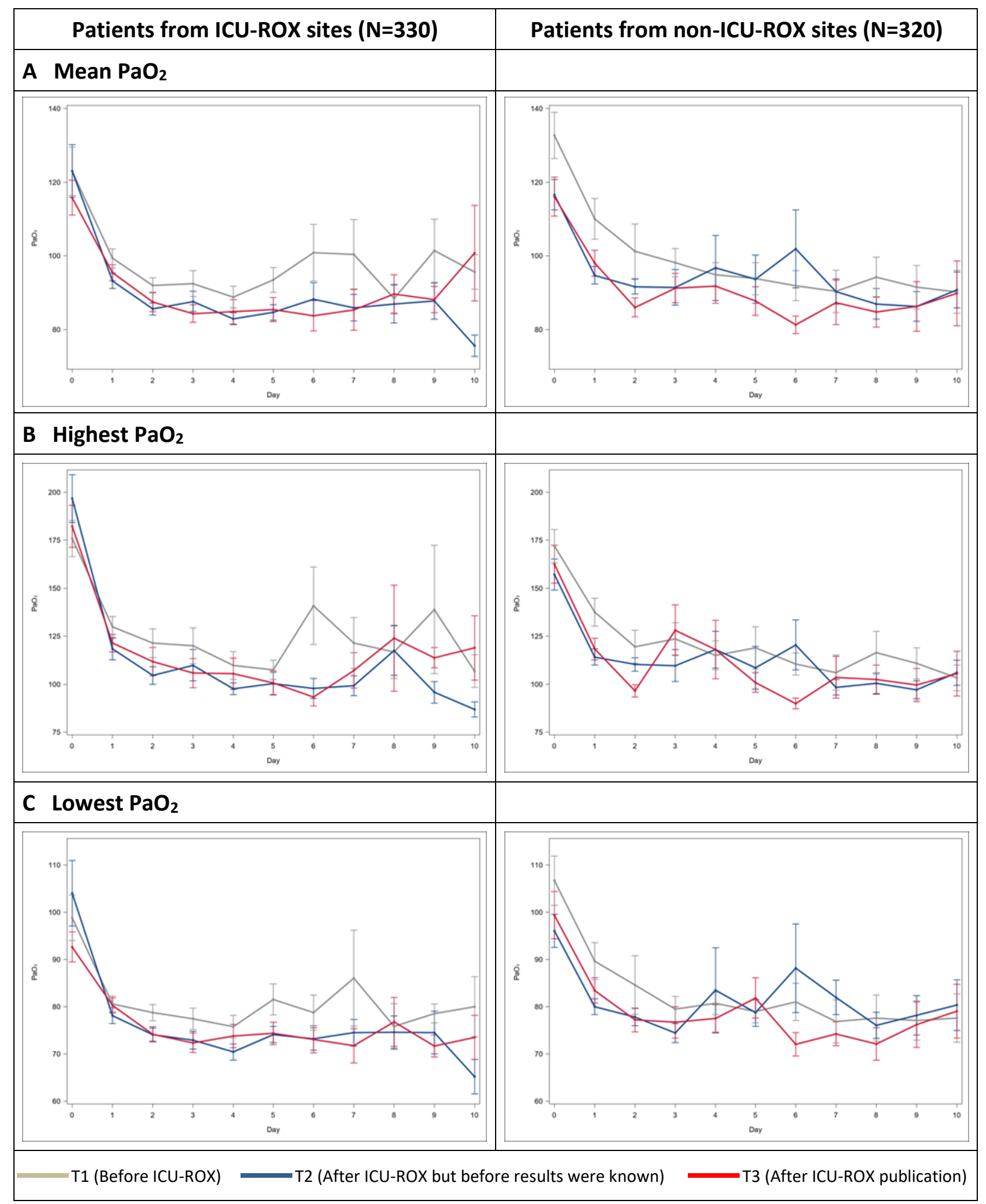

Figure $25 \mathrm{PaO}_{2}$ while ventilated in ICU by time and exposure group (A: mean, B: highest, C: lowest)

Abbreviations: $\mathrm{PaO}_{2}$ : partial arterial oxygen; ICU-ROX trial: Intensive Care Unit Randomised Trial Comparing Two Approaches to Oxygen therapy; ICU-ROX sites: ICUs that participated in ICU-ROX; non-ICU-ROX sites: ICUs that did not participate in ICU-ROX. 


\subsubsection{Primary and secondary outcomes}

There was no statistically significant difference in the estimated percentage of hours patients from ICU-ROX sites spent ventilated with an $\mathrm{F}_{1} \mathrm{O}_{2}$ of 0.21 , over time (Table 35). Specifically, after publication of the ICU-ROX results compared to before the sites commenced ICU-ROX, there was no difference (relative rate (RR) 0.85; 95\% confidence interval (Cl) 0.54 to 1.50, $P=0.45)$. However, for patients from non-ICU-ROX sites there was a difference in the percentage of hours spent with an $\mathrm{F}_{1} \mathrm{O}_{2}$ of 0.21 while mechanically ventilated in an ICU after publication of ICU-ROX compared to 'before ICU-ROX' (RR 3.63; 95\% Cl 1.39 to 9.47, P=0.01). No differences were seen in patients from ICU-ROX or non-ICU-ROX sites for the period 'after ICU-ROX, but before results were known' compared to 'after ICU-ROX publication'.

After the sensitivity analysis was conducted, a difference was seen in the percentage of time spent at $\mathrm{F}_{1} \mathrm{O}_{2}$ of 0.21 while ventilated, over time, in both ICU-ROX and non-ICU-ROX sites (Table 42). After accounting for the pre-specified potential confounding variables of age, APACHE II score, ICU-ROX eligibility, emergency versus elective admission, respiratory admission diagnosis, respiratory comorbidity, sex, site, suspected hypoxic ischaemic encephalopathy, there was a statistically significant decrease in percentage of hours patients from ICU-ROX sites were ventilated at $\mathrm{F}_{1} \mathrm{O}_{2}$ of 0.21 after ICU-ROX was conducted, compared to before ICU-ROX started (RR; 0.88; $95 \% \mathrm{Cl} 0.78$ to 0.99, P=0.03). Similarly, for non-ICU-ROX site patients there was a statistically significant increase in percentage of time for the same period once the sensitivity analysis was conducted ( $R R$ 2.97; 95\% $\mathrm{Cl} 2.30$ to 3.83, $\mathrm{P}<0.001$ ). There was no difference for the ICU-ROX cohort of patients for the period after ICU-ROX publication compared to after ICU-ROX finished, but before results were known; however, for the non-ICU-ROX cohort there was a modest increase for this period (RR $1.21 ; 95 \% \mathrm{Cl} 1.00$ to $1.45, \mathrm{P}=0.05)$.

\subsubsection{Subgroup analysis}

There was no evidence of a difference in percentage of time ventilated at $\mathrm{F}_{1} \mathrm{O}_{2}$ of 0.21 for the pre-specified subgroup analysis of patients who met eligibility criteria for the ICU-ROX trial (Table 43). No difference was apparent for the percentage of time spent ventilated at $\mathrm{F}_{1} \mathrm{O}_{2}$ of 0.21 for time period or for exposure (patients who would have been eligible for ICU-ROX) and no further analyses were conducted for this subgroup of patients. For patients from ICU-ROX sites who were not eligible for the ICU-ROX trial, there was no difference over time, at either 
time period in the percentage of time spent at $\mathrm{F}_{1} \mathrm{O}_{2}$ of 0.21 . For non-ICU-ROX patients who did not meet the eligibility criteria for ICU-ROX, there was a statistically significantly increase in time spent ventilated at $\mathrm{F}_{1} \mathrm{O}_{2}$ of 0.21 after publication, both compared to before ICU-ROX started (RR 17.77, 95\% Cl (3.47 to 91.05), P<0.001), and after ICU-ROX was completed (but before results were known) ( $\mathrm{RR} 7.91,95 \% \mathrm{Cl}, 2.44$ to $25.60, \mathrm{P}<0.001)$.

Table 42 Sensitivity analysis for percentage of time $\mathrm{F}_{1} \mathrm{O}_{2}$ is 0.21 while ventilated in ICU

\begin{tabular}{|c|c|c|c|}
\hline Time period & $\begin{array}{c}\text { Percentage of time } \\
\mathrm{F}_{1} \mathrm{O}_{2} 0.21 \\
(95 \% \mathrm{Cl})^{*}\end{array}$ & $\begin{array}{c}\text { Relative rate } \mathrm{F}_{1} \mathrm{O}_{2} \text { is } 0.21 \\
(95 \% \mathrm{Cl})^{*}\end{array}$ & P value* \\
\hline \multicolumn{4}{|l|}{ ICU-ROX sites } \\
\hline \multirow[t]{2}{*}{ T1: Before ICU-ROX } & $8.3 \%$ (3.9 to $17.7 \%)$ & & \\
\hline & & T1 to T3: 0.88 (0.78 to 0.99$)$ & 0.03 \\
\hline \multirow[t]{2}{*}{ T2: After ICU-ROX } & $6.7 \%$ (3.2 to $14.2 \%$ ) & & \\
\hline & & T2 to T3: 1.10 (0.98 to 1.23$)$ & 0.11 \\
\hline T3: After ICU-ROX publication & $7.3 \%$ (3.4 to $15.6 \%)$ & & \\
\hline \multicolumn{4}{|l|}{ Non-ICU-ROX sites } \\
\hline \multirow[t]{2}{*}{ T1: Before ICU-ROX } & $1.5 \%$ (0.7 to $3.3 \%)$ & & \\
\hline & & T1 to T3: 2.97 (2.30 to 3.83 ) & $<0.001$ \\
\hline \multirow[t]{2}{*}{ T2: After ICU-ROX } & $3.7 \%$ (1.7 to $7.9 \%)$ & & \\
\hline & & T2 to T3: 1.21 (1.00 to 1.45$)$ & 0.05 \\
\hline T3: After ICU-ROX publication & $4.4 \%$ (2.1 to $9.5 \%)$ & & \\
\hline
\end{tabular}

* Adjusted for age, APACHE II score, ICU-ROX eligibility, emergency vs elective admission, respiratory admission diagnosis, respiratory comorbidity, sex, site and suspected hypoxic ischaemic encephalopathy. Abbreviations: $\mathrm{F}_{1} \mathrm{O}_{2}$ : fraction of inspired oxygen; ICU-ROX sites: ICUs that participated in ICU-ROX; non-ICU-ROX sites: ICUs that did not participate in ICU-ROX; T1: before ICU-ROX started; T2: after ICU-ROX completed but before results were known; T3: after publication of ICU-ROX results. 
Table 43 Subgroup analyses: and relative rate $\mathrm{F}_{1} \mathrm{O}_{2}$ is 0.21 while ventilated in ICU

\begin{tabular}{|c|c|c|}
\hline & $\begin{array}{c}\text { Relative rate } \mathrm{F}_{1} \mathrm{O}_{2} \text { is } 0.21 \\
(95 \% \mathrm{Cl})\end{array}$ & $P$ value \\
\hline \multicolumn{3}{|c|}{ Met eligibility criteria for ICU-ROX } \\
\hline T3 vs T1, ICU-ROX sites & $\mathrm{np}$ & $\mathrm{np}$ \\
\hline T3 vs T1, Non-ICU-ROX sites & $\mathrm{np}$ & $\mathrm{np}$ \\
\hline \multicolumn{3}{|c|}{ Did not meet eligibility criteria for ICU-ROX } \\
\hline \multicolumn{3}{|l|}{ ICU-ROX sites } \\
\hline T3 vs T1 & 1.69 (0.76 to 3.75$)$ & 0.20 \\
\hline T3 vs T2 & 0.73 (0.37 to 1.46$)$ & 0.37 \\
\hline \multicolumn{3}{|l|}{ Non-ICU-ROX sites } \\
\hline T3 vs T1 & 17.77 (3.47 to 91.05$)$ & $<0.001$ \\
\hline T3 vs T2 & 7.91 (2.44 to 25.60$)$ & $<0.001$ \\
\hline
\end{tabular}

Abbreviations: ICU-ROX sites: ICUs that participated in ICU-ROX; np: statistical analysis was not possible to complete; non-ICU-ROX sites: ICUs that did not participate in ICU-ROX; T1: before ICU-ROX started; T2: after ICU-ROX completed but before results were known; T3: after publication of ICU-ROX results.

\subsubsection{Other outcomes}

\subsubsection{Outcome measures for $\mathrm{SpO}_{2} \geq 97 \%$}

Initial analysis indicated the percentage of time patients spent ventilated with an $\mathrm{SpO}_{2} \geq 97 \%$ was higher before ICU-ROX, than after the ICU-ROX results were published (Table 37). However, the time period by treatment (site participation or non-participation) interaction term was non-significant $(P=0.21)$, indicating there was no evidence that the time period differed for ICU-ROX versus. non-ICU-ROX sites. No further analyses were conducted for change in $\mathrm{SpO}_{2} \geq 97 \%$ for this time period. There was no difference for either ICU-ROX or non-ICU-ROX sites patients after ICU-ROX publication, compared to after ICU-ROX enrolment completed.

\subsubsection{Outcome measures for $\mathrm{SpO}_{2}<91 \%$}

There was no difference in the percentage of time ICU-ROX site patients had an $\mathrm{SpO}_{2}<91 \%$ while ventilated, after the ICU-ROX results were published compared to before ICU-ROX started (Table 39). For patients from non-ICU-ROX sites, however, there was a statistically significant higher percentage of time with $\mathrm{SpO}_{2}<91 \%$ after ICU-ROX was published than before ICU-ROX started (RR 2.33; 95\% $\mathrm{Cl} 1.43$ to 3.78, $\mathrm{P}<0.001$ ). There was no difference in either group after publication compared to after ICU-ROX finished, but before results were known. 


\subsubsection{Outcome measures for $\mathrm{SpO}_{2}<88 \%$}

There was no evidence of a difference in percentage of time spent at $\mathrm{SpO}_{2}<88 \%$ for patients from ICU-ROX sites, after the ICU-ROX results were published compared to before ICU-ROX (Table 41). However, there was a statistically significant increase in the percentage of time with an $\mathrm{SpO}_{2}<88 \%$ for patients from non-ICU-ROX sites (RR 3.38; 95\% Cl 1.87 to 6.09, $\mathrm{P}<0.001)$ between these two time points. There was no difference in either group of patients after publication compared to after ICU-ROX finished, but before results were known. 


\subsection{Discussion}

In this multicentre inception cohort study, the key finding was that there was no difference in the estimated percentage of hours patients from ICU-ROX trial sites spent ventilated at $\mathrm{F}_{1} \mathrm{O}_{2}$ 0.21, after ICU-ROX was published relative to before ICU-ROX started or after it was completed (but before results were known). However, there was a significant increase in the percentage of hours patients from the non-ICU-ROX sites spent ventilated at $\mathrm{F}_{1} \mathrm{O}_{2}$ of 0.21 after publication of ICU-ROX compared to before ICU-ROX started. Once adjusted for potentially confounding differences, there was also a statistically significant difference for patients from the ICU-ROX sites after ICU-ROX publication, compared to before ICU-ROX commenced. However, the percentage of time ventilated at $\mathrm{F}_{1} \mathrm{O}_{2}$ was small at both ICU-ROX and non-ICUROX sites and overall the change was not considered clinically important.

The finding that ICU participation in a research trial did not change practice was similar to several other studies (Khera et al., 2018; Majumdar et al., 2002; Shah et al., 2010). ICU-ROX showed no difference in the primary outcome of ventilator-free days at 28 days after randomisation, restoring equipoise about the optimal level of oxygen to use (Mackle et al., 2020; Young, Bagshaw et al., 2019). The ICU-ROX results did not provide a strong rationale for changing practice. In contrast, Majumdar et al. reviewed the effects of participation in an RCT that showed a significant difference in use of captopril at hospital discharge following myocardial infarction; however trial-sites were no more likely to change practice than nontrial sties. Likewise, with Khera et al., in which trial sites failed to use the findings of an RCT that showed bone marrow transplant had less adverse effects than peripheral blood transplant, any more than non-trial sites. This research is most similar to Shah et al. (2010), that found no change in practice after site participation in an RCT that showed that low molecular weight heparin was not inferior to unfractionated heparin.

The change in non-ICU-ROX site patients' time spent at $\mathrm{F}_{1} \mathrm{O}_{2}$ of 0.21 was unexpected, and not seen previously in the literature. One explanation might be that there was a 'general' increasing avoidance of hyperoxia due to increasing evidence that hyperoxia may be harmful (Livingston et al., 2020) or the influence of two publications showing a mortality benefit of conservative oxygen therapy (Chu et al., 2018; Girardis et al., 2016). At the beginning of the research, use of $\mathrm{F}_{1} \mathrm{O}_{2}$ of 0.21 while ventilated was much lower in non-ICU-ROX sites compared 
to the ICU-ROX sites (0.9\% and $8.2 \%$, respectively). Overall, the percentage of time at $\mathrm{F}_{1} \mathrm{O}_{2}$ of 0.21 was considerably less than the $36.6 \%$ of time seen in the conservative arm of the ICUROX, but similar to the usual care arm (8.8\%) (Mackle et al., 2020). When comparing the oxygenation data from ICU-ROX with data obtained in this study, it is important to note that there were differences in the way data were recorded. In the current research, $\mathrm{F}_{1} \mathrm{O}_{2}$ was only recorded while patients were ventilated, whereas in the ICU-ROX trial the time included after post-extubation. The small percentage of time ventilated at $\mathrm{F}_{1} \mathrm{O}_{2}$ of 0.21 was also similar to previous observational studies that indicate that ICU patients spend most of their time ventilated with an $\mathrm{F}_{1} \mathrm{O}_{2}$ between 0.3 and 0.5 (Panwar et al., 2013; Suzuki et al., 2013).

This study had limitations. Primarily, the percentage of time patients spent ventilated at $\mathrm{F}_{1} \mathrm{O}_{2}$ of 0.21 was low, meaning there were too few data to gain meaningful insights. Additionally, not all analyses could be conducted as intended due to the risk of type I error, meaning that there is no analysis between 'before ICU-ROX' and 'after ICU-ROX, but before the results were known'. Due to the low percentage of time spent at $\mathrm{F}_{1} \mathrm{O}_{2}$ of 0.21 and time spent ventilated, it is unlikely this would have affected the overall results. The observed group of 650 patients was a small proportion of the many hundreds of patients who were mechanically ventilated in the study ICUs each year (Table 11). There was a difference between the two groups of patients regarding admission source, with more in the non-ICU-ROX group admitted following emergency surgery, and more in the ICU-ROX cohort admitted following elective surgery (Table 32). As the overall total admitted from the operating theatre were similar, it is unlikely that this baseline difference would have affected the results. The two groups of patients were otherwise similar to each other (Table 32), and similar age and sex to the ICU-ROX trial (Mackle et al., 2020). Risk of inclusion bias was mitigated by protocolising that site research staff include consecutive, eligible patients. As the sample is similar to ICU-ROX, which was randomised, it appears this was not a major issue. Two patients were included who were ventilated for less than the 12-hour eligibility window; however, this is unlikely to have materially impacted on the study results.

The inception cohort study suggests more conservative practice in ICU-ROX sites but also that practice did not change substantially over time. While there was a statistically significant change in $\mathrm{F}_{1} \mathrm{O}_{2}$ of 0.21 use in ventilated patients in non-ICU-ROX sites over time, this practice 
remains uncommon. Any changes observed were not considered to be clinically important, because the difference in time spent ventilated at $\mathrm{F}_{1} \mathrm{O}_{2}$ of 0.21 was very small. 


\section{CHAPTER 8 RETROSPECTIVE COHORT STUDY}

In this chapter the hypotheses, design, methods, results and discussion for the retrospective cohort study are outlined. The chapter begins with the aims and objectives for the study, then an overview of the Australian New Zealand Intensive Care Society (ANZICS) Adult Patient Database (APD), from which the data for this study are sourced, then describes the study design and methods, and finally the results and discussion specific to this study. Permission to use data from the APD was approved by ANZICS Centre for Outcome and Resource Evaluation (CORE) prior to data download (Appendix 7).

\subsection{Aims and objectives}

The aim of this study was to gain an understanding of oxygen management practices in Australian and New Zealand (ANZ) ICUs over time, and establish if there was any change in relation to the publication of the ICU-ROX results.

The main objective of this retrospective cohort study was to determine whether a practice change regarding oxygen management was seen in ANZ ICUs over time, when reviewed 11 months after publication of the ICU-ROX results, compared to 11 months prior to publication, and whether this was different between ICU-ROX and non-ICU-ROX sites. Specifically, the study assessed whether there was a difference between oxygen management practices in ICUs that participated in ICU-ROX compared to ICUs that did not, and whether those practices changed after publication of the ICU-ROX trial results, using data from the ANZICS CORE database.

\subsection{Hypotheses}

The hypotheses for the retrospective cohort study were:

1a In the event that ICU-ROX demonstrates that conservative oxygen therapy improved outcomes, implementation of this therapy would be more rapid in ANZ ICUs that participated in ICU-ROX than those that did not.

1b In the event of no difference or harm from conservative oxygen therapy, or usual oxygen therapy improves outcomes, all ANZ ICUs will show similar patterns of oxygen use over time. 


\subsection{ANZICS Adult Patient Database}

The adult patient database (APD) is a registry administered by the Australian and New Zealand Intensive Care Society Centre for Outcome Resource Evaluation (ANZICS-CORE) in Melbourne, Australia (ANZICS CORE, n.d.) . This registry contains de-identified information about patients primarily for benchmarking of ICU outcomes. The ICUs that contribute to the APD can access and receive reporting from this benchmarking. In addition, this repository of data about ICU patients can be used for research projects. There are over 2 million patient episodes in the APD, of which 200,000 were in 2020 (ANZICS, 2021). Each episode refers to an individual ICU admission (rather than individual patient) as some patients are admitted to ICU more than once during their hospitalisation.

\subsubsection{Data available}

Data are collected for the first 24 hours of ICU stay for all patients from contributing sites (ANZICS CORE, 2021). The APD minimum dataset contains 105 variables which are collected by trained staff at each ICU and submitted quarterly. All data that were used for this study are defined in Table 44; data that require further explanation because of their particular relevance to the current research are $\mathrm{F}_{1} \mathrm{O}_{2}, \mathrm{PaO}_{2}$ and Acute Physiology and Chronic Health Evaluation (APACHE-III-J) score.

Table 44 Data descriptions from ANZICS APD

\begin{tabular}{|l|l|l|}
\hline Data variable & Definition/ Explanation & Options (if applicable) \\
\hline Age & Years (at admission date) & \\
\hline Sex & & $\begin{array}{l}\text { Male } \\
\text { Female } \\
\text { Intersex/Indeterminate } \\
\text { Unknown }\end{array}$ \\
\hline ANZROD & $\begin{array}{l}\text { Australian and New Zealand Risk of } \\
\text { Death model (primary risk prediction } \\
\text { model) }\end{array}$ & $\begin{array}{l}\text { Calculated automatically using } \\
\text { the variables entered into the } \\
\text { APD }\end{array}$ \\
\hline APACHE III-J score & $\begin{array}{l}\text { Acute Physiology and Chronic Health } \\
\text { Evaluation } \\
\text { Severity of illness score }\end{array}$ & $\begin{array}{l}\text { Calculated automatically using } \\
\text { the variables entered into the } \\
\text { APD }\end{array}$ \\
\hline $\begin{array}{l}\text { Chronic health } \\
\text { evaluation } \\
\text { (Respiratory) }\end{array}$ & $\begin{array}{l}\text { Exists prior to hospital admission and } \\
\text { meets the following definition: } \\
\text { "Chronic restrictive, obstructive disease } \\
\text { resulting in severe exercise restriction } \\
\text { (unable to climb stairs or perform } \\
\text { household duties); or documented } \\
\text { chronic hypoxia, hypercannia, } \\
\text { secondary polycythaemia, severe }\end{array}$ & $\begin{array}{l}\text { Yes for co-morbidity } \\
\text { No for co-morbidity does not } \\
\text { exist }\end{array}$ \\
\hline
\end{tabular}




\begin{tabular}{|c|c|c|}
\hline Data variable & Definition/ Explanation & Options (if applicable) \\
\hline & $\begin{array}{l}\text { pulmonary hypertension (mean }>40 \\
m m H \text { ); or ventilator dependency.", } \\
\text { p. } 96 \text {, (ANZICS Centre for Outcome and } \\
\text { Resource Evaluation, 2021) }\end{array}$ & \\
\hline Country & & $\begin{array}{l}\text { Australia } \\
\text { New Zealand }\end{array}$ \\
\hline $\begin{array}{l}\text { Elective or } \\
\text { emergency }\end{array}$ & $\begin{array}{l}\text { Elective indicates a planned admission } \\
\text { to ICU. These are usually following } \\
\text { elective surgery, but may also be when } \\
\text { patients are transferred from another } \\
\text { ICU or other reason }\end{array}$ & $\begin{array}{l}\text { Yes for elective or no for non- } \\
\text { elective }\end{array}$ \\
\hline $\mathrm{F}_{1} \mathrm{O}_{2}$ & $\begin{array}{l}\text { Fraction of inspired oxygen from ABG } \\
\text { with worst APACHE-III J score }\end{array}$ & \\
\hline Hospital type & $\begin{array}{l}\text { Including location and management } \\
\text { type }\end{array}$ & $\begin{array}{l}\text { Rural/ Regional } \\
\text { Metropolitan Tertiary/Teaching } \\
\text { Private }\end{array}$ \\
\hline ICU admission date & $\begin{array}{l}\text { Date when patient was physically } \\
\text { admitted to the ICU }\end{array}$ & \\
\hline $\begin{array}{l}\text { ICU admission } \\
\text { diagnosis }\end{array}$ & APACHE-III J diagnostic code & $\begin{array}{l}\text { List provided in APD data } \\
\text { dictionary } \\
\text { Categories: non-operative } \\
\text { (respiratory } 201-213 \text { ) and } \\
\text { post-operative (respiratory } \\
\text { 1301-1304) }\end{array}$ \\
\hline $\begin{array}{l}\text { Invasive ventilation } \\
\text { status }\end{array}$ & & $\begin{array}{l}\text { Yes for invasively mechanically } \\
\text { ventilated or No for not } \\
\text { invasively mechanically } \\
\text { ventilated }\end{array}$ \\
\hline $\mathrm{PaO}_{2}$ & $\begin{array}{l}\text { Partial pressure of arterial oxygen from } \\
\text { ABG with worst APACHE-III J score }\end{array}$ & \\
\hline $\begin{array}{l}\text { Treatment goals for } \\
\text { admission }\end{array}$ & $\begin{array}{l}\text { Used to ascertain patients admitted for } \\
\text { palliative care) }\end{array}$ & $\begin{array}{l}\text { Full active treatment } \\
\text { Treatment limitation order } \\
\text { Palliative are of a dying patient } \\
\text { Potential organ donation } \\
\text { Unknown }\end{array}$ \\
\hline Site ID & $\begin{array}{l}\text { Each contributing ICU has a unique site } \\
\text { ID }\end{array}$ & \\
\hline $\begin{array}{l}\text { Source of ICU } \\
\text { admission }\end{array}$ & Place patient was admitted from & $\begin{array}{l}\text { Operating theatre/ recovery } \\
\text { Emergency Department } \\
\text { Ward } \\
\text { ICU, same hospital } \\
\text { Other hospital } \\
\text { ICU other hospital } \\
\text { Direct admission from home }\end{array}$ \\
\hline
\end{tabular}

Adapted from APD Data Dictionary (ANZICS Centre for Outcome and Resource Evaluation, 2021)

Abbreviations: ABG: arterial blood gas; APD: Adult Patient Database; ANZROD: Australian and New Zealand Risk of Death model; APACHE III-J score: Acute Physiology and Chronic Health Evaluation severity of illness score; $\mathrm{F}_{1} \mathrm{O}_{2}$ : fraction of expired oxygen; ICU: Intensive Care Unit; ID: identification number; $\mathrm{mmHg}$ : millimetres of mercury; $\mathrm{PaO}_{2}$ : partial pressure of arterial oxygen. 
The available variables related to oxygenation in the APD are $\mathrm{F}_{1} \mathrm{O}_{2}$ and $\mathrm{PaO}_{2}$. The $\mathrm{F}_{1} \mathrm{O}_{2}$ and $\mathrm{PaO}_{2}$ variables are from the same arterial blood gas (ABG) and are considered the 'worst' in the first 24 hours of ICU stay (ANZICS CORE, 2021). All ABG results for the first 24 hours of a patient's ICU admission are reviewed, and the $\mathrm{F}_{1} \mathrm{O}_{2}$ and $\mathrm{PaO}_{2}$ from the $A B G$ that produces the highest APACHE III-J score, indicating the worst oxygenation, are entered into the database, along with partial pressure of carbon dioxide $\left(\mathrm{PaCO}_{2}\right)$, and arterial $\mathrm{pH}$. For patients who are intubated and receive an $\mathrm{F}_{1} \mathrm{O}_{2}$ of 0.5 or more, the $\mathrm{PaO}_{2}$ associated with the widest (highest) alveolar-arterial (A-a) gradient is used for the score (Table 45). For patients who are not intubated, or who are intubated, but receive an $\mathrm{F}_{1} \mathrm{O}_{2}$ less than 0.5 , the lowest $\mathrm{PaO}_{2}$ is used. The APACHE-III-J score is a severity of illness scoring system, with 'J' representing the $10^{\text {th }}$ iteration of APACHE-III score. APACHE-III-J scores range from 0-299, with higher scores associated with an increased risk of hospital death (Knaus et al., 1991). The potential score for the oxygenation component is 0 to 15 points (in grouped ranges), with 15 being the worst possible score (ANZICS CORE, 2021).

Table 45 Determining the worst APACHE-III-J score for oxygenation variables

\begin{tabular}{|c|c|c|c|c|c|c|c|c|c|}
\hline \multirow[b]{2}{*}{$\begin{array}{c}\text { A-a } \\
\text { gradient }\end{array}$} & \multicolumn{4}{|c|}{$\begin{array}{c}\text { If patient is not intubated or they are } \\
\text { intubated and } \mathrm{F}_{1} \mathrm{O}_{2}<0.5, \\
\text { use } \mathrm{PaO}_{2}(\mathrm{mmHg})\end{array}$} & \multicolumn{5}{|c|}{$\begin{array}{l}\text { If patient is intubated and } \mathrm{F}_{1} \mathrm{O}_{2} \geq 0.5 \text {, } \\
\text { use } A-\text { a gradient* }\end{array}$} \\
\hline & & & & & $<100$ & $100-249$ & $250-349$ & $350-499$ & $\geq 500$ \\
\hline $\mathrm{PaO}_{2}$ & $\leq 49$ & $50-69$ & $70-79$ & $\geq 80$ & & & & & \\
\hline $\begin{array}{l}\text { APACHE } \\
\text { III-J score }\end{array}$ & 15 & 5 & 2 & 0 & 0 & 7 & 9 & 11 & 14 \\
\hline
\end{tabular}

Adapted from Knaus et al. (1991) and APD Data Dictionary (ANZICS Centre for Outcome and Resource Evaluation, 2021)

$*$ A-a gradient $=\left(713 \times \mathrm{F}_{1} \mathrm{O}_{2}\right)-\mathrm{PaO}_{2}-\left(\mathrm{PaCO}_{2} / 0.8\right)$

Abbreviations: A-a gradient: alveolar-arterial gradient; APACHE III-J score: Acute Physiology and Chronic Health Evaluation severity of illness score; $\mathrm{mmHg}$ : millimetres of mercury; $\mathrm{PaO}_{2}$ : partial pressure of arterial oxygen.

\subsubsection{Adult Patient Database validity strategies}

There are a number of validity strategies employed by the ANZICS CORE, to ensure accuracy of APD data. Firstly, data are collected by trained staff from the contributing ICUs, and all variables are defined in the data dictionary (ANZICS CORE, 2021). Data can be uploaded via a software system called Core Outcome Measurement and Evaluation Tool (COMET) or alternatively using their own site-specific database. If data are uploading using COMET, internal validity measures for critical errors must be applied before submission. For ICUs that 
do not use COMET, the system they are using must be validated prior to approval for use (ANZICS CORE, 2018).

\subsection{Design and methods}

The design was a retrospective cohort study using data from the ANZICS CORE adult patient database.

\subsubsection{Study population}

Patients were eligible for inclusion if they were mechanically ventilated and admitted to a public or private ICU, that contributed to the ANZICS APD within the study period. Patients who were admitted to ICU for palliative care were excluded, as were patients from the ICUROX trial pilot study.

The study population was initially divided into two cohorts, based on whether the ICUs had participated in the ICU-ROX trial or not. Each of these cohorts was divided into two further cohorts, based on the patients' ICU admission dates. The pre-publication cohorts were for patients admitted from 1 November 2018 to 30 September 2019, and the post-publication cohorts were for patients admitted from 1 November 2019 to 30 September 2020.

\subsubsection{Exposures and Comparator}

There are two exposure and comparators in this study. The main exposure was participation as an ICU in the ICU-ROX trial (ICU-ROX sites) and the comparator was ICU non-participation in ICU-ROX (non-ICU-ROX sites). In addition, patients admitted before the ICU-ROX publication (pre-publication comparator), on 14 October, 2019 (Mackle et al., 2020) were compared to those admitted after the publication (post-publication exposure).

\subsubsection{Outcomes}

The primary outcome was the difference over time in average weekly $\mathrm{F}_{1} \mathrm{O}_{2}$, between the ICUs that participated in the ICU-ROX trial and those that did not. Secondary outcomes were the difference in $\mathrm{F}_{1} \mathrm{O}_{2}$ before and after ICU-ROX publication for the ICU-ROX and non-ICU-ROX sites; overall difference in $\mathrm{PaO}_{2}$ between ICU-ROX and non-ICU-ROX sites; and the difference in $\mathrm{PaO}_{2}$ before and after the ICU-ROX publication for the ICU-ROX and non-ICU-ROX sites. 


\subsubsection{Data management}

Permission was sought in advance from ANZICS CORE (Appendix 7), and the variables outlined in Table 44 were extracted from the ANZICS APD in November 2020, and managed by an independent study statistician. Patients who were not ventilated, receiving palliative care and those from ICUs that participated in the ICU-ROX trial pilot study were removed from the dataset prior to analysis.

\subsubsection{Data analysis}

The main comparisons were between the pre-ICU-ROX trial publication cohort and the postICU-ROX trial publication cohort as a whole, and additionally, for both ICU-ROX and non-ICUROX sites. The decision to compare these cohorts for 11 months before and after publication was based on data available in the ANZICS APD after the ICU-ROX publication in October 2019, matched with the same before ICU-ROX publication. Patient characteristics were compared between pre-publication and post-publication for patients admitted from ICU-ROX sites and non-ICU-ROX sites. The analysis was planned to be conducted by months; however, initial review of the data found that with only 11 months there were insufficient data points to obtain a clear result. This was overcome by averaging the data weekly, so that there are 48 weeks before and after ICU-ROX publication.

Comparisons between groups were performed using chi-square tests for equal proportion, Student $t$-test for normally distributed data and Wilcoxon rank sum tests otherwise, with results reported as numbers (\%), means \pm standard deviations (SD) or medians [interquartile range (IQR)], respectively.

Data were initially analysed using hierarchical mixed linear modelling with patients nested within sites and sites treated as a random effect. Additional analyses were then conducted adjusting for any baseline difference in age, gender, illness severity (Australian and New Zealand Risk of Death [ANZROD]), ICU admission diagnosis of respiratory pathology, respiratory co-morbidity, admission source and emergency versus elective admission. An interrupted time series analysis was then conducted from November 2018 to September 2020, using a linear regression model, with data aggregated at a weekly level. The interruption or intervention in this study is defined as the publication of the ICU-ROX trial 
results, and the month of publication (October 2019) was excluded from analysis. Segmented regression was used to evaluate whether there was a stepwise change in the value of each outcome of interest associated with the ICU-ROX trial publication. Stepwise change is a change that occurs immediately at a specified time point (in this case, the ICU-ROX publication). Additionally, analysis comparing the pre-intervention slope with the postintervention slope by calculating the mean level difference in slopes (between pre-publication and post-publication) was conducted. The slope is a measure of rate of change over time. For the current study, a stepwise change would have indicated an immediate change in average weekly $\mathrm{F}_{1} \mathrm{O}_{2}$ or $\mathrm{PaO}_{2}$ after ICU-ROX publication, and a change in slope would be indicative a change in the rate of change in average $\mathrm{F}_{1} \mathrm{O}_{2}$ or $\mathrm{PaO}_{2}$, over time. Where autocorrelation was present, this was adjusted for and the analyses adjusted for autocorrelation were used. The analysis was completed as raw data, then adjusting for ANZROD.

All analyses were performed by a statistician, in collaboration with the researcher, using SAS version 9.4 (SAS Institute, Inc., Cary, NC). A two-sided P value of 0.05 was used to indicate statistical significance. 


\subsection{Results}

\subsubsection{Patients}

Of 340,649 ICU admission episodes from 194 ICUs, between November 2018 and September 2020 (excluding October 2019), 99,021 patients fulfilled the eligibility criteria of being mechanically ventilated and not admitted for palliative care, in a non-pilot study ICU (Figure 26). There were 9294 patients in the pre-publication cohort and 8443 in the post-publication cohort, from 15 non-pilot ICU-ROX sites. From the 188 non-ICU-ROX ICUs, there were 42,204 patients in the pre-publication cohort and 39,080 in the post-publication cohort.

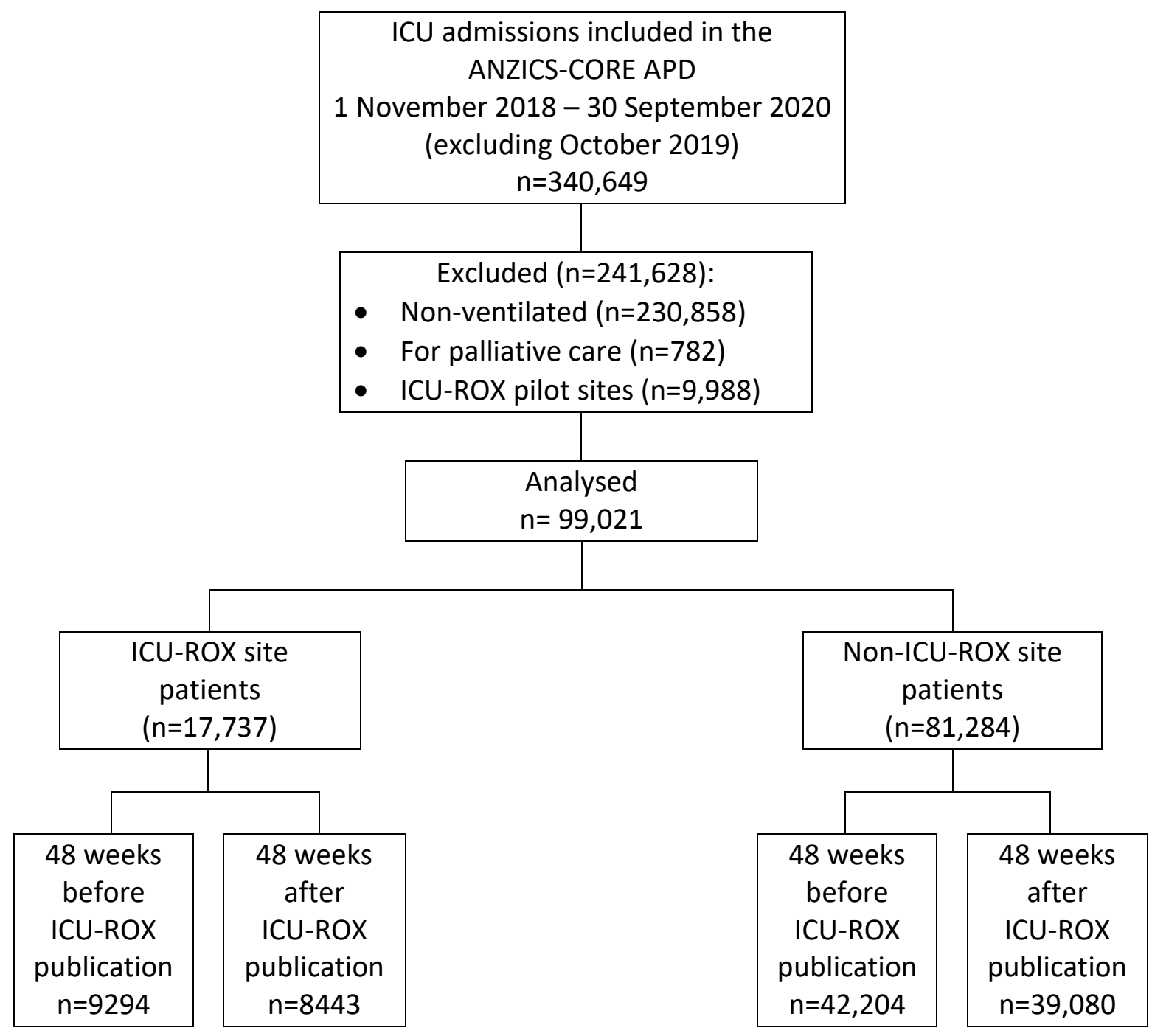

Figure 26 Flow of patients for retrospective cohort study

Abbreviations: ANZICS-CORE APD: Australian and New Zealand Intensive Care Society Centre for Outcome and Resource Evaluation Adult Patient Database; ICU-ROX: Intensive Care Unit Randomised Trial Comparing Two Approaches to Oxygen therapy trial; ICU-ROX sites: patients from ICUs that participated in the ICU-ROX trial; non-ICU-ROX sites: patients from ICUs that did not participate in the ICU-ROX trial. 
While broadly similar, there were some differences in baseline characteristics between cohorts. For patients from the ICU-ROX sites, patients before the publication were statistically significantly older, less likely to be male or admitted from the emergency department, and more likely to be admitted from another hospital than the post-publication cohort (Table 46). For patients from the non-ICU-ROX sites, patients were statistically significantly younger in the pre-publication cohort, more likely to have a respiratory co-morbidity and admission diagnosis, and be admitted from another hospital, less likely to be admitted from an emergency department, and have higher risk of death [ANZROD score] than the postpublication cohort (Table 47). In addition, pre-publication they were more likely to be from New Zealand and from a private ICU, and post-publication they were more likely to be from Australia, and from a metropolitan ICU. Data were not available about the exact number of patients who were COVID-19 positive and mechanically ventilated in ANZ ICUs during the post-publication period, but the number is thought to be a very small percentage of the overall sample.

\subsubsection{Oxygen exposure}

The $\mathrm{F}_{1} \mathrm{O}_{2}$ in the first 24 hours of ICU stay changed in ICU-ROX sites after publication of the ICUROX results and did not in non-ICU-ROX sites (Table 48). Hierarchical linear modelling showed an interaction for $\mathrm{F}_{1} \mathrm{O}_{2}$ over time, between ICU-ROX and non-ICU-ROX sites ( $\left.\mathrm{P}=0.003\right)$, that was materially unchanged when adjusted for baseline differences $(P<0.001)$. For patients from ICU-ROX sites, the median $\mathrm{F}_{1} \mathrm{O}_{2}$ in the first 24 hours in ICU for pre-and post-publication was 0.6 [IQR 0.4 to 1.0] and 0.6 [0.38 to 1.0] respectively (estimated difference $0.011[95 \% \mathrm{Cl}$, 0.003 to 0.02 ]; $P=0.001$ ). For patients from the non-ICU-ROX sites, the median $\mathrm{F}_{1} \mathrm{O}_{2}$ in the first 24 hours in ICU for pre and post ICU-ROX publication was 0.5 [IQR 0.35 to 0.6 ] and 0.5 [0.35 to 0.6 ] respectively (estimated difference $-0.002[95 \% \mathrm{Cl},-0.005$ to 0.002$] ; \mathrm{P}=0.31$ ).

Segmented regression analysis demonstrated there was no statistically significant stepwise change in $\mathrm{F}_{1} \mathrm{O}_{2}$ in the first 24 hours in ICU, associated with publication of the ICU-ROX trial for ICU-ROX sites (Figure 27) or non-ICU-ROX sites (Figure 28). The rate of change (slope) for $\mathrm{F}_{1} \mathrm{O}_{2}$ in the first 24 hours in ICU was statistically significant after publication of the ICU-ROX results in the ICU-ROX sites ( $P=0.01$ ) (Figure 27). There was no difference in slope between the preand post-publication cohorts for the non-ICU-ROX ICUs ( $P=0.49$ ) (Figure 28). The results were unchanged when adjusted for ANZROD (Figure 27, Figure 28). 
Table 46 Characteristics of patients in the ICU-ROX trial site cohort (pre-ICU-ROX publication vs post publication)

\begin{tabular}{|c|c|c|c|}
\hline Characteristics & $\begin{array}{l}\text { Pre-publication } \\
\mathrm{n}=9294^{*}\end{array}$ & $\begin{array}{l}\text { Post-publication } \\
n=8443^{*}\end{array}$ & $P$ value \\
\hline & $n=8440$ & $n=8440$ & \\
\hline Age, years, median [IQR] & $60.6[45.5-71]$ & $59.6[44.7-70.1]$ & 0.001 \\
\hline \multirow[t]{2}{*}{ Male, sex, n (\%) } & $6059(65.2)$ & $5661(67.0)$ & 0.009 \\
\hline & $n=9292$ & $n=8443$ & \\
\hline \multirow[t]{2}{*}{ APACHE III score, mean (SD) } & $61.7 \pm 28$ & $61.3 \pm 27.8$ & 0.32 \\
\hline & $n=9292$ & $n=8443$ & \\
\hline ANZROD, median [IQR] & $0.035[0.00771-0.173]$ & $0.034[0.00794-0.166]$ & 0.74 \\
\hline Respiratory co-morbidity, n (\%) & $485(5.2)$ & $430(5.1)$ & 0.71 \\
\hline Respiratory admission diagnosis, $\mathrm{n}(\%)$ & $10 \%(931)$ & $9.5 \%(806)$ & 0.29 \\
\hline \multicolumn{4}{|l|}{ Admission type, $n(\%)$} \\
\hline Elective admission & $3089(33.2)$ & $2722(32.2)$ & 0.16 \\
\hline \multicolumn{4}{|l|}{ Country of admission, $\mathrm{n}(\%)$} \\
\hline Australia & $6691(72)$ & $6016(71.3)$ & 0.28 \\
\hline New Zealand & $2603(28)$ & $2427(28.7)$ & 0.28 \\
\hline \multicolumn{4}{|l|}{ Type of ICU admitted to, $\mathrm{n}(\%)$} \\
\hline Tertiary & 8259 (88.9) & $7463(88.4)$ & 0.32 \\
\hline Metropolitan & $543(5.8)$ & $540(6.4)$ & 0.12 \\
\hline Rural & $435(4.7)$ & $365(4.3)$ & 0.25 \\
\hline Private & $57(0.613)$ & $75(0.888)$ & 0.03 \\
\hline Source of admission to ICU, n (\%) & $n=9294$ & $n=8443$ & \\
\hline Emergency department & $2672(28.7)$ & $2587(30.6)$ & 0.006 \\
\hline Hospital ward & $943(10.1)$ & $871(10.3)$ & 0.71 \\
\hline Transfer from another ICU & $251(2.7)$ & $240(2.8)$ & 0.57 \\
\hline $\begin{array}{l}\text { Transfer from another hospital } \\
\text { (except from another ICU) }\end{array}$ & $607(6.5)$ & $475(5.6)$ & 0.01 \\
\hline $\begin{array}{l}\text { Operating theatre following } \\
\text { elective surgery }\end{array}$ & $3051(32.8)$ & $2682(31.8)$ & 0.13 \\
\hline $\begin{array}{l}\text { Operating theatre following } \\
\text { emergency surgery }\end{array}$ & $1767(19)$ & $1583(18.7)$ & 0.66 \\
\hline Home & $0(0.0)$ & $3(0.036)$ & 0.11 \\
\hline Unknown & $3(0.032)$ & $2(0.024)$ & 1.00 \\
\hline
\end{tabular}

* unless otherwise stated

Abbreviations: ANZROD: Australian and New Zealand Risk of Death model; APACHE III score: Acute Physiology and Chronic Health Evaluation Severity of illness score; ICU: Intensive Care unit; IQR: Interquartile range; $\mathrm{n}$ : number; SD: standard deviation. 
Table 47 Characteristics of patients in the non-ICU-ROX trial site cohort (pre-ICU-ROX publication vs post publication)

\begin{tabular}{|c|c|c|c|}
\hline Characteristics & $\begin{array}{l}\text { Pre-publication } \\
\text { n=42,204* }\end{array}$ & $\begin{array}{l}\text { Post-publication } \\
n=39,080^{*}\end{array}$ & $P$ value \\
\hline & $n=42,203$ & $n=39,078$ & \\
\hline Age, years, mean (SD) & $60.9 \pm 17.5$ & $60.9 \pm 17.6$ & $<0.001$ \\
\hline \multirow[t]{2}{*}{ Male, sex, n (\%) } & $27,185(64.4)$ & $25,311(64.8)$ & 0.29 \\
\hline & $\mathrm{n}=42,190$ & $n=39,069$ & \\
\hline \multirow[t]{2}{*}{ APACHE III score, mean (SD) } & $59.8 \pm 27.1$ & $59.5 \pm 26.8$ & 0.12 \\
\hline & $n=42,190$ & $n=39,069$ & \\
\hline ANZROD, median (IQR) & $0.025[0.0064-0.128]$ & $0.023[0.0062-0.121]$ & 0.002 \\
\hline Respiratory co-morbidity, n (\%) & $2599(6.2)$ & $2215(5.7)$ & 0.003 \\
\hline Respiratory admission diagnosis, $\mathrm{n}(\%)$ & $4575(10.8)$ & 3881 (9.9) & $<0.001$ \\
\hline Admission type, $n(\%)$ & $n=42,073$ & $\mathrm{n}=38,831$ & \\
\hline Elective admission & $15,688(37.3)$ & $14,366(37.0)$ & 0.39 \\
\hline \multicolumn{4}{|l|}{ Country of admission, $\mathrm{n}(\%)$} \\
\hline Australia & $40,582(96.2)$ & $37,741(96.6)$ & 0.002 \\
\hline New Zealand & $1622(3.8)$ & $1339(3.4)$ & 0.002 \\
\hline \multicolumn{4}{|l|}{ Type of ICU admitted to, $n$ (\%) } \\
\hline Tertiary & $20,468(48.5)$ & $18,961(48.5)$ & 0.95 \\
\hline Metropolitan & $5875(13.9)$ & $5858(15)$ & $<0.001$ \\
\hline Rural & $4429(10.5)$ & $4093(10.5)$ & 0.92 \\
\hline Private & $11,432(27.1)$ & $10,168(26)$ & 0.001 \\
\hline \multicolumn{4}{|l|}{ Source of admission to ICU $-\mathrm{n}(\%)$} \\
\hline Emergency department & $10,336(24.5)$ & $10,246(26.2)$ & $<0.001$ \\
\hline Hospital ward & $4340(10.3)$ & $3842(9.8)$ & 0.03 \\
\hline Transfer from another ICU & $931(2.2)$ & $919(2.4)$ & 0.16 \\
\hline $\begin{array}{l}\text { Transfer from another hospital } \\
\text { (except from another ICU) }\end{array}$ & $2351(5.6)$ & $1900(4.9)$ & $<0.001$ \\
\hline $\begin{array}{l}\text { Operating theatre following } \\
\text { elective surgery }\end{array}$ & $15,621(37)$ & $14,312(36.6)$ & 0.25 \\
\hline $\begin{array}{l}\text { Operating theatre following } \\
\text { emergency surgery }\end{array}$ & $8535(20.2)$ & $7754(19.8)$ & 0.17 \\
\hline Home & $49(0.116)$ & $46(0.118)$ & 0.95 \\
\hline Unknown & $6(0.014)$ & $2(0.005)$ & 0.19 \\
\hline
\end{tabular}

* unless otherwise stated

Abbreviations: ANZROD: Australian and New Zealand Risk of Death model; APACHE III score: Acute Physiology and Chronic Health Evaluation Severity of illness score; ICU: Intensive Care unit; IQR: Interquartile range; $\mathrm{n}$ : number; SD: standard deviation. 


\subsubsection{Arterial oxygen levels}

There was a difference between ICU-ROX and non-ICU-ROX site patients for $\mathrm{PaO}_{2}$ in the first 24 hours in ICU. The interaction $\mathrm{P}$ value of $\mathrm{PaO}_{2}$ was 0.01 , and when adjusted for confounding variables was 0.001 , indicating a difference in the change in $\mathrm{PaO}_{2}$ level coinciding with the publication of the ICU-ROX trial for the ICU-ROX cohort compared with the non-ICU-ROX cohort (Table 48). Patients from ICU-ROX sites had a median $\mathrm{PaO}_{2}$ of $125 \mathrm{mmHg}$ [IQR 83 to 220] pre-publication, and $120 \mathrm{mmHg}$ [IQR 79 to 214] post-publication (estimated difference 3.39 [95\% Cl, 0.53 to 6.25]; $\mathrm{P}=0.001$ ). For patients from non-ICU-ROX sites, the median $\mathrm{PaO}_{2}$ pre and post publication was $105 \mathrm{mmHg}$ [IQR 77 to 162] and 106mmHg [IQR 77 to 162], respectively (estimated difference -0.57 [95\% $\mathrm{Cl},-1.92$ to 0.78]; $\mathrm{P}=0.66$ ).

Segmented regression analysis demonstrated a stepwise change in $\mathrm{PaO}_{2}$ for ICU-ROX site patients $(P=0.01)$ (Figure 29). There was no stepwise change in $\mathrm{PaO}_{2}$ for non-ICU-ROX-site patients $(\mathrm{P}=0.98)$ (Figure 30). The rate of change over time (slope) for $\mathrm{PaO}_{2}$ did not significantly differ for either ICU-ROX or non-ICU-ROX site patients in relation to publication of the ICU-ROX trial ( $\mathrm{P}=0.08$ and 0.51 , respectively) (Figure 29, Figure 30). Results were materially unchanged when adjusted for ANZROD. 
Table $48 \mathrm{~F}_{1} \mathrm{O}_{2}$ and $\mathrm{PaO}_{2}$ levels in patients' first 24 hours of ICU admission for ICU-ROX vs non-ICU-ROX sites

\begin{tabular}{|c|c|c|c|c|}
\hline & $\begin{array}{c}\text { Pre-ICU-ROX } \\
\text { publication cohort }\end{array}$ & $\begin{array}{c}\text { Post ICU-ROX } \\
\text { publication cohort }\end{array}$ & Between group (pre vs post) difference & Interaction $\mathbf{P}$ value \\
\hline ICU-ROX sites & $n=8925$ & $n=8109$ & & \multirow{5}{*}{$\begin{array}{l}\text { Unadjusted } 0.003 \\
\text { Adjusted }^{\dagger}<0.001\end{array}$} \\
\hline Mean (SD) & $0.628 \pm 0.274$ & $0.617 \pm 0.283$ & \multirow{2}{*}{$\begin{array}{c}\text { Unadjusted } \\
0.011(95 \% \mathrm{Cl}[0.003 \text { to } 0.018], \mathrm{P}=0.001) \\
\text { Adjusted }^{+} \\
0.015(95 \% \mathrm{Cl}[0.009 \text { to } 0.022], \mathrm{P}<0.001)\end{array}$} & \\
\hline Median [IQR] & $0.6[0.4$ to 1.0$]$ & $0.6[0.38$ to 1.0$]$ & & \\
\hline Mean (SD) & $0.536 \pm 0.243$ & $0.537 \pm 0.245$ & \multirow{2}{*}{$\begin{array}{c}\text { Unadjusted } \\
-0.002(95 \% \mathrm{Cl}[-0.005 \text { to } 0.002], \mathrm{P}=0.31) \\
\text { Adjusted }^{+} \\
0.003(95 \% \mathrm{Cl}[-0.006 \text { to } 0.001], \mathrm{P}=0.11)\end{array}$} & \\
\hline Median [IQR] & $0.5[0.35$ to 0.6$]$ & $0.5[0.35$ to 0.6$]$ & & \\
\hline \multicolumn{5}{|l|}{$\mathrm{PaO}_{2}(\mathrm{mmHg})^{*}$} \\
\hline Median [IQR] & 125 [82 to 220$]$ & 120 [79 to 214$]$ & $\begin{array}{c}\text { Unadjusted } \\
3.39(95 \% \mathrm{Cl}[0.53 \text { to } 6.25], \mathrm{P}=0.001) \\
\text { Adjusted }^{+} \\
5.09(95 \% \mathrm{Cl}[2.53 \text { to } 7.65], \mathrm{P}<0.001)\end{array}$ & \multirow{4}{*}{$\begin{array}{l}\text { Unadjusted } 0.01 \\
\text { Adjusted }^{+} 0.001\end{array}$} \\
\hline Non-ICU-ROX sites & $n=39738$ & $n=36678$ & & \\
\hline Mean (SD) & $136 \pm 90$ & $136 \pm 90.6$ & \multirow{2}{*}{$\begin{array}{c}\text { Unadjusted } \\
-0.57(95 \% \mathrm{Cl}[-1.92 \text { to } 0.78], \mathrm{P}=0.66) \\
\text { Adjusted }^{+} \\
0.17(95 \% \mathrm{Cl}[-1.04 \text { to } 1.38], \mathrm{P}=0.78)\end{array}$} & \\
\hline Median [IQR] & 105 [77 to 162$]$ & 106 [77 to 162$]$ & & \\
\hline
\end{tabular}

${ }^{*} \mathrm{~F}_{\mathrm{i}} \mathrm{O}_{2}$ and $\mathrm{PaO}_{2}$ from the arterial blood gas taken during the first 24 hours of the ICU admission that produces the highest (worst) score using the APACHE III-J scoring algorithm + adjusted for age, ANZROD score, elective vs emergency admission, ICU source, respiratory diagnosis, chronic respiratory comorbidity, sex and site

Abbreviations: ANZROD: Australian and New Zealand Risk of Death model; Cl: confidence interval; FiO2: fraction of expired oxygen; ICU: Intensive Care Unit; ICU-ROX sites: ICUs that participated in the ICU-ROX trial, IQR: Interquartile range; n: number; non-ICU-ROX sites: ICUs that did not participate in the ICU-ROX trial; $\mathrm{PaO}{ }_{2}$ : partial pressure of arterial oxygen; mmHg: millimetres of mercury; SD: standard deviation. 


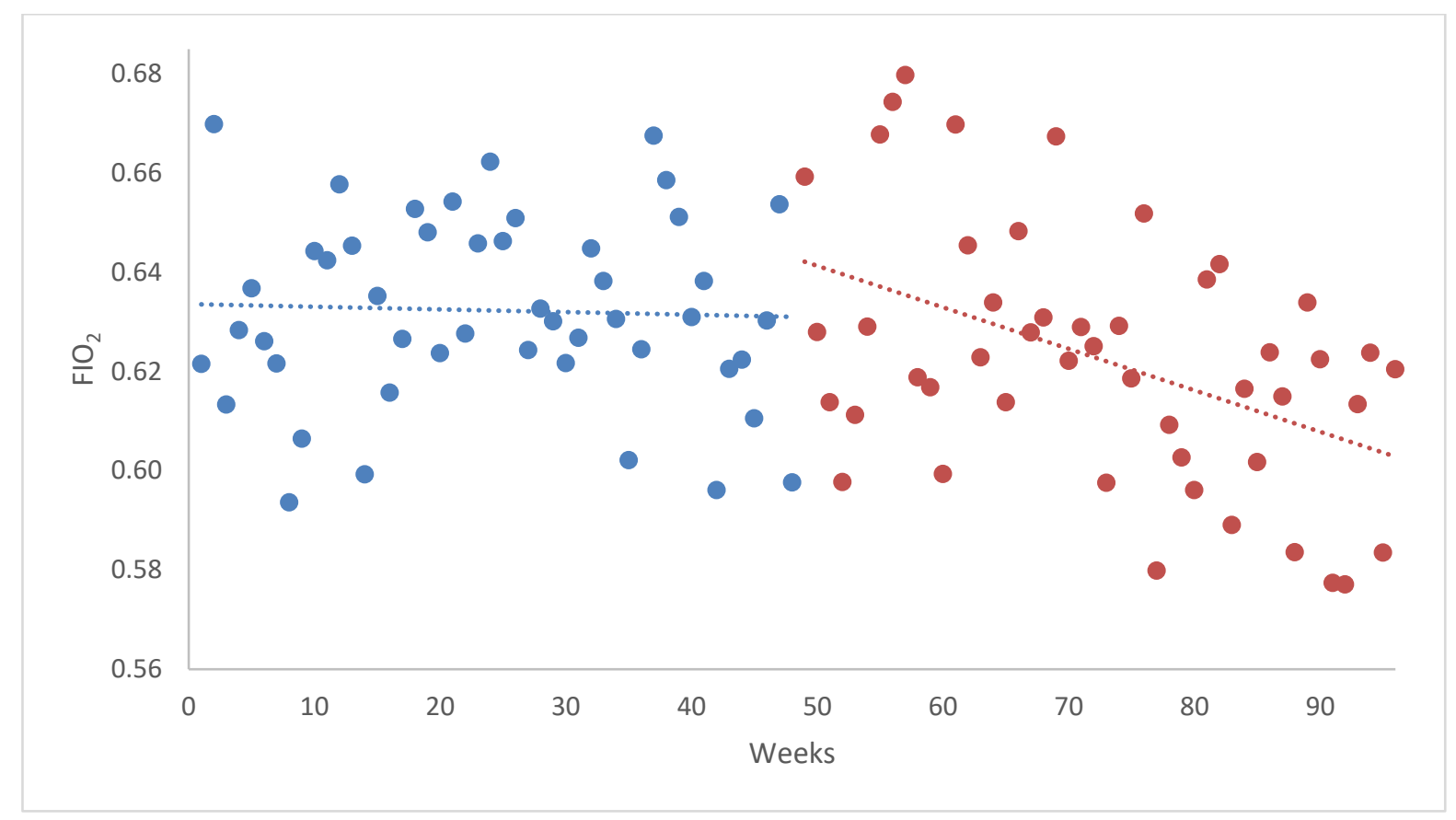

Figure $27 \mathrm{~F}_{1} \mathrm{O}_{2} *$ in the first 24 hours in the ICU by week (ICU-ROX sites) ${ }^{\dagger}$

* $\mathrm{F}_{1} \mathrm{O}_{2}$ is the $\mathrm{F}_{1} \mathrm{O}_{2}$ from the arterial blood gas taken during the first 24 hours of the ICU admission that produces the highest (worst) score using the APACHE III-J scoring algorithm. Data points represent the average $\mathrm{F}_{1} \mathrm{O}_{2}$ for eligible patients by week. The blue dots represent these data for the period 1 November 2018 to 30 September 2019 (prior to publication of the ICU-ROX trial results) and the red dots represent these data for the period 1 November 2019 to 30 September 2020 (after publication of the ICU-ROX trial results).

${ }^{+} \mathrm{P}=0.18$ for stepwise change

$P=0.20$ for stepwise change (adjusted for ANZROD)

$\mathrm{P}=0.01$ for change in slope

$\mathrm{P}=0.02$ for change in slope (adjusted for ANZROD)

Abbreviations: APACHE-III-J: Acute Physiology and Chronic Health Evaluation; $\mathrm{F}_{1} \mathrm{O}_{2}$ : fraction of inspired oxygen; ICU: Intensive Care Unit; ICU-ROX sites: ICUs that participated in the ICU-ROX trial. 


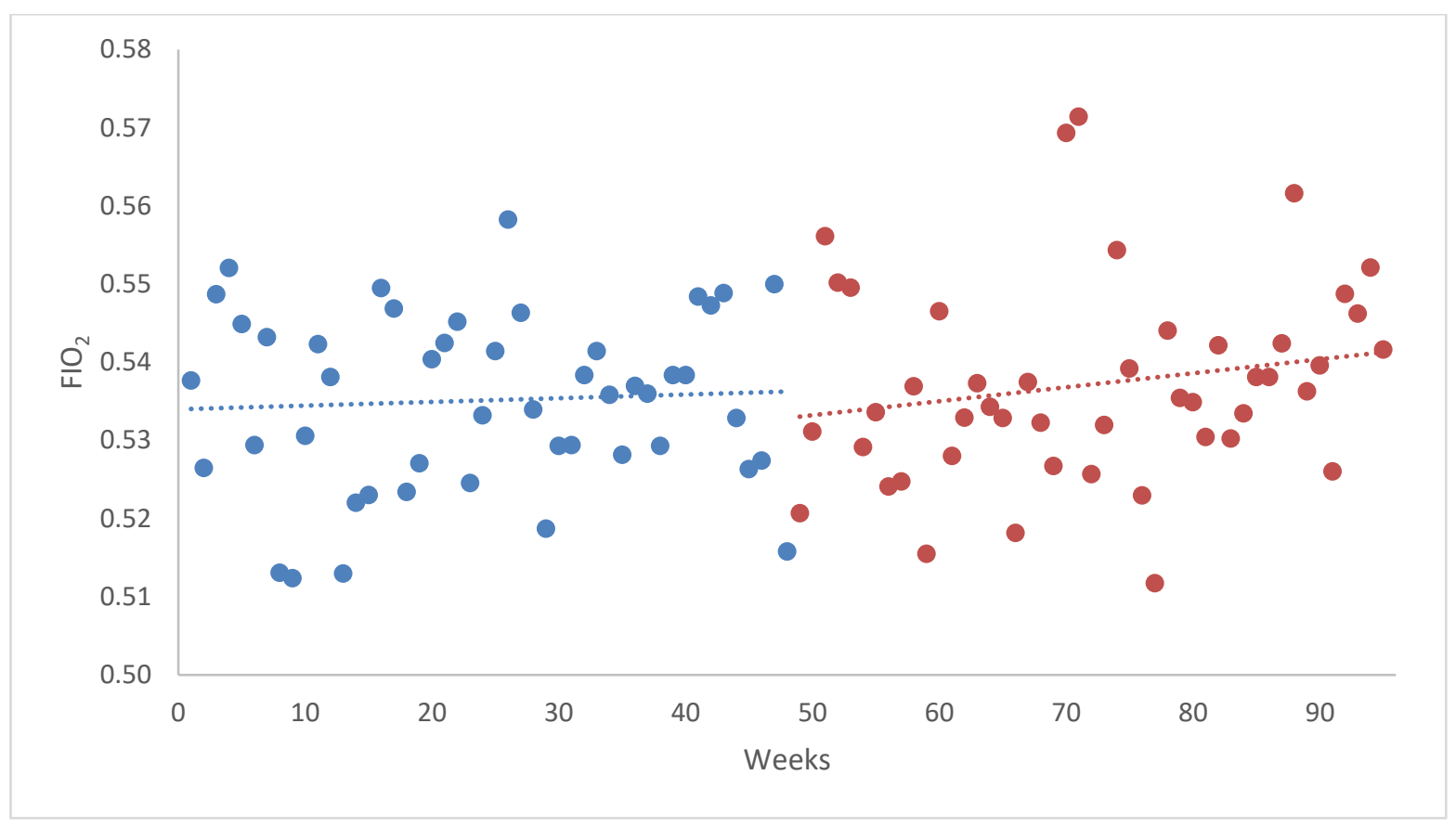

\section{Figure $28 \mathrm{~F}_{1} \mathrm{O}_{2}{ }^{*}$ in the first 24 hours in the ICU by week (non-ICU-ROX sites) ${ }^{\dagger}$}

* $\mathrm{F}_{1} \mathrm{O}_{2}$ is the $\mathrm{F}_{1} \mathrm{O}_{2}$ from the arterial blood gas taken during the first 24 hours of the ICU admission that produces the highest (worst) score using the APACHE III-J scoring algorithm. Data points represent the average $\mathrm{F}_{1} \mathrm{O}_{2}$ for eligible patients by week. The blue dots represent these data for the period 1 November 2018 to 30 September 2019 (prior to publication of the ICU-ROX trial results) and the red dots represent these data for the period 1 November 2019 to September 2020 (after publication of the ICU-ROX trial results).

${ }^{+} \mathrm{P}=0.59$ for stepwise change

$P=0.40$ for stepwise change (adjusted for ANZROD)

$\mathrm{P}=0.49$ for change in slope

$\mathrm{P}=0.60$ for change in slope (adjusted for ANZROD)

Abbreviations: APACHE-III-J: Acute Physiology and Chronic Health Evaluation; $\mathrm{F}_{1} \mathrm{O}_{2}$ : fraction of inspired oxygen; ICU: Intensive Care Unit; ICU-ROX sites: ICUs that participated in the ICU-ROX trial. 


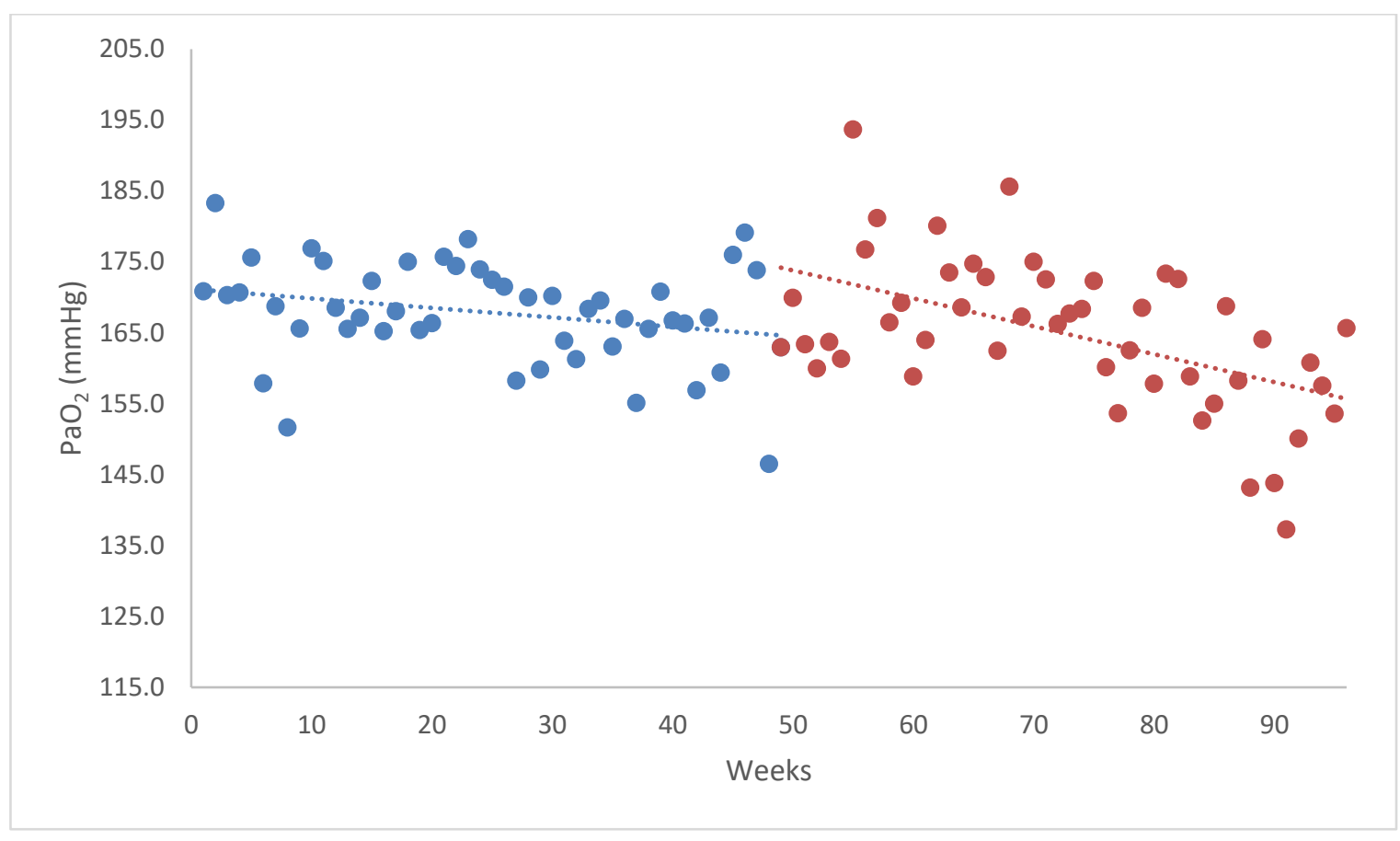

\section{Figure $29 \mathrm{PaO}_{2}{ }^{*}$ in the first 24 hours in the ICU by week (ICU-ROX sites) ${ }^{\dagger}$}

* $\mathrm{PaO}_{2}$ is the $\mathrm{PaO}_{2}$ from the arterial blood gas taken during the first 24 hours of the ICU admission that produces the highest (worst) score using the APACHE III-J scoring algorithm. Data points represent the average $\mathrm{PaO}_{2}$ for eligible patients by week. The blue dots represent these data for the period 1 November 2018 to 30 September 2019 (prior to publication of the ICU-ROX trial results) and the red dots represent these data for the period 1 November 2019 to September 2020 (after publication of the ICU-ROX trial results).

${ }^{+} \mathrm{P}=0.01$ for stepwise change $P=0.02$ for stepwise change (adjusted for ANZROD) $\mathrm{P}=0.08$ for change in slope $\mathrm{P}=0.10$ for change in slope (adjusted for ANZROD)

Abbreviations: APACHE-III-J: Acute Physiology and Chronic Health Evaluation; ICU: Intensive Care Unit; ICUROX sites: ICUs that participated in the ICU-ROX trial; $\mathrm{mmHg}$ : millimetres of mercury; $\mathrm{PaO}_{2}$ : partial pressure of arterial oxygen. 


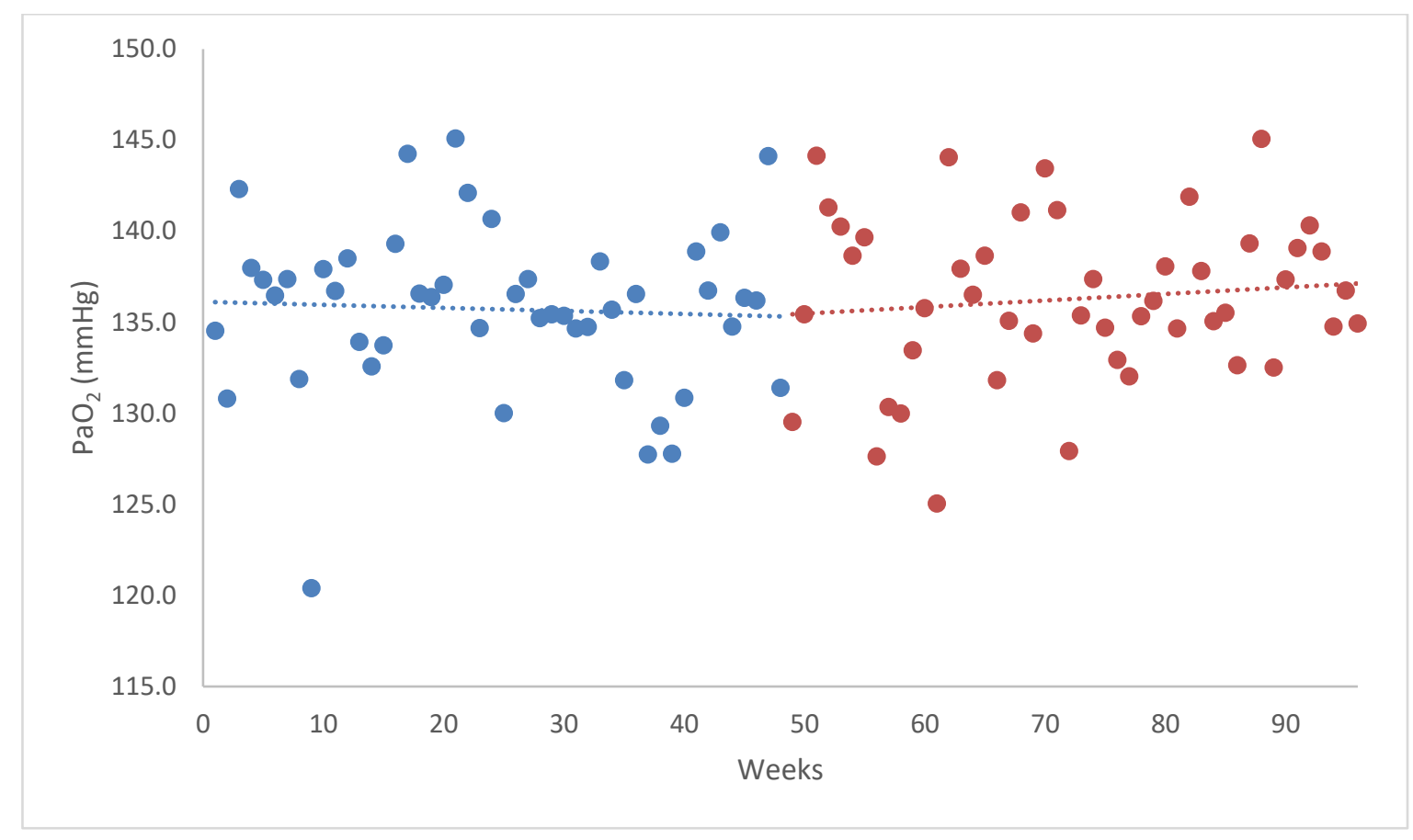

\section{Figure $30 \mathrm{PaO}_{2}{ }^{*}$ in the first 24 hours in the ICU by week (non-ICU-ROX sites) ${ }^{\dagger}$}

* $\mathrm{PaO}_{2}$ is the $\mathrm{PaO}_{2}$ from the arterial blood gas taken during the first 24 hours of the ICU admission that produces the highest score using the APACHE III-J scoring algorithm. Data points represent the average $\mathrm{PaO}_{2}$ for eligible patients by week. The blue dots represent these data for the period 1 November 2018 to 30 September 2019 (prior to publication of the ICU-ROX trial results) and the red dots represent these data for the period 1 November 2019 to September 2020 (after publication of the ICU-ROX trial results).

${ }^{+} \mathrm{P}=0.98$ for stepwise change $P=0.41$ for stepwise change (adjusted for ANZROD) $\mathrm{P}=0.51$ for change in slope $\mathrm{P}=0.81$ for change in slope (adjusted for ANZROD)

Abbreviations: APACHE-III-J: Acute Physiology and Chronic Health Evaluation; ICU: Intensive Care Unit; $\mathrm{mmHg}$ : millimetres of mercury; non-ICU-ROX sites: ICUs that did not participate in the ICU-ROX trial; $\mathrm{PaO}_{2}$ : partial pressure of arterial oxygen. 


\subsection{Discussion}

The main finding of this retrospective cohort study was that average weekly $\mathrm{F}_{1} \mathrm{O}_{2}$ (slope) decreased in ICU-ROX but not non-ICU-ROX sites after publication of the ICU-ROX trial. No stepwise change in $\mathrm{F}_{1} \mathrm{O}_{2}$ was seen in either ICU-ROX or non-ICU-ROX sites. There was a stepwise increase in $\mathrm{PaO}_{2}$ levels in ICU-ROX sites but not in non-ICU-ROX sites; however, no change in average weekly (slope) $\mathrm{PaO}_{2}$ was found in either cohort. It appears that the differences, while statistically significant for the ICU-ROX sites, are very small.

No other studies have reviewed oxygen over time in relation to participation in a research trial. However, Eastwood, Bellomo et al. (2012) used the APD to analyse $\mathrm{F}_{1} \mathrm{O}_{2}$ between 2000 and 2009. The mean $\mathrm{F}_{1} \mathrm{O}_{2}$ of 0.62 was similar to the mean $\mathrm{F}_{1} \mathrm{O}_{2}$ in the ICU-ROX cohorts in the current study, although the mean was lower in the non-ICU-ROX cohorts.

The findings that participation in ICU-ROX changed the average $\mathrm{F}_{1} \mathrm{O}_{2}$ in ICU-ROX sites was similar to registry-based studies in the knowledge translation literature (Corrigan \& Glass, 2005; Ketley \& Woods, 1993; Kizer et al., 1999). Corrigan and Glass (2005) who using existing registry data, showed physicians who had participated in at least one RCT about new drugs, were more likely to prescribe new drugs three months after the drug launch. Conversely, a number of studies showed no change in practice following site participation in an RCT, despite compelling evidence (Khera et al., 2018; Majumdar et al., 2002; Shah et al., 2010). The finding in the current study is complicated by ICU-ROX not showing a difference in the primary outcome of ventilator-free days at Day 28 , thus neither confirming nor refuting current knowledge translation evidence.

Methodologically, this study was very similar to two studies set in ANZ ICUs (Kaukonen et al., 2013; Salter et al., 2018). The current research shows a decrease in the average weekly $\mathrm{F}_{1} \mathrm{O}_{2}$ over time in the ICU-ROX trial sites. However, unlike the research by Salter et al. (2018) which suggested that knowledge translation of a publication about targeted temperature management following cardiac arrest was rapidly adopted in ANZ ICUs, no immediate change (step) in practice was seen after publication of the ICU-ROX results. One explanation for this is that the evidence from ICU-ROX did not show a significant difference in ventilatorfree days between conservative and usual oxygen therapy, whereas the publication 
regarding temperature management, examined in Salter et al. showed strong evidence that cooling patients to $36^{\circ} \mathrm{C}$ had a similar outcome to cooling them to $33^{\circ} \mathrm{C}$. Another explanation is that the practice change in Salter et al.'s study was easier to implement than the alternative, as cooling patients to $33^{\circ} \mathrm{C}$ requires significantly more resources and closer monitoring than cooling to $36^{\circ} \mathrm{C}$ (Nielsen et al., 2013).

Inherent in researching existing databases, this study has limitations. Primarily, the oxygenrelated data available in the $A P D$ is the $\mathrm{F}_{1} \mathrm{O}_{2}$ and $\mathrm{PaO}_{2}$ from the arterial blood gas associated with the worst APACHE III-J score in the first 24 hours of ICU stay. The primary purpose of these measures is to calculate the illness severity and risk of death, for benchmarking. While these data are used to measure the oxygen exposure the patient experiences, they are an imperfect measure to overall exposure. All ABGs recorded in the first 24 hours are assessed but only the worst is recorded in the APD, and there is no component which measures the time of exposure to a given $\mathrm{F}_{1} \mathrm{O}_{2}$. This means that even if a patient had one high scoring $A B G$, and the for remainder of their first 24 hours in $\mathrm{ICU}$, they were receiving $\mathrm{F}_{1} \mathrm{O}_{2}$ below 0.5 , this would not be reflected. In addition, if a patient is on an $\mathrm{F}_{1} \mathrm{O}_{2}$ above 0.5 , the score will be very different than if the $\mathrm{F}_{1} \mathrm{O}_{2}$ is below 0.5. As an example, if a patient was intubated and receiving an $\mathrm{F}_{1} \mathrm{O}_{2}$ of 0.51 with a $\mathrm{PaO}_{2}$ of $80 \mathrm{mmHg}$ and $\mathrm{PaCO}_{2}$ of $40 \mathrm{mmHg}$, their $\mathrm{A}$-a gradient would be 233.6 and they would score 7 points (out of a maximum 14). If another patient was ventilated on $\mathrm{F}_{1} \mathrm{O}_{2}$ of 0.49 , with a $\mathrm{PaO}_{2}$ of $80 \mathrm{mmHg}$, they would score 0 (out of a maximum 15 for this category). However, even though the data were not a perfect match for the research question, the availability of these data allowed analysis on a scale that would be very difficult by any other means.

In addition, while there are validity strategies for the ANZICS adult patient database, as outlined in section 8.3.2, the findings of this study are only as reliable as the data in the database. Obtaining these data require the data collector to review all available $A B G$ results for the first 24 hours of ICU stay, and select the worst. Electronic tools are available to assist; however, this process is still subject to human error. 
Unlike the findings of Das et al. (2008) and Kizer et al. (1999), no patients included in the ICU-ROX cohort were subject to the protocolised study treatment, as enrolment finished in May 2018; five months prior to the data analysed for this retrospective cohort study

The timeframe for data analysis was constrained by the data available. Data are entered into the APD quarterly, and it is not uncommon for sites to be at least one quarter (of a year) behind in data entry. In order to conduct this study in the available timeframe, the end date was 30 September 2020 (11 months after ICU-ROX publication), which was matched with 11 months before publication of ICU-ROX in October 2019.

In conclusion, similar to the inception cohort study, differences in oxygen therapy practice changes for ICU-ROX compared to non-ICU-ROX sites in the retrospective cohort study were small, and likely to be of limited clinical importance. 


\section{CHAPTER 9 INTEGRATED DISCUSSION AND CONCLUSION}

The overall purpose of this research was to increase the understanding of knowledge translation in the ICU setting. Specifically, the aim was to determine whether participating in the ICU-ROX trial changed the attitudes and practices of healthcare practitioners' who worked in an ICU that participated in the ICU-ROX trial, compared to those from an ICU that did not participate. Additionally, whether there was a difference between the attitudes of ICU specialist doctors and ICU nurses in relation to conservative oxygen therapy was explored. Finally, an evaluation of the effectiveness of the ICU-ROX dissemination plan was conducted.

Three studies, using different methods were conducted to answer the question about whether participating in research about conservative oxygen therapy changed attitudes and practices, compared to non-participation in ICU-ROX. The first was a survey over time to assess whether attitudes changed in relation to participation in the ICU-ROX trial. The second was an inception cohort study to review oxygen management practices in the same ICUs, over time. The third was a retrospective cohort study to review temporal trends regarding oxygen management in ANZ ICUs before and after ICU-ROX publication, and between ICUs that participated in ICU-ROX compared to ICUs that did not. This overall design was chosen to capture knowledge translation from different perspectives.

\subsection{Summary of key findings}

The findings from the three studies were inconsistent, and complicated by the ICU-ROX trial result showing no difference between conservative and usual oxygen therapy; thereby restoring equipoise to the clinical question of how much oxygen to use for mechanically ventilated patients in ICU.

The survey showed that there was no difference in the baseline attitudes about conservative oxygen therapy (using $\mathrm{F}_{1} \mathrm{O}_{2}$ 0.21) of health practitioners from the ICUs which participated in ICU-ROX (ICU-ROX sites), compared to those from ICUs that did not participate (non-ICUROX sites). There was, however, a statistically significant difference between the health practitioners' attitudes from ICU-ROX sites compared to non-ICU-ROX sites after ICU-ROX was conducted. Once the ICU-ROX results were published, this difference in attitudes 
remained for most patient admission categories. Both groups reported more likelihood of using a conservative approach over time, and this was statistically significant in both groups after ICU-ROX was conducted, compared to before ICU-ROX. The ICU-ROX site health practitioners reportedly became even more conservative after ICU-ROX publication, a change not seen in the non-ICU-ROX respondents.

Nurses reported they were less likely to use an $\mathrm{F}_{1} \mathrm{O}_{2}$ of 0.21 than doctors, before ICU-ROX started. This difference remained after ICU-ROX was completed but before the results were known. After ICU-ROX was published, there was a difference in attitudes between doctors and nurses for post-operative surgical patients, those with a suspected hypoxic encephalopathy and patients with pneumonia. In all cases, the doctors were reportedly more likely to use an $\mathrm{F}_{1} \mathrm{O}_{2}$ of 0.21 than nurses.

The inception cohort study showed a difference in oxygen management practices between the ICU-ROX and non-ICU-ROX sites before ICU-ROX started. The ICU-ROX sites were more conservative, using an $\mathrm{F}_{1} \mathrm{O}_{2}$ of 0.21 in patients while ventilated, a higher percentage of the time. Oxygen management practices in ICU-ROX sites did not change over time, in relation to participation in ICU-ROX or publication of the ICU-ROX results. However, there was a significant increase in the percentage of time patients were ventilated at $\mathrm{F}_{1} \mathrm{O}_{2}$ of 0.21 in the non-ICU-ROX sites after ICU-ROX publication, compared to before ICU-ROX started. Overall, the use of conservative oxygen therapy in mechanically ventilated ICU patients was uncommon. There are many reasons the practices with regard to oxygen management did not change at the ICU-ROX sites, despite the change in attitudes. Previous research has reported that what clinicians say their attitudes to oxygen management is not necessarily reflected in practice (Helmerhorst et al., 2014). An alternative explanation is that there are many staff involved in decision-making at a site level, and these decisions would have been made by more than just the practitioner attitudes survey respondents. The influence of publications about oxygen management in ICU, other than the ICU-ROX results is unknown.

In contrast to the inception cohort study, the retrospective cohort study showed a change in oxygen practices of ICU-ROX sites, but not non-ICU-ROX sites. Specifically, there was a difference in the trend of the average $\mathrm{F}_{1} \mathrm{O}_{2}$ in mechanically ventilated patients in the ICU- 
ROX sites, and not in the non-ICU-ROX sites, after publication of ICU-ROX. This study incorporated all ANZ ICUs (which contribute to the ANZICS adult patient database), so showed a broader view of practices than the inception cohort study. The data examined differed from those examined in the inception cohort study, as only routinely collected data could be used. Overall, the magnitude of the differences in oxygen therapy practice changes for ICU-ROX compared to non-ICU-ROX sites shown in the inception cohort and retrospective cohort studies were small and of uncertain clinical importance.

There was no difference between ICU-ROX and non-ICU-ROX site health practitioners in their knowledge of the ICU-ROX trial, indicating the ICU-ROX dissemination plan reached the target audience. However, analysis by role showed that the dissemination plan had worked for ICU specialist doctors but not ICU nurses. The ICU doctors were statistically significantly more likely to have heard the ICU-ROX results and to have read the ICU-ROX publication than the nurses. This finding is important, because ICU nurses were reportedly the role most likely to adjust oxygen at the bedside. Dissemination of the ICU-ROX results was by multiple modes but on review of the literature (Ebenezer, 2015; Marshall et al., 2011; Thompson et al., 2001), all methods employed were more suited to doctors than nurses, such as reading published papers, attending conferences and following social media. Additionally, while results were disseminated to participating sites' research teams, modes of dissemination at an ICU level to clinical staff varied between ICUs.

Most ICUs reported having a research champion and knowledge leader in their ICU. These included (usually senior) doctors and nurses, and often included the research teams. Over half of ICUs surveyed had a process for change management. However; these varied in formality between ICUs and the time to implement a change were variable, depending on the nature of the change and the ICU.

\subsection{Contribution to knowledge}

This research has contributed to the literature by conducting a study about the knowledge translation effects of participation in an ICU trial about oxygen, in Australia and New Zealand. It has shown that participation changed attitudes to conservative oxygen therapy but did not consistently change practices. Both doctors and nurses were informants in this 
research in similar numbers; with nurses previously underrepresented in the literature. Additionally, a knowledge translation study reviewing the impact of participating in a trial about oxygen has not previously been conducted. Using multiple methods was uncommon in the knowledge translation literature about participation in research trials. Comparing participation and non-participation in a clinical trial, while not new, had not prospectively been researched before. The approach of beginning the research before a trial began, and following through until after the trial results were published and disseminated has not previously been undertaken in the literature. Open-ended questions provided unexpected depth and insights into knowledge translation and oxygen management practices in ICU. Of significant importance, this research has provided evidence of the importance of targeting future dissemination plans.

The ICU-ROX trial found that conservative oxygen therapy did not decrease alive, ventilatorfree days to day 28 , thus restoring equipoise to the overall issue. It is common to find no difference between the intervention and comparator in ICU studies (Chapman et al., 2018; Young et al., 2015); however, dissemination of research findings is important regardless of the result. ICU-ROX also showed that conservative oxygen therapy was not more harmful than standard (liberal) care, which is a finding worthy of disseminating to health practitioners, and may influence some health practitioner's attitudes. This research has shown research results should be disseminated in a way that all health practitioners understand. For research which does not indicate a change in practice, emphasising the 'implications of research' and 'what the research means for their clinical practice' are important aspects of dissemination, especially when the research findings are not clear-cut.

Attitudes to oxygen management in ICU had previously been reported in the literature. The findings in this research add to review of self-reported opinion about oxygen management of ANZ ICU specialists and ICU nurses (Eastwood et al., 2011; Eastwood, Reade, et al., 2012), conducted ten years ago. That health practitioners' attitudes do not always translate into practice was similar to Helmerhorst et al. (2014) which reported attitudes were more conservative than practices and highlights how complex this issue is. The finding that nurses' were less likely to use conservative oxygen management than doctors aligned with the 
finding by Grim, (2020) which found nurses were less accepting of low $\mathrm{SaO}_{2}$ and $\mathrm{PaO}_{2}$ targets than doctors.

This research has added to the literature about nurses learning new information from people, rather than written evidence, such as publications. This knowledge was well-stated in the literature, but not previously considered in respect to new research findings (Ebenezer, 2015; Marshall et al., 2011; Thompson et al., 2001). The work by Marshall et al., who previously conducted qualitative research in a single Australian ICU, has been extended on by including multiple ICUs and comparing nurses' ways of finding out the ICU-ROX results with doctors'. Furthermore, the current research has combined the findings about how nurses receive information, with findings about how trial results were disseminated at an ICU level. The concept of targeting dissemination to the audience, and that publishing results is insufficient to translate knowledge into practice is not new, and was previously stated in the literature, and in official guidance documents almost 20 years ago (Ministry of Health, 2002). The current research, however, provides empirical evidence that more can be done to improve targeted dissemination.

Only one small study has previously reported about how results were disseminated, following participation in a study (Sweeting et al., 2020), beyond diffusion by publication and conference presentation. This study has included how the ICU-ROX results were disseminated and reviewed the effectiveness of that plan, by reviewing the knowledge of the results by the end users - ICU doctors and nurses. In addition, the current research has examined dissemination, knowledge translation and change management practices in ANZ ICUs, which had not previously been reported. While limited findings were obtained, this is a useful addition to understanding how to disseminate research findings in the future.

\subsection{Overall strengths and limitations}

The contemporaneity of this study was both a strength and limitation. Knowledge translation research is usually conducted when the evidence is already known. At the start of this study, the evidence that ICU-ROX would produce was not known. However, conducting this study in a 'real world' way mirrors the difficulty that researchers applying 
for grants for RCTs and similar studies encounter; anticipating how they will translate knowledge several years in advance of having that knowledge.

All three studies were observational and the survey was self-reported; these were the best designs to use. There were few other ways the research questions could have been answered, and conducting the research using multiple methods added to the robustness of the findings. Another key strength was the validity measures used. Specifically, biases were acknowledged and mitigated where possible. In addition, validity measures were used and reported; including validation and piloting of the survey by an expert group, using prespecified statistical analysis plans for all analyses and de-identification of respondents prior to analysis. Every effort was made to ensure that in my role as ICU-ROX project manager, I had no influence on ICU-ROX results.

The limitations of this research include the purposeful recruitment of sites and survey respondents as this may reduce the generalisability of the study findings. Additionally, ICUs that participated in these two studies were likely to already have a strong research culture, as they were recruited via the ANZICS-CTG network. It is unknown whether a strong research culture exists in other ANZ ICUs, although inclusion of the retrospective cohort study means the practices of all ICUs that contribute to the ANZICS APD were included. The research was also limited to review of oxygen practices and the impact of the ICU-ROX trial to ANZ ICUs, however, the overall concepts could be extended to other clinical trials in ICU.

\subsection{Future research}

Further investigation is required to fully understand the relevance of the differences between ICU doctors and nurses in finding out research results. It is difficult to speculate whether disseminating in a way that the literature indicates nurses are more receptive to, would have changed their knowledge of the ICU-ROX results. However, an alternative way to address this issue in a more conclusive manner would be to randomise dissemination strategies according to site, and then reviewing the knowledge by role.

Communicating to nurses is important, and the literature about how nurses learn and understand new information suggests that targeted approaches have not previously been 
adequate. Review of the literature found that nurses commonly learn from a person they trust, as was seen in this research. A future important addition to the current research, would be investigation of who the "someone at work" that people heard the ICU-ROX results from, was. Knowing who this person or people were has important implications for future dissemination plans, so that key influential people can be targeted and supported.

Future research could include undertaking a study of ICU nurses' views about the best way to learn about research results. The current research showed that only one nurse heard about the ICU-ROX results from social media. Understanding why this medium did not work for nurses is important. Questions about which forms of social media (if any) nurses prefer, and what factors would increase their use of social media (e.g., following nurse leaders, rather than medical) may be important for future research dissemination plans.

This research showed that nurses learn clinical skills and knowledge differently to doctors. Future knowledge translation research should always include all key practitioners to whom the research findings will be implemented, as well as key informants such as change agents, (e.g., research co-ordinators and nurse educators). In almost every clinical specialty, nurses are significantly involved, and should be considered when dissemination is planned. Little research was found about allied health professions, such as dietitians, physiotherapists and pharmacists in relation to knowledge translation. These roles hold a key place in ICU and other clinical areas, often work in isolation, and understanding their view would be an important addition to future research.

ICU research has rarely used a framework when disseminating research findings, or reviewing barriers and facilitators to implement research. The PARIHS framework was found to be fit for purpose for this study; specifically, the components of Evidence, Context and Facilitation were relevant. Facilitation usually refers to a role or person in PARIHS, but in the current research was used to include the dissemination of the ICU-ROX plan, which was a useful interpretation. There are, however, many other available frameworks not reviewed in-depth in this thesis and future ICU knowledge translation research should consider not only using a framework to assess barriers and facilitators of knowledge translation, but assessing which framework would work best. The findings of this thesis showed strategies 
that worked and did not work for the dissemination of one ICU trial, and could be used as a starting point in consideration of barriers and facilitators in future ICU research dissemination.

One area not covered by this research was the patient experience. Part of ethical approval in New Zealand includes sending research results to participants (unless they choose to not receive them). Provision of results is usually one paragraph in a letter posted or emailed, and invites participants to phone the local research team if they have any questions. Future knowledge translation studies could review this practice and investigate other ways of including patients in research at an earlier stage.

Given the continuous nature of new research emerging that impacts ICU, health practitioners need to balance between being overwhelmed with information and knowing when change should be made in relation to research. Synthesising vast amounts of research is difficult and beyond the skills and time of most individuals. There are, however, useful critical review websites that do this such as 'The Bottom Line' or Critical Care Review. Following key trusted people on social media is another way to keep abreast of change. Consideration in the future needs to include how we communicate to all ICU staff that new research exists on a specific topic. Culture was identified in the literature as a major factor for whether research was used or not (Bringsvor et al., 2014; A. Grant \& Coyer, 2019; Livingston et al., 2020; Sauro et al., 2019). Culture can be difficult to change, but should be recognised, when assessing barriers to knowledge translation.

\subsection{Implications for practice}

This research has implications for the way research results are disseminated in the future. While set around oxygen management in ICU, these results may apply to future ICU research or other clinical areas. The implications of this research fall into four main categories: increasing participation in research; role of funding and research organisations such as HRC and ANZICS CTG, researchers and ICU clinicians. Specifically, funders, researchers and clinicians must think differently and be aware of the different vehicles of dissemination that work for different roles. 
The results from this study showed that participation changed the attitudes of the health practitioners from ICUs that participated in ICU-ROX. That participating in research opened the minds of nurses in relation to oxygen management was a useful finding, and could still lead to change in practice, or increased awareness of future research results. ICUs should be encouraged to participate in research trials. However, funding ICU research remains an issue and to enable more ICUs to participate in research involves increased funding opportunities to support research coordinator and project coordination time. The loss of one site before the end of this study, because their research coordinator resigned and was not replaced, highlighted the tenuous place research holds in the ICU infrastructure. Many research staff are on temporary contracts, and working in their own time to maintain a research presence (Eastwood et al., 2013; Mackle \& Nelson, 2018). This is despite clear guidelines for ICUs that they must maintain a research programme and Level II and III ICUs must employ a full-time research coordinator (CICM, 2016).

The Health Research Council of NZ already requires researchers applying for grants to submit a plan for dissemination of research results. The timing of these plans remains problematic, as it may not be possible to identify the target audience for research results at the time a funding application is submitted. Identification of these key stakeholders, and adjusting dissemination to suit their learning needs is imperative to ensure the right people receive the right information in a way they understand. Researchers need to report on how they addressed these issues in the final HRC report. Ensuring that HRC funded research findings have been disseminated takes time and money, and researchers need to be supported to do this.

Similarly, the ANZICS CTG need to consider how research they endorse is disseminated to all key stakeholders, addressing the specific needs of ICU nurses, who are the predominant workforce in ICU. The current research highlights that a comprehensive and multifaceted dissemination plan did not reach ICU nurses, and this needs to be considered in the future dissemination of research in the ANZ ICU setting.

This research has significant implications for researchers, and recognition that different ways of disseminating research results may need to be added to future plans. It is clear that 
publication, even in a high impact journal, and presentation at worldwide conferences is insufficient as a plan for dissemination of results. These methods generally target doctors, and do not address the learning needs of all ICU health practitioners. Reading new information takes time, and many doctors and nurses may not have the time or resources to read published papers or keep up with recent research. The literature has previously suggested the use of 'one-page' summaries, similar to the tweetorial used for the ICU-ROX trial; these may be a useful addition to the toolkit of targeting all health practitioners. Additionally, video explanation or use of infographics may assist in the dissemination of research findings. Researchers may need to provide clear direction to ICU research staff about the best way to deliver new results to all ICU staff although it is recognised that even a targeted response, may not change the information the end-user receives, understands or uses.

At an ICU level, senior doctors and nurses need to be aware that current research dissemination avenues are not working for ICU nurses. Senior nurses need to be more involved in this, and journal clubs need to not only 'allow' nurses, but encourage their attendance. Research teams in ICUs are well-placed to not only know about current research findings, but to disseminate these findings in ways that all staff understand.

\subsection{Recommendations}

Recommendations to address the implications are provided for four groups: funders such as HRC, ANZICS CTG, researchers and ICU clinical staff. The recommendations from this research are about increasing participation in research and dissemination of research results, and specifically targeting results to key stakeholders.

\subsubsection{Health Research Council of NZ (and other funders)}

Funders, such as the HRC, are in a unique position to affect change, and the following are recommended.

- Provide a template offering prompts of different dissemination methods and for planned dissemination, similar to the one designed by the Canadian Institute of Health Research (CIHR, 2012). 
- Require researchers to include in specific terms in their final report to HRC about how the research was disseminated and follow-up with investigators if the dissemination was not considered adequate.

- Increase funding so that all ICUs can participate in research.

- Increase resourcing of researchers to enable dissemination to be prioritised.

\subsubsection{ANZICS-CTG}

Like the HRC, ANZICS-CTG is in a unique position to affect change in ICU research, as it reviews CTG-endorsement applications. The following could be incorporated into the process:

- Require researchers to include an acceptable dissemination plan, which includes identification of the target audience. This should include specific information about communicating to nurses, and other non-medical roles, when applying for CTG endorsement.

- Review whether results have been disseminated to an acceptable level when the final report is submitted.

- Encourage ICUs to participate in research, enhancing a research culture.

- A translation into research practice group already exists; however, this could be further developed and should at least determine whether a shared framework should be adopted for knowledge translation in the ANZ ICU setting.

\subsubsection{Researchers}

Dissemination plans need to be detailed and consider the following:

- Assess and identify the target audience/ key stakeholders. For most ICU research this would be doctors and nurses, but may also include dietitians, pharmacists, physiotherapists and speech therapists.

- Recognise that different stakeholders have different learning styles, and that most people find out the result from 'someone at work'.

- Use multiple modes to diffuse and disseminate research results.

- Circulate published papers of significance in full to all ICUs (whether they participated in the research or not). 
- Recognise that dissemination of research results is at two levels: from the researchers to the sites; and from the site research staff to their clinical staff. Give direction to sites about how to actively disseminate the findings.

- Provide ICUs with a one-page plain language summary and/or infographics, including potential impact on practice, and encourage them to send this to all of their clinical staff.

- Educate research staff about different learning styles of doctors and nurses.

- Ensure that ICU senior doctors and nurses are sent the full publication, along with slide set or video of the findings, including interpretation of the research, so that they can present these findings to other staff.

- Encourage discussion of research findings at ICU journal clubs (including the abovementioned tools to assist ICUs).

\subsubsection{ICU level recommendations}

Researchers cannot disseminate research results alone, and ICUs research staff and senior management need to take some responsibility for ensuring that results are targeted to the people who need them, in a way they understand. Recommendations include:

- Engage senior nurses, such as nurse educators, as well as doctors, in decisions about how to disseminate research results.

- Conduct in-service or education sessions when new research is published.

- Encourage all staff to access critical appraisal sites such as The Bottom line.

- Encourage all staff to sign up for updates on the latest research, such as emails from Critical Care Reviews.

- Use existing knowledge translation strategies and processes, such as research champions and change managers to disseminate research findings to staff.

- Support senior nurses to remain up to date with latest research, and provide resources (time) for them to do this, so they can communicate findings to staff.

- Encourage nurses to be involved in journal clubs.

- Use interactions such as shift handover, multi-disciplinary meetings and bedside discussions at ward rounds to communicate important research findings. 
- Provide funding for nurses to attend conferences. This is more achievable now that many conferences have an on-line option and there is often a cheaper option for nurses to attend.

\subsection{Dissemination plan for this research}

The findings of this research will be disseminated to the target audience, in a way they understand. The preliminary findings of this study have already been presented to three audiences: fellow PhD and Master's students at a recent research school; the ANZICS CTG Winter meeting, with an audience mostly comprised of ICU research coordinators and doctors, many of whom were from ICUs that participated in the research and finally the College of Intensive Care Medicine Annual Scientific meeting, that had very few nurse attendees. Further presentation of the more detailed findings about the effectiveness of the dissemination plan, in relation to ICU-ROX participation and role, will target research teams within the ICU community.

The findings will be published in journal articles as three separate publications: practitioner attitudes survey, inception cohort study and retrospective cohort study. As one of the conditions of the inception cohort study being ANZCIS-CTG-endorsed, all participating site research staff will be included as authors (in a supplementary appendix) and the article approved by the ANZICS-CTG prior to journal submission. In addition, a summary of the overall findings will be sent to the ANZICS-CTG.

The target audience for this research is ICU researchers, research doctors and nurses, senior doctors, senior nurses and managers. The research teams will be sent a one-page summary of the findings, outlining the importance of active dissemination to key stakeholders in a way they understand.

\subsection{Conclusion}

This thesis has successfully achieved the overall aim of increasing understanding about knowledge translation in the ICU setting. This research makes a unique contribution to the literature, evaluating the effect of trial participation and role on knowledge translation in the ICU setting, and assessing the effectiveness of existing dissemination strategies. 
Dissemination practices of research results using traditional modes are only partially successful, failing to reach ICU nurses who hold a key role in oxygen management in ICU. For successful translation of research into clinical practice, researchers need to be aware of who the target audience is, how different stakeholders learn about research findings, and tailor their dissemination strategies to meet the target audience, whoever they are. 


\section{APPENDICES}

\section{Appendix 1 ICU-ROX publication}

\section{Permission}

ICU-ROX was published in the New England Journal of Medicine, initially electronically in October 2019, and in print in March 2020 (Mackle et al., 2020). The supervising and coordinating author is my supervisor, Dr Paul Young. All members of the ICU-ROX Management Committee are named authors and ICU-ROX was published on behalf of the ICU-ROX Investigators and the Australian and New Zealand Intensive Care Society Clinical Trials Group. The publication is presented, as submitted for publication.

From: New England Journal of Medicine, Mackle, D., Bellomo, R., Bailey, M., Beasley, R., Deane, A., Eastwood, G., Finfer, S., Freebairn, R., King, V., Linke, N., Litton, E., McArthur, C., McGuinness, S., Panwar, R., Young, P., \& ICU-ROX Investigators and the Australian and New Zealand Intensive Care Society Clinical Trials, Conservative Oxygen Therapy during Mechanical Ventilation in the ICU, 382(11), 989-998. https://doi.org/10.1056/nejmoa19032972020)., Copyright (C (2020) Massachusetts Medical Society. Reprinted with permission. 


\section{Conservative Oxygen Therapy for Ventilated Adults in Intensive Care}

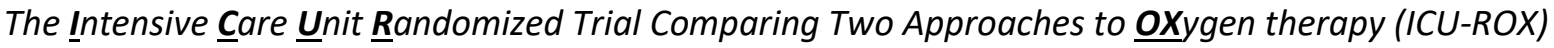
Diane Mackle ${ }^{1}$, M.N., Rinaldo Bellomo ${ }^{2,3,4}$, M.D., Michael Bailey ${ }^{2,4}$, Ph.D., Richard Beasley ${ }^{1}$, M.D., D. Sc., Adam Deane ${ }^{4,5}$ M.D., Ph.D., Glenn Eastwood ${ }^{3}$, R.N., Ph.D., Simon Finfer ${ }^{6,7}$, M.D., Ross Freebairn ${ }^{1,8}$, M.D., Victoria King ${ }^{2}$, R.N., Natalie Linke ${ }^{2}$, R.N., Edward Litton ${ }^{9}$, M.D., Ph.D. Colin McArthur ${ }^{1,10}$, M.D., Shay McGuinness ${ }^{1,11}$, M.D., Rakshit Panwar ${ }^{12,13}$, M.D., Paul Young ${ }^{1,14}$, M.D., Ph.D., for the ICU-ROX Investigators and the Australian and New Zealand Intensive Care Society Clinical Trials Group*

${ }^{1}$ Medical Research Institute of New Zealand, Wellington, New Zealand

${ }^{2}$ Australian and New Zealand Intensive Care Research Centre, Monash University, Melbourne, Victoria, Australia

${ }^{3}$ Intensive Care Unit, Austin Hospital, Heidelberg, Victoria, Australia

${ }^{4}$ University of Melbourne, Parkville, Victoria, Australia

${ }^{5}$ Intensive Care Unit, Royal Melbourne Hospital, Parkville, Victoria, Australia

${ }^{6}$ Division of Critical Care and Trauma, The George Institute for Global Health, Sydney, New South Wales, Australia

${ }^{7}$ Malcolm Fisher Department of Intensive Care Medicine, Royal North Shore Hospital, St Leonards, New South Wales, Australia

8 Intensive Care Unit, Hawkes Bay Hospital, Hastings, New Zealand

${ }^{9}$ Intensive Care Unit, Fiona Stanley Hospital, Murdoch, Western Australia, Australia

${ }^{10}$ Department of Critical Care Medicine, Auckland City Hospital, Auckland, New Zealand

${ }^{11}$ Cardiothoracic and Vascular Intensive Care Unit, Auckland City Hospital, Auckland, New Zealand

12 Intensive Care Unit, John Hunter Hospital, New Lambton Heights, New South Wales, Australia

${ }^{13}$ School of Medicine and Public Health, University of Newcastle, Newcastle, Australia ${ }^{14}$ Intensive Care Unit, Wellington Hospital, Wellington, New Zealand

Address reprint requests to:

Dr. Paul Young, M.D., Ph.D.

Intensive Care Unit, Wellington Regional Hospital

Private Bag 7902

Wellington South, New Zealand

paul.young@ccdhb.org.nz.

*A complete list of investigators in the Intensive Care Unit Randomized Trial Comparing Two Approaches to OXygen therapy (ICU-ROX) is provided in the Appendix. 


\section{ABSTRACT}

BACKGROUND

Mechanically ventilated intensive care unit (ICU) patients often receive a high fraction of inspired oxygen $\left(\mathrm{F}_{1} \mathrm{O}_{2}\right)$ and have high arterial oxygen tensions. Conservative use of oxygen may reduce oxygen exposure, diminish lung and systemic oxidative injury, and thereby increase ventilator-free days (days alive and free from mechanical ventilation).

METHODS

We randomly assigned 1000 adult ICU patients anticipated to require mechanical ventilation beyond the calendar day after recruitment to conservative or usual oxygen therapy. The primary outcome was ventilator-free days from randomization until day 28 .

\section{RESULTS}

The number of ventilator-free days did not differ significantly between the conservative and usual oxygen therapy groups: 21.3 days (interquartile range, 0 to 26.3) vs. 22.1 days (interquartile range, 0 to 26.2); absolute difference,

-0.3 days; $95 \%$ confidence interval $[\mathrm{Cl}],-2.1-1.6$ days; $\mathrm{P}=0.80$. The conservative oxygen group spent more time in the ICU receiving an $\mathrm{F}_{1} \mathrm{O}_{2}$ of 0.21 than the usual oxygen group: 29 hours (interquartile range, 5 to 78 ) vs. 1 hour (interquartile range, 0 to 17); absolute difference, 28 hours; $95 \% \mathrm{Cl}, 22-34$, and less time with arterial oxygen saturations measured by pulse oximetry $\geq 97 \%$ : 27 hours (interquartile range, 11 to 63.5 ) vs. 49 hours (interquartile range, 22 to 112); absolute difference, 22 hours; $95 \% \mathrm{Cl}, 14-30.170$ of 476 conservative oxygen patients (35.7\%) and 164 of 475 usual oxygen patients (34.5\%) had died by day 180 (relative risk; $1.03 ; 95 \% \mathrm{Cl}, 0.87-1.23$ ).

\section{CONCLUSIONS}

In adults requiring mechanical ventilation in ICU, conservative oxygen therapy did not significantly affect ventilator free days compared with usual oxygen therapy.

(Funded by the New Zealand Health Research Council; ICU-ROX Australian and New Zealand Clinical Trials Registry number, ACTRN12615000957594.) 
Provision of supplemental oxygen to intensive care unit (ICU) patients who require invasive ventilation often exposes them to a high fraction of inspired oxygen $\left(\mathrm{F}_{1} \mathrm{O}_{2}\right)$ and higher than normal arterial oxygen partial pressure $\left(\mathrm{PaO}_{2}\right) \cdot{ }^{1-3}$

Among invasively ventilated adults, hyperoxemia has been associated with increased mortality ${ }^{4,5}$ and fewer ventilator-free days. ${ }^{5}$ In a meta-analysis of randomized trials in acutely ill adults, oxygen use without limitation due to achieved arterial oxygen saturation was reported to increase mortality compared with more restrictive approaches. ${ }^{6}$ A single center ICU trial, ${ }^{7}$ in which around two-thirds of the participants were receiving invasive mechanical ventilation at randomization, showed that conservative oxygen therapy, a therapeutic regimen which sought to minimize exposure to high levels of oxygen, reduced ICU mortality and increased ventilator-free days compared with usual oxygen therapy. As supplemental oxygen is used commonly, such findings suggest that establishing regimens for oxygen use could be of value. Despite this, there are not good clinically directive data examining strategies of oxygen administration in mechanically ventilated adults. ${ }^{7-9}$ Accordingly, we conducted a multicenter, randomized clinical trial to test the hypothesis that, compared with usual oxygen therapy, conservative oxygen therapy would increase ventilator-free days (days alive and free from invasive mechanical ventilation) in mechanically ventilated adult ICU patients expected to be ventilated beyond the day after recruitment.

\section{METHODS}

\section{TRIAL DESIGN}

We conducted a 1000-participant, investigator-initiated, multicenter, prospective, parallelgroup, randomized clinical trial. The management committee designed the trial, which was endorsed by the Australian and New Zealand Intensive Care Society Clinical Trials Group. The Medical Research Institute of New Zealand (Wellington, New Zealand) and Australian and New Zealand Intensive Care Research Centre (Melbourne, Australia) managed the trial and monitored data quality. The trial began with a 100-participant vanguard pilot phase ${ }^{10}$ leading to minor changes to the protocol for the subsequent 900 participants (Table S1, Supplementary Appendix). An independent data and safety monitoring committee oversaw the trial and reviewed planned interim analyses after 100 and 500 patients had reached 28 days of follow-up. 
The protocol (available at NEJM.org), which was reported before enrollment was completed,

${ }^{11}$ was approved by the Health Research Ethics Committee responsible for each participating institution. Written informed consent for enrollment, or consent to continue and to use patient data, was obtained from each patient or from a legal surrogate. Where a patient died before consent to continue could be obtained, data were included if allowed by local regulations and approved by the relevant ethics committee. The listed authors vouch for the accuracy and completeness of the data and analyses, and for the fidelity of this report to the study findings.

\section{PATIENTS}

Mechanically ventilated patients 18 years of age or older who were expected to remain mechanically ventilated in ICU beyond the calendar day after recruitment were eligible for inclusion. The exclusion criteria are provided in the Supplementary Appendix. Enrollment was restricted to patients who had received less than two hours of invasive mechanical ventilation and/or non-invasive ventilation in an ICU. Eligible patients who were not enrolled within the two-hour time window were categorized as missed, rather than excluded, for the purposes of describing participant flow.

\section{RANDOMIZATION AND STUDY TREATMENT}

We randomly assigned patients to conservative oxygen therapy (conservative oxygen) or usual oxygen therapy (usual oxygen) using a secure, centralized, web-based, randomization interface. The allocation sequence was generated by the study statistician using computergenerated random numbers with variable block randomization in a 1:1 ratio and stratification by center.

In both groups, the acceptable lower limit for oxygen saturation measured by pulse oximetry $\left(\mathrm{SpO}_{2}\right)$ was monitored with an alarm set at an $\mathrm{SpO}_{2}$ of $90 \%$ or less. This alarm limit could be altered at the discretion of the treating clinician. If an arterial blood gas showed $\mathrm{PaO}_{2}$ of less than $60 \mathrm{mmHg}$ or an arterial oxygen saturation $\left(\mathrm{SaO}_{2}\right)$ lower than the acceptable $\mathrm{SpO}_{2}$, $\mathrm{F}_{1} \mathrm{O}_{2}$ could be increased, irrespective of $\mathrm{SpO}_{2}$, at the discretion of the treating clinician.

In patients assigned to conservative oxygen, the $\mathrm{F}_{1} \mathrm{O}_{2}$ was decreased to 0.21 , or supplemental oxygen was discontinued in patients who had been extubated provided the $\mathrm{SpO}_{2}$ was above the acceptable lower limit. We sought to minimize exposure to an $\mathrm{SpO}_{2}$ of $97 \%$ or higher in 
patients assigned to conservative oxygen by mandating use of an upper $\mathrm{SpO}_{2}$ alarm limit, set to sound when $\mathrm{SpO}_{2}$ was $97 \%$ or higher, whenever supplemental oxygen was administered in the ICU (Fig. 1).

In patients assigned to usual oxygen, no specific measures limited $\mathrm{F}_{1} \mathrm{O}_{2}$ or $\mathrm{SpO}_{2}$ and use of upper alarm limits for $\mathrm{SpO}_{2}$ was prohibited by the protocol. Use of $\mathrm{F}_{1} \mathrm{O}_{2}$ less than 0.3 during invasive ventilation was discouraged (Fig. 1).

In both treatment groups, use of high $\mathrm{F}_{1} \mathrm{O}_{2}$, irrespective of $\mathrm{SpO}_{2}$, was permitted in some specific circumstances (see the Supplementary Appendix). Other aspects of care, including ventilator weaning and extubation practices were at the discretion of the treating clinician.

Patients received their assigned oxygen therapy strategy until discharge from the ICU or 28 days from randomization, whichever was sooner. Treatment assignment was known to clinical staff but not disclosed to participants or next-of-kin.

\section{OUTCOME MEASURES}

The primary outcome was ventilator-free days to day $28 .{ }^{12}$ We defined ventilator-free days as the total number of calendar days or part calendar days of unassisted breathing during the first 28 days after randomization; all patients who died by day 28 were assigned zero ventilator-free days.

Secondary endpoints were death from all causes at 90 and 180 days after randomization, duration of survival, the proportion of patients in paid employment at baseline who were unemployed at day 180, and cognitive function and health-related quality of life at day 180 . Cause-specific mortality was also recorded..$^{13}$ Cognitive function was assessed using the Telephone Interview for Cognitive Status (TICS) questionnaire; scores on this questionnaire range from 0 to 41 with a higher number indicating a better outcome. Categories of cognitive function based on the TICS score were severe (scores20); mild (score 21-25); ambiguous (score 28-32); and non-impaired (score $\geq 33$ ). ${ }^{14}$ Quality of life was assessed using the five-level EuroQol five dimensions (EQ-5D-5L) questionnaire; this scale evaluates mobility, personal care, usual activities, pain/discomfort, and anxiety/depression and categorises each of these dimensions into five levels that range from no problems to extreme problems. ${ }^{15}$ For patients with acute brain pathologies at randomization, functional 
outcome at day 180 was also assessed using the Extended Glasgow Outcome Scale (GOS-E); this scale ranges from 1 to 8 , with a higher number indicating a better outcome. ${ }^{16}$ Centralized assessors masked to study-group assignments undertook day 180 assessments of cognitive status, quality of life, and function.

Additional details of study outcomes and subgroups are in the Supplementary Appendix.

\section{STATISTICAL ANALYSIS}

The statistical analysis plan was reported before enrollment completion. ${ }^{11}$ We assumed a mean of $16.4 \pm 11.3$ ventilator-free days in the usual oxygen group. ${ }^{8,17}$ Allowing for a $15 \%$ inflation in sample size to account for a rank-based test ${ }^{18}$ and an additional inflation of 80 participants to account for drop-outs, and interim analyses, a sample size of 1000 patients provided $90 \%$ power to detect an absolute difference of 2.6 ventilator-free days at day 28 after randomization with a two-sided type 1 error rate (alpha) of $0.05 .^{8}$

Analyses were conducted on an intention-to-treat basis. We defined the intention-to-treat population as all enrolled patients except those who withdrew consent for use of data. We did not impute missing values.

For the primary analysis, we used a Wilcoxon rank-sum test with differences between medians calculated using quantile regression employing a simplex algorithm with 95\% confidence intervals $(\mathrm{Cl})$ calculated using the inversion method ${ }^{19}$ and adjusted for site. We also analyzed the primary end point using quantile regression adjusting for site, patient age, gender, and risk of death as assessed by the Acute Physiology and Chronic Health Evaluation (APACHE) II mode ${ }^{20}$ and performed an unadjusted analysis.

For pre-specified subgroups, we performed quantile regression analysis and tested for heterogeneity between subgroups in ventilator-free days by fitting an interaction between treatment and subgroup. Analyses were conducted using SAS statistical software, version 9.4 (SAS Institute). Statistical significance was determined using a two-sided hypothesis test with an alpha of 0.05 . We did not correct for multiplicity when comparing secondary or other outcomes. Thus, such results are exploratory and reported as point estimates with $95 \% \mathrm{Cl}$. Additional details of statistical analyses are in the Supplementary Appendix. 


\section{RESULTS}

\section{PATIENT CHARACTERISTICS}

From September 2015 through May 2018, we enrolled 1000 patients in 21 adult medicalsurgical ICUs in Australia and New Zealand, with 499 patients assigned to conservative oxygen and 501 to usual oxygen (Fig. 2). Consent was withdrawn by 35 participants, resulting in an intention-to-treat population of 965, of whom 484 were assigned to conservative oxygen and 481 to usual oxygen. The characteristics of the 100-patient vanguard pilot vs. main study patients, and of the patients who were randomized vs. those eligible for enrollment but not randomized are shown in the Supplementary Appendix (Table S2 and Table S3). Data on the primary outcome were available for the entire intention to treat population. The study groups had similar characteristics at baseline (Table 1, and Tables S4 to S7 in the Supplementary Appendix).

\section{OXYGENATION AND PROCESS OF CARE}

Patients allocated to conservative oxygen spent more time receiving an $\mathrm{F}_{1} \mathrm{O}_{2}$ of 0.21 in the ICU than patients allocated to usual oxygen (29 hours [interquartile range (IQR), 5 to 78] vs. 1 hour [IQR, 0 to 17]; absolute difference, 28 hours; $95 \%$ Cl, 22-34), and less time in the ICU with an $\mathrm{SpO}_{2}$ of $97 \%$ or higher (27 hours [IQR, 11 to 63.5] vs. 49 hours [IQR, 22 to 112]; absolute difference, 22 hours; $95 \% \mathrm{Cl}, 14-30)$. The number and percentage of hours with $\mathrm{SpO}_{2}$ less than $91 \%$ and with $\mathrm{SpO}_{2}$ less than $88 \%$ were similar between groups (Table $\mathrm{S} 8$ in the Supplementary Appendix). Mean $\mathrm{F}_{1} \mathrm{O}_{2}$ during the first 10 mechanical ventilation days, and lowest and highest $\mathrm{F}_{1} \mathrm{O}_{2}$ while ventilated in ICU until day 28 are shown in Fig. S1 (Supplementary Appendix) with all $\mathrm{F}_{1} \mathrm{O}_{2}$ indices being lower in the conservative oxygen group. Similarly, time-weighted mean $\mathrm{PaO}_{2}$ during the first 10 ventilated ICU days and the lowest and highest $\mathrm{PaO}_{2}$ while ventilated until ICU day 28 are shown in Fig. S2 (Supplementary Appendix) with all $\mathrm{PaO}_{2}$ indices being lower in the conservative oxygen group. Additional physiological descriptors and process of care measures are shown in Table 2 and Fig. S3 to Fig. S6, and Table S9 of the Supplementary Appendix.

PRIMARY OUTCOME 
Ventilator-free days to day 28 did not differ significantly between the conservative oxygen group and the usual oxygen group: 21.3 days (IQR, 0 to 26.3) for patients assigned to conservative oxygen and 22.1 days (IQR, 0 to 26.2) for patients assigned to usual oxygen (absolute difference, -0.3 days; $95 \% \mathrm{Cl},-2.1$ to 1.6; $\mathrm{P}=0.80$ ) (Table 2 and Fig $\mathrm{S} 7$ of the Supplementary Appendix).

\section{SECONDARY OUTCOMES}

Final study follow-up to assess secondary outcomes occurred a median of 186 days after randomization [IQR, 181 to 197 days]. A total of 170 of 476 patients (35.7\%) assigned to conservative oxygen and 164 of 475 patients (34.5\%) assigned to usual oxygen had died by day 180 (relative risk; $1.03 ; 95 \% \mathrm{Cl}, 0.87$ to 1.23 ; hazard ratio; $1.05 ; 95 \% \mathrm{Cl}, 0.85$ to 1.30 ) (Table 2 and Fig 3). Cause-specific mortality by treatment group is shown in the Supplementary Appendix (Table S10).

Among survivors, we found no evidence of difference between groups in employment status (among participants in paid employment at baseline); of such patients 77 of 112 (68.8\%) conservative oxygen patients and 66 of 108 (61.1\%) usual oxygen patients were in paid employment at final follow-up (Table S11 in the Supplementary Appendix). Cognitive function was similar by treatment group with 5 of 203 (2.5\%) conservative oxygen patients and 6 of 206 (2.9\%) usual oxygen patients having severe cognitive impairment at final followup (Table S12 in the Supplementary Appendix). In mobility and personal-care components of the quality of life assessment, our estimates indicate a relatively greater proportion of patients had moderate problems and a relatively smaller proportion of patients had severe problems in the conservative group. We found no evidence of differences in other domains of the quality of life assessment (Table S13 in the Supplementary Appendix).

\section{SUBGROUP ANALYSIS}

There was substantial heterogeneity in the effect of conservative oxygen on ventilator-free days in patients with vs. without suspected hypoxic ischemic encephalopathy but not in other pre-specified subgroups (Table S14 in the Supplementary Appendix). Among patients with suspected hypoxic ischemic encephalopathy, median ventilator free days to day 28 were 21.1 (IQR, 0 to 26.1) and 0 (IQR, 0 to 26) for conservative oxygen and usual oxygen 
groups respectively (absolute difference, 21.1 days; 95\% Cl, 10.4 to 28.0). In post-hoc analyses of the suspected hypoxic ischemic encephalopathy subgroup, 37 of 86 (43\%) conservative oxygen patients and 46 of 78 (59\%) usual oxygen patients had died by day 180 (relative risk; $0.73 ; 95 \% \mathrm{Cl}, 0.54$ to 0.99 ; hazard ratio; $0.67 ; 95 \% \mathrm{Cl}, 0.43$ to 1.03 ) with 43 of $78(55.1 \%)$ and 49 of $72(68.1 \%)$ respectively having an unfavorable outcome on the GOS-E (relative risk; $0.81 ; 95 \% \mathrm{Cl}, 0.63$ to 1.05 ). Details of post-hoc analyses are shown in the Supplementary Appendix.

\section{ADVERSE EVENTS}

One patient allocated to conservative oxygen was reported to have hypoxemia with a $\mathrm{PaO}_{2}$ of $33.5 \mathrm{mmHg}$ as an adverse event and a second had a low $\mathrm{SpO}_{2}$ reported. One patient allocated to usual oxygen had an ischemic stroke reported as an adverse event. Details of adverse events are provided in the Supplementary Appendix.

\section{DISCUSSION}

In this binational multicenter randomized clinical trial of adults receiving mechanical ventilation in the ICU, conservative oxygen therapy did not alter ventilator-free days compared with usual oxygen therapy. We did not find evidence of significant betweengroup differences in 90-day mortality, 180-day mortality, or survival.

Our findings are at variance with a previous single center trial. ${ }^{7}$ In that trial, which was stopped early following an unplanned interim analysis, conservative oxygen was associated with a greater number of ventilator-free days and a markedly lower ICU mortality rate than usual oxygen therapy. ${ }^{7}$ The control group in the previous trial was more liberal than our usual oxygen therapy group, allowing for a $\mathrm{PaO}_{2}$ of up to $150 \mathrm{mmHg}$ and suggesting use of an $\mathrm{F}_{1} \mathrm{O}_{2}$ greater than 0.40 ; however, observed $\mathrm{PaO}_{2}$ levels in their control group were similar to those in our study. Our study is much larger and provides more precise and robust treatment effect estimates. ${ }^{21}$

We achieved clear separation in oxygen exposure. Conservative oxygen markedly reduced hours with an $\mathrm{SpO}_{2}$ of $97 \%$ or more and increased hours breathing $21 \%$ oxygen.

Our data are suggestive of a possible benefit of conservative oxygen therapy in patients with suspected hypoxic ischemic encephalopathy. It is biologically plausible that conservative oxygen therapy reduces secondary brain damage after resuscitation from cardiac arrest, ${ }^{22}$ 
and observational data suggest exposure to hyperoxemia in this setting may be harmful. ${ }^{23,24}$ However, these findings should be considered hypothesis-generating.

Our study has limitations. Clinicians and research staff could not be blinded to treatment allocation; however, to mitigate ascertainment bias, blinded centralized assessors conducted day 180 assessments. Some outcome variables (e.g., employment status) were compared only among survivors. Because survival is a post-randomization event, these are not randomized comparisons and may be subject to bias. Some data, particularly related to quality of life and cognition, were missing. These data may not be missing at random because patients with better (or worse) outcomes might be harder to contact or less likely to complete interviews. Despite this, given that problems with mobility and personal care are common after critical illnesses, ${ }^{25,26}$ our finding that relatively fewer survivors had severe problems in these domains in the conservative group is potentially important. We compared the characteristics of trial participants with those of eligible but non-enrolled patients. Eligible, non-enrolled patients had lower illness acuity and mortality rates. Accordingly, our findings may not apply to low acuity patients. As we did not protocolize weaning or extubation, changes in $\mathrm{F}_{1} \mathrm{O}_{2}, \mathrm{SpO}_{2}, \mathrm{PaO}_{2}$, and $\mathrm{PaCO}_{2}$ that occurred because of treatment assignment may have affected clinicians' decisions to wean and extubate particular patients. We allowed clinicians to increase oxygen in both groups in some specific circumstances. This may have exposed patients in both groups to hyperoxemia and thereby reduced our ability to find a difference in outcomes between groups.

A recent systematic review and meta-analysis reported that a conservative oxygen strategy reduced mortality in acutely ill adults compared with liberal oxygen. ${ }^{6}$ Many of the liberal oxygen interventions in the trials included in this meta-analysis were considerably more liberal than the oxygen regimen used in our usual care group and relatively few of the patients included were critically ill ${ }^{6}$. Our trial does not preclude the possibility of benefit or harm with more liberal oxygen regimens than used in our usual oxygen group. Different results might also be found with different conservative oxygen therapy regimens. Our findings decrease the probability that using our conservative oxygen therapy protocol in this study population markedly reduces mortality compared with usual oxygen therapy. However, the confidence intervals around our mortality estimates are sufficiently wide that important effects of our conservative oxygen regimen on mortality are not excluded. 
In conclusion, conservative oxygen therapy did not significantly affect ventilator free days compared with usual oxygen therapy in mechanically ventilated adults.

Disclosure forms provided by the authors are available with the full text of this article at NEJM.org.

\section{REFERENCES}

1. Young PJ, Beasley RW, Capellier G, et al. Oxygenation targets, monitoring in the critically ill: a point prevalence study of clinical practice in Australia and New Zealand. Crit Care Resusc 2015;17:202-7.

2. Helmerhorst HJ, Schultz MJ, van der Voort PH, et al. Self-reported attitudes versus actual practice of oxygen therapy by ICU physicians and nurses. Ann Intensive Care 2014;4:23.

3. Panwar R, Capellier G, Schmutz N, et al. Current oxygenation practice in ventilated patients-an observational cohort study. Anaesth Intensive Care 2013;41:505-14.

4. Helmerhorst HJ, Roos-Blom MJ, van Westerloo DJ, de Jonge E. Association Between Arterial Hyperoxia and Outcome in Subsets of Critical Illness: A Systematic Review, MetaAnalysis, and Meta-Regression of Cohort Studies. Crit Care Med 2015;43:1508-19.

5. Helmerhorst HJ, Arts DL, Schultz MJ, et al. Metrics of Arterial Hyperoxia and Associated Outcomes in Critical Care. Crit Care Med 2017;45:187-95.

6. Chu DK, Kim LH, Young PJ, et al. Mortality and morbidity in acutely ill adults treated with liberal versus conservative oxygen therapy (IOTA): a systematic review and metaanalysis. Lancet 2018;391:1693-705.

7. Girardis M, Busani S, Damiani E, et al. Effect of Conservative vs Conventional Oxygen Therapy on Mortality Among Patients in an Intensive Care Unit: The Oxygen-ICU Randomized Clinical Trial. JAMA 2016;316:1583-9.

8. Panwar R, Hardie M, Bellomo R, et al. Conservative versus Liberal Oxygenation Targets for Mechanically Ventilated Patients. A Pilot Multicenter Randomized Controlled Trial. Am J Respir Crit Care Med 2016;193:43-51.

9. Asfar P, Schortgen F, Boisrame-Helms J, et al. Hyperoxia and hypertonic saline in patients with septic shock (HYPERS2S): a two-by-two factorial, multicentre, randomised, clinical trial. Lancet Respir Med 2017;5:180-90.

10. Young PJ, Mackle DM, Bailey MJ, et al. Intensive care unit randomised trial comparing two approaches to oxygen therapy (ICU-ROX): results of the pilot phase. Crit Care Resusc 2017;19:344-54.

11. Mackle DM, Bailey MJ, Beasley RW, et al. Protocol summary and statistical analysis plan for the intensive care unit randomised trial comparing two approaches to oxygen therapy (ICU-ROX). Crit Care Resusc 2018;20:22-32.

12. Schoenfeld DA, Bernard GR, Network A. Statistical evaluation of ventilator-free days as an efficacy measure in clinical trials of treatments for acute respiratory distress syndrome. Crit Care Med 2002;30:1772-7. 
13. Ridgeon E, Bellomo R, Myburgh J, et al. Validation of a classification system for causes of death in critical care: an assessment of inter-rater reliability. Crit Care Resusc 2016;18:50-4.

14. Davydow DS, Zatzick D, Hough CL, Katon WJ. In-hospital acute stress symptoms are associated with impairment in cognition 1 year after intensive care unit admission. Ann Am Thorac Soc 2013;10:450-7.

15. Badia X, Diaz-Prieto A, Gorriz MT, et al. Using the EuroQol-5D to measure changes in quality of life 12 months after discharge from an intensive care unit. Intensive Care Med 2001;27:1901-7.

16. Weir J, Steyerberg EW, Butcher I, et al. Does the extended Glasgow Outcome Scale add value to the conventional Glasgow Outcome Scale? J Neurotrauma 2012;29:53-8.

17. Suzuki S, Eastwood GM, Glassford NJ, et al. Conservative oxygen therapy in mechanically ventilated patients: a pilot before-and-after trial. Crit Care Med 2014;42:1414-22.

18. Lehmann EL. Nonparametrics: statistical methods based on rank; revised ed. Springer: 2006

19. Koenker RdO, V. Computing Regression Quantiles. Applied Statistics 1993;43:410-4. 20. Knaus WA, Draper EA, Wagner DP, Zimmerman JE. APACHE II: a severity of disease classification system. Crit Care Med 1985;13:818-29.

21. Ferguson ND. Oxygen in the ICU: Too Much of a Good Thing? JAMA 2016;316:15534.

22. Pilcher J, Weatherall M, Shirtcliffe P, Bellomo R, Young P, Beasley R. The effect of hyperoxia following cardiac arrest - A systematic review and meta-analysis of animal trials. Resuscitation 2012;83:417-22.

23. Roberts BW, Kilgannon JH, Hunter BR, et al. Association Between Early Hyperoxia Exposure After Resuscitation From Cardiac Arrest and Neurological Disability: Prospective Multicenter Protocol-Directed Cohort Study. Circulation 2018;137:2114-24.

24. Kilgannon JH, Jones AE, Shapiro NI, et al. Association between arterial hyperoxia following resuscitation from cardiac arrest and in-hospital mortality. JAMA 2010;303:216571.

25. Herridge MS, Tansey CM, Matte A, et al. Functional disability 5 years after acute respiratory distress syndrome. N EngI J Med 2011;364:1293-304.

26. Hodgson CL, Bailey M, Bellomo R, et al. A Binational Multicenter Pilot Feasibility Randomized Controlled Trial of Early Goal-Directed Mobilization in the ICU. Crit Care Med 2016;44:1145-52. 


\section{FIGURES}

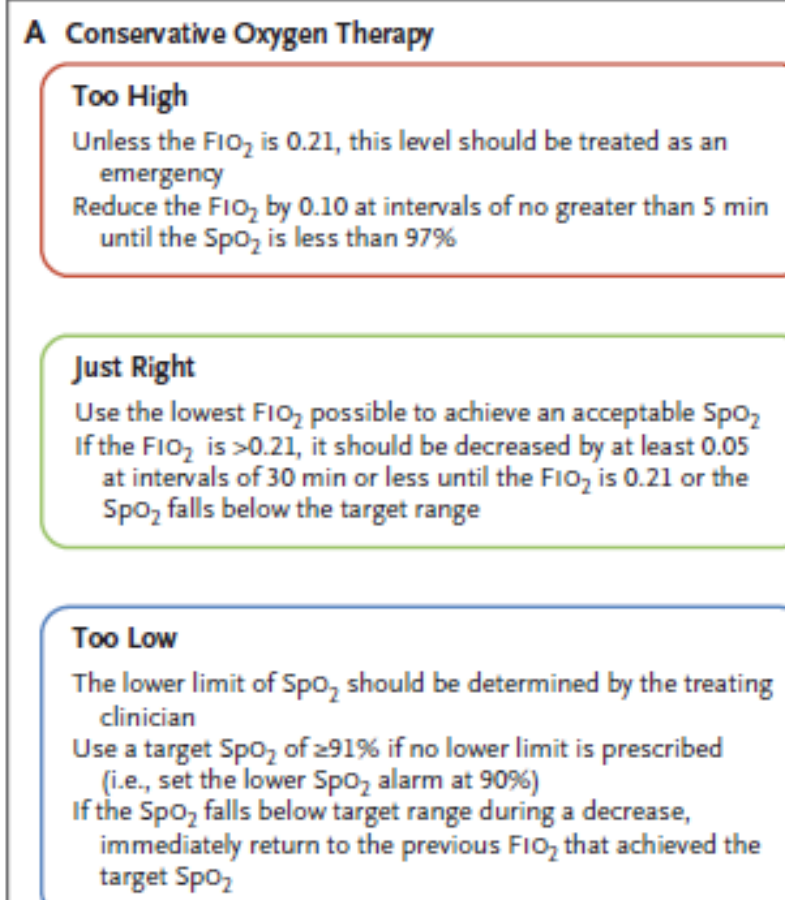

Unless the $\mathrm{FIO}_{2}$ is 0.21 , this level should be treated as an emergency

Reduce the $\mathrm{FIO}_{2}$ by 0.10 at intervals of no greater than 5 min until the $\mathrm{SpO}_{2}$ is less than $97 \%$

Just Right

Use the lowest $\mathrm{FIO}_{2}$ possible to achieve an acceptable $\mathrm{SpO}_{2}$

If the $\mathrm{FIO}_{2}$ is $>0.21$, it should be decreased by at least 0.05 at intervals of 30 min or less until the $\mathrm{FIO}_{2}$ is 0.21 or the $\mathrm{SpO}_{2}$ falls below the target range

\section{Too Low}

The lower limit of $\mathrm{SpO}_{2}$ should be determined by the treating clinician

Use a target $\mathrm{SpO}_{2}$ of $291 \%$ if no lower limit is prescribed (i.e., set the lower $\mathrm{SpO}_{2}$ alarm at $90 \%$ )

If the $\mathrm{SpO}_{2}$ falls below target range during a decrease, immediately return to the previous $\mathrm{FIO}_{2}$ that achieved the target $\mathrm{SpO}_{2}$

\section{B Usual Oxygen Therapy}

\section{Upper Limit of $\mathrm{SpO}_{2}$}

There is no protocol-defined upper limit of $\mathrm{SpO}_{2}$

Do not use upper-limit $\mathrm{SpO}_{2}$ alarms

\section{Target Range}

Any $\mathrm{SpO}_{2}$ greater than the lower limit is acceptable The use of an $\mathrm{FIO}_{2}$ of $<0.3$ is discouraged

\section{Lower Limit of $\mathrm{SpO}_{2}$}

The lower limit of $\mathrm{SpO}_{2}$ should be determined by the treating clinician

Use a target $\mathrm{SpO}_{2}$ of $291 \%$ if no lower limit is prescribed

(i.e., set the lower $\mathrm{SpO}_{2}$ alarm at $90 \%$ )

Upper limit of $\mathrm{SpO}_{2}$

$\mathrm{SpO}_{2}$ below target range
Target range

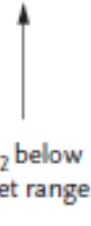

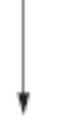

Target range

Lower limit of $\mathrm{SpO}_{2}$

Figure 1. Treatment Administered to Patients Receiving Conservative Oxygen Therapy or Usual Oxygen Therapy.

In the two groups, the acceptable lower limit for oxygen saturation as measured by pulse oximetry $\left(\mathrm{SpO}_{2}\right)$ was monitored with an alarm set at a level of $90 \%$. This alarm limit could be altered at the discretion of the treating clinician. If a measurement of arterial blood gas showed a partial pressure of oxygen $\left(\mathrm{PaO}_{2}\right)$ of less than $60 \mathrm{~mm} \mathrm{Hg}$ or an arterial oxygen saturation $\left(\mathrm{SaO}_{2}\right)$ lower than the acceptable $\mathrm{SpO}_{2}$, the fraction of inspired oxygen $\left(\mathrm{F}_{10} \mathrm{O}_{2}\right)$ could be increased at the discretion of the treating clinician.

Abbreviations: $\mathrm{F}_{1} \mathrm{O}_{2}$ : fraction of inspired oxygen; ICU-ROX: intensive care unit randomized trial comparing two approaches to oxygen therapy; $\mathrm{PaO}_{2}$ : arterial partial pressure of oxygen. $\mathrm{SaO}_{2}$ : arterial oxygen saturation. $\mathrm{SpO}_{2}$ : arterial oxygen saturation measured by peripheral pulse oximetry. 


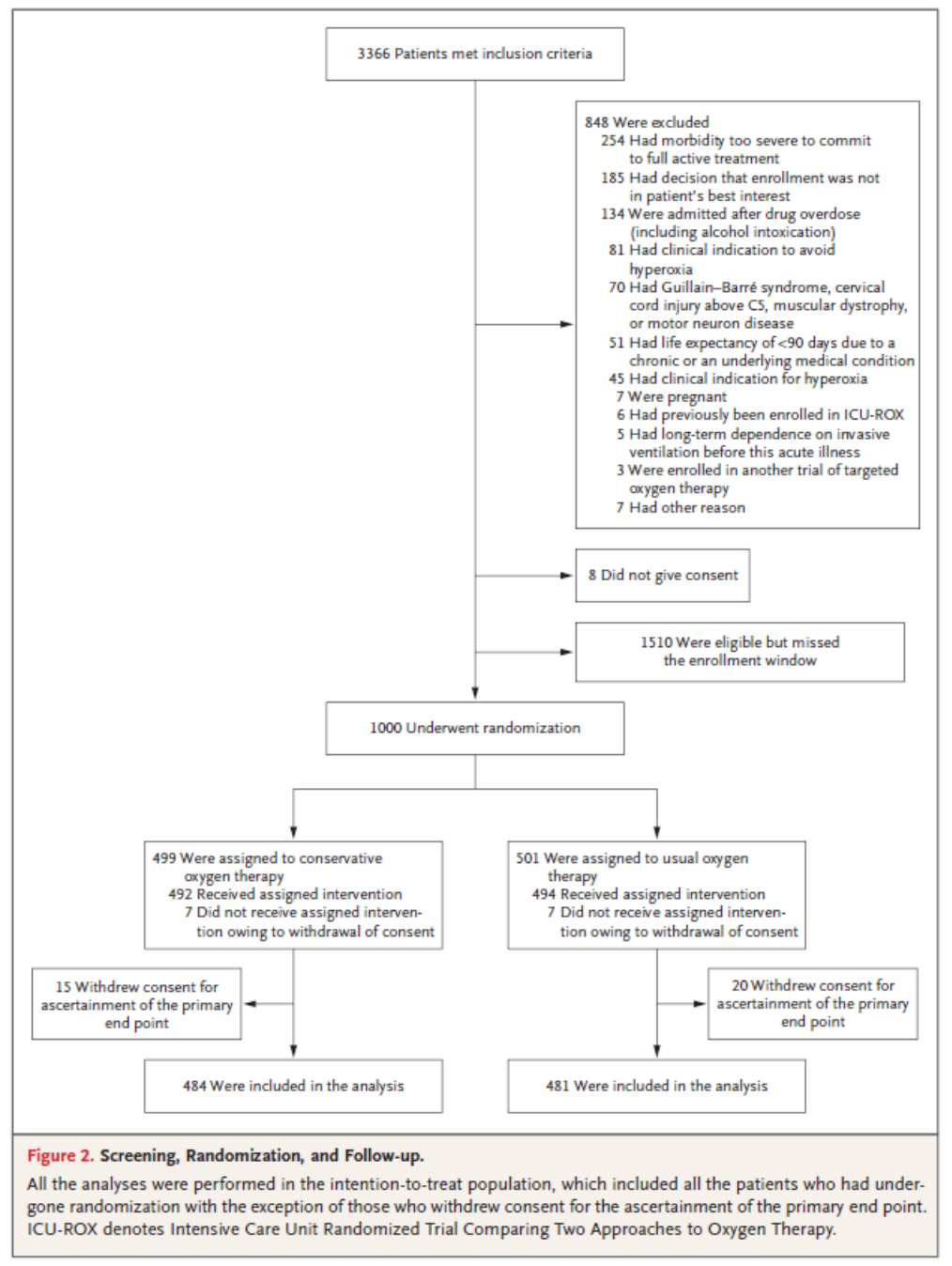

Abbreviations: ICU-ROX: intensive care unit randomized trial comparing two approaches to oxygen therapy.

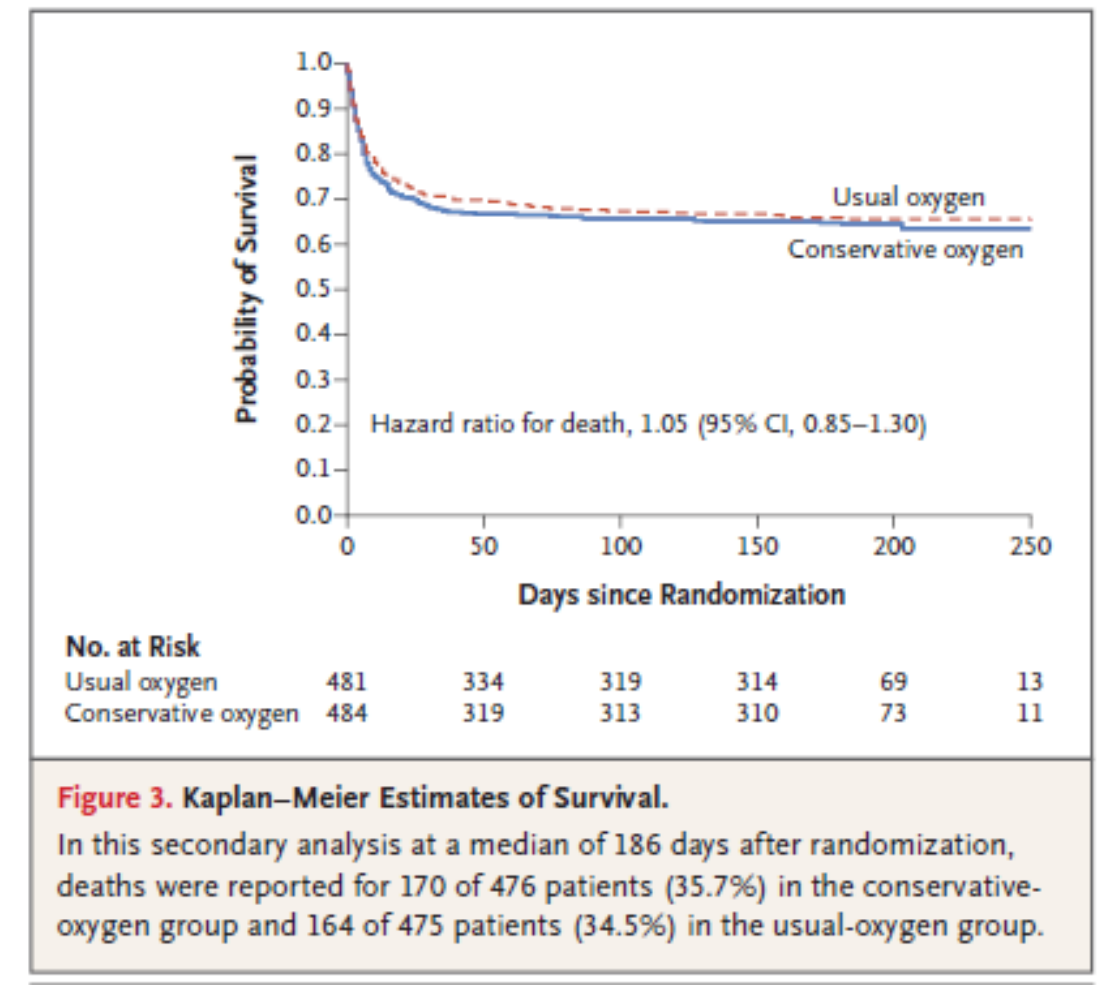


TABLES

Table 1: Characteristics of the Patients at Baseline*

\begin{tabular}{|c|c|c|}
\hline Characteristic & $\begin{array}{l}\text { Conservative } \\
\text { oxygen } \\
(n=484)\end{array}$ & $\begin{array}{l}\text { Usual } \\
\text { oxygen } \\
(n=481)\end{array}$ \\
\hline Age - yr & $58.1 \pm 16.2$ & $57.5 \pm 16.1$ \\
\hline Male sex - no. (\%) & $306(63.2 \%)$ & $302(62.8 \%)$ \\
\hline \multicolumn{3}{|l|}{ Source of admission to ICU - no. (\%) } \\
\hline Emergency department & 187 (38.6\%) & $212(44.1 \%)$ \\
\hline Hospital ward & 107 (22.1\%) & $82(17.0 \%)$ \\
\hline Transfer from another ICU & $2(0.4 \%)$ & $5(1.0 \%)$ \\
\hline $\begin{array}{l}\text { Transfer from another hospital (except from another } \\
\text { ICU) }\end{array}$ & $39(8.1 \%)$ & $36(7.5 \%)$ \\
\hline From OR following elective surgery & $31(6.4 \%)$ & 39 (8.1\%) \\
\hline From OR following emergency surgery & $118(24.4 \%)$ & $107(22.2 \%)$ \\
\hline $\begin{array}{l}\text { Hours from initiation of invasive ventilation to } \\
\text { randomization; median [IQR] }\end{array}$ & $3.2[1.6-5.4]$ & $3.0[1.5-5.3]$ \\
\hline APACHE-II score $¥$ & $23.6 \pm 9.3$ & $23.3 \pm 9.4$ \\
\hline \multicolumn{3}{|l|}{ Subgroups - no. (\%) } \\
\hline Admitted following surgery & $149(30.8 \%)$ & $146(30.4 \%)$ \\
\hline Acute brain pathology & $199(41.1 \%)$ & $184(38.3 \%)$ \\
\hline $\mathrm{PaO}_{2}: \mathrm{FIO}_{2}$ ratio $<300 \mathrm{mmHg}$ & $304(65.9 \%)$ & $319(71.2 \%)$ \\
\hline Suspected hypoxic ischemic encephalopathy & $87(18.0 \%)$ & $79(16.4 \%)$ \\
\hline \multicolumn{3}{|l|}{ Physiology and physiological support§ } \\
\hline $\mathrm{SpO}_{2}-\%$ & $97.1 \pm 3.1$ & $96.7 \pm 3.7$ \\
\hline $\mathrm{PaO}_{2}-\mathrm{mmHg}$; median [IQR] & $\begin{array}{c}110 \\
{[83.1-177]}\end{array}$ & $\begin{array}{c}112 \\
{[82.3-167]}\end{array}$ \\
\hline $\mathrm{PaO}_{2}: \mathrm{FIO}_{2}$ ratio $-\mathrm{mmHg}$ & $259 \pm 146$ & $245 \pm 138$ \\
\hline $\mathrm{PEEP}-\mathrm{cmH}_{2} \mathrm{O}$; median [IQR] & $6[5-10]$ & $6[5-10]$ \\
\hline
\end{tabular}

* Plus-minus values are expressed as mean \pm SD.

+ Includes hours of ventilation prior to ICU admission

¥ Scores on the APACHE II range from 0 to 71, with higher scores indicating more severe disease and a higher risk of death.

$\S \mathrm{SpO}_{2}$ data were available for 481 conservative oxygen patients and 476 usual oxygen patients; $\mathrm{PaO}_{2}$ and $\mathrm{PaO}_{2} / \mathrm{FIO}_{2}$ ratio data were available for 461 conservative oxygen patients and 448 usual oxygen patients; and $\mathrm{PaCO}_{2}$ data were available for 469 conservative oxygen patients and 441 usual oxygen patients. $\mathrm{FIO}_{2}$ data were only available for 483 conservative oxygen patients; PEEP data were only available for 477 conservative oxygen patients and 470 usual oxygen patients.

Abbreviations: APACHE: Acute Physiology And Chronic Health Evaluation; ICU: Intensive Care Unit; OR: operating room; $\mathrm{SpO}_{2}$ : arterial oxygen saturation on pulse oximetry; $\mathrm{PaO}_{2}$ : arterial partial pressure of oxygen; 
$\mathrm{FIO}_{2}$ : fraction of inspired oxygen; $\mathrm{PaCO}_{2}$ : arterial partial pressure of carbon dioxide; PEEP: positive end expiratory pressure. 


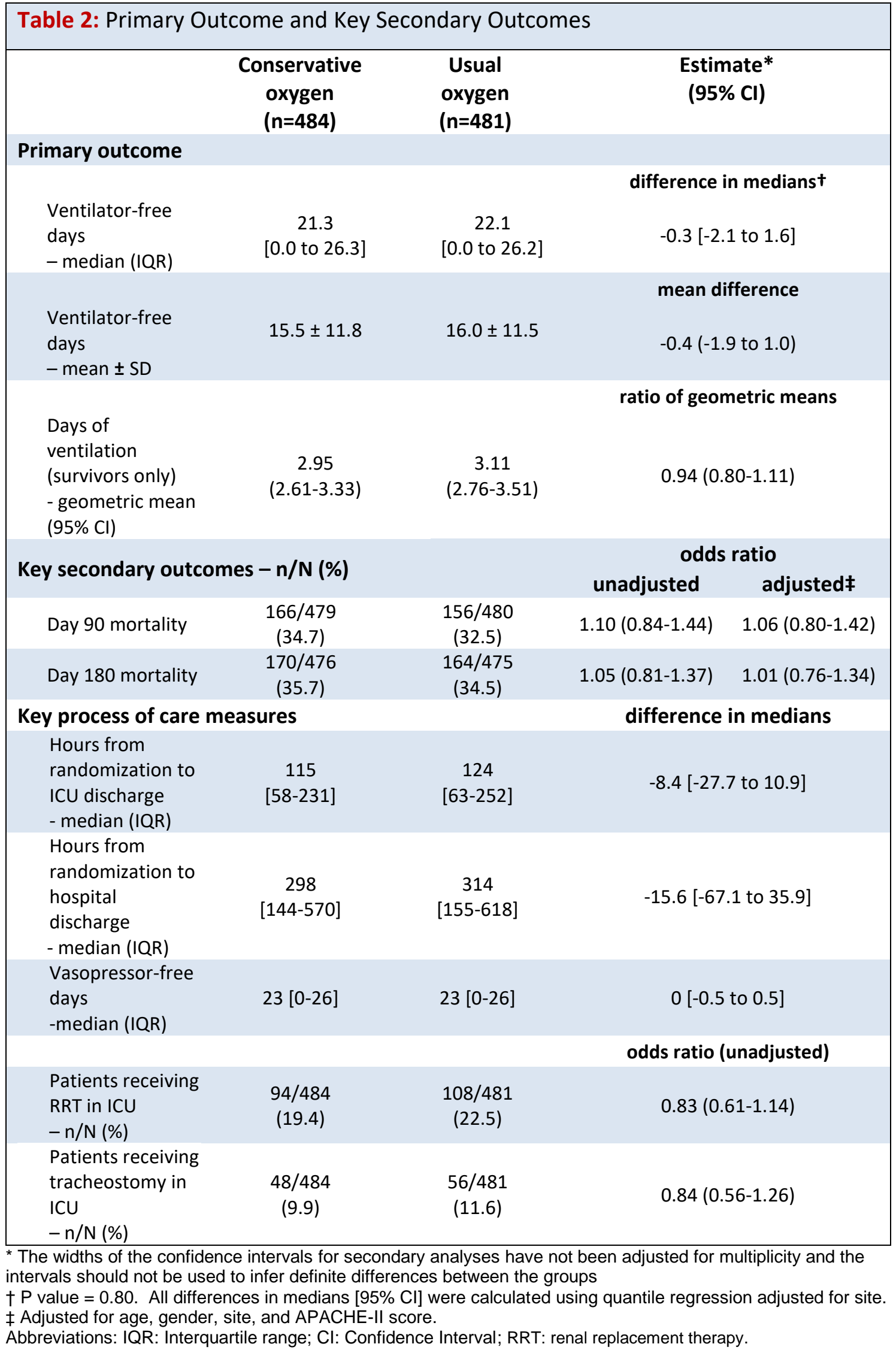




\section{Appendix 2 New Zealand ethics approvals}

\section{Victoria University of Wellington ethics approval}

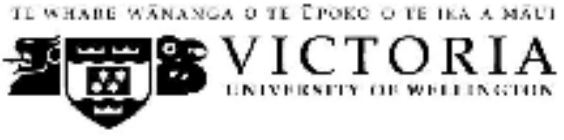

MEMORANDUM
This content is unavailable

\begin{tabular}{l|l}
\hline TO & Dr Kathy Nelson \\
\hline COPY TO & $\begin{array}{l}\text { Diane Mackle } \\
\text { Prof Richard Beasley }\end{array}$ \\
\hline FROM & AProf Susan Corbett, Convener, Human Ethics Committee \\
\hline \multicolumn{2}{|l}{} \\
\hline DATE & 27 October 2016 \\
\hline PAGES & 1 \\
\hline \multicolumn{2}{|l}{} \\
\hline SUBJECT & $\begin{array}{l}\text { Ethics Approval: 23657 } \\
\text { Practitioner attitudes to oxygen management in ICU survey }\end{array}$
\end{tabular}

Thank you for your application for ethical approval, which has now been considered by the Standing Committee of the Human Ethics Committee.

Your application has been approved from the above date and this approval continues until 31 December 2020. If your data collection is not completed by this date you should apply to the Human Ethics Committee for an extension to this approval.

Best wishes with the research.

Kind regards

This content is unavailable

Susan Corbett

Convener, Victoria University Human Ethics Committee 
Health

and

Disability

Ethics

Commillees
Health and Disability Ethica Committers Ministry of Health 133 Molesworth Street PO Box 5013 Wellington 6011

08004 ETHICS ndecsegmoh.govt.nz

08 December 2016

Ms Diane Mackle

This content is unavailable

Dear Ms Mackle

\begin{tabular}{|c|c|c|}
\hline \multirow[t]{2}{*}{ Re: } & Ethics ref: & 16/NTA/177 \\
\hline & Study title: & $\begin{array}{l}\text { The Intensive Care Unit Randomised Trial Comparing Two } \\
\text { Approaches to OXygen therapy: Translating Research into Practice } \\
\text { Study }\end{array}$ \\
\hline
\end{tabular}

I am pleased to advise that this application has been approved by the Northern A Health and Disability Ethics Committee. This decision was made through the HDEC-Full Review pathway.

\section{Summary of ethical issues (resolved)}

The main ethical issues considered by the Committee and addressed by the Researcher are as follows.

- The Committee noted that presented in this way the prospective study is collecting retrospectively routinely collected data (but more detailed data than available in the international database) about a prospective cohort of patients. This would not be in conflict with right 7(4).

- This application is instead approved under Observational guidelines 6.43 permit health information in observational studies to be collected without consent.

\section{Conditions of $\mathrm{HDEC}$ aporoval}

HDEC approval for this study is subject to the following conditions being met prior to the commencement of the study in New Zealand. It is your responsibility, and that of the study's sponsor, to ensure that these conditions are met. No further review by the Northem A Health and Disability Ethics Committee is required.

Standard conditions:

1. Before the study commences at any locality in New Zealand, all relevant regulatory approvals must be obtained.

2. Before the study commences at a given locality in New Zealand, it must be authorised by that locality in Online Forms. Locality authorisation confirms that 
the locality is suitable for the safe and effective conduct of the study, and that local research governance issues have been addressed.

\section{After HDEC review}

Please refer to the Standard Operating Procedures for Health and Disability Ethics Committees (available on www.ethics.health.govt.nz) for HDEC requirements relating to amendments and other post-approval processes.

Your next progress report is due by 8 December 2017.

\section{Participantaccess to $\mathrm{ACC}$}

The Northem A Health and Disability Ethics Committee is satisfied that your study is not a clinical trial that is to be conducted principally for the benefit of the manufacturer or distributor of the medicine or item being trialled. Participants injured as a result of treatment received as part of your study may therefore be eligible for publicly-funded compensation through the Accident Compensation Corporation (ACC).

Please don't hesitate to contact the HDEC secretariat for further information. We wish you all the best for your study.

Yours sincerely,

This content is unavailable

Dr Brian Fergus

Chairperson

Northem A Health and Disability Ethics Committee

Encl: appendix A: documents submitted appendix B: statement of compliance and list of members 


\section{Appendix A}

Documents submitted

\begin{tabular}{|l|l|l|}
\hline Document & Version & Date \\
\hline CV for Cl: CV - D Mackle & 1.0 & 29 June 2016 \\
\hline CVs for other Investigators: CV - Paul Young & 1 & 29 June 2016 \\
\hline CVs for other Investigators: CV - Richard Beasley & 1 & 29 June 2016 \\
\hline CVs for other Investigators: CV - Kathy Nelson & 1 & 29 June 2016 \\
\hline $\begin{array}{l}\text { Evidence of scientific review: Acceptance for HRC Clinical Training } \\
\text { Fellowship }\end{array}$ & 1 & 14 October 2016 \\
\hline Protocol: ICU-ROX TRIPS protocol & 1.0 & 18 October 2016 \\
\hline $\begin{array}{l}\text { Survey/questionnaire: Practitioner Attitudes Survey (approved by } \\
\text { Victoria University Ethics) }\end{array}$ & 1.0 & 30 September 2016 \\
\hline Application & 1 & - \\
\hline Response letter to provisional approval & 1.0 & 28 November 2016 \\
\hline Response to Request for Further Information & 1 & - \\
\hline
\end{tabular}




\section{Appendix B}

Statement of compliance and list of members

\section{Statement of compliance}

The Northem A Health and Disability Ethics Committee:

- is constituted in accordance with its Terms of Reference

- operates in accordance with the Standard Operating Procedures for Health and Disability Ethics Committees, and with the principles of international good clinical practice (GCP)

- is approved by the Health Research Council of New Zealand's Ethics Committee for the purposes of section 25(1)(c) of the Health Research Council Act 1990

— is registered (number 00008714) with the US Department of Health and Human Services' Office for Human Research Protection (OHRP).

\section{List of members}

\begin{tabular}{|l|l|l|l|}
\hline Name & Category & Appointed & Term Expires \\
\hline Dr Brtan Fergus & $\begin{array}{l}\text { Lay (consumerlcommunity } \\
\text { perspectives) }\end{array}$ & $11 / 11 / 2015$ & $11 / 11 / 2018$ \\
\hline Ms Rosemary Abbott & Lay (the law) & $15 / 03 / 2016$ & $15 / 03 / 2019$ \\
\hline Dr Karen Bartholomew & Non-lay (intervention studles) & $13 / 05 / 2016$ & $13 / 05 / 2019$ \\
\hline Dr Charis Brown & Non-lay (observational studles) & $11 / 11 / 2015$ & $11 / 11 / 2018$ \\
\hline Dr Christine Crooks & Non-lay (intervention studles) & $11 / 11 / 2015$ & $11 / 11 / 2018$ \\
\hline Dr Catherine Jackson & $\begin{array}{l}\text { Non-lay (health/disabilty service } \\
\text { provislon) }\end{array}$ & $11 / 11 / 2016$ & $11 / 11 / 2019$ \\
\hline Ms Tonl Millar & $\begin{array}{l}\text { Lay (consumericommunity } \\
\text { perspectives) }\end{array}$ & $11 / 11 / 2016$ & $11 / 11 / 2019$ \\
\hline Dr Kate Parker & Non-lay (observational studes) & $11 / 11 / 2015$ & $11 / 11 / 2018$ \\
\hline
\end{tabular}

Unless members resign, vacate or are removed from their office, every member of HDEC shall continue in office until their successor comes into office (HDEC Terms of Reference) 


\section{Appendix 3 Australian state and territory ethics approvals}

\section{Australian Capital Territories ethics approval}

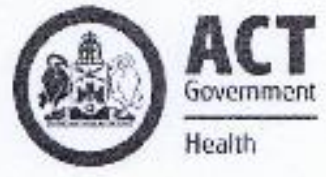

ACT Health

Research Ethics and Governance Office

Low Risk Sub-Committee

A/Professor Frank van Haren

This content is unavailable

Dear AiProfessor van Haren

ETHLR.17.117

The ACT Health Human Research Ethics Committee's Low Risk Sub-Committee received notification of the proposed study:

The Intensive Care Unit Randomised Trial Comparing Two Approaches to Oxygen therapy: Translating Research into Practice at its meeting of 30 May 2017

I am pleased to inform you that, following further correspondence, your application has been approved.

The Sub-Committee agreed that the application is for low risk research and determined that the research meets the requirements of the National Statement on Ethical Conduct in Human Research and is ethically acceptable.

I attach for your records an Outcome of Consideration of Protocol form.

I confirm that the ACT Health Human Research Ethics Committee is constituted according to the National Statement on Ethical Conduct in Human Research 2007 and is certified for single review of multi-centre clinical trials. ACT Health HREC operates in compliance with applicable regulatory requirements and the International Conference on Harmonization Guidelines on Good Clinical Practice.

Yours sincerely

This content is unavailable

Louise Morauta PSM PhD

Chair

ACT Health Human Research Ethics Committee

Low Risk Sub-Committee

29 Junte 2017

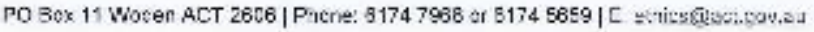




\section{Cabrini Health ethics approval}

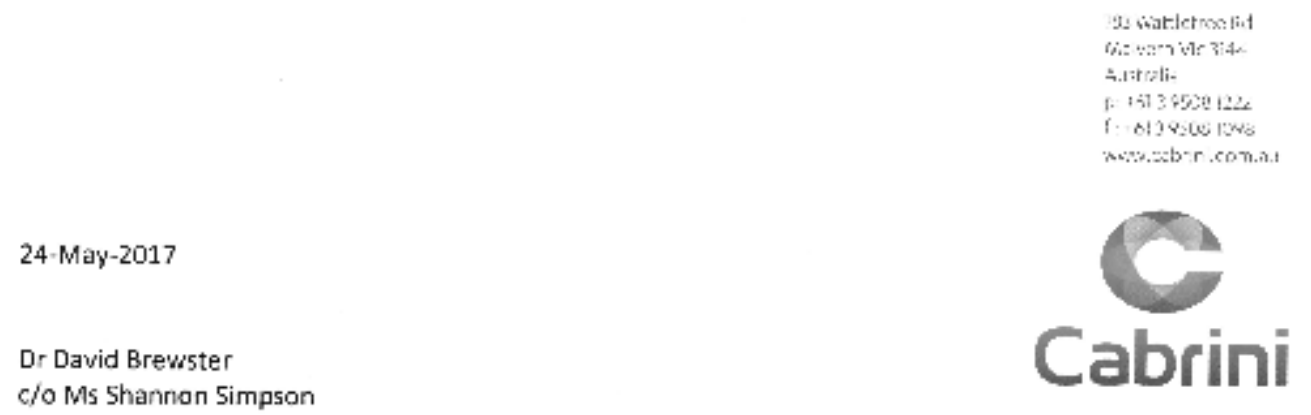

Dear David

14-15-05-17

ICU ROX TRIPS: The Intensive Care Unit Randomised Trial Comparing Two Approaches to Oxygen Therapy: Translating Research into Practice.

This study is now approved. This approval recognises the approval of The Alfred HREC Human Research Ethics Committee, reference number HR.EC/16/Alfred/175 (Local ref: Project 553/16), dated 11-Jan-2017 and the documents listed. The gavernance aspects are now approved by Cabrini.

If the study does not commence before the anniversary of approval, approval will lapse. Approval is ongoing for the life of the project, subject to satisfactary compliance and reporting.

In accordance with the NHMRC's Notional Statement on Ethical Conduct in Human Research, 2007, you are abliged to:

- provide the CHREC with annual reports on the anniversary of this approval;

- provide the CHREC with a final report on study completion;

- be available for audits/site visits/interviews as requested.

In addition, you are obliged to inform the CHREC of:

- any change to the protocol or participant information or consent form;

- any adverse events that occur during the process of this trial as per guidelines in the CHREC handbook;

- any changes to the research team of investigators;

- study completion:

- any change in the financial arrangements regarding the study.

Cabrini should be acknowledged in all papers developed from this study.

We wish you well with your project.

Yours sincerely

This content is unavailable

Jerinifer Burden

Manager

Cabrini Human Rescarch Ethics Committee 


\title{
New South Wales ethics approval
}

\author{
Regearch Difice \\ Kolling Building, Level 13 \\ Roval North Shore Hosstol \\ St Leonards NSW' 206 \\ Tel (02) 99264590 Fax (02) 99266179
}

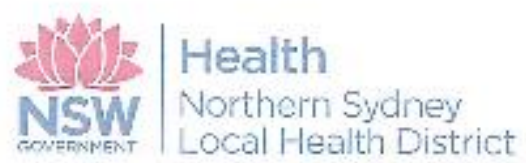

14 Fabruany 2017

Ms Diane Meckle

\section{This content is unavailable}

Dear Diane.

MSLHO reference: RESP/1S/3s0

95udy Thle: Tho Intsnsive Gave Uni: Rendemised trial comperting wo approathes to Oxygen therspy:

Translating Research Into Practice study

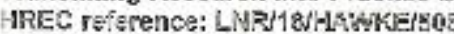

Thank you for submitting the above project for review. This HREC nas been acuiedited by NSW Miniztor of Hoalth as a Lead HREC under the model for single ethical ard scientific revienv and Certified by the NHMRC under the Mational model for Harmonisation of Rulticente Ethical Review HoMERi. This lead HREC is constituted and operates in accordance with the National Health and isedica Research Council's Netorna' Stajement on Ethical Conduct in Human Ressarch and the CPWPACH Noto for Guidancs on Ccod Clinica! Practice. No HREC members with a conflict of interest were prasent far review of this project.

1 am pleased to advise that the HREC at a meeting on 13 Fsisuary 3017 has granted ethical and scientific approve of the atove multi sentre project.

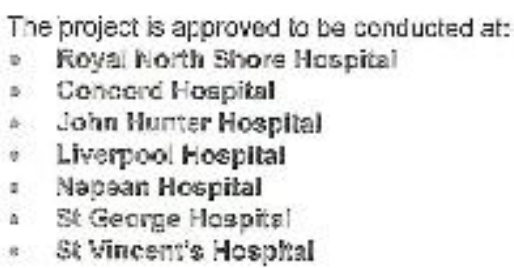

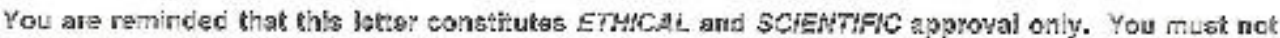
commence this research project until you receive notification of Sitte Spcciflc hesecsmant Authcrieation from the Resesch Govemance Officer, The LNRGSA submitted wilth this LWR application has been fonwrcied to the N\&LHD Rssadrch Governgnce Oficer for restew.

If a new siteis) is to be added please inform the HREC in witing and submit a Site Speciic Assassment Form (SSA) to the Research Governance Officer at the neys site.

The following documentelion hes beer reviewed and aporoved by the HREC:

\begin{tabular}{|l|l|l|}
\hline Decument & Verzion & Dake \\
\hline Study Protoool & 1.0 & 18 Octooer 2016 \\
\hline Study Protocel Appendix One: Practitioner Attitudas Survay & 1.0 & 18 October 2016 \\
\hline Information Sheat and Inv tation Letter for Implied Consent & 1 & 25 January 2017 \\
\hline Data Colection Form: Form 1 Beseline & 1.0 & 8 September 2016 \\
\hline Data Colection Form: Form 2 Daily Data Days 0-10 & 1.0 & 8 September 2016 \\
\hline
\end{tabular}

The fallawing documents have been noted:

- Letter of SLpport from CORE Management Committes, ANZICS CORE dated 2 December 20.16 


\section{South Australia ethics approval}

Approval Date: 12 December 2016

HREC Reference number. HREC/16/RAH/538

CALHN Reference number: R20161130

Ms Diane Mackle

Intensive Care Unit

MEDICAL RESEARCH INSTITUTE OF NEW ZEALAND

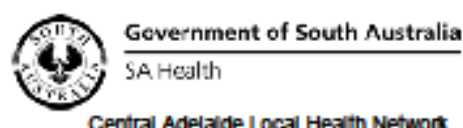

Royal Adelalde Hospital Human Research Ethics Committee Level 4, Women's Health Centre Royal Adelaide Hospit: North Terrace

Adelaide, South Australla, 5000 Telephone: +61882224139
Emall: Heath.CALHNResearchEthics $85 a . g 0 v . a u$

\section{Dear Ms Mackle}

Project Title: The Intensive Care Unit Randomised Trial Comparing Two Approaches to OXygen therapy: Translating Research Into Practice Study.

- Study 1 Inception Cohort Study

- Study 2 Practitioner Attitudes Survey

- Study 3 Retrospective cohort study of oxygen management over time

Thank you for submitting the above project for ethical review. This project was considered by the Chairman of the Royal Adelaide Hospital Human Research Ethics Committee. I am pleased to advise that your protocol has been granted full ethics approval and meets the requirements of the National Statement on Ethical Conduct in Human Research (2007) incorporating all updates. The documents reviewed and approved include:

\begin{tabular}{|l|c|l|}
\hline Document & Version & Date \\
\hline LNR Application (AU/15/943A212) & - & - \\
\hline Protocol & 1.0 & 18 October 2016 \\
\hline Patient Information Sheet & 1 & 22 November 2016 \\
\hline Ethics Approval Letter from Victoria University of Wellington & - & - \\
\hline
\end{tabular}

Sites covered by this approval:

- Lyell McEwin Hospital, SA : CPI - Ms Diane Mackle

- Queen Elizabeth Hospital, SA : CPI - Ms Diane Mackle

- Flinders Medical Centre, SA : CPI - Ms Diane Mackle

\section{GENERAL TERMS AND CONDITIONS OF ETHICAL APPROVAL:}

- Adequate record-keeping is important. If the project involves signed consent, you should retain the completed consent forms which relate to this project and a list of all those participating in the project, to enable contact with them in the future if necessary. The duration of record retention for all clinical research data is 15 years.

- You must notify the Research Ethics Committee of any events which might warrant review of the approval or which warrant new information being presented to research participants, including:

(a) serious or unexpected adverse events which warrant protocol change or notfication to research participants,

(b) changes to the protocol,

(c) premature termination of the study.

- The Committee must be notified within 72 hours of any serious adverse event occurring at this site.

- Approval is valid for $\mathbf{5}$ years from the date of this letter, after which an extension must be applied for.

- Confidentiality of the research participants shall be maintained at all times as required by law.

- Investigators are responsible for providing an annual review to the RAH REC Executive Officer each anniversary of the above approval date, within 10 workings days, using the Annual Review Form available at: https://www.rahresearchfund.com. au/rah-research-institute/for-researchers/human-research-ethics/

- The REC must be advised with a report or in writing within 30 days of completion. 
Should you have any queries about the HREC's consideration of your project, please contact Ms Heather O'Dea on 0882224139 , or Health.CALHNResearchEthics(6)sa.oov.au.

You are reminded that this letter constitutes ethical approval only. You must not commence this research project at a SA Health site until governance authorisation at that site has been obtained. Please contact the CALHN Research Office Health.CALHNResearchLIRRmsangraul

This Committee is constituted in accordance with the NHMRC's National Statement on the Ethical Conduct of Human Research (2007)

The HREC wishes you every success in your research.

Yours sincerely.

This content is unavailable

A/Prof A Thornton

CHAIRMAN

RESEARCH ETHICS COMMITTEE 


\section{Tasmania ethics approval}

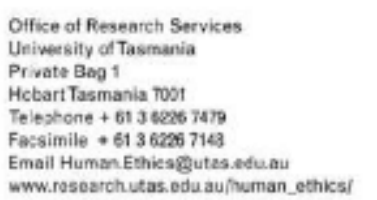

19 September 2017

Dr Matthew Brain

Ci- Launceston General Hospital

Sent via email

Dear Dr Brair

REF NO: H0016433

TITLE: ICU ROX TRIPS The Intensive Care Unit Randomised Trial comparing two Approaches lo Oxygen therapy

\begin{tabular}{|c|c|c|}
\hline Document & Version & Date \\
\hline \multicolumn{3}{|l|}{ Alfred Health HREC application } \\
\hline Alfred Health HRFC approval letter & & 18 Oct 2016 \\
\hline Prior Approval & & 22 Mar 2017 \\
\hline Privacy Fom & & 22 Mar 2017 \\
\hline ICU-ROX TRIPS form 1 Baseline & Version 0.1 & 08 Sep 2016 \\
\hline ICU-ROX TRIPS form 2 daily data days 0-10 & Version 0.1 & $08 \operatorname{Scp} 2016$ \\
\hline ICU-ROX TRIPS Protoco| & Version 0.1 & 18 Oct 2016 \\
\hline ICU-ROX TRIPS MASTER Information Sheet & Version 1 & 25 May 2017 \\
\hline ICU-ROX TRIPS H0016433 LGH Information Sheet & Version 1 & 25 May 2017 \\
\hline ICU-ROX-TRIPS LGH Supporting Document & & \\
\hline
\end{tabular}

The Tasmanian Health and Medical Human Research Ethics Committee considered and approved the above documentation on 04 September 2017 to be conducted at the following site(s):

\section{Launceston General Hospital}

Please ensure that all investigators involved with this project have cited the approved versions of the dociuments lister: within this lefter and use tonly these versions in conducting this research project.

This approval constitutes ethical clearance by the Health and Medical HREC. The decision and authority to commence the associated rescarch may bo dopendent on factors beyond the remit of the ethics review process. For example, your research may need ethics clearance from other organisaticns or review by your research governance coordinator or Heac of Department. It is ycur responsibility to find out if the approvals of other bodies or authorities are required. It is recommended that the proposed research should not commence until you have satisfied these requirements. 
All committees operating under the Human Research Ethics Committee (Tasmania)

Network are registered and required to comply with the National Statement on the Ethical Conduct in Hurman Research (NHMRC 2007 updalec 2014).

Therefore, the Chief Investigator's responsibility is to ensure that:

(1) The individual researcher's protocol complies with the HREC approvec protocal.

(2) Modifications to the protocol do not proceed until approval is obtained in writing from the HREC. Please note that all requests for changes to approved documents must include a version number and date when submitted for review by the HREC.

(3) Section 5.5 .3 of the National Statement states:

Researchers have a significant responsibility in monitoring approved research as they are in the best position to observe any adverse events or unexpected outcomes. They should report such events or outcomes promptly to the relevant institutionis and ethical review bndylies and take prompt steps to deal with any unexpected risks.

The appropriate forms for reporting such events in re ation to clinica and non-clinical trials and innovations can be located at the website below. All adverse events must be reported regardless of whether or not the ovent, in your opinion, is a dircet effect of the therapeutic goods being tested. http:ihww.utas edu auiresearch-adminiresearchintegrity-and-ethics-unit-rieu'human-ethics/human-research-ethics-reviewprocessihealth-and-med cal-hrecimanaging-your-approved-project

(4) All research participants must he provided with the current Patient Information Sheet and Consent Form, unless otherwise approved by the Committee.

(5) The Committee is notified if any investigators are added to, or cease involverrent with, the project.

(6) This study has approval for four ycars contingent upon annual rovicw. A Progress Report is to be provided on the anniversary date of your approval. Your first report is due 4 September 2018. You will be sent a courtesy reminder closer to this due date.

(7) A Final Report and a copy of the published malerial, either in full on abstract, must be provided at the end of the project.

Should you have any queries please do not hesitate to contact me on (Uj) 62262764 .

Yours sincerely

This content is unavailable

Jude Vienna-Hallam

Ethics Administration Officer 


\section{Victoria ethics approval}

\section{0 \\ theAlfred}

\section{ETHICS COMMITTEE CERTIFICATE OF APPROVAL}

This is to certify that

Project No: HREC/16/Alfred/175 (Local Reference Project 553/16)

Project Title: The Intensive Care Unit Randomised Trial Comparing Two Approaches to Oxygen therapy: Translating Research Into Practice (ICU-ROX TRIPS)

Principal Researcher: A/Professor Andrew Udy

Project Proposal Version 1 dated: 18-10-2016

was considered for Low Risk Review under the Victorian Streamlined Ethical Review Process (SERP), meets the requirements of the National Statement on Ethical Conduct in Human Research (2007) and was APPROVED on 11-01-2017

It is the Principal Researcher's responsibility to ensure that all researchers associated with this project are aware of the conditions of approval and which documents have been approved.

The Principal Researcher is required zo nouty ute Secrerary of the Euthics Commimee, via amendment or progress report, of

- Any signincant change to the project and the reason for that change, including an indlcaton of ethical implications (II any

Senlous adverse effects on participants and the action taken to address those effects;

- Any other unforeseen events or unexpected developments that mert notitcation;

- The inability of the Principal Researcher to continue in that role, or any other change in research personnel Involved In the project.

- Any expiry of the insurance coverage provided with respect to commerclally sponsored projects and proof of re-

Insurance;

- A delay of more than 12 months in the commencement of the project, and,

- Temination or closure of the project.

Additionally, the Principal Researcher is required to submin

- A Progress Report on the anniversary of approval and on completion of the project forms to be provided); The Etrics Committee may conduct an audit at any time.

All research subject to the Alfred Hospital Ethics Committee review must be conducted In accordance with the National Statement on Ethical Conduct in Human Research (2007).

The Alfred Hospital Ethics Committee is a properly consttuted Human Research Ethics Committee in accordance with the National Statement on Etrical Conduct in Human Research (2007)

SPECIAL CONDITIONS

None

\section{CONSENT WAIVER}

In accordance with the Omce of the Health Services Commlssioner's Stautory Guldelines on Research issued for the

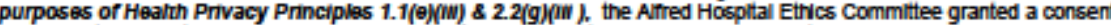
walver for the collection, use and dsclosure of participants' health and personal information . 


\section{Approved documents}

Documents reviewed and approved at the meeting were:

\begin{tabular}{|l|c|c|}
\hline Document & Version & Date \\
\hline Project Protocol & 1 & $18-$ Oct-2016 \\
\hline Practitioner Attitude Survey & 1 & $18-$ Oct-2016 \\
\hline Information Statement master (practitioners) & 1 & $22-$ Nov-2016 \\
\hline Email for survey master & 1 & $24-$ Nov-2016 \\
\hline CRF Baseline & 1 & $08-S e p t-2016$ \\
\hline CRF Daily Data & 1 & $08-S e p t-2016$ \\
\hline
\end{tabular}

\section{Approved sites}

Approval is given for this research project to be conducted at the following sites and campuses:

- Alfred Health - The Alfred

- Royal Melbourne Hospital

- St Vincent's Hospital (Melbourne)

- Sunshine Hospital

- Footscray Hospital

- Monash Hospital

- Dandenong Hospital

- Bendigo Health

The Alfred Hospital Ethics Committee has approved the study but does not take responsibility for research governance processes at the participating sites. It is the responsibility of each participating site to create and implement research governance practices to adequately authorise, monitor and oversee the conduct of the study at their site.

\section{Site-Specific Assessment (SSA)}

SSA authorisation is required at all sites participating in the study, SSA must be authorised at a site before the research proiect can commence.

The completed Site-Specific Assessment Form and a copy of this ethics approval letter must be submitted to the Research Governance Officer for authorisation by the Chief Executive or delegate. This applies to each site participating in the research.

The HREC wishes you and your colleagues every success in your research.

SIGNED:

This content is

unavailable

Protessor John J. McNell

Chalr, Ethics Committee

Please quote project number and utule in all correspondence 


\section{Appendix 4 Information sheets}

Information sheet for ICU-ROX TRIPS (v 1.0_25 October 2016)
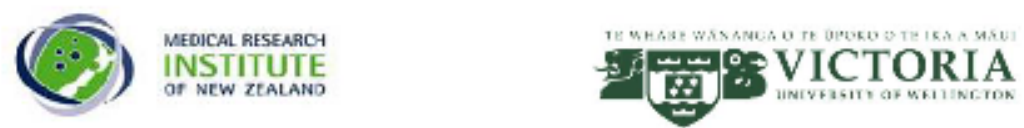

\section{ICU-ROX TRIPS Practitioner Attitudes Survey}

\section{INFORMATION SHEET}

Thank you for your interest in this project. Please read this information before deciding whether or not to take part. If you decide to participate, thank you. If you decide not to take part, thank you for considering my request.

Who am I?

My name is Diane Mackle and I am a Doctoral student in PhD (Health) at Victoria University of Wellington. This research project will contribute to my thesis.

What is the aim of the project?

This survey is part of an overall project investigating the knowledge translation of the ICU-ROX (Intensive Care Unit Randomised Trial Comparing Two Approaches to Oxygen therapy) trial. ICUROX Translation of Research into Practice Study (TRIPS) is designed to assess the attitudes of health practitioners with regard to oxygen management in invasively mechanically ventilated patients in ICU. The study aims to see whether these attitudes change over time in practitioners working in ICUs which participate in the ICU-ROX trial compared to those working in ICUs which do not participate.

This research has been approved by the Victoria University of Wellington Human Ethics Committee [provide approval number].

How can you help?

You will be sent a link to an on-line survey about oxygenation practices in invasively mechanically ventilated ICU patients. You will be asked to complete the survey at 3 timepoints:

- before ICU-ROX starts

- after the ICU-ROX trial is completed (but before it is published), and

- 6-12 months after the publication of the results

Because we want to see whether your views about oxygenation practice change over time, we require your email address, so that we can contact you for the subsequent surveys. Participation is optional and consent will be implied by completion of the survey.

What will happen to the information you give?

This research is confidential. This means that the researchers' named below will be aware of your identity as a respondent. The research data will be aggregated for analysis and your identity will not be disclosed in any reports, presentations, or public documentation. 
Only my supervisors and I will have access to the data from the on-line survey.

What will the project produce?

The information from my research will be used in my $\mathrm{PhD}$ dissertation, and the findings will be published.

If you accept this invitation, what are your rights as a research participant?

You do not have to accept this invitation if you don't want to. If you do decide to participate, you have the right to:

- choose not to answer any question;

- withdraw from the study within a month of completion of each of the surveys;

- $\quad$ ask any questions about the study at any time;

If you have any questions or problems, who can you contact?

If you have any questions, either now or in the future, please feel free to contact:

Student:

Name: Diane Mackle

This content is unavailable
Supervisors:

Kathy Nelson (Senior Lecturer, GSNMH)

This content is unavailable

Richard Beasley (Director, MRINZ)

This content is unavailable Paul Young (ICU Specialist, Wellington Hospital)

This content is unavailable

Human Ethics Committee information

If you have any concerns about the ethical conduct of the research you may contact the Victoria University HEC Convener: Associate Professor Susan Corbett. Email

This content is unavailable 

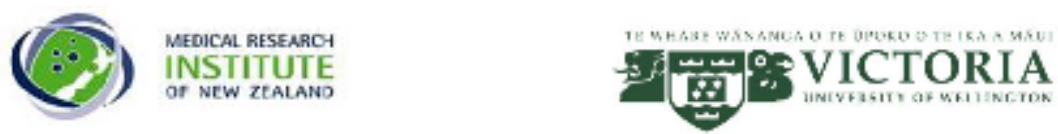

\section{ICU-ROX TRIPS Practitioner Attitudes Survey INFORMATION SHEET}

Thank you for your interest in this project. Please read this information before deciding whether or not to take part. If you decide to participate, thank you. If you decide not to take part, thank you for considering my request.

Who am I?

My name is Diane Mackle and I am a Doctoral student in PhD (Health) at Victoria University of Wellington. This research project will contribute to my thesis.

What is the aim of the project?

This survey is part of an overall project investigating the knowledge translation of the ICU-ROX (Intensive Care Unit Randomised Trial Comparing Two Approaches to Oxygen therapy) trial. ICUROX Translation of Research into Practice Study (TRIPS) is designed to assess the attitudes of health practitioners with regard to oxygen management in invasively mechanically ventilated patients in ICU. The study aims to see whether these attitudes change over time in practitioners working in ICUs which participate in the ICU-ROX trial compared to those working in ICUs which do not participate.

This research has been approved by the Victoria University of Wellington Human Ethics Committee (reference 23657).

\section{How can you help?}

You will be sent a link to an on-line survey about oxygenation practices in invasively mechanically ventilated ICU patients. You will be asked to complete the survey at 3 timepoints:

- before ICU-ROX starts

- after the ICU-ROX trial is completed (but before it is published), and

- 1-12 months after the publication of the results

Because we want to see whether your views about oxygenation practice change over time, we require your email address, so that we can contact you for the subsequent surveys. Participation is optional and consent will be implied by completion of the survey.

What will happen to the information you give?

This research is confidential. This means that the researchers' named below will be aware of your identity as a respondent. The research data will be aggregated for analysis and your identity will not be disclosed in any reports, presentations, or public documentation.

ICU-ROX TRIPS Information Sheet v1.2 dated 29 August 2019 
Only my supervisors and I will have access to the data from the on-line survey.

What will the project produce?

The information from my research will be used in my $\mathrm{PhD}$ dissertation, and the findings will be published.

If you accept this invitation, what are your rights as a research participant?

You do not have to accept this invitation if you don't want to. If you do decide to participate, you have the right to:

- choose not to answer any question;

- withdraw from the study within a month of completion of each of the surveys;

- $\quad$ ask any questions about the study at any time;

If you have any questions or problems, who can you contact?

If you have any questions, either now or in the future, please feel free to contact:

Student:

Name: Diane Mackle

This content is unavailable
Supervisors:

Kathy Nelson (Senior Lecturer, GSNMH)

This content is unavailable

Richard Beasley (Director, MRINZ)

This content is unavailable

Paul Young (ICU Specialist, Wellington Hospital)

This content is unavailable

Human Ethics Committee information

If you have any concerns about the ethical conduct of the research you may contact the Victoria University HEC Convenor This content is unavailable

This content is unavailable 


\section{Appendix 5 Supplemental table}

Table S1 Survey response rate by question over time (denominators)

\begin{tabular}{|c|c|c|c|c|c|c|}
\hline & \multicolumn{2}{|c|}{ T1: Before ICU-ROX } & \multicolumn{2}{c|}{ T2: After ICU-ROX } & \multicolumn{2}{c|}{ T3: After publication } \\
\hline $\mathrm{F}_{\mathrm{IO}}$ level & $\begin{array}{c}\text { ICU-ROX } \\
\text { respondents } \\
(\mathrm{n}=142)\end{array}$ & $\begin{array}{c}\text { Non-ICU-ROX } \\
\text { respondents } \\
(\mathrm{n}=122)\end{array}$ & $\begin{array}{c}\text { ICU-ROX } \\
\text { respondents } \\
(\mathrm{n}=127)\end{array}$ & $\begin{array}{c}\text { Non-ICU-ROX } \\
\text { respondents } \\
(\mathrm{n}=109)\end{array}$ & $\begin{array}{c}\text { ICU-ROX } \\
\text { respondents } \\
(\mathrm{n}=111)\end{array}$ & $\begin{array}{c}\text { Non-ICU-ROX } \\
\text { respondents } \\
(\mathrm{n}=82)\end{array}$ \\
\hline
\end{tabular}

Post-operative surgical patient

\begin{tabular}{|l|l|l|l|l|l|l|}
\hline 0.21 & 142 & 122 & 125 & 108 & 111 & 82 \\
\hline 0.25 & 140 & 119 & 126 & 107 & 108 & 80 \\
\hline 0.3 & 140 & 115 & 119 & 104 & 107 & 79 \\
\hline 0.4 & 137 & 114 & 121 & 102 & 108 & 76 \\
\hline 0.6 & 138 & 113 & 117 & 107 & 106 & 79 \\
\hline 1.0 & 139 & 114 & 124 & 107 & 107 & 80 \\
\hline
\end{tabular}

\section{Patient with admission diagnosis of acute brain pathology}

\begin{tabular}{|l|l|l|l|l|l|l|}
\hline 0.21 & 141 & 121 & 127 & 109 & 110 & 82 \\
\hline 0.25 & 140 & 119 & 126 & 107 & 108 & 80 \\
\hline 0.3 & 140 & 115 & 120 & 104 & 107 & 79 \\
\hline 0.4 & 137 & 114 & 122 & 101 & 108 & 77 \\
\hline 0.6 & 138 & 113 & 119 & 106 & 106 & 79 \\
\hline 1.0 & 138 & 115 & 126 & 108 & 107 & 80 \\
\hline
\end{tabular}

Patient with a suspected hypoxic brain injury following a cardiac arrest

\begin{tabular}{|l|l|l|l|l|l|l|}
\hline 0.21 & - & - & - & - & 111 & 82 \\
\hline 0.25 & - & - & - & - & 108 & 80 \\
\hline 0.3 & - & - & - & - & 107 & 78 \\
\hline 0.4 & - & - & - & - & 108 & 76 \\
\hline 0.6 & - & - & - & - & 105 & 79 \\
\hline 1.0 & - & - & - & - & 107 & 78 \\
\hline
\end{tabular}

\section{Patient with pneumonia}

\begin{tabular}{|l|l|l|l|l|l|l|}
\hline 0.21 & 140 & 122 & 127 & 109 & 111 & 82 \\
\hline 0.25 & 140 & 119 & 125 & 106 & 108 & 79 \\
\hline 0.3 & 140 & 115 & 119 & 104 & 107 & 79 \\
\hline 0.4 & 137 & 114 & 121 & 102 & 108 & 77 \\
\hline 0.6 & 138 & 113 & 118 & 107 & 106 & 79 \\
\hline 1.0 & 140 & 115 & 125 & 107 & 107 & 80 \\
\hline
\end{tabular}

ICU patient with none of the above and no indication for low or high $\mathrm{F}_{1} \mathrm{O}_{2}$

\begin{tabular}{|c|c|c|c|c|c|c|}
\hline 0.21 & 142 & 122 & 127 & 109 & 111 & 82 \\
\hline 0.25 & 139 & 119 & 125 & 107 & 108 & 80 \\
\hline 0.3 & 138 & 114 & 119 & 104 & 107 & 79 \\
\hline 0.4 & 137 & 114 & 122 & 102 & 107 & 76 \\
\hline 0.6 & 137 & 113 & 118 & 104 & 105 & 77 \\
\hline
\end{tabular}




\begin{tabular}{|c|c|c|c|c|c|c|}
\hline & \multicolumn{2}{|c|}{ T1: Before ICU-ROX } & \multicolumn{2}{c|}{ T2: After ICU-ROX } & \multicolumn{2}{c|}{ T3: After publication } \\
\hline $\mathrm{F}_{1} \mathrm{O}_{2}$ level & $\begin{array}{c}\text { ICU-ROX } \\
\text { respondents } \\
(\mathrm{n}=142)\end{array}$ & $\begin{array}{c}\text { Non-ICU-ROX } \\
\text { respondents } \\
(\mathrm{n}=122)\end{array}$ & $\begin{array}{c}\text { ICU-ROX } \\
\text { respondents } \\
(\mathrm{n}=127)\end{array}$ & $\begin{array}{c}\text { Non-ICU-ROX } \\
\text { respondents } \\
(\mathrm{n}=109)\end{array}$ & $\begin{array}{c}\text { ICU-ROX } \\
\text { respondents } \\
(\mathrm{n}=111)\end{array}$ & $\begin{array}{c}\text { Non-ICU-ROX } \\
\text { respondents } \\
(\mathrm{n}=82)\end{array}$ \\
\hline 1.0 & 139 & 115 & 125 & 107 & 106 & 79 \\
\hline
\end{tabular}

Abbreviations: $\mathrm{F}_{\mathrm{O}}$ : fraction of inspired oxygen; ICU-ROX: Intensive Care Unit Randomised Trial Comparing Two Approaches to Oxygen therapy; ICU-ROX respondents: respondents from ICUs that participated in ICUROX; non-ICU-ROX respondents: respondents from ICUs that did not participate in ICU-ROX; T1: before ICUROX; T2: after ICU-ROX (but before results known); T3: after ICU-ROX publication. 


\title{
Appendix 6 ANZICS Clinical Trials Group endorsement
}

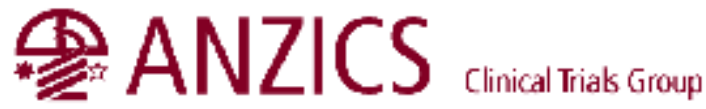

$3^{\text {rd }}$ April 2017

Dianne Mackle

This content is unavailable

Dear Dianne,

Re: Application for CTG Endorsement: ANZICS CTG Study Endorsement Number CTG1617-008

Thank you for submitting " ICU-ROX TRIPS : Study 1 Inception Cohort Study " for CTG endorsement. I am pleased to inform you that your application has been reviewed in accordance with the CTG Terms of Reference and approved.

You are invited to refer to this project as a CTG-endorsed study subject to the conditions of endorsement set out in the CTG Terms of Reference, Conflict of Interest and Competing Studies Policies, as amended periodically (current versions attached). Among these, please note that any formal agreement with a commercial body to provide support for the study will need to be governed by a contract which will need to be submitted for CTG approval prior to being signed.

Please also note above your study number and please use this number whenever sending correspondence to the CTG office. Could you please also forward your study twitter handle to the CTG office if you have not already done so and remember to include the ANZICS CTG twitter handle @ANZICS_CTG on all of your tweets.

Finally, please ensure that copies of all relevant study documents and project updates are submitted 6-monthly prior to the CTG committee meeting. Submission requirements and deadlines will be notified by email.

On behalf of the CTG and the CTG Committee, congratulations on an excellent study proposal.

Yours sincerely,

This content is unavailable

Prof Craig French

Chair, ANZICS Clinical Trials Group

\author{
Level 3, 10 levers Terrace CARLTON VIC 3053 \\ PO Box 164 Cariton South AUSTRALIA 3053 \\ Ph +61 393403455 Fax +61 93403499 Email ctg @anzics.com.au \\ www.anzics.com.au/clínical-trials-group
}




\section{Appendix 7 ANZICS Centre for Outcome and Resource Evaluation approval}

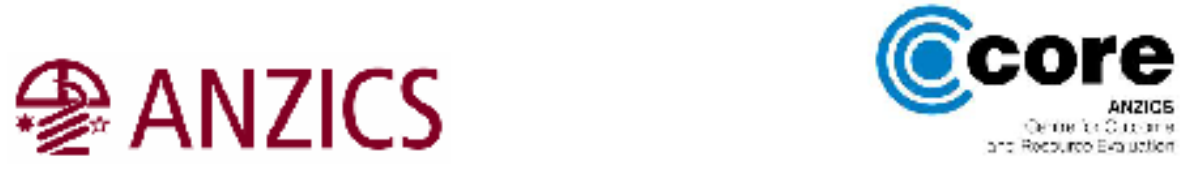

Diane Mackle

This content is unavailable

$2^{\text {nd }}$ December 2016

Dear Ms Mackle,

The CORE Management Committee encourages the use of data from the Intensive Care registries data held by ANZICS CORE to support research. We and look forward to your data request related to the 'ICU-ROX TRIPS' study (an observational study based on the ICU-ROX trial). Please note all data requests are subject to approval from the CORE Management Committee and comply with the 'ANZICS CORE Data Access and Publication Policy'.

Thank you for your ongoing support of ANZICS CORE, we look forward to seeing the outputs from your research. If you have any further questions please do not hesitate to contact us.

Regards

This content is unavailable

Manager, ANZICS CORE

On behalf of the CORE Management Committee

Australian AND NeW Zealand InTENSIVE CARE SOCIETY: CENTRE FOR OUTCOME AND RESOURCE EVALUATION 


\section{REFERENCE LIST}

Acute Respiratory Distress Syndrome Network. (2000). Ventilation with Lower Tidal Volumes as Compared with Traditional Tidal Volumes for Acute Lung Injury and the Acute Respiratory Distress Syndrome. New England Journal of Medicine, 342(18), 11301-11308. https://doi.org/10.1097/00132586-200102000-00017

Adler, M. W. (1978). Changes in local clinical practice following an experiment in medical care: Evaluation of evaluation. Journal of Epidemiology and Community Health, 32, 143-146.

Adler, M. W., Waller, J. J., Creese, A., \& Thorne, S. C. (1978). Randomised controlled trial of early discharge for inguinal hernia and varicose veins. Journal of Epidemiology and Community Health, 32(2), 136-142. https://doi.org/10.1136/jech.32.2.136

AIMS Trial Study Group. (1988). Effect of intravenous ASPAC on mortility after acute myocardial infarction: Preliminary report of a placebo-controlled clinical trial. Lancet, 331(8585), 545-549. https://doi.org/10.1016/S0140-6736(88)91350-5

Anasetti, C., Logan, B. R., Lee, S. J., Waller, E. K., Weisdorf, D. J., Wingard, J. R., Cutler, C. S., Westervelt, P., Woolfrey, A., Couban, S., Ehninger, G., Johnston, L., Maziarz, R. T., Pulsipher, M. A., Porter, D. L., Mineishi, S., McCarty, J. M., Khan, S. P., Anderlini, P., ... Confer, D. L. (2012). Peripheral-blood stem cells versus bone marrow from unrelated donors. New England Journal of Medicine, 367(16), 1487-1496.

https://doi.org/10.1056/nejmoa1203517

Andersen, M., Kragstrup, J., \& Søndergaard, J. (2006). How conducting a clinical trial affects physicians' guideline adherence and drug preferences. JAMA, 295(23), 2759-2764. https://doi.org/10.1001/jama.295.23.2759

Antman, E. M. (1996). Hirudin in acute myocardial infarction: Thrombolysis and thrombin inhibition in myocardial infarction (TIMI) 9B trial. Circulation, 94(5), 911-921. https://doi.org/10.1161/01.CIR.94.5.911

ANZICS Centre for Outcome and Resource Evaluation. (n.d.). Adult Patient Database (APD) - ANZICS. Retrieved March 28, 2021, from https://www.anzics.com.au/adult-patientdatabase-apd/

ANZICS Centre for Outcome and Resource Evaluation. (2018). Guidelines for validating APD data submission extracts generated by non-COMET systems. http://www.anzics.com.au/Pages/CORE/data-tools.aspx 
ANZICS Centre for Outcome and Resource Evaluation. (2020). Centre for Outcome and Resource Evaluation 2019 report. https://www.anzics.com.au/annual-reports/ ANZICS Centre for Outcome and Resource Evaluation. (2021). ANZICS CORE APD data dictionary version 5.12. https://www.anzics.com.au/wpcontent/uploads/2021/03/ANZICS-APD-Dictionary.pdf

ANZICS Clinical Trials Group. (2016). ANZICS CTG terms of reference. https://www.anzics.com.au/wp-content/uploads/2018/09/ANZICS-CTG-Terms-ofReference-June-2016.pdf

ANZICS Clinical Trials Group. (2018). ANZICS Clinical Trials Group Activity Report 2017 2018. https://www.anzics.com.au/wpcontent/uploads/2019/05/ANZICS_CTG_Activity_Report_201718.pdf

Australian and New Zealand Intensive Care Society. (2021). ANZICS 2020 annual report. https://www.anzics.com.au/wp-content/uploads/2020/11/ANZI_3422_AnnualReport.pdf

Bakker, J. J. H., Verhoeven, C. J. M., Janssen, P. F., van Lith, J. M., van Oudgaarden, E. D., Bloemenkamp, K. W. M., Papatsonis, D. N. M., Mol, B. W. J., \& van der Post, J. A. M. (2010). Outcomes after Internal versus External Tocodynamometry for Monitoring Labor. New England Journal of Medicine, 362(4), 306-313. https://doi.org/10.1056/NEJMoa0902748

Bayram, N., Van Wely, M., Kaaijk, E. M., Bossuyt, P. M. M., \& Van Der Veen, F. (2004). Using an electrocautery strategy or recombinant follicle stimulating hormone to induce ovulation in polycystic ovary syndrome: Randomised controlled trial. British Medical Journal, 328(7433), 192-195. https://doi.org/10.1136/bmj.328.7433.192

Beasley, R., Chien, J., Douglas, J., Eastlake, L., Farah, C., King, G., Moore, R., Pilcher, J., Richards, M., Smith, S., \& Walters, H. (2015). Thoracic Society of Australia and New Zealand oxygen guidelines for acute oxygen use in adults: Swimming between the flags. Respirology, 20(8), 1182-1191. https://doi.org/10.1111/resp.12620

Beasley, R., Mackle, D., \& Young, P. (2019). Oxygen: a new look at an old therapy. Journal of the Royal Society of New Zealand, 49(2). https://doi.org/10.1080/03036758.2019.1566154

Bennett, W. O., Bird, J. H., Burrows, S. A., Counter, P. R., \& Reddy, V. M. (2012). Does academic output correlate with better mortality rates in NHS trusts in England? Public 
Health, 126(0), S40-S43. https://doi.org/10.1016/j.puhe.2012.05.021

Birken, S. A., Powell, B. J., Shea, C. M., Haines, E. R., Alexis Kirk, M., Leeman, J., Rohweder, C., Damschroder, L., \& Presseau, J. (2017). Criteria for selecting implementation science theories and frameworks: Results from an international survey. Implementation Science, 12(1), 1-9. https://doi.org/10.1186/s13012-017-0656-y

Boaz, A., Hanney, S., Jones, T., \& Soper, B. (2015). Does the engagement of clinicians and organisations in research improve healthcare performance: a three-stage review. BMJ Open, 5(12), e009415. https://doi.org/10.1136/bmjopen-2015-009415

Boers, K. E., Vijgen, S. M. C., Bijlenga, D., Van Der Post, J. A. M., Bekedam, D. J., Kwee, A., Van Der Salm, P. C. M., Van Pampus, M. G., Spaanderman, M. E. A., De Boer, K., Duvekot, J. J., Bremer, H. A., Hasaart, T. H. M., Delemarre, F. M. C., Bloemenkamp, K. W. M., Van Meir, C. A., Willekes, C., Wijnen, E. J., Rijken, M., ... Scherjon, S. A. (2011). Induction versus expectant monitoring for intrauterine growth restriction at term: Randomised equivalence trial (DIGITAT). BMJ, 342(7787), 35. https://doi.org/10.1136/bmj.c7087

Bringsvor, H. B., Bentsen, S. B., \& Berland, A. (2014). Sources of knowledge used by intensive care nurses in Norway: An exploratory study. Intensive and Critical Care Nursing, 30(3), 159-166. https://doi.org/10.1016/j.iccn.2013.12.001

Cabana, M. D., Rand, C. S., Powe, N. R., Wu, A. W., Wilson, M. H., Abboud, P.-A. C., \& Rubin, H. R. (1999). Why don't physicians follow a framework for improvement. JAMA, Vol 282, 1458-1465. https://doi.org/10.1001/jama.282.15.1458

Campbell, D. T., \& Fiske, D. W. (1959). Convergent and discriminant validation by the multitrait-multimethod matrix. Psychological Bulletin, 56(2), 81-105. https://doi.org/10.1037/h0046016

Canadian Institutes of Health Research. (2012). Guide to knowledge translation planning at CIHR: Integrated and end-of-grant approaches. http://www.cihr.ca/e/45321.html Canadian Institutes of Health Research. (2020). Knowledge Translation. https://cihrirsc.gc.ca/e/29529.html

Cannon, C. P., McCabe, C. H., Diver, D. J., Herson, S., Greene, R. M., Shah, P. K., Sequeira, R. F., Leya, F., Kirshenbaum, J. M., Magorien, R. D., Palmeri, S. T., Davis, V., Gibson, C. M., Poole, W. K., \& Braunwald, E. (1994). Comparison of front-loaded recombinant tissuetype plasminogen activator, anistreplase and combination thrombolytic therapy for 
acute myocardial infarction: Results of the thrombolysis in myocardial infarction (TIMI) 4 trial. Journal of the American College of Cardiology, 24(7), 1602-1610. https://doi.org/10.1016/0735-1097(94)90163-5

Cannon, C. P., McCabe, C. H., Henry, T. D., Schweiger, M. J., Gibson, R. S., Mueller, H. S., Becker, R. C., Kleiman, N. S., Haugland, J. M., Anderson, J. L., Sharaf, B. L., Edwards, S. J., Rogers, W. J., Williams, D. O., \& Braunwald, E. (1994). A pilot trial of recombinant desulfatohirudin compared with heparin in conjunction with tissue-type plasminogen activator and aspirin for acute myocardial infarction: Results of the Thrombolysis in Myocardial Infarction (TIMI) 5 trial. Journal of the American College of Cardiology, 23(5), 993-1003. https://doi.org/10.1016/0735-1097(94)90581-9

Chalmers, I., Hedges, L. V, Boruch, B., Clarke, M., Glass, G., Horn, J., Jull, A., Lewis, S., Macfarlane, A., Marks, H., Mosteller, F., Davey, G., Petticrew, M., \& Sandercock, P. (2002). A brief history of research synthesis. Evaluation and the Health Professions, 25(1), 12-37.

Chapman, M., Peake, S. L., Bellomo, R., Davies, A., Deane, A., Horowitz, M., Hurford, S., Lange, K., Little, L., Mackle, D., O’Connor, S., Presneill, J., Ridley, E., Williams, P., \& Young, P. (2018). Energy-dense versus routine enteral nutrition in the critically ill. New England Journal of Medicine, 379(19). https://doi.org/10.1056/NEJMoa1811687

Chesebro, J. H., Knatterud, G., Roberts, R., Borer, J., Cohen, L. S., Dalen, J., Dodge, H. T., Francis, C. K., Hillis, D., \& Ludbrook, P. (1987). Thrombolysis in myocardial infarction (TIMI) trial, phase I: A comparison between intravenous tissue plasminogen activator and intravenous streptokinase. Clinical findings through hospital discharge. Circulation, 76(1), 142-154. https://doi.org/10.1161/01.CIR.76.1.142

Chester, R., Jerosch-Herold, C., Lewis, J., \& Shepstone, L. (2018). Psychological factors are associated with the outcome of physiotherapy for people with shoulder pain: A multicentre longitudinal cohort study. British Journal of Sports Medicine, 52(4), 269275. https://doi.org/10.1136/bjsports-2016-096084

Chu, D. K., Kim, L. H., Young, P. J., Zamiri, N., Almenawer, S. A., Jaeschke, R., Szczeklik, W., \& Schünemann, H. J. (2018). Mortality and morbidity in acutely ill adults treated with liberal versus conservative oxygen therapy (IOTA): A systematic review and metaanalysis. Lancet, 391(10131), 1693-1705. https://doi.org/10.1016/S01406736(18)30479-3 
Clarke, M., \& Loudon, K. (2011). Effects on patients of their healthcare practitioner's or institution's participation in clinical trials: a systematic review. Trials, 12(1), 16. https://doi.org/10.1186/1745-6215-12-16

Cochrane. (2016). Strategy to 2020.

https://www.cochrane.org/sites/default/files/public/uploads/pdf/strategy_to_2020_ 22june2016.pdf

College of Intensive Care Medicine. (2016). IC-1 Minimum Standards for Intensive Care. https://www.cicm.org.au/CICM_Media/CICMSite/CICM-

Website/Resources/Professional Documents/IC-1-Minimum-Standards-for-IntensiveCare-Units_2.pdf

Colquhoun, H. L., Letts, L. J., Law, M. C., MacDermid, J. C., \& Missiuna, C. A. (2010). A scoping review of the use of theory in studies of knowledge translation. Canadian Journal of Occupational Therapy. https://doi.org/10.2182/cjot.2010.77.5.3

Corrigan, M. H., \& Glass, H. E. (2005). Physician participation in clinical studies and subsequent prescribing of new drugs. Pharmacy and Therapeutics, 30(1), 60-66.

Creswell, J. (2014). Research design. Qualitative, quantitative and mixed methods approaches (4th ed.). Sage Publications, Inc.

Crotty, M. (1998). The foundations of social research: meaning and perspective in the research process. Sage Publications, Inc.

Custers, I. M., Flierman, P. A., Maas, P., Cox, T., Van Dessel, T. J. H. M., Gerards, M. H., Mochtar, M. H., Janssen, C. A. H., Van Der Veen, F., \& Mol, B. W. J. (2009). Immobilisation versus immediate mobilisation after intrauterine insemination: Randomised controlled trial. BMJ (Online), 339(7729), 1065. https://doi.org/10.1136/bmj.b4080

Cuthbertson, B. H., Campbell, M. K., MacLennan, G., Duncan, E. M., Marshall, A. P., Wells, E. C., Prior, M. E., Todd, L., Rose, L., Seppelt, I. M., Bellingan, G., \& Francis, J. J. (2013). Clinical stakeholders' opinions on the use of selective decontamination of the digestive tract in critically ill patients in intensive care units: An international Delphi study. Critical Care, 17(6), no pagination. https://doi.org/10.1186/cc13096

Das, D., Ishaq, S., Harrison, R., Kosuri, K., Harper, E., DeCaestecker, J., Sampliner, R., Attwood, S., Barr, H., Watson, P., Moayyedi, P., \& Jankowski, J. (2008). Management of Barrett's esophagus in the UK: Overtreated and underbiopsied but improved by the 
introduction of a national randomized trial. American Journal of Gastroenterology, 103(5), 1079-1089. https://doi.org/10.1111/j.1572-0241.2008.01790.x

Davies, P., Walker, A. E., \& Grimshaw, J. M. (2010). A systematic review of the use of theory in the design of guideline dissemination and implementation strategies and interpretation of the results of rigorous evaluations. Implementation Science, 5(1), 16. https://doi.org/10.1186/1748-5908-5-14

Davis, D. F., Golicic, S. L., \& Boerstler, C. N. (2010). Benefits and challenges of conducting multiple methods research in marketing. Journal of the Academy of Marketing Science, 38(3), 467-479. https://doi.org/10.1007/s11747-010-0204-7

De Graaff, A. E., Dongelmans, D. A., Binnekade, J. M., \& De Jonge, E. (2011). Clinicians' response to hyperoxia in ventilated patients in a Dutch ICU depends on the level of FiO2. Intensive Care Medicine, 37(1), 46-51. https://doi.org/10.1007/s00134-0102025-z

de Jonge, E., Peelen, L., Keijzers, P. J., Joore, H., de Lange, D., van der Voort, P. H., Bosman, R. J., de Waal, R. AL, Wesselink, R., \& de Keizer, N. F. (2008). Association between administered oxygen, arterial partial oxygen pressure and mortality in mechanically ventilated intensive care unit patients. Critical Care, 12(6), R156. https://doi.org/10.1186/cc7150

Eastwood, G. M., Bellomo, R., Bailey, M., Taori, G., Pilcher, D., Young, P., \& Beasley, R. (2012). Arterial oxygen tension and mortality in mechanically ventilated patients. Intensive Care Medicine, 38(1), 91-98. https://doi.org/10.1007/s00134-011-2419-6

Eastwood, G. M., Parke, R., \& Mackle, D. (2013). Funding for intensive care research coordinators: An online survey. Australian Critical Care, 26(2), 91.

https://doi.org/10.1016/j.aucc.2013.02.032

Eastwood, G. M., Reade, M. C., Peck, L., Baldwin, I., Considine, J., \& Bellomo, R. (2012). Critical care nurses' opinion and self-reported practice of oxygen therapy: A survey. Australian Critical Care, 25(1), 23-30. https://doi.org/10.1016/j.aucc.2011.05.001

Eastwood, G. M., Reade, M. C., Peck, L., Jones, D., \& Bellomo, R. (2011). Intensivists' opinion and self-reported practice of oxygen therapy. Anaesthesia and Intensive Care, 39(1), 122-126. https://doi.org/10.1177/0310057×1103900120

Ebenezer, C. (2015). Nurses' and midwives' information behaviour: A review of literature from 1998 to 2014. New Library World, 116(3-4), 155-172. 
https://doi.org/10.1108/NLW-07-2014-0085

Eccles, M., Grimshaw, J., Walker, A., Johnston, M., \& Pitts, N. (2005). Changing the behavior of healthcare professionals: The use of theory in promoting the uptake of research findings. Journal of Clinical Epidemiology, 58(2), 107-112. https://doi.org/10.1016/j.jclinepi.2004.09.002

Estabrooks, C. A., Thompson, D. S., Lovely, J. J. E., \& Hofmeyer, A. (2006). A guide to knowledge translation theory. Journal of Continuing Education in the Health Professions, 26(1), 25-36. https://doi.org/10.1002/chp.48

Ferguson, J., Califf, R., Antman, E., Cohen, M., Grines, C., Goodman, S., Kereiakes, D., Langer, A., Mahaffey, K., Nessel, C., Armstrong, P., Avezum, A., Aylward, P., Becker, R., Biasucci, L., Borzak, S., Col, J., Frey, M., Fry, E., ... Investigators, S. T. (2004). Enoxaparin vs unfractionated heparin in high-risk patients with non-ST-segment elevation acute coronary syndromes managed with an intended early invasive strategy: Primary results of the SYNERGY randomized trial. Journal of the American Medical Association, 292(1), 45-54. https://doi.org/10.1001/jama.292.1.45

Finfer, S., Liu, B., Taylor, C., Bellomo, R., Billot, L., Cook, D., \& Du, B. (2010). Resuscitation fluid use in critically ill adults: An international cross-sectional study in 391 intensive care units. Critical Care, 14(R185), 1-12. http://ccforum.com/content/14/5/R185

Florczak, K. L. (2013). In the Zeal to Synthesize. Nursing Science Quarterly, 26(3), 220-225. https://doi.org/10.1177/0894318413489157

Girardis, M., Busani, S., Damiani, E., Donati, A., Rinaldi, L., Marudi, A., Morelli, A., Antonelli, M., \& Singer, M. (2016). Effect of conservative vs conventional oxygen therapy on mortality among patients in an intensive care unit. JAMA, 316(15), 1583. https://doi.org/10.1001/jama.2016.11993

GISSI. (1987). Long-term effects of intravenous thrombolysis in acute myocardial infarction: final report of the GISSI study. Lancet, 330(8564), 871-874. https://doi.org/10.1016/S0140-6736(87)91369-9

Graham, I. D., Logan, J., Harrison, M. B., Straus, S. E., Tetroe, J., Caswell, W., \& Robinson, N. (2006). Lost in knowledge translation: time for a map? Journal of Continuing Education in the Health Professions, 26(1), 13-24. https://doi.org/10.1002/chp.47 Grant, A., \& Coyer, F. (2019). Contextual factors to registered nurse research utilisation in an Australian adult metropolitan tertiary intensive care unit: A descriptive study. 
Australian Critical Care, xxxx. https://doi.org/10.1016/j.aucc.2019.01.001

Grant, M. J., \& Booth, A. (2009). A typology of reviews: An analysis of 14 review types and associated methodologies. Health Information and Libraries Journal, 26(2), 91-108. https://doi.org/10.1111/j.1471-1842.2009.00848.x

Grim, C. C. A. (2020). Attitudes of dutch intensive care unit clinicians towards oxygen therapy. Netherlands Journal of Medicine, 78(4), 167-174.

Grim, C. C. A., Helmerhorst, H. J. F., Schultz, M. J., Winters, T., van der Voort, P. H. J., van Westerloo, D. J., \& de Jonge, E. (2020). Changes in attitudes and actual practice of oxygen therapy in icus after implementation of a conservative oxygenation guideline. Respiratory Care, 65(10), 1502-1510. https://doi.org/10.4187/respcare.07527

Grimshaw, J. M., Eccles, M. P., Lavis, J. N., Hill, S. J., \& Squires, J. E. (2012). Knowledge translation of research findings. Implementation Science, 7(1), 50. https://doi.org/10.1186/1748-5908-7-50

Grimshaw, J. M., Freemantle, N., Wallace, S., Russell, I., Hurwitz, B., Watt, I., Long, A., \& Sheldon, T. (1995). Developing and implementing clinical practice guidelines. Quality in Health Care : QHC, 4(1), 55-64. https://doi.org/10.1136/qshc.4.1.55

Grol, R. (2001). Successes and failures in the implementation of evidence-based guidelines for clinical practice. Medical Care, 39(8 Suppl 2), II46-154. https://doi.org/10.1097/00005650-200108002-00003

Grol, R., \& Grimshaw, J. (2003). From best evidence to best practice: effective implementation of change in patients' care. Lancet, 362(9391), 1225-1230. https://doi.org/10.1016/S0140-6736(03)14546-1

Guba, E. G. (1990). The Paradigm Dialog (G. Guba, Egon (Ed.)). SAGE publications, Inc. Gupta, D. M., Boland, R. J., \& Aron, D. C. (2017). The physician's experience of changing clinical practice: A struggle to unlearn. Implementation Science, 12(1), 1-11. https://doi.org/10.1186/s13012-017-0555-2

Hammond, N. E., Taylor, C., Finfer, S., Machado, F. R., An, Y. Z., Billot, L., Bloos, F., Bozza, F., Cavalcanti, A. B., Correa, M., Du, B., Hjortrup, P. B., Li, Y., Mclntryre, L., Saxena, M., Schortgen, F., Watts, N. R., \& Myburgh, J. (2017). Patterns of intravenous fluid resuscitation use in adult intensive care patients between 2007 and 2014: An international cross-sectional study. PLOS ONE, 12(5), 1-21. https://doi.org/10.1371/journal.pone.0176292 
Harding, K. E., Porter, J., Horne-Thompson, A., Donley, E., \& Taylor, N. F. (2014). Not enough time or a low priority? Barriers to evidence-based practice for allied health clinicians. Journal of Continuing Education in the Health Professions, 34(4), 224-231. https://doi.org/10.1002/chp.21255

Harvey, G., \& Kitson, A. (2016). PARIHS revisited: From heuristic to integrated framework for the successful implementation of knowledge into practice. Implementation Science, 11(1). https://doi.org/10.1186/s13012-016-0398-2

Harvey, G., Loftus-Hills, A., Rycroft-Malone, J., Titchen, A., Kitson, A., McCormack, B., \& Seers, K. (2002). Getting evidence into practice: The role and function of facilitation. Journal of Advanced Nursing, 37(6), 577-588. https://doi.org/10.1046/j.13652648.2002.02126.x

Health Research Council of New Zealand. (n.d.). Update on NZ Health Delivery changes I Health Research Council of New Zealand 20 Sep 2019. Retrieved May 10, 2021, from https://www.hrc.govt.nz/news-and-events/update-nz-health-delivery-changes Health Research Council of New Zealand. (2020). 2020 Peer review manual (Issue August 2020).

Helfrich, C. D., Damschroder, L. J., Hagedorn, H. J., Daggett, G. S., Sahay, A., Ritchie, M., Damush, T., Guihan, M., Ullrich, P. M., \& Stetler, C. B. (2010). A critical synthesis of literature on the promoting action on research implementation in health services (PARIHS) framework. Implementation Science, 5(1). https://doi.org/10.1186/1748$5908-5-82$

Helmerhorst, H. J. F., Arts, D. L., Schultz, M. J., Van Der Voort, P. H. J., Abu-Hanna, A., De Jonge, E., \& Van Westerloo, D. J. (2017). Metrics of arterial hyperoxia and associated outcomes in critical care. Critical Care Medicine, 45(2), 187-195. https://doi.org/10.1097/CCM.0000000000002084

Helmerhorst, H. J. F., Roos-Blom, M. J., Van Westerloo, D. J., \& De Jonge, E. (2015). Association between arterial hyperoxia and outcome in subsets of critical illness: A systematic review, meta-analysis, and meta-regression of cohort studies. Critical Care Medicine, 43(7), 1508-1519. https://doi.org/10.1097/CCM.0000000000000998

Helmerhorst, H. J. F., Schultz, M. J., van der Voort, P. H. J., Bosman, R. J., Juffermans, N. P., de Jonge, E., \& van Westerloo, D. J. (2014). Self-reported attitudes versus actual practice of oxygen therapy by ICU physicians and nurses. Annals of Intensive Care, 
4(1), 1-9. https://doi.org/10.1186/s13613-014-0023-y

Helmerhorst, H. J. F., Schultz, M. J., Van Der Voort, P. H. J., Bosman, R. J., Juffermans, N. P., De Wilde, R. B. P., Van Den Akker-Van Marle, M. E., Van Bodegom-Vos, L., De Vries, M., Eslami, S., De Keizer, N. F., Abu-Hanna, A., Van Westerloo, D. J., \& De Jonge, E. (2016). Effectiveness and Clinical Outcomes of a Two-Step Implementation of Conservative Oxygenation Targets in Critically III Patients: A before and after Trial. Critical Care Medicine, 44(3), 554-563.

https://doi.org/10.1097/CCM.0000000000001461

Henderwood, M. (2015). ICU nurses' role in ventilation. Kai Tiaki Nursing New Zealand, 21(7), 14-15.

Herrera-Perez, D., Haslam, A., Crain, T., Gill, J., Livingston, C., Kaestner, V., Hayes, M., Morgan, D., Cifu, A. S., \& Prasad, V. (2019). A comprehensive review of randomized clinical trials in three medical journals reveals 396 medical reversals. ELife, 8, 1-19. https://doi.org/10.7554/eLife.45183

Hess-Biber, S., \& Johnson, R. B. (Eds.). (2015). The Oxford Handbook of Multimethod and Mixed Methods Research Inquiry. Oxford University Press.

Hill, J. N., Guihan, M., Hogan, T. P., Smith, B. M., LaVela, S. L., Weaver, F. M., Anaya, H. D., \& Evans, C. T. (2017). Use of the PARIHS Framework for Retrospective and Prospective Implementation Evaluations. Worldviews on Evidence-Based Nursing, 14(2), 99-107. https://doi.org/10.1111/wvn.12211

Houghton, C., Hunter, A., \& Meskell, P. (2012). Linking aims, paradigm and method in nursing research. Nurse Researcher, 20(2), 34-39.

https://doi.org/10.7748/nr2012.11.20.2.34.c9439

Hunt, T. D., Ramanathan, S. A., Hannaford, N. A., Hibbert, P. D., Braithwaite, J., Coiera, E., Day, R. O., Westbrook, J. I., \& Runciman, W. B. (2012). CareTrack Australia: Assessing the appropriateness of adult healthcare: Protocol for a retrospective medical record review. BMJ Open, 2(1). https://doi.org/10.1136/bmjopen-2011-000665

ISIS-2 Collaborative Group. (1988). Randomised trial of intravenous streptokinase, oral aspirin, both or neither among 17,187 cases of suspected myocardiaL infarction: ISIS2. Lancet, 332(8607), 349-360. https://doi.org/10.1016/S0140-6736(88)92833-4 Jankowski, J. A. Z., de Caestecker, J., Love, S. B., Reilly, G., Watson, P., Sanders, S., Ang, Y., Morris, D., Bhandari, P., Attwood, S., Ragunath, K., Rameh, B., Fullarton, G., Tucker, A., 
Penman, I., Rodgers, C., Neale, J., Brooks, C., Wise, A., ... Moayyedi, P. (2018).

Esomeprazole and aspirin in Barrett's oesophagus (AspECT): a randomised factorial trial. Lancet, 392(10145), 400-408. https://doi.org/10.1016/S0140-6736(18)31388-6 Janni, W., Kiechle, M., Sommer, H., Rack, B., Gauger, K., Heinrigs, M., Steinfeld, D., Augustin, D., Simon, W., Harbeck, N., \& Friese, K. (2006). Study participation improves treatment strategies and individual patient care in participating centers. Anticancer Research, 26(5 B), 3661-3667.

Kaandorp, S. P., Goddijn, M., van der Post, J. A. M., Hutten, B. A., Verhoeve, H. R., Hamulyák, K., Mol, B. W., Folkeringa, N., Nahuis, M., Papatsonis, D. N. M., Büller, H. R., van der Veen, F., \& Middeldorp, S. (2010). Aspirin plus Heparin or Aspirin Alone in Women with Recurrent Miscarriage. New England Journal of Medicine, 362(17), 1586-1596. https://doi.org/10.1056/NEJMoa1000641

Kaukonen, K. M., Bailey, M., Pilcher, D., Orford, N., Finfer, S., \& Bellomo, R. (2013). Glycaemic control in Australia and New Zealand before and after the NICE-SUGAR trial: A translational study. Critical Care, 17(5). https://doi.org/10.1186/cc13030 Kessler, R. S., Purcell, E. P., Glasgow, R. E., Klesges, L. M., Benkeser, R. M., \& Peek, C. J. (2013). What does it mean to "employ" the RE-AIM model? Evaluation and the Health Professions, 36(1), 44-66. https://doi.org/10.1177/0163278712446066 Ketley, D., \& Woods, K. L. (1993). Impact of clinical trials on clinical practice: example of thrombolysis for acute myocardial infarction. Lancet, 342(8876), 891-894. http://www.ncbi.nlm.nih.gov/pubmed/8105166

Khera, N. (2015). From evidence to clinical practice in blood and marrow transplantation. Blood Reviews, 29(6), 351-357. https://doi.org/10.1016/j.blre.2015.04.001 Khera, N., Mau, L. W., Denzen, E. M., Meyer, C., Houg, K., Lee, S. J., Horowitz, M. M., \& Burns, L. J. (2018). Translation of clinical research into practice: An impact assessment of the results from the blood and marrow transplant Clinical Trials Network Protocol 0201 on unrelated graft source utilization. Biology of Blood and Marrow Transplantation, 24(11), 2204-2210. https://doi.org/10.1016/j.bbmt.2018.06.028 Kitson, A., Harvey, G., \& McCormack, B. (1998). Enabling the implementation of evidence based practice: a conceptual framework. Quality and Safety in Health Care, 7(3), 149158. https://doi.org/10.1136/qshc.7.3.149

Kitson, A., Rycroft-Malone, J., Harvey, G., McCormack, B., Seers, K., \& Titchen, A. (2008). 
Evaluating the successful implementation of evidence into practice using the PARiHS framework: Theoretical and practical challenges. Implementation Science, 3(1). https://doi.org/10.1186/1748-5908-3-1

Kizer, J. R., Cannon, C. P., McCabe, C. H., Mueller, H. S., Schweiger, M. J., Davis, V. G., Perritt, R., \& Antman, E. M. (1999). Trends in the use of pharmacotherapies for acute myocardial infarction among physicians who design and/or implement randomized trials versus physicians in routine clinical practice: The MILIS-TIMI experience. American Heart Journal, 137(1), 79-92. https://doi.org/10.1016/S00028703(99)70462-X

Knaus, W. A., Wagner, D. P., Draper, E. A., Zimmerman, J. E., Bergner, M., Bastos, P. G., Sirio, C. A., Murphy, D. J., Lotring, T., Damiano, A., \& Harrell, F. E. (1991). The APACHE III prognostic system: Risk prediction of hospital mortality for critically III hospitalized adults. Chest. https://doi.org/10.1378/chest.100.6.1619

Kok, M., Bais, J. M., van Lith, J. M., Papatsonis, D. M., Kleiverda, G., Hanny, D., Doornbos, J. P., Mol, B. W., \& van der Post, J. A. (2008). Nifedipine as a Uterine Relaxant for External Cephalic Version. Obstetrics \& Gynecology, 112(2), 271-276. https://doi.org/10.1097/AOG.0b013e31817f1f2e

Koopmans, C. M., Bijlenga, D., Groen, H., Vijgen, S. M., Aarnoudse, J. G., Bekedam, D. J., van den Berg, P. P., de Boer, K., Burggraaff, J. M., Bloemenkamp, K. W., Drogtrop, A. P., Franx, A., de Groot, C. J., Huisjes, A. J., Kwee, A., van Loon, A. J., Lub, A., Papatsonis, D. N., van der Post, J. A., ... van Pampus, M. G. (2009). Induction of labour versus expectant monitoring for gestational hypertension or mild pre-eclampsia after 36 weeks' gestation (HYPITAT): a multicentre, open-label randomised controlled trial. Lancet, 374(9694), 979-988. https://doi.org/10.1016/S0140-6736(09)60736-4

Krzyzanowska, M. K., Kaplan, R., \& Sullivan, R. (2011). How may clinical research improve healthcare outcomes? Annals of Oncology, 22(SUPPL.7), 10-15. https://doi.org/10.1093/annonc/mdr420

Lavis, J. N., Robertson, D., Woodside, J. M., McLeod, C. B., \& Abelson, J. (2003). How can research organizations more effectively transfer research knowledge to decision makers? Milbank Quarterly, 81(2), 221-248. https://doi.org/10.1111/1468-0009.t01$1-00052$

Lee, L. V., \& TIMI 6 Investigators. (1995). Initial experience with hirudin andstreptokinase in 
acute myocardial infarction: Results of the thrombolysis in myocardial infarction (TIMI) 6 trial. American Journal of Cardiology, 75(1), 7-13.

https://doi.org/10.1016/S0002-9149(99)80517-7

Letourneau, N., \& Allen, M. (1999). Post-positivistic critical multiplism: A beginning dialogue. Journal of Advanced Nursing, 30(3), 623-630.

https://doi.org/10.1046/j.1365-2648.1999.01133.x

Liang, L., Bernhardsson, S., Vernooij, R. W. M., Armstrong, M. J., Bussières, A., Brouwers, M. C., Gagliardi, A. R., Alhabib, S., Fleuren, M., Fortino, M., Mazza, D., O’Rourke, N., \& Willson, M. (2017). Use of theory to plan or evaluate guideline implementation among physicians: A scoping review. Implementation Science.

https://doi.org/10.1186/s13012-017-0557-0

Litjens, R. J. N. T. M., Oude Rengerink, K., Danhof, N. A., Kruitwagen, R. F. P. M., \& Mol, B. W. J. (2013). Does recruitment for multicenter clinical trials improve dissemination and timely implementation of their results? A survey study from the Netherlands. Clinical Trials, 10(6), 915-923. https://doi.org/10.1177/1740774513504150

Livingston, A. E., Hutchinson, A. F., \& Brooks, L. A. (2020). Use of excessive supplemental oxygen in mechanically ventilated patients is based on unit culture. A multiplemethods study in a regional intensive care unit. Australian Critical Care, 33(4), 343349. https://doi.org/10.1016/j.aucc.2019.07.001

MacGuire, J. M. (1990). Putting nursing research findings into practice: a research utilization as an aspect of the management of change. Journal of Advanced Nursing, $15,614-620$.

Mackle, D., Bailey, M. J., Beasley, R. W., Bellomo, R., Bennett, V. L., Deane, A. M., Eastwood, G. M., Finfer, S., Freebairn, R. C., Litton, E., Linke, N. J., McArthur, C. J., McGuinness, S. P., Panwar, R., Young, P., \& Group, A. and N. Z. I. C. S. C. T. (2018). Protocol summary and statistical analysis plan for the intensive care unit randomised trial comparing two approaches to oxygen therapy (ICU-ROX). Critical Care and Resuscitation, 20(1), 22-32. http://www.ncbi.nlm.nih.gov/pubmed/29458318 Mackle, D., Bellomo, R., Bailey, M., Beasley, R., Deane, A., Eastwood, G., Finfer, S., Freebairn, R., King, V., Linke, N., Litton, E., McArthur, C., McGuinness, S., Panwar, R., Young, P., \& ICU-ROX Investigators and the Australian and New Zealand Intensive Care Society Clinical Trials. (2020). Conservative Oxygen Therapy during Mechanical 
Ventilation in the ICU. New England Journal of Medicine, 382(11), 989-998.

https://doi.org/10.1056/nejmoa1903297

Mackle, D., \& Nelson, K. (2018). Research nurses in New Zealand intensive care units: A qualitative descriptive study. Australian Critical Care, 1-7.

https://doi.org/10.1016/j.aucc.2018.03.005

Majumdar, S. R., Chang, W. C., \& Armstrong, P. W. (2002). Do the investigative sites that take part in a positive clinical trial translate that evidence into practice? American Journal of Medicine, 113(2), 140-145. https://doi.org/10.1016/S0002-9343(02)01166$\mathrm{X}$

Majumdar, S. R., Mcalister, F. A., Ms, C., \& Furberg, C. D. (2004). From Knowledge to Practice in Chronic Cardiovascular Disease : A Long and Winding Road. 43(10). https://doi.org/10.1016/j.jacc.2003.12.043

Marshall, A. P., West, S. H., \& Aitken, L. M. (2011). Preferred information sources for clinical decision making: Critical care nurses' perceptions of information accessibility and usefulness. Worldviews on Evidence-Based Nursing, 8(4), 224-235. https://doi.org/10.1111/j.1741-6787.2011.00221.x

Massachusetts Medical Society. (2021). About NEJM. New England Journal of Medicine. https://www.nejm.org/about-nejm/about-nejm?query=footer

McCambridge, J., Witton, J., \& Elbourne, D. R. (2014). Systematic review of the Hawthorne effect: New concepts are needed to study research participation effects. Journal of Clinical Epidemiology. https://doi.org/10.1016/j.jclinepi.2013.08.015

McCormack, B., Kitson, A., Harvey, G., Rycroft-Malone, J., Titchen, A., \& Seers, K. (2002). Getting evidence into practice: The meaning of "context." Journal of Advanced Nursing, 38(1), 94-104. https://doi.org/10.1046/j.1365-2648.2002.02150.x

McGlynn, E. A., Asch, S. M., Adams, J., Keesey, J., Hicks, J., DeCristofaro, A., \& Kerr, E. A. (2003). The quality of health care delivered to adults in the United States. New England Journal of Medicine, 348(26), 2635-2645. https://doi.org/10.1056/NEJMsa022615

McHugh, M. L. (2013). The Chi-square test of independence. Biochemia Medica, 23(2), 143-149. https://doi.org/10.11613/BM.2013.018

Mckillop, A., Crisp, J., \& Walsh, K. (2012). Barriers and Enablers to Implementation of a New Zealand-Wide Guideline for Assessment and Management of Cardiovascular Risk 
in Primary Health Care: A Template Analysis. Worldviews on Evidence-Based Nursing, 9(3), 159-171. https://doi.org/10.1111/j.1741-6787.2011.00233.x

McNett, M., O’Mathúna, D., Tucker, S., Roberts, H., Mion, L. C., \& Balas, M. C. (2020). A Scoping Review of Implementation Science in Adult Critical Care Settings. Critical Care Explorations, 2(12), e0301. https://doi.org/10.1097/cce.0000000000000301

Meineche-Schmidt, V., Hauschildt Juhl, H., Østergaard, J. E., Luckow, A., \& Hvenegaard, A. (2004). Costs and efficacy of three different esomeprazole treatment strategies for long-term management of gastro-oesophageal reflux symptoms in primary care. Alimentary Pharmacology and Therapeutics, 19(8), 907-915. https://doi.org/10.1111/j.1365-2036.2004.01916.x

Meineche-Schmidt, V., Hvenegaard, A., \& Juhl, H. H. (2006). Participation in a clinical trial influences the future management of patients with gastro-oesophageal reflux disease in general practice. Alimentary Pharmacology and Therapeutics, 24(7), 1117-1125. https://doi.org/10.1111/j.1365-2036.2006.03046.x

Ministry of Business, Innovation and Employment, \& Ministry of Health. (2017). NZ Health Research Strategy 2017-2027.

Ministry of Health, \& Ministry of Business Innovation and Employment. (2017). New Zealand Health Research Strategy 2017-2027.

Ministry of Health, N. (n.d.). New Zealand Guidelines Group. Retrieved May 10, 2021, from https://www.health.govt.nz/about-ministry/ministry-health-websites/new-zealandguidelines-group

Ministry of Health, N. (2002). Toward Clinical Excellence: An Introduction to Clinical Audit, Peer Review and Other Clinical Practice Improvements. http://www.health.govt.nz/system/files/documents/publications/moh_tce_2002.pdf

Moll, E., Bossuyt, P. M. M., Korevaar, J. C., Lambalk, C. B., \& Van Der Veen, F. (2006). Effect of clomifene citrate plus metformin and clomifene citrate plus placebo on induction of ovulation in women with newly diagnosed polycystic ovary syndrome: Randomised double blind clinical trial. British Medical Journal, 332(7556), 1485-1488. https://doi.org/10.1136/bmj.38867.631551.55

Morris, Z. S., wooding, S., \& Grant, J. (2011). The answer is 17 years, what is the question: Understanding time lags in translational research. Journal of the Royal Society of Medicine, 104(12), 510-520. https://doi.org/10.1258/jrsm.2011.110180 
Morse, J. (2010). Procedures and practice of mixed method design. In A. Tashakkori \& C. Teddlie (Eds.), Sage handbook of mixed methods in social and behavioural research (2nd ed., pp. 339-352). SAGE publications, Inc.

Mourits, M. J. E., Bijen, C. B., Arts, H. J., ter Brugge, H. G., van der Sijde, R., Paulsen, L., Wijma, J., Bongers, M. Y., Post, W. J., van der Zee, A. G., \& de Bock, G. H. (2010). Safety of laparoscopy versus laparotomy in early-stage endometrial cancer: A randomised trial. The Lancet Oncology, 11(8), 763-771.

https://doi.org/10.1016/S1470-2045(10)70143-1

Myburgh, J. A., Finfer, S., Bellomo, R., Billot, L., Cass, A., Gattas, D., Glass, P., Lipman, J., Liu, B., McArthur, C., McGuinness, S., Rajbhandari, D., Taylor, C. B., \& Webb, S. A. R. (2012). Hydroxyethyl starch or saline for fluid resuscitation in intensive care. New England Journal of Medicine, 367(20), 1901-1911. https://doi.org/10.1056/nejmoa1209759

NEJM. (2020). Notable Articles of 2020. New England Journal of Medicine, 1-51.

New Zealand Nurses Organisation. Critical Care Nurses' Section. (2014). New Zealand standards for critical care nursing practice. 1-8. https://www.nzno.org.nz/

Nielsen, N., Wetterslev, J., Cronberg, T., Erlinge, D., Gasche, Y., Hassager, C., Horn, J., Hovdenes, J., Kjaergaard, J., Kuiper, M., Pellis, T., Stammet, P., Wanscher, M., Wise, M. P., Åneman, A., Al-Subaie, N., Boesgaard, S., Bro-Jeppesen, J., Brunetti, I., ... Friberg, H. (2013). Targeted temperature management at $33^{\circ} \mathrm{C}$ versus $36^{\circ} \mathrm{C}$ after cardiac arrest. New England Journal of Medicine, 369(23), 2197-2206. https://doi.org/10.1056/nejmoa1310519

Nilsen, P. (2015). Making sense of implementation theories, models and frameworks. Implementation Science, 10(1). https://doi.org/10.1186/s13012-015-0242-0 Niven, D. J., Rubenfeld, G. D., Kramer, A. A., \& Stelfox, H. T. (2015). Effect of published scientific evidence on glycemic control in adult intensive care units. JAMA Internal Medicine, 175(5), 801-809. https://doi.org/10.1001/jamainternmed.2015.0157

O’Driscoll, B. R., Howard, L. S., \& Davison, A. G. (2008). BTS guideline for emergency oxygen use in adult patients. Thorax, 63(SUPPL. 6). https://doi.org/10.1136/thx.2008.102947

O’Keefe-McCarthy, S., Santiago, C., \& Lau, G. (2008). Ventilator-associated pneumonia bundled strategies: An evidence-based practice. Worldviews on Evidence-Based Nursing, 5(4), 193-204. https://doi.org/10.1111/j.1741-6787.2008.00140.x 
Omoigui, N. A., Silver, M. J., Rybicki, L. A., Rosenthal, M., Berdan, L. G., Pieper, K., King, S. V, Califf, R. M., \& Topol, E. J. (1998). Influence of a randomized clinical trial on practice by participating investigators : Lessons from the Coronary Angioplasty Versus Excisional Atherectomy Trial (CAVEAT ). Journal of the American College of Cardiology, 31(2), 265-272. https://doi.org/10.1016/S0735-1097(97)00498-1

Oxford Centre for Triple Value Healthcare. (n.d.). CASP Checklists - CASP - Critical Appraisal Skills Programme. Retrieved April 28, 2021, from https://casp-uk.net/casp-toolschecklists/

Ozdemir, B. A., Karthikesalingam, A., Sinha, S., Poloniecki, J. D., Hinchliffe, R. J., Thompson, M. M., Gower, J. D., Boaz, A., \& Holt, P. J. E. (2015). Research activity and the association with mortality. PLOS ONE, 10(2), 1-15.

https://doi.org/10.1371/journal.pone.0118253

Panwar, R., Capellier, G., Schmutz, N., Davies, a, Cooper, D. J., Bailey, M., Baguley, D., Pilcher, V., \& Bellomo, R. (2013). Current oxygenation practice in ventilated patientsan observational cohort study. Anaesthesia and Intensive Care, 41(4), 505-514. http://www.ncbi.nlm.nih.gov/pubmed/23808511

Panwar, R., Hardie, M., Bellomo, R., Barrot, L., Eastwood, G. M., Young, P. J., Capellier, G., Harrigan, P. W. J., \& Bailey, M. (2016). Conservative versus liberal oxygenation targets for mechanically ventilated patients: A pilot multicenter randomized controlled trial. American Journal of Respiratory and Critical Care Medicine, 193(1), 43-51. https://doi.org/10.1164/rccm.201505-10190C

Pfeffer, M. A., Braunwald, E., Moyé, L. A., Basta, L., Brown, E. J., Cuddy, T. E., Davis, B. R., Geltman, E. M., Goldman, S., Flaker, G. C., Klein, M., Lamas, G. A., Packer, M., Rouleau, J., Rouleau, J. L., Rutherford, J., Wertheimer, J. H., \& Hawkins, C. M. (1992). Effect of captopril on mortality and morbidity in patients with left ventricular dysfunction after myocardial infarction. New England Journal of Medicine, 327(10), 669-677. https://doi.org/10.1056/nejm199209033271001

Phillips, D., \& Burbules, N. C. (2000). Postpositivism and educational research. Rowman and Littlefield Publishers, Inc.

Pilcher, J., Ploen, L., McKinstry, S., Bardsley, G., Chien, J., Howard, L., Lee, S., Beckert, L., Swanney, M., Weatherall, M., \& Beasley, R. (2020). A multicentre prospective observational study comparing arterial blood gas values to those obtained by pulse 
oximeters used in adult patients attending Australian and New Zealand hospitals. BMC Pulmonary Medicine, 20(1), 3-11. https://doi.org/10.1186/s12890-019-1007-3

Polit, D. F., \& Beck, C. T. (2017). Nursing research: generating and assessing evidence for nursing practice (10th editi). Wolters Kluzer.

Powrie, S. L., Danly, D., Corbett, C. F., Purath, J., \& Dupler, A. (2014). Using implementation science to facilitate evidence-based practice changes to promote optimal outcomes for orthopaedic patients. Orthopaedic Nursing, 33(2), 109-114.

https://doi.org/10.1097/NOR.0000000000000036

Prasad, V., Vandross, A., Toomey, C., Cheung, M., Rho, J., Quinn, S., Chacko, S. J., Borkar, D., Gall, V., Selvaraj, S., Ho, N., \& Cifu, A. (2013). A decade of reversal: An analysis of 146 contradicted medical practices. Mayo Clinic Proceedings, 88(8), 790-798. https://doi.org/10.1016/j.mayocp.2013.05.012

Qin, X., Yu, P., Li, H., Hu, Y., Li, X., Wang, Q., Lin, L., \& Tian, L. (2020). Integrating the "best" evidence into nursing of venous thromboembolism in ICU patients using the i-PARIHS framework. PLoS ONE, 15(8 August), 1-13. https://doi.org/10.1371/journal.pone.0237342

Rabin, B. A., Brownson, R. C., Haire-Joshu, D., Kreuter, M. W., \& Weaver, N. L. (2008). A glossary for dissemination and implementation research in health. Journal of Public Health Management and Practice, 14(2), 117-123.

https://doi.org/10.1097/01.PHH.0000311888.06252.bb [doi]

Rapport, F., Clay-Williams, R., Churruca, K., Shih, P., Hogden, A., \& Braithwaite, J. (2018). The struggle of translating science into action: Foundational concepts of implementation science. Journal of Evaluation in Clinical Practice, 24(1), 117-126. https://doi.org/10.1111/jep.12741

Rickard, C. M., Roberts, B. L., Foote, J., \& McGrail, M. R. (2006). Intensive care research coordinators: who are they and what do they do? Results of a binational survey. Dimensions of Critical Care Nursing, 25(5), 234-242. https://doi.org/10.1097/00003465-200609000-00014

Roberts, R., Croft, C., Gold, H. K., Hartwell, T. D., Jaffe, A. S., Muller, J. E., Mullin, S. M., Parker, C., Passamani, E. R., Poole, W. K., Raabe, D. S., Rude, R. E., Stone, P. H., Turi, Z. G., Sobel, B. E., Willerson, J. T., \& Braunwald, E. (1984). Effect of Propranolol on Myocardial-Infarct Size in a Randomized Blinded Multicenter Trial. New England 
Journal of Medicine, 311(4), 218-225.

https://doi.org/10.1056/NEJM198407263110403

Rogers, E. M. (1995). Diffusion of innovations. In Macmillian Publishing Co.

https://doi.org/citeulike-article-id:126680

Rose, L., Nelson, S., Johnston, L., \& Presneill, J. J. (2008). Workforce profile, organisation structure and role responsibility for ventilation and weaning practices in Australia and New Zealand intensive care units. Journal of Clinical Nursing, 17(8), 1035-1043. https://doi.org/10.1111/j.1365-2702.2007.02129.x

Rycroft-Malone, J. (2004). The PARIHS Framework - A framework for guiding the implementation of evidence-based practice. Journal of Nursing Care Quality, 19(4), 297-304. https://doi.org/10.1097/00001786-200410000-00002

Rycroft-Malone, J., Harvey, G., Kitson, A., McCormack, B., Seers, K., \& Titchen, A. (2002). Getting evidence into practice: ingredients for change. Nursing Standard (Royal College of Nursing (Great Britain) : 1987), 16(37), 38-43. https://doi.org/10.7748/ns2002.05.16.37.38.c3201

Rycroft-Malone, J., Harvey, G., Seers, K., Kitson, A., McCormack, B., \& Titchen, A. (2004). An exploration of the factors that influence the implementation of evidence into practice. Journal of Clinical Nursing, 13(8), 913-924. https://doi.org/10.1111/j.13652702.2004.01007.x

Rycroft-Malone, J., Kitson, A., Harvey, G., McCormack, B., Seers, K., Titchen, A., \& Estabrooks, C. (2002). Ingredients for change: Revisiting a conceptual framework. Quality and Safety in Health Care, 11(2), 174-180.

Rycroft-Malone, J., Seers, K., Chandler, J., Hawkes, C., Crichton, N., Allen, C., Bullock, I., \& Strunin, L. (2013). The role of evidence, context, and facilitation in an implementation trial: Implications for the development of the PARIHS framework. Implementation Science, 8(1), 1-13. https://doi.org/10.1186/1748-5908-8-28

Rycroft-Malone, J., Seers, K., Titchen, A., Harvey, G., Kitson, A., \& McCormack, B. (2004). What counts as evidence in evidence-based practice? In Journal of Advanced Nursing. https://doi.org/10.1111/j.1365-2648.2004.03068.x

Sackett, D. L. (1989). Rules of evidence and clinical recommendations on the use of antithrombotic agents. Chest, 95(2 Suppl), 2S-4S.

Sackett, D. L., Rosenberg, W. M., Gray, J. A., Haynes, R. B., \& Richardson, W. S. (1996). 
Evidence based medicine: what it is and what it isn't. BMJ (Clinical Research Ed.), 312(7023), 71-72. https://doi.org/10.1136/bmj.312.7023.71

Salter, R., Bailey, M., Bellomo, R., Eastwood, G., Goodwin, A., Nielsen, N., Pilcher, D., Nichol, A., \& ANZICS-CORE. (2018). Changes in temperature management of cardiac arrest patients following publication of the Target Temperature Management Trial. Critical Care Medicine, 46(11), 1722-1730. https://doi.org/10.1097/CCM.0000000000003339

Sauro, K., Bagshaw, S. M., Niven, D., Soo, A., Brundin-Mather, R., Parsons Leigh, J., Cook, D. J., \& Stelfox, H. T. (2019). Barriers and facilitators to adopting high value practices and de-adopting low value practices in Canadian intensive care units: A multimethod study. BMJ Open, 9(3), 1-10. https://doi.org/10.1136/bmjopen-2018-024159

Schjørring, O. L., Klitgaard, T. L., Perner, A., Wetterslev, J., Lange, T., Siegemund, M., Bäcklund, M., Keus, F., Laake, J. H., Morgan, M., Thormar, K. M., Rosborg, S. A., Bisgaard, J., Erntgaard, A. E. S., Lynnerup, A.-S. H., Pedersen, R. L., Crescioli, E., Gielstrup, T. C., Behzadi, M. T., ... Rasmussen, B. S. (2021). Lower or higher oxygenation targets for acute hypoxemic respiratory failure. New England Journal of Medicine, 384(14), 1301-1311. https://doi.org/10.1056/nejmoa2032510

Schoenfeld, D. a, \& Bernard, G. R. (2002). Statistical evaluation of ventilator-free days as an efficacy measure in clinical trials of treatments for acute respiratory distress syndrome. Critical Care Medicine, 30(8), 1772-1777. https://doi.org/10.1097/00003246-200208000-00016

Seddon, M. E., Marshall, M. N., Campbell, S. M., \& Roland, M. O. (2001). Systematic review of studies of quality of clinical care in general practice in the UK, Australia and New Zealand. Quality in Health Care, 10(3), 152-158. https://doi.org/10.1136/qhc.0100152..

Shah, B. R., Peterson, E. D., Chen, A. Y., Mahaffey, K. W., Delong, E. R., Ohman, E. M., Pollack, C. V., Gibler, W. B., \& Roe, M. T. (2010). Influence of clinical trial participation on subsequent antithrombin use. Clinical Cardiology, 33(3), 49-55. https://doi.org/10.1002/clc.20581

Sinuff, T., Muscedere, J., Adhikari, N. K. J., Stelfox, H. T., Dodek, P., Heyland, D. K., Rubenfeld, G. D., Cook, D. J., Pinto, R., Manoharan, V., Currie, J., Cahill, N., Friedrich, J. O., Amaral, A., Piquette, D., Scales, D. C., Dhanani, S., \& Garland, A. (2013). Knowledge 
translation interventions for critically ill patients: a systematic review*. Critical Care Medicine, 41(11), 2627-2640. https://doi.org/10.1097/CCM.0b013e3182982b03

Smith, J. K. (1983). Quantitative Versus Qualitative Research: An Attempt to Clarify the Issue. Educational Researcher, 12(3), 6-13. https://doi.org/10.3102/0013189X012003006

Souza, M. T. de, Silva, M. D. da, \& Carvalho, R. de. (2010). Integrative review: what is it? How to do it? Einstein (São Paulo), 8(1), 102-106. https://doi.org/10.1590/s167945082010 rw1134

Squires, J. E., Estabrooks, C. A., Gustavsson, P., \& Wallin, L. (2011a). Individual determinants of research utilization by nurses: A systematic review update. Implementation Science, 6(1), 1-20. https://doi.org/10.1186/1748-5908-6-1

Squires, J. E., Estabrooks, C. A., Gustavsson, P., \& Wallin, L. (2011b). Individual determinants of research utilization by nurses: A systematic review update. Implementation Science, 6(1). https://doi.org/10.1186/1748-5908-6-1

Stetler, C. B., Damschroder, L. J., Helfrich, C. D., \& Hagedorn, H. J. (2011). A guide for applying a revised version of the PARIHS framework for implementation: Contextual readiness for targeted EBP implementation element. Implementation Science, 6(99), 1-10.

Suzuki, S., Eastwood, G. M., Glassford, N. J., Peck, L., Young, H., Garcia-Alvarez, M., Schneider, A. G., \& Bellomo, R. (2014). Conservative oxygen therapy in mechanically ventilated patients: A pilot before-and-after trial. Critical Care Medicine, 42(6), 14141422. https://doi.org/10.1097/CCM.0000000000000219

Suzuki, S., Eastwood, G. M., Peck, L., Glassford, N. J., \& Bellomo, R. (2013). Current oxygen management in mechanically ventilated patients: A prospective observational cohort study. Journal of Critical Care, 28(5), 647-654. https://doi.org/10.1016/j.jcrc.2013.03.010

Sweeting, D., Daniell, H., \& Chester, R. (2020). Clinicians' involvement in data collection for portfolio research: Impact on their clinical practice and perception of research. International Journal of Therapy and Rehabilitation, 27(6). https://doi.org/10.12968/ijtr.2018.0130

Taylor, C. B., Hammond, N. E., Laba, T. L., Watts, N., Thompson, K., Saxena, M., Micallef, S., Finfer, S., \& Myburgh, J. (2017). Drivers of choice of resuscitation fluid in the intensive 
care unit: A discrete choice experiment. Critical Care and Resuscitation, 19(2), 134141.

The Multicenter Diltiazem Postinfarction Trial Research Group. (1988). The effect of diltiazem on mortality and reinfarction after myocardial infarction. New England Journal of Medicine, 319(7), 385-392.

https://doi.org/10.1056/NEJM198808183190701

The NICE-SUGAR Investigators. (2009). Intensive versus conventional glucose control in critically ill patients. New England Journal of Medicine, 360(13), 1283-1297.

The NICE-SUGAR Investigators. (2012). Hypoglycemia and risk of death in critically ill patients. New England Journal of Medicine, 367(12), 1108-1118. https://doi.org/10.1056/nejmoa1204942

The TIMI Study Group. (1989). Comparison of invasive and conservative strategies after rreatment with intravenous tissue plasminogen activator in acute myocardial infarction. New England Journal of Medicine, 320(10), 618-627. https://doi.org/10.1056/nejm198903093201002

Thompson, C., McCaughan, D., Cullum, N., Sheldon, T. A., Mulhall, A., \& Thompson, D. R. (2001). Research information in nurses' clinical decision-making: What is useful? Journal of Advanced Nursing, 36(3), 376-388. https://doi.org/10.1046/j.13652648.2001.01985.x

Topol, E. J., Leya, F., Pinkerton, C. A., Whitlow, P. L., Hofling, B., Simonton, C. A., Masden, R. R., Serruys, P. W., Leon, M. B., Williams, D. O., King, S. B., Mark, D. B., Isner, J. M., Holmes, D. R., Ellis, S. G., Lee, K. L., Keeler, G. P., Berdan, L. G., Hinohara, T., \& Califf, R. M. (1993). A Comparison of directional atherectomy with coronary angioplasty in patients with coronary artery disease. New England Journal of Medicine, 329(4), 221227. https://doi.org/10.1056/nejm199307223290401

Torraco, R. J. (2005). Writing Integrative Literature Reviews: Guidelines and Examples. Human Resource Development Review, 4(3), 356-367. https://doi.org/10.1177/1534484305278283

Tranmer, J. E., Lochhaus-Gerlach, J., \& Lam, M. (2002). The effect of staff nurse participation in a clinical nursing research project. Canadian Journal of Nursing Leadership, 15(1), 18-26.

Tranmer, J. E., Minard, J., Fox, L. A., \& Rebelo, L. (2003). The sleep experience of medical 
and surgical patients. Clinical Nursing Research, 12(2), 159-173.

https://doi.org/10.1177/1054773803012002004

Tricco, A. C., Cardoso, R., Thomas, S. M., Motiwala, S., Sullivan, S., Kealey, M. R., Hemmelgarn, B., Ouimet, M., Hillmer, M. P., Perrier, L., Shepperd, S., \& Straus, S. E. (2016). Barriers and facilitators to uptake of systematic reviews by policy makers and health care managers: a scoping review. Implementation Science, 11, 4. https://doi.org/10.1186/s13012-016-0370-1

Van den Berghe, G., Wilmer, A., Hermans, G., Meersseman, W., Wouters, P. J., Milants, I., Van Wijngaerden, E., Bobbaers, H., \& Bouillon, R. (2006). Intensive insulin therapy in the medical ICU. New England Journal of Medicine, 354(5), 449-461.

Weaver, K., \& Olson, J. K. (2006). Understanding paradigms used for nursing research. Journal of Advanced Nursing, 53(4), 459-469. https://doi.org/10.1111/j.13652648.2006.03740.x

Webster, J., Bucknall, T., Wallis, M., McInnes, E., Roberts, S., \& Chaboyer, W. (2017). Does participating in a clinical trial affect subsequent nursing management? Post-trial care for participants recruited to the INTACT pressure ulcer prevention trial: A follow-up study. International Journal of Nursing Studies, 71, 34-38.

https://doi.org/10.1016/j.ijnurstu.2017.02.022

Wilcox, R. G., Olsson, C. G., Skene, A. M., Von Der Lippe, G., Jensen, G., Hampton, J. R., \& For The Asset Study Group. (1988). Trial of tissue plaminogen activator for mortality reduction in acute myocardial infarction. Anglo-Scandinavian Study of Early Thrombolysis (ASSET). Lancet, 332(8610), 525-530. https://doi.org/10.1016/S01406736(88)92656-6

Woolf, S. H. (2008). The meaning of translational research and why it matters. JAMA, 299(2), 211-213. https://doi.org/10.1001/jama.2007.26

Young, P., Bailey, M., Bellomo, R., Bernard, S., Dicker, B., Freebairn, R., Henderson, S., Mackle, D., McArthur, C., McGuinness, S., Smith, T., Swain, A., Weatherall, M., \& Beasley, R. (2014). Hyperoxic therapy or normoxic therapy after out-of-hospital cardiac arrest (HOT OR NOT): A randomised controlled feasibility trial. Resuscitation, 85(12). https://doi.org/10.1016/j.resuscitation.2014.09.011

Young, P.J., Bailey, M., Bellomo, R., Bernard, S., Bray, J., Jakkula, P., Kuisma, M., Mackle, D., Martin, D., Nolan, J. P., Panwar, R., Reinikainen, M., Skrifvars, M. B., \& Thomas, M. 
(2020). Conservative or liberal oxygen therapy in adults after cardiac arrest: An individual-level patient data meta-analysis of randomised controlled trials.

Resuscitation, 157. https://doi.org/10.1016/j.resuscitation.2020.09.036

Young, P.J., Beasley, R. W., Capellier, G., Eastwood, G. M., \& Webb, S. A. R. (2015).

Oxygenation targets and monitoring in the critically ill : a point prevalence study of clinical practice in Australia and New Zealand. Critical Care and Resuscitation, 17(3), 202-207.

Young, P.J., Mackle, D. M., Bailey, M. J., Beasley, R. W., Bennett, V. L., Deane, A. M., Eastwood, G. M., Finfer, S., Freebairn, R. C., Litton, E., Linke, N. J., McArthur, C. J., McGuinness, S. P., Panwar, R., \& Bellomo, R. (2017). Intensive care unit randomised trial comparing two approaches to oxygen therapy (ICU-ROX): results of the pilot phase. Critical Care and Resuscitation, 19(4).

Young, Paul. (2019). ICU-ROX trial thread reader. https://threadreaderapp.com/thread/1183875137243762688.html

Young, Paul J., Bagshaw, S. M., Bailey, M., Bellomo, R., Mackle, D., Pilcher, D., Landoni, G., Nichol, A., \& Martin, D. (2019). O2, do we know what to do? Critical Care and Resuscitation, 21(4), 230-232.

Young, Paul J., Bagshaw, S. M., Forbes, A. B., Nichol, A. D., Wright, S. E., Bailey, M., Bellomo, R., Beasley, R., Brickell, K., Eastwood, G. M., Gattas, D. J., Van Haren, F., Litton, E., Mackle, D. M., McArthur, C. J., McGuinness, S. P., Mouncey, P. R., Navarra, L., Opgenorth, D., ... Rowan, K. M. (2020). Effect of stress ulcer prophylaxis with proton pump inhibitors vs histamine-2 receptor blockers on in-hospital mortality among ICU patients receiving invasive mechanical ventilation: The PEPTIC randomized clinical trial. JAMA. https://doi.org/10.1001/jama.2019.22190

Young, Paul, Mackle, D., Bellomo, R., Bailey, M., Beasley, R., Deane, A., Eastwood, G., Finfer, S., Freebairn, R., King, V., Linke, N., Litton, E., McArthur, C., McGuinness, S., \& Panwar, R. (2020a). Conservative oxygen therapy for mechanically ventilated adults with sepsis: a post hoc analysis of data from the intensive care unit randomized trial comparing two approaches to oxygen therapy (ICU-ROX). Intensive Care Medicine, 46(1), 17-26. https://doi.org/10.1007/s00134-019-05857-x

Young, Paul, Mackle, D., Bellomo, R., Bailey, M., Beasley, R., Deane, A., Eastwood, G., Finfer, S., Freebairn, R., King, V., Linke, N., Litton, E., McArthur, C., McGuinness, S., \& 
Panwar, R. (2020b). Conservative oxygen therapy for mechanically ventilated adults with suspected hypoxic ischaemic encephalopathy. Intensive Care Medicine, 46, 2411-2422. https://doi.org/10.1007/s00134-020-06196-y

Young, Paul, Saxena, M., Bellomo, R., Freebairn, R., Hammond, N., van Haren, F., Holliday, M., Henderson, S., Mackle, D., McArthur, C., McGuinness, S., Myburgh, J., Weatherall, M., Webb, S., \& Beasley, R. (2015). Acetaminophen for Fever in Critically III Patients with Suspected Infection. New England Journal of Medicine, 373(23), 2215-2224. https://doi.org/10.1056/nejmoa1508375 